\title{
TITLE:
}

\section{Record of the 242 species in each quadrat, 1985?2010}

\section{$\operatorname{AUTHOR}(S)$ :}

OHGAKI, SHUN-ICHI; KOMEMOTO, KEN-ICHI; FUNAYAMA, NOBUTAKA

\section{CITATION:}

OHGAKI, SHUN-ICHI ... [et al]. 10. Record of the 242 species in each quadrat, 1985?2010. Publications of the Seto Marine Biological Laboratory. Special Publication Series 2011, 11 : 199-311

\section{ISSUE DATE:}

2011

URL:

http://hdl.handle.net/2433/159490

RIGHT: 
10. Record of the 242 species in each quadrat, 1985-2010

Table 9.

The abundance of species in each quadrat in each year. The data of all of the species found during the course of the study are shown. Only the presence $(+)$ of a species was recorded from 1985 to 1994 . Subsequently, the maximum density (no. $/ \mathrm{m}^{2}$ ) of a species was evaluated and assigned to one of the following classes: 1, (1-9); 2 (10-99); 3, (100-999); 4, (1000-5000). (D) denotes that only one individual was encountered in a quadrat. An Italic numeral in the first column corresponds to the species code shown in the List of recorded species (Section 2). A1 to F10 in the first and second rows indicate the quadrat codes (see Fig. 1d). 


\begin{tabular}{|c|c|c|c|c|c|c|c|c|c|c|c|c|c|c|c|c|c|c|c|c|c|c|c|c|c|c|c|c|c|c|c|c|c|c|c|}
\hline \multicolumn{36}{|c|}{ Table 9, 1985-1 } \\
\hline & \multicolumn{8}{|c|}{$\mathrm{A}$} & & & & & $\mathrm{B}$ & & & & & & & & & & & & & C & & & & & & & & D & \\
\hline & 1 & 2 & 3 & 4 & 5 & 6 & 7 & 8 & 1 & 2 & 36 & 4 & 5 & 6 & 7 & 8 & $\begin{array}{l}9 \\
\end{array}$ & 0 & 1 & 2 & 3 & 4 & 5 & 6 & 7 & 8 & 9 & & 11 & & & 14 & & 2 & 3 \\
\hline 2 & & & & & & & & & & & & & + & & & & & & & & & & & & & & & & & & + & + & & & \\
\hline 3 & & & & & & & & & & & & & & & & & & & & & & & & & & & & & & & & & & & \\
\hline 4 & + & + & + & + & + & + & + & + & + & + & +- & + & + & + & + & +- & + & + & + & + & + & + & + & + & + & + & + & + & + & + & + & & & + & + \\
\hline 6 & & & & & & & & + & & & & & & & & & & + & & & & & & & 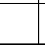 & & & & + & + & & + & & & \\
\hline 7 & & & + & & + & & + & + & & + & - & + & + & + & & +- & + & + & & & + & & & & + & + & + & + & + & + & + & + & & & \\
\hline 10 & & & & & & & & & & & & & & & & & & & & & & & & & & & & & + & & + & + & & & \\
\hline 11 & & & & & & & & & & & & & & & & & & & & & & + & & & & & & & & & & & & & \\
\hline 12 & + & + & + & + & + & + & + & + & + & + & + & & & + & & & + & + & + & + & + & + & + & & + & + & + & + & & & & & & & \\
\hline 13 & + & & & + & + & + & & + & & + & +- & + & + & + & & +- & + & + & + & & & + & & & 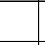 & & + & + & + & + & + & + & & & \\
\hline 14 & & & & & & & & & + & + & + & + & + & + & + & + & & & + & + & + & + & + & + & + & + & & & & & & & & + & + \\
\hline 15 & + & + & + & + & + & + & + & + & + & + & + & + & + & + & + & + & + & + & + & + & + & + & + & + & + & + & + & + & & + & + & & + & + & + \\
\hline 16 & & & & & & & & & & & & & & & & & & + & & & & & & & & & + & + & & + & + & + & & & \\
\hline 17 & + & + & + & & + & & + & & + & & & & + & & & + & + & & + & + & + & & & & + & + & + & + & + & + & + & + & & & \\
\hline 20 & & & & & & & & + & + & + & - & + & & & & + & + & + & & & & + & & & & & & + & & & & & & & \\
\hline 23 & & & & & & & & & & & & & & & & & & & & & & & & & & & & & & & & & & & \\
\hline 26 & & & & & & & & & & & & & & & & & 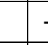 & + & & & & & & & & & & & & & & + & & & \\
\hline 27 & & & & & & & & & & & & & & & & & & & & & & & & & & & & & & + & + & + & & & \\
\hline 28 & + & & & + & & + & & + & & & & + & + & & & & + & + & + & & + & & & & & & & + & & + & + & & & & + \\
\hline 29 & & & & & & & & & & & & & & & & & & & & + & & & & & & & & & & & + & & & & \\
\hline 31 & & & & & & & & & & & & & & & & & & & & & & & & & & & & & & & & & & & \\
\hline 33 & & & & & & & & & & & & & & & & & + & & & & & & & & & & & & & & & & & & \\
\hline 37 & & & & & & & & & & & & & & & & & & & & & & & & & & & & & & & + & + & & & \\
\hline 39 & & & & & & & & & & & & & & & & & & & & & & & & & & & & & & & & & & & \\
\hline 41 & & & & & & & & + & & & & & & & & & & & & & & & & & & & & & & & & & & & \\
\hline 44 & + & + & + & + & + & & + & + & + & + & + & + & + & + & + & & + & + & + & + & + & + & + & + & + & + & + & + & & . & & 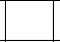 & + & + & + \\
\hline 45 & + & & & & & & & & & & & & & & & & & & & & & & & & & & & & & & & & & & \\
\hline 48 & & & & & & & & + & & & & & & & & & + & + & & & & & & & & + & + & & + & + & + & + & & & \\
\hline 49 & & & & & & & & & & & & & & & & & & & & & 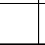 & & & 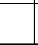 & & 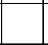 & 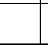 & & 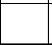 & 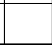 & & + & & & \\
\hline 53 & & & & + & & & & & & & & & & & & & & & & & & & & & & & 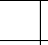 & & + & + & + & + & & & \\
\hline 57 & & & & & & & & & & & & & & & & & & & & & & & & & & & & & & & & + & & & \\
\hline 58 & & & & & & & & & & & & & & & & & & & & & & & & & & & & & & & & & & & \\
\hline 60 & + & + & + & + & + & & & & + & + & + & + & + & & & & & & & & 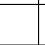 & + & & & & 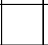 & 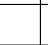 & & & & & & & & \\
\hline 61 & & & & & & & & & & & & & & & & & & & & & & & & & & . & & & & + & + & + & & & \\
\hline 63 & + & + & + & & + & + & & + & + & & + & + & & & & & & & + & + & + & & & & & + & + & & & & & & & & \\
\hline 64 & & & & + & + & + & + & + & & & & + & + & + & + & + & & & + & & & + & + & + & + & + & + & & & & & & & + & \\
\hline 70 & & & 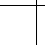 & & & & & & & & 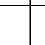 & & & & & & & & + & & 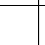 & 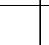 & & & & 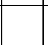 & & & & & & & & 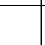 & \\
\hline 71 & & & + & + & + & + & + & & & + & + & + & + & & . & 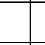 & & & + & & 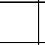 & 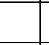 & 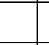 & & . & 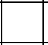 & 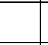 & & & & + & & & - & \\
\hline 73 & + & + & + & & & & & & + & + & & & . & , & & + & & & + & & + & + & + & & . & 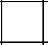 & 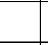 & & & & & & + & + & + \\
\hline 77 & + & + & + & + & + & & & + & & & & & + & + & + & + & + & & + & & & + & + & + & + & + & + & + & & & & & + & + & + \\
\hline 78 & + & + & + & + & + & + & + & $t$ & & & & + & + & + & + & + & + & & + & & + & + & + & + & + & + & + & + & & & & & + & + & + \\
\hline 79 & & & & & & + & + & & & & & & + & + & + & & & & + & & + & + & + & + & + & & 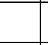 & & & - & & & + & & + \\
\hline 80 & & & & & & & & & & & & + & & & & & & & & & 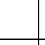 & 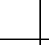 & & & & 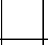 & 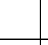 & & & & & & 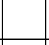 & . & \\
\hline 81 & + & + & + & + & + & + & + & + & + & + & + & + & + & + & + & + & + & & + & + & + & + & + & + & + & + & + & + & & & & & + & + & + \\
\hline 82 & & + & + & & + & & & & + & + & + & + & + & & & & & & + & & & & & & & & 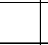 & & & & & & & & + \\
\hline 85 & & & & & & & & & & & & & & & & & & & & & & & & & & & & & & & & & & & \\
\hline 86 & & & & & & & & & & & & & & & & & & + & & & & & & & & & & & + & & + & + & & & \\
\hline 87 & & & & & & & & & & & & & & & & & & + & & & & & & & & & & & & & & + & & & \\
\hline 88 & & & & & & & & & & & & & & & & & & & & & & & & & & & + & & & & + & + & & & \\
\hline 89 & & & & & & & & + & & & & & & & & & & & & & & & & & & & & & & & + & + & & & \\
\hline 91 & & & & & & & & & & & & & & & & & & + & & & & & & & & & & & & + & + & + & & & \\
\hline 94 & & & + & & & + & & + & & & + & + & & + & & & & & & & & & & & & & & & + & + & + & + & & & \\
\hline 97 & & & & & & & & & & & & & & & & & & & & & & & & & & & & & & & & & & & \\
\hline 100 & & & & & & & & + & & & & & & & & & & & & & & & & & & & & & & & & & & & \\
\hline 102 & & & & & & & & & & & & & & & & & & & & & & & & & & & & & & & & & & & \\
\hline 103 & & & & & & & & & & & & & & & & & & & & & & & & & & & & & + & + & & + & & & \\
\hline
\end{tabular}




\begin{tabular}{|c|c|c|c|c|c|c|c|c|c|c|c|c|c|c|c|c|c|c|c|c|c|c|c|c|c|c|c|c|c|c|c|c|c|c|}
\hline \multicolumn{35}{|c|}{ Table 9, 1985-2 } \\
\hline & \multicolumn{11}{|c|}{ D } & \multicolumn{13}{|c|}{$\mathrm{E}$} & & & & & $\mathrm{F}$ & & & & & \\
\hline & 4 & 5 & 6 & 7 & & \begin{tabular}{l|l}
9 & 1 \\
\end{tabular} & 01 & & 12 & & & 1 & 2 & 3 & 4 & 5 & 6 & 7 & 8 & 9 & 10 & 11 & 12 & 13 & 1 & 2 & 3 & 4 & & 6 & 7 & 8 & & 10 \\
\hline 2 & & & & & & & & & & & & & & & & & & & & & & & & + & & & & & & & & & & + \\
\hline 3 & & & & & & & & & & & & & & & & & & & & & & & & & & & & & & & & & & + \\
\hline 4 & + & + & + & + & & + & + & + & + & & & & & & + & + & + & + & + & + & + & + & & & & & & + & + & + & + & + & & + \\
\hline 6 & & & & + & & & & + & & + & & & & & & & & & & & & + & + & + & & & & 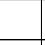 & 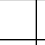 & & & & & \\
\hline 7 & & & & & & & + & + & + & + & + & & & & & + & & + & + & + & + & + & + & + & & & & & & & & & & + \\
\hline 10 & & & & & & & & & & & + & & & & & & & & & & & & & + & & & & & & & & & & \\
\hline 11 & & & & & & & & & & & & & & & & & & + & & & & & & & & & & & & & & + & & \\
\hline 12 & & + & + & + & & + & + & & & & & & & & & + & + & + & + & + & + & & & & & & & + & & & + & + & & \\
\hline 13 & & + & & + & & & + & + & + & + & + & & & & & + & + & + & & + & + & + & & + & & & & + & + & + & + & + & + & + \\
\hline 14 & + & + & + & & & + & + & + & + & & + & & & & & & & + & & & & + & & + & & & + & + & + & + & & & & \\
\hline 15 & + & + & + & + & & - & + & + & & 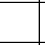 & & & & + & + & + & + & + & + & + & + & + & + & & + & + & + & + & + & + & + & + & & \\
\hline 16 & & & & & & + & & + & + & + & + & & & & & 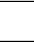 & 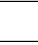 & 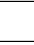 & & + & + & & + & + & & 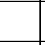 & 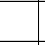 & 4 & 4 & 1 & & & & + \\
\hline 17 & & + & & + & & + & + & & + & + & + & & & & + & + & + & + & + & + & + & & & + & & + & + & + & & 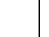 & 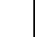 & & & \\
\hline 20 & & + & + & + & & + & & & & & & & & & & + & + & + & + & & & & 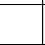 & & + & & & + & & & & & & \\
\hline 23 & & & & & & & & & & & & & & & & + & & & & & & & & & & & & & 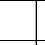 & & + & & & \\
\hline 26 & & & & & & & & & & & + & & & & & & & & & & & + & & + & & & & . & . & . & & & & \\
\hline 27 & & & & & & & & & + & + & + & & & & & 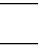 & & 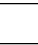 & & & & & + & + & & & & 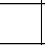 & & & & & & \\
\hline 28 & & & & & & + & + & + & + & & & & & & & . & & + & & & & + & & & & 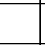 & 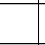 & 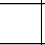 & + & + & + & + & & \\
\hline 29 & & & & & & & & & & + & & & & & & & & & & & & & & & & & & 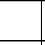 & & + & & & & + \\
\hline 31 & & & & & & & & & & & & & & & & & & & & & & & & & & & & 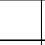 & & & & & & \\
\hline 33 & & & + & + & & & + & & & & & & & & + & & + & + & & & & & 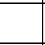 & & & + & & + & + & & & & & \\
\hline 37 & & & & & & & & & & & & & & & & & & & & & & & & & & & & & & . & & & & \\
\hline 39 & & & & & & & & & & & & & & & & & 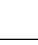 & & & & & & + & & & & & 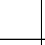 & 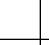 & & & & & + \\
\hline 41 & & & & & & & & & & & & & & & & & & & & & & & & & & & & . & - & . & & + & & + \\
\hline 44 & + & + & + & + & & + & + & + & & & & & & & + & + & + & + & + & + & + & & 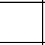 & 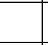 & + & + & + & + & + & + & + & & & \\
\hline 45 & & & & & & & & & & & & & & & & & & & & & & & & & & & & & & & & & & \\
\hline 48 & & & & & & + & + & + & + & + & + & & & & & 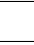 & 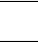 & 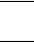 & + & + & + & + & + & + & & & & 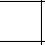 & & & & + & & + \\
\hline 49 & & & & & & & & & & & + & & & & & & & & & & & & + & + & & & & 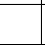 & & & & & & \\
\hline 53 & & 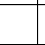 & & & & + & & & 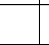 & + & + & & & & & 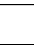 & & + & & + & + & & + & + & & & & + & & & & & & \\
\hline 57 & & & & & & & & & + & & & & & & & + & & & & 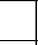 & . & & + & & & & & 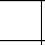 & & & & & & \\
\hline 58 & & + & & & & & & & & & & & & & & & & + & & & & & & & & & & 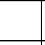 & & + & & & & \\
\hline 60 & & + & & & & & & & & & & & & & & + & & & & & & & & & + & & & + & & 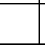 & 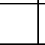 & & & \\
\hline 61 & & & & & & 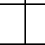 & & & + & + & & & & & . & - & + & & 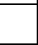 & 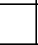 & . & + & + & + & & 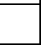 & ( & + & 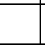 & 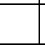 & + & & + & + \\
\hline 63 & & + & & & - & + & + & + & & & & & & & + & + & + & + & + & + & + & & & & . & 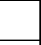 & & + & + & + & + & + & & . \\
\hline 64 & + & + & + & + & & & & & & & & + & & + & + & & & & & & & & & & + & + & + & + & & & & & & 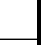 \\
\hline 70 & & & & & & & & & & & & & & & & & & & & & & & & & & & & & & & & & & \\
\hline 71 & & & & & & & & & & & & & & . & 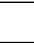 & 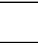 & & + & + & & + & & & & 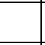 & & & + & & . & . & & + & + \\
\hline 73 & + & + & + & + & & & & & & & & & + & + & + & + & + & + & + & & & & & & + & + & + & + & & & & & & \\
\hline 77 & + & + & + & + & & + & + & & & & & + & + & + & + & + & + & + & + & & & & & & + & + & + & + & & & & & & \\
\hline 78 & + & + & + & & & + & + & & & & & + & + & + & + & + & & & & & & & & & + & + & + & + & & & & & & \\
\hline 79 & & & & & & & & & & & & + & + & + & + & & & 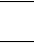 & & & & & & & + & & + & & & & & & & \\
\hline 80 & & & & & & & & & & & & & & & & & & & & & & & & & & & & & & & & & & \\
\hline 81 & + & + & + & + & 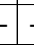 & + & + & & & & & + & + & + & + & + & + & + & & & & & & & + & + & + & + & & & & & & \\
\hline 82 & & & & + & & & & & & & & & & & & & & + & & & & & & & & & & & & & & & & \\
\hline 85 & & & & & & & & & & & & & & & & & & & & & & & & & & & & & & & & & + & \\
\hline 86 & & & & & & + & & & & + & + & & & & & & & & & & & & + & + & & & & + & & & & & & \\
\hline 87 & & & & & & & & + & + & + & + & & & & & & & & & & & & + & + & + & & & + & & & & & & + \\
\hline 88 & & & & & & & & & + & & + & & & & & & & & & & & + & & + & & & & & + & + & + & & & 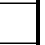 \\
\hline 89 & & & & & & & & & & & + & & & & & & & & & & & + & + & + & & & & & & & & 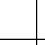 & & + \\
\hline 91 & & & & & & & & & & + & + & & & & & & & & & & & & & & & & & & & & & & & \\
\hline 94 & & & & + & & + & + & + & + & + & & & & & & & & & & & & + & + & + & & & & & & & + & + & + & + \\
\hline 97 & & & & & & & & & & + & & & & & & & & & & & & & & + & & & & & & & & & & \\
\hline 100 & & & & & & & & & & & & & & & & & & & & & & & & & & & & & & & & & & \\
\hline 102 & & & & & & & & & + & & & & & & & & & & & & & & & & & & & & & & & & & \\
\hline 103 & & & & & & & & & & & + & & & & & & & & & & & & + & & & & & & & & & & & \\
\hline
\end{tabular}




\begin{tabular}{|c|c|c|c|c|c|c|c|c|c|c|c|c|c|c|c|c|c|c|c|c|c|c|c|c|c|c|c|c|c|c|c|c|c|c|}
\hline \multicolumn{35}{|c|}{ Table 9, 1985-3 } \\
\hline & \multicolumn{8}{|c|}{$\mathrm{A}$} & \multicolumn{9}{|c|}{ B } & \multicolumn{14}{|c|}{ C } & \multicolumn{3}{|c|}{ D } \\
\hline & 1 & 2 & 3 & 4 & & 6 & & 8 & 1 & 2 & 3 . & 45 & 56 & 7 & 8 & 9 & 10 & 1 & 2 & 3 & 4 & 5 & 6 & 7 & 8 & 9 & & 11 & 12 & & 14 & & & 3 \\
\hline 115 & & & & & & & & & & & & & & & & & & & & & & & & & & & & & + & & & & & \\
\hline 119 & & & & & & & & & & & & & & & & & & & & & & & & & & & & & & & & & & \\
\hline 122 & & & & & & & & & & & & & & & & & & & & & & & & & & & & & & & & & & \\
\hline 123 & & & & & & & & + & & & & & & & & & & & & & & & & & & + & & + & + & + & + & & & \\
\hline 124 & + & + & + & + & + & + & & + & + & + & +- & +-1 & ++ & ++ & + & & & + & + & + & + & + & + & + & + & & & 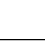 & 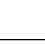 & & & & + & + \\
\hline 130 & & & & & & & & & & & & & & & & & & & & & & & & & & & & & & & & & & \\
\hline 132 & & & & & & & & + & & & & & & & & & + & & & & & & & & & + & & + & & & + & & & \\
\hline 141 & & & & & & & & & & & & & & & & & & & & & & & & & & & & + & & + & + & & & \\
\hline 142 & & & + & & + & & & + & & & & & + & + & & + & + & + & & + & & & 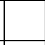 & & + & + & + & + & + & + & + & & & + \\
\hline 143 & & & & & & & & & & & & & & & & & & & & & & & & & & & & + & + & & + & & & \\
\hline 144 & & & & & & & & & & & & & & & & & & & & & & & & & & & & & & + & & & & \\
\hline 145 & & & & & & & & + & & & & & & & & & & & & & & & & & & & & + & + & + & + & & & \\
\hline 148 & & & & & & & & & & & & & & & & & & & & & & & & & & & & & & & & & & \\
\hline 149 & & & & & & & & & & & & & & & & & & & & & & & & & & & & & & + & & & & \\
\hline 151 & & & & & & & & & & & & & & & & & & & & & & & & & & & & & & & & & & \\
\hline 156 & & & & & + & & & & & & & & & & & & & & & & & & & & & & & & & & & & & \\
\hline 158 & & & + & & & & + & & & & & & + & & 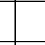 & & & & & + & + & & 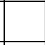 & & & 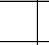 & & & & & & & & \\
\hline 161 & & & & & & & & & & & & & & & & & & & & & & & & & & & & & & & & & & \\
\hline 171 & & & & & & & & & & & & & & 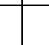 & 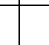 & & & & & & & & 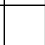 & & & 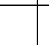 & & & & & & & & \\
\hline 198 & & & & & & + & & & & & & & & + & +5 & & & & & 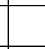 & & & + & + & & 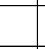 & & . & & & & & & \\
\hline 199 & + & & + & & + & + & + & + & & & & & t & ++ & $+t$ & + & + & & + & + & + & + & + & + & + & + & + & + & & & & + & & + \\
\hline 200 & & & & & + & + & & + & & & & & & & & & & & & & & & & & & + & & + & + & + & & & & \\
\hline 203 & & & & & & & & & & & & & & & & & & & & & & & & & & & & & & & + & & & \\
\hline 207 & & + & & & & & & + & & & & & & & & & + & + & & + & + & + & & + & + & + & & + & + & + & + & & & \\
\hline 209 & & & & & & & & & & & & & & & & & & & & & & & & & & & & & & & + & & & \\
\hline 210 & & & & & & & & & & & & & & & & & & & & & & & & & 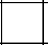 & 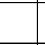 & & & & & & & & \\
\hline 211 & + & + & + & + & + & + & + & + & & & & & ++ & ++ & +5 & $-1+$ & & + & & 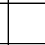 & + & + & + & + & + & + & + & & . & 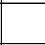 & 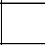 & + & + & + \\
\hline 212 & & & & & & & & & & & & & & & & & & & & & & & & & + & & & & & & & & & \\
\hline 213 & & & & & & & & + & & & & & & & 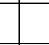 & & + & & & 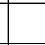 & 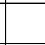 & 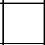 & 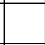 & 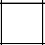 & 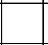 & + & & + & + & + & + & & & \\
\hline 214 & & + & + & & & + & + & + & & & & + & ++ & ++ & +1 & -1 & + & & + & + & + & + & + & + & + & + & + & + & & + & + & & + & + \\
\hline 216 & & & & + & & & & & & & & & & & & & & + & & & & & & & & & & & & & & & & \\
\hline 220 & & & & & & & & & & & & & & & & & & & & & & & & & & & & & + & + & + & & & \\
\hline 222 & & & & & & & & & & & & & & & & & & & & & & & & & 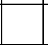 & 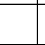 & & & & + & & & & \\
\hline 227 & & & & & & & & & & & & & & & & & & & & & & & & & 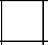 & 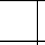 & & & & & & & 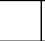 & \\
\hline 229 & + & + & + & + & + & + & + & + & + & + & + & + & -1 & ++ & $+t$ & -1 & + & + & + & + & + & & + & + & + & + & + & + & + & & & + & + & + \\
\hline 232 & & & & & & & & & & & & & & & + & & & & + & & & & & & + & & & & & & & & & + \\
\hline 233 & & & & & & & & & & & & & & & & & & & & & & & & & & + & & & + & + & & & & \\
\hline 234 & & & & & & & & & & & & & & & & & & & & & & & & & & & & & & & + & & & \\
\hline 235 & & & & & & & & + & & & + & & & & & & & & & & & & & & & & & + & & + & + & & & \\
\hline 240 & & & & & & & & & & & & & & & & & & & & & & & & & & & & & & & & & & 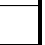 \\
\hline
\end{tabular}




\begin{tabular}{|c|c|c|c|c|c|c|c|c|c|c|c|c|c|c|c|c|c|c|c|c|c|c|c|c|c|c|c|c|c|c|c|c|c|c|c|}
\hline \multicolumn{36}{|c|}{ Table 9, 1985-4 } \\
\hline & \multicolumn{11}{|c|}{ 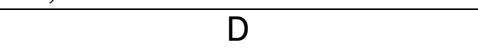 } & \multicolumn{14}{|c|}{$\mathrm{E}$} & \multicolumn{10}{|c|}{$\mathrm{F}$} \\
\hline & 4 & 5 & 6 & 7 & 8 & 9 & 10 & 11 & & & 14 & & 12 & 25 & 36 & 4 & 5 & \begin{tabular}{l|l}
6 & 7 \\
\end{tabular} & 78 & \begin{tabular}{l|l}
8 & 9 \\
\end{tabular} & 9 & 10 & 11 & 12 & & 1 & 2 & 3 & 4 & 5 & 6 & 7 & & & 10 \\
\hline 115 & & & & & & & & + & & & & & & & & & & & & & & & + & & & & & & & + & & & & & \\
\hline 119 & & & & & & & & & & & & & & & & & & & & + & & & & + & & & & & & & & & & + & + \\
\hline 122 & & & & & & & & & & & & & & & & & & & & + & & & & & & & & & & + & + & & & 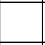 & \\
\hline 123 & & + & + & & & + & + & + & + & & + & & & & & & + & ++ & + & + & + & + & + & + & + & & & & + & + & + & + & + & + & + \\
\hline 124 & + & + & + & + & + & + & & & & & & & & & & + & + & $+t$ & + & & & & & & & + & & & + & + & + & & & & \\
\hline 130 & & & & & & & & & & & & & & & & & & & & & & & & & & & & & & & + & & & 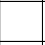 & \\
\hline 132 & & & + & + & & + & + & + & & + & & & & & & & & ++ & ++ & ++ & + & + & & & + & & & & + & + & & & + & + & \\
\hline 141 & & & & & & + & & & + & + & + & & & & & & & & & & & & & + & + & & & & & & & & + & + & \\
\hline 142 & & + & + & + & + & + & + & + & + & + & & & & & & & & $+t$ & + & ++ & + & + & + & + & + & & & & + & + & + & + & + & + & + \\
\hline 143 & & & & & & & & & & & & & & & & & & & & & & & & & + & & & & & & & & & & \\
\hline 144 & & & & & & + & & & & & & & & & & & & & & & & & & 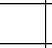 & 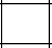 & & & & 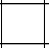 & & & & 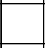 & & + \\
\hline 145 & & & + & & + & + & + & + & + & + & + & & & & & & & & & + & & & + & + & + & & & & + & & & & + & + & + \\
\hline 148 & & & & & & & & & & & & & & & & + & & & & & & & & & & & & & & & & & & & \\
\hline 149 & & & & & & & & & & & & & & & & & & & & & & & & & & & & & & & & & & & \\
\hline 151 & & & & & & & & & & & & & & & & & & & & & & & & & & & & & + & & & & & & \\
\hline 156 & & & & & & & & & & & & & & & & & & & & & & & & & & & & & & & & & & & \\
\hline 158 & & + & + & & & + & & & & & & & & & & & & + & + & + & & & 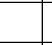 & + & & & & & & & & & & & \\
\hline 161 & & & & & & & & & & & & & & & & & & & & & & & & & & & & & & & & & & + & \\
\hline 171 & & & & & & & & & & & & & & & & & & & & & & & & & & & & & + & & & & & & \\
\hline 198 & & & + & & & & & & & & & & & & + & & & & & & & & & & & & & & 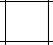 & & & & & 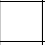 & \\
\hline 199 & + & + & + & + & + & + & + & & & & & & & & & + & + & ++ & ++ & + & + & + & + & & + & + & + & & + & + & + & + & & 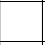 & + \\
\hline 200 & & & & & + & + & + & + & + & + & & & & & & & & & + & ++ & & & + & + & + & & + & + & + & + & + & + & + & & + \\
\hline 203 & & & & & & & & & & & & & & & & & & & & & & & & & + & & & & & & & & & + & \\
\hline 207 & & & & & + & & + & + & + & + & + & & & & & & & + & & & & + & + & + & + & & 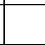 & 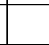 & + & + & + & + & & + & + \\
\hline 209 & & & & & & & & & & & + & & & & & & & & & & & & & & & & & & & & & & & & \\
\hline 210 & & & & & & & & & & & & & & & & & & & & & & 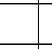 & & 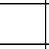 & + & & & & 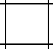 & 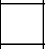 & & & & 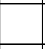 & \\
\hline 211 & + & + & + & + & + & + & + & & & & & + & +- & + & +- & + & + & ++ & + & + & & & & & & & + & + & + & & & & & 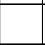 & \\
\hline 212 & & + & + & & & & & & + & + & & & & & + & & & 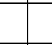 & + & + & & + & & + & & & 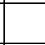 & & + & + & & 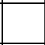 & & + & \\
\hline 213 & & + & + & & & + & + & + & + & + & + & & & & & & & + & + & + & + & + & + & + & + & & 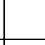 & 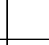 & 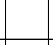 & + & + & + & + & + & + \\
\hline 214 & + & + & + & + & + & + & + & + & + & + & + & & & & & + & + & ++ & $++t$ & + & & + & & + & & + & + & & + & + & + & + & + & + & \\
\hline 216 & & & & & & & & & + & & + & & & & & & & & + & + & & & & & + & & & & & + & & & & & \\
\hline 220 & & & & & + & + & + & & + & & & & & & & & & & + & ++ & + & + & + & + & & & 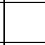 & 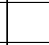 & & + & & & + & + & + \\
\hline 222 & & & & & & & & & & & & & & & & & & & & & & & & & & & & & & & & & & & \\
\hline 227 & & & & & & & & & & & & & & & + & & & & & & & & & & & & & & & & & & & & \\
\hline 229 & + & + & + & + & + & + & + & & & & & + & & + & + & + & + & + & ++ & ++ & + & + & & & & + & + & + & + & + & & & & & \\
\hline 232 & + & & & & + & & + & & & & & + & +1 & + & & + & + & + & + & ++ & & + & & & & & + & + & + & & & & & 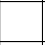 & \\
\hline 233 & & & & & & & & & + & + & & & & & & & & & & & & & & + & + & & & & + & + & + & & & + & \\
\hline 234 & & & & & & & & & & + & + & & & & & & & & & & & & & & & & & & & + & & & & & \\
\hline 235 & & & & + & & & & & & & & & & & & & & & & + & & + & & & & & & & & & & & & & \\
\hline 240 & & & & & & & & & & & + & & & & & & & & & & & & & & & & & & & & & & & & \\
\hline
\end{tabular}




\begin{tabular}{|c|c|c|c|c|c|c|c|c|c|c|c|c|c|c|c|c|c|c|c|c|c|c|c|c|c|c|c|c|c|c|c|c|c|c|}
\hline \multicolumn{35}{|c|}{ Table 9, 1986-1 } \\
\hline & \multicolumn{7}{|c|}{$\mathrm{A}$} & \multicolumn{10}{|c|}{$\mathrm{B}$} & & & & & & & $c$ & 5 & & & & & & & & D & \\
\hline & \begin{tabular}{l|l}
12 \\
1
\end{tabular} & 3 & 4 & & 6 & & 8 & 1 & 2 & 3 & 4 & 5 & 6 & & 8 & 9 & 10 & 1 & 2 & 3 & 4 & 5 & 6 & & $8 \mathrm{~s}$ & \begin{tabular}{l|l|}
9 & 1 \\
\end{tabular} & \begin{tabular}{l|l}
0 & 1 \\
\end{tabular} & 11 & 121 & 13 & 14 & 1 & & 3 \\
\hline 2 & & & & & & & + & & + & & + & & & & & & & & & & + & & & & & & & & & + & & & & \\
\hline 4 & + & + & + & + & + & + & + & & + & + & + & + & + & + & + & + & + & + & + & + & + & + & + & +- & + & ++ & + & + & + & + & + & & + & + \\
\hline 6 & & & & & & & + & & & & & & & & & & & & & & & & & & & & & + & + & & + & & & \\
\hline 7 & & + & & + & & & + & & & & + & + & + & & + & + & + & & & & & & & & & $+t+$ & & + & + & + & + & & & \\
\hline 10 & & & & & & & & & & & & & & & & & & & & & & & & & & & & & & + & + & & & \\
\hline 11 & & & & & & + & + & & & 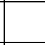 & 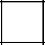 & & 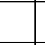 & & & + & + & & & & 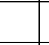 & ( & & +- & + & + & + & & & & 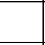 & & & \\
\hline 12 & + & + & + & + & + & + & + & + & + & + & + & + & + & + & + & + & & & & + & + & + & + & + & + & + & + & + & & & & & + & \\
\hline 13 & + & & & + & + & + & + & & + & + & + & + & + & + & + & + & + & & + & + & + & + & & + & + & + & & + & + & + & + & & & + \\
\hline 14 & & & & & & & + & & & & + & & & & + & & & + & + & + & + & + & & & 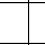 & . & & & & & & & - & \\
\hline 15 & + & + & + & + & + & + & + & + & + & + & + & + & + & + & + & + & + & + & + & + & + & + & + & +1 & + & + & + & + & + & + & + & + & + & + \\
\hline 16 & & & & & & & & & & & & & 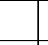 & & 7 & & + & & & 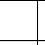 & 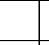 & 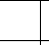 & & & & + & & & + & + & + & & & \\
\hline 17 & + & + & + & & + & + & + & + & + & + & & + & + & + & + & + & + & + & + & & + & + & + & +1 & + & + & + & + & + & + & + & & + & \\
\hline 20 & + & + & & & & & + & & + & + & + & + & & & & & + & + & + & + & + & & & 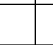 & . & + & + & + & & & & & & + \\
\hline 21 & & & & & & & & & & & & & & & & & & & & & & & & & & & & & & & & & & \\
\hline 22 & & & & & & & & & & & & & & & & & & & & & & & & & & & & & & & & & & \\
\hline 23 & & & & & & & & & & & + & & & & & & & & & & & & & & & & & & & & & & & \\
\hline 25 & & & & & & & & & & & & & & & & & & & & & & & & & & & & & & & & & & \\
\hline 26 & & & & & & & & & & & & & & & & & & & & & & & & & & & & + & + & & + & & & \\
\hline 27 & & & & & & & . & & & & & & & & & & & & & & 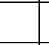 & & & & & & & + & + & + & & & & \\
\hline 28 & & + & & & & & + & & & & & + & & & & & + & + & & & + & & & - & + & & + & + & + & & & & & \\
\hline 29 & & & & & & & & & & & & & & & & & & & & & & & & & & & & + & + & & & & & \\
\hline 31 & & & & & & & & & & & & & & & & & & & & & & & & & & & & & & & & & & \\
\hline 34 & & & & & & & & & & & & & & & & & & & & & & & & & & & & & & & & & & \\
\hline 39 & & & & & & & & & & & & & & & & & & & & & & & & & & & & + & + & & & & & \\
\hline 41 & & & & & & & & & & & & & & & & & & & & & & & & & & & & & & + & & & & \\
\hline 42 & & & & & & & + & & & 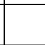 & & & & & & & & & & & & & & & & & & & & & & & & \\
\hline 43 & & & & & & & + & & & & & & & & & & & & & & & & & & & & & & + & & & & & \\
\hline 44 & + & + & + & + & + & + & + & + & + & + & + & + & + & + & + & + & + & + & + & + & + & + & + & + & + & +- & + & + & & & & + & + & + \\
\hline 48 & & & & & & & + & & & & & & & & & + & + & & & & & & & & & & & + & + & + & + & & & \\
\hline 49 & & & & & & & & & & & & & & & & & & & & & & & & & & & & & & & + & & & \\
\hline 52 & & & & & & & & & & & & & & & & & & & & & & & & & & & & & + & & 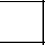 & & & \\
\hline 53 & & & + & & & & + & & & & & & & & & & & & & + & & & & & & & & & + & + & + & & & \\
\hline 58 & & & & & & & & & & & & & & & & & & & & & & & & & & & & & & & & & & \\
\hline 60 & + & + & + & + & + & & 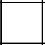 & & + & + & + & + & & & & & & + & + & + & & & & & & & & & & & & & & \\
\hline 61 & & & & & & & + & & & & & & & & & & & & & & & & & & & & & & + & + & & & & \\
\hline 63 & + & + & + & + & + & + & + & & + & + & + & & & + & + & + & & + & + & & & + & + & + & +- & + & + & & & & & & & \\
\hline 64 & & & + & + & + & + & + & & 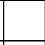 & 4 & 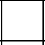 & + & + & + & + & + & & 1 & & + & + & + & + & + & + & & & & & & & + & & + \\
\hline 70 & & & & & & & & & & & & & & & & & & + & & & & & & & & & & & & & & & & \\
\hline 71 & & & + & + & + & & & & + & + & + & + & + & & & & & & & 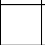 & . & 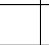 & 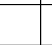 & & & & & & & & & & & \\
\hline 73 & + & + & & & + & + & & + & + & & + & + & & + & & & & + & + & & + & + & + & + & & 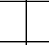 & & & & & & + & + & + \\
\hline 77 & + & + & + & + & + & + & + & & & & + & + & + & + & + & + & & + & & + & + & + & + & + & + & + & + & + & + & & & + & + & + \\
\hline 78 & + & + & + & + & + & + & + & & & & + & + & + & + & + & + & & + & & + & + & + & + & + & + & + & + & & & & & + & + & + \\
\hline 79 & & & & + & + & & & & & & & + & + & + & & + & & & & + & + & + & + & + & & & & & & & & + & & \\
\hline 80 & & & & & & & & & & & & & & & & & & & & & & & & & & & & & & & & & & \\
\hline 81 & + & + & + & + & + & + & + & + & + & + & + & + & + & + & + & + & & + & + & + & + & + & + & + & + & + & & & & & & + & + & + \\
\hline 82 & + & + & + & & + & + & & & + & + & + & + & & & & & & + & & + & + & & & & & & & & & & & & & \\
\hline 86 & & & & & & & + & & & & & & & & & & & & & & & & & + & & & & + & + & & & & & \\
\hline 87 & & & & & & & & & + & & & & & & & & & + & & & & & & & & & & + & + & + & + & & & \\
\hline 88 & & & & & & & & & & & & & & & & & & & & & & & & & & 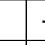 & + & & & + & + & & & \\
\hline 89 & & & & & & & + & & & & & & & & & & & & & & & & & & & & & + & + & + & + & & & \\
\hline 91 & & & & & & & & & & & & & & & & & + & & & & & & & & & & & & + & + & + & & & \\
\hline 93 & & & & & & & & & & & & & & & & & & & & & & & & & & & & & & & & & & \\
\hline 94 & & & & & + & & + & & & & & & + & & & & + & & & & & & & & & & & + & + & + & & & & \\
\hline 97 & & & & & & & & & & & & & & & & & & & & & & & & & & & & & & & & & & \\
\hline 103 & & & & & & & & & & & & & & & & & & & & & & & & & & & & + & & + & & & & \\
\hline
\end{tabular}




\begin{tabular}{|c|c|c|c|c|c|c|c|c|c|c|c|c|c|c|c|c|c|c|c|c|c|c|c|c|c|c|c|c|c|c|c|c|c|c|}
\hline \multicolumn{35}{|c|}{ Table 9, 1986-2 } \\
\hline & \multicolumn{11}{|c|}{$\mathrm{D}$} & \multicolumn{13}{|c|}{$E$} & & & & & $\mathrm{~F}$ & & & & & \\
\hline & 4 & 56 & 6 & 7 & 8 & 9 & 10 & & 12 & 13 & & & 2 & 3 & 4 & 5 & 6 & 7 & 8 & 9 & 10 & & & 13 & 1 & 2 & 3 & 4 & $5 \mid$ & \begin{tabular}{l|l}
6 & 7 \\
7
\end{tabular} & & & \begin{tabular}{l|l}
9 & $1 \mathrm{C}$ \\
\end{tabular} & \\
\hline 2 & & & & & & & & & + & & & & & & & & & & & & & & & + & & & & & + & & + & $t$ & & \\
\hline 4 & + & +-1 & + & + & + & + & + & + & + & + & + & & & & + & + & + & + & + & + & & + & + & + & & & + & + & + & +-1 & $+1-1$ & + & ++ & \\
\hline 6 & & & & & & & & & + & + & + & & & & & & & & & & + & & & + & & & & & & & & & & \\
\hline 7 & & & & & + & + & + & + & + & + & + & & & & & + & + & + & + & + & + & + & + & + & & & & & & + & -1 & + & ++ & \\
\hline 10 & & & & & & & & & & & & & & & & & & & & & & & & & & & & & & & & & & \\
\hline 11 & & + & & & + & & + & & & & & & & & & & & & & + & & & & & & & & & & & & & & \\
\hline 12 & & + & + & + & + & + & + & + & & & & & & & + & + & + & + & + & + & + & & & & + & & & + & & -1 & + & + & ++ & \\
\hline 13 & + & +- & + & + & + & + & + & + & + & + & + & & & 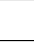 & & & + & + & + & + & + & + & & + & & & & + & + & + & $+1-$ & + & ++ & \\
\hline 14 & + & + & & & + & & & & & & & & & & & & & & + & & & & & & & & & & + & & & & + & \\
\hline 15 & & +- & + & + & + & + & + & + & + & & & & + & + & + & + & + & + & + & + & + & + & + & + & + & + & + & + & + & +7 & +- & ++ & ++ & \\
\hline 16 & & & & & + & & + & + & + & + & + & & & & & 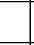 & & 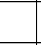 & 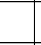 & & + & & + & + & & & & + & & 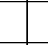 & & & + & \\
\hline 17 & & + & + & + & + & + & + & + & + & + & + & & & & + & + & + & + & + & + & + & + & + & + & + & + & + & + & + & + & + & + & ++ & \\
\hline 20 & + & + & + & & + & + & & & & & & & & & & & + & + & & & & & & & & & & & & + & & & & \\
\hline 21 & & & & & & & & + & & & & & & & & & & & & & & + & + & + & & & & & & & -1 & + & & \\
\hline 22 & & & & & & & & & & & & & & & & & & & & & & & + & & & & & & & & & & & \\
\hline 23 & & & & & + & & & & & & & & & & & & & & & & & & & & & & & & + & & & & & \\
\hline 25 & & & & & & & & & & & & & & & & & & & & & & & & + & & & & & & & & & & \\
\hline 26 & & & & & + & & & & & & + & & & & & & & & & & & + & & & & & & & & & & & & \\
\hline 27 & & & & & & & & & + & + & & & & & & & & & 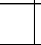 & & & + & + & + & & & & & & & & & & \\
\hline 28 & & + & & & + & + & + & + & + & + & & & & & & & + & & + & + & + & + & & + & & & & & & +- & ++ & ++ & ++ & \\
\hline 29 & & & & & & & & + & & & & & & & & & & & & & & + & + & + & & & & & & & & \pm & + & \\
\hline 31 & & & & + & & & & & & & & & & & & & & & & & & & & & & & & & & & & & & \\
\hline 34 & & & & & & & & & & & & & & & & & & & & & & & + & & & & & & + & & -1 & + & & \\
\hline 39 & & & & & & & & & & & & & & & & & & & & + & & & + & & & & & & & & & & & \\
\hline 41 & & & & & & & & + & + & & & & & & & & & 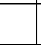 & & 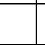 & & + & + & & & & 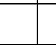 & & & & + & + & & \\
\hline 42 & & & & & & & & & & & & & & & & & & & & 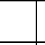 & & & 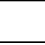 & & & & & & & & & & & \\
\hline 43 & & & & & + & & & & & & & & & & 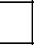 & & & + & & 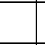 & & & 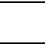 & & 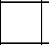 & & & + & & +-1 & + & & & \\
\hline 44 & + & + & + & + & + & & + & & + & & & + & + & + & + & + & + & + & & + & + & & 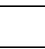 & & + & + & + & + & + & + & + & & & \\
\hline 48 & & & & & & + & + & + & + & + & + & & & & & & & + & + & + & + & + & + & + & & & & & + & & +1 & $t$ & $t$ & \\
\hline 49 & & & & & & & & & & & + & & & & & & & & & & & & & & & & & & & & & & & \\
\hline 52 & & & & & & & & & & & & & & & & & & & & & & & & & & & & & & & & & & \\
\hline 53 & & & 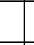 & & + & + & + & + & & & + & & & & 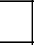 & 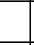 & & & + & + & + & & & & & & & & + & + & & & + & \\
\hline 58 & & & + & & & & & & & & & & & & & & & & & & & & & & & & & & & & & & & \\
\hline 60 & & & & & + & & & & & & & & & & + & + & & + & & & & + & & & & & & + & + & + & & & + & \\
\hline 61 & & & & & & & & + & + & & & & & & & 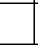 & & & & & + & + & + & + & & & & & 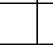 & & $t$ & +1 & ++ & \\
\hline 63 & & & + & + & + & + & & & & & & & & & & + & & + & + & + & & & & & & + & & & + & + & + & & & \\
\hline 64 & + & + & + & + & + & & & & & & & & + & & & + & + & + & & & & 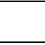 & & & + & & + & + & & & & & & \\
\hline 70 & & & & & & & & & & & & & & & & & & & & & & & & & & & & & & & & & & \\
\hline 71 & & & & + & & & & & & & & & & & & & & & & & & & & & & & 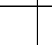 & & + & & & & & \\
\hline 73 & + & + & + & + & & & & & & & & & + & & + & + & + & + & & & & & & & + & + & + & + & + & & & & + & \\
\hline 77 & + & + & + & + & + & + & + & & & & & + & + & + & + & + & + & + & + & + & + & + & & & + & + & + & + & + & +1 & + & & + & \\
\hline 78 & + & + & + & + & + & & + & & 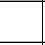 & & & + & + & + & + & + & + & + & & & + & & & & + & & + & + & + & & & & & \\
\hline 79 & & & & & & & & & & & & & & & & & & & & & & & & & + & & & & & & & & & \\
\hline 80 & & & & & & & & & & & & & & & & & & & & & & & & & & + & & & & & & & & \\
\hline 81 & + & + & + & + & + & + & & & & & & + & + & + & + & + & + & + & & & & & & & + & + & + & + & + & + & & & & \\
\hline 82 & & & & + & + & & + & & + & & & & & & & + & & + & & & & & & & & & & + & + & & & & & \\
\hline 86 & & & & & & & & + & + & & + & & & & & & & & & & & & + & & & & & & & & -1 & + & + & \\
\hline 87 & & & & & & & & & + & & + & & & & & & & & & & & & & & & & & & + & + & & $+t$ & ++ & \\
\hline 88 & & & & & & & & & + & & + & & & & & & & & & & & + & + & & & & & & + & + & + & & + & \\
\hline 89 & & & & & & & & & + & & & & & & & & & & & & & + & + & + & & & & & & & & & + & \\
\hline 91 & & & & & & & & + & + & + & & & & & & & & & & & & & & + & & & & & & & & & & \\
\hline 93 & & & & & & & & & & & & & & & & & & & & & & & & & & & & & + & & & & & \\
\hline 94 & & & & + & + & & & + & + & + & & & & & & + & & & & + & + & & + & + & & & & & + & & +1 & & $+t$ & \\
\hline 97 & & & & & & & & & & & & & & & & & & & & & & + & + & & & & & & & & & & & \\
\hline 03 & & & & & & & & & + & & & & & & & & & & & & & & & + & & & & & & & & & & \\
\hline
\end{tabular}




\begin{tabular}{|c|c|c|c|c|c|c|c|c|c|c|c|c|c|c|c|c|c|c|c|c|c|c|c|c|c|c|c|c|c|c|c|c|c|c|c|}
\hline \multicolumn{36}{|c|}{ Table 9, 1986-3 } \\
\hline & & \multicolumn{10}{|c|}{$\mathrm{B}$} & \multicolumn{14}{|c|}{ C } & \multicolumn{3}{|c|}{$\mathrm{D}$} \\
\hline & 1 & 2 & 3 & 4 & & 6 & 7 & 8 & 1 & 2 & 3 & 4 & 5 & 6 & 7 & 8 & 9 & 10 & 1 & 2 & 3 & 4 & 5 & 6 & & 8 & 9 & & 11 & & 13 & 14 & & & 3 \\
\hline 115 & & & & & & & & & & & & & & & & & & & & & & & & & & & & & & & & & & & \\
\hline 116 & & & & & & & & & & & & & & & & & & & & & & & & & & & & & + & & & & & & \\
\hline 122 & & & & & & & & + & & & & & & & & & & & & & & & & & & & & & & & & & & & \\
\hline 123 & & & & & & + & & + & & + & & & & & & & & & & + & & & & & & & & & + & + & & + & & & \\
\hline 124 & & + & + & + & + & + & + & + & & + & + & + & + & + & + & + & + & & + & + & + & & + & + & + & + & & & & 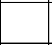 & & & & & + \\
\hline 130 & & & & & & & & & & & & & & & & & & & & & & & & & & & & & & + & & & & & \\
\hline 131 & & & & & & & & & & & & & & & & & & & & & & & & & & & & & & & & & & & \\
\hline 132 & & & & & & & & + & & & & & & & & & + & + & & & & & & & & . & + & + & + & & & & & & \\
\hline 141 & & & & & & & & & & & & & & & & & & & & & & & & & + & & & & + & + & + & + & & & \\
\hline 142 & & & & & & + & & + & & + & & + & & & + & + & + & + & & + & + & + & & & & + & + & + & + & + & + & & & & + \\
\hline 143 & & & & & & & & & & & & & & & & & & & & & & & & 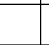 & & & & & + & + & & & & & \\
\hline 144 & & & & & & & & + & & & & & & & & & & & & & & & & & & & & & & + & & & & & \\
\hline 145 & & & & & & + & & + & & & & & + & & & & & & & & & & & & & & & & + & + & + & + & & & \\
\hline 149 & & & & & & & & & & & & & & & & & & & & & & & & & & & & & & & & & & & \\
\hline 156 & & & & & & + & & & & & & & & & & & & & & + & & & & & & & & & & & & & & & \\
\hline 158 & & & & & + & + & & & & & & & + & & & & & & & & + & + & + & & & & & & & & & & & & \\
\hline 161 & & & & & & & & & & & & & & & & & & & & & & & & 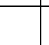 & & & & & & & & & & & \\
\hline 175 & & & & & & & & & & & & & & & & & & & & & + & & & & & & & & & & & & & & \\
\hline 198 & & & + & & & + & + & & & & & & + & & + & + & + & & & & & + & + & + & + & + & + & + & & & & & & & + \\
\hline 199 & & + & + & + & + & + & + & + & & & & & + & + & + & + & + & + & & & + & + & + & + & + & + & + & + & + & + & & & & & + \\
\hline 200 & & + & & & & & + & + & & & & & & & & + & & & & & & & + & & + & + & + & + & + & + & + & & & & \\
\hline 203 & & & & & & & & & & & & & & & & & & & & 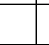 & - & & & 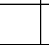 & & 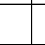 & & & + & + & & & & & \\
\hline 207 & & + & & & & & & & + & + & + & + & & + & & 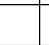 & & + & + & + & + & & & 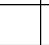 & & . & & + & + & + & & + & & & 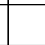 \\
\hline 209 & & & & & & & & & & & & & & & & & & & & & & & & & & & & & & + & & & & & \\
\hline 210 & & & & & & & & & & & & & & & & & & & & & & & & & & & & & & + & & & & & \\
\hline 211 & & + & + & + & + & + & + & + & & & & + & & + & + & + & + & + & + & + & & + & + & + & + & + & + & + & & . & 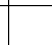 & 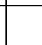 & + & + & + \\
\hline 212 & & & & & & & & & & & & & & & & & & & & & & & & & & & & & & + & + & + & & & \\
\hline 213 & & & & & & & & + & & & & & & & & & & + & & & & & & 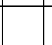 & & 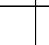 & & & + & + & + & + & & & \\
\hline 214 & & + & & & + & & + & + & & + & + & + & + & + & + & + & + & + & + & + & + & & + & + & + & + & + & + & + & + & + & + & & + & \\
\hline 216 & & + & & & & & & & & & & & & & & & & & & & & & & & & & & & & & & & & & \\
\hline 220 & & & + & & & + & & + & & & + & + & & & & & & & & + & & & & 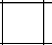 & & . & - & 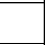 & + & + & + & + & & & \\
\hline 222 & & & & & & & & & & & & & & & & & & & & & & & & . & & & & & & + & + & & & & \\
\hline 227 & & & & & & & & + & & & & & & & & + & + & & & & & & & + & + & + & + & + & & & + & & & & \\
\hline 229 & & + & + & + & + & + & + & + & + & + & + & + & & + & + & + & + & + & + & + & + & + & + & + & + & + & + & + & + & + & & & + & & + \\
\hline 232 & & & & & & & & + & & & & + & & & & + & + & + & + & & & & & & + & + & + & + & + & + & & & & + & \\
\hline 233 & & & & & & & & + & & & & & & & & & & & & & & & & & & & & & + & + & + & & & & \\
\hline 234 & & & & & & & & & & & & & & & & & & & & & & & & & & & & & & & & + & & & \\
\hline 235 & & & & & & & & + & & & & + & & & & & + & + & + & + & & & & & + & & & + & & & & & & & \\
\hline 242 & & & & & & & & & & & & & & & & & & & & & & & & & & & & & & & & & & & \\
\hline
\end{tabular}




\begin{tabular}{|c|c|c|c|c|c|c|c|c|c|c|c|c|c|c|c|c|c|c|c|c|c|c|c|c|c|c|c|c|c|c|c|c|c|c|c|}
\hline \multicolumn{36}{|c|}{ Table 9, 1986-4 } \\
\hline & \multicolumn{11}{|c|}{$\mathrm{D}$} & \multicolumn{14}{|c|}{$\mathrm{E}$} & \multicolumn{10}{|c|}{$\mathrm{F}$} \\
\hline & 4 & 5 & 6 & 7 & 8 & 9 & 10 & 11 & 12 & & 14 & 1 & & 2 & 32 & 45 & 6 & 7 & 8 & 9 & 10 & & & & $13 \mid$ & & 2 & 3 & 45 & \begin{tabular}{l|l}
5 & 6 \\
\end{tabular} & & $7 \varepsilon$ & 89 & & 10 \\
\hline 115 & & & & & & & & & & & & & & & & & & & & & & + & & & & & & & & & & & & & \\
\hline 116 & & & & & & & & & & & & & & & & & & & & & & & & & & & & & & & & & & & \\
\hline 122 & & & & & & & & & & & & & & & & & & & + & -+ & + & & & & & & & & & ++ & + & & $+t$ & & + \\
\hline 123 & & + & + & & + & + & & + & + & & & & & & & + & + & + & + & + & & + & -+ & $+t$ & + & & & & & ++ & + & +-1 & ++ & & + \\
\hline 124 & + & + & + & + & + & + & & & & & & & & & $-t$ & -1 & + & + & + & & & & & & & & + & & $+1-1$ & ++ & + & & & + & \\
\hline 130 & & & & & & & & & & & & & & & & & & & & & & & & & & & & & & & & & & & \\
\hline 131 & & & & & & & & & & & & & & & & & & & + & & & & & & & & & & & & & & & & \\
\hline 132 & & + & + & + & + & + & + & + & + & + & & & & & & 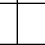 & 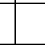 & + & & + & + & & + & + & & & & & $+t$ & ++ & + & & $+t$ & & + \\
\hline 141 & & & & & & & & + & + & + & + & & & & & & & & & & & + & & & + & & & & & & & + & & & + \\
\hline 142 & & + & + & + & + & + & + & + & + & + & & + & & & & + & + & + & + & + & t & + & $+t$ & +1 & + & & . & & + & ++1 & + & + & $t$ & & + \\
\hline 143 & & & & & & & & & & & & & & & & + & & & & & & & & & & & & & & & & & & & \\
\hline 144 & & & & & & & & & & & & & & & & & & & & & & & & & & & & & & & & & & & \\
\hline 145 & & + & + & & + & + & & + & + & & & & & & & & & & + & -+ & + & $-1+$ & + & $+t$ & + & & & & & + & & & & + & + \\
\hline 149 & & & & & & & & & & & & & & & & & & & & & & & + & + & & & & & & & & & & & \\
\hline 156 & & & & & & & & & & & & & & & & & & & & & & & & & & & & & & & & & & & \\
\hline 158 & & + & + & & & & & & & & & & & & & & & & & & & & & & & & & & & & & & & & \\
\hline 161 & & & & & & & & & & & & & & & & & & & & & & + & & & & & & & & & & & & & \\
\hline 175 & & & & & & & & & & & & & & & & & & & & & & & & & & & & & & & & & & & \\
\hline 198 & + & + & & + & + & & & & & & & & & +- & +- & + & & & & & & & & & & + & + & + & + & & & + & & & \\
\hline 199 & + & + & + & + & + & + & + & + & & & & & & 7 & +1 & $+t$ & $-1+$ & & + & -+ & + & & & & & + & + & + & + & $+t$ & + & & & 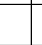 & \\
\hline 200 & & & & & + & + & + & + & + & + & & & & & 7 & -1 & & + & + & + & + & $-1+$ & + & & + & & & & + & ++ & + & +1 & & + & + \\
\hline 203 & & & & & & & & & & & & & & & & & & & & & & & & & & & & & & & & & & & \\
\hline 207 & & & + & & + & & & + & + & + & + & & & & & & + & + & & & + & $-1+$ & ++ & +7 & + & & 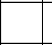 & & + & ++ & + & + & & + & + \\
\hline 209 & & & & & & & & & & + & & & & & & & & & & & & & & & & & & & & & & & & & \\
\hline 210 & & & & & & & & & & + & & & & & & & & & & & & & & & & & & & & & & & & & \\
\hline 211 & + & + & + & + & + & + & + & & & & & + & & +- & + & + & + & + & & + & & + & & & & & + & + & + & & & & & & \\
\hline 212 & & & & & & & + & & + & & + & & & & & & & & & & & + & & & + & & & & & & & & & & \\
\hline 213 & & & & & + & + & + & + & + & + & + & & & & & + & + & + & + & $-1+$ & + & +1 & $+t$ & +1 & + & & . & & + & $+t$ & + & & & + & + \\
\hline 214 & + & + & + & + & + & & + & + & + & + & & & & & -1 & ++ & -+ & + & + & + & + & + & +1 & +1 & $+1-$ & + & + & & & ++ & + & +1 & & + & + \\
\hline 216 & & & & & & & & & + & & & & & & -1 & -1 & & & & & & & & & & & & & & & & & & & \\
\hline 220 & & & & & + & + & & & + & + & & & & & & & & + & + & -+ & & + & + & & & & & & & & & & & + & + \\
\hline 222 & & & & & & & & & & & & & & & & & & & & & & & & & & & & & & & & & & & \\
\hline 227 & & & & & + & & + & & & & & & & & $t$ & - & & & & & & & & & + & & . & & + & & & & & & \\
\hline 229 & + & + & + & + & + & + & + & + & + & + & & + & $-1-$ & +- & +1 & $-1+$ & +1 & & & & & & & & & + & + & + & + & + & & & & & \\
\hline 232 & + & + & & + & + & + & + & & & & & & & & & & + & + & & & + & & & & & & + & + & + & $+t$ & + & + & & + & \\
\hline 233 & & & & & & & & & & + & & & & & & & & & & & & + & $t$ & + & + & & & & & + & & & + & & + \\
\hline 234 & & & & & & & & & & & + & & & & & & & & & & & & & & + & & & & & + & & & & & + \\
\hline 235 & & + & & & + & + & + & + & & + & & & & & & & & & & & & & $t$ & + & + & & & & & + & & + & & & \\
\hline 242 & & & & & & & & & & & & & & & & & & + & & & & & & & + & & & & & & & & & & \\
\hline
\end{tabular}




\begin{tabular}{|c|c|c|c|c|c|c|c|c|c|c|c|c|c|c|c|c|c|c|c|c|c|c|c|c|c|c|c|c|c|c|c|c|c|c|}
\hline \multicolumn{35}{|c|}{ Table 9, 1987-1 } \\
\hline & \multicolumn{7}{|c|}{$\mathrm{A}$} & \multicolumn{10}{|c|}{ B } & & & & & & & & j & & & & & & & & D & \\
\hline & \begin{tabular}{l|l}
1 & 2 \\
\end{tabular} & 3 & 4 & & 6 & 7 & 8 & 1 & & 3 & 4 & 5 & 6 & 7 & 8 & 9.1 & 10 & 1 & 2 & 3 & 4 & 5 & 6 & 7 & 8 & $\begin{array}{l}9 \\
\end{array}$ & & 11. & 12 & 13 & 14 & 1 & & 3 \\
\hline 2 & & & + & & & & & + & + & & + & & & & t & & & & & & & & & & & & & & & & + & & & 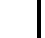 \\
\hline 3 & & & & & & & & & & & & & & & & & + & & & & & & & & & & & & & & + & & & \\
\hline 4 & + & + & + & + & + & + & + & + & + & + & + & + & + & + & + & + & + & + & + & + & & + & & + & & & + & + & + & & + & & & + \\
\hline 6 & & & & & & & & & & & & & & & & & + & & & & & & & & & & & + & & + & + & & & \\
\hline 7 & & & + & + & + & + & + & & & + & & + & + & & + & + & + & & + & & & & & & +-1 & + & + & + & + & + & + & & & \\
\hline 8 & & & & & & & & & & & & & & & & & & & & & & & & & & & & & + & & + & & & \\
\hline 9 & & & & & & & & & & & & & & & & & & & & & & & & & & & & & & & & & & \\
\hline 10 & & & & & & & & & & & & 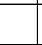 & & & & & + & & & & & & & & & & & & & + & & & & \\
\hline 11 & & & & & & & + & & & & & 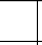 & & & + & + & & & & & & & & & + & & & & & & & & & \\
\hline 12 & + & + & + & + & + & + & + & + & + & + & + & + & + & + & + & + & & + & & + & + & & + & + & +-1 & & & & & & & & & \\
\hline 13 & + & + & + & + & + & + & + & + & + & + & + & + & + & + & + & + & + & + & + & & & + & & + & + & + & + & + & + & & + & & + & + \\
\hline 14 & + & + & + & + & + & + & & + & & + & + & + & + & + & + & + & + & + & + & + & & + & & + & + & + & $t$ & + & + & + & & + & + & \\
\hline 15 & + & + & + & + & + & + & + & + & + & + & + & + & + & + & + & + & + & & + & + & + & & + & + & +-1 & + & $t$ & & + & + & & + & + & + \\
\hline 16 & & & & & & & & & & & & & & & + & + & + & & & $\square$ & 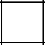 & 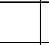 & 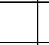 & & 7 & & + & + & + & + & + & & & 7 \\
\hline 17 & & + & + & & & + & + & & & 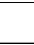 & & + & + & + & + & + & + & & + & + & + & + & + & + & +1 & + & + & + & + & + & & & & + \\
\hline 20 & + & + & & + & + & + & & + & & + & + & + & & & & + & + & + & + & + & & + & & & 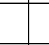 & & & & + & & & + & + & + \\
\hline 21 & & & & & & & & & & & & & & & & & + & & & & & & & & & & & + & & & & & & \\
\hline 22 & & & + & & & & & & & & & & & & & & & + & & & & & & & & & & & & & & & & \\
\hline 23 & + & & & & & & & + & + & & & & & & & & & & & & & & & & & & & & & & & & & \\
\hline 25 & & & & & & & & & & & & & & & & & & & & & & & & & & & & & & & & & & \\
\hline 26 & & & & & & & & & & & & & & & & & & & & & & & & & & & & + & & + & + & & & \\
\hline 27 & & & & & & & & & & & & & & & + & + & & & & & & & & & & & & + & + & + & & & & 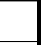 \\
\hline 28 & & & & & + & + & + & & + & & & & & & & + & + & & & & & & & & +- & + & & + & + & & & & & 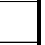 \\
\hline 29 & & & & & & & & & & & & & & & & & & & & & & & & & & & & + & + & + & + & & & \\
\hline 31 & & & & & 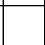 & & + & & & & & & & & 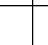 & & & & & & + & & & & - & & & & & & & & & \\
\hline 33 & & & + & + & + & + & + & & & & & & & & + & + & & & & & & & & & & & & & & & & & & 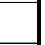 \\
\hline 35 & & & & & & & & & + & & & & & & & & & & & & & & & & & & & & & & & & & \\
\hline 38 & & & & & & & & & & & & & & & & & & & & & & & & & & & & & & & & & & \\
\hline 39 & & & & & & & + & & & & & & & & & & + & & & & & & & & & & & + & & & & & & \\
\hline 41 & + & & & & & & & & & + & & & & & & & + & & & & & & & & & & & + & & & + & & & \\
\hline 42 & & & & & & & & & & & & & & & & & & & & & & & & & & & & & & & & & & \\
\hline 43 & & & & & & & + & & & & + & & & & + & + & + & & & & & & & + & & & + & & & & & + & & \\
\hline 44 & + & + & + & + & + & + & + & + & + & + & + & + & + & + & + & + & + & + & + & + & + & + & + & + & + & + & + & + & & & & + & + & + \\
\hline 45 & & & & & & & & & & & & & & & & & & + & & & & & & & & & & & & & & & + & \\
\hline 48 & & & & & & & + & & & & & & & & + & + & + & & & & & & & & + & + & & + & + & + & + & & & \\
\hline 49 & & & & & & & & & & & & & & & & & & & & & & & & & & & & & & & + & & & \\
\hline 52 & & & & & & & & & & & & & & & & & & & & & & & & & & & & & & & & & & \\
\hline 53 & + & & & & + & + & + & & & & & & & & + & + & & & & & & & & + & + & 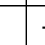 & + & + & + & + & + & & & \\
\hline 57 & & & & & & & & & & & & & & & & & & & & & & & & & & & & & & & & & & \\
\hline 58 & & & & & & & & + & & & & & & & & & & & & & & 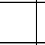 & & & & & & & & & & & & \\
\hline 60 & + & + & + & + & + & + & & + & + & + & + & + & & + & & & & + & + & + & & + & & & & & & & & & & + & + & + \\
\hline 61 & & & & + & & + & + & & & & & & & & & + & + & & & & & & & & & & & + & & & + & & & \\
\hline 62 & & & & & & & & & & & & & & & & & & & & & & & & & & & & & & & & & & \\
\hline 63 & & & & + & + & + & & & & + & + & & + & + & + & + & & + & & & + & + & & & + & & & & & & & 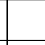 & & \\
\hline 64 & + & + & + & + & + & + & & 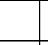 & & & + & + & + & + & + & & & & & + & + & + & + & + & & & & & & & & + & & + \\
\hline 70 & & & & & & & & & & & & & & & & & & & + & & & & & & & & & & & & & & & \\
\hline 71 & + & + & + & + & + & + & & + & + & + & + & + & + & & & & & & + & + & + & & & + & & & & & & & & & + & + \\
\hline 72 & & & & & & & & & & & & & & & & & & & & & & 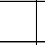 & & & & & & & & & & & & \\
\hline 73 & + & + & + & + & + & + & & + & + & & + & + & + & + & + & & & + & + & + & + & + & + & & & & & & & & & + & + & + \\
\hline 75 & & & & & & & & & & & & & & & & & & + & & & & 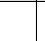 & & & & & & & & & & & & \\
\hline 77 & & + & + & + & & + & + & & & & + & + & + & + & + & + & + & + & + & + & + & + & + & + & & & + & & & & & + & + & + \\
\hline 78 & & + & + & + & + & + & + & & & & + & + & + & + & + & + & & + & + & & + & + & + & + & + & + & $t$ & & & & & + & + & + \\
\hline 79 & & & & & & & & & & & & + & + & + & & + & & + & & + & + & + & & + & + & & & & & & & + & & \\
\hline 80 & & & & & & & & & & & & & + & & + & & & & & + & + & + & & & & & & & & & & & & \\
\hline 81 & + & $-1+$ & + & + & + & + & + & & & & + & + & + & + & + & + & & & + & + & + & + & + & + & + & + & + & & & & & & & + \\
\hline
\end{tabular}




\begin{tabular}{|c|c|c|c|c|c|c|c|c|c|c|c|c|c|c|c|c|c|c|c|c|c|c|c|c|c|c|c|c|c|c|c|c|c|c|c|c|c|c|c|}
\hline Tabl & e 9 & 1 & 198 & 87 & & & & & & & & & & & & & & & & & & & & & & & & & & & & & & & & & & & \\
\hline & & & & & & [ & 5 & & & & & & & & & & & & & $\mathrm{E}$ & & & & & & & & & & & & & $\mathrm{F}$ & $E$ & & & & & \\
\hline & 4 & 5 & 6 & 7 & 8 & 9 & 10 & & 1 & 12 & 13 & 14 & 1 & 2 & 3 & & 4 & 5 & 6 & 7 & 8 & 9 & 10 & & 11 & 12 & 13 & 1 & 2 & 2 & 3 & 4 & 5 & 6 & 7 & 8 & 9 & & 10 \\
\hline 2 & & & & & & & & & + & & & & & & & & & & & & & & & & + & + & + & & & & & & & + & & + & & & \\
\hline 3 & & & & & & & & & & & & & & & & & & & & & & & & & & + & & & & & & & & & & & & & \\
\hline 4 & + & + & + & + & + & + & + & & + & + & + & + & & & & & + & + & + & + & + & + & + & & & + & & & & & & + & + & + & + & + & + & & + \\
\hline 6 & & & & & & & & & & & + & + & & & & & & & & & & & & & + & + & + & & & & & & & & & & + & & + \\
\hline 7 & & + & & & & & + & & + & + & + & + & & & & & + & + & & + & + & + & + & & + & + & + & & & & & & & + & + & + & + & & + \\
\hline 8 & & & & & & & & & & & + & & & & & & & & & & + & & & & + & & & & & & & & & & & & + & & \\
\hline 9 & & & & & & & & & & & & & & & & & & & & & & & & & & & & & & & & & & + & & & & & \\
\hline 10 & & & & & & + & & & & & & + & & & & & & & & & & & & & & & & & & & & & & & & & & & \\
\hline 11 & + & & + & & & & + & & & & & & & & & & + & & + & + & + & & & & & & & & & & & + & & & & + & & & \\
\hline 12 & + & + & & + & + & + & + & & + & & & & & & & &.+ & + & + & + & + & + & & & & & & & & & & + & & & + & + & & & \\
\hline 13 & + & + & + & + & + & + & + & & + & + & + & + & & & & & + & + & + & + & + & + & + & & + & + & & & + & + & & + & + & + & + & + & + & & + \\
\hline 14 & + & + & & & + & & + & & & & & & & & & & + & + & + & + & + & & + & & & & + & & + & + & & & & + & + & + & + & & + \\
\hline 15 & + & + & + & + & + & + & + & & + & + & & & + & & + & & + & + & + & + & + & + & + & & + & + & & + & + & +1 & + & + & + & + & + & + & + & & + \\
\hline 16 & & & & + & & & + & & + & + & + & + & & & & & & & + & & + & + & + & & + & + & + & & & & & & & & & + & & & + \\
\hline 17 & + & + & + & + & + & & + & & + & + & + & + & & & + & & + & + & + & + & + & + & + & & + & + & & + & & & & + & + & + & + & + & + & & + \\
\hline 20 & & + & + & + & & & & & & + & & & & & & & + & + & + & + & + & & & & & & & & & & & & + & & & & + & & \\
\hline 21 & & & & & & & & & + & & & & & & & & & & & & & & + & & + & & & & & & & & & & & + & + & & \\
\hline 22 & & & & & & & & & & & & & & & & & & & & & & & & & & & & & & & & & & & & & & & \\
\hline 23 & & & & & & & & & & & & & & & & & & & & & & & & & & & & & & & & & & + & + & & & & \\
\hline 25 & & & & & & & & & & & & & & & & & & & & & & & & & & + & & & & & & & & & & & & & \\
\hline 26 & & & & & & & & & & + & & + & & & & & & & & & & & & & & & + & & & & & & & & & + & & & \\
\hline 27 & & & & & & & & & & + & + & + & & & & & & & & & & & & & + & + & + & & & & & & & & & & & & + \\
\hline 28 & & + & + & & + & + & + & & + & + & & & & & & & & & + & + & + & + & + & & + & + & & & & & & & & + & + & + & + & & \\
\hline 29 & & & & & & + & & & + & & & & & & & & & & & & + & + & + & & + & + & + & & & & & & & + & + & & & & + \\
\hline 31 & & & & & & & + & & & & & & & & & & & & & & & & & & & & & & & & & & & & & & & & \\
\hline 33 & & + & & + & & & & & & & & & & & & & & & & & & & & & & & & & & & & & & & & & + & & \\
\hline 35 & & & & & & & & & & & & & & & & & & & & & & & & & & & & & & & & & & & & & & & \\
\hline 38 & & & & & & & & & & & & & & & & & & & & & + & & & & & & & & & & & & & & & + & & & \\
\hline 39 & & & & & & + & & & + & & & + & & & & & & & & & & & & & & + & + & & & & & & & & & & & & \\
\hline 41 & & & & & + & & & & + & & & & & & & & & & & & & & + & & & & & & & & & & & + & & + & & & + \\
\hline 42 & & & & & & & & & & & & & & & & & & & & & & & & & & & & & & & & & & + & & & & & \\
\hline 43 & & & & & & + & & & + & & & & & & & & & & & & + & & & & & & + & & & & & + & & + & + & + & + & & + \\
\hline 44 & + & + & + & + & + & + & & & & & & & + & + & + & & + & + & + & + & + & + & + & & & & & + & + & +- & + & + & + & + & + & + & & & \\
\hline 45 & & & & & & & & & & & & & & & & & & & & & & & & & & & & & & & & & & & & . & & & \\
\hline 48 & & & & & + & + & + & & + & + & + & + & & & & & & & & + & + & + & + & & + & + & + & & & & & & & + & + & + & + & & + \\
\hline 49 & & & & & & & & & & & & + & & & & & & & & & & + & & & & & + & & & & & & & & & & & & + \\
\hline 52 & & & & & & & & & & & & & & & & & & & & & & & & & & & + & & & & & & & & & & & & \\
\hline 53 & & + & + & + & + & + & + & & + & + & + & + & & & & & & + & + & + & + & + & + & & & + & + & & & & & & & + & + & + & + & & + \\
\hline 57 & & & & & & & & & & & & & & & & & & & + & + & & & & & & & & & & & & & & + & & & & & \\
\hline 58 & & + & & & & & & & & & & & & & & & & & & & & & & & + & & + & & & & & & & + & & & & & + \\
\hline 60 & & + & & + & & & & & & & & & & & & & & + & + & + & & & & & & & & & & & & & + & + & & + & & & \\
\hline 61 & & & & & & & + & & + & + & + & & & & & & & & & & + & + & + & & + & + & + & & & & & & & + & + & + & + & & + \\
\hline 62 & & & & & & & & & & & & & & & & & & & + & & & & & & & & & & & & & & & & & & & & \\
\hline 63 & & + & & + & & + & & & & & & & & & & & & + & + & + & + & + & & & & & & & & & & & & + & & & & & \\
\hline 64 & & + & + & + & & & & & & & & & & & & & + & & & & & & & & & & & & & & & & & & & & & & \\
\hline 70 & & & & & & & & & & & & & & & & & & & & & & & & & & & & & & & & & & & & & & & \\
\hline 71 & & & & & & & & & & & & & & & & & & + & + & & & & & & + & & & & & & & + & + & & & & & & \\
\hline 72 & & & & & & & & & & & & & & & & & & + & & & & & & & & & & & & & & & & & & & & & \\
\hline 73 & + & + & + & + & + & & & & & & & & + & & + & & + & + & + & + & + & & & & & & & + & + & + & + & + & + & & & & & & \\
\hline 75 & & & & & & & & & & & & & & & & & & & & & & & & & & & & & & & & & & & & & & & \\
\hline 77 & + & + & + & + & + & + & + & & & & & & + & + & + & & + & + & + & + & + & + & + & & & & & + & + & + & + & + & + & + & & + & + & & \\
\hline 78 & + & + & + & + & + & & + & & & & & & + & + & + & & + & + & + & + & & + & + & & & & & + & & & + & + & & & & & & & \\
\hline 79 & & & & & & & & & & & & & + & & + & & & & & & & & & & & & & + & & & & & & & & & & & \\
\hline 80 & & & & & & & & & & & & & & & & & & & & & & & & & & & & + & + & + & + & + & & & & & & & \\
\hline 81 & + & + & + & + & + & & & & & & & & + & + & + & & & & & & & & & & & & & + & + & + & + & + & + & & + & & & & \\
\hline
\end{tabular}


Table 9, 1987-3

\begin{tabular}{|c|c|c|c|c|c|c|c|c|c|c|c|c|c|c|c|c|c|c|c|c|c|c|c|c|c|c|c|c|c|c|c|c|c|}
\hline & \multicolumn{7}{|c|}{$A$} & \multicolumn{10}{|c|}{$B$} & & & & & & & C & & & & & & & & $\mathrm{D}$ & \\
\hline & \begin{tabular}{l|l}
1 & 2 \\
\end{tabular} & \begin{tabular}{l|l}
2 & 3 \\
\end{tabular} & 4 & & 6 & 7 & 8 & 1 & 2 & 3 & 4 & $\begin{array}{lll}5 & 6\end{array}$ & \begin{tabular}{l|l}
6 & 7 \\
\end{tabular} & 8 & 9 & 10 & 01 & 2 & 3 & 4 & 5 & 6 & 7 & 8 & 9 & & 11 & 12 & & 14 & 1 & & 3 \\
\hline 82 & + & ++ & + & + & & & + & + & + & + & + & ++ & $t$ & & & & + & + & + & & & & & & & & & & & & & + & + \\
\hline 85 & & & & & & & & & & & & & & & & & & & & & & & & & & & & & & & & & \\
\hline 86 & & & + & + & + & & + & & & & & - & $t$ & & & + & & & & & & & & & & & + & & & + & & & \\
\hline 87 & & & & & + & & & & & & & & & & & + & & & & & & & & & & & & & & + & & & + \\
\hline 88 & & & & & & & & & & & & & & & & & & & & & & & & & & & & & & + & & & \\
\hline 89 & & & & & & & & & & & & & & & & & & & & & & & & & & & + & & + & + & & & \\
\hline 91 & & & & & & & & & & & & & & & & & & & & & & & & & & & & + & + & + & & & \\
\hline 94 & & + & & & + & & + & & & & + & - & + & & 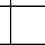 & + & & & & & & & & & & & + & + & + & + & & & \\
\hline 99 & & & & & & & & & & & & & & & & & & & & & & & & & & & & & & & & & \\
\hline 103 & & & & & & & & & & & & & & & + & & & & & & & & & & & & + & & & & & & \\
\hline 115 & & & & & & & & & & & & & & & & & & & & & + & & & & & & & & & & & & \\
\hline 116 & & & & & & & & & & & & & & & & & & & & & & & & & & & & & & & & & \\
\hline 117 & & & & & & & & & & & & & & & 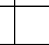 & & & & & & & & & & & & & & & & & & \\
\hline 119 & & & & & & & & & & & & & & & 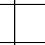 & & & & & & & & & & & & & & & & & & \\
\hline 122 & & & & & & + & & & & & & & & & & & & & & & & & & + & & & & & & & & & \\
\hline 123 & + & ++ & + & + & + & + & + & + & & + & + & +- & + & $+t$ & + & & & + & + & + & + & & & + & & & + & & + & + & & & \\
\hline 124 & + & ++ & + & + & + & + & + & + & + & + & + & +- & +7 & +1 & + & & + & +1 & + & + & + & + & + & + & & & 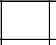 & + & & & & + & + \\
\hline 125 & & & & & & & & & & & & & & & & & & & & & & & & & & & & & & & & & \\
\hline 130 & & & & & & & & & & & & & & & & & & & & & & & & & & & + & & & & & & \\
\hline 132 & & & & & & + & + & & & & & 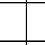 & & + & + & + & & 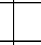 & 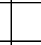 & 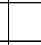 & 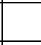 & 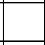 & 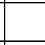 & 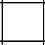 & 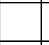 & & + & + & & & & & \\
\hline 141 & & & & & & & & & & & & & & & & & & & & & & & & & & & + & & & + & & & \\
\hline 142 & & + & & + & + & & + & & & + & & - & $+1-$ & $-1+$ & + & + & & + & & . & + & + & + & + & + & + & + & + & & + & & + & + \\
\hline 143 & & & & & & & & & & & & & & & + & $t$ & & & & & & & & & & & + & + & + & + & & & \\
\hline 144 & & & & & & & & & & & & & & & & & & & & & & & & & & & & & & & & & \\
\hline 145 & & & & + & + & + & + & & & & + & +1 & $t$ & & + & + & & & & 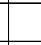 & 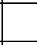 & + & & & & & + & + & & & & & \\
\hline 146 & & & & & & & & & & & & & & & & & & & & & & & & & & & & & & & & & \\
\hline 148 & & & & & & & & & & & & & & & & & & & & & & & & & & & & & & & & & \\
\hline 156 & + & + & & & & & & & & & & & & & & & & & & . & 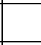 & & & & & & & & & & & & \\
\hline 158 & & + & & + & + & + & & & & + & + & + & & & & & & & & 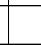 & 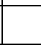 & & & & & & 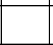 & & & & & & \\
\hline 161 & & & & & & & & & & & & & & & & & & & & & & & & & & & & & & & & & \\
\hline 168 & & & & & & & & & & & & & & & & & & & & & 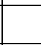 & & & & & & & & & & & & \\
\hline 176 & & & & & & & & & & & & & & & & & & & & & & & & & & & & & & & & & \\
\hline 198 & & & + & & + & + & & & & & & & -1 & 5 & & & & & & + & + & + & + & & & + & & & & & & & \\
\hline 199 & + & $1+$ & + & + & + & + & + & & & & & +- & +5 & +1 & + & + & & & + & + & + & + & + & + & + & + & & + & & & + & + & + \\
\hline 200 & & & + & & & + & + & & & & & & & & + & + & & & + & & + & & + & & + & + & + & + & + & + & & & \\
\hline 202 & & & & & & + & & & & & & & & & & & & & & & & & & & & & & & & & & & \\
\hline 203 & & & & & & & & & & & & & & & & & & & & & & & & & & & + & & & + & & & \\
\hline 205 & & & & & & & & & & & & & & & & & & & & & & & & & & & & & & + & & & \\
\hline 207 & + & & & & & & & + & + & + & + & & & & + & + & & & + & + & & & & + & & & + & + & + & + & & & \\
\hline 208 & & & & & & & & & & & & & & & & & & & & & & & & & & & & & & & & & \\
\hline 209 & & & & & & & & & & & & & & & & & & & & & & & & & & & & & & & & & \\
\hline 210 & & & & & & + & & & & & & & & & & & & & & & . & & & & & & & & & & & & \\
\hline 211 & + & $-1+$ & & + & + & + & + & & & + & + & + & + & ++ & +1 & + & & & & & + & + & & + & + & + & & & & & + & + & + \\
\hline 212 & & & & + & & & + & & & & & & & & + & + & & & + & & & & & & & & & & & + & & & \\
\hline 213 & & + & + & & & & + & & & & + & & & & + & + & & & . & & + & & & 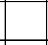 & 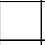 & & + & + & + & + & & & + \\
\hline 214 & + & + & & + & & & + & + & + & + & + & + & + & +1 & $+1+$ & $t$ & & + & + & + & + & + & + & + & + & + & + & + & + & + & + & + & + \\
\hline 216 & & & & & & & & & & & & & & & & & & & & & & & & & & & & & & & & & \\
\hline 220 & & + & + & + & + & + & & & & + & + & - & + & & & + & & + & + & & & & & & & + & + & + & + & + & & & \\
\hline 227 & & & & & & & & & & & + & & $1-1$ & $+1+$ & $+t$ & & & & & & & + & & & & + & & & & & & & \\
\hline 229 & + & $-1+$ & + & + & + & + & + & + & + & + & + & & -1 & ++ & $+t$ & + & + & + & & + & + & + & + & + & + & + & + & & & & + & + & + \\
\hline 230 & & & & & & & & & & & & & & & & & & & & & & & & & & & & & & & & & \\
\hline 232 & & & & & + & + & + & & & & & & -1 & +1 & $+1+$ & + & & + & + & + & + & + & & & + & + & + & & & & + & + & + \\
\hline 233 & & & & & & & & & & & & & & & & & & & & & & & & & & & + & + & + & & & & \\
\hline 234 & & & & & & & & & & & & & & & & & & & & & & & & & & & & & & & & & \\
\hline 235 & & + & & & & & + & & & + & + & + & & + & ++ & + & & & & & & & & & & & + & & & & & & \\
\hline 239 & & & & & & & & + & & & & & & & & & & & & & & & & & & & & & & & & & \\
\hline 240 & & & & & & & & & & + & & & & & & & & & + & & & & & & & & & & & & & & \\
\hline 242 & & & & & & & & & & & & & & & & & & & & & & & & & & & & & + & & & & \\
\hline
\end{tabular}




\begin{tabular}{|c|c|c|c|c|c|c|c|c|c|c|c|c|c|c|c|c|c|c|c|c|c|c|c|c|c|c|c|c|c|c|c|c|c|c|}
\hline \multicolumn{35}{|c|}{ Table 9, 1987-4 } \\
\hline & & & & & & D & & & & & & & & & & & & $\mathrm{E}$ & $E$ & & & & & & & & & & & $\mathrm{~F}$ & & & & \\
\hline & 4 & & 6 & & 8 & & 10 & 11 & 12 & 13 & $\mid 12$ & & & 3 & 4 & 5 & 6 & & 8 & 9 & & 11 & 12 & 13 & \begin{tabular}{l|l}
3 & 1 \\
\end{tabular} & 2 & 3 & 4 & & 6 & 7 & 8 & & 10 \\
\hline 82 & & + & + & + & + & + & & & & & & & & & & + & + & & & + & & & & & & & & + & + & + & & & & \\
\hline 85 & & & & & & & & & & & & & & & & & & & & & & & & & & & & & & & & + & & \\
\hline 86 & & & & & & + & & + & + & & + & & & & & + & + & + & & & + & & + & & & & & & & & + & + & + & \\
\hline 87 & & & & & & & & + & & + & + & & & & & & & & + & & & + & + & + & & & & & & & + & + & + & + \\
\hline 88 & & & & & & & & + & & & + & & & & & & & & + & & & & + & + & & & & & & + & + & & & + \\
\hline 89 & & & & & & & & & & & + & & & & & & & & & & & + & + & + & & & & & & & & & & + \\
\hline 91 & & & & & & & & & & + & & & & & & & & & & & & & & + & & & & & & & & & & \\
\hline 94 & & & & + & & & + & + & + & + & & & & & & & & + & & + & & & + & + & & & & & & & 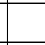 & + & + & + \\
\hline 99 & & & & & & & & & & & & & & & & & & & & & & & & + & & & & & & & & & & + \\
\hline 103 & & & & & & & & & & & & & & & & & & & & & & + & + & + & & & & & & & + & & & + \\
\hline 115 & & & & & & & & + & & & & & & & & & & & & & & + & + & & & & & & & & & & & + \\
\hline 116 & & & & & & & & & & & & & & & & & + & & & & + & & & & & & & & & & & & & \\
\hline 117 & & & & & & & & & & & & & & & & & + & & & & & & & & & & & & & & & & & \\
\hline 119 & & & & & & & & & & & + & & & & & 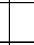 & 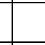 & . & & 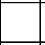 & & + & + & & & & & & & & 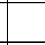 & . & + & \\
\hline 122 & & & & & & & & & & & & & & & & & & + & & + & & & & & & & & & & + & + & + & + & + \\
\hline 123 & & + & + & + & + & + & + & + & & 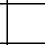 & & & & & & + & + & + & + & + & + & + & + & + & & & & & & + & + & + & + & + \\
\hline 124 & + & + & + & + & + & & & + & & 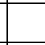 & & & & & & + & + & + & + & & & & & & & + & & + & + & & + & & & + \\
\hline 125 & & & & & & & & & & & & & & & & & & & & & & + & + & & & & & & & & & & & \\
\hline 130 & & & & & & & & & & & & & & & & & & & & & & & + & + & & & & & & + & & & & + \\
\hline 132 & & + & & & & + & & + & & 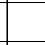 & 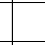 & & & & & + & + & + & & + & + & & & + & & & & & + & + & + & + & + & \\
\hline 141 & & & & & & & & + & & + & + & & & & & & & & & & & + & + & + & & & & 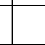 & & + & & 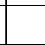 & 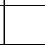 & \\
\hline 142 & + & + & + & + & + & + & + & + & + & + & & & & & & & + & + & + & + & + & + & + & & & & & + & + & + & + & + & + & + \\
\hline 143 & & & & & & & & + & + & + & + & & & & & 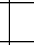 & & & & & & + & + & + & & & & & & + & & 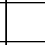 & + & + \\
\hline 144 & & & & & & & & & & & & & & & & & & . & & & & & + & & & & & & & & & 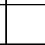 & & \\
\hline 145 & & & & & + & + & & + & + & + & & & & & & & & + & + & & + & + & + & + & & & & & & 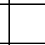 & 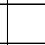 & + & + & + \\
\hline 146 & & & & & & & & & & & & & & & & + & & & & & & & & & & & & & & & & & & \\
\hline 148 & & & + & & & & & & & & & & & & & & & & & & & & & & & & & & & & & & & + \\
\hline 156 & & & & & & & & & & & & & & & & & & & & & & & & & & & & & & & & & & \\
\hline 158 & & + & + & & & + & & & & & & & & & & & & & & & & & & & & & & & & & & & & \\
\hline 161 & & & & & & & & & & & & & & & & & & & & & & & & + & & & & & & & & + & & \\
\hline 168 & & & & & & & & & & & & & & & & & & & & & & & & + & & & & 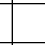 & & & & 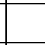 & & \\
\hline 176 & & & & & & & & & & & & & & & & & & 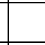 & & & + & & & & & & & & & 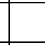 & & L & & \\
\hline 198 & + & & & & & & & & & & & + & & $t$ & -1 & & . & 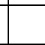 & 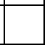 & & & & 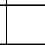 & & & + & + & + & & 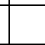 & 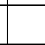 & 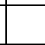 & & \\
\hline 199 & + & + & + & + & + & + & + & + & + & & & + & & $7-$ & $7-$ & + & + & + & + & & + & & & & + & $+1+$ & + & + & & + & + & + & + & + \\
\hline 200 & + & & + & + & + & + & + & + & & + & + & & & & & & 5 & + & + & + & + & + & + & + & & + & & + & & + & + & + & + & + \\
\hline 202 & & & & & & & & & & & & & & & & & & & & & & & & & & & & & & & & & & \\
\hline 203 & & & & & & & & & & & & & & & & & & & & & & & & & & & & & & & 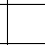 & 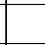 & + & \\
\hline 205 & & & & & & & & & & & & & & & & & & & & & & & & & & & & & & & & + & & \\
\hline 207 & & & + & + & + & + & + & + & + & + & & & & & & & + & + & & + & + & + & + & + & & & & & + & & + & + & & + \\
\hline 208 & & & & & & & & & & & + & & & & & & & & & & & & & & & & & & & & & & & \\
\hline 209 & & & & & & & & & & & + & & & & & & & & & & & + & & & & & & & & & & & & \\
\hline 210 & & & & & & & & & + & & & & & & & & & & & & & & & & & & & & & & & & & \\
\hline 211 & + & & + & + & + & + & + & + & & & & + & & $+t$ & $-t$ & + & & + & & 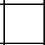 & & & 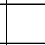 & . & + & +1 & + & + & + & & 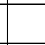 & 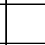 & 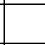 & + \\
\hline 212 & & & & & & & & + & + & + & + & & & & & & + & + & & & + & & + & + & & & & & + & + & 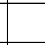 & + & & \\
\hline 213 & & & & & + & & + & + & + & + & + & & & & & & & + & + & + & & & + & + & & & & & & + & + & + & + & + \\
\hline 214 & + & + & + & + & + & + & + & + & + & + & + & + & & -1 & -1 & + & + & + & + & + & + & & & + & $-1+$ & +1 & + & + & + & + & + & + & + & + \\
\hline 216 & & & & & & & & + & & & & & & & & & & & & & & & & + & & & & & & & & & & + \\
\hline 220 & & + & & & + & + & + & + & & + & & & & & & & & + & + & + & + & + & + & + & & & & & & + & + & + & + & + \\
\hline 227 & & & & & & & & & & & & & & & & & & & & & & & & & & & & & & & & & & \\
\hline 229 & + & + & + & + & + & + & + & + & & & & + & & -1 & -1 & + & + & + & & + & + & + & & & + & $+t$ & + & + & + & + & & & & + \\
\hline 230 & & + & & & & & & & & & & & & & & & & & & & & & & & & & & & & & & & & \\
\hline 232 & + & + & & & & & & + & & & & & & & & & & & & & & & & & + & $+t$ & + & + & + & + & + & & + & + \\
\hline 233 & & & + & & & & & + & + & + & & & & & & & + & + & + & & + & + & & & & & & & & & + & & + & + \\
\hline 234 & & & & & & & & + & & & + & & & & & & & & & & & & & + & & & & & & & & & & + \\
\hline 235 & & & & & & & & & + & & + & & & & & & & & & & & & + & + & & & & & + & & + & & + & \\
\hline 239 & & & & & & & & & & & & & & & & & & & & & & & & & & & & & & & & & & \\
\hline 240 & & & & & & & & & & & & & & & & & & & & & & & & & & & & & & & & & & \\
\hline 242 & & & & & & & & & & & & & & & & & & & & & & & & & & & & & & & & & & \\
\hline
\end{tabular}




\begin{tabular}{|c|c|c|c|c|c|c|c|c|c|c|c|c|c|c|c|c|c|c|c|c|c|c|c|c|c|c|c|c|c|c|c|c|c|c|c|}
\hline \multicolumn{36}{|c|}{ Table 9, 1988-1 } \\
\hline & \multicolumn{8}{|c|}{$\mathrm{A}$} & & & & & B & & & & & & & & & & & & C & & & & & & & & & $\mathrm{D}$ & \\
\hline & 1 & 2 & 3 & 4 & & 6 & 7 & 8 & 1 & 2 & 3 & 4 & 56 & \begin{tabular}{l|l}
6 & 7 \\
\end{tabular} & 8 & 9 & 10 & 01 & 2 & & $3 \mid 2$ & 45 & 56 & 7 & 8 & 9 & & 11 & 11 & \begin{tabular}{l|l}
21 & 1 \\
$r$
\end{tabular} & \begin{tabular}{l|l}
3 & 1 \\
\end{tabular} & & & & 3 \\
\hline 2 & & & & & + & & & + & & & & & & & & & & & & & & & & & & & & & $+t$ & & & & & & \\
\hline 3 & & & & & & & & & & & & & & & & & & & & & & & & & & & & & & & & & & & \\
\hline 4 & & + & + & + & + & + & + & + & + & + & + & + & +- & $+-1-$ & +1 & + & + & & + & -1 & +-1 & +-1 & ++ & $-1+$ & + & + & + & 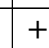 & + & - & + & & & & \\
\hline 6 & & & & & & & & & & & & & & & & + & & & & & & & & & & & & & & & + & & & & \\
\hline 7 & & & & + & + & & & + & & & & + & +- & + & + & + & + & & & & & - & + & + & + & + & + & + & + & $-1-1$ & $t$ & & & & \\
\hline 8 & & & & & & & & & & & & & & & & & & & & & & & & & 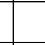 & . & & + & & & & & & & \\
\hline 10 & & & & & & & & & & & & & 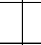 & & & + & & & & 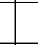 & 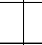 & L & t & & & & & + & & & & & & & \\
\hline 11 & & & & & & & + & + & & 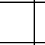 & & & & -1 & 5 & + & & & & & & & & & + & & + & & & & & & & & \\
\hline 12 & & + & + & & & + & & + & + & + & + & & +- & + & + & + & & + & + & & - & +-1 & ++ & $t+$ & + & + & & & & & & & & & \\
\hline 13 & + & + & + & + & + & + & + & + & + & + & + & + & +- & $+1-1$ & $+t$ & + & & + & + & & +- & $+1-1$ & ++ & $-1+$ & + & & + & + & + & - & + & & . & & + \\
\hline 14 & + & & + & + & + & + & & 7 & + & + & + & + & + & & $t$ & + & & + & + & & 5 & 7 & + & + & + & + & + & & -1 & -1 & + & & + & & \\
\hline 15 & & + & + & + & + & + & + & + & & + & + & + & + & + & $1+$ & + & + & $t$ & +1 & -1 & + & +1 & ++ & $-1+$ & + & + & + & + & $t$ & & & & + & & + \\
\hline 16 & & & & & & & & + & & & & & & & I & 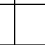 & + & & & & & & & 1 & 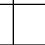 & + & + & + & $t$ & - & + & & & & \\
\hline 17 & & + & + & + & + & + & + & + & & + & + & - & +1 & +7 & $1+$ & + & & & + & & +1 & +1 & ++ & $-1+$ & + & + & + & + & + & - & $t$ & & - & + & \\
\hline 20 & + & + & + & + & & & & + & + & + & + & & & & + & & & & + & & + & 7 & + & & + & & & & & & & & & & \\
\hline 22 & & & + & & & & & & & & & & & & & & & & & & + & -1 & + & & & & 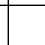 & & & & & & & & \\
\hline 25 & & & & & & & & & & & & & & & & & & & & & & & & & & & & + & & & & & & & \\
\hline 26 & & & & & & & & & & & & & & & & + & & & & & & & & & & & & & & - & + & & + & & \\
\hline 27 & & & & & & & & & & & & & & & & & & & & & & & & & & 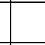 & & & + & -1 & & & & & \\
\hline 28 & & & & & + & & & + & & & + & + & + & & & & + & & & 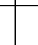 & & & & & & + & + & & $t$ & -1 & + & & & & \\
\hline 29 & & & & & & & & & & & & & & & & & & & & & & & & & & & & & & & & & & & \\
\hline 31 & + & & & & & & & & & & & & & & & & & & & & & & & & & + & & & & & & & & & \\
\hline 33 & & & + & & & & & & & + & & & & & & & & & + & - & + & & & & & & & & & & & & & & \\
\hline 35 & & & & & & & & & & & + & & & & & & & & & & & & & & & & & & & & & & & & \\
\hline 38 & & & & & & & & & & & & & & & & & & & & & & & & & & & & & & & & & & & \\
\hline 39 & & & & & & & & & & & & & & & & & & & & - & + & & & & & & & & & & & & & & \\
\hline 41 & + & & & & & & & & & & & & & & & & & & & & & & & & & & & & & & & & & & \\
\hline 42 & & & & & & & & & + & & & & & & & & & & & & & & & & & & & & & & & & & & \\
\hline 43 & & & & & & & & + & & + & + & & & 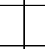 & . & & & & & & & & . & & & & + & + & $-t$ & & & & & & \\
\hline 44 & + & + & + & + & + & + & + & + & + & + & + & + & + & +-1 & ++ & + & & + & +1 & & + & +- & ++ & $-1+$ & + & + & + & & & & & & + & & + \\
\hline 47 & & & & & & & & & & & & & & & & & & & & & & & & & & & & & $t$ & & & & & & \\
\hline 48 & & & & & & & & + & & & & & & & + & + & + & & & & & & & & & + & + & + & + & $-1-$ & + & & & & \\
\hline 49 & & & & & & & & & & & & & & & & & & & & & & & & & & & & & & & 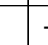 & & & & \\
\hline 53 & & & + & + & + & + & + & + & & + & & + & +- & $t$ & & & & & & & & & & & & + & + & & + & -1 & + & & & & \\
\hline 58 & & & & & & + & & & . & 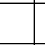 & & + & & & 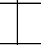 & & & & . & $t$ & + & & & & & & & & & & & & & & \\
\hline 60 & + & + & + & + & + & + & + & & + & + & + & + & +1 & $t$ & & & & + & $+1+$ & & +- & & & & & & & & & & & & & & \\
\hline 61 & & & & & & & & & & & & & & & & & & & & & & & & & & & & & $t$ & & & & & & \\
\hline 63 & & & & + & + & + & + & & & + & + & + & + & +- & ++ & & & $t$ & +1 & & + & + & + & & & + & & & & & & & & & \\
\hline 64 & & & & & + & + & + & + & & & & & & $t$ & $-1+$ & & & & & 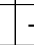 & + & +1 & $+t$ & $-1+$ & & + & & & & & & & & + & \\
\hline 70 & + & & & & & & & & & & & & & & & & & & + & & & & & & & & & & & & & & & & \\
\hline 71 & + & + & & + & + & + & + & & + & + & + & + & + & + & & & & & 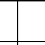 & + & + & & & & & & & & & & & & & & \\
\hline 72 & + & + & + & & & & & & + & & & & & & & & & & & & & & & & & & & & & & & & & & \\
\hline 73 & + & + & + & + & + & & + & & + & + & + & + & + & + & ++ & +1 & & + & +1 & & + & +7 & ++ & $+1+$ & & 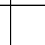 & 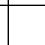 & & & & & & & & + \\
\hline 77 & + & + & + & + & + & + & + & + & + & + & + & + & + & + & $1+$ & +1 & & + & + & & + & +1 & ++ & $-1+$ & + & + & + & & & -1 & + & & + & & + \\
\hline 78 & & & & + & + & + & + & + & & & & & +1 & + & $1+$ & +1 & & + & & - & +- & +1 & $+t$ & $-1+$ & + & + & + & & & & & & + & + & + \\
\hline 79 & & & & & + & & + & & & & & & & -1 & & & & & & & + & +1 & ++ & & & & 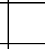 & & & & & & + & & \\
\hline 80 & & & & & & & & & & & & & & & & & & & & & & & & & & & + & & & & & & & & \\
\hline 81 & + & + & + & + & + & + & + & + & + & & + & + & + & +-1 & $-1+$ & +1 & & + & +1 & & + & +- & +1 & $+1+$ & + & + & + & & & & & & + & & + \\
\hline 82 & + & + & + & + & + & + & & & + & + & + & + & + & +1 & & & & & & & & & & & & & & & & & & & & & \\
\hline 84 & + & & & & & & & & & & & & & & & & & & & & & & & & & & & & & & & & & & \\
\hline 86 & & & & & + & & & + & & & + & & & & & + & & & & & & & & & & & & + & $-t$ & & & & & & \\
\hline 87 & & & & + & & & & & & + & + & + & & & & & & & + & & + & & & & & & & & & $t$ & & & & & \\
\hline 88 & & & & & & & & & & & & & & & & & & & & & & & & & & & & & & & 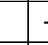 & & & & \\
\hline 89 & & & & & & & & & & & & + & & & & & & & & & & & & & & & & & & & & & & & \\
\hline 91 & & & & & & & & & & & & & & & & & + & & & & & & & & & & & & & & & & & & \\
\hline
\end{tabular}




\begin{tabular}{|c|c|c|c|c|c|c|c|c|c|c|c|c|c|c|c|c|c|c|c|c|c|c|c|c|c|c|c|c|c|c|c|c|c|c|}
\hline \multicolumn{35}{|c|}{ Table $9,1988-2$} \\
\hline & \multicolumn{11}{|c|}{$\mathrm{D}$} & \multicolumn{13}{|c|}{$\mathrm{E}$} & & & & & 1 & & & & & \\
\hline & 4 & 5 & 6 & 7 & 8 & 9 & 10 & & 12 & 13 & & 1 & 2 & 3 & 4 & 5 & 6 & 7 & 8 & 9 & 10 & & & 13 & 1 & 2 & 3 & 4 & 5 & \begin{tabular}{|l|}
6 \\
\end{tabular} & & 8 & & 10 \\
\hline 2 & & & & & & & & & & & & & & & & & + & + & & & & & & & & & & & + & + & + & & & \\
\hline 3 & & & & & & & & & & & + & & & & & & & & & & & & & + & & & & & & & & & & \\
\hline 4 & + & + & + & + & + & + & + & + & + & + & + & & & & + & + & + & + & + & + & + & + & + & + & & + & + & + & + & + & + & + & + & + \\
\hline 6 & & & & & & & & + & + & + & + & & & & & & & & & & & & & & & & & & & & & & & \\
\hline 7 & & +- & + & + & + & + & + & + & + & + & + & & & & & + & + & + & + & + & + & + & + & + & & & + & + & + & + & + & + & + & + \\
\hline 8 & & & & & & & & & & + & + & & & & & & & & & & & & & & & & & & & & & & & \\
\hline 10 & & & & & + & & & & & & + & & & & & & & & & & & & & & & & & & & & & & & \\
\hline 11 & & + & & + & & + & & & & & & & & & & & + & & & + & & & & & & & & & & & & & & \\
\hline 12 & + & + & + & + & + & + & + & & & & & & & & + & + & + & + & + & + & & & & & & & + & & & & & + & & \\
\hline 13 & + & + & + & + & + & + & + & + & + & + & + & & & & + & + & + & + & + & + & + & + & + & + & & & + & + & + & + & + & + & + & + \\
\hline 14 & + & - & + & + & + & + & + & + & + & & + & & & & & + & + & 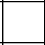 & 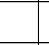 & 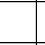 & & + & + & + & & & + & & & + & + & & & + \\
\hline 15 & + & + & + & + & + & + & + & + & + & + & & & & & + & + & + & + & + & + & + & + & & & + & + & + & & + & + & + & + & + & + \\
\hline 16 & & - & + & & + & + & + & + & & + & + & & & & . & & + & + & & & + & + & + & + & & & + & & & + & & + & & + \\
\hline 17 & + & + & + & + & + & + & + & + & + & + & + & & & & + & + & + & & + & + & + & + & + & + & & + & + & + & + & + & + & + & + & \\
\hline 20 & & + & + & + & + & + & + & & & & + & & & & & + & + & & + & & & & & & + & & + & & + & & & & & \\
\hline 22 & & & & & & & & & & & & & & & & & & & & & & & & & & & & 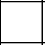 & & & 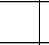 & & & \\
\hline 25 & & & & & & & & & & & & & & & & & & & & & & & & & & & & & & & 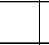 & & & \\
\hline 26 & & & & & & & & & & + & + & + & & & & & & & 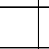 & & & & + & + & & & & & & & 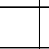 & & & + \\
\hline 27 & & & & & & + & & & + & + & + & & & & & & & & 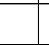 & & & + & & + & & & 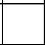 & & & & & & & \\
\hline 28 & & + & + & + & & & + & + & + & & + & & & & & & & & + & + & + & + & + & & & & + & + & & & + & & & + \\
\hline 29 & & & & & & & & & & & + & & & & & & & & & & & & & & & & + & & + & & 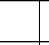 & & & \\
\hline 31 & & & & & & & & & & & & & & & & & & & & & & & & & & & & & & & & & & \\
\hline 33 & & & & & & & & & & & & & & & & & & + & & & & & & & & & & & & & & & & \\
\hline 35 & & & & & & & & & & & & & & & & & & & & & & & & & & & & & & & & & & \\
\hline 38 & & & & & & & & & & & + & & & & & & & - & 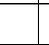 & & & & & & & & . & & & & & & & \\
\hline 39 & & & & & & & + & & & + & & & & & & & & + & + & & & & & + & & & 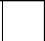 & . & & & & & & \\
\hline 41 & & & & & & & & & & & & & & & & & & & & & & & & + & & & & & + & & & & & \\
\hline 42 & & & & & & & & & & & & & & & & & & & & . & & & & & & & & & & & & & & \\
\hline 43 & & & & + & + & + & + & & + & & & & & & & + & + & & 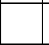 & + & + & & & & & & . & & & & & & & \\
\hline 44 & + & + & + & + & + & + & & & & & & + & + & + & + & + & + & + & + & & & & & & + & + & + & & & & . & + & & + \\
\hline 47 & & & & & & & & & & & & & & & & 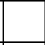 & 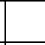 & & & 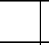 & & & & & & & 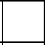 & & & & & 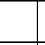 & & \\
\hline 48 & & & & & & + & + & + & + & + & + & & & & & + & + & + & + & + & + & + & + & + & & & & + & & + & + & & + & + \\
\hline 49 & & & & & & & & & & & + & & & & & & & & & 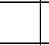 & & & & + & & & 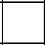 & & & & $\square$ & 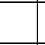 & & + \\
\hline 53 & & & & + & + & + & & + & + & + & + & & & & & + & + & + & + & & + & + & & + & & & + & + & + & + & + & + & & + \\
\hline 58 & & & & & & & & & & & + & & & & & + & & & + & & & & & + & & & 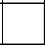 & 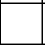 & & 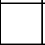 & 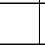 & & & \\
\hline 60 & & & & & & & & & & & & & & & & + & + & + & & & & 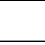 & & & + & & + & + & + & + & + & + & & \\
\hline 61 & & & & & & & & & + & + & + & & & & & 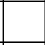 & 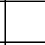 & 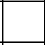 & & & & + & + & + & & & 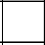 & + & + & + & & + & + & \\
\hline 63 & & & & & + & & & & & & & & & & & + & + & + & & & & & & & & & & & & & & + & & \\
\hline 64 & & + & + & + & & & & & & & & & & & & + & & & & & & & & & + & & & & & & & & & \\
\hline 70 & & & & & & & & & & & & & & & & & & & & & & & & & & & & & & & & & & \\
\hline 71 & & & & & + & & & & & & & & & & & + & & & + & & & & & & 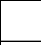 & & + & + & + & + & + & + & & + \\
\hline 72 & & & & & & & & & & & & & & & & & & & & & & & & & & & & & & & & & & \\
\hline 73 & + & + & + & + & & & & & & & & + & + & + & + & + & + & + & & & & & & & + & + & + & & & & & & & \\
\hline 77 & + & + & + & + & + & + & + & + & + & & & & + & + & + & + & + & + & + & + & + & & + & & + & + & + & + & + & + & & & & \\
\hline 78 & + & + & + & + & + & + & & & & & & + & + & + & + & + & + & + & & + & & & & & + & + & + & & & & & & + & \\
\hline 79 & & & & & & & & & & & & & + & & + & & & & & & & & & & + & & + & & & & & & & \\
\hline 80 & & & & & & & & & & & & & & & & & & & & & & & & & & & & & & & & & & \\
\hline 81 & + & +- & + & + & + & + & + & & & & & + & & + & + & + & + & + & & & & & & & + & + & + & & & & & & & \\
\hline 82 & & & & + & + & & & & & & & & & & & + & & + & & & & & & & & & & & & & & & & \\
\hline 84 & & & & & & & & & & & & & & & & & & & & & & & & & & & & & & & & & & \\
\hline 86 & & + & & + & + & & & & + & & & & & & & & + & + & & & & & & & & & + & & + & + & + & & + & \\
\hline 87 & & + & & & & & & & + & & + & & & & & + & & & + & & + & & + & & & & + & & & & + & & & + \\
\hline 88 & & & & & & & & & + & & + & & & & & & & & + & & & & & & & & + & + & & + & + & & & + \\
\hline 89 & & & & & + & & & & & + & + & & & & & & & & & & + & & + & + & & & & & & + & & & & + \\
\hline & & & & & & & & & & & + & & & & & & & & & & & & & & & & & & & & & & & \\
\hline
\end{tabular}




\begin{tabular}{|c|c|c|c|c|c|c|c|c|c|c|c|c|c|c|c|c|c|c|c|c|c|c|c|c|c|c|c|c|c|c|c|c|c|c|}
\hline \multicolumn{35}{|c|}{ Table 9, 1988-3 } \\
\hline & \multicolumn{8}{|c|}{$\mathrm{A}$} & \multicolumn{9}{|c|}{$\mathrm{B}$} & \multicolumn{14}{|c|}{$\mathrm{C}$} & \multicolumn{3}{|c|}{ D } \\
\hline & 1 & 2 & 3 & 4 & & 6 & $7 \mid \varepsilon$ & & 1 & 2 & 3 & 45 & & $\begin{array}{ll}6 & 7 \\
\end{array}$ & 8 & 9 & 10 & 01 & 2 & 3 & 4 & 5 & 6 & 7 & 8 & 9 & & 11 & 12 & 13 & 14 & 1 & \begin{tabular}{l|l}
2 &
\end{tabular} & 3 \\
\hline 92 & & & & & & & & & & & & & & & & & & & & & & & & & & & & & & & + & & & \\
\hline 93 & & & & & & & & & & & & & & & & & + & & & & & & & & & & & + & & & & & & \\
\hline 94 & & & & & & & & & & & + & & + & + & & + & & & & & & & & & & & & & + & + & & & & \\
\hline 115 & & & & & & & & & & & & & & & & & & & & & & & & & & & & + & & & & & & \\
\hline 116 & + & & & & & & & & & & & + & & & & & & & & + & & & & & & & & + & & & & & & \\
\hline 119 & & & & & & & & & & & & & & & & & & & & & & & & & & & & & & & & & & \\
\hline 122 & & & & & & + & & & & & & & & & & & & & & & & & & & & & + & & & & & & & \\
\hline 123 & & + & + & + & + & + & + & + & & + & + & + & + & & & + & & & + & + & + & + & & & & & + & & & & & & & \\
\hline 124 & + & + & + & + & + & + & & + & + & + & + & + & $+t+$ & $+1-1$ & $t+$ & + & & + & + & + & + & + & + & + & + & + & + & & & & & + & + & + \\
\hline 125 & & & & & & & & & & & & & & & & & & & & & & & & & & & & & & & & & & \\
\hline 126 & & & & & & & & & & & & & & & & & & & & & & & & & & & & & & & & & & \\
\hline 130 & & & & & & & & & & & & & & & & & & & & & . & & & & & & + & + & & & & & & \\
\hline 132 & & & & & & + & + & + & & & & & & -1 & + & + & + & & & & 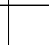 & & & & 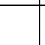 & + & + & + & & & & & & \\
\hline 141 & & & & & & + & & & & & & & & & & + & & & & & & & & & 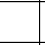 & & + & + & + & + & + & & & \\
\hline 142 & & & & & & & + & + & & & + & & & -1 & 5 & + & + & & + & & & & & & + & + & + & + & + & + & + & & & + \\
\hline 143 & & & & & & & & & & & & & & & & + & & & & & & & & & & & & & & & & & & \\
\hline 144 & & & & & & & & & & & & & & & & & & & & & & & & & & & & + & & & & & & \\
\hline 145 & & & + & + & + & + & + & & & & + & + & + & & & & & & + & + & & + & & & & & & & & + & & & & \\
\hline 146 & & & & & & & & & & & & & & & & & & & & & & & & & & & & & & + & & & & \\
\hline 148 & + & & + & & & & & & & & & & & & & & & & & & & & & & & & & & & & & & & \\
\hline 158 & & & & & + & + & & & & & &. & + & & & & & & + & + & + & & & & & & & & & & & & & \\
\hline 161 & & & & & & & & & & & & & & & & & & & & & & & & & & & & & & & & & & \\
\hline 171 & & & & & & & & & & & & & & & & & & & & & & & & & & & & & & & & & & \\
\hline 198 & & & & & & + & + & & & & & & +1 & + & ++ & ++ & & & 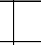 & & + & + & + & + & + & & & 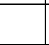 & + & & & & & \\
\hline 199 & & & + & + & + & + & + & + & + & & & & + & -1 & +1 & +1 & & & + & + & + & + & + & + & + & + & + & & & & & + & + & + \\
\hline 200 & & & & & & + & & + & & & & + & & -1 & $+t$ & & & + & & $\sqrt{2}$ & + & & & + & + & + & + & + & + & + & + & & & + \\
\hline 201 & & & & & & & & & & & & & & & & & & & & & & & & & & & & & & & & & & \\
\hline 202 & & & & & & & & & & & & & & & & & & & & & & & & & & & & & & + & & & & \\
\hline 203 & & & & & & & & & & & & & & & & & & & & & & & & & & & & & + & & & & & \\
\hline 207 & & & + & & & & & & & + & & + & + & + & & & + & & + & + & & & & & 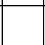 & 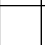 & + & + & + & + & + & & & \\
\hline 209 & & & & & & & & & & & & & & & & & & & & & & & & & & & & & & & & & & \\
\hline 210 & & & & & & & & & & & & & & & & + & & & & + & & & + & & & & & & & + & + & & & \\
\hline 211 & + & & + & + & & + & + & + & & & & & +1 & + & +1 & +1 & & + & + & + & + & + & + & + & + & + & + & & 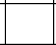 & & & + & + & + \\
\hline 212 & & & & & & & & & & & & & & & & & & & & & & & & + & & & & & + & + & + & & & \\
\hline 213 & & & & + & & & & + & & & & & & 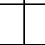 & & + & $-1+$ & & 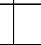 & 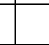 & 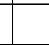 & & 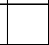 & 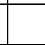 & 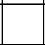 & & + & + & + & + & + & & & \\
\hline 214 & & & + & + & + & & +1 & + & & + & + & + & +1 & $+1-$ & $5+$ & $+t$ & $-1+$ & + & + & + & + & & & + & + & + & + & + & + & + & + & + & + & + \\
\hline 216 & & & & & & & & & & & + & + & & & & & & & & & & & 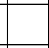 & & & & & & 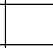 & & & & & \\
\hline 220 & & & & & & & & + & + & & + & + & - & + & & & & & & & & & & & & & & & + & + & & & & \\
\hline 227 & & & & & & & & & & & & & & & $t$ & 1 & & & & & & & + & + & + & & + & & & + & & & & \\
\hline 229 & & + & + & + & + & & + & & + & + & + & + & + & -1 & $1+$ & ++ & & + & + & + & + & + & + & + & + & + & + & + & + & & & + & + & + \\
\hline 230 & & & & & & & & & & & & & & & & & & & & & & & & & & & & & & & & & & \\
\hline 232 & & & + & & & & & & + & + & + & & & & $t$ & & + & & & & & & & + & + & + & + & + & + & + & & + & & \\
\hline 233 & & & & + & & & & & & & & + & & & & & & & & & & & & & & & & & & & & & & \\
\hline 234 & & & & & & & & & & & & & & & & & & & & & & & & & & & & & + & & & & & \\
\hline 235 & + & & + & & & & & & & & & & & & & & + & $-1+$ & ++ & & & & & & & + & & + & + & & & + & & \\
\hline
\end{tabular}




\begin{tabular}{|c|c|c|c|c|c|c|c|c|c|c|c|c|c|c|c|c|c|c|c|c|c|c|c|c|c|c|c|c|c|c|c|c|c|c|}
\hline \multicolumn{35}{|c|}{ Table 9, 1988-4 } \\
\hline & \multicolumn{11}{|c|}{ D } & \multicolumn{13}{|c|}{$E$} & \multicolumn{10}{|c|}{$\mathrm{F}$} \\
\hline & 4 & 5 & 6 & 7 & 8 & $9 \mid 1$ & & & 12 & 13 & & 1 & 2 & 3 & 4 & 5 & 6 & 7 & 8 & 9 & 10 & & & 13 & 1 & 2 & 3 & & 5 & 6 & & \begin{tabular}{l|l}
8 & 5
\end{tabular} & & 10 \\
\hline 92 & & & & & & & & & & & & & & & & & & & & & & & & & & & & & & & & & & \\
\hline 93 & & & & & & & & & & & & & & & & & & & & & & & & & & & & & & & & & & \\
\hline 94 & & & & & + & + & & + & + & + & + & & & & & + & & & & & & + & + & & & & & & + & + & & + & & + \\
\hline 115 & & & & & & & & + & + & & & & & & & & + & + & & & & + & + & & & & & & & & & & & \\
\hline 116 & & & & & & & + & + & + & & & & & & & & + & + & & & + & + & & & & & & + & + & + & + & & & \\
\hline 119 & & & & & & & + & & + & & & & & & & & & & & & & & & & & & & & & & & & + & \\
\hline 122 & & & & & & & & & & & & & & & & & & & & & & & & & & & & + & + & + & + & + & + & + \\
\hline 123 & & + & & & + & + & + & & + & & & & & & & + & + & + & + & & + & + & + & & & & + & + & + & + & + & + & + & + \\
\hline 124 & + & + & + & + & + & + & & & & & & & & + & + & + & + & + & + & & & + & & & + & & + & + & + & + & + & + & + & \\
\hline 125 & & & & & & + & & & & & & & & & & + & & & & & & & & & & & & & & & & & & \\
\hline 126 & & & & & & & & & & & & & & & & & & & & & & & & + & & & & & & & & & & \\
\hline 130 & & & & & & & & + & + & & & & & & & & & & & & & & & + & & & & & & & & & + & + \\
\hline 132 & & + & & & + & + & + & + & + & & & & & & 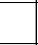 & & + & + & + & + & + & + & & & & & + & + & + & + & + & + & + & \\
\hline 141 & & & & & + & & & + & & + & + & & & & 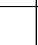 & & + & & + & & & & + & & & & & & + & & 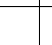 & & + & \\
\hline 142 & & + & + & + & + & + & + & + & + & + & & & & & + & + & + & + & + & + & + & + & + & + & & + & + & + & + & + & + & + & & + \\
\hline 143 & & & & & & & & & & + & & & & & & & & & & & & + & & + & & & & & & & & & & \\
\hline 144 & & & & & & & & & & & & & & & & & & & & & & & & & & & & & & & & & + & \\
\hline 145 & & + & & & + & + & + & & + & & & & & & & + & + & + & + & & & + & & & & & & & + & + & + & + & + & + \\
\hline 146 & & & & & & & + & + & & & & & & & & & & & & & & & & & & & & & & & & & & \\
\hline 148 & + & & & & & & + & & & & & & & & & & & & & & & + & & + & & & & & & & & & & \\
\hline 158 & & & & & & + & & & & & & & & & & & & & & & & & & & & & & & & & & & & \\
\hline 161 & & & & & & & & & & & & & & & & & & & & & & & & & & & & & + & & & & & \\
\hline 171 & & & & & & & & & & & & & & & & & & & 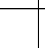 & & & & & & & & & & + & & & & & \\
\hline 198 & + & & & & & & & & & & & + & + & & + & & & & 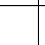 & & & & & & & + & & & & & & & & \\
\hline 199 & + & + & + & + & + & + & + & & & & & & & & + & + & + & + & + & + & + & & & & + & + & + & & & + & + & & & \\
\hline 200 & + & + & & + & & + & & + & + & + & + & & & & & + & & & + & + & + & + & + & + & & + & + & + & + & + & + & + & + & + \\
\hline 201 & & & & & & & & & & & & & & & & & & & & & & & & & & & & & + & + & & & & \\
\hline 202 & & & & & & & & & & & & & & & & & & & & & & & & & & & & & & & & & & \\
\hline 203 & & & & & & & & & & & & & & & & & & & & & & & & + & & & & & & & & & & \\
\hline 207 & & + & & & + & + & & & + & + & + & & & & & + & + & & 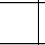 & + & + & + & + & + & & & + & + & + & + & + & & & + \\
\hline 209 & & & & & & & & & & & + & & & & & & & & & & & & & & & & & & & & & & & \\
\hline 210 & & & & & & & & & & + & + & & & & & & & & & & & & & + & & & & & & & & & & \\
\hline 211 & + & + & + & + & & + & + & & & & & + & + & + & + & + & + & + & + & & & & & & + & + & + & & & 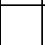 & & & & \\
\hline 212 & & & & & & & & & & + & + & & & & & & + & & & & & & & + & & & + & + & + & + & + & & & \\
\hline 213 & & & & & + & & + & & + & & + & & & & & + & + & & + & + & + & + & + & + & & & & + & + & + & + & + & & \\
\hline 214 & + & & + & + & + & + & + & + & + & + & + & & & & + & + & + & + & + & + & + & + & + & + & + & + & + & + & + & + & + & & & + \\
\hline 216 & & & & & & & & & + & & & & & & & & & & & & & + & & & & & & & & + & & & & \\
\hline 220 & & & & & & +1 & + & & & & + & & & & & & + & & + & + & & & & & & & + & & & & + & + & & \\
\hline 227 & & & & & & & + & & + & & & & & & & & & & & & & & & & & & & & & & & & & \\
\hline 229 & + & + & + & + & + & + & + & & & & & & + & + & + & + & + & + & + & + & + & + & & & + & + & + & + & & & + & & & + \\
\hline 230 & & & & & & & & + & & & + & & & & & & & & & + & & & & & & & & & & & & & & \\
\hline 232 & + & & + & + & + & + & + & & & & & & + & + & + & + & & + & + & & & & & & + & + & + & & & & 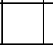 & & & \\
\hline 233 & & + & & & & & & & & + & + & & & & & & & + & & & + & + & & + & & & & + & & + & + & & & \\
\hline 234 & & & & & & & & & & & & & & & & & & & & & & & & & & & & & & & & & & \\
\hline 235 & & & & & & & & + & & & + & & & & & & + & + & + & & + & & & & & & & & + & & + & & & \\
\hline
\end{tabular}




\begin{tabular}{|c|c|c|c|c|c|c|c|c|c|c|c|c|c|c|c|c|c|c|c|c|c|c|c|c|c|c|c|c|c|c|c|c|c|c|c|}
\hline \multicolumn{36}{|c|}{ Table 9, 1989-1 } \\
\hline & \multicolumn{8}{|c|}{$\mathrm{A}$} & & & & & & & & & & & & & & & & & $\mathrm{C}$ & c & & & & & & & & 5 & \\
\hline & 1 & 2 & 3 & & & 6 & 7 & 8 & 1 & 2 & 3 & 4 & & 6 & 7 & 8 & 9 & 10 & 1 & 2 & 3 & 4 & 5 & 6 & & $8 \mathrm{~s}$ & \begin{tabular}{l|l}
9 & 1 \\
\end{tabular} & & & & \begin{tabular}{l|l}
13 & 1
\end{tabular} & 14 & & & 3 \\
\hline 1 & & & & & & & & & & & & & & & & & & & & + & & & & & & & & & & & & & & & \\
\hline 2 & & & + & & & & + & & + & & & & & & & & & & & + & & + & & & & & & & & & & & & & \\
\hline 3 & & & & & & & & & & & & & & & & & & & & & & & & & & & & & + & & & + & & & \\
\hline 4 & & + & + & + & + & + & + & + & & + & + & + & & + & + & + & + & + & + & + & + & + & + & &.+ & +-1 &.+ & + & + & + & + & & + & & \\
\hline 5 & & & & & & & & + & & & & & & & & + & & & & & & & & & & & & & & + & & & & & \\
\hline 6 & & & & & & & + & + & & & & & & & & & & + & & & & & & & & & & & + & + & + & + & & & \\
\hline 7 & & + & + & & & + & + & + & & & & + & + & & & & + & + & & & & + & & & + & &.+ & & + & + & + & + & & & \\
\hline 8 & & & & & & & & & & & & & & & & & & & & & & & & & & & & & & & & & & & \\
\hline 9 & & & & & & & + & & & & & & & & & & & & & & & & & & & & & & & & & & & & \\
\hline 10 & & & & & & & & & & & & & & & & & + & & & & & & & & & & & & & & & & & & \\
\hline 11 & & & & & & + & & + & & & & & + & & & & + & & & & & & & & & & & & + & + & + & & & & \\
\hline 12 & + & + & + & + & + & + & + & + & & + & & + & + & + & & & + & & + & + & + & + & + & & + & +- & & & & & & & & & \\
\hline 13 & & + & + & + & + & + & + & + & + & & + & + & + & + & & + & + & + & + & & + & & + & & + & -1 & + & & & + & & + & & & + \\
\hline 14 & & + & + & & & + & + & + & + & + & & & & & & + & + & + & & & + & + & & & + & + & & + & + & + & + & + & & & + \\
\hline 15 & + & + & + & + & + & + & + & + & & + & & + & + & + & + & + & + & + & + & + & + & + & + & + & + & + & + & $t$ & + & & & & + & & + \\
\hline 16 & & & & & & + & & + & & & & & & & & & & + & & + & & & 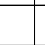 & & & 4 & & & & + & + & & & & \\
\hline 17 & + & + & + & + & + & + & + & + & & + & + & + & + & + & + & + & + & + & + & + & + & + & + & + & + & + & + & + & + & + & + & + & & & + \\
\hline 20 & + & + & + & & + & & & & + & + & + & + & + & & & & & & + & + & + & & + & & & + & & & & & & & & & + \\
\hline 21 & & & & & + & & + & & & & & & & & & & & & & + & & & & & & & & & & & & & & & \\
\hline 22 & + & & & & & & & & & & & & & & & & & & & & + & + & & & & & & & & & & & + & & \\
\hline 23 & & & & & & & 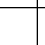 & & + & + & & + & & & & & & & & & + & + & & & & + & & & & & & & & & \\
\hline 26 & & & & & & & + & & & & & & & & & & & & & & & & & & & & & & & & & & & & \\
\hline 27 & & & & & & & & & & & & & & & & & & + & & & & & & & & & & & & + & + & & & & \\
\hline 28 & & & & & & & + & + & & & & + & & + & & & + & + & & & & & & & & - & +1. & + & + & + & & & & & \\
\hline 29 & & & & & & & & & & & & & & & & & & & & & & & & & & & & & & & & & & & \\
\hline 31 & & & & & & & + & & & & & & & & & & & & & & & & & & & & & & & & & & & & \\
\hline 33 & & & & + & & & & & & & & & & & & & + & & & & & & & & & & + & & & & & & & & \\
\hline 34 & & & & & & & & & & & & & & & & & & & & & & & & & & & & & & & & & & & \\
\hline 35 & & & & & & & & & & & & & & & & & & & & & & & & & & & & & & & & & & & \\
\hline 38 & & & & & & & & & & & & & & & & & & & & & & & & & & & & & & & & & & & \\
\hline 39 & & & & & & & + & + & & & & 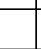 & & & & & & + & & & & & & & & & & & + & & + & & & & \\
\hline 41 & & & & & & & & + & & & & & & & & & & & & & & & & & & & & & & & & & & & \\
\hline 42 & & & & & & & & & & & & & & & & & & & & & & & & & & & & & & & & & & & \\
\hline 43 & & + & & & & & + & + & & & + & & & & & & & & & & & & & & & . & + & & & & & & & & \\
\hline 44 & + & + & + & + & + & + & + & + & + & + & + & + & + & + & + & + & + & + & + & + & + & + & + & +1 & + & + & + & + & + & & & & + & & + \\
\hline 46 & & & & & & & & + & & & & & & & & & & & & & & & & & & & & & & & & & & & \\
\hline 48 & & & & & & & + & + & & & & & & & & & + & + & & & & & & & & & + & + & & + & + & + & & & \\
\hline 49 & & & & & & & + & & & & & 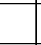 & & & & & & & & & & & & & & & & & + & & & + & & & \\
\hline 53 & & & & & + & + & + & + & + & & & + & & & & & & + & & & + & + & & & & & & & + & + & + & + & & & \\
\hline 55 & + & & & & & & & & & & & & & & & & & & & & & & & & & & & & & & & & & & \\
\hline 58 & & & & & & + & + & + & & & & & & & & & & & & & & & & & & & & & & & & & & & \\
\hline 60 & & + & + & + & + & & 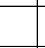 & & + & + & + & + & + & & & & & & + & + & + & + & & & & + & & & & & & & & & + \\
\hline 61 & & & & & & & + & & & & & & & & & & & & & & & & & & & & & & & & + & & & & \\
\hline 63 & & + & & & + & + & & & & + & + & + & + & + & & & & & & + & + & & & & & & & & & & & & & & \\
\hline 64 & & & & & + & + & & & & & & & + & + & + & + & & & & & + & + & + & + & + & + & & & & & & & + & & + \\
\hline 71 & & & + & + & + & + & & + & + & + & + & + & + & + & & & & & + & + & + & & & & & & & & & & & & + & & + \\
\hline 72 & & + & + & + & + & + & + & & + & + & + & + & + & + & + & & & & + & + & + & & + & & & & & & & & & & + & & + \\
\hline 73 & + & + & + & + & + & & & + & & + & + & + & & + & + & + & & & + & + & + & + & + & + & + & & & & & & & & + & & + \\
\hline 76 & + & & & & & & & & & & & & & & & & & & 4 & & & & & & & & & & & & & & & & \\
\hline 77 & + & +1 & + & + & + & + & + & + & & & + & + & + & + & & + & + & & + & + & + & + & + & + & + & + & + & + & & & & & + & & + \\
\hline 78 & & & + & + & + & + & & + & & & & & + & + & + & + & + & & + & + & + & + & + & + & + & + & + & + & & & & & + & & + \\
\hline 79 & & & & & + & + & + & & & & & & + & + & + & & & & + & & + & + & + & + & + & & & & & & & & + & & + \\
\hline 80 & & & & & & & & & & & & & & & & & & & & & & & & & & & & & & & & & & & \\
\hline 81 & & & & & & & & & & & & + & + & + & & & + & & & & + & & & + & + & & & & & & & & & & + \\
\hline 82 & & + & & & & & & & & & & & & & & & & & & & & & & & & & & & & & & & & & \\
\hline
\end{tabular}




\begin{tabular}{|c|c|c|c|c|c|c|c|c|c|c|c|c|c|c|c|c|c|c|c|c|c|c|c|c|c|c|c|c|c|c|c|c|}
\hline \multicolumn{33}{|c|}{ Table 9, 1989-2 } \\
\hline & \multicolumn{11}{|c|}{$\mathrm{D}$} & \multicolumn{12}{|c|}{$E$} & \multicolumn{9}{|c|}{$\mathrm{F}$} \\
\hline & 4 & 5 & 6 & 7 & 8 & 9 & & & 12 & 13 & & 1 & 2 & 3 & 4 & 5 & \begin{tabular}{l|l}
6 & -1 \\
6
\end{tabular} & $\begin{array}{l}7 \mid \varepsilon \\
\varepsilon\end{array}$ & $\begin{array}{l}30 \\
9\end{array}$ & \begin{tabular}{l|l}
910 \\
10
\end{tabular} & \begin{tabular}{l|l|l}
0 & 11 \\
\end{tabular} & \begin{tabular}{l|l}
112 \\
12
\end{tabular} & & 1 & 2 & \begin{tabular}{l|l}
3 & \\
\end{tabular} & \begin{tabular}{l|l}
45 & 5 \\
4
\end{tabular} & & & 8 & & 10 \\
\hline 1 & & & & & & & & & & & & & & & & & & & & & & & & & & & & & & & & \\
\hline 2 & & & & & & & & & & & & & & & & & & & & + & & + & + & & & & & $+t+$ & + & + & + & \\
\hline 3 & & & & & & & & & & + & & & & & & & & & & & & + & & & & & & & & & & \\
\hline 4 & + & + & + & & + & + & + & + & + & + & + & & & & + & + & + & $+1+$ & $+t$ & + & +1 & + & & + & + & + & + & + & + & + & + & + \\
\hline 5 & & + & + & + & & & + & + & & & & & & & + & + & + & 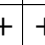 & t & & + & & & & & & & & & & & \\
\hline 6 & & & & & & & + & + & + & + & + & & & & & & & t & $t$ & & + & + & + & & & & & & & & + & + \\
\hline 7 & & + & + & + & & + & + & + & + & + & + & & & & & + & + & $+1+$ & $+t$ & + & + & + & + & & & & $+1-1$ & + & + & & + & + \\
\hline 8 & & & & & & & & & & & + & & & & & & & & & & + & + & + & & & & & & & & & \\
\hline 9 & & & & & & & & & & & & & & & & & & & & & & & & & & & & & & & & \\
\hline 10 & & & & & & & & + & & + & & & & & & & & & & + & & + & + & & & & & & & & & \\
\hline 11 & & & + & + & & + & + & & & & & & & & & & & $+t$ & ++ & 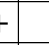 & . & & & & & + & -1 & + & 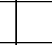 & & & + \\
\hline 12 & & + & + & + & + & + & + & & & 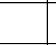 & & & & & + & + & + & $+1+$ & $+t+$ & + & & . & & + & + & + & + & + & + & + & + & \\
\hline 13 & & + & + & + & + & + & + & + & + & + & + & & & & & & + & + & $t$ & + & + & + & & t. & & & + & + & + & + & + & + \\
\hline 14 & & + & + & + & & & + & & & + & + & & & & & + & & +1 & $t$ & + & + & & & & + & + & + & + & + & & & + \\
\hline 15 & + & + & + & + & + & + & + & + & & & + & & + & + & + & + & + & $+t$ & $t$ & + & + & + & + & + & + & + & + & + & $-1+$ & + & + & + \\
\hline 16 & & & & + & & + & + & + & + & + & + & & & & & & & & + & + & +1 & + & + & & & & & + & + & + & + & + \\
\hline 17 & + & + & + & + & + & + & + & & + & + & & & & & + & + & + & +1 & +1 & +1 & +1 & + & + & + & + & + & + & +1 & + & + & + & + \\
\hline 20 & & + & + & + & & & + & & & & & & & & + & + & + & +1 & + & & & & 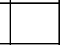 & & & & + & + & + & & + & \\
\hline 21 & & & + & & & & & & & & & & & & & & & & & & & & + & & & & & +1 & $-1+$ & + & + & \\
\hline 22 & + & + & & & & & & & & & & & & & & & & & & & & & & & & & & & 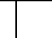 & & & \\
\hline 23 & & + & & & & & & & & & & & & & & + & & & & & & & & & & & & +7 & $1+$ & + & + & 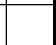 \\
\hline 26 & & & & & & & & & & & + & & & & & & & 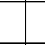 & 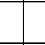 & . & & & + & + & & & & & & & & 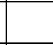 \\
\hline 27 & & & 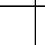 & & & & & + & + & + & + & & & & & & & & 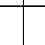 & & & & + & & & & & & 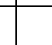 & 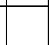 & & + \\
\hline 28 & & + & + & + & & + & + & + & + & & & & & & & & & 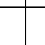 & $t$ & +1 & $+1+$ & + & & 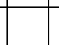 & & & -1 & +1 & . & + & & 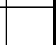 \\
\hline 29 & & & & & & & & & & & & & & & & & & & & & & + & & & & & & $t$ & & 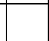 & & \\
\hline 31 & & & & & & & & & & & & & & & & & . & + & & & & & & & & & & & 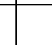 & & & \\
\hline 33 & & & & & & & & & & & & & & & & & & & & & & & & + & & & & + & $1+$ & + & & \\
\hline 34 & & & & & & & & & & & & & & & & & & & & + & & & & & & & & & & & + & \\
\hline 35 & & & & & & & & & & & & & & & & & & & & & & & & & & & & & & + & & \\
\hline 38 & & & & & & & & & & & & & & & & & & & & & & & + & & & & & & & & 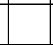 & \\
\hline 39 & & & & & & & & & & + & + & & & & & & & & & + & & + & + & & & & & & & & + & + \\
\hline 41 & & & & & & & & & & & & & & & & & & - & + & & & & + & & & & & & & & & \\
\hline 42 & & & & & & & & & & & & & & & & & & & & & + & & & & & & & & & & & \\
\hline 43 & & + & & & + & & & & & & & & & & + & + & + & +7 & $+1+$ & + & & & & 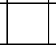 & & 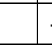 & + & + & & & & \\
\hline 44 & + & + & + & + & + & + & + & & & & & + & + & + & + & + & + & +1 & ++ & +1 & & & & + & + & + & +7 & +1 & & & & \\
\hline 46 & & & & & & & & & & & & & & & & & & & & & & & + & & & & & & & & & \\
\hline 48 & & & & & + & + & + & + & + & + & + & & & & & & & - & +1 & ++ & \begin{tabular}{l|l}
+ & +
\end{tabular} & -1 & + & & & & & & & + & + & + \\
\hline 49 & & & & & & & & & & + & + & & & & & & & 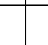 & 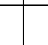 & & + & & + & & & & 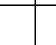 & 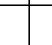 & 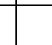 & & + & 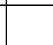 \\
\hline 53 & & + & & & + & + & & + & + & + & & & & & & & + & +7 & +7 & $+1+$ & \begin{tabular}{l|l}
+ & +
\end{tabular} & + & + & & & & +1 & +1 & + & + & + & + \\
\hline 55 & & & & & & & & & & & & & & & & & & & & & & & & & & & & & & & & \\
\hline 58 & & + & & & & & & & & & & & & & & + & & - & $t$ & & & & & & & & & & & & & \\
\hline 60 & & + & + & & & & & & & & & & & & + & + & + & +- & + & & & & & + & & + & + & +1 & $-1+$ & & 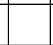 & \\
\hline 61 & & & & & & & & & + & & & & & & & & & & & & & & & & & & & & & + & + & + \\
\hline 63 & + & + & & + & + & & & & & & & & & & & + & + & & $t$ & + & & & & & & + & & + & & & & \\
\hline 64 & + & + & + & + & & & & & & & & & & & + & & & & & & & & & + & & & +7 & + & & & & \\
\hline 71 & & + & & 7 & + & & + & & & & & & & & & & & +1 & + & + & & & & . & & & + & - & $-1+$ & + & + & \\
\hline 72 & & + & & + & + & & & & & & & & & & & + & + & + & & & & & & + & & & +7 & + & & + & + & \\
\hline 73 & + & + & + & + & & & & & & & & + & + & + & + & + & + & & & & & & & + & + & + & + & & & & & \\
\hline 76 & & & & & & & & & & & & & & & & & & & & & & & & & & & & & & & & \\
\hline 77 & + & + & + & + & + & + & + & & & & & + & + & + & + & + & + & + & +-1 & + & & & & + & + & + & + & +1 & $-1+$ & & & \\
\hline 78 & + & + & + & + & + & + & + & & & & & + & + & + & + & + & + & +1 & $t$ & & & & & + & + & + & +7 & + & & & & \\
\hline 79 & + & & + & & & & & & & & & + & + & + & + & & & & & & & & & & & + & & & & & & \\
\hline 80 & & & & & & & & & & & & & & & & & & & & & & & & & + & & & & & & & \\
\hline 81 & +1 & + & + & + & & & + & & & & & + & + & + & + & + & & & & & & & & + & + & + & & & & & & \\
\hline 82 & & & & & & & & & & & & & & & & & & & & & & & & + & & & & & & & & \\
\hline
\end{tabular}




\begin{tabular}{|c|c|c|c|c|c|c|c|c|c|c|c|c|c|c|c|c|c|c|c|c|c|c|c|c|c|c|c|c|c|c|c|c|c|c|}
\hline \multicolumn{35}{|c|}{ Table 9, 1989-3 } \\
\hline & \multicolumn{8}{|c|}{$\mathrm{A}$} & \multicolumn{9}{|c|}{ B } & & & & & & & & C & & & & & & & & D & \\
\hline & 1 & 2 & & 4 & & $\begin{array}{ll}67 \\
\end{array}$ & & & 1 & 23 & 34 & 45 & 6 & 7 & 8 & 9 & 10 & & 2 & 3 & 4 & 5 & 6 & 7 & 8 & 9 & & 11 & & 13 & 14 & 1 & 2 & 3 \\
\hline 83 & & & & & & & & & & & & & & & & & & & & & & & & & & & & & & & & & & \\
\hline 86 & & & & + & & +-1 & + & + & & -1 & + & & & & & & + & & + & & & & & & & & & + & & & + & & & \\
\hline 87 & & & & & & & & + & & & & & + & & & & + & & & & & & & & & & & + & + & + & + & & & \\
\hline 88 & & & & & & & & & & & & & & & & & & & & & + & & & & & & & & + & + & & & & \\
\hline 89 & & & & & & - & & & & & & & & & & & & & & & & & & & & & & & & + & & & & \\
\hline 90 & & & & & & - & & & & & & & & & & & & & & & & & & & & & & & & & & & & \\
\hline 91 & & & & & & & & & & & & & & & & & + & & & & & & & & & & & & + & + & + & & & \\
\hline 94 & & & & & & - & + & + & & & & & + & & & & & & & & & & & & & & & + & + & + & + & & & \\
\hline 95 & & & & & & - & & & & & & & & & & & & & & & & & & & & & & & & & & & & \\
\hline 97 & & & & & & & & & & & & & & & & & & & & & & & & & & & & + & & & & & & \\
\hline 99 & & & & & & - & + & & & & & & & & & & + & & & & & & & & & & & + & + & + & & & & \\
\hline 100 & & & & & & & & & & & & & & & & & & & & & & & & & & & & & + & & & & & \\
\hline 103 & & & & & & & & & & & & & & & & & & & & & & & & & & & & & & + & & & & \\
\hline 105 & & & & & & & & & & & & & & & & & & & & & & & & & & & & & & & & & & \\
\hline 107 & & & & & & & & & & & & & & & & & & & & & & & & & & & & & & & & & & \\
\hline 115 & & & & & & - & & & & & & & & & & & & & & & & & & & & & & & & & & & & \\
\hline 116 & & & & & & - & + & + & & & & & & & & & & & & & & & & & & & & & + & + & & & & \\
\hline 119 & & & & & & & & & & & & & & & & & & & & & & & & & & & & & & & & & & \\
\hline 122 & & & & & & - & + & + & & & & & & & & & & & & & & & & D & & & & & 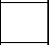 & & & & & \\
\hline 123 & & + & & + & + & - & + & + & & & & & & & & + & + & & + & + & & + & & & & & & + & + & & & & & \\
\hline 124 & & + & + & + & + & + & & + & + & $+7-1$ & +-1 & +5 & + & + & + & + & + & + & + & + & + & + & + & + & + & + & & + & 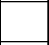 & 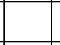 & & & + & + \\
\hline 125 & & & & & & & & + & & & & & & & & & & & & & & & & & & & & + & & & & & & \\
\hline 129 & & & & & & & & & & & & & & & & & & & & & & & & & & & & + & & & & & & \\
\hline 130 & & & & & & & & + & & & & & & & & & & & & & & & & & & & & & + & & & & & \\
\hline 131 & & & & & & & & & & & & & & & & & & & & & & & & & & & & & & & & & & \\
\hline 132 & & & & & + & ++ & & + & & & & & & & 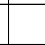 & & & & & & & & & & & + & + & + & & 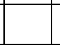 & & & & \\
\hline 140 & & & & & & & & & & & & & & & & & & & & & & & & & & & & + & & & & & & \\
\hline 141 & & & & & & - & + & + & & & & & & & & & + & & & & & & & & & 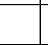 & & + & + & + & + & & & \\
\hline 142 & & & & & & - & + & + & & & & & & + & + & + & + & & & & & & & & & + & + & + & + & + & + & & & \\
\hline 143 & & & & & & & & + & & & & & & & & & & & & & & & & & & & & & 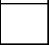 & + & & & & \\
\hline 144 & & & & & & - & + & & & & & & & & & & & & & & & & & & & & & & + & & + & & & \\
\hline 145 & & & & + & & +- & + & & & & +- & $-1+$ & & & & & & & & & & & & & & & & & + & & & & & \\
\hline 146 & & & & & & - & + & & & & & & & & & & & & & & & & & & & & & + & + & + & & & & \\
\hline 148 & & & & & & & & & & + & & & & & & & & & & & & & & & & & & & & & & & & \\
\hline 149 & & & & & & & & & & & & & & & & & & & & & & & & & & & & & . & + & & & & \\
\hline 154 & & & & & & & & & & & & & & & & & & & & & & & & & & & & & & & & & & \\
\hline 158 & & & + & & + & + & & & & & & $t$ & & & & & & & & + & + & & & & & & & & & & & & & \\
\hline 161 & & & & & & & & + & & & & & & & & & & & & & & & & & & & & & & & & & & \\
\hline 171 & & & & & & - & & & & & & & & & & & & & & & & & & & & & & & & & & & & \\
\hline 196 & & & & & & & & & & & & & & & & & & & & & & & & & & & & + & & & & & & \\
\hline 197 & & & & & & & & & & & & & & & & & & & & & & & & & & & & & + & & & & & \\
\hline 198 & & & & & + & + & & & & & & & & + & + & + & & & & + & + & + & + & + & & 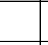 & & 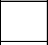 & $\square$ & . & & & 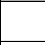 & + \\
\hline 199 & + & + & + & + & + & +- & & + & & + & + & + & + & + & + & + & + & + & & & + & + & + & + & + & + & + & . & + & & + & + & + & + \\
\hline 200 & & & & & + & $+1-$ & + & & & & & & & + & & & + & & & & & & + & + & + & + & + & + & + & + & + & & & \\
\hline 201 & & & & & & & & & & & & & & & & & & & & & & & & & & & & & & & & & & \\
\hline 202 & & & & & & & & & & & & & & & & & & & & & & & & & & & & & & & & & & \\
\hline 203 & & & & & & & & & & & & & & & & & & & & & & & & & & & & & & & & & & \\
\hline 205 & & & & & & & & + & & & & & & & & & & & & & & & & & & & & & & & & & & \\
\hline 207 & & + & & + & + & -1 & + & + & & + & +-1 & + & & & + & + & + & + & + & + & & & & & + & + & + & + & + & + & & & & \\
\hline 209 & & & & & & & & & & & & & & & & & & & & & & & & & & & & & & & & & & \\
\hline 210 & & & & & & & & & & & & & & & & & & & & & & & & & & & & & & & & & & \\
\hline 211 & + & + & + & + & + & +- & + & + & & & +-1 & ++ & + & + & + & + & & + & + & + & + & + & + & + & + & + & + & & & & & + & + & + \\
\hline 212 & & & & + & & -1 & & + & & & & & & & & & & + & & & & & & & & & & & + & + & + & & & \\
\hline 213 & & & & & & - & + & + & & & & & & & & + & + & & & + & & & & & & + & + & + & + & + & + & & & \\
\hline 214 & + & + & + & + & & & & + & + & & +1 & ++ & $-1+$ & + & + & + & + & + & + & + & + & + & + & + & + & + & + & + & + & + & + & + & + & + \\
\hline
\end{tabular}




\begin{tabular}{|c|c|c|c|c|c|c|c|c|c|c|c|c|c|c|c|c|c|c|c|c|c|c|c|c|c|c|c|c|c|c|c|c|c|c|c|}
\hline \multicolumn{36}{|c|}{ Table 9, 1989-4 } \\
\hline & \multicolumn{12}{|c|}{ D } & & & & & & & $\mathrm{E}$ & & & & & & & & & & & & & & & & \\
\hline & 4 & 5 & 6 & 7 & 8 & 9 & 10 & & & & & & & 2 & & & & \begin{tabular}{l|l}
6 & 7 \\
\end{tabular} & & 8 & \begin{tabular}{|l|l}
9 &
\end{tabular} & 10 & 11 & & 13 & 1 & 2 & 3 & & 5 & 6 & & 8 & & 10 \\
\hline 83 & & & & & & & & & & & & & & & & & & & & & & & & & & & & & & & & & + & & \\
\hline 86 & & + & + & & + & & + & & + & & - & & & & & & & & & + & & & + & & + & & & & & + & + & + & & + & + \\
\hline 87 & & + & & & & & & & & + & - & & & & & & & & & + & & & + & + & + & & & & & + & + & & + & + & + \\
\hline 88 & & & & & & & & & + & + & - & & & & & & & & & & & & & + & + & & & & + & + & + & + & + & + & + \\
\hline 89 & & & & & & & + & $\begin{array}{llll}+ & & l & -1\end{array}$ & & & - & & & & & & & & & + & & & + & + & + & & & & & & & & & + & + \\
\hline 90 & & & & & & & & & & & & & & & & & & & & & & & & & & & & & & & & & & & \\
\hline 91 & & & & & & & & & + & + & - & & & & & & & & & & & & & & & & & & & & & & & & \\
\hline 94 & & & & & + & & & + & + & & - & & & & & & & & & & & + & + & + & & & & & & & & & & & + \\
\hline 95 & & & & & & & & & & & & & & & & & & & & & & & & & & & & & & & & & & & \\
\hline 97 & & & & & & & & & & & & & & & & & & & & & & & & & & & & & & & & & & & \\
\hline 99 & & & & & & & & & + & + & & & & & & & & & & & & & & & + & & & & & & & & & & + \\
\hline 100 & & & & & & & & & & & & & & & & & & & & & & & & & + & & & & & & & & & & \\
\hline 103 & & & & & & & & + & & & & & & & & & & & & & & & & & & & & & & & & & & + & \\
\hline 105 & & & & & & & & & & & & & & & & & & & & & & & & & & & & & & & & & & & + \\
\hline 107 & & & & & & & & & & & - & & & & & & & & & & & & & & & & & & & & & & & & \\
\hline 115 & & & & & & & + & & & & & & & & & & & & + & + & & & + & & + & & & & & & & + & & + & + \\
\hline 116 & & & & + & & + & + & + & + & & & & & & & & & & + & + & & & & + & + & & & & 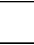 & & 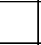 & 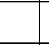 & & + & \\
\hline 119 & & & & & & & & & & & & & & & & & & & & & & & 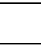 & & & & & & & & & & + & & \\
\hline 122 & & & & & & & & & & & & & & & & & & & & + & & & & & & & & & & & + & + & + & +- & + \\
\hline 123 & & + & + & & + & + & + & + & + & & & & & & & & & & + & + & + & + & + & + & & & & 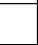 & + & + & + & + & + & + & + \\
\hline 124 & + & + & + & + & + & + & + & & & & & & + & + & + & & & + & \pm & + & + & & & & 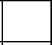 & + & + & + & + & + & + & + & & & \\
\hline 125 & & & & & & & & + & + & & & & & & & & & & & + & & + & + & & & & & & & & & & & & + \\
\hline 129 & & & & & & & & & & & & & & & & & & & & & & & + & & + & & & & & & & 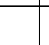 & & & \\
\hline 130 & & & & & + & & & + & + & & & & & & & & & & & . & & + & & & + & & & & 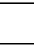 & & & 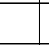 & & & \\
\hline 131 & & & 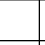 & & + & & & & & & & & & & & & & & & 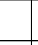 & & & . & & & & & & 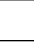 & & . & 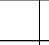 & & - & + \\
\hline 132 & & + & + & & & + & + & + & + & & & & & & & 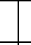 & & + & + & + & + & + & 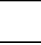 & 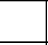 & + & & & & + & + & + & + & & + & \\
\hline 140 & & & & & & & & & & & & & & & & & & & & & & & & & & & & & & & & & & & \\
\hline 141 & & & + & + & & & + & + & + & + & - & & & & & & & & & + & & + & + & + & + & & & & & & & + & + & + & + \\
\hline 142 & & + & + & + & + & + & + & + & + & + & & & & & & & 7 & + & + & + & + & + & + & + & + & & & & + & + & + & + & + & + & + \\
\hline 143 & & & & & & & & & & & & & & & & & & & & & & & + & + & + & & & & & & & . & 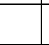 & & \\
\hline 144 & & + & & & & & & & & & & & & & & & & & & 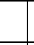 & & & & + & & & & & & & & 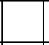 & . & & \\
\hline 145 & & + & + & & + & & & + & + & & & & & & & & F & & & + & & + & + & + & & & & & & & & + & + & + & + \\
\hline 146 & & & & & & & + & & + & & - & & & & & & & & & & & & + & + & + & & & & & & & & & + & + \\
\hline 148 & & & & & & & & & & & & & & & & & & & & & & & & & & & & & & & & & & & \\
\hline 149 & & & & & & & & & + & & & & & & & & & & & & & & + & & + & & & & & & & . & 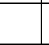 & & \\
\hline 154 & & & & & & & & & & & & & & & & & & & & & & & & & & & & & & & & & + & & \\
\hline 158 & & & + & & & & & & & & & & & & & & & & & & & & & & & & & & & & & & + & & \\
\hline 161 & & & & & & & & & & & & & & & & & & & & & & + & + & + & & & & & & & & 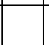 & + & & \\
\hline 171 & & & & & & & & & & & & & & & & & & . & + & & & & & & & & & & & & + & + & + & & \\
\hline 196 & & & & & & & & & & & & & & & & & & & & & & & & & & & & & & & & & & & \\
\hline 197 & & & & & & & & & & & & & & & & & & - & + & & & & & & & & & & & & & & & & \\
\hline 198 & + & & + & & & & & & & & & & + & + & & & & & & 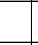 & & & & & & + & & & & & 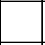 & 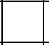 & & & \\
\hline 199 & + & + & + & + & + & + & + & & & & & & + & & & & & + & + & + & + & + & + & & & + & + & + & + & + & + & + & & & \\
\hline 200 & & & & & + & + & + & + & & $t$ & & & + & & & & &.+ & + & + & + & + & + & + & + & & & & + & + & + & + & + & + & + \\
\hline 201 & & & & & & & & & & & & & & & & & & & & & & & & & & & & & & & & + & & & \\
\hline 202 & & & & & & & & & & & & & & & & & & & & & & & & & & & & & & & & & & + & \\
\hline 203 & & & & & & & & & & & - & & & & & & & & & & & & & + & + & & & & & & + & & & & \\
\hline 205 & & & & & & & & & + & & & & & & & & & & & & & & & & & & & & & & & & & & \\
\hline 207 & & + & + & + & & + & + & + & + & $t$ & & & & & & & & + & + & + & & + & + & + & + & & & & + & + & + & + & & + & + \\
\hline 209 & & & & & & & & & & & - & & & & & & & & & & & & & & & & & & & & & & & & \\
\hline 210 & & & & & & & & & & & & & & & & & & & & & & & & & & & & & + & & + & & & & \\
\hline 211 & + & + & + & + & + & + & + & & & & & & + & + & & & & + & + & + & + & & & & & + & + & + & + & + & + & + & & & \\
\hline 212 & & + & & + & & & & + & + & + & & & & & & & & & & + & & & + & + & + & + & & & & + & & + & + & + & \\
\hline 213 & & & + & & + & + & + & + & + & $t$ & & & & & & & & & + & + & + & + & + & + & + & & & & & & & & & + & + \\
\hline 214 & & & & & & & + & & & & & & & & & & & & & & & & & & & & & & & & & & & & + \\
\hline
\end{tabular}




\begin{tabular}{|c|c|c|c|c|c|c|c|c|c|c|c|c|c|c|c|c|c|c|c|c|c|c|c|c|c|c|c|c|c|c|c|c|c|c|}
\hline \multicolumn{35}{|c|}{ Table 9, 1989-5 } \\
\hline & \multicolumn{8}{|c|}{$\mathrm{A}$} & \multicolumn{9}{|c|}{ B } & \multicolumn{14}{|c|}{ C } & \multicolumn{3}{|c|}{ D } \\
\hline & 12 & 2 & & 4 & & 6 & 7 & & 12 & is & 4 & 5 & 6 & 7 & 8 & 9 & 10 & 1 & 2 & 3 & 4 & 5 & 6 & 78 & & & & & 12 & $13 \mid 1$ & 4 & 1 & 2 & 3 \\
\hline 216 & & & & & & & & & & & & & & & & & & + & & & & & & & & & & & & & & & & \\
\hline 220 & & & & & t & & + & + & & & & & + & & & & + & & + & & & & & & & & & + & + & + & & & & \\
\hline 221 & & & & & & + & & & & & & & & & & & & & & & & & & & & & & & & & & & & \\
\hline 224 & & & & & & & & & & & & & + & & & & & & & & & & & & & & & & & & & & & \\
\hline 227 & & & & & & & + & & 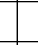 & & & & + & & + & + & + & & . & . & & & $+-1-5$ & + & & & & & + & + & + & & & \\
\hline 229 & & + & + & + & & + & + & & & & 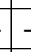 & & + & + & + & + & + & + & + & + & + & + & + & + & & +-- & + & + & & & & + & + & + \\
\hline 230 & & & & & & & & & & & & & & & & & & & & & & & & - & & $F$ & & & & & & & & \\
\hline 232 & $+t$ & + & & & & + & + & + & -1 & & $-1-$ & +1 & + & + & + & + & + & & & + & + & & + & + & & +- & + & + & & & & & + & + \\
\hline 233 & & & & & & & + & + & & & & & + & & & & 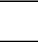 & 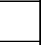 & & & & & & & & & & & + & & & & & \\
\hline 234 & & & & & & & & & & & & & + & & & & & & & & & & & & & & & & & & & & & \\
\hline 235 & $t$ & + & & & & & & + & -1 & & & & & & + & & + & & & + & & & & & & + & & & & & & & & \\
\hline 238 & & & & & & & & & & & & & & & & & & & & & & & & & & & & & & & & & & \\
\hline 239 & & & & & & & & & & & & & & & & & & & & & + & & & & & & & & & & & & & \\
\hline 240 & & & & & & & & & & & & & & & & & + & & & & & & & & & & & & & & & & & \\
\hline 242 & & & & & & & & & & & & & & & & & & & & & & & & & & & & & & & & & & \\
\hline
\end{tabular}

\begin{tabular}{|c|c|c|c|c|c|c|c|c|c|c|c|c|c|c|c|c|c|c|c|c|c|c|c|c|c|c|c|c|c|c|c|c|c|c|}
\hline \multicolumn{35}{|c|}{ Table 9, 1989-6 } \\
\hline & \multicolumn{11}{|c|}{ (2) } & \multicolumn{13}{|c|}{$E$} & \multicolumn{10}{|c|}{$F$} \\
\hline & 4 & 5 & 6 & 7 & 8 & \begin{tabular}{ll|}
9 &
\end{tabular} & 10 & & 12 & 13 & 14 & & 2 & 3 & 4 & 5 & 6 & 7 & 8 & 9 & 10 & 11 & 12 & 13 & 1 & 2 & 3 & 4 & \begin{tabular}{l|l}
5 & \\
\end{tabular} & \begin{tabular}{l|l}
6 & \\
\end{tabular} & & & \begin{tabular}{l|l}
9 & 1 \\
\end{tabular} & \\
\hline 216 & & & & & & & + & & & & & & & & & 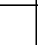 & 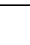 & & & & & & & & & & & & & & & & & \\
\hline 220 & & & & & + & + & + & & & + & & & & & & & & + & + & + & + & + & + & & & & & & + & + & + & + & $t$ & + \\
\hline 221 & & & & & & & & & & & & & & & & & & & & & & & & & & & & & & & & & & \\
\hline 224 & & & & & & & & & & & & & & & & & & & & 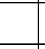 & & & & & & & & & & & & & & \\
\hline 227 & & + & & & & & & & & & & & & & + & + & & & + & + & + & & & & + & & & + & & & & & & \\
\hline 229 & + & + & + & + & + & + & + & + & & & & + & + & + & + & + & + & + & + & + & + & + & & & + & + & + & + & + & + & + & & & \\
\hline 230 & & & & & & & & & & & + & & & & & & & & & 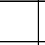 & & & & & & & & & & & & & & \\
\hline 232 & + & + & + & + & + & + & + & & & & & + & + & + & + & + & + & + & + & + & + & + & + & & + & + & + & + & + & + & & & & \\
\hline 233 & & & & & & & & + & + & + & & & & & 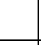 & 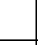 & 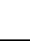 & & - & - & & + & & & & - & & & + & & & + & + & + \\
\hline 234 & & & & & & & & + & & & & & & & & & & & & & & & & & & & & & & & & & & \\
\hline 235 & & + & & + & + & & + & + & & & + & & & & + & + & + & & + & & & & + & & & + & & + & + & + & & & & \\
\hline 238 & & & & & & & & & & & & & & & & & & & & & & & & & & & & & & & & & & \\
\hline 239 & & & & & & & & & & & & & & & & & & & & & & & & & & & & & & & & & & \\
\hline 240 & & & & & & & & & & & & & & & & & & & & & & & & & & & & & & + & & & & \\
\hline 242 & & & & & & & & & & & + & & & & & & & & & & & & & & & & & & & & & & & \\
\hline
\end{tabular}




\begin{tabular}{|c|c|c|c|c|c|c|c|c|c|c|c|c|c|c|c|c|c|c|c|c|c|c|c|c|c|c|c|c|c|c|c|c|c|c|c|c|c|}
\hline \multicolumn{38}{|c|}{ Table 9 , } \\
\hline & & & & A & & & & & & & & & & & $B$ & & & & & & & & & & & & & $\mathrm{C}$ & & & & & & & & D & \\
\hline & 1 & 2 & 3 & 4 & 5 & 6 & 7 & 8 & 1 & 2 & 2 & 3 & 4 & 5 & 6 & 7 & 8 & 9 & 10 & 1 & 2 & 2 & 3 & 4 & 5 & 6 & 7 & 8 & 9 & 10 & 11 & 12 & 13 & 14 & 1 & 2 & 3 \\
\hline 2 & & & & & & & & + & & & & + & & + & & & & & & & + & 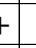 & & & & & & & & & & & & + & & & \\
\hline 3 & & & & & & & & + & & & & & & & & & & & & & & & & & & & & & & & & & + & & & & \\
\hline 4 & + & + & + & + & + & + & + & + & & + & + & + & + & + & & & + & + & + & + & + & + & + & + & + & & + & + & + & + & + & + & + & & & & + \\
\hline 5 & & & & & & & & & & & & & & & + & & & + & & & & & & & + & & + & & & & & & & & & & \\
\hline 6 & & & + & & & & & + & & & & & & & & & & + & & & & & & & & & & & & & + & & + & + & & & \\
\hline 7 & & + & & & & & + & + & & + & + & & & & & & + & + & + & & & & & & + & & + & & + & & + & + & + & + & & & \\
\hline 8 & & & & & & & & & & & & & & & & & & & & & & & & & & & & & & & + & + & + & & & & \\
\hline 10 & & & & & & & & & & & & & & & & & & & & & & & & & & & & & & & & & + & & & & \\
\hline 11 & & & & & & & & + & + & & & & & & + & & + & + & + & & & & & & & + & + & + & + & + & + & & & & & & \\
\hline 12 & + & + & + & + & + & + & + & + & + & + & + & + & + & + & + & & + & + & & + & + & + & + & + & + & + & + & + & + & & & & & & + & + & + \\
\hline 13 & + & + & + & + & + & + & + & + & + & + & + & + & + & + & + & & + & + & + & & + & & + & + & + & & + & + & + & + & + & + & & + & & + & + \\
\hline 14 & + & + & & & + & + & + & & + & + & + & & + & + & & & & + & & + & + & & + & + & + & + & + & & + & & + & & & & & + & + \\
\hline 15 & + & + & + & + & + & + & + & + & & + & + & + & + & + & + & + & + & + & + & + & + & + & + & + & + & + & + & + & + & + & + & + & & & + & + & + \\
\hline 16 & & & & & & & & & & & & & & & & & & & + & & & & & & & & & & + & & + & + & + & + & & & \\
\hline 17 & + & + & & + & & + & + & + & & + & + & + & + & + & + & + & + & + & + & & & & + & + & + & + & + & + & + & + & + & + & + & + & & & + \\
\hline 18 & & & & & & & & & & & & + & & & & & & & & & & & & & & & & & & & & & & & & & \\
\hline 20 & + & + & + & + & + & & & + & + & + & + & + & & + & & & & & & + & + & & + & & + & & + & & & & + & & & & + & & \\
\hline 21 & & & & & & & & + & & & & & & & & & & & & & & & & & & & & & & & & & & & & & \\
\hline 22 & & & & & & & & & + & & & & & & & & & & & & + & & & & & & & & & & & & & & & & \\
\hline 23 & + & & + & & & & & + & & & & & & & & & & & & + & & & & & + & & & & & & & & + & & & & \\
\hline 25 & & & & & & & & & & & & & & & & & & & & & & & & & & & & & & & & + & + & & & & \\
\hline 26 & & & & & & & & & & & & & & & & & & & & & & & & & & & & & & & + & & & & & & \\
\hline 27 & & & & + & & & & & & & & & & & & & & & & & & & & & & & & & & & + & + & + & + & & & \\
\hline 28 & & & & & & & & + & & & & & & & & & & + & + & & & & + & & + & & & & & + & + & + & & & & & \\
\hline 29 & & & & & & & & + & & & & & & & & & & & & & & & & & & & & & & & + & & & + & & & \\
\hline 30 & & & & & & & & & & & & & & & & & & & & & & & & & & & & & & & & & & & & & \\
\hline 31 & & & & & & & & & & & & & & & & & & & & & & & & & & & & & & & & + & & & & & \\
\hline 34 & + & & & & & + & & + & & & & & & & & & & & & & & & & & & & & & & & + & & & & & & \\
\hline 35 & & & & & & & & & & & & & & & & & & & & & & & & & & & & & & & & & & & & & \\
\hline 36 & & & & & & & & & & & & & & & & & & & & & & & + & & & & & & & & & & & & & & \\
\hline 38 & & + & & & & & & & & & & & & & & & & & & & & & & & & & & & & & & & & & & & \\
\hline 39 & & & & & & & & + & & & & & & & & & & & & & & & & & & & & & & & + & & & + & & & \\
\hline 41 & & & & & & & & + & & & & & & & & & & + & & & & & & & & & & & & & & & & & & & \\
\hline 43 & + & & & + & & & & + & & & & & & + & & & & & & & & & & & & & & & + & + & & & & & & & \\
\hline 44 & + & + & + & + & + & + & + & + & + & + & + & + & + & + & + & + & + & + & + & + & + & + & + & + & + & + & + & + & + & + & + & & & & + & + & + \\
\hline 45 & & & & & & & & & & & & & & & & & & & & & & & & & & & & & & & & & & & & & \\
\hline 48 & & & & & & & & + & & & & & & & & & & + & + & & & & & & & & & & + & + & + & + & + & + & & & \\
\hline 49 & & & & & & & & & & & & & & & & & & & & & & & & & & & & & & & + & & & + & & & \\
\hline 53 & & & & & & + & & + & & & & & + & + & & & + & & & & & & & & & & & & & & + & + & + & + & & & \\
\hline 57 & & & & & & & & & & & & & & & & & & & & & & & + & & & & & & & & & & + & & & & \\
\hline 58 & & & + & & & & & & & + & + & & + & & & & & & & & + & & & & + & & & & & & & & & & & & \\
\hline 60 & + & + & + & + & + & + & & & + & + & + & + & + & + & & & & & & + & + & & + & + & + & & & & & & & & & & + & + & + \\
\hline 61 & & & & & & & & + & & & & & & & & & & & & & & & & & & & & + & & & & & + & & & & \\
\hline 62 & & & & & & & & & & & & & & & & & & & & & & & & & & & & & & & & + & & & & & \\
\hline 63 & + & + & + & + & & + & + & + & + & + & + & + & + & + & + & & + & + & & + & + & & + & + & + & + & + & & + & & & & & & & + & + \\
\hline 64 & & + & + & + & + & + & + & & & + & + & & + & + & + & + & + & & & + & + & & + & + & + & + & + & + & & & & & & & + & & + \\
\hline 69 & & & & & & & & & & & & & & & & & & & & & & & & & & & & & & & & & & & & & \\
\hline 70 & & & & & & & & & & & & & & & & & & & & & + & & & & & & & & & & & & & & & & \\
\hline 71 & + & + & + & + & + & + & + & & + & + & + & + & + & + & + & & & & & + & & & + & + & & & & & & & & & & & & & \\
\hline 72 & & + & & & + & + & + & & + & + & + & + & + & & + & & & & & + & + & & + & + & & & & & & & & & & & & & \\
\hline 73 & + & + & + & + & + & + & + & & + & + & + & + & + & + & + & + & & & & + & + & & + & + & + & + & + & & & & & & & & + & + & + \\
\hline 75 & & & & & + & & & & & & & & & & & & & & & & & & & & & & & & & & & & & & & & \\
\hline 76 & & & & & & & & & & & & & & & & & & & & + & & & & & & & & & & & & & & & & & \\
\hline 77 & + & + & + & + & + & + & + & + & & & & & + & + & + & + & + & + & & + & + & & + & + & + & + & + & + & + & + & & & & & + & + & + \\
\hline 78 & + & + & + & + & + & + & + & + & & & & & + & + & + & + & + & + & & + & & & + & + & + & + & + & + & + & + & & & & & + & + & + \\
\hline
\end{tabular}




\begin{tabular}{|c|c|c|c|c|c|c|c|c|c|c|c|c|c|c|c|c|c|c|c|c|c|c|c|c|c|c|c|c|c|c|c|c|c|}
\hline \multicolumn{34}{|c|}{ Table 9, 1990-2 } \\
\hline & \multicolumn{11}{|c|}{$\mathrm{D}$} & \multicolumn{13}{|c|}{$E$} & & & & & $\mathrm{~F}$ & & & & \\
\hline & \begin{tabular}{|l|l|}
4 &
\end{tabular} & $5 \mid$ & $\begin{array}{ll}6 & 7 \\
6\end{array}$ & $\begin{array}{ll}7 \varepsilon & \varepsilon \\
\end{array}$ & \begin{tabular}{|l|l}
3 & 9 \\
\end{tabular} & & & & 12 & 13 & & 1 & 2 & 3 & 4 & 5 & 6 & 7 & 8 & 9 & 10 & & 12 & & 1 & 2 & \begin{tabular}{l|l}
3 & 4 \\
\end{tabular} & \begin{tabular}{l|l}
4 & 5 \\
\end{tabular} & \begin{tabular}{l|l}
5 & 6 \\
\end{tabular} & & 8 & & 10 \\
\hline 2 & & & & & & & & & & & & & & & & & & & & & & & + & & & & & & & & & + & \\
\hline 3 & & & & & & & & + & + & & + & & & & & & & & & & & & + & + & & & & & & & & + & \\
\hline 4 & + & + & +-1 & $+1+$ & t & & + & + & + & + & & & & & + & + & + & + & + & + & + & + & + & + & & & & + & $+t+$ & + & + & & + \\
\hline 5 & & & & & t & & + & + & & & & & & & & & + & & + & + & & & & & & & & & & & + & & \\
\hline 6 & & + & & & & & & + & + & + & + & & & & & & & & & & + & + & + & + & & & & & & & & + & + \\
\hline 7 & & + & -1 & $+1+$ & $-1+$ & & + & + & + & + & + & & & & & & 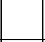 & & + & + & + & + & + & + & & & & + & + & & + & & + \\
\hline 8 & & & & & & & & + & + & + & + & & & & & & & & & & & + & & + & & & & & & & & & \\
\hline 10 & & & & & & & & & & & + & & & & & & & & 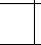 & & & & & & & & & & & & & & \\
\hline 11 & & +- & +-1 & + & t & & + & & & & & & & & + & & + & & & & & & & & & & & + & & & & & \\
\hline 12 & + & + & $+1-1$ & $+t+2$ r a & $t+$ & & & 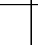 & & & & & & & + & + & + & + & + & + & & . & & & + & + & 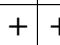 & $+t$ & + & + & & & \\
\hline 13 & & +- & $+1-1$ & + & +1 & & + & + & + & + & + & & & & 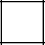 & + & + & + & + & + & + & + & + & + & & 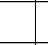 & & $++t$ & + & + & + & + & + \\
\hline 14 & + & + & & $t$ & & & & + & & + & & & & & + & + & & + & & + & & & & & + & . & & + & $t$ & $1+$ & + & & \\
\hline 15 & + & + & +1 & $+t$ & $t$ & & + & + & + & + & & + & & & + & + & + & + & + & + & + & & + & & + & + & ++ & ++ & + & $-1+$ & + & + & + \\
\hline 16 & & & & + & & & + & + & + & + & + & & & & & 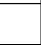 & 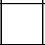 & & + & + & + & + & & + & & & & & & 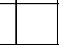 & & & + \\
\hline 17 & & + & +1 & $+t$ & $-1+$ & & + & + & + & + & + & & & & + & + & + & + & + & + & + & & & + & & & & ++ & + & $-1+$ & + & + & + \\
\hline 18 & & & & & & & & & & & + & & & & & & + & + & & & & & & & & & & & & & + & & \\
\hline 20 & & + & +7 & $t$ & \pm & & & & & & & & & & & + & + & + & + & & & & & & & & & $+t$ & + & - & $t$ & 4 & \\
\hline 21 & & & & & & & & & & & + & & & & & & & & & & + & & & & & & & & & & + & + & + \\
\hline 22 & & & & & & & & & & & & & & & & & & & & & & & & & & & & & & & & & \\
\hline 23 & & & & & & & & & & & & & & & & & & & & & & & & & & & & & & & & & \\
\hline 25 & & & & & & & & & & & & & & & & & & & & & & & & & & & & & & & & & \\
\hline 26 & & & & & & & & & & & + & & & & & & & & 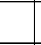 & & & + & & + & & & & & & 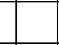 & & & \\
\hline 27 & & & & & & & & & + & + & + & & & & & & & & 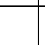 & & & & + & + & & & & & & & & & \\
\hline 28 & & & & & $t$ & & & + & + & & & & & & & & & & 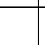 & 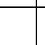 & + & + & + & + & & & & $t$ & + & + & & + & + \\
\hline 29 & & & & & & & & + & & & & & & & & & & & & + & & + & + & & & & & & & 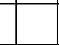 & + & & + \\
\hline 30 & & & & & & & & & & & + & & & & & & & & & & & & & & & & & & & & & & \\
\hline 31 & & & & & & & & & & & & & & & & & & & & & & & & & & & & & & & & & \\
\hline 34 & & & & & & & & & & & & & & & & & & & & & & & & & & & & & & & & & \\
\hline 35 & & & & & & & & & & & & & & & & & & & & & + & & + & & & & & & & & & & \\
\hline 36 & & & & & & & & + & & & & & & & & & & & 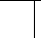 & & & & 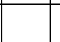 & & & & & & & & & & \\
\hline 38 & & & & & & & & & & & + & & & & & & & & 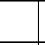 & & & & & & & & & & & & + & & \\
\hline 39 & & & & & + & & & + & & & & & & & & & & & + & & & & + & + & & & & & & 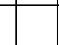 & + & + & + \\
\hline 41 & & 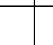 & 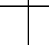 & & & & & + & + & & & & & & & & & & + & & + & + & + & + & & & & & & + & + & & \\
\hline 43 & & + & +7 & + & $t$ & & + & + & + & & & & & & . & 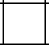 & & + & + & + & & & 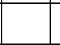 & & & & & + & & + & 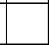 & & \\
\hline 44 & + & + & +7 & +1 & $1+$ & & + & + & & & & + & + & + & + & + & + & + & + & + & + & & + & & + & + & $+t$ & $+t$ & + & + & + & & \\
\hline 45 & & & & & & & & & & & & & & & & & & & & & & & & & + & & & & & 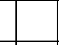 & 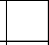 & & \\
\hline 48 & & & - & +1 & $-1+$ & & + & + & + & + & + & & & & & & & & & + & + & + & + & + & & & & + & + & + & + & + & + \\
\hline 49 & & & & & & & & & & + & + & & & & & & 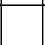 & 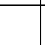 & 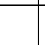 & 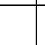 & & & & + & & & & 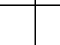 & & 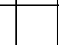 & & . & \\
\hline 53 & & & & $t$ & $+1+$ & & & + & + & + & + & & & & & & + & + & + & + & + & + & & & & & & ++ & + & $+1+$ & & + & \\
\hline 57 & & & & & & & & & & + & & & & & & & & & & & & & & & & & & & - & $-1+$ & & & \\
\hline 58 & & + & & $t$ & $-1+$ & & & & & & & & & & & + & & & + & & & & & & & & & + & +1 & -1 & & & \\
\hline 60 & & + & +7 & $+t$ & & & & & & & & & & & & + & + & + & + & + & & & + & & & & +1 & + & + & $-1+$ & + & & + \\
\hline 61 & & & & & & & & + & + & + & + & & & & & & & & + & + & + & + & + & + & & & & & $t$ & $-1+$ & + & + & + \\
\hline 62 & & & & & & & & & & & & & & & & & & & & & & & & & & & & & & & & & \\
\hline 63 & + & & +7 & +1 & $-1+$ & & & & & & & & & & + & + & + & + & + & + & & & & & & & & + & & + & + & & \\
\hline 64 & + & + & +7 & + & $+t$ & & & & & & & & + & & + & & & & & & & & & & + & + & $+t$ & + & & 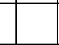 & & & \\
\hline 69 & & & & & & & & & & & & & & & & & & & & & & & & & & & & & & + & & & \\
\hline 70 & & & & & & & & & & & & & & & & & & & & & & & & & & & & & & & & & \\
\hline 71 & & & & & & & & & & & & & & & & & + & & & & & & & & + & & & & & + & & & \\
\hline 72 & & & & & & & & & & & & & & & & & & & & & & & & & & & & & & & & & \\
\hline 73 & + & +- & +-7 & + & & & & & & & & + & + & + & + & + & + & & & & & & & & + & + & $+t$ & ++ & + & & & & \\
\hline 75 & & & & & & & & & & & & & & & & & & & & & & & & & & & & & & & & & \\
\hline 76 & & & & & & & & & & & & + & & & & & & & & & & & & & & & & & & & & & \\
\hline 77 & + & +- & +- & + & $+1+$ & & + & & & & & + & $1+$ & + & + & + & + & + & + & + & & & & & + & + & + & + & & + & & & \\
\hline 78 & + & & & ++ & & & & & & & & & & & & & & + & & & & & & & & & & & & & & & \\
\hline
\end{tabular}




\begin{tabular}{|c|c|c|c|c|c|c|c|c|c|c|c|c|c|c|c|c|c|c|c|c|c|c|c|c|c|c|c|c|c|c|c|c|c|c|c|}
\hline \multicolumn{36}{|c|}{ Table 9, 1990-3 } \\
\hline & \multicolumn{8}{|c|}{$\mathrm{A}$} & & & & & $E$ & & & & & & & & & & & & & C & & & & & & & & D & \\
\hline & 1 & 2 & 3 & 4 & & 6 & & 8 & 1 & 2 & 3 & 4 & & 6 & & 8 & 9 & 10 & 1 & 2 & 3 & 4 & 5 & 6 & & 8 & & 10 & 11 & 12 & 13 & 14 & & & 3 \\
\hline 79 & & & & & & & & & & & & + & + & + & + & & & & + & & + & + & + & + & & & & & & & & & + & & \\
\hline 80 & & + & & & & & & & & & & & + & & & & & & & & 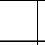 & & + & & & & & & & & & & & & \\
\hline 81 & & + & + & + & + & + & + & + & & & + & + & + & + & + & + & + & & + & + & + & + & + & + & + & + & + & + & & & & & + & + & + \\
\hline 82 & + & + & + & + & + & + & + & & + & & + & + & + & + & & & & & + & + & + & & + & & & & & & & & & & + & + & \\
\hline 86 & & & + & + & & & & + & & & & & & & & & & + & & & & + & + & & & & & & + & + & + & + & & & \\
\hline 87 & & & & & & & & & & & & & & & & & & & & + & & & & & & & & & + & & & & & & \\
\hline 88 & & & & & & & & & & & & & & & & & & & & & & & & & & & & & & & & & + & & \\
\hline 89 & & & & & & & & + & & & 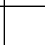 & & & & & & & & & & & & & & & & & & & + & & & & & \\
\hline 91 & & & & & & & & & & & & & & & & & & & & & & & & & & & & & & + & + & + & & & \\
\hline 94 & & & & & & & & + & & & + & & & + & & & & + & & & & & & & & & & & + & + & + & & & & \\
\hline 97 & & & & & & & & & & & & & & & & & & & & & & & & & & & & & & & & & & & \\
\hline 99 & & & & & & & & & & & & & & & & & & & & & & & & & & & & & + & & + & & & & \\
\hline 103 & & & & & & & & & & & 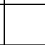 & & & & & & $\begin{array}{llll}+ & & l & -1\end{array}$ & & & & & & & & & & & & & + & & & & & \\
\hline 106 & & & & & & & & & & & & & & & & & & & & & & & & & & & & & & & & & & & \\
\hline 111 & & & & & & & & & & & & & & & & & & & & & & & & & & & & & & & & & & & \\
\hline 115 & & & & & & & & + & & & + & & & & & & & & & & & & & & & & & & & & & & & & \\
\hline 116 & & & & & + & & & + & & & 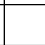 & & & & & & & & & & & & & & & & & & & & & & & & \\
\hline 119 & & & & & & & & & & & & & & & & & & & & & & & & & & & & & + & + & & & & & \\
\hline 122 & & & & & & & & + & & & & & & & & 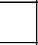 & & & & & 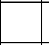 & 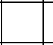 & $t$ & & & & & & & & & & & & \\
\hline 123 & & + & & + & t & & & + & & + & + & & 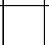 & 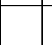 & & + & & & & + & + & + & + & & & & & + & + & + & & & & & \\
\hline 124 & + & + & + & + & + & + & + & + & + & + & + & + & + & + & & + & + & & + & + & + & + & + & + & + & + & + & & - & & & & + & + & + \\
\hline 125 & & & & & & & & + & & & & & & & & & & & & & & & + & & & & & & + & & & & & & \\
\hline 126 & & & & & & & & & & & & & & & & & & & & & & & & & & & & & & & & & & & \\
\hline 127 & & & & & & & & & & & & & & & & & & & & & & & & & & & & & & & & & & & \\
\hline 129 & & & & & & & & + & & & & & & & & & & & & & & & & & & & & & & & & & & & \\
\hline 130 & & & & & & & & + & & & & & & & & & & & & & & & & & & & & & & & & & & & \\
\hline 131 & & & & & & & & & & & & & & & & & & & & & & & & & & & & & & & & & & & \\
\hline 132 & & & & & & & & + & & & & & & & & & + & + & & & & & 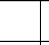 & & + & & & & + & & & & & & \\
\hline 134 & & & & & & & & & & & & & & & & & & & & & & & & & & & & & & & & & & & \\
\hline 140 & & & & & & & & + & & & 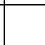 & & & & & & 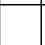 & & & & & & 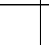 & & & & & & & & & & & & \\
\hline 141 & & & & & & & & + & & & & & & & & & & + & & & & & + & & & & & & + & + & + & + & & & \\
\hline 142 & + & & & + & + & & & + & & + & + & & & & & + & + & + & + & + & + & & + & & & & + & + & + & + & + & & & & \\
\hline 143 & & & & & & & & & & & & & & & & & & & & & & & & & & & & & + & & & & & & \\
\hline 144 & & & & & & & & & & & & & & & & & & & & & & & & & & & & & & & & + & & & \\
\hline 145 & & & & & & + & + & + & & & & + & & & & & & & & & 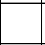 & & & & & & & & 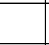 & & 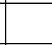 & & & & \\
\hline 146 & & & & & & & & + & & & & & & & & & & & & & & & & & & & & & + & & + & & & & \\
\hline 149 & & & & & & & & & & & & & & & & & & & & & & & & & & & & & & & & & & & \\
\hline 158 & & + & & & & & + & & & & & & & & & & & & & & + & + & + & & & & & & & & & & & & \\
\hline 161 & & & & & & & & + & & & & & & & & & & & & & & & & & & & & & & & & & & & \\
\hline 176 & & & & & & & & + & & & & & & & & & & & & & & & & & & & & & & & & & & & \\
\hline 198 & & & . & & & + & & 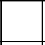 & & & & & & & + & & 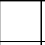 & & & & & & & + & + & & 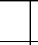 & & . & & & & & & + \\
\hline 199 & + & + & + & + & & + & + & + & & & & & & & + & + & + & + & & & & & + & + & + & + & + & + & + & & & & + & & + \\
\hline 200 & & & & & & & & + & & + & & & & & & & + & & & & & & & & + & + & + & & + & + & & + & + & & \\
\hline 202 & & & & & & & & + & & & & & & & & & & & & & & & & & & & & & & & & & & & \\
\hline 203 & & & & & & & & & & & & & & & & & & & & & & & & & & & & & & & & & & & \\
\hline 205 & & & & & & & & & & & & & & & & & & & & & & & & & & & & & & & & & & & \\
\hline 207 & & + & & + & + & & & + & + & + & & + & & + & & + & + & + & & + & + & & & & + & + & + & + & + & + & + & + & & & \\
\hline 210 & & & & & & & & & & & & & & & & & & & & & & & & & & & & & & & & & & & \\
\hline 211 & + & + & + & + & + & + & + & + & & + & & + & + & + & + & + & + & & + & + & + & + & + & + & + & + & + & + & & & 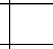 & & + & + & + \\
\hline 212 & & & & & & & + & + & & & + & & & & & & & & & & + & & & & & & & - & + & + & + & & + & & + \\
\hline 213 & & & & & & & & + & & & & & & & & & & + & & + & & & 7 & & & & & & + & + & & + & & & \\
\hline 214 & + & + & + & + & + & & & + & + & + & + & + & + & & + & + & + & + & + & + & + & + & + & + & + & + & + & + & + & + & + & + & & + & + \\
\hline 216 & & & & & & & & & & & & & & & & & & & & & & & & & & & & & & & & & & & \\
\hline 220 & & & + & + & + & + & & + & + & & + & & & & + & & & + & & + & & & & & & & & & & + & + & & & & \\
\hline 227 & & & & & & & & & + & + & & & & & + & & + & + & + & + & + & & & & + & & & + & & & & & & & \\
\hline
\end{tabular}




\begin{tabular}{|c|c|c|c|c|c|c|c|c|c|c|c|c|c|c|c|c|c|c|c|c|c|c|c|c|c|c|c|c|c|c|c|c|c|c|}
\hline \multicolumn{35}{|c|}{ Table $9,1990-4$} \\
\hline & \multicolumn{11}{|c|}{$\mathrm{D}$} & \multicolumn{13}{|c|}{$E$} & & & & & & $F$ & & & & \\
\hline & 4 & 5 & 6 & 7 & 8 & 9 & 10 & & 12 & & & 1 & 2 & 3 & 4 & 5 & 6 & & 8 & 9 & 10 & & & & 1 & 2 & 3 & 4 & 5 & \begin{tabular}{|c|}
6 \\
\end{tabular} & & 8 & & 10 \\
\hline 79 & + & & & & & & & & & & & + & & & + & & & & & & & & & & + & & & & & & & & & \\
\hline 80 & & & & & & & & & & & & & & & + & & & & & & & & & & & & & & & & & & & \\
\hline 81 & + & + & + & + & + & & + & & & & & + & + & + & + & + & & & & & & & & & + & + & + & + & & & & & & \\
\hline 82 & & & & & + & & & & & & & & & & & + & + & + & & & & & & & + & & & + & + & + & & & & \\
\hline 86 & & + & & & + & & & + & & & + & & & & & & & & & & & + & + & + & & & & + & + & + & + & + & +- & + \\
\hline 87 & & & & & & & & + & + & & + & & & & & & & & & & & & & + & & & & & + & + & + & & + & \\
\hline 88 & & & & & & & & + & & + & + & & & & & & & & + & & & & & + & & & & & + & + & & + & & \\
\hline 89 & & & & & & & & & & & & & & & & & & & & & & + & + & + & & & & & & & + & & +- & + \\
\hline 91 & & & & & & & & & + & + & + & & & & & & & & & & & + & & + & & & & & & & & & & \\
\hline 94 & & & & & & & & + & + & + & & & & & & & & & & & + & & + & + & & & & & & & & & & + \\
\hline 97 & & & & & & & & & & & & & & & & & & & & & & & & + & & & & & & & & & & \\
\hline 99 & & & & & & & & + & + & & & & & & & + & & + & & & & + & + & + & & & & & & & & & & \\
\hline 103 & & & & & & & & & & & & & & & & & & & & & & + & + & & & & & & & & & & & \\
\hline 106 & & & & & & & & & & & & & & & & & & & & & & & + & & & & & & & & & & & \\
\hline 111 & & & & & & & & & & & & & & & & & & & & & & + & & & & & & & & & & & & \\
\hline 115 & & & & & & & & + & & + & & & & & & & & & & & & + & & & & & & & & & & + & + & \\
\hline 116 & & & & & & & & + & + & & & & & & & & & & & + & & & + & + & & & & & + & & + & + & & 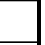 \\
\hline 119 & & & & & & & & & & & & & & & & & & & & & & & & + & & & & & & & & & & \\
\hline 122 & & & & & & & & & & & & & & & & & & & + & + & + & & 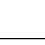 & & & & & & + & + & + & + & +- & + \\
\hline 123 & & + & + & + & + & + & & + & + & + & & & & & & + & + & + & + & + & + & + & + & & & & & + & + & + & + & + & + & + \\
\hline 124 & + & + & + & + & + & + & + & & & & & & & & & + & + & + & + & + & & 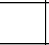 & + & & & & & + & + & + & + & + & & 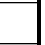 \\
\hline 125 & & + & & & & & & & & & & & & & & & & & & & & + & & & & & & & & & 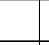 & & 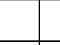 & 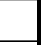 \\
\hline 126 & & & & & & & & + & & & & & & & & & & & & & & & & + & & & & & & & & & & \\
\hline 127 & & & & & & & & & & & & & & & & & & & & + & & & & & & & & & & & & & & \\
\hline 129 & & & & & & & & & & & & & & & & & & & & & & & & & & & & & & & & & & \\
\hline 130 & & & & & & & & & + & + & & & & & & & & & 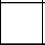 & 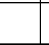 & & + & & + & & & & 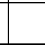 & & & 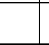 & - & & \\
\hline 131 & & & & & & & & + & & & & & & & & . & & & & 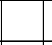 & & & + & + & & & & 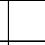 & & & 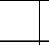 & & & + \\
\hline 132 & & + & + & + & + & + & + & + & + & + & & & & & & + & + & + & + & + & + & & 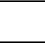 & - & & & & + & + & & + & + & & \\
\hline 134 & & & & & & & & & & & & & & & & & & & & & & & & & & & & & & & + & & & \\
\hline 140 & & & & & & & & & & & & & & & & & & & & & & & & & & & & & & & & & & \\
\hline 141 & & & + & & + & & & + & + & + & + & & & & & 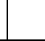 & & 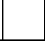 & 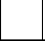 & 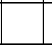 & & + & + & + & & & & 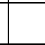 & 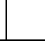 & 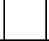 & 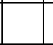 & + & + & + \\
\hline 142 & & & + & + & + & + & + & + & + & + & + & & & & & + & + & + & + & + & + & + & + & + & & & & & + & + & + & + & + & + \\
\hline 143 & & & & & & & & & & & & & & & & & & & & & & + & & & & & & & & & & & & \\
\hline 144 & & & & & & & & & & & & & & & & & & & & & & & & & & & & & & & & & & \\
\hline 145 & & & & & + & & & + & + & + & & & & & & & & & + & & & + & + & + & & & & & + & & + & + & + & + \\
\hline 146 & & & & & + & & & + & + & & + & & & & & & & & & + & & + & + & + & & & & & & & & + & + & + \\
\hline 149 & & & & & & & & + & & & & & & & & & & & & & & & & & & & & & & & & 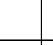 & + & \\
\hline 158 & & + & + & & & & & & & & & & & & & & & & & & & & & & & & & & & & & . & 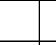 & \\
\hline 161 & & & & & & & & + & & & & & & & & & & & & + & & & & & & & & & & + & & + & + & \\
\hline 176 & & & & & & & & & & & & & & & & & & & & & & & & & & & & & & & & & & \\
\hline 198 & + & & & & & & & & & & & + & + & + & & & & & & . & & & & & + & & & & & & 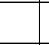 & 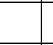 & . & \\
\hline 199 & + & + & + & + & + & + & + & + & & & & & & & & + & + & + & + & + & + & & & & & + & + & + & + & + & + & + & + & \\
\hline 200 & & + & + & + & + & + & + & + & + & + & + & & & & & & & + & + & + & + & + & + & + & & & & 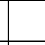 & + & + & + & + & + & + \\
\hline 202 & & & & & & & & & + & & & & & & & & & & & & & & & & & & & & & & & & & \\
\hline 203 & & & & & & & & & & & & & & & & & & & & & & & & & & & & & & & & + & & \\
\hline 205 & & & & & & & & & & & & & & & & & & & & & & & & + & & & & & & & & & - & \\
\hline 207 & & & + & + & + & & & + & + & + & + & & & & & & + & + & + & & & + & + & + & & & & + & + & + & + & + & + & + \\
\hline 210 & & & & & & & & & & & & & & & & & & & & & & & & & & & & & & & & & + & \\
\hline 211 & + & + & + & + & + & + & + & & & & & + & + & + & + & + & + & + & + & & + & & & & + & + & + & + & + & + & + & + & & \\
\hline 212 & & & & & & & & + & + & + & + & & & & & + & & & + & & & + & + & + & & & & + & + & + & + & + & + & + \\
\hline 213 & & & & & & & + & + & + & + & & & & & & & & + & + & + & + & + & + & + & & & & + & & + & + & + & + & + \\
\hline 214 & + & + & + & + & + & + & + & + & + & + & + & + & + & & + & + & + & + & + & + & + & + & + & + & + & + & & + & + & + & + & + & + & \\
\hline 216 & & & & & & & & & + & + & & & & & & & & & & & & & & + & & & & & & & & + & & \\
\hline 220 & & & + & + & + & + & + & & + & & & & & & & & & + & + & + & + & & + & & & & & + & + & + & + & + & + & + \\
\hline 227 & & + & & + & & & + & & + & & & & & + & & & + & & + & & & & & & & & & & & & + & & & \\
\hline
\end{tabular}




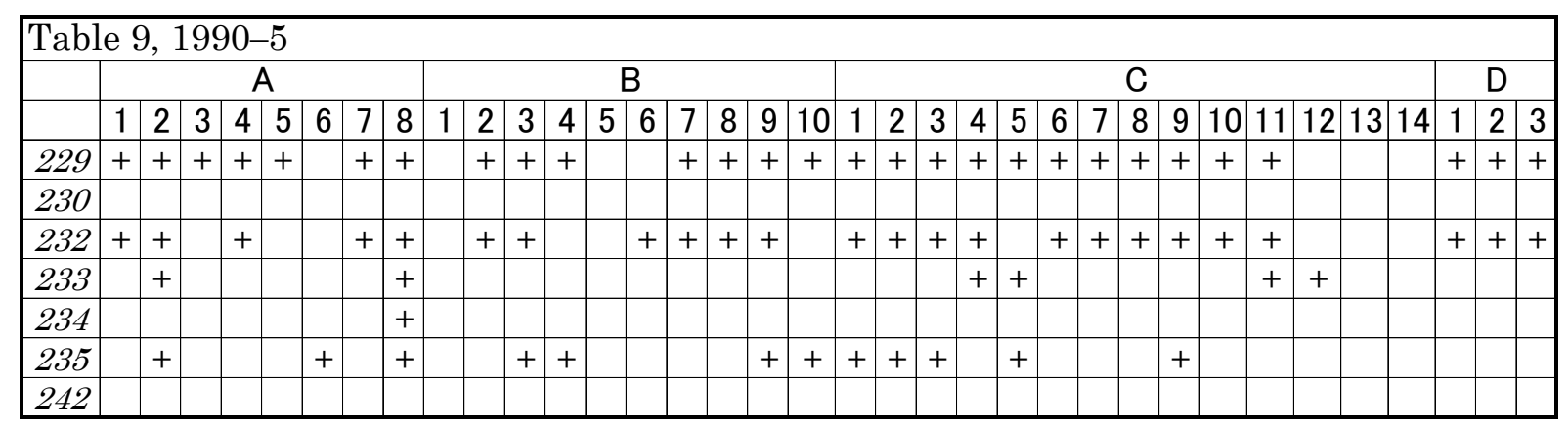

\begin{tabular}{|c|c|c|c|c|c|c|c|c|c|c|c|c|c|c|c|c|c|c|c|c|c|c|c|c|c|c|c|c|c|c|c|c|c|}
\hline \multicolumn{34}{|c|}{ Table 9, 1990-6 } \\
\hline & \multicolumn{11}{|c|}{ D } & \multicolumn{12}{|c|}{$E$} & \multicolumn{10}{|c|}{$\mathrm{F}$} \\
\hline & 4 & 5 & 6 & 7 & 8 & 9 & 10 & & 12 & & & 1 & 2 & 3 & 4 & 5 & 6 & 7 & 8 & 9 & 0 & 1112 & $\begin{array}{l}213 \\
\end{array}$ & 1 & 2 & 3 & 4 & 5 & 6 & 7 & 8 & & 10 \\
\hline 229 & + & + & + & + & + & + & + & + & & & & + & + & + & + & + & + & + & + & + & + & + & & + & + & + & + & + & + & & & & + \\
\hline 230 & & & & & & + & & + & & & & & & & & & & & & + & & & & & & & & & + & & & & \\
\hline 232 & + & + & + & + & + & + & & + & & & & + & + & + & + & + & + & + & + & + & & & & + & + & + & + & + & + & & & & \\
\hline 233 & & & & & & & & + & + & & + & & & & & & & & + & & + & + & + & & & & & & & & & + & \\
\hline 234 & & & & & & & & & + & & & & & & & & & & & & & & & & & & & & & & & + & \\
\hline 235 & & & & & & + & & + & & & + & & & & & & + & + & + & & + & & + & & & & & & & & + & & \\
\hline 242 & & & & & & & & & & & & & & & & & & & & & + & & & & & & & & & & & & \\
\hline
\end{tabular}




\begin{tabular}{|c|c|c|c|c|c|c|c|c|c|c|c|c|c|c|c|c|c|c|c|c|c|c|c|c|c|c|c|c|c|c|c|c|c|c|c|}
\hline \multicolumn{36}{|c|}{ Table 9, 1991-1 } \\
\hline & \multicolumn{8}{|c|}{$\mathrm{A}$} & & & & & $\mathrm{b}$ & & & & & & & & & & & & & $\mathrm{C}$ & & & & & & & & D & \\
\hline & 1 & 2 & 3 & 4 & & 6 & 7 & 8 & 1 & 2 & 3 & 4 & 5 & 6 & 7 & 8 & 9 & 10 & 1 & 2 & 3 & 4 & 5 & 6 & 7 & 8 & \begin{tabular}{l|l}
9 & 1 \\
\end{tabular} & 101 & 11 & 12 & 13 & 14 & & & 3 \\
\hline 2 & & & & & & & & & & + & & & & & & & + & & + & & & & & & & & & & & & & & & & \\
\hline 4 & & + & + & + & + & + & + & + & + & + & + & + & + & + & + & + & + & + & + & & + & + & + & & + & + & + & + & + & + & + & + & & & + \\
\hline 5 & & & & & + & + & + & + & & & & & & & & & & & & & & & & & + & & & & & & & + & & & \\
\hline 6 & & & & & & & & & & & & & & & & & & & & & & & & & & & & & + & + & + & + & & & \\
\hline 7 & & + & + & & & & & + & & & & & & & & & + & & & & & & & & & + & +1. & + & + & + & + & + & & & \\
\hline 8 & & & & & & & & & & & & & & & & & & & & & & & & & & & & & + & & & & & & \\
\hline 10 & & & & & & & & & & & & & & & & & & & & & & & & & & & & & & + & + & + & & & \\
\hline 11 & & & & & + & & & + & & & & & & 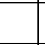 & & + & + & & & + & & . & & & & + & & + & & & + & & & & \\
\hline 12 & + & + & + & + & + & + & + & + & + & + & + & + & + & + & + & + & & & + & + & + & + & + & + & + & + & & + & & & & & + & + & \\
\hline 13 & + & + & + & + & + & + & + & + & + & + & + & + & + & + & & + & + & + & + & + & + & + & + & + & + & + & +1 & + & + & + & + & + & & + & + \\
\hline 14 & + & & + & & + & + & + & + & & + & + & & + & & + & + & & & & & + & & & + & + & + & & & & & & & & & \\
\hline 15 & + & + & + & + & + & + & + & + & & + & + & & + & + & + & + & + & + & + & + & + & + & + & + & + & + & + & + & + & + & + & & + & + & + \\
\hline 16 & & & & & & & & & & & & & & & & & & & & & & & & & & & + & & + & + & + & + & & & \\
\hline 17 & & + & + & + & + & + & + & + & + & & & & & & + & + & + & + & + & + & & + & + & + & + & + & + & + & + & + & + & + & + & + & + \\
\hline 20 & + & + & & & & + & + & + & & & & & & & & & & & & + & + & & + & & & & & & & & & & & & \\
\hline 21 & & & & & & & & & & & & & & & & & & & & & & & & & & & & & + & & & & & & \\
\hline 22 & & & & & & & & & & & & & & & & & & & & & & & & & & & & & & & & & & & \\
\hline 23 & & & & & & & & & & + & + & & & & & + & & & & & & & & & & & & & & & & & & & \\
\hline 25 & & & & & & & & & & & & & & & & & & & & & & & & & & & & & & & + & & & & \\
\hline 26 & & & & & & & & & & & & & & & & & + & & & & & & & & & 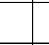 & & & & & + & + & & & \\
\hline 27 & & & & & & & & & & & & & & & & & & & & & & & & & & & & & & + & + & + & & & \\
\hline 28 & & & & & & & & + & & & & & & & & & & & & & & & & & & & & + & + & & & & & & \\
\hline 31 & & & & & & & & & & & & & & & & & & & & & & & & & & & & & & & & & & & \\
\hline 33 & & & & & & & & & & & + & & & & & & & & & & & & & & & & & & & & & & & & \\
\hline 34 & & + & & & & & & & & & 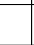 & & & & & & & + & & & & & & & & & & & & & & & & & \\
\hline 35 & & & & & & & & & & & + & & & & & & & & & + & & & & & & & & & & & & & & & \\
\hline 36 & & & & + & & & & & & & & & & & & & & & & & & & & & & & & & & & & & & & \\
\hline 38 & & & & & & & & & & & & & & & & & & & & & & & & & & & & & & & + & & & & \\
\hline 39 & & & & & & & & + & & & & & & & & & & + & & & & & & & & & & & & & + & + & & & \\
\hline 41 & & & & & & & & & & & & & & & & & + & & & & & & & & & & & & + & + & & + & & & \\
\hline 43 & & & & & & & + & + & & & & & & & & & & & & & & + & & + & + & & & & + & & & & & & \\
\hline 44 & + & + & + & + & + & + & + & + & + & + & + & + & + & + & + & + & + & + & + & + & + & + & + & + & + & + & + & + & & & & & + & + & + \\
\hline 46 & & & & & & & & & & & & & & & & & & & & & & & & & & & & & & & & & & & \\
\hline 47 & & & & & & & & & & & & & & & & & & & & & & & & & & & & & & & & & & & \\
\hline 48 & & & & & & + & & + & & & & & & & & & + & + & & & & & & & & + & +1 & + & + & + & + & + & & & \\
\hline 49 & & & & & & & & & & & & & & & & & & & & & & & & & & & & & & & & & & & \\
\hline 52 & & & & & & & & & & & & & & & & & & & & & & & & & & & & & & & & & & & \\
\hline 53 & & & + & + & & & & & & + & & + & + & + & & & & & & & & & & & & & & & + & + & + & + & & & \\
\hline 57 & & & & & & & & & & & & & & & & & & & & & & & & & & & & & & & & & & & \\
\hline 58 & & + & + & & + & & & & & + & + & + & + & & & & & & & & & & 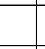 & & & & & & & & & & & . & \\
\hline 60 & + & + & + & & & & & & + & + & + & + & + & & & & & & + & + & + & & + & & & & & & & & & & & & + \\
\hline 61 & & & & & & & & & & & & & & & & & & & & & & & & & & & & & & + & & + & & 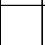 & \\
\hline 63 & + & + & + & & + & + & + & & + & + & + & + & & & + & + & & & + & + & + & + & + & + & + & + & $t$ & & & & & & & + & + \\
\hline 64 & & & + & + & + & + & + & + & & & & + & + & + & + & + & & & + & & + & + & + & + & + & + & & & & & & & + & + & + \\
\hline 70 & & & & & & & & & & & & & & & & & & & + & & & & & & & & & & & & & & & & \\
\hline 71 & + & + & + & & & & & & + & + & + & + & + & & & & & & + & & & & & & & & & & & & & & & & \\
\hline 72 & + & + & + & + & & & 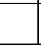 & 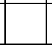 & + & + & + & + & + & + & & & & & + & & + & & 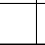 & & & & & & & & & & & + & \\
\hline 73 & + & + & + & + & + & + & + & $1+$ & + & + & + & + & + & & + & + & & & + & + & + & + & + & + & + & + & & & & & & & + & + & + \\
\hline 76 & & & & & & & & & + & & & & & & & & & & + & & & & & & & & & & & & & & & & \\
\hline 77 & + & & + & + & + & + & + & + & & & & & + & + & + & + & + & + & + & + & + & + & + & + & + & + & + & + & & & & & + & + & + \\
\hline 78 & + & & + & + & + & + & + & + & + & & & & + & + & + & + & + & + & + & + & + & + & + & + & + & + & + & + & & & & & + & + & + \\
\hline 79 & + & & & & + & + & + & & & & & & & & & & & & + & & + & + & + & + & + & & & & & & & & + & & \\
\hline 80 & & & + & & + & + & & + & & & & & & & & & & & & & & & & & & + & & & & & & & + & & + \\
\hline 81 & + & & + & + & + & + & + & + & + & & + & & + & + & & + & + & & + & + & + & + & + & + & + & + & + & + & & & & & + & + & + \\
\hline 82 & & + & + & + & & & & & + & + & + & + & & + & & & & & + & & & & & & & & & & & & & & & & \\
\hline
\end{tabular}




\begin{tabular}{|c|c|c|c|c|c|c|c|c|c|c|c|c|c|c|c|c|c|c|c|c|c|c|c|c|c|c|c|c|c|c|c|c|c|c|}
\hline \multicolumn{35}{|c|}{ Table 9, 1991-2 } \\
\hline & \multicolumn{11}{|c|}{ D } & \multicolumn{13}{|c|}{$E$} & & & & & $\mathrm{~F}$ & & & & & \\
\hline & 4 & $5 \mid 6$ & 6 & 7 & 8 & 9 & & & 12 & 13 & & 1 & 2 & 3 & 4 & 5 & 6 & & 39 & & & & 12 & & 1 & 2 & 3 & 4 & $5 \mid$ & 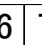 & & \begin{tabular}{l|l}
8 & 9
\end{tabular} & 10 & \\
\hline 2 & & & & & & & & & + & & & & & & & & & & & & & & + & + & & & & + & & & & + & & \\
\hline 4 & & + & + & + & + & + & + & + & + & + & + & & & & + & + & + & +- & + & - & + & + & + & & & & + & + & + & $+1-$ & + & ++ & + & \\
\hline 5 & & & + & + & + & + & + & + & & & & & & & & & & & & & & + & & & & & & + & & & & & & \\
\hline 6 & & & & & & & & + & + & + & + & & & & & & & & & -1 & $t$ & + & + & + & & & & & & & & + & + & \\
\hline 7 & & & + & + & + & + & + & + & & + & & & & & & & + & +-1 & ++ & t & t & + & + & + & & & & + & + & + & + & + & + & \\
\hline 8 & & & & & & & & & + & + & + & & & & & & & & 1 & +1 & t. & + & + & + & & & & & & & & & & \\
\hline 10 & & & & + & & & & + & & + & + & & & & 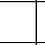 & & & 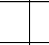 & & & & & + & + & & & & & & & & & & \\
\hline 11 & & +- & + & & + & + & + & & & & & & & & & & & - & $t$ & -1 & & & & & & & & + & & & & & & \\
\hline 12 & + & +- & + & + & + & + & + & & & & & & & + & + & + & + & +- & ++ & 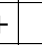 & & & & & + & + & + & + & + & - & + & & & \\
\hline 13 & + & + & + & + & + & & + & + & + & + & + & & & & & + & + & +1 & $+t$ & t & t & + & + & & & & & + & + & + & + & + & $+t$ & \\
\hline 14 & & & & + & + & & + & & & + & + & & & & + & + & + & & & & & $t$ & + & & 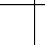 & 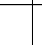 & & + & & + & + & + & + & \\
\hline 15 & + & + & + & + & + & + & + & + & + & & & & & + & + & + & + & +1 & +7 & 5 & 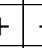 & + & + & & + & + & + & + & + & & +7 & + & $+t$ & \\
\hline 16 & & & & & + & & & + & + & + & + & & & & 4 & & & 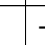 & $t$ & -1 & 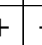 & + & & + & & & & & & & & & & \\
\hline 17 & + & + & + & + & + & + & + & + & & + & + & & & & + & + & + & + & $t$ & 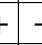 & 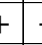 & + & + & + & + & + & + & + & + & + & + & + & $+t$ & \\
\hline 20 & + & & + & + & + & + & & & & & & & & & & & + & - & +1 & F & & & & & & & & + & & & & & & \\
\hline 21 & & & & & & & & & & & & & & & & & & + & & & & + & + & & 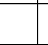 & & & & & & $+t$ & ++ & $t$ & \\
\hline 22 & & + & & & & & & & & & & & & & & & & & & & & & & & & & & & & & & & & \\
\hline 23 & & & & & & & & & & & & & & & & & + & 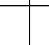 & & & & & & & + & & & + & & & -1 & + & & \\
\hline 25 & & & & & & & & & & & & & & & & & & 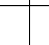 & & & & & & & & & & & & & & & & \\
\hline 26 & & & & & & & & & & & + & & & & & & & 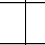 & & & & & + & + & & & & & & & & & + & \\
\hline 27 & & & & & & & & & & + & & & & + & & & & 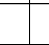 & 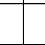 & & & & + & + & & & & & & & & & & \\
\hline 28 & & & + & + & & + & & + & + & & & & & & & & & + & +7 & +7 & + & + & + & & & & & & + & + & & & & \\
\hline 31 & & & & & & & & & & & & & & & & & & & & & & + & + & & & & & & & & & & & \\
\hline 33 & & & & & & & & & & & & & & & & & & & & & & & & & & & & & & & & & & \\
\hline 34 & & & & & & + & & & & & & & & & & & & & & & & & & & & & & & & & & & & \\
\hline 35 & & & & & & & & & & & & & & & & & & & & & & & & & & & & & & & -1 & + & & \\
\hline 36 & & & & & & & & & & & & & & & & & & & & & & & & & & & & & & & & & & \\
\hline 38 & & & & & & & & & & & & & & & & & & & & & & & & + & & & & & & & & & & \\
\hline 39 & & & & & & + & & + & + & & & & & & & & & 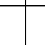 & & & & & + & & & & & & & & $t$ & ++ & & \\
\hline 41 & & & & & & + & & & & & & & & & & + & & & & & & + & & + & & & & & & & ++ & ++ & $+t$ & \\
\hline 43 & & + & & + & & + & & & & & & & & & + & + & . & +- & +1 & 5 & & + & & & 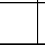 & & & + & + & + & & - & & \\
\hline 44 & +1 & + & + & + & + & + & + & & & & & + & + & + & + & + & + & + & + & & & & & & + & + & & + & + & 7 & ++ & + & & \\
\hline 46 & & & & & & & + & & & & & & & & & & & & & & & & & & & & & & & & & & & \\
\hline 47 & & & & & & & & + & & & & & & & & & & & & & & & & & & & & & & & & & & \\
\hline 48 & & & & & + & + & + & + & + & + & + & & & & + & & & - & + & 5 & + & + & + & & & & & + & + & 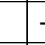 & + & + & $+1+$ & \\
\hline 49 & & & & & & & & & & & + & & & & & & & & & & & & + & & & & & & & & 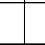 & & & \\
\hline 52 & & & & & & & & & & & & & & & & & & & & & & & & + & & & & & & & & & & \\
\hline 53 & & & & + & +1 & + & & + & + & & + & & & & & & 7 & +1 & $+t$ & +7 & +1 & + & + & & & & & + & + & -1 & ++ & + & $t$ & \\
\hline 57 & & & & & & + & & & & & & & & & & & & & & & & & & & & & & & & & & 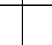 & & \\
\hline 58 & & + & & & + & & & & & & & & & & & & & - & + & & & & & & & & & + & & & $t$ & +1 & & \\
\hline 60 & & + & + & & & & & & & & & & & & & + & + & + & & & & & & & & & & + & + & + & + & 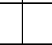 & & \\
\hline 61 & & & & & & & & + & + & & & & & & & & & & $t$ & + & & + & & + & & & & 4 & 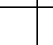 & & -1 & +1 & & \\
\hline 63 & & & & + & + & + & & & & & & & & & & + & +1 & + & ++ & $+t$ & + & & & & + & + & & + & + & + & + & ++ & $+t$ & \\
\hline 64 & + & + & + & + & & & & & & & & & + & + & + & + & & & & & & & & & + & + & + & + & & & & & & \\
\hline 70 & & & & & & & & & & & & & & & & & & & & & & & & & & & & & & & & & & \\
\hline 71 & & + & & & & & & + & & & & & & & & & & + & & & & & & & + & & & & & & & & & \\
\hline 72 & & & + & + & & & & & & & & & & & & & 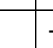 & + & & & & & & & + & & & & & & & & & \\
\hline 73 & +1 & + & + & + & & & & & & & & + & + & & + & + & + & & & & & & & & + & + & + & + & + & & & & & \\
\hline 76 & & & & & & & & & & & & & & & & & & & & & & & & & & & & & & & & & & \\
\hline 77 & + & + & + & + & + & + & + & + & + & & & + & + & + & + & + & + & + & + & $5-7$ & & + & & & + & + & + & + & + & - & + & + & + & \\
\hline 78 & + & & + & & + & + & + & & & & & + & + & + & + & & + & + & $t$ & & & & & & + & + & + & + & & & & & & \\
\hline 79 & + & & & & & & & & & & & + & + & + & + & & & & & & & & & & + & + & & & & & & & & \\
\hline 80 & + & + & + & & & & & & & & & 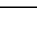 & & & -1 & & & & & & & & & & & + & + & + & + & & & & & \\
\hline 81 & + & +1 & + & + & + & + & + & & & & & + & + & + & + & + & + & + & & & & & & & + & + & + & + & + & & & & & \\
\hline 821 & & + & & + & & & & & & & & & & & & & & & & & & & & & & & & & & & & & & \\
\hline
\end{tabular}




\begin{tabular}{|c|c|c|c|c|c|c|c|c|c|c|c|c|c|c|c|c|c|c|c|c|c|c|c|c|c|c|c|c|c|c|c|c|c|c|c|}
\hline \multicolumn{36}{|c|}{ Table 9, 1991-3 } \\
\hline & \multicolumn{8}{|c|}{$\mathrm{A}$} & & & & & & & & & & & & & & & & & & C & & & & & & & & D & \\
\hline & 1 & 2 & 3 & 4 & & 6 & 7 & 8 & 1 & 2 & 3 & 4 & & 6 & 7 & 8 & 9 & 10 & 1 & 2 & 3 & 4 & 5 & 6 & & & 9 & & 11 & 12 & & 14 & & & 3 \\
\hline 86 & & & & & & & & & & & & & & & & & & & & & & & & & & & & & + & & + & + & & & \\
\hline 88 & + & & & & & & & & & & & & + & & & & & & & & & & & & & & & & + & & & + & & & \\
\hline 89 & & & & & & & & & & & & & & & & & & & & & & & & & & & & & + & & & & & & \\
\hline 91 & & & & & & & & + & & & & & & & & & & & & & & & & & & & & & & + & & + & & & \\
\hline 93 & & & & & & & & & & & & & & & & & & & & & & & & & & & & & & & & & & & \\
\hline 94 & & & & & & & & + & & & & & & + & & & & & & + & & & & & & & & & + & + & + & + & & & \\
\hline 100 & & & & & & & & & & & & & & & & & & & & & & & & & & & & & & & & & & & \\
\hline 103 & & & & & & & & & & & & & & & & & & & & & & & & & & & & & & & & & & & \\
\hline 115 & & & & & & & & + & & & & & & & & & & & & & & & & & & & & & & & & & & & \\
\hline 116 & & & & & & & & + & & & & & & - & & & & & & & & & + & & & & & & & + & & & & & \\
\hline 119 & & & & & & & & & & & & & & & & & & & & & & & & & & & & & & & & & & & \\
\hline 122 & & & & & & & & + & & & & & & & & & & & & & & & & 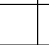 & & & & & & & & & & & \\
\hline 123 & & & & & & + & & + & + & + & & & & 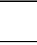 & 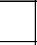 & 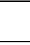 & & & + & & + & + & + & & & & & + & & & & & & & \\
\hline 124 & + & + & + & + & + & + & + & + & + & + & + & + & + & + & + & + & & & + & + & + & + & + & + & + & + & + & & & 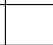 & . & & & + & + \\
\hline 125 & & & & & & + & & & & & & & & & & & & & & + & & & & & & & & & & & & & & & \\
\hline 126 & & & & & & & & & & & & & & & & & & & & & & & & & & & & & & & & & & & \\
\hline 130 & & & & & & & & & & & & & & & & & & & & & & & & & & & & & & + & & & & & \\
\hline 131 & & & & & & & & & & & & & & & & & & & & & & & & & & & & & & & & & & & \\
\hline 132 & & & & & & & + & + & & & & & & 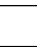 & & & + & + & & & & & & & & & + & + & + & + & & & & & \\
\hline 140 & & & & & & & & & & & & & & & & & & & & & & . & 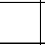 & & & & & & & & + & & & & \\
\hline 141 & & & & & & & & + & & & & & & & & & . & + & & & & 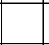 & & & & & + & & + & + & + & + & & & \\
\hline 142 & + & & & + & & + & & + & & & & & & 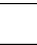 & & + & + & + & & & & & & & & + & + & + & + & + & + & + & & & \\
\hline 143 & & & & & & & & & & & & & & 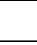 & & & & & & & & & & & & & & & & & & + & & & \\
\hline 144 & & & & & & & & . & & & & & & & & & & & & & & & & & & & & & & + & & & & & \\
\hline 145 & & & & & & & & + & & & & & & & & & & + & & & & & & & & & & & & & & & & & \\
\hline 146 & & & & & & & & + & & & & & & & & & & & & & & & & & & & & & 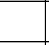 & + & & & & & \\
\hline 148 & & & & & & & & & & & & & & + & & & & & & & & & & & & & + & & & & & & & & \\
\hline 149 & & & & & & & & + & & & & & & & & & & + & & & & & & & & & & & & & & & & & \\
\hline 158 & & & + & & & & & & & & & & & & & & & & & & & + & & & & & & & & & & & & & \\
\hline 161 & & & & & & & & & & & & & & & & & & & & & & & & & & & & & & & & & & & \\
\hline 171 & & & & & & & & & & & & & & & & & & & & & & & & & & & & & 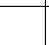 & & & & & & \\
\hline 198 & & & & & & + & + & & & & & & & 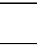 & + & + & & & & 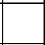 & & + & & + & + & & 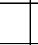 & & ( & & 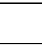 & 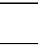 & + & + & + \\
\hline 199 & + & & & + & & + & + & + & + & & & & & & + & + & + & + & & + & + & + & & + & + & + & + & + & + & & & & + & + & + \\
\hline 200 & & & & & & + & + & + & & & & & & + & + & + & + & & & & + & + & & + & + & & + & & + & + & + & + & & & 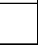 \\
\hline 201 & & & & & & & & & & & & & & & & & & & & & & & & & & & & & & & 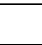 & & & & \\
\hline 202 & & & & & & & & & & & & & & & & & & & & & & & & & & & & & + & & 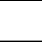 & & & & \\
\hline 203 & & & & & & & & & & & & & & & & & & & & & & & & & & & & & & & & & & & \\
\hline 207 & + & & + & + & & + & & & & + & & + & + & + & & & + & + & & + & + & & & & . & + & + & + & + & + & + & + & & & \\
\hline 209 & & & & & & & & & & & & & & & & & & & & & & & & & & & & & & & & & & & \\
\hline 210 & & & & & & & & & & & & & & + & & & & & & & & & 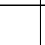 & 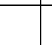 & & & & & & & & & & & \\
\hline 211 & + & & + & + & + & + & + & + & & & & & + & & + & + & + & & + & + & & + & + & + & + & + & + & + & & & & & & + & + \\
\hline 212 & & & & & & & & + & & & & + & & & & & & & & & & & & & & & & + & & + & + & + & & & \\
\hline 213 & & & & & + & & & + & & & & 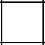 & & & 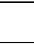 & & + & + & & 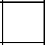 & . & 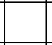 & 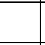 & 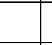 & . & 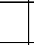 & 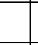 & & + & + & + & & & 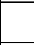 & \\
\hline 214 & + & & + & & + & + & & + & + & + & + & + & + & & + & + & + & + & + & + & + & + & + & + & + & + & + & + & + & + & + & + & + & + & + \\
\hline 215 & & & & & & & & & & & & & & & & & & & & & & & & & & & & & & & & & & & \\
\hline 216 & & & & & & & & & & & & & & & & & & & & & & & & & & & & & & & & & & & \\
\hline 217 & & & & & & & & & & & & & & & & & & & & & & & & & & & & & & & & + & & & \\
\hline 220 & & & & & & & + & & & & & + & & & & & & & + & + & & & & & & & & & & & & & & & \\
\hline 227 & + & & & & & + & + & + & & & & & + & & & + & + & & & & & & & + & & & + & + & & & & + & & + & \\
\hline 229 & + & + & + & + & + & & + & + & + & + & + & + & & & + & + & + & + & + & + & + & + & + & + & + & + & + & + & + & & & & + & + & + \\
\hline 230 & & & & & & & & & & & & & & & & & + & & & & & & & & & & & & & & & & & & \\
\hline 232 & + & + & & + & & & & + & & & & & & & + & + & + & + & & + & & & + & + & + & & + & + & + & & & & + & + & \\
\hline 233 & & & & & & & & & & & & & + & & & & & & & + & & & & & & & & & + & & & & & & \\
\hline 234 & & & & & & & & & & & & & & + & & & & & & & & & & & & & & & & & & & & & \\
\hline 235 & & & & & & & & & & & & & & & & & & & & + & + & & & & & & & & & & & & & & \\
\hline
\end{tabular}




\begin{tabular}{|c|c|c|c|c|c|c|c|c|c|c|c|c|c|c|c|c|c|c|c|c|c|c|c|c|c|c|c|c|c|c|c|c|}
\hline \multicolumn{33}{|c|}{ Table 9, 1991-4 } \\
\hline & \multicolumn{11}{|c|}{ D } & \multicolumn{12}{|c|}{$E$} & \multicolumn{9}{|c|}{$\mathrm{F}$} \\
\hline & 4 & $5 \mid$ & $\begin{array}{ll}6 & 7 \\
6\end{array}$ & \begin{tabular}{l|l}
7 & $\varepsilon$ \\
\end{tabular} & \begin{tabular}{|l|l}
9 & 9 \\
\end{tabular} & & \begin{tabular}{l|l}
0 & 1
\end{tabular} & & 2 & 13 & & 1 & 2 & 3 & 45 & 5 & & & & \begin{tabular}{l|l|}
9 & 10 \\
\end{tabular} & 11 & 12 & & 1 & 2 & $3 \mid$ & 45 & & & & \begin{tabular}{l|l}
8 & 9 \\
\end{tabular} & 10 \\
\hline 86 & & + & + & +1 & & & -1 & + & + & + & + & & & & & & + & 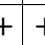 & $+1+$ & + & + & + & + & & & & 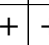 & + & & & + & \\
\hline 88 & & & & & & & & + & & & & & & & & & & & & & + & + & + & & & & & + & & & + & \\
\hline 89 & & & & & & & & & + & & + & & & & & & & & & + & + & + & + & & & & & & & & + & \\
\hline 91 & & & & & & & & & + & + & + & & & & & & & & & & & & + & & & & & & & & & \\
\hline 93 & & & & & & & & & & & & & & & & & & & & & & + & & & & & & & & & & \\
\hline 94 & & & & & & + & $+1-$ & + & + & + & & & & & & & & & & ++ & + & + & + & & & & & & & & & + \\
\hline 100 & & & & & & & & & & & & & & & & & & & & & & & + & & & & & & & & & \\
\hline 103 & & & & & & & - & + & & & & & & & & & & & & & & & & & & & & & & & & \\
\hline 115 & & & & & + & & - & + & + & + & & & & & & & - & + & & & & + & & & & & & & & t & & \\
\hline 116 & & & & & & & + & & & & & & & & & & & $+1+$ & ++ & ++ & & + & & + & & & +-1 & + & & & & \\
\hline 119 & & & & & & & - & + & & & & & & & & & & 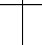 & 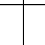 & & & . & & & & & & & & & & \\
\hline 122 & & & & & & & & & & & & & & & & & & $+1-$ & $+1+$ & + & + & & & & & & & + & $+1-$ & ++ & ++ & + \\
\hline 123 & & & +-1 & ++ & $-1+$ & & - & + & + & & & & & & & & & +- & + & + & + & & + & & & & + & + & + & + & + & \\
\hline 124 & + & + & $+1-1$ & $+t+$ & t & $t$ & & & & & & & + & + & + & + & + & +- & $t$ & + & & + & & + & + & + & + & + & + & + & + & \\
\hline 125 & & & + & & & & - & + & & & & & & & & & + +. & $+1-$ & $t$ & & & & & & & & + & & & + & + & \\
\hline 126 & & & & & & & & & & & & & & & & & & & & & & + & + & & & & & & 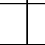 & 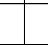 & . & \\
\hline 130 & & & & & & & - & + & & & & & & & & & & 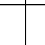 & & & & + & + & & & & & $t$ & + & -1 & + & \\
\hline 131 & & & & & & + & - & + & & & & & & & & & & $+1-$ & +1 & ++ & + & + & + & & & & & & & & + & $+t$ \\
\hline 132 & & & +-1 & +1 & $-1+$ & + & +7 & + & + & + & & & & & & + & . & +- & $t$ & + & & & + & & & & +1 & + & +1. & $+t$ & ++ & \\
\hline 140 & & & & & & & & & & & & & & & & & & & & & & & & & & & & & & & & \\
\hline 141 & & & & $t$ & $t$ & & - & + & + & + & + & & & & & & & +1 & + & + & + & + & + & & & & & & & ++ & ++ & + \\
\hline 142 & & & 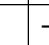 & $+t$ & +1 & + & + & + & + & + & + & & & & + & + & + & +- & +1 & ++ & + & + & + & & & & + & + & + & ++ & ++ & $+t$ \\
\hline 143 & & & & & & & & & & & + & & & & & & & & & & & + & & & & & & & & & & \\
\hline 144 & & & & & & & & & & & & & & & & & & 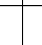 & & + & & & & & & & & & & . & 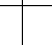 & \\
\hline 145 & & & + & $t$ & $-1+$ & & - & + & + & & & & & & & & & $t$ & + & & + & + & & & & & + & & & + & + & +1 \\
\hline 146 & & & & & & + & $t$ & + & + & + & & & & & & & . & $+t$ & + & + & + & + & + & & & & & & & t & ++ & + \\
\hline 148 & & & & & & & & + & & & & & & & & & & & & + & & & & & & & & & & & & \\
\hline 149 & & & & & & & 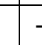 & $t$ & & & & & & & & & & + & & & & & + & & & & & & & & & + \\
\hline 158 & & & & & & & & & & & & & & & & & & & & & & & & & & & & & & & & \\
\hline 161 & & & & & & & & & & & & & & & & & & & & & & & & & & & & + & & & + & \\
\hline 171 & & & & & & & & & & & & & & & & & & 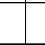 & & & & & & & + & & + & & & & 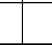 & \\
\hline 198 & + & & & & & & & & & & & + & + & + & + & & & & & & 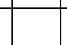 & & & + & + & + & + & & & & 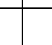 & \\
\hline 199 & + & + & +1 & +1 & $1+$ & $1+$ & +7 & + & + & & & + & & + & + & + & +1. & +7 & +7 & + & + & & & + & + & + & +1 & + & + & + & + & \\
\hline 200 & & & & $+t$ & $1+$ & $1+$ & $-1-$ & + & + & + & + & & & & + & & +1. & + & $+t$ & ++ & + & + & & & & & +1 & +1 & + & $+t$ & $+t$ & $+t$ \\
\hline 201 & & & & & & & & & & & & & & & & & & & & & & & & & & & & + & & & & \\
\hline 202 & & & & & & & & & & & & & & & & & & & & & & & & & & & & & & & & \\
\hline 203 & & & & & & & - & + & & & & & & & & & & & & & + & + & + & & & & & + & & & & \\
\hline 207 & & & 7 & +1 & $1+$ & $1+$ & & + & + & + & & & & & & + & +1 & +1 & +7 & ++ & + & + & + & & & & +1 & + & + & + & + & +1 \\
\hline 209 & & & & & & & & & & + & & & & & & & & & & & & & & & & & & & & & & \\
\hline 210 & & & & $t$ & & & & & & & + & & & & & & & & & & & & & & & & & & & & & \\
\hline 211 & + & + & +1 & $+t$ & $1+$ & $-1+$ & & & & & & + & + & + & + & & +1. & +1 & +1 & ++ & + & & & + & + & + & + & $t$ & + & 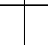 & & \\
\hline 212 & & & + & 7 & & & - & $t$ & + & & + & & & & & & + & & & & + & + & + & + & & & +1 & + & + & +1 & ++ & +1 \\
\hline 213 & & & 7 & $+t$ & $1+$ & $t$ & + & + & + & & + & & & 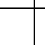 & & + & 7 & +7 & +7 & ++ & + & + & + & & 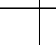 & & + & + & + & + & + & + \\
\hline 214 & + & + & +1 & +1 & $1+$ & $1+$ & & + & + & + & + & + & & + & + & + & +1. & +1 & $t$ & + & + & + & + & + & + & & +1 & + & & -1 & + & + \\
\hline 215 & & & & & & & & & & + & & & & & & & & & & & & & & & & & & & & & & \\
\hline 216 & & & & & & & & & & & & & & & & & & & & & & & + & & & & & & & & & \\
\hline 217 & & & + & + & $+t$ & & & + & & + & & & & & & & 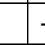 & + & & + & & & & & & & & & & & & + \\
\hline 220 & & & & 7 & $1+$ & & & & + & & + & & & & & & & +1 & +1 & + & & + & & & & & + & + & & + & +1 & ++ \\
\hline 227 & + & + & + & & $t$ & + & & & & & & & & & & & & & & & & & & + & & + & + & & & & & \\
\hline 229 & + & & + & $+1+$ & $1+$ & $t$ & $-1-$ & + & + & + & & & + & + & + & + & +1 & +1 & +1 & ++ & + & + & & + & + & + & +1 & + & + & + & & + \\
\hline 230 & & & -1 & + & + & $-1+$ & t & & & & & & & & + & & +1. & + & $t$ & ++ & & & & & & & & +1 & + & & & \\
\hline 232 & + & + & +1 & +1 & $+t$ & $1+$ & t & & + & + & & & & & + & & & - & +1 & + & + & & & & & + & + & + & + & & & \\
\hline 233 & & & & & & & - & + & & + & + & & & & & & & & & + & & + & + & & & & & + & + & + & & \\
\hline 234 & & & & + & $t$ & & & & & & + & & & & & & & & & + & & & & & & & & & & & & \\
\hline 235 & & & +1 & & & & & & + & & & & & & & & + & & & & + & & & & + & & & & & & + & + \\
\hline
\end{tabular}




\begin{tabular}{|c|c|c|c|c|c|c|c|c|c|c|c|c|c|c|c|c|c|c|c|c|c|c|c|c|c|c|c|c|c|c|c|c|c|c|}
\hline \multicolumn{35}{|c|}{ Table 9, 1992-1 } \\
\hline & \multicolumn{8}{|c|}{$\mathrm{A}$} & \multicolumn{9}{|c|}{$\mathrm{B}$} & & & & & & & & C & & & & & & & & D & \\
\hline & 1 & 2 & 3 & 4 & & 6 & $\begin{array}{ll}78 \\
\end{array}$ & $\begin{array}{ll}31 \\
\end{array}$ & \begin{tabular}{l|l}
12 \\
\end{tabular} & 3 & 4 & 5 & 6 & 7 & 8 & 9 & 10 & 1 & 2 & 3 & 4 & 5 & \begin{tabular}{l|l}
6 \\
\end{tabular} & & & \begin{tabular}{l|l}
9 & 1 \\
\end{tabular} & & 11 & 12 & & 14 & & & 3 \\
\hline 2 & & & & & + & & $1+$ & + & & & & & & & & & & & & & & & & & & & & & & & & & & \\
\hline 3 & & & & & & & & & & & & & & & & & & & & & & & & & & & & & & & & & & \\
\hline 4 & + & + & + & + & + & + & $+1+$ & ++ & ++ & -1 & + & + & + & + & + & + & + & + & + & + & + & + & & +- & +-1 & 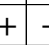 & + & + & + & + & & & + & + \\
\hline 5 & & & & & & + & & ++ & & & & & & & + & + & & & & & & & & & & & & & & & & & + & \\
\hline 6 & & & & & & & & & & & & & & & & & & & & & & & & & & & & + & & + & + & & & \\
\hline 7 & & + & & & + & & + & ++ & ++ & & + & & + & & & + & + & & & & + & & + & & +-1 & + & & + & + & + & + & & & \\
\hline 8 & & & & & & + & & & & & & & & & & & & & & & & & & & & & & + & & & & & & \\
\hline 10 & & & & & & & & & & & & & & & & & & & & & & & & & & & & & & & + & & & \\
\hline 11 & & + & & & & & $+1+$ & & & + & & & . & 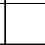 & & + & & & & & 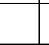 & & + & +1 & $+1-1$ & $+-1-$ & + & + & & & + & & & \\
\hline 12 & + & + & + & + & + & + & + & ++ & ++ & + & + & + & + & + & + & + & & + & + & + & + & + & + & + & +-1 & + & & & & & & + & + & \\
\hline 13 & + & + & + & + & + & + & + & ++ & ++1 & + & + & + & + & + & + & + & + & + & + & + & + & + & + & + & +7 & + & + & + & + & + & + & & + & + \\
\hline 14 & + & + & + & + & + & + & + & $+t$ & $+1+$ & + & + & + & & + & & & + & & + & + & + & + & & & 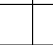 & & & & + & & + & + & + & + \\
\hline 15 & + & + & + & + & + & + & +1 & ++ & $+1+$ & $+t$ & + & + & + & + & + & + & + & + & + & + & + & + & + & + & + & + & + & + & & & + & + & + & + \\
\hline 16 & & & & & + & & & & & & & & & 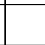 & & & & & & & + & & & & + & & & & + & + & + & & & \\
\hline 17 & & & + & & + & + & $+1-$ & ++ & & + & + & + & & + & + & + & + & & & + & + & + & + & + & + & + & + & + & + & + & + & + & + & + \\
\hline 20 & + & + & & & + & & + & + & & $t$ & & & - & & & & & + & & + & + & & & & + & & & & & & & + & & \\
\hline 21 & & & & & & & & & & & & & & & & & & & & & & & & & & & + & & & & & & & \\
\hline 22 & & & & & & & & + & & + & & & & & & & & & & + & & & & & 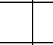 & & & & & & & & & \\
\hline 23 & & & & & & & & & & & & & & & & & & + & & & & & & & & & & & & & & & & \\
\hline 24 & & & & & & & & & & & & & & & & & & & & & & & & & & & & & & & & & & \\
\hline 25 & & & & & & & & & & & & & & & & & & & & & & & & & & & & & & & & & & \\
\hline 26 & & & & & & & & & & & & & & & & & & & & & & & & & & & & + & & & + & & & \\
\hline 27 & & & & & & & & & & & & & & & & 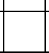 & & & & & & & & & & & & + & + & & + & & & \\
\hline 28 & & & & & & + & $t$ & + & & & & & & & & + & + & & & & & & & & & & & & & & & & & \\
\hline 29 & & & & & & & & & & & & & & & & & & & & & & & & & & & & & & & & & & \\
\hline 33 & & & & & & & $t$ & + & & & & & & & & & & & & & & & & & & & & & & & & & & \\
\hline 34 & & & & & & & & & & & & & & & & & & & & & & & & & & & & & & & & & & \\
\hline 36 & & & & & & & & & & & & & & & & & & & & & & & & & & & & & & & & & & \\
\hline 39 & & & & & & & & + & & & & & & & & & & & & & . & & & & & & & & & & + & & & \\
\hline 41 & & & & & & & & & & & & & & & + & & & & & & & & & & & & + & & & & & & & \\
\hline 43 & & & & & + & & & + & & + & + & & & 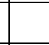 & 5 & & & & & & & . & & & - & + & & & + & & & & & \\
\hline 44 & + & + & + & + & + & + & + & $t$ & + & $t$ & + & + & + & + & + & + & + & + & + & + & + & + & + & + & + & + & + & + & & & & + & + & + \\
\hline 47 & & & & & & & & & & & & & & & & & & & & & & & & & & & & & & & & & & \\
\hline 48 & & & & & & & & + & & & & & & & & + & + & & & & & & & & + & + & + & + & + & + & + & & & \\
\hline 49 & & & & & & & & & & & & & & & & & & & & & & & & & & & & & & & + & & & \\
\hline 50 & & & & & & & & & & & & & & & & & & & & & & & & & & & & & & & & & & \\
\hline 52 & & & & & & & & & & & & & & & & & & & & & & & & & & & & & & & & & & \\
\hline 53 & + & + & & + & + & & & + & $t$ & $-1+$ & + & + & + & & & & & & & 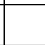 & 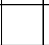 & & & & + & & & + & + & & + & & & \\
\hline 58 & & & & & & & + & & & & & & & & & & & & + & & & & & & & & & & & & & & + & \\
\hline 60 & + & + & + & + & + & + & & -1 & ++1 & $-1+$ & + & + & & & . & & & + & + & + & + & + & & & + & & & & & & & + & + & + \\
\hline 61 & & & & & & & & + & & & + & & & & & & & & & & & & & & & & & + & + & + & + & & & \\
\hline 62 & & & 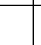 & & & & + & & 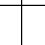 & & & 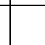 & & 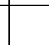 & 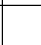 & & & 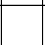 & & + & & . & 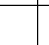 & & & & & & & & & & & \\
\hline 63 & + & + & + & + & + & + & +1 & +7 & $+1+$ & $1+$ & + & & & + & + & + & & + & + & & & + & + & + & + & + & + & & & & & + & + & + \\
\hline 64 & + & & + & & & + & + & & & & + & + & + & + & + & & & + & & + & + & + & + & + & + & + & & & & & & + & + & + \\
\hline 71 & + & + & + & & + & & & -1 & $+1+$ & t & + & + & & & & & & + & + & & & & & & & & & & & & & & & \\
\hline 72 & + & + & + & + & + & & + & - & $+t$ & +1 & + & + & & 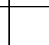 & & & & + & + & & 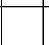 & 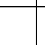 & & & & & & & & & & & & \\
\hline 73 & + & + & + & + & + & + & + & -1 & $++t$ & +1 & + & + & + & + & & & & + & + & + & + & + & + & + & & & & & & & & + & + & + \\
\hline 77 & + & & + & + & + & + & + & -1 & + & & + & + & + & + & + & + & & + & & + & + & + & + & + & + & + & + & & & & & + & + & + \\
\hline 78 & + & & & + & + & + & + & + & & & + & + & + & + & + & + & & + & & + & + & + & + & + & + & + & + & & & & & + & + & + \\
\hline 79 & + & & 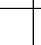 & & + & + & + & & & & & + & + & + & & & & & & + & + & + & & & & & & & & & & + & & \\
\hline 80 & & & + & & & & & & & & & & & & & & & & & & & & & & & & & & & & & & 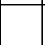 & \\
\hline 81 & + & & + & + & + & + & + & & & + & + & + & + & $1+$ & + & + & & + & + & + & + & + & + & + & + & +1 & + & & & & & + & + & + \\
\hline 82 & + & + & + & + & + & & & -1 & ++ & $-1+$ & + & + & & & & & & + & + & + & & & & & & & & & & & & & & \\
\hline 86 & & & & & & & & + & & & & & & & & & & & + & + & & & & & & & & & & & + & & & \\
\hline 87 & & & & & & & & & & & & & & & & & & & & & & & & & & & & & & & & & & \\
\hline
\end{tabular}




\begin{tabular}{|c|c|c|c|c|c|c|c|c|c|c|c|c|c|c|c|c|c|c|c|c|c|c|c|c|c|c|c|c|c|c|c|}
\hline \multicolumn{32}{|c|}{ Table 9, 1992-2 } \\
\hline & \multicolumn{10}{|c|}{ D } & \multicolumn{12}{|c|}{$E$} & \multicolumn{9}{|c|}{$\mathrm{F}$} \\
\hline & \begin{tabular}{l|l}
4 & 5 \\
\end{tabular} & 56 & \begin{tabular}{l|l}
6 & 7 \\
\end{tabular} & \begin{tabular}{l|l}
7 & 8
\end{tabular} & \begin{tabular}{|l|l}
9 \\
\end{tabular} & & \begin{tabular}{l|l|}
0 & 11 \\
\end{tabular} & 12 & 13 & & 1 & 2 & 3 & 4 & $5 \mid$ & 6 & \begin{tabular}{l|l}
7 & 8 \\
\end{tabular} & \begin{tabular}{|l|l|}
8 & 9 \\
\end{tabular} & 10 & 11 & & & 1 & 2 & \begin{tabular}{l|l}
3 & 2 \\
\end{tabular} & 4 & \begin{tabular}{l|l}
5 & 6 \\
\end{tabular} & & 8 & & 10 \\
\hline 2 & & & & & & & & & & & & & & & & & & & + & + & + & & & & & & + & & & & + \\
\hline 3 & & & & & & & & & & & & & & & & & & & & & & & & & & & & & & & + \\
\hline 4 & + & +1 & ++ & ++ & + & + & + & + & + & + & & & & + & + & + & ++ & ++ & + & + & + & + & & & +- & + & ++ & + & + & + & + \\
\hline 5 & & & & & & & + & & & & & & & & & & & & + & + & + & & & & & + & + & + & & & \\
\hline 6 & & & & & & & + & + & + & + & & & & & & & & 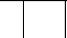 & & + & + & + & & & & & & 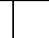 & & & + \\
\hline 7 & & & ++ & $+t$ & $-1+$ & + & + & + & + & + & & & & & & & ++ & ++ & + & + & & + & & & & + & & + & + & + & + \\
\hline 8 & & & & & & & + & & + & + & & & & & & & & + & & & + & & & & & & & 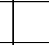 & & & \\
\hline 10 & & & & & & & + & & & & & & & & & & & & & & & & & & & & & & & & + \\
\hline 11 & ++ & ++ & ++ & $+t$ & + & + & + & + & & & & & & + & & + & ++ & & & & & & & + & + & & & + & & & \\
\hline 12 & $+t+r a t e$ & $+t$ & ++ & ++ & + & + & & & & & & & & +- & + & + & $+t$ & ++ & & & & & + & + & +- & + & + & + & & & \\
\hline 13 & + & & ++ & + & + & + & + & + & + & + & & & & + & + & + & $+t$ & ++ & + & + & & & 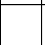 & 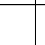 & - & & ++ & & + & + & + \\
\hline 14 & + & & & + & & & + & + & & & & & & + & + & & 1 & + & + & & & & + & & & + & & & + & + & + \\
\hline 15 & ++ & ++ & ++ & ++ & + & + & + & + & & & & + & + & + & + & + & ++ & ++ & + & + & + & & + & + & + & + & ++ & $-1+$ & + & + & + \\
\hline 16 & & & & & + & & + & + & + & + & & & & 7 & 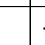 & + & & + & & & & & & & & & & & & + & \\
\hline 17 & + & + & ++ & ++ & $-1+$ & + & -1 & + & + & + & & + & + & + & + & + & $+t$ & ++ & + & & & + & + & + & +1 & + & ++ & $-1+$ & + & & + \\
\hline 20 & & & ++ & + & & & & & & & & & + & & + & + & $+t$ & ++ & & & & & + & & & + & & & & & \\
\hline 21 & & & & & & & & & & & & & & & & & + & & & & + & & & & & & & & + & & + \\
\hline 22 & & & & & & & & & & & & & & & & & & & & & & & & & & & & & & & \\
\hline 23 & & & & & & & & & & & & & & & & & & & & & & & & & & & & & & & \\
\hline 24 & & & & & & & & & & + & & & & & & & & & & & & & & & & & & & & & \\
\hline 25 & & & & & & & & & & + & & & & & & & & & & & & & & & & & & & & & \\
\hline 26 & & & & & & & + & & & + & & & & & & & 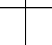 & & + & + & + & + & & & & & & & & & \\
\hline 27 & & & & & & & & + & & + & & & & & & & 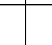 & & & & & + & & & & & & & & & \\
\hline 28 & & + & & & + & & + & + & & & & & & & & & & + & + & + & & & & & & & ++ & & + & + & + \\
\hline 29 & & & & & & & & + & & + & & & & & & & & & & & & + & & & & & + & & & + & \\
\hline 33 & & & & & & & & & & & & & & & & & & & & & & & & & & & & & & & \\
\hline 34 & & & & & & & & & & & & & & & & & & & & & & & & & & & & & & & + \\
\hline 36 & & & & & & & & & & & & & & & & & & & & & & & & & & & + & & & & + \\
\hline 39 & & & & & & & & & + & + & & & & & & & 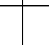 & 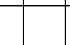 & + & + & + & + & & & & & & & & & + \\
\hline 41 & & & & & & & & & & & & & & & & & & & & & & + & & & & & & & & & \\
\hline 43 & & & + & + & & & & & & & & & & 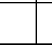 & &. & ++ & ++ & + & & & & 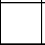 & & & + & 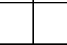 & 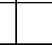 & & + & + \\
\hline 44 & ++ & +7 & ++ & ++ & +1 & + & + & & & & + & + & + & + & + & + & ++ & ++ & & & & & + & + & + & + & ++ & $-1+$ & & & 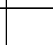 \\
\hline 47 & & & & & & & + & & + & & & & & & & + & & 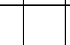 & & & & & & & & & & 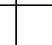 & & & \\
\hline 48 & & & & + & $+1+$ & $-1+$ & -1 & + & + & + & & & & & & & ++ & ++ & + & + & + & + & & & & & + & + & + & + & + \\
\hline 49 & & & & & & & & & & + & & & & & & & & & & & + & & & & & & & & & & \\
\hline 50 & & & & & & & & & & & & & & & & & & & & & & & & & & & + & & & & \\
\hline 52 & & + & & & & & & & & + & & & & & & & & & & & & & & & & & & & & & \\
\hline 53 & & & + & + & & + & $-1+$ & & + & + & & & & & & +1 & + & + & +1 & + & + & + & & & & + & + & $-1+$ & + & + & + \\
\hline 58 & & & + & & & & & & & & & & & & & & & & & & & & & & & 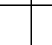 & + & & + & & \\
\hline 60 & + & & ++ & + & & & & & & & & & & & + & 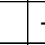 & + & & & & & & + & & & + & + & & & & \\
\hline 61 & & & & & & + & $-1+$ & + & & + & & & & & & & & + & + & + & + & + & & & & 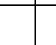 & & + & & + & + \\
\hline 62 & & & & & & & + & & & & & & & & & & & & & & & & & & & & & & & & + \\
\hline 63 & ++ & +7 & +1 & ++ & $+1+$ & & + & & & & & & 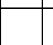 & & + & +1 & +1 & ++1 & & & & & + & + & + & + & ++ & $+1+$ & + & & \\
\hline 64 & ++ & +1 & +1 & + & & & & & & & + & + & + & + & + & + & & & & & & & + & + & +1 & + & & & & & \\
\hline 71 & & & & & & & & & & & & & & & & & & & & & & & + & & & & + & & & & \\
\hline 72 & & & & & & & & & & & & & & & & & & & & & & & & & & & & & & & \\
\hline 73 & + & +1 & $+1+$ & $t$ & & & & & & & + & + & + & + & + & +1 & + & & & & & & + & + & + & + & & & & & \\
\hline 77 & + & + & +1 & ++ & $+1+$ & + & $-1+$ & & & & + & + & + & + & + & +1 & ++ & ++1 & + & & & & + & + & + & + & + & + & & + & \\
\hline 78 & ++ & +7 & $+7+$ & +1 & + & + & & & & & + & + & + & + & + & +1 & + & & & & & & + & + & +1 & + & + & & & & \\
\hline 79 & & & & + & & & & & & & + & & & & & & & & & & & & & & & & & & & & \\
\hline 80 & & & & & & & & & & & & & & & & & & & & & & & & & & & & & & & \\
\hline 81 & + & & & + & $+1+$ & $-1+$ & & & & & + & + & + & + & + & & & & & & & & + & + & + & & & & & & \\
\hline 82 & & & & & & & & & & & & & & & & & & & & & & & & & & & & & & 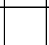 & 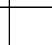 \\
\hline 86 & & $-t$ & + & & & & + & & & & & & & & & & ++ & ++ & & & + & + & & & & + & $+1+$ & & & + & + \\
\hline $87 \mid$ & & & & & & & & & & + & & & & & & & + & & & + & & & & & & & + & & & & \\
\hline
\end{tabular}


Table 9, 1992-3

\begin{tabular}{|c|c|c|c|c|c|c|c|c|c|c|c|c|c|c|c|c|c|c|c|c|c|c|c|c|c|c|c|c|c|c|c|c|c|c|}
\hline & & A & & & & \multicolumn{10}{|c|}{ B } & & & & & & & & 5 & & & & & & & & D & \\
\hline & 1 & 2 & 3 & 4 & & $\begin{array}{ll}6 & 7 \\
\end{array}$ & $\begin{array}{l}7 \\
7 \\
\varepsilon\end{array}$ & 8 & 12 & 23 & 4 & 5 & 6 & & 8 & 9 & 10 & 1 & 2 & 3 & 4 & 5 & \begin{tabular}{l|l}
6 \\
\end{tabular} & 7 | & 8 & & 0 & 11 & 12 & 13 & 14 & & & 3 \\
\hline 88 & & & & & & & & & & & & & & & & & & & & & & & & & & & & & & & & & & \\
\hline 89 & & & & & & & $-t$ & + & & & & & & & & & & & & & & & & & & & & & & & + & & & \\
\hline 91 & & & & & & & & & & & & & & & & & & & & & & & & & & & & & + & & + & & & \\
\hline 94 & & & & & & & & + & & & & & + & & & & & & & & & & & & & & & + & + & & & & & \\
\hline 97 & & & & & & & & & & & & & & & & & & & & & & & & & & & & & & & & & & \\
\hline 98 & & & & & & & & & & & & & & & & & & & & & & & & & & & & & & + & & & & \\
\hline 99 & & & & & & & & & & & & & & & & & & & & & & & & 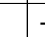 & + & & & + & & + & + & & & \\
\hline 100 & & & & & & & & & & & & & & & & & & & & & & & & & & & & & & & & & & \\
\hline 103 & & & & & & + & & & & & & & & & & & & & & & & & & & & & & & + & + & & & & \\
\hline 106 & & & & & & & & & & & & & & & & & & & & & & & & & & & & & & & & & & \\
\hline 107 & & & & & & & & & & & & & & & & & & & & & & & & & & & & & & + & & & & \\
\hline 111 & & & & & & & & & & & & & & & & & & & & & & & & & & & & & + & & & & & \\
\hline 112 & & & & & & & & & & & & & & & & & & & & & & & & & & & & & & & & & & \\
\hline 115 & + & & & & & & & & & & & & & & & & & & & & & & & & & & & + & & & & & & \\
\hline 116 & & & & & & & & & & & & & & & & & & & & & & & & & & & & & & & & & & \\
\hline 118 & & & & & & & & & & & & & & & & & & + & + & & & & & & & & & & & & & & + & \\
\hline 119 & & & & + & & & & & & \pm & & + & & 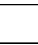 & & & 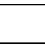 & & & & & & & & & & & & + & & 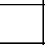 & & & \\
\hline 122 & + & & & & & & & & & & & & . & & & & & & & & & & & & & & & & & & & & 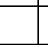 & \\
\hline 123 & & & & + & & +- & + & & & + & + & + & & + & & + & + & & & + & + & + & & + & + & + & + & + & + & & + & & & \\
\hline 124 & + & + & + & + & + & +- & $+1-$ & +-1 & ++ & $+t$ & + & + & + & + & + & + & + & + & + & + & + & + & + & + & + & + & & 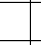 & & & & + & + & + \\
\hline 125 & & & & & & + & & & & + & & & & & & & & & & & & & & & & & & & & & & & & \\
\hline 130 & & & & & & & & & & & & & & & & & 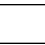 & & & & & & & & & & & & & & & & & \\
\hline 131 & & & & & & & & & & & & & 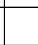 & & & & & & & & & & & & & & & + & & & & & & \\
\hline 132 & & & & & & +- & + & + & & & & 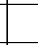 & 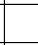 & & & + & + & & & + & & & & & & +- & + & + & & & & & & \\
\hline 141 & & & & & & & & & & & & & & & & & + & & & & & & & & + & & & + & + & + & + & & + & \\
\hline 142 & & & & + & + & & + & +7 & $+5+$ & & & + & & + & & + & + & & & & & + & & & + & + & $t$ & + & + & + & + & & & \\
\hline 143 & & & & & & & & & & & 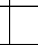 & & & & & & & & & & & & & & & & & & & + & + & & & \\
\hline 144 & & & & & & & & & & & & & & & & & & & & & & & & & & & & + & & & & & & \\
\hline 145 & & & & & + & + & + & & & & + & & & & & & + & & & & & & & & & & & + & & & & & & \\
\hline 146 & & & & + & & & & & & & & & 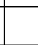 & & & & + & & & & & & & & & & & + & + & + & + & & & \\
\hline 148 & & & & & & & & & & & & & & & & & & & & & & & & & & & & & & & & & & \\
\hline 149 & & & & & & & & & & & & & + & & & & & & & & & & & & & & & & + & & & & & \\
\hline 154 & & & & & & & & & & & & + & & & & & & & & & & & & & & & & & & & & & & \\
\hline 156 & & & & & & & & & + & & & & & & & & & & & & & & & & & & & & & & & & & \\
\hline 158 & & & & & + & + & & & & & + & & & & & & & & & + & + & & & & & & & & & & & & & \\
\hline 176 & & & & & & & & + & & & & & & & & & & & & & & & & & & & & & & & & & & \\
\hline 198 & & & & & + & + & + & & & & & & & + & + & & & & & & + & + & + & & & & & & & & & & & + \\
\hline 199 & & & & & + & + & + & & & & & & & + & + & + & & & & & + & + & + & + & + & + & + & & & & & + & + & + \\
\hline 200 & & & & & & & $+1-$ & + & & & & & & & + & + & + & & & & 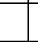 & & + & & + & + & & + & & & + & & & \\
\hline 202 & & & & & & & & & & & & & & & & & & & & & & & & & & & & & & & & & & \\
\hline 203 & & & & & & & & & & & & & & & & & & & & & & & & & & & & & & & + & & & \\
\hline 204 & & & & & & & & & & & & & & & & & & & & & & & & & & & & & & & + & & & \\
\hline 205 & & & & & & & & & & & & & & & & & & & & & & & & & & & & & & & & & & \\
\hline 207 & & & + & & + & & & & & & + & & + & & + & + & + & & + & + & & + & & + & & + & + & + & + & + & + & & & \\
\hline 209 & & & & & & & & & & & & & & & & & & & & & & & & & & & & & & & & & & \\
\hline 210 & & & & & & & & & & & & & & & & & & & & & & & & & & & & & & & & & & \\
\hline 211 & + & & + & + & + & + & + & -1 & t & & & & & + & + & + & & & & + & + & + & + & + & + & . & + & & & & & + & + & + \\
\hline 212 & & & & & & & & +-1 & $t$ & & & & & & & & & & + & & & & & & & & & + & + & + & + & & + & \\
\hline 213 & & & & & & & - & + & & & + & & & & & + & & & & & & & & & & 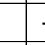 & $t$ & + & & & & & & \\
\hline 214 & + & + & + & & + & + & + & -1 & ++1 & +1 & + & + & + & + & + & + & + & & + & + & + & + & + & + & + & + & + & + & + & + & + & + & & + \\
\hline 216 & & & & & & & & & & & & & & & & & & & & & & & & & & & & & + & & & & & \\
\hline 220 & & & & & & + & & & & & & & & & & & & & & + & & & & & & & & + & + & & & . & & \\
\hline 227 & + & & & & & + & + & & & & & & & + & + & + & + & & & & + & & + & & + & + & + & & + & + & & + & & + \\
\hline 229 & + & + & + & + & + & & + & +-1 & ++ & $+1+$ & + & & & + & + & + & + & + & + & + & + & + & + & + & + & + & $t$ & + & & & & + & + & + \\
\hline 230 & & & & & & & + & + & & & & & & & & + & & & & & & & & & 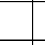 & \pm & $t$ & & & & & & & \\
\hline 232 & + & + & + & + & + & & & - & + & + & + & + & + & + & + & + & + & & + & + & & & + & + & + & + & $t$ & & & & & + & + & + \\
\hline 233 & & & & & & & & & & + & & & & & & & & & & & & & & & & & & + & & & + & & & \\
\hline 235 & & & & & & & & - & + & & + & & & & & + & & & + & & & + & & & & & + & & & & + & & & \\
\hline
\end{tabular}




\begin{tabular}{|c|c|c|c|c|c|c|c|c|c|c|c|c|c|c|c|c|c|c|c|c|c|c|c|c|c|c|c|c|c|c|c|c|c|c|}
\hline \multicolumn{35}{|c|}{ Table 9, 1992-4 } \\
\hline & & & & & & $\mathrm{D}$ & & & & & & & & & & & & $\mathrm{E}$ & & & & & & & & & & & 1 & & & & & \\
\hline & 4 & 5 & 6 & 7 & 8 & 9 & 10 & & & & & 1 & 2 & 3 & 4 & 5 & 6 & & 8 & 9 & 10 & 11 & 12 & & 1 & 2 & 3 & 4 & 5 & & & 8 & & 10 \\
\hline 88 & & & & & & & & & & & + & & & & & & & + & & & & & + & & & & & & + & & & & & + \\
\hline 89 & & & & & & & & & & & + & & & & & & & & & & + & & + & 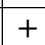 & & & & & & & & & & \\
\hline 91 & & & & & & & & & + & + & + & & & & & & & & & & & & & & & & & & & & & & & \\
\hline 94 & & & & & & & + & + & + & + & & & & & & & & & & & + & + & & & & & & & & & & & & \\
\hline 97 & & & & & & & & & & & & & & & & & & & & & & & & & & & & & & & & & & + \\
\hline 98 & & & & & & & & & & & & & & & & & & & & & & & & & & & & & & & & & & \\
\hline 99 & & & & & & & & + & & & + & & & & & & & & & & & + & & + & & & & & & & & & & + \\
\hline 100 & & & & & & & & + & & & & & & & & & & & & & & + & & & & & & & & & & & & \\
\hline 103 & & & + & & & & & & + & & & & & & & & & & & & & & & & & & & & & & & & & + \\
\hline 106 & & & & & & & & & + & & & & & & & & & & & & & & & & & & & & & & & & & + \\
\hline 107 & & & & & & & & & & & & & & & & & & & & & & & & & & & & & & & & & & \\
\hline 111 & & & & & & & & + & & & & & & & & & & & & & & & & & & & & & & & & & & \\
\hline 112 & & & & & & & + & & & & + & & & & & & & & & & & & & & & & & & & & & & & \\
\hline 115 & & & & & & & & & & & & & & & & + & & & & + & & & & & & & & & & + & + & & & \\
\hline 116 & & & & & & & & + & & & & & & & & + & & & & & & & & & & & & & & & & & + & \\
\hline 118 & & & & & & & & & & & & & & & & + & & & & & & & & & & & & & & & & & & \\
\hline 119 & & & & & & & & & & & & & & & & & & & & & & & & & & & & & & & & + & & \\
\hline 122 & & & & & & & & & & & & & & & & & & & + & + & & & & & & & & & + & & & + & & \\
\hline 123 & & & + & + & + & + & & + & & & & & & & & + & & + & + & + & + & + & + & & & & & + & + & + & + & + & + & + \\
\hline 124 & + & + & + & + & + & + & + & & 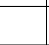 & & & & + & + & + & + & + & + & + & + & + & & & & + & + & + & + & + & & + & & & \\
\hline 125 & & & & & & & & & & & & & & & & & & & & & & & & & & & & & & & & & & \\
\hline 130 & & & & & & & & + & + & & & & & & & & & + & & & + & & + & & & & & 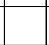 & & & & & & \\
\hline 131 & & & + & & + & & & + & & . & & & & & 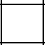 & 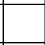 & 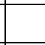 & 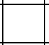 & 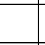 & + & & + & + & + & & & & & & 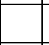 & + & & & + \\
\hline 132 & & + & + & + & + & + & + & + & + & & & & & & & + & & + & + & + & + & & 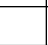 & & & & & + & + & & + & & + & \\
\hline 141 & & + & + & & & & & + & + & + & + & & & & + & & & & + & + & + & + & + & + & + & & & & + & + & + & + & & + \\
\hline 142 & & & + & + & & + & + & + & + & + & + & + & & + & & + & + & + & + & + & + & + & + & + & & + & + & + & + & + & + & + & + & + \\
\hline 143 & & & & & & & + & + & & & + & & & & & & & & & & & & & & & & & & & & & & & \\
\hline 144 & & & & & & & & & & & & & & & & & & & & & & & & & & & & & & & & & & \\
\hline 145 & & & & & + & & & + & & + & & & & & & + & & & + & + & + & + & & + & & & & & + & + & + & + & & + \\
\hline 146 & & & & & & & + & + & + & + & + & & & & & & & & + & + & + & + & + & + & & & & & & & + & + & + & + \\
\hline 148 & & & & & & & & & & & & & & + & & & & & & & & & & & & & & & & & & & & \\
\hline 149 & & & & & & & + & & & & & & & & & & & & & & + & & + & + & & & & & & & & & & \\
\hline 154 & & & & & & & & & & & & & & & & & & & & & & & & & & & & & & & & & & \\
\hline 156 & & & & & & & & & & & & & & & & & & & & & & & & & & & & & & & & & & \\
\hline 158 & & + & + & & & & & & & & & & & & & & + & & & & & & & & & & & & & & & & & \\
\hline 176 & & & & & & & & & & & & & & & & & & & & & & & & & & & & & & & & & & \\
\hline 198 & + & & & & & & & + & & & & & + & + & + & & & & & & & & & & + & + & + & & + & & & & & \\
\hline 199 & + & + & + & + & + & + & + & & + & & & + & + & + & + & & & & & + & & & & & + & + & + & & + & + & & & & \\
\hline 200 & & & + & & + & + & + & + & + & + & & & & & + & + & + & + & + & + & + & + & + & + & & & & & + & & + & + & + & + \\
\hline 202 & & & & & & & & & & & & & & & & & & & & & & & + & & & & & & & & & & & \\
\hline 203 & & & & + & & & & & & & & & & & & & & & & & & & & & & & & & & & & & & + \\
\hline 204 & & & & & & & & & & & & & & & & & & & & & & & & & & & & & & & & & & \\
\hline 205 & & & & & & & & & & & & & & & & & & & & & & & + & & & & & & & & & & & \\
\hline 207 & & + & + & + & + & + & + & + & + & + & + & & & & & + & + & & + & & + & & + & + & & & & + & + & + & + & & + & + \\
\hline 209 & & & & & & & & & & & + & & & & & & & & & & & & & & & & & & & & & & & \\
\hline 210 & & & & & & & & & & & & & & & & & & & & & & & & & & & & + & & & & & & \\
\hline 211 & + & & & & & + & + & & & & & + & & + & + & + & + & + & + & & & & & & + & + & + & & & 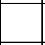 & 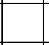 & & + & \\
\hline 212 & + & + & & & & & & + & & + & + & & & + & & + & + & + & & & + & + & + & + & & & & + & + & + & + & + & & \\
\hline 213 & & & + & & + & + & + & + & & + & + & & & & & 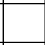 & + & & + & + & + & + & + & + & & & & & + & & $\mid$ & & + & + \\
\hline 214 & + & + & + & + & + & + & + & + & + & + & + & + & & + & + & + & + & + & + & + & + & + & + & + & + & + & & & + & + & + & + & + & + \\
\hline 216 & & & & & & & & & & & + & & & & & & & & & & & & & & & & & & & & & & & \\
\hline 220 & & & & & + & & + & & & + & + & & & & & & & & + & & + & & & + & & & & & & & + & & + & + \\
\hline 227 & + & + & & + & & & & & & & & & + & + & + & + & & & + & & + & & & & & & & & & & & & & \\
\hline 229 & + & + & + & + & + & + & + & + & + & & & & + & + & + & + & + & + & + & + & + & + & & & + & + & + & + & + & & + & & & + \\
\hline 230 & & & + & & + & + & + & & & & & & & & & + & + & + & & & & & & & & & & & + & & + & + & & \\
\hline 232 & + & + & + & + & ++ & + & & + & + & & & & + & + & + & + & + & + & + & + & + & & & & & & + & + & + & & & + & + & + \\
\hline 233 & & + & & & & & & + & & + & & & & & & & & & & & & & + & & & & & & + & + & & & & \\
\hline 235 & & & & & + & & & + & & & & & & & & & & & & + & & & & & & & + & + & + & & & & & \\
\hline
\end{tabular}




\begin{tabular}{|c|c|c|c|c|c|c|c|c|c|c|c|c|c|c|c|c|c|c|c|c|c|c|c|c|c|c|c|c|c|c|c|c|c|c|c|}
\hline \multicolumn{36}{|c|}{ Table 9, 1993-1 } \\
\hline & \multicolumn{8}{|c|}{$\mathrm{A}$} & & & & & $\mathrm{E}$ & & & & & & & & & & & & ( & C & & & & & & & & D & \\
\hline & 1 & 2 & 3 & 4 & & 6 & 7 & 8 & 1 & 2 & 3 & 4 & & 6 & 7 & 8 & 9 & 10 & 1 & 2 & 3 & 4 & 5 & 6 & & 8 & 9 & 10 & 11 & 12 & & 14 & & & 3 \\
\hline 2 & & & + & & + & + & & & & & & & & & & & & & & & & & & & & & & & + & & + & + & & & \\
\hline 3 & & & & & & & & & & & & & & & & & & & & & & & & & & & & & & & + & & & & \\
\hline 4 & & + & + & + & + & + & + & + & + & + & + & + & + & + & & + & + & + & & & & + & + & & + & + & + & + & + & & & + & & & \\
\hline 5 & & & & & & & & & & & & & & & & + & & & & & & & & & & & & & & & & & & & \\
\hline 6 & & & & & & & & & & & & & & & & & & + & & & & & & & & & & & + & & + & + & & & \\
\hline 7 & & & & & & & & + & + & + & + & + & & & & & + & + & & & & & & & & & + & + & + & + & + & + & & & \\
\hline 8 & & & & & & & & & & & & & & & & & & & & & & & & & D & & & & + & & & & & & \\
\hline 10 & & & & & & & & & & & & & & & & & & + & & & & & & & & & & & + & & & + & & & \\
\hline 11 & + & & & & & & & + & & 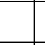 & & + & & + & + & + & + & & & & & & + & + & + & + & + & + & + & + & & & & & \\
\hline 12 & + & + & + & + & + & + & & + & + & + & + & + & + & + & + & + & + & & + & + & + & + & + & & + & + & + & & & & & & & & \\
\hline 13 & + & + & + & + & + & + & & + & & + & + & + & & + & & + & + & + & + & + & & + & + & & + & + & + & & + & & & + & & & \\
\hline 14 & & & + & & + & & & & + & + & + & + & & & . & 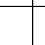 & 4 & & + & + & & & & . & & 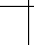 & 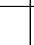 & & & & & & & & \\
\hline 15 & + & + & + & + & + & + & + & + & + & + & + & + & + & + & + & + & + & & & & + & + & + & + & + & + & + & + & & + & & & + & + & + \\
\hline 16 & & & & & & & & & & & & & & & & & + & & & 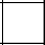 & & & & & & & + & & + & & + & + & & & \\
\hline 17 & + & + & + & + & & + & + & + & & 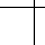 & & + & + & & + & + & + & & + & + & & 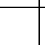 & + & & + & + & + & + & + & + & + & + & & & \\
\hline 20 & + & & + & & & & & & & + & & + & & & & & + & & & + & + & & & & & & 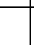 & & & & & & & & \\
\hline 21 & & & & & & & & & & & & & & & & & & & & & & & & & & & & & & & & & & & \\
\hline 23 & & & & & & + & & & & & & & & & & & & & + & & & & & & & & & & & & & & & & \\
\hline 25 & & & & & & & & & & & & & & & & & & & & & & & & & & & & & & & + & & & & \\
\hline 26 & & & & & & & & + & & & & & & & & & & + & & & & & & & & & 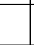 & . & + & + & + & + & & & \\
\hline 27 & & & & & & & & & & & & & & & & & & & & & & & & & & & & & & & + & & & & \\
\hline 28 & & & & & & + & & + & & & & & & & & & & + & & & & & & & & & + & + & 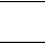 & + & + & & & & \\
\hline 29 & & & & & & & & & & & & & & & & & & & & & & & & & & & & & 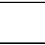 & & + & + & & & \\
\hline 30 & & & & & & & & & & & & & & & & & & & & & & & & & & & & & & & & & & & \\
\hline 36 & & & & & & & & & & & & & + & & & & & & & & & & & & & & & & & & & & & & \\
\hline 39 & & & & & & & & & & & & & & & & & & & & & & & & & & & & & + & + & + & + & & & \\
\hline 41 & & & & + & & & & & & & & & & & & . & & + & & & & & 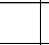 & & & & 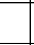 & & + & + & + & + & & & \\
\hline 43 & & & & & & & & & & & & & & & & + & + & & & & & 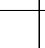 & . & 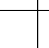 & & & 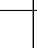 & & & & & + & & & \\
\hline 44 & + & + & + & + & & + & + & + & + & + & + & + & + & + & + & + & + & & + & + & + & + & + & + & + & + & + & + & + & & . & - & + & + & + \\
\hline 47 & & & & & & & & & & & & & & & & & & & & & & & & & & & & & & & & & & & \\
\hline 48 & & & & & & & & + & & & & & & & & & + & + & & & & & & & & & + & + & + & + & + & + & & & \\
\hline 49 & & & & & & & & & & & & & & & & & & & & & & & & & & & & & & & & + & & & \\
\hline 53 & & & & + & & & & & & & & & & + & & & & & & & & & & & & & + & & + & + & & & & & \\
\hline 53 & & & & & & & & & & & & & & & & & & & & & & & & & & & & & & & & & & & \\
\hline 54 & & & & & & & & & & & & & & & & & & & & & & & & & & & & & & & & & & & \\
\hline 57 & & & & & & & & & & & & & & & & & & & & & & & & & & & & & & & & & & & \\
\hline 58 & & & & & & & & & & & & & & & & & & & & & & & & & & & & & & & & & & & \\
\hline 60 & + & + & & & + & & & & & + & + & + & + & & & + & & & + & + & & & & & & & & & & & & & & & \\
\hline 61 & & & & & & & & & & & & & & & & & & & & & & & & & & & & & & & + & & & & \\
\hline 62 & & & & & & & & & & & & & & & & . & & & & & & 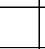 & 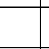 & & & & & & & & & & & & \\
\hline 63 & + & + & + & + & + & + & & & + & + & + & + & + & & + & + & + & & + & + & & + & + & + & + & + & & + & & & & & & + & + \\
\hline 64 & + & & & & & + & + & + & & & & + & + & + & + & + & & & + & & + & + & + & + & + & + & + & & & & & & & + & + \\
\hline 70 & & & & & & & & & & & - & & & & & & & & + & & & & & & & & & & . & & & & & & \\
\hline 71 & & & & + & + & + & & & & + & + & + & & + & & & & & + & & & & 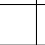 & & & & & & & & & & & & \\
\hline 72 & & & & + & & & & & 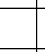 & + & + & + & & + & & 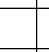 & & & 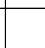 & & & 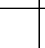 & - & & & & & & & & & & & & \\
\hline 73 & + & + & + & + & + & + & & & + & + & + & + & + & + & + & + & & & + & & & + & + & + & + & + & & & & & & & + & + & + \\
\hline 76 & + & & & & & & & & + & & & & & & & & & & & & & & & & & & & & & & & & & & \\
\hline 77 & & & & + & + & + & + & + & & & & & + & + & + & + & + & & & & . & 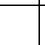 & 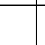 & & + & & + & + & + & & & & & + & \\
\hline 78 & & & & + & + & & + & + & + & & & & + & + & + & + & + & + & + & & + & + & + & + & + & + & + & & + & & & & + & & + \\
\hline 79 & + & & & & + & + & + & & + & & & & + & & + & + & + & & + & & + & + & + & + & + & & + & & & & & & + & & \\
\hline 80 & & & & & & & & & & & & & & & + & & & & & & & & & & & & & & & & & & & & \\
\hline 81 & + & & + & + & & & + & + & + & & & & + & & + & + & + & & + & & + & + & + & & & & + & & & & & & + & & \\
\hline 82 & & + & & + & & & & & & & & + & + & + & & & & & + & & & & & & & & & & & & & & & & \\
\hline 86 & & & & + & & + & & & & & & & & & & & & & & & & & & & & & & & + & & + & + & & & \\
\hline 87 & & & & & & & & & & & & & & & & & & + & & & & & & & & & & & + & & + & + & & & \\
\hline
\end{tabular}




\begin{tabular}{|c|c|c|c|c|c|c|c|c|c|c|c|c|c|c|c|c|c|c|c|c|c|c|c|c|c|c|c|c|c|c|c|c|}
\hline \multicolumn{33}{|c|}{ Table 9, 1993-2 } \\
\hline & \multicolumn{11}{|c|}{ D } & \multicolumn{12}{|c|}{$E$} & \multicolumn{9}{|c|}{$\mathrm{F}$} \\
\hline & 4 & 5 & 6 & 7 & 8 & 9 & & & 12 & 13 & & 1 & 2 & 3 & 4 & $5 \mid$ & $\begin{array}{ll}6 & 7 \\
6\end{array}$ & & \begin{tabular}{|l|l}
8 & 9
\end{tabular} & 10 & 11 & 12 & 13 & 1 & 2 & \begin{tabular}{l|l}
3 & 4 \\
4
\end{tabular} & 4 & & \begin{tabular}{l|l}
6 & 7
\end{tabular} & 8 & & 10 \\
\hline 2 & & & & & & & & & & & & & & & & & & & & & & + & & & & & & & & & & \\
\hline 3 & & & & & & & & & & & & & & & & & & & & & & & & & & & & & & & & \\
\hline 4 & + & + & + & + & + & + & + & + & + & + & + & & & & + & + & + & $+t$ & $+t$ & + & + & & + & & & $+t+$ & + & + & ++ & + & + & + \\
\hline 5 & & & & & & & + & & & & & & & & & & & & & & & & & & & & & & & & & \\
\hline 6 & & & & & & & & + & + & + & + & & & & & & & & & & & & & & & & & & & + & & + \\
\hline 7 & & & & & & + & + & + & + & + & + & & & & & & + & $+t$ & + & + & & + & & & & & & + & + & & + & \\
\hline 8 & & & & & & & & & & & + & & & & & & & 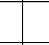 & & & & + & + & & & & & & 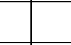 & & & \\
\hline 10 & & & & & + & & & + & & & & & & & & & & + & & & + & & & & & & & & & & + & \\
\hline 11 & & + & + & + & + & + & + & & & & & & & & + & &.+ & + & & . & & & & & & & + & + & + & & & \\
\hline 12 & & + & + & + & + & + & + & & & & & & & & +- & + & + & $+t$ & & 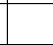 & & & & + & + & $+t+r a t s$ & + & + & & + & & \\
\hline 13 & & + & + & + & + & + & + & + & + & + & + & & & & & + & + & $+t$ & $+t$ & + & & & & 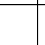 & 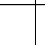 & +1 & + & ++ & ++ & + & + & + \\
\hline 14 & & + & & & & & & & & & & & & & & & & & & & & & & & & & & & & & & \\
\hline 15 & + & + & + & + & + & + & + & + & & & & & & + & + & + & +1. & $+t$ & +1 & + & + & + & & + & + & +1 & + & + & ++ & + & + & + \\
\hline 16 & & & & & & & & + & + & + & + & & & & & & & + & + & & & & + & & & & & & + & + & + & \\
\hline 17 & & & & + & + & +1 & + & + & + & + & + & & & & & & +1 & $+t$ & +1 & + & + & & + & + & & $+t$ & + & + & + & + & + & + \\
\hline 20 & & & + & & + & + & & & & & & & & & & + & +1. & + & & & & & & + & & $+t$ & + & & ++ & & & \\
\hline 21 & & & & & & & & & & & & & & & & & & & & & + & & & & & & & & & & & \\
\hline 23 & & & & & & & & & & & & & & & & & + & 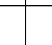 & & & & & & & & & & & & & & \\
\hline 25 & & & & & & & & & & & & & & & & & & & & & & & & & & & & & & & & \\
\hline 26 & & & & & & & & + & & & + & & & & & & & - & & & + & + & + & & & & & & & & & \\
\hline 27 & & & & & & & & & & + & & & & & & & & 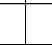 & - & & + & & & & & & & & 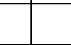 & & & \\
\hline 28 & & + & & & & + & & + & + & & & & & & & + & + & 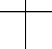 & + & & 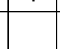 & . & & & & & & + & + & & + & + \\
\hline 29 & & & & & & & & & & & & & & & & & & + & & & + & & & & & & & & 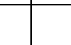 & & & \\
\hline 30 & & & & & & & & & & & & & & & & & & & & & & + & & & & & & & & & & \\
\hline 36 & & & & & & & & & & & & & & & & & & + & & & & & & & & & & & & & & \\
\hline 39 & & & & & & + & & & & & & & & & & 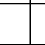 & & & & & + & & + & & & & & & & & + & \\
\hline 41 & & & & & & & & + & & . & & & & & . & & & 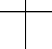 & & & + & + & + & & & 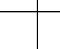 & & & & + & + & + \\
\hline 43 & & & 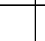 & & & & + & & & + & + & & 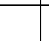 & & + & + & + & 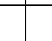 & & + & & 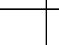 & & 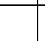 & & +7 & + & & & 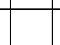 & 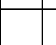 & \\
\hline 44 & + & + & + & + & + & + & + & + & & & & + & + & + & + & + & +1. & ++ & & + & & & & + & + & $+t$ & + & + & & 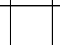 & & \\
\hline 47 & & & & & & & & & & & & & & & & & & & & & & + & & & & & & & & & & \\
\hline 48 & & & & & + & + & + & + & + & + & + & & & & & & 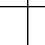 & + & +1 & + & + & + & + & & & & & $+1+$ & ++ & + & + & + \\
\hline 49 & & & & & & & & & & + & + & & & & & & & & & & & & & & & & & & & & & \\
\hline 53 & & & & & + & & & & + & & + & & & & & & +1 & ++ & & & & + & + & & & & & & + & & + & \\
\hline 53 & & & & & & & & & & & + & & & & & & & & & & & & & & & & & & & & & \\
\hline 54 & & & & & & & & & & & & & & & & & & & & & & & & & & & & & & & + & \\
\hline 57 & & & & & & & & & & & & & & & & & + & & & & & & & & & & & & & & 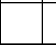 & \\
\hline 58 & & & & & & & & & & & & & & & & + & & & & & & & & & & & & & & + & + & \\
\hline 60 & & & & & & + & & & & & & & & & & + & 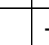 & + & & & & & & & & 7 & + & + & + & . & 4 & \\
\hline 61 & & & & & & & + & + & & + & + & & & & & & & + & & + & + & + & + & & & & & & + & & + & \\
\hline 62 & & & & & & & & & & & & & & & & & & & & & & + & & & & & & & & & & \\
\hline 63 & + & + & + & + & + & + & + & & & & & & & & + & + & + & ++ & + & & & & & + & + & +1 & + & +1 & ++ & + & & \\
\hline 64 & + & + & + & + & & & & & & & & + & + & + & + & & +1 & & + & & & & & + & + & $+t$ & + & + & & & & \\
\hline 70 & & & & & & & & & & & & & & & & & & & & & & & & & & & - & & & & & \\
\hline 71 & & + & & & & & & & & & & & & & & & & & & & & & & & & & & & & & & \\
\hline 72 & & & & & & & & & & & & & & & & & & & & & & & & & & & & & & & & \\
\hline 73 & + & + & + & + & & & & & & & & + & + & + & + & + & +1. & + & & & & & & + & + & $+t$ & + & + & & & & \\
\hline 76 & & & & & & & & & & & & & & & & & & & & & & & & & & & & & & & & \\
\hline 77 & + & & + & + & + & & & & & & & + & + & + & + & + & +1 & + & + & & & & & + & + & $+t$ & + & + & & & & \\
\hline 78 & + & + & + & & + & + & + & & & & & + & + & + & + & + & +1 & + & & & & & & + & + & $+t$ & + & + & & & & \\
\hline 79 & + & & & & & & & & & & & & + & + & + & & & & & & & & & + & + & & & & & & & \\
\hline 80 & & + & & & & & & & & & & & & & & & + & & & & & & & & & & & & & & & \\
\hline 81 & + & & & & & & & & & & & + & + & & + & & . & + & & & & & & + & + & $+t$ & + & + & & & & \\
\hline 82 & & + & & & & & & & & & & & & & & & & + & & & & & & + & & & & & & & & \\
\hline 86 & & & & & & + & & + & & & + & & & & & + & & 7 & $t$ & & + & + & + & & & & & + & 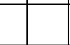 & 7 & 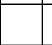 & \\
\hline 87 & & & & & & & & + & & + & + & & & & & & & + & & & & + & & & & & & + & & & & \\
\hline
\end{tabular}


Table 9, 1993-3

\begin{tabular}{|c|c|c|c|c|c|c|c|c|c|c|c|c|c|c|c|c|c|c|c|c|c|c|c|c|c|c|c|c|c|c|c|c|c|c|}
\hline & & & & A & & & & & & & & & $B$ & & & & & & & & & & & & C & & & & & & & & $\bar{D}$ & \\
\hline & 1 & 2 & 3 & 4 & 5 & \begin{tabular}{l|l}
6 \\
\end{tabular} & & & 1 & & 3 & 4 & $\begin{array}{l}5 \\
5\end{array}$ & \begin{tabular}{l|l}
6 & 7
\end{tabular} & 78 & 9 & 10 & 01 & 2 & 3 & 4 & 5 & 6 & 7 & 8 & 9 & & 11 & 12 & & 14 & \begin{tabular}{|l|}
1 \\
\end{tabular} & & 3 \\
\hline 88 & & & & & & & & & & & & & & & & & & & & & & & & & & & & & & & & & & \\
\hline 89 & & & & & & & & & & & & & & & & & & & & & & & & & & & & & & & & & & \\
\hline 90 & & & & & & & & & & & & & & & & & & & & & & & & & & & & & + & & & & & \\
\hline 91 & & & & & & & & & & & & & & & & & & & & & & & & & & & & & & & & & & \\
\hline 93 & & & & & & & & & & & & & & & & & & & & & & & & & & & & & & & & & & \\
\hline 94 & & & & & & & & + & & & & & & $t$ & & & + & & & & & & & & & & & & & & & & & \\
\hline 99 & & & & & & & & & & & & & & & & & & & & & & & & & & & & + & & + & & & & \\
\hline 100 & & & & & & & & & & & & & & & & & & & & & & & & & & & & & & & & & & \\
\hline 103 & & & & & & & & & & & & & & & & & & & & & & & & & & & & & & & & & & \\
\hline 114 & & & & & & & & & & & & & & & & & & & & & & & & & & & & & & & & & & \\
\hline 115 & & & & & & & & & & & & & & & & & & & & & & & & & & & & & & & & & & \\
\hline 116 & & & & & & & & & & + & + & & & & & & 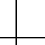 & & & & & & & & . & & & & & & & & & \\
\hline 118 & & & & & & & & & & & & & & & & & & + & & & 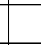 & & & & & & & & & & & & & \\
\hline 119 & & & & & & & & & & & & + & + & & & & 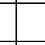 & & & & & & & & & & & + & + & + & & & & \\
\hline 121 & & & & & & & & & & 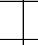 & & & & & & & ( & & & & & & & & & & & & + & & & & & \\
\hline 122 & & & & & & + & & & & & & & & & D & 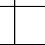 & & & & 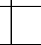 & 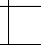 & & & D & 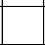 & 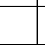 & & 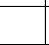 & & & & & & \\
\hline 123 & & & + & + & & + & & + & + & + & + & +1 & +1 & $t$ & 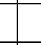 & 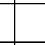 & + & & + & 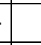 & & + & & 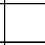 & 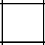 & & & + & + & + & & & & \\
\hline 124 & + & + & + & + & + & + & + & + & + & + & + & + & +7 & +7 & $5+$ & + & & + & + & + & + & + & + & + & + & + & & + & & & & + & & + \\
\hline 125 & & & & & & & & & & & & + & & & & & & & & & & & & & & & + & & & & & & & \\
\hline 128 & & & & & & & & & & & & & & & & & & & & & & & & & & & & & & & & & & \\
\hline 129 & & & & & & & & & & & & & & & & & & & & & & & & & 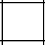 & 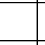 & & & & & & & & \\
\hline 130 & & & & & & & & & & & & & & & & & + & & & & & 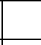 & & & & & & + & & & & & & \\
\hline 131 & & & & & & & & & & & & & & & & & & & & 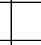 & & & & & & & & & + & & & & & \\
\hline 132 & & & & & & + & & + & & & & & & & & + & $-t$ & & & & 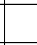 & + & & & + & + & + & + & + & & & & & \\
\hline 141 & & & & & & & & & & & & & & -1 & $1+$ & & + & & & 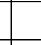 & 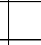 & & + & & + & & + & + & + & + & + & & & \\
\hline 142 & + & & & & & & & + & & + & + & + & & & & & $t$ & & & & & & & & 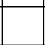 & & + & + & + & + & + & & & \\
\hline 143 & & & & & & & & & & & & & & & & & & & & & & & & & & & & & & + & & & & \\
\hline 144 & & & & & & & & & & & & & & & & & & & & & & & & & & & & & & + & & & & \\
\hline 145 & & & & + & & + & & & & & & - & + & & & & & & 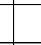 & & & & & & & & & 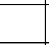 & & + & & & & \\
\hline 146 & & & & & & & & & & & & & & & & & & & & & & & & & & & & + & + & + & & & & \\
\hline 149 & & & & & & & & & & & & & & & & & & & & & & & & & & & & & & & & & & \\
\hline 156 & & & & & & & & & & + & & & & & & & & & + & & & & & & & & & & & & & & & \\
\hline 158 & & & & & & & & & & & & & & & & & & & & & & & & & & & & & & & & & & \\
\hline 159 & & & & & & & & & & & & & & & & & & & & & & & & & & & & & & & & & & \\
\hline 161 & & & & & & & & & & & & & & & & & & & & & & & & & & & & & + & & & & & \\
\hline 177 & & & & & & & & & & & & & & & & & & & & & & & & & & & & & & & & & & \\
\hline 198 & & & & & & & + & & & & & & + & & & + & & & & & & & + & & & & . & & & & & & & \\
\hline 199 & & & & & & + & + & + & & & & . & + & -1 & $t$ & + & & & & & & + & & & + & & & + & & 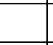 & & & & \\
\hline 200 & & & & & & & + & + & & & & & & & & + & $t$ & & & & & & & & & + & + & + & + & & + & & & \\
\hline 202 & & & & & & & & & & & & & & & & & & & & & & & & & & + & & & & & & & & \\
\hline 205 & & & & & & & & & & & & & & & & & & & & & & & & & & & & & & & & & & \\
\hline 207 & + & + & + & & & & & - & & & & & & & & + & -1 & & & 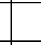 & & & 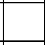 & & + & + & + & + & + & + & + & & & \\
\hline 210 & & & & & & & & & & & & & - & + & & & & & & & & & & & & & & & & & & & & \\
\hline 211 & & & & & & & + & + & & & & & & - & +1 & ++ & & & & + & & & & + & + & + & & 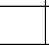 & & & & & & + \\
\hline 212 & & & & & & & & + & & & & & & & & & & & + & & & 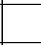 & 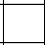 & & & & & & & & & & & \\
\hline 213 & & & + & & & & & + & & & & & + & & & & + & & & & & & & & & & & & + & & & & & \\
\hline 214 & + & + & & & + & & & + & + & + & + & + & +1 & +-1 & +1 & $+t$ & $-1+$ & + & + & + & + & + & + & + & + & + & + & + & + & + & + & + & & + \\
\hline 216 & & & & & + & & & & & & & & & & & & & & & & & & & & & & & & & & & & & \\
\hline 220 & & & + & & & & & + & & + & + & & + & & & & + & & & & & & & & & & & & + & + & + & & & \\
\hline 222 & & & & & & & & & & & & & & & & & & & & & & & & & & & & + & & & & & & \\
\hline 223 & & & & & & & & & & & & & & & & & + & & & & & & & & & & & & & & & & & \\
\hline 227 & & & & & & & & & & & & & & & + & ++ & & & & & & & & & + & + & & & & & & + & & \\
\hline 229 & + & + & + & & + & + & + & & + & + & + & + & & -1 & $5+$ & $+t$ & -1 & + & + & & & & + & + & + & + & + & + & & 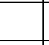 & & + & & + \\
\hline 230 & & & & & & & + & + & & & & & & & & + & & & & & & & & + & + & + & & + & & & & & & \\
\hline 232 & & & & + & & & & + & & & & & - & +-1 & +1 & $+t$ & -1 & & & & & & & + & + & + & + & + & + & + & & + & & + \\
\hline 233 & & & & & & & & & & & & & & & & & & & & & & & & & & & & + & + & & & & & \\
\hline 235 & & & & & & & & & & & & & & & & + & & & & & & & & & & & & & & & & & & \\
\hline 240 & & & & & & & & & & & & & & & & & & & & & & & & & & & & & & & & & & L \\
\hline
\end{tabular}




\begin{tabular}{|c|c|c|c|c|c|c|c|c|c|c|c|c|c|c|c|c|c|c|c|c|c|c|c|c|c|c|c|c|c|c|c|c|c|c|}
\hline \multicolumn{35}{|c|}{ Table 9, 1993-4 } \\
\hline & & & & & & D & & & & & & & & & & & & $\mathrm{E}$ & & & & & & & & & & & 1 & & & & & \\
\hline & 4 & 5 & 6 & 7 & 8 & 9 & & & & & & 1 & 2 & 3 & 4 & 5 & 6 & & 8 & 9 & & & & & 1 & 2 & 3 & 4 & 5 & 6 & & 8 & & 10 \\
\hline 88 & & & & & & & & + & & & & & & & & & & & & & & & & + & & & & & + & & + & + & & \\
\hline 89 & & & & & & & & & & & & & & & & & & & & & & + & + & + & & & & & & & & & & + \\
\hline 90 & & & & & & & & & & & & & & & & & & & & & & & & & & & & & & & & & & \\
\hline 91 & & & & & & & & & + & + & & & & & & & & & & & & & & & & & & & & & & & & \\
\hline 93 & & & & & & & & & & & & & & & & & & & & & & + & & & & & & & & & & & & + \\
\hline 94 & & & & & & & & & & & & & & & & & & & & & + & & & & & & & & & & & & + & \\
\hline 99 & & & & & & & & & + & & & & & & & & & & & & & & & & & & & & & & & & & + \\
\hline 100 & & & & & & & & & & & & & & & & & & & & & & + & & & & & & & & & & & & \\
\hline 103 & & & & & & + & & & & & & & & & & & & & & & & & + & & & & & & & & + & & & \\
\hline 114 & & & & & & & & & & & & & & & & & & & & & & + & & & & & & & & & & & & \\
\hline 115 & & & & & & & & + & & & & & & & & & + & + & & & & & & & & & & + & & + & + & & & \\
\hline 116 & & & & & & & & & + & & & & & & & & & & & & + & + & + & & + & & & + & + & & & + & & \\
\hline 118 & & & & & & & & & & & & & & & & & & & & & & & & & & & & + & + & & & & & \\
\hline 119 & & & & & & & & & & + & + & & & & & & & & & & & & & & & & & & & & & + & & \\
\hline 121 & & & & & & & & & & & & & & & & & & & & & & & & & & & & & & & & & & \\
\hline 122 & & & & & & + & & & & & & & & & & & & & + & & & & & & & & & & + & & + & + & + & + \\
\hline 123 & & & + & & + & + & & + & + & & & & & & + & + & & + & + & + & & + & + & & & + & & + & + & + & + & + & + & + \\
\hline 124 & + & + & + & + & + & + & + & & & & & & + & & 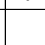 & 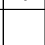 & + & + & + & + & & & 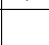 & & + & + & + & + & + & + & 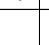 & 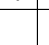 & $\rightarrow$ & 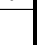 \\
\hline 125 & & & & & & & & & & & & & & & + & + & & & & & & & + & & & & & & & & & & & + \\
\hline 128 & & & & & & & & & 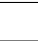 & & & & & & & + & & & 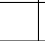 & & & & & & & & & & & & & & & \\
\hline 129 & & & & & & & & & & & & & & & & & & & & & & + & & & & & & & & & & & & \\
\hline 130 & & & & & & & & + & & & & & & & & & & & + & + & + & & + & & & & & & + & & + & & + & \\
\hline 131 & & & & & & & & & & & & & & & & & & & + & & & & & & 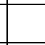 & & & & & & & + & + & + \\
\hline 132 & & & + & & + & + & + & + & + & & & & & & & + & + & + & + & + & + & & 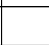 & & & 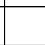 & 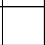 & + & + & + & + & + & + & \\
\hline 141 & & & & & + & + & & + & + & + & + & & & & & + & & & & + & + & + & + & + & & & 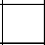 & & + & + & + & + & + & + \\
\hline 142 & & & & + & + & + & + & + & + & + & + & & & & & & + & + & + & + & + & + & + & + & & & 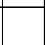 & + & + & + & + & + & + & + \\
\hline 143 & & & & & & & & & & & & & & & & & & & & & & & & & & & & & & & & & & \\
\hline 144 & & & & & & & & & & & & & & & & & & & & & & + & & & & & & & & & & + & & \\
\hline 145 & & & & & & + & & & & & & & + & & & + & & & + & & 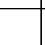 & + & & & & & & + & & & + & + & + & \\
\hline 146 & & & & & & & + & & & + & & & & & & & & & & & + & & 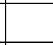 & + & & & & & & & & + & + & + \\
\hline 149 & & & & & & & & & & & & & & & & & & & & & + & + & & & & & & & & & & & & \\
\hline 156 & & & & & & & & & & & & & & & & & & & & & & & & & & & & & & & & & & \\
\hline 158 & & & & & & & & & & & & & & & & + & & & & & & & & & & & & & & & & & & \\
\hline 159 & & & & & & & & + & & & & & & & & & & & & & & & & & & & & & & & & & & \\
\hline 161 & & & & & & & & & & & & & & & & & + & & + & & & & & & & & & & & + & & + & & \\
\hline 177 & & & & & & & & & & & & & & & & & & & & & & + & & & & & & & & & & & & \\
\hline 198 & & & + & & & & & & & & & & & + & & & & & 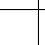 & & & & & & + & + & & & & & & & & \\
\hline 199 & & & & + & + & & & & & + & & & & & + & & + & & & + & + & & & & + & + & + & & + & + & + & + & & \\
\hline 200 & & & & & + & + & + & + & & + & & & & & 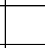 & & & + & + & + & + & + & + & + & & & & & + & + & + & + & + & + \\
\hline 202 & & & & & & + & & & & & & & & & & & & & & & & & & & & & & & & & & & & + \\
\hline 205 & & & & & & & & & & & & & & & & & & & & & & & + & & & & & & & & 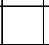 & 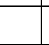 & & \\
\hline 207 & & & + & + & + & & & + & + & + & + & & & & + & + & + & & + & & & + & + & + & & & & + & + & & + & + & + & + \\
\hline 210 & & & & & & & & & & + & & & & & & & + & & & & & & & & & & & & & & & & & \\
\hline 211 & + & + & + & + & + & & & & 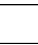 & & & + & + & + & + & + & & + & & + & & 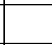 & 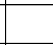 & & & + & + & + & + & & + & & & \\
\hline 212 & & & & & & & & & + & + & & & & & & & + & + & + & + & & + & + & + & & & & 4 & & + & & & + & \\
\hline 213 & & & & & + & + & + & + & + & + & & & & & & + & & 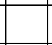 & + & & + & + & & & + & & 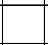 & & + & + & + & + & & \\
\hline 214 & + & + & + & + & + & + & + & + & + & + & + & & & & + & + & + & + & + & + & + & + & + & + & + & + & + & + & + & & + & & + & \\
\hline 216 & & & & & & & & & & & & & & & & & & & & & & & & & & & & & & & & + & & \\
\hline 220 & & & & & + & + & & + & + & + & & & & & & + & + & + & + & & + & & + & & & & & & & & & + & & \\
\hline 222 & & & & & & & & & & & & & & & & & & & & & & & & & & & & & & & & & & \\
\hline 223 & & & & & & & & & & & & & & & & & & & & & & & & & & & & & & & & & & \\
\hline 227 & + & + & & & + & & & & + & & & & & & + & & & + & + & & & & & + & & & & & + & & & & & \\
\hline 229 & + & + & & + & + & + & + & & + & & & & & + & + & + & + & + & & + & + & + & & & + & + & + & + & + & + & & & & + \\
\hline 230 & & & + & & + & + & + & & & & & & & & & & + & + & + & + & + & & & & 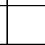 & 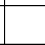 & & + & & + & & + & + & + \\
\hline 232 & + & + & + & + & + & + & + & + & & & & & + & & + & + & + & + & & + & + & & & & + & + & + & + & + & & & & & \\
\hline 233 & & & & & & & & & & & & & & & & & & + & & & & & + & & & & & & + & & & & & + \\
\hline 235 & & & + & + & & & & + & & & & & & & & + & + & & & & + & & & + & & & & & & & & & & \\
\hline 240 & & & & & & & & & & & & & & & & & + & & & & & & & & & & & & & & & & 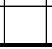 & \\
\hline
\end{tabular}




\begin{tabular}{|c|c|c|c|c|c|c|c|c|c|c|c|c|c|c|c|c|c|c|c|c|c|c|c|c|c|c|c|c|c|c|c|c|c|c|c|}
\hline \multicolumn{36}{|c|}{ Table 9, 1994-1 } \\
\hline & \multicolumn{8}{|c|}{$\mathrm{A}$} & & & & & $E$ & & & & & & & & & & & & & $\mathrm{C}$ & & & & & & & & D & \\
\hline & 1 & 2 & & 4 & & 6 & 7 & 8 & 1 & 2 & 3 & 4 & 5 & 6 & 7 & 8 & 9 & 10 & 1 & 2 & 3 & 4 & \begin{tabular}{|l|l|}
5 \\
\end{tabular} & \begin{tabular}{l|l}
6 \\
\end{tabular} & & 8 & \begin{tabular}{l|l}
9 & 1 \\
\end{tabular} & 101 & 11 & $12 \mid 1$ & & 14 & & & 3 \\
\hline 2 & & & & & 1 & & 1 & & & & & & & & & & & 2 & & & & & & & & & & & 2 & 1 & 2 & & & & \\
\hline 3 & & & & & & & & & & & & & & & & & & & & & & & & & & & & & & 1 & 1 & & & & \\
\hline 4 & 1 & 1 & 1 & 1 & 2 & 1 & 2 & & & 1 & 2 & 2 & 2 & 2 & 2 & 2 & 2 & 2 & & 1 & 1 & 1 & 2 & 1 & 2 & 2 & 2 & & & & & 1 & & & \\
\hline 5 & & & & & & & & & & & & & & 1 & 1 & 2 & & & & & 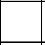 & & & & 1 & 1 & 1 & & & & & & & & \\
\hline 6 & & & & & & & & 1 & & & & & & & & & & 1 & & & & 1 & & & & & & & & & 1 & 1 & & & \\
\hline 7 & 1 & & & & & & & 1 & & & & 1 & & & & 1 & 2 & 2 & & & & & & & 1 & 1 & 2 & 2 & 2 & & 2 & 2 & & & \\
\hline 8 & & & & & & & & & & & & & & & & & & 1 & & & & & & & & & & & & & & & & & \\
\hline 10 & & & & & & & & & & & & & & & & & 1 & 1 & & & & & & & & & & & & & & & & & \\
\hline 11 & & & & 1 & & & & 1 & & & & & & & 1 & 1 & 2 & 1 & & & & & & & 2 & 1 & 25 & 2 & 1 & 1 & & & & & \\
\hline 12 & 2 & 2 & 2 & 1 & 1 & 1 & & & 1 & 1 & 1 & 2 & 2 & & 1 & 2 & 1 & & 1 & 1 & 1 & 2 & 2 & & 2 & 2 & 1 & & & & & & 1 & 1 & 1 \\
\hline 13 & 2 & 2 & 2 & 2 & 2 & 2 & 2 & 2 & 1 & 1 & 1 & 2 & 2 & 2 & 2 & 3 & & & 2 & 2 & 2 & 1 & \begin{tabular}{|l|}
1 \\
\end{tabular} & 2 & 2 & 3 & 3 & & 1 & & & 2 & & 2 & 1 \\
\hline 14 & & 2 & & & & & 2 & 2 & & & & & 1 & & 2 & 2 & & & & & & & \begin{tabular}{|l|}
1 \\
\end{tabular} & 2 & 2 & 2 & 2 & & & & & & 1 & 1 & 1 \\
\hline 15 & 3 & 3 & 3 & 2 & 2 & 2 & 3 & 3 & 1 & 1 & 2 & 1 & 2 & 3 & 3 & 3 & 1 & & 2 & 2 & 2 & 2 & \begin{tabular}{|l|}
2 \\
\end{tabular} & 2 & 3 & 3 & 3 & & & & & & & 2 & 2 \\
\hline 16 & & & & & & & & 2 & & & & & & & & & & & & & & & \begin{tabular}{|l|} 
\\
\end{tabular} & & & & 2 & & & & & 3 & & & \\
\hline 17 & 2 & 2 & 2 & 1 & & & 2 & 3 & & & 1 & 1 & 1 & 1 & 2 & 2 & 2 & 1 & 1 & 1 & & 1 & 1 & 1 & 2 & 2 & 3 & 3 & 3 & 2 & 2 & 2 & 1 & & \\
\hline 20 & 2 & 2 & 1 & & & & & & 1 & & & & & & & & & & 2 & 2 & 2 & \begin{tabular}{|l|}
1 \\
\end{tabular} & \begin{tabular}{|l|l|}
2 \\
\end{tabular} & 1 & & 1 & 1 & & & & & & \begin{tabular}{|l|}
1 \\
\end{tabular} & & \\
\hline 21 & & & & & & & & & & & & & & & & & & & & & & & & & & & & & & & & & & & \\
\hline 23 & & & & & & & & & & & & & & & & & & & & & & & & & & & & & & & & & & & \\
\hline 25 & & & & & & & & & & & & & & & & & & & & & & & & & & | & & & & & & & & & \\
\hline 26 & & & & & & & & & & & & & & & & & & & & & & & & & & | & & & & 1 & & 1 & & & \\
\hline 27 & & & & & & & & & & & & & & & & & & & & & & & & & & - & & & 1 & 1 & 1 & & & & \\
\hline 28 & & & & & 1 & & & 1 & & & & 1 & & & & & 2 & 1 & 1 & & & 1 & 1 & & & & 1 & 2 & & & 3 & & & & \\
\hline 29 & & & & & & & & & & & & & & & & & & & & & & & & & & & & & & & & & & & \\
\hline 32 & & & & & & & & & & & & & & & & & & & 1 & & & & & & & & & & & & & & & & \\
\hline 33 & & & & & & & & 1 & & & & & & & & & & & & & & & & & & & & & & & & & & & \\
\hline 34 & & & & & & & & 1 & & 1 & 1 & & & & & & & & & & & & & & & & & & & & & & & & \\
\hline 36 & & 1 & 2 & & & & & & & & & & & & & & & & & 1 & & & & & & & & & & & & & & & \\
\hline 38 & & & & & & & & & & & & & & & & & & & & & & & & & & & & & 1 & 1 & & 1 & & & \\
\hline 39 & & & & & & & & 1 & & & & & & & & & & & & & & & & & & & & & & & & & & & \\
\hline 41 & & & & & & & 1 & 1 & & & & 1 & & & & & & & & & 1 & & & & & & & 1 & & 1 & 1 & 1 & & & \\
\hline 43 & & & & & & & & & & & & & & & & & & & & & & & & & & & 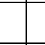 & 1 & & & 3 & & & & \\
\hline 44 & 3 & 2 & 2 & 2 & 1 & 1 & 2 & & 2 & & 2 & 2 & 2 & & 2 & 2 & 2 & & 2 & 1 & 3 & 2 & 2 & & 2 & 2 & 1 & 2 & 1 & & & & 2 & 2 & 2 \\
\hline 47 & & & & & & & & & & & & & & & & & & & & & & & & & & & & & & & & & & & \\
\hline 48 & & & & & & & & 2 & & & & & & & & 1 & 2 & 2 & & & & & & & & 1 & 2 & 3 & 3 & 3 & 3 & 3 & & & 1 \\
\hline 49 & & & & & & & & & & & & & & & & & & & & & & & & & & & & & & & & 2 & & & \\
\hline 53 & & & & 2 & & & 2 & 2 & & & & & & & & + & & & & 2 & 2 & & & & & 3 & 1 & & & & & 3 & & & \\
\hline 54 & & & & & & & & & & & & & & & & & & & & & & & & & & & & & & & & & & & \\
\hline 57 & & & & & & & & & & & & & & & & & & & & & & & & & & & & & & & & & & & \\
\hline 58 & & & & 1 & & & & & & & & & 1 & & & & & 1 & & 1 & & & & & & & & & & & & & & & \\
\hline 60 & 2 & 2 & 2 & 1 & 1 & & & & 1 & 2 & 2 & & 1 & & & & & & 1 & 1 & & & & & & & & & & & & & & 1 & 1 \\
\hline 61 & & & & 1 & & & & 1 & & 1 & & & & & & & & & & & & & & & & & & & & 1 & 1 & 1 & & & \\
\hline 62 & & & & & & & & & & & & & & & & & & & & & & & & & & & & & & & & & & & \\
\hline 63 & 2 & 2 & 3 & 2 & 2 & 2 & 2 & 2 & 2 & 1 & 2 & 2 & 1 & 2 & 2 & 2 & 1 & & 2 & 2 & 2 & 1 & 1 & 2 & 2 & 1 & 1 & 1 & & & & & 2 & & \\
\hline 64 & 2 & 1 & 1 & 2 & 1 & 2 & 1 & 2 & 2 & & & & 2 & 2 & 2 & 2 & & & 2 & & 2 & 1 & \begin{tabular}{|l|l|}
2 \\
\end{tabular} & 2 & 2 & 2 & & & & & & & 2 & 2 & 2 \\
\hline 70 & & & & & & & & & & & & & & & & & & & & 2 & & & & & & & & & & & & & & & \\
\hline 71 & 2 & 2 & 1 & 2 & 2 & 2 & 3 & 3 & & 1 & & & 2 & & & & & & & & & & & & & & & & & & & & & & \\
\hline 72 & & 1 & 1 & & & & & & & & & & 2 & 2 & 3 & & & & & & & & & & & & & & & & & & & 2 & \\
\hline 73 & 3 & 3 & 3 & 2 & 2 & 3 & & & 4 & 3 & & 3 & 2 & & 2 & 2 & & & 3 & & 2 & 2 & 2 & 2 & 2 & 2 & & & & & & & 3 & 3 & 3 \\
\hline 75 & & & & & & & & & & & & & & & & & & & 1 & & & & & & & & & & & & & & & & \\
\hline 77 & & 1 & & 3 & 4 & 4 & 4 & 3 & & & & 1 & 4 & 4 & 4 & 4 & 2 & 3 & & 2 & 2 & 3 & 3 & 3 & 3 & 3 & 3 & 3 & & & & & 1 & 3 & \\
\hline 78 & 44 & & & 3 & 2 & 4 & 4 & 3 & 3 & & & & 4 & 4 & 4 & 4 & 3 & & 2 & & 4 & 4 & 4 & 4 & 3 & 3 & 3 & 3 & & & & & 4 & 1 & 3 \\
\hline 79 & & & & & & & 2 & & 1 & & & & 2 & 2 & 2 & 2 & & & & & 2 & 2 & \begin{tabular}{|l|l|}
3 \\
\end{tabular} & 2 & 2 & 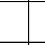 & 1 & & & & & & 2 & & 2 \\
\hline 80 & & & & & & & & & 1 & & & & & & & 1 & & & & & & & & & & & & & & & & & & & \\
\hline 81 & 3 & & 1 & 2 & 2 & 2 & 2 & 2 & 3 & & & & 2 & 3 & 3 & 3 & & & 3 & 1 & 1 & 2 & \begin{tabular}{|l|l|}
2 \\
\end{tabular} & 2 & 2 & 2 & 2 & & & & & & 2 & & 2 \\
\hline 82 & 3 & 2 & 2 & 3 & 3 & 2 & 3 & & & & & & & 2 & & & & & & & & & & & & & & & & & & & & & \\
\hline
\end{tabular}




\begin{tabular}{|c|c|c|c|c|c|c|c|c|c|c|c|c|c|c|c|c|c|c|c|c|c|c|c|c|c|c|c|c|c|c|c|c|}
\hline \multicolumn{33}{|c|}{ Table 9, 1994-2 } \\
\hline & \multicolumn{11}{|c|}{ D } & \multicolumn{12}{|c|}{$E$} & \multicolumn{9}{|c|}{$\mathrm{F}$} \\
\hline & 4 & $5 \mid$ & $\begin{array}{ll}6 & 7 \\
6\end{array}$ & \begin{tabular}{l|l}
7 & $\varepsilon$ \\
\end{tabular} & \begin{tabular}{|l|l}
9 \\
\end{tabular} & & \begin{tabular}{l|l}
0 & 1
\end{tabular} & & 2 & 13 & & 1 & 2 & 3 & 4 & 5 & 6 & \begin{tabular}{l|l}
7 & $\varepsilon$ \\
\end{tabular} & \begin{tabular}{l|l}
8 & 9 \\
\end{tabular} & 10 & 11 & 12 & & 1 & 2 & \begin{tabular}{l|l}
3 & 4 \\
4
\end{tabular} & 45 & & & 8 & \begin{tabular}{|l|l|}
9 & 1 \\
\end{tabular} & 10 \\
\hline 2 & & & 1 & & & & & & 1 & 1 & 1 & & & & & & 1 & & & & 1 & 1 & & & & & 2 & 1 & & & 1 & \\
\hline 3 & & & & & & 1 & 1 & & & & & & & & & & & & & & & 1 & & & & & & & & & 1 & \\
\hline 4 & 1 & $1:$ & 25 & $2 \sqrt{2}$ & 2 & & 2 & 1 & & & & & & & 1 & 2 & 2 & 25 & \begin{tabular}{l|l}
2 & 2 \\
\end{tabular} & 2 & 2 & & 1 & & & \begin{tabular}{l|l}
12 &
\end{tabular} & 2 & 2 & \begin{tabular}{l|l|}
2 & 2
\end{tabular} & 2 & 2 & 2 \\
\hline 5 & & & & & & & & & & & & & & & & & & & & & & & & & & & & & & & & \\
\hline 6 & & & & & & & & & & & 1 & & & & & & & & & 1 & 1 & & & & & & & & & & 1 & \\
\hline 7 & 1 & & & 1 & 1 & & & 1 & 2 & 2 & 3 & & & & & 1 & 1 & 2 & \begin{tabular}{l|l}
2 & 1 \\
\end{tabular} & $\begin{array}{ll}1 & 1 \\
1\end{array}$ & 1 & 1 & 2 & & & & 2 & 2 & 2 & & 2 & 2 \\
\hline 8 & & & & & & & & 1 & 1 & 1 & & & & & & & & & \begin{tabular}{l|l}
1 & 1 \\
\end{tabular} & & & 1 & & & & & & & \begin{tabular}{l|l|}
1 & 1 \\
\end{tabular} & & 2 & 2 \\
\hline 10 & & & & & & & 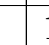 & 1 & 1 & & & & & & & & & & 1 & & & & & & & & & & & & & \\
\hline 11 & & 1 : & $1]$ & $1]$ & 1 & 1 & & & & 1 & & & & & 1 & & 1 & 1 [: & 1 & & & & & & & $1] 1$ & 1 & 1 & & & & \\
\hline 12 & 1 & 25 & 25 & $2 \sqrt{2}$ & 1 & & & & & & & & & & 1 & 2 & 2 & 2 & & & & & & 1 & 1 & \begin{tabular}{l|l}
2 & 2 \\
2
\end{tabular} & 2 & 1 & \begin{tabular}{l|l|}
2 & 1 \\
\end{tabular} & & & \\
\hline 13 & & 25 & 25 & $22^{2}$ & 2 & 2 & 2 & 1 & 1 & 2 & 2 & & & & 1 & 2 & 2 & $3:$ & $\begin{array}{ll}22 & 2 \\
\end{array}$ & 2 & & 1 & & 1 & & \begin{tabular}{l|l}
2 & 2 \\
2
\end{tabular} & 2 & 2 & \begin{tabular}{l|l}
3 & 3
\end{tabular} & 3 & 2 & 2 \\
\hline 14 & 1 & & & 71 & & & & & & & & & & 1 & \begin{tabular}{|l|l|}
3 \\
\end{tabular} & 2 & 1 & 2 & 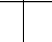 & & 2 & 1 & & 1 & & 12 & \begin{tabular}{l|l|}
2 \\
\end{tabular} & & \begin{tabular}{l|l}
2 & 2
\end{tabular} & 2 & \begin{tabular}{|l|l|}
3 \\
\end{tabular} & \\
\hline 15 & 2 & 35 & $2:$ & \begin{tabular}{l|l}
3 & 2
\end{tabular} & 2 & 2 & 2 & 1 & & & & 1 & 1 & 2 & 3 & 3 & 2 & 3 & 22 & 22 & 2 & & & 1 & 2 & \begin{tabular}{l|l}
2 & 4
\end{tabular} & 4 & 2 & \begin{tabular}{|l|l}
4 & 3
\end{tabular} & 3 & 3 & 2 \\
\hline 16 & & & & & & & 1 & 1 & 2 & 1 & 3 & & & & & & & & 2 & \begin{tabular}{|l|l}
2 & 1
\end{tabular} & \begin{tabular}{|l|l} 
\\
\end{tabular} & 1 & & & & & & & & 1 & 2 & 2 \\
\hline 17 & & 2 & 16 & 25 & 2 & 2 & & 2 & 3 & 2 & 1 & & 1 & 1 & 3 & 3 & 3 & 36 & 2 & 2 & 2 & 2 & 2 & & & 12 & 2 & 2 & \begin{tabular}{l|l}
2 & 3
\end{tabular} & 2 & 3 & 3 \\
\hline 20 & & 1 & 21 & $1]$ & 1 & & & 1 & & & & & & & 1 & & 2 & & & & - & & & 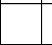 & & & & 1 & 1 & & & \\
\hline 21 & & & & & & & & & & & 1 & & & & & & & & & & & & & & & & 1 & & & 1 & $\begin{array}{ll} \\
\end{array}$ & \\
\hline 23 & & & & & & & & & & & & & & 1 & & & 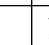 & 1 & & & & & & & & & & 1 & & & & \\
\hline 25 & & & & & & & & & & & & & & & & & & & & & & 1 & & & & & & & & & & \\
\hline 26 & & & & & & & & & & 1 & 1 & & & & & & & & 1 & \begin{tabular}{l|l}
1 & 1
\end{tabular} & 1 & & 1 & & & & & & & & & \\
\hline 27 & & & & & & 1 & 1 & 1 & 1 & 2 & & & & & & & & 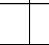 & & & 1 & 1 & & & & & & & & & 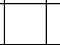 & \\
\hline 28 & & 1 & 11 & 1 & & & & & & & & & & & & & & & 1 & \begin{tabular}{|l|l}
1 & 1
\end{tabular} & \begin{tabular}{|l|}
1 \\
\end{tabular} & & & & & & $\begin{array}{ll}2 & 1\end{array}$ & 1 & & & 1 & 2 \\
\hline 29 & & & & & & 1 & & & & & & & & & & & & & & & 1 & & & & & & & & & & & \\
\hline 32 & & & & & & & & & & & & & & & & & & & & & & & & & & & & & & & & \\
\hline 33 & & & & & & & & & & & & & & & & & & & & & 1 & & & & & & & & & & & \\
\hline 34 & & & & & & & & & & & & & & & & & & & & & & & & & & & & 1 & 1 & 1 & & \\
\hline 36 & & & & & & & & & & & & & & & & & & & & & & & & & & & & & & & & \\
\hline 38 & & & & & & & & & & & & & & & & & & & & & & & & & & & & & & & & \\
\hline 39 & & & & & & & & & & & 1 & & & & & & & & & 1 & 1 & & & & & & & & & & 1 & \\
\hline 41 & & & & & & & 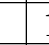 & 1 & 1 & & & & & & & 1 & & & & & 1 & 1 & 1 & & & & & 1 & & 1 & 1 & 1 \\
\hline 43 & & & & 1 & & & & & & & & & & & & & & & & & 1 & & 3 & & & & & & & & 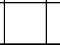 & \\
\hline 44 & \begin{tabular}{|l|}
2 \\
\end{tabular} & 2 & 25 & 22 & 1 & 1 & & & & & & 2 & 1 & \begin{tabular}{|l|}
2 \\
\end{tabular} & 2 & 2 & 2 & 2 & 1 & 2 & & & & 2 & 2 & 22 & 2 & & & & & \\
\hline 47 & & & & & & & & & & & & & & & & & & & & & & 1 & & & & & & & & & & \\
\hline 48 & & & & 12 & 2 & 2 & 25 & 2 & 2 & 3 & 3 & & & & 1 & & & 1 & \begin{tabular}{l|l}
12 \\
\end{tabular} & $\begin{array}{ll}2 \\
\end{array}$ & 3 & & 2 & & & & & 2 & & & 1 & 2 \\
\hline 49 & & & & & & & & & & & 2 & & & & & & & & \begin{tabular}{l|l} 
& 1
\end{tabular} & 1 & \begin{tabular}{|l|} 
\\
\end{tabular} & 1 & 2 & & & & & 1 & & & & \\
\hline 53 & & 2 & & 22 & 2 & 2 & 2 & & & & 3 & & & & & & & & 2 & 2 & 3 & & & & & & & & 1 & & & 3 \\
\hline 54 & & & & & & & & & & & & & & & & & & & & & & & & & & & 1 & & & & \begin{tabular}{l|l} 
\\
\end{tabular} & \\
\hline 57 & & & & & & & & & & & & & & & & & & & & & & & & & & & 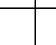 & & 2 & & & \\
\hline 58 & & 1 & 1 & & 1 & & & & & & & & & & & & & & & & & & & & & & 1 & & 1 & & & \\
\hline 60 & & 1 & & & & & & & & & & & 1 & & & 1 & & & 1 & 1 & & & & & & & 1 & 1 & & & & \\
\hline 61 & & & & & & 1 & 1 & 1 & & & & & & & & 1 & 1 & & & & 1 & 1 & 1 & & & & 1 & & 1 & & 1 & 1 \\
\hline 62 & & & & & & & & & & & & & & & & & & & & & & 1 & 1 & & & & & & & & & \\
\hline 63 & & 25 & 26 & 28 & 21 & 1 & 1 & & & & & & 1 & 1 & 1 & 2 & 28 & 28 & 21 & \begin{tabular}{|l|l}
1 &
\end{tabular} & & & & 2 & 1 & & 2 & 2 & 2 & 1 & & \\
\hline 64 & 2 & 2 & 25 & 2 & & & & & & & & 2 & 2 & 2 & 2 & & 7 & 2 & & & & & & 2 & 1 & 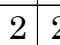 & 2 & & & & & \\
\hline 70 & & & & & & & & & & & & & & & & & & & & & & & & & & & & & & & & \\
\hline 71 & & 1 & & & & & & & & & & & & & & & & & & & & & & 2 & & & & & & 1 & & \\
\hline 72 & & & & & & & & & & & & & & & & & & & & & & & & & & & & & & & & \\
\hline 73 & 3 & & 22 & 41 & & & & & & & & 3 & 2 & 2 & 3 & 2 & 25 & 2 & & & & & & 3 & 3 & 3 & 2 & 2 & & & & \\
\hline 75 & & & & & & & & & & & & & & & & & & & & & & & & & & & & & & & & \\
\hline 77 & 4 & 4 & 4 & 44 & 14 & 3 & 3 & 1 & & & & 3 & 4 & 3 & 3 & & 3 & 2 & & + & & & & 3 & 3 & 32 & 2 & 1 & & & & \\
\hline 78 & 2 & 2 & 36 & 22 & 3 & & 3 & & & & & 4 & 4 & 3 & 3 & & & & & & & & & 4 & 2 & & 4 & & & & & \\
\hline 79 & & 2 & & & & & & & & & & 3 & 2 & & & & & & & & & & & 2 & & & 2 & & & & & \\
\hline 80 & & & & 1 & & & & & & & & & & & . & & . & 1 & & & & & & & & 1 & & & & & & \\
\hline 81 & \begin{tabular}{|l|}
2 \\
\end{tabular} & 1 & 25 & 22 & 25 & & & & & & & 2 & 2 & 3 & 2 & & & & & & & & & 2 & 2 & 2 & 2 & & & & & \\
\hline 82 & & & & & & & & & & & & & & & & & & & & & & & & 2 & & & & & & & & \\
\hline
\end{tabular}




\begin{tabular}{|c|c|c|c|c|c|c|c|c|c|c|c|c|c|c|c|c|c|c|c|c|c|c|c|c|c|c|c|c|c|c|c|c|c|c|c|}
\hline \multicolumn{36}{|c|}{ Table 9, 1994-3 } \\
\hline & \multicolumn{8}{|c|}{$\mathrm{A}$} & & & & & B & & & & & & & & & & & & C & & & & & & & & $\mathrm{D}$ & & \\
\hline & 1 & 2 & 3 & $4 !$ & & 6 & $7 \mid \varepsilon$ & 3 & 1 & 2 & 3 & 4 & 56 & \begin{tabular}{l|l}
6 & 7 \\
\end{tabular} & 78 & 9 & 10 & 01 & 2 & 3 & 4 & 5 & 6 & 7 & 8 & 9 & & 11 & 12 & & 14 & & & 3 & \\
\hline 84 & & & & & & & & & & & & & & & & & & & & & & & & & & & & & & & & & & & \\
\hline 86 & & & & & 1 & & & 1 & & & & & & & & & & & 1 & & & 1 & & & & 1 & & 1 & & 2 & & & & & \\
\hline 87 & & & & & & & & & & & & & & & & & & & & & & & & & & & & & & & 1 & & & & \\
\hline 88 & & & 1 & & & & & & & & & & & & & & & & & & & & & & & & & & & & & & & & \\
\hline 89 & & & & & & & & & & & & & & & & & & & & & & & & & & & 1 & & 1 & 1 & & & & & \\
\hline 90 & & & & & & & & & & & & & & & & & & & & & & & & & & & & & & & & & & & \\
\hline 91 & & & & & & & & & & & & & & & & & & & & & & & & & & & & & & & & & & & \\
\hline 93 & & & & & & & & & & & & & & & & & 1 & & & & & & & & & & & & & & & & & & \\
\hline 94 & & & & & & & & 1 & & & 1 & & 1 & 1 & & & & & & & & & & & & & & & & & 1 & & & & \\
\hline 97 & & & & & & & & & & & & & & & & & & & & & & & & & & & & & & 1 & & & & & \\
\hline 99 & & & & & & & & & & & & & & & & & & & & & & & & & & & & 1 & 1 & 1 & & & & & \\
\hline 100 & & & & & & & & & & & & & & & & & & & & & & & & & & & & 1 & & & & & & & \\
\hline 103 & & & & & & & & & & & & & & & & & & & & & & & & & & & & & & & 1 & & & & \\
\hline 107 & & & & & & & & & & & & & & & & & & & & & & & & & & & & & & 1 & & & & & \\
\hline 111 & & & & & & & & & & & & & & & & & & & & & & & & & & & & & & & & & & & \\
\hline 115 & & & & & & & & & & & & 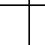 & 1 & & & & & & & & & & & & & & & & & & & & & & \\
\hline 116 & & & & & & & 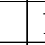 & 1 & & & & & & & & 1 & & & & & & & & & & & & & & & & & & & \\
\hline 118 & 1 & 2 & & & & & & & & & 1 & & & & & & & & L & . & & & & & 1 & 1 & & & & & & & & & \\
\hline 119 & & & 1 & & & & & & & & 1 & 1 & & & & & & & & & & & & & & & & & & & & & & & \\
\hline 120 & & & & & & & & & & & & & & & & & & & & & & & & & & & & & & & & & & & \\
\hline 122 & & & & 1 & & 1 & 2 & 2 & & & & & & & & & & & & & & & & & & & & & & & & & & & \\
\hline 123 & & 1 & 2 & 1 & 1 & 1 & 2 & 2 & 1 & 1 & 1 & 2 & 2 & & 1 & 2 & 2 & 1 & 1 & 1 & 1 & 1 & & & 1 & & 2 & 2 & 2 & 2 & 1 & & & 1 & \\
\hline 124 & 2 & 2 & 2 & 2 & 1 & 2 & & & 1 & 1 & 1 & 2 & $2 \sqrt{2}$ & $2] 2$ & $2 \sqrt{2}$ & 1 & & 2 & 2 & 2 & 1 & 2 & 2 & 2 & 2 & 2 & 1 & & & & & 2 & 2 & 2 & \\
\hline 125 & & & & & 1 & & & & & & & & 1 & & & & & & & & & & & & . & & & & & & & & & & \\
\hline 129 & & & & & & & & & & & & & & & & 1 & 1 & & & & 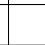 & 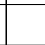 & & & . & 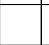 & . & 1 & 1 & 1 & & & & & \\
\hline 130 & & & & & & & & & & & & & & & & & & & & & & . & & & & & & & & & & & & & \\
\hline 131 & & & & & & & & & & & & & & & & & & & & & & 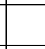 & & & & & & & & & & & & & \\
\hline 132 & & & & & & & 18 & 2 & & & & 1 & 1 & & & 2 & 2 & & & & 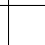 & & & 1 & 1 & 2 & 2 & 2 & & & & & & & \\
\hline 141 & & & & & & & & 1 & & & & 1 & & & 1 & 1 & 1 & & & & 1 & 1 & & & 1 & 2 & & 3 & 2 & 3 & 2 & & & & \\
\hline 142 & 1 & & & & & & & 2 & & 1 & & 1 & & 1 & 1 & 2 & 2 & & & & & & & 1 & 1 & 1 & 2 & 2 & 2 & 3 & 1 & & & & \\
\hline 143 & & & & & & & & & & & & & & & & & & & & & & & & & & & 1 & & & & & & & & \\
\hline 145 & & & 2 & 2 & 2 & 2 & 2 & & & 1 & & 3 & 35 & 2 & & $x$ & & & 1 & & & & & & & & & 1 & 1 & & & & & & \\
\hline 146 & & & & & & &. & 1 & & & & & & & & 1 & & & & & & & & & & & & 1 & \begin{tabular}{|l|l}
1 \\
\end{tabular} & \begin{tabular}{|l|l}
1 \\
\end{tabular} & & & & & \\
\hline 148 & & & & & & & & & & & & & & & & & & & & & & & & & & & & & & & & & & & \\
\hline 149 & & & & & & & 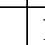 & 1 & & & & & & & & & & & & & & & & & & & & & & & & & & & \\
\hline 156 & 1 & 1 & 1 & & & & & & & & & & & & & & & & & & & & & & & & & & & & & & & 1 & \\
\hline 158 & & & & & & 2 & & & & & & 1 & & & & & & & & 1 & & & & & & & & & & & & & & & \\
\hline 161 & & & & & & & 1 & & & & & & & & & & & & & & & & & & & & & & & & & & & & \\
\hline 175 & 2 & & & & & & & & & 1 & & & & & & & & & & & & . & & & & & & & & & & & & & \\
\hline 176 & & 1 & & & & & & & & & & & & & & & & & & & & & & & & & & & & & & & & & \\
\hline 198 & & & & & & 2 & 2 & & & & & & 2 & $3:$ & 3 & 1 & & & & 1 & 1 & 1 & 1 & & & & & & & 2 & & 1 & & 1 & \\
\hline 199 & & & & & & 2 & 2 & 2 & & & 2 & & 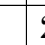 & 25 & 26 & 2 & 2 & & & & & 1 & 1 & 1 & 2 & 2 & 3 & 1 & & & & 1 & 1 & 1 & \\
\hline 200 & & & & & & 1 & 1 & 1 & & & & & & 7 & 1 & 2 & 1 & & & & & & 1 & & & 2 & 2 & & & & 1 & & & & \\
\hline 201 & & & & & & & & & & & & & & & & & & & & 1 & & & & & & & & & & & & & & & \\
\hline 203 & & & & & & & & & & & & & & & & & & & & & & & & & & & & & 1 & 1 & 1 & & & & \\
\hline 207 & & 1 & 1 & & & & & & & & & 1 & & & & 2 & 2 & 21 & 1 & 1 & 1 & 1 & & 1 & 2 & 2 & 2 & 2 & 2 & 2 & & & & & \\
\hline 209 & & & & & & & & & & & & & & & & & & & & & & & & & & & & & & & & & & & \\
\hline 210 & & & & & & & & & & & & & & & & & & & & & & & & & & & & & & & & & & & \\
\hline 211 & & & & 2 & 2 & 2 & $2:$ & 3 & & & & & 2 & 15 & 2 & & & & & & 2 & 1 & 2 & 2 & 3 & 3 & & & & 3 & & & 2 & 2 & \\
\hline 212 & & & & & 4 & & 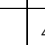 & 4 & & & & 4 & 4 & & & & & & 4 & 1 & & & & & & & & & & & 1 & & & & \\
\hline 213 & & & & & 1 & & 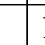 & 1 & & & & & & & & & & & & & & 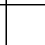 & 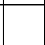 & & & & 1 & 1 & & & & & & & \\
\hline 214 & 2 & 2 & 1 & & 1 & & 1 & & & 1 & 1 & & & $\begin{array}{lll}1 & 1\end{array}$ & 1 & 12 & 3 & 1 & 1 & 1 & 1 & 1 & 1 & 2 & 3 & 3 & 4 & 4 & 4 & $\mid$\begin{tabular}{|l|l} 
\\
\end{tabular} & 4 & 1 & 1 & 1 & \\
\hline 216 & & & & & & & & & & & & & & & & & & & & & & & & & & & & & & & & & & & \\
\hline 217 & & & 1 & & & & & & & & & & & & & & & & & & & & & & & & & & & & & & & & \\
\hline 220 & & 1 & 2 & 1 & 1 & 1 & 1 & 1 & 1 & 1 & 1 & 1 & 1 & 1 & & & & 1 & 11 & & & & & & & & & 1 & & & 1 & & & 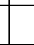 & \\
\hline
\end{tabular}




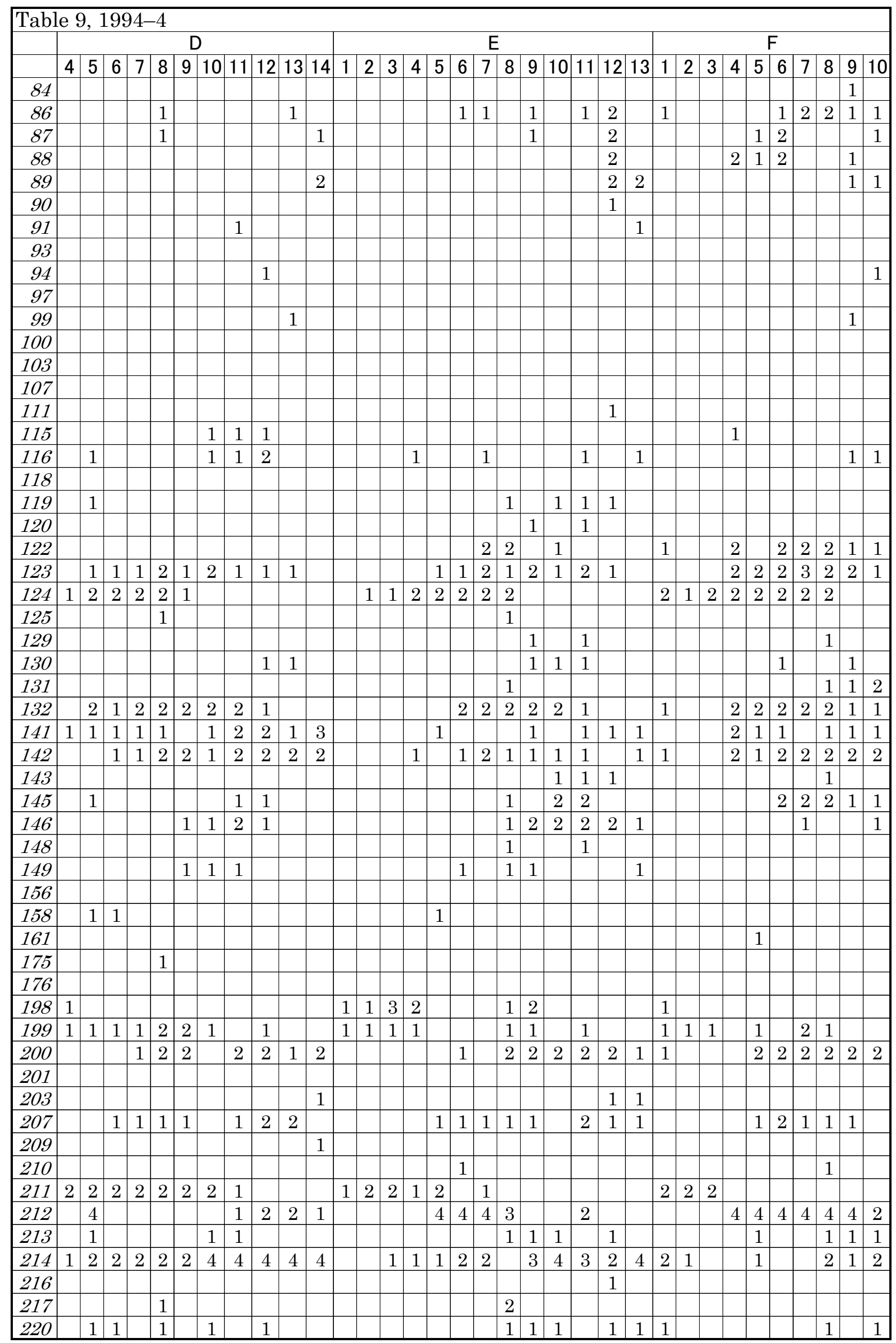




\begin{tabular}{|c|c|c|c|c|c|c|c|c|c|c|c|c|c|c|c|c|c|c|c|c|c|c|c|c|c|c|c|c|c|c|c|c|c|}
\hline \multicolumn{34}{|c|}{ Table 9, 1994-5 } \\
\hline & \multicolumn{8}{|c|}{$\mathrm{A}$} & \multicolumn{9}{|c|}{$\mathrm{B}$} & \multicolumn{14}{|c|}{ C } & \multicolumn{2}{|c|}{ D } \\
\hline & 1 & 2 & 3 & 4 & & & 7 & 8 & 1 & 2 & $3 \mid<$ & & 6 & 7 & 8 & 9 & 10 & 1 & 2 & 3 & 4 & 5 & 6 & & 8 & 91 & 01 & 11. & 12 & \begin{tabular}{l|l}
13 & 14 \\
\end{tabular} & & & 3 \\
\hline 227 & & & & & & 1 & 1 & 2 & & & & & & 1 & 1 & & & & & & & & & 1 & 1 & 1 & & & & & 1 & & \\
\hline 229 & 2 & 2 & 2 & 1 & 1 & 1 & 1 & 2 & 1 & & 1 & 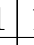 & & 1 & 2 & 2 & 2 & 1 & 1 & 1 & & 1 & 1 & 2 & 2 & 2 & 3 & 3 & 1 & & & 1 & 1 \\
\hline 230 & & & & & & & 1 & 2 & & & & & & & & & & & & & & & & & & & & & & & & & \\
\hline 232 & + & + & + & + & & + & + & + & & & - & & + & + & + & & + & & . & & + & + & + & + & + & + & + & + & + & & + & + & + \\
\hline 233 & & & & & & & & 1 & & & & & & & & & & & 1 & & & & & & & & & & 1 & 1 & & & \\
\hline 234 & & & & & & & & & & & & & 1 & & & & & & & & & & & & & & & & & & & & \\
\hline 235 & & & & & & & & 1 & & & & & & & & & 1 & & & & & & & & & & & & & & & & \\
\hline 240 & & & & & & & & & & & & & & & & & & & & & & & & & & & & & & 1 & & & \\
\hline
\end{tabular}

\begin{tabular}{|c|c|c|c|c|c|c|c|c|c|c|c|c|c|c|c|c|c|c|c|c|c|c|c|c|c|c|c|c|c|c|c|c|c|}
\hline \multicolumn{34}{|c|}{ Table 9, 1994-6 } \\
\hline & \multicolumn{11}{|c|}{ D } & \multicolumn{12}{|c|}{$E$} & \multicolumn{10}{|c|}{$F$} \\
\hline & 4 & 5 & 6 & 7 & 8 & 9 & & & 12 & 13 & & 1 & & & & 56 & & 8 & 9 & & \begin{tabular}{|l|l}
0 & 1
\end{tabular} & & \begin{tabular}{l|l}
12 & 1
\end{tabular} & \begin{tabular}{|l|l}
3 & 1
\end{tabular} & 2 & 3 & 4 & 5 & & & & & 10 \\
\hline 227 & 1 & & & 1 & 1 & & 1 & 1 & 1 & & & & & & & 1 & 2 & 1 & & & 1 & & & 1 & 1 & 1 & 1 & & & & 1 & & \\
\hline 229 & 1 & 2 & 2 & 2 & 2 & 2 & 2 & 2 & 2 & 1 & & & 1 & & & 2 & 2 & 1 & 1 & 2 & 2 & & & 1 & 1 & 2 & 2 & 2 & 2 & 2 & 1 & 1 & 1 \\
\hline 230 & & 1 & 1 & 1 & 1 & 1 & 1 & 1 & & 1 & & & & & . & 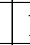 & 2 & 2 & 2 & 1 & & & & & & 1 & 1 & 1 & 1 & 1 & 1 & 1 & \\
\hline 232 & + & + & + & + & + & + & & & & & & + & + & & & 1 & $-1-$ & + & & $t$ & & & & + & & $t$ & + & + & + & + & + & + & + \\
\hline 233 & & 1 & & 1 & & & & 1 & & & & & & & & & & & & & 1 & & 1 & & & & & 1 & & & & & 1 \\
\hline 234 & & & & & & 1 & & & & 1 & & & & & & & & & & & & & & & & & & & & & & & \\
\hline 235 & & & & & & & & & & & & & & & & & 1 & 1 & & & & & & & & & 2 & 1 & 2 & 1 & & & \\
\hline 240 & & & & & & & & & & & & & & & & & & & & & & & & & & & & & & & & & \\
\hline
\end{tabular}




\begin{tabular}{|c|c|c|c|c|c|c|c|c|c|c|c|c|c|c|c|c|c|c|c|c|c|c|c|c|c|c|c|c|c|c|c|c|c|c|}
\hline \multicolumn{35}{|c|}{ Table 9, 1995-1 } \\
\hline & \multicolumn{8}{|c|}{$\mathrm{A}$} & \multicolumn{9}{|c|}{ B } & & & & & & & & C & & & & & & & & $\mathrm{D}$ & \\
\hline & 1 & 2 & & 4 & & 6 & $7 \mid \varepsilon$ & \begin{tabular}{l|l}
8 & 1 \\
1
\end{tabular} & 1 & & \begin{tabular}{l|l}
3 & 2 \\
3 & 2
\end{tabular} & & 56 & \begin{tabular}{l|l}
6 & 7 \\
\end{tabular} & 78 & 9 & 10 & 01 & 2 & 3 & 4 & 5 & 6 & 7 & & 9 & & 11 & 12 & & 14 & & & 3 \\
\hline 2 & & & & & & & & 1 & & & & & & & & & & 1 & & & & & & & & & & & & & & & & \\
\hline 3 & & & & & & & & & & & & & & & & & & & & & & & & & & & & & & & 1 & & & \\
\hline 4 & & 1 & 1 & 2 & 2 & 2 & 15 & 2 & & & 2 & 21 & 1 & \begin{tabular}{l|l}
1 & 1 \\
1
\end{tabular} & 12 & 2 & 2 & & 1 & 1 & 1 & 1 & & 1 & & 2 & 2 & 2 & 2 & 2 & & & & 1 \\
\hline 5 & & & & 1 & & & & 1 & & & 1 & & & 2 & 2 & 2 & 2 & & & & & & 1 & & 1 & 1 & 1 & & 1 & & & & & 1 \\
\hline 6 & & & & & & & & & & & & & & & & 1 & & & & & & & & & & & & 1 & 1 & 1 & 1 & & & \\
\hline 7 & & & & & & 1 & 2 & 2 & & & 1 & & & 1 & 2 & 1 & 2 & & & 1 & & & 1 & & 2 & 1 & 2 & 2 & 2 & 2 & 2 & & & \\
\hline 8 & & & & & & & & & & & & & & & & & & & & & & & & & & & & 1 & & 1 & & & & \\
\hline 10 & & & & & & & & & & & & & & & & 1 & 1 & & & & & & & & & & & 1 & 1 & & 1 & & & \\
\hline 11 & & & & & & & 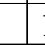 & 1 & & & & & & & 1 & 1 & & & & & & 1 & 1 & 1 & & 1 & 1 & 1 & & & & & & \\
\hline 12 & 2 & 2 & 2 & 1 & 1 & 1 & & 1 & 1 & 2 & $1: 2$ & 21 & 1 & 1 & 1 & & & 1 & 1 & 1 & 1 & 2 & & 2 & 2 & 1 & & & & & & 1 & 1 & 1 \\
\hline 13 & 1 & 1 & 1 & 2 & 2 & 2 & $2 \mid$ & 2 & & 1 & 1 & & 2 & $2 \sqrt{2}$ & 2 & 2 & 2 & 1 & 2 & 1 & 1 & & & 2 & 2 & 2 & 2 & 1 & 1 & & 2 & & & 1 \\
\hline 14 & 2 & 1 & 2 & 1 & & 2 & 1 & 2 & & 1 & 1 & & 1 & & 1 & 1 & & 1 & 1 & 1 & 1 & 1 & 1 & & 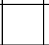 & & & & & & & 2 & 2 & 2 \\
\hline 15 & 2 & 2 & 2 & 2 & 2 & 2 & 2 & 3 & & & 15 & 26 & 2 & \begin{tabular}{l|l}
4 & 4
\end{tabular} & 14 & 43 & 3 & 2 & 2 & 2 & 3 & 3 & 3 & 3 & 3 & 3 & 3 & 2 & 2 & 2 & 1 & 2 & 1 & 3 \\
\hline 16 & & & & & & & & 1 & & & & & & & & & 2 & & & & & & & & & & 1 & 1 & 1 & 1 & 3 & & & \\
\hline 17 & & 2 & 2 & & & & 2 & \begin{tabular}{l|l}
3 & 1
\end{tabular} & & & & & & 1 & 11 & 12 & 1 & & & 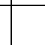 & 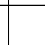 & & 1 & 1 & 1 & 2 & 3 & 1 & 1 & 1 & & 2 & & \\
\hline 18 & & & & & & & & & & & & & & & 1 & 1 & 1 & & & & & & & 1 & & 11 & 1 & 1 & 2 & & 1 & & & \\
\hline 19 & & & & & & & & & & & & & & & & & & & & & & & & & & & & & & & & & & \\
\hline 20 & & 1 & 2 & 1 & 1 & 1 & & & & & 1 & & & & & & & 1 & & 1 & 1 & 1 & & & & & & & & & & & & \\
\hline 21 & & & & & & & & & & & & & & & & & & & & & & & & & & & & & & & & & & \\
\hline 22 & & & & & & & & & & & & & & & & & & 1 & & & & & & & & & & & & & & & & \\
\hline 23 & & & & & & & & & & & & & & & & & & & & & & & & & & & & & & & & & & \\
\hline 25 & & & & & & & & & & & & & & & & & & & & & & & & & & & & & & & & & & \\
\hline 26 & & & & & & & 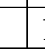 & 1 & & & 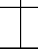 & & & & & & 1 & & & & & & & & & & & 1 & 1 & & 1 & & & \\
\hline 27 & & & & & & & & & & & & & & & & & & & & & & & & & & & & 1 & 1 & 1 & & & & \\
\hline 28 & & & & & & 1 & 15 & 2 & & & 1 & 1 & 1 & & 2 & 2 & 2 & & & & & & & 1 & 1 & 1 & 1 & 1 & 1 & & & & & \\
\hline 29 & & & & & & & & & & & & & & & & & & & & & & & & & & & & 1 & & & & & & \\
\hline 36 & & & & & & & & & & & & & & & & & & & & & & & & & & & & & & & & & & \\
\hline 38 & & & & & & & & & & & & & & & & & & & & & & & & & & & & & & & & & & \\
\hline 39 & & & & & & & & & & & & & & & & 1 & & & & & & & & & & & & & & 1 & & & & \\
\hline 41 & & & & & & & 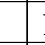 & 1 & & & 1 & & & & & & 2 & & & & & & & & & & 1 & & 1 & & & & & \\
\hline 43 & & 1 & & & & 2 & & & & & & & & & & 1 & 2 & & & & & & & & & & & & & & & & & \\
\hline 44 & 2 & 2 & 2 & 2 & 1 & 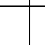 & 1 & 25 & 2 & 2 & 2 & 26 & 2 & 2 & 2 & 22 & 1 & 12 & 2 & 2 & 1 & 2 & 2 & 2 & 2 & 2 & 1 & & & & & 2 & 2 & 2 \\
\hline 48 & & & & & & & & 2 & & & & & & 1 & & 2 & 2 & & & & & & & & & 2 & 2 & 2 & 2 & 2 & 3 & & & \\
\hline 49 & & & & & & & & & & & & & & & & & & & & & & & & & & & & 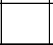 & & 1 & & & & \\
\hline 52 & & & & & & & & & & & & & & & & & & & & & & & & & & & & 1 & & & & & & \\
\hline 53 & & & & & & & & & & & & & & & & & & & & & & & & & & & & & & & & & & \\
\hline 54 & & & & & & & & & & & & & & & & & & & & & & & & & & & & & & & & & & \\
\hline 57 & & & & & & & & & & & & & & & & & & & & & & & & & & & & & & & & & & \\
\hline 58 & & & 1 & & & & & 1 & & & & 1. & 1 & & & & & & & & & & & 1 & & & & & & & & & & \\
\hline 60 & & 1 & 2 & 1 & 1 & & & 2 & 2 & & 21 & 17 & 1 & & & & & 2 & 2 & 1 & 1 & 1 & & & & & & & & & & & 2 & 1 \\
\hline 61 & & & & & & & & 1 & & & & & & & & & & & & & 1 & & & & & & & 1 & 1 & 1 & & & & \\
\hline 62 & & & & & & & & & & & & & & & & & & & & & & . & & & & & & & & & & & & \\
\hline 63 & 2 & 2 & 2 & 2 & 2 & 2 & & 2 & 2 & & 2 & 26 & 2 & 2 & 2 & 21 & 1 & 2 & 2 & 2 & 1 & 1 & 1 & \begin{tabular}{|l|l} 
\\
\end{tabular} & 2 & 1 & 1 & & & & & 2 & 2 & 2 \\
\hline 64 & 1 & 2 & 2 & 1 & 2 & 1 & 1 & 25 & 2 & & 7 & 26 & 25 & 25 & 22 & & & 2 & & 2 & 2 & 2 & 2 & 2 & 1 & & & & & & & 2 & & \\
\hline 70 & & & & & & & & & & & & & & & & & & & & & & & & & & & & & & & & & 1 & \\
\hline 71 & & & & & & 1 & & & & & 38 & & 2 & 4 & & & & 2 & 2 & & & & & & & & & & & & & & & \\
\hline 72 & & & & & & & & & & 3 & 2 & & & & & & & & & & & & & & & & & & & & & & & \\
\hline 73 & 3 & 3 & 3 & 3 & & 3 & 1 & $\vdots$ & 3 & 4 & $3 !$ & 3 & 25 & 2 & 3 & 1 & & 3 & 3 & 3 & 3 & 3 & 2 & 2 & 2 & & & & & & & 3 & 3 & 3 \\
\hline 77 & & 3 & 2 & 3 & 3 & 3 & 3 & 4 & & & & 24 & 4 & 4 & 4 & 44 & 2 & 1 & & 2 & 3 & 3 & 3 & 3 & 3 & 3 & 3 & & & & & + & + & + \\
\hline 78 & 3 & 2 & & 3 & 4 & 4 & 4 & $\begin{array}{l}3: \\
3\end{array}$ & 3 & & & & 4 & 42 & 44 & 44 & & 3 & & 4 & 4 & 4 & 3 & 3 & 3 & 3 & 3 & & & & & & & 3 \\
\hline 79 & 2 & & & 1 & 1 & 2 & 2 & 2 & 2 & & & & & & & & & 2 & & 2 & 2 & 2 & 1 & & & & & & & & & 2 & & \\
\hline 80 & & & & 1 & 2 & & 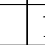 & 1 & & & & & & & & & & & & 1 & & & 1 & & & & & & & & & & & \\
\hline 81 & 2 & & 1 & 2 & 2 & 2 & 2 & 12 & 2 & & & & 25 & $\begin{array}{l}2 \\
2\end{array}$ & 22 & 2 & & 2 & & 2 & 2 & 1 & 2 & 2 & 2 & 2 & 1 & & & & & 2 & & 2 \\
\hline 82 & & & & & & & & & & & 1 & & & 4 & & & & 1 & 2 & & & & & & & & & & & & & & & \\
\hline 86 & & & & & & & & & & & 1 & & & & & 1 & 1 & & & & & & & & 1 & & & 1 & & & & & & \\
\hline
\end{tabular}




\begin{tabular}{|c|c|c|c|c|c|c|c|c|c|c|c|c|c|c|c|c|c|c|c|c|c|c|c|c|c|c|c|c|c|c|c|c|}
\hline \multicolumn{33}{|c|}{ Table 9, 1995-2 } \\
\hline & \multicolumn{11}{|c|}{, $1000-$} & \multicolumn{12}{|c|}{$E$} & \multicolumn{9}{|c|}{$\mathrm{F}$} \\
\hline & 4 & $5 \mid$ & 67 & \begin{tabular}{l|l}
78 & 8 \\
\end{tabular} & \begin{tabular}{|l|l}
9 \\
\end{tabular} & & \begin{tabular}{l|l}
0 & 1
\end{tabular} & & & 13 & & 1 & 2 & 3 & 4 & $5 \mid$ & & & \begin{tabular}{|l|l|}
8 & 9 \\
\end{tabular} & 10 & & 12 & & 1 & 2 & \begin{tabular}{l|l}
3 & 4 \\
\end{tabular} & \begin{tabular}{l|l}
4 & 5
\end{tabular} & & 7 & 8 & \begin{tabular}{l|l}
9 & 1 \\
\end{tabular} & 10 \\
\hline 2 & & & & & & & & & & & & & & & & & & & & & & & & & & & 1 & 2 & 1 & 1 & & \\
\hline 3 & & & & & & 1 & & & & 1 & & & & & & & & & & & & & & & & & & & & & & 1 \\
\hline 4 & 1 & $1:$ & $1]$ & $1]$ & 1 & & 1 & 2 & 1 & 1 & & & & & 2 & 2 & 2 & \begin{tabular}{l|l}
22 & 2 \\
\end{tabular} & \begin{tabular}{|l|l|}
2 & 2 \\
\end{tabular} & & 2 & 2 & & & & \begin{tabular}{l|l}
1 & 2 \\
\end{tabular} & \begin{tabular}{l|l}
2 & 1 \\
\end{tabular} & 2 & 1 & 1 & 2 & 2 \\
\hline 5 & & & & & & & & & & & & & & & & 1 & 1 & 1 & 1 & 1 & & 1 & & & & & & & 1 & & 1. & 1 \\
\hline 6 & & & & & & & & 1 & 1 & & 1 & & & & & & & & 1 & & & & 1 & & & & & & & & & 1 \\
\hline 7 & & & 1 & 1 & 1 & 1 & 1 & 2 & 1 & 2 & 2 & & & & & 1 & 2 & $\begin{array}{ll}22 \\
\end{array}$ & \begin{tabular}{|l|l|}
2 & 2 \\
\end{tabular} & 2 & 2 & 2 & 2 & & & & 1 & 2 & 1 & 2 & 2 & 2 \\
\hline 8 & & & & & & & & & & & & & & & 1 & & & & & & 1 & & & & & & & & +5 & & & 1 \\
\hline 10 & & & & & & & & & & & & & & & & & & & & & & & & & & & & & & & 1. & 1 \\
\hline 11 & & & 1 & 1 & 1 & 1 & 1 & 1 & 1 & 1 & & & & & 1 & & 1 & \begin{tabular}{l|l|l}
1 & 1 \\
\end{tabular} & \begin{tabular}{l|l}
1 & 1 \\
\end{tabular} & & & & & & & & \begin{tabular}{l|l}
1 & 1 \\
\end{tabular} & & & 1 & & 1 \\
\hline 12 & 1 & 1 : & 1 & 1 & 1 & 1 & & & & & & & & & 2 & 1 & 1 & \begin{tabular}{l|l|}
2 & 1 \\
\end{tabular} & \begin{tabular}{l|l}
1 & 1 \\
\end{tabular} & & & & & 1 & 1 & \begin{tabular}{l|l}
1 & 2 \\
\end{tabular} & \begin{tabular}{l|l|}
2 & 1 \\
\end{tabular} & & 1 & & & \\
\hline 13 & & 1 : & $1]$ & 11 & 1 & 1 & & & & & 1 & & & & 1 & 1 & 1 & \begin{tabular}{l|l}
2 & 2 \\
\end{tabular} & 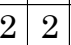 & 2 & & & 1 & 1 & & & \begin{tabular}{l|l}
1 & 2 \\
\end{tabular} & 1 & 2 & 2 & 2 & 2 \\
\hline 14 & \begin{tabular}{|l|l|}
2 \\
\end{tabular} & 1 & & & & & & & & & & & & & & 1 & 2 & \begin{tabular}{l|l}
2 & 2 \\
\end{tabular} & \begin{tabular}{l|l}
2 & 2 \\
\end{tabular} & & & & & & & & 1 & 1 & \begin{tabular}{|l|}
1 \\
\end{tabular} & & \begin{tabular}{l|l|}
2 & \\
\end{tabular} & \\
\hline 15 & 2 & 1 & 1 & & 1 & 1 & & & & & 1 & & 2 & 3 & 3 & 3 & 3 & \begin{tabular}{l|l}
2 & 3
\end{tabular} & \begin{tabular}{l|l}
3 & 3
\end{tabular} & 3 & 3 & 1 & & 2 & 2 & \begin{tabular}{l|l}
3 & 2
\end{tabular} & \begin{tabular}{l|l}
2 & 2
\end{tabular} & 2 & 2 & 3 & 3 & 3 \\
\hline 16 & & & 1 & $\begin{array}{lll}1 & 1\end{array}$ & 1 & 1 & 1 & 1 & 1 & & 3 & & & & & & & & 1 & 1 & & & 3 & & & & & 1 & 1 & & 1 & 1 \\
\hline 17 & 2 & 1 & & & 1 & 1 & 1 & 1 & & 1 & & & & & & & 2 & 2 & 2 & 2 & & & & 1 & 1 & \begin{tabular}{l|l}
2 & 2 \\
\end{tabular} & \begin{tabular}{l|l}
2 & 2 \\
\end{tabular} & 2 & 2 & 2 & 3 & 3 \\
\hline 18 & & & 1 & & & & & & 1 & & 2 & & & & & 1 & 1 & & 1 & & & & 1 & & & & & 1 & & 1 & & 1 \\
\hline 19 & & & & & & & & & & & & & & & & & & & & 1 & & & & & & & & & & & & \\
\hline 20 & 1 & & 1 & & & & & & & & & & & & & 1 & 2 & \begin{tabular}{l|l}
1 &
\end{tabular} & 2 & & & & & & & & 1 & 2 & & & & \\
\hline 21 & & & & & & & & & & & & & & & & & & & & & & & & & & & & & & & & 1 \\
\hline 22 & & & & & & & & & & & & & & & & & & & & & & & & & & & & & & & & \\
\hline 23 & & & & & & & & & & & & & & & & & & & & & & & & 2 & & & & 2 & & & & \\
\hline 25 & & & & & & & & & & & & & & & & & & & & & & & & & & & & & & 1 & & \\
\hline 26 & & & & & & & 1 & 1 & & 1 & & & & & & & & & 1 & & 1 & & & & & & & & & & $\begin{array}{ll}1 \\
\end{array}$ & 1 \\
\hline 27 & & & & & & & 1 & 1 & 1 & & & & & & & & & & 1 & 1 & 1 & & 1 & & & & & & & & & 1 \\
\hline 28 & & & $1]$ & $\begin{array}{lll}1 & 1 \\
\end{array}$ & 1 & 2 & 2 & 2 & 1 & 1 & & & & & & & 1 & & \begin{tabular}{l|l}
1 & 1 \\
\end{tabular} & 1 & & 1 & & & & & 2 & 2 & 1 & 1 & 2 & 2 \\
\hline 29 & & & & & & & 1 & 1 & & & & & & & & & & & 1 & & 1 & & & & & & & & & 1 & 1 & 1 \\
\hline 36 & & & & & & & & & & & & & & & & & & 1 & & 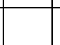 & & & & & & & & & & & & \\
\hline 38 & & & & & & & & & & & & & & & & & & & & 1 & & & & & & & & & & & & \\
\hline 39 & & & & & & & & & & & & & & & & & & & & 1 & & & & & & 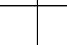 & & & & & 1 & \\
\hline 41 & & & . & 1 & 1 & & 1 & 1 & 1 & & & & & & & 1 & 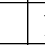 & 1 & 1 & & 1 & 1 & 1 & & & & & 1 & 1 & 1 & 1 & 1 \\
\hline 43 & & & & & & & & & & & & & & & & & 1 & & 1 & + & & & & & & ++ & ++ & & 2 & & \begin{tabular}{l|l}
2 \\
\end{tabular} & \\
\hline 44 & 2 & 1 & 1 & $\begin{array}{ll}1 & 1\end{array}$ & 1 & & 1 & & & & & 2 & 2 & 2 & 2 & 2 & $\frac{1}{2}$ & \begin{tabular}{l|l}
2 & 1
\end{tabular} & \begin{tabular}{|l|l|}
1 & 1
\end{tabular} & & & & & 2 & 2 & 22 & \begin{tabular}{|l|l}
2 & 2
\end{tabular} & & 1 & & 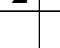 & \\
\hline 48 & & & & & 1 & & $\begin{array}{ll}25 \\
\end{array}$ & 2 & 2 & 2 & 2 & & & & & & & & 3 & 2 & 2 & 2 & 2 & & & & 1 & & 2 & \begin{tabular}{|l|}
1 \\
\end{tabular} & 28 & 2 \\
\hline 49 & & & & & & & & & & & & & & & & & & & & & & 2 & & & & & & & 4 & 1 & 1 & \\
\hline 52 & & & & & & & & & & & & & & & & & & & & & & & & & & & & & & & & \\
\hline 53 & & & & & & & & & & & & & & & & & & & & & & & 1 & & & + & + & 1 & & & 2 & 2 \\
\hline 54 & & & & & & & & & & & & & & & & & & & & & & & & & & & & & & 1 & & \\
\hline 57 & & & & & & & & & & & & & & & & & 1 & 1 & 1 & & & & & & & & & & 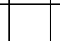 & 1 & 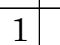 & \\
\hline 58 & & & 1 & & & & & & & & & & & & & 1 & 1 & 1 & & & & & & & & & \begin{tabular}{l|l}
1 & 1 \\
\end{tabular} & & \begin{tabular}{|l|l|} 
& \\
\end{tabular} & 1 & & \\
\hline 60 & & & & & & & & & & & & & & & & 1 & 1 & & & & & & & & & & & 1 & & & & \\
\hline 61 & & & & & & & & 1 & 1 & 1 & & & & & & & & 2 & \begin{tabular}{|l|l}
2 & 1 \\
\end{tabular} & 1 & 2 & 2 & 2 & & & & & & & 1 & 1 & 2 \\
\hline 62 & & & & & & & & & & & & & & & & & & & & & 1 & & & & & & & & & & & \\
\hline 63 & 2 & 1 & \begin{tabular}{l|l}
1 &
\end{tabular} & 15 & $2 \sqrt{2}$ & & \begin{tabular}{l|l}
1 & 1
\end{tabular} & 1 & & & & & & 1 & 2 & 2 & 2 & \begin{tabular}{l|l}
2 &
\end{tabular} & \begin{tabular}{|l|l}
2 & 1
\end{tabular} & 1 & & & & 1 & 1 & \begin{tabular}{l|l}
1 &
\end{tabular} & \begin{tabular}{l|l}
2 &
\end{tabular} & \begin{tabular}{|l|l}
2 & 1
\end{tabular} & 2 & \begin{tabular}{|l|}
1 \\
\end{tabular} & 1 & \\
\hline 64 & \begin{tabular}{|l|}
1 \\
\end{tabular} & 1 & 1 & & & & & & & & & 2 & 1 & 2 & 2 & & & & & & & & & & 2 & \begin{tabular}{l|l}
1 & 2 \\
\end{tabular} & \begin{tabular}{|l|l|}
2 & \\
\end{tabular} & & & & & \\
\hline 70 & & & & & & & & & & & & & & & & & & & & & & & & & & & & & & & & \\
\hline 71 & & & & & & & & & & & & & & & & & & & & & & & & 1 & & & & & & & & \\
\hline 72 & & & & & & & & & & & & & & & & & & & & & & & & & & & & & & & & \\
\hline 73 & 3 & 2 & $\begin{array}{ll}36 \\
\end{array}$ & 2 & & & & & & & & 2 & 2 & 2 & 3 & 3 & 2 & 2 & & & & & & 3 & 3 & \begin{tabular}{l|l}
3 & 2
\end{tabular} & \begin{tabular}{l|l}
2 & 3
\end{tabular} & & & & 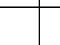 & \\
\hline 77 & 4 & 3 & 28 & 25 & 1 & & 1 & & & & & + & + & 4 & \begin{tabular}{|l|}
3 \\
\end{tabular} & 4 & +1 & ++ & ++ & & & & 2 & 1 & 3 & \begin{tabular}{l|l}
4 & 4 \\
\end{tabular} & \begin{tabular}{l|l}
4 & 3 \\
\end{tabular} & \begin{tabular}{|l|l}
3 \\
\end{tabular} & & & $\begin{array}{ll}2 & \\
\end{array}$ & + \\
\hline 78 & 3 & 3 & 28 & 25 & \begin{tabular}{l|l}
2 \\
\end{tabular} & & 1 & & & & & 4 & 4 & 4 & 4 & 3 & 3 & & & & & & & 4 & 3 & \begin{tabular}{l|l}
3 \\
\end{tabular} & 1 & & & & & \\
\hline 79 & 1 & & & & & & & & & & & 2 & 1 & & & & & & & & & & & 3 & 1 & & & & & & & \\
\hline 80 & \begin{tabular}{|l|}
1 \\
\end{tabular} & & & & & & & & & & & & & & & & 2 & & & & & & & & & & & & & & & \\
\hline 81 & 2 & & & & & & & & & & & 3 & & 2 & & & & & & & & & & 2 & 2 & 1 & & & & & & \\
\hline 82 & & & & & & & & & & & & & & & & & & & & & & & & & & & & & & & & \\
\hline 86 & & & 1 & & & & & & & & & & & & & & & 1 & & & & & & & & & & & 1 & & 1 & \\
\hline
\end{tabular}




\begin{tabular}{|c|c|c|c|c|c|c|c|c|c|c|c|c|c|c|c|c|c|c|c|c|c|c|c|c|c|c|c|c|c|c|c|c|c|c|c|}
\hline \multicolumn{36}{|c|}{ Table 9, 1995-3 } \\
\hline & \multicolumn{8}{|c|}{$\mathrm{A}$} & & & & & $\mathrm{E}$ & $B$ & & & & & & & & & & & & C & & & & & & & & D & \\
\hline & 1 & 2 & 3 & 4 & & 6 & 7 & 8 & 1 & 2 & 3 & 4 & 5 & & 7 & 8 & 9 & 10 & 1 & 2 & 3 & 4 & 5 & 6 & 7 & 8 & 9 & & 11 & 12 & & 14 & & & 3 \\
\hline 87 & & & & & & & & & & & & & & & & & & & & 1 & & & & & & & & & & & & 1 & & & \\
\hline 88 & & & & & & & & & & & & 1 & 1 & & & & & & & & & & & & & & & & & & 1 & & & & \\
\hline 89 & & & & & & & & & & & & & & & & & & & & & & & & & & & & & & & 1 & & & & \\
\hline 91 & & & & & & & & & & & & & & & & & & & & & & & & & & & & & & 1 & & & & & \\
\hline 93 & & & & & & & & 1 & & & & & & & & & & & & & & & & & & & & & & & & & & & \\
\hline 94 & & & & & & & & 1 & & & & & & 1 & & & & 1 & & & & & & & & & & & & & & & & & \\
\hline 95 & & & & & & & & 1 & & & & & & & & & & & & & & & & & & & & & & & & & & & \\
\hline 99 & & & & & & & & & & & & & & & & & & & & & & & & & & & & & 1 & & 1 & & & & \\
\hline 101 & & & & & & & & & & & & & & & & & & & & & & & & & & & & & & & & & & & \\
\hline 103 & & & & & & & & 1 & & & & & & & & & & & & & & & & & & & & & 1 & 1 & 1 & & & & \\
\hline 106 & & & & & & & & & & & & & & & & & & & & & & & & & & & & & & & & & & & \\
\hline 107 & & & & & & & & & & & & & & & & & & & & & & & & & & & & & 1 & & & & & & \\
\hline 112 & & & & & & & & & & & & & & & & & & & & & & & & & & & & & & & & & & & \\
\hline 115 & & & & & & & & & & & & & & & & & & & & & & & & & & & & & & & & & & & \\
\hline 116 & & & & & & & & & & & & & & & & & & & & & & & & & & & & & & & & & & & \\
\hline 119 & & & & & & & & & & & & & & & & & & & & & & & & & & & & & 1 & & 1 & & & & \\
\hline 122 & & & & & 1 & 1 & & & & & & & & & & & & & & & & & & & & & & & & & & & & & \\
\hline 123 & & & 1 & 1 & & & 1 & 1 & & 1 & 1 & 1 & 1 & & 1 & & 1 & 1 & 1 & 1 & 1 & 1 & 1 & & 1 & & 1 & 1 & 1 & & & & & & \\
\hline 124 & 2 & 2 & 2 & 2 & 2 & 2 & & & \begin{tabular}{|l|} 
\\
\end{tabular} & & 2 & 2 & 2 & 2 & 2 & 2 & & & 2 & 2 & 2 & & 2 & 2 & 2 & 2 & 2 & & & & . & . & 1 & 2 & 2 \\
\hline 125 & & & & & & & & & & & & & & & & & & & & & & & & & & & & & & & & & & & \\
\hline 126 & & & & & & & & & & & & & & & & & & & & & & . & & & & & . & & & & 1 & & & & \\
\hline 129 & & & & & & & & & & & & & & & & & & & & & & & . & & & & . & & & & & & & & \\
\hline 130 & & & & & & & & & & & & & & 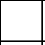 & & & & & & & 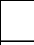 & . & . & & & & 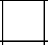 & & & 1 & 1 & & & & \\
\hline 131 & & & & & & & & & & & & & & & & & & & & & & & . & & & & & & & & & & & & \\
\hline 132 & & & & & & 2 & 1 & 2 & & & & & 1 & & & 1 & & 2 & & & & & & & & & 2 & 2 & 1 & 1 & & & & & \\
\hline 141 & & & & & & & & & & & & & & & & 1 & & & & & & & & & & & 1 & & 2 & 1 & 1 & 2 & & & \\
\hline 142 & & & & & & 1 & & 1 & & 1 & 1 & 1 & & & 2 & & & 3 & & & 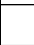 & & . & & & 1 & 2 & 1 & 1 & 2 & 1 & 1 & & & \\
\hline 143 & & & & & & & & 2 & & & & & & & & & & & & & & & & & & & & & & & & & & & \\
\hline 144 & & & & & & & & & & & & & & & & & & & & & & & & & & & & & 1 & & & & & & \\
\hline 145 & & & 1 & 2 & 2 & 2 & & 1 & & & 1 & 2 & 3 & 1 & & 1 & 2 & & & & & & & & & & & & 1 & 1 & & & & & \\
\hline 146 & & & & & & & & 2 & & & & & & & & & & 1 & & & & & & & & & & & 1 & 1 & 1 & & & & \\
\hline 149 & & & & & & & & & & & & & & & & & & & & & & & & & & & & & & & \begin{tabular}{|l|} 
\\
\end{tabular} & & & & \\
\hline 156 & & & 1 & & & & & & & & & & & & & & & & 1 & 1 & 1 & & & & & & & & & & & & & & \\
\hline 158 & & & & & & 1 & & & & & & 1 & & & & & & & & & 1 & & & & & & & & & & & & & & \\
\hline 159 & & & & & & & & & & & & & & & & & & & & & & & & & & & & & & & & & & & \\
\hline 161 & & & 1 & & & & & & & & & & & & & & & & & & & & & & & & & & & & & & & & \\
\hline 168 & & & & & & & & & & & 1 & & & & & & & & & & & & & & & & & & & & & & & & \\
\hline 169 & & & & & & & & & & & & & & & & & & & & & & & & & & & & & & & & & & & \\
\hline 173 & & & & & & & & & & & & & & & & & & & & & & & & & & & & & 1 & & & & & & \\
\hline 176 & & & & & & & & & & & & & & & & & & 1 & & & & & & & & & & & & & & & & & \\
\hline 180 & & & & & 2 & & & 1 & & 1 & & & & & & & & 1 & & & & & & & & & & & & 1 & & & & & \\
\hline 181 & & & 1 & & & & & 1 & & & 1 & & 1 & & 1 & & & & & & & & & & & & & & & & & & & & \\
\hline 182 & & & 1 & & & & & & & & & & & 1 & & & & 1 & 1 & & & & & & & & & & & & & & & & \\
\hline 183 & & & & & & & & & & & & & & & & & & & & & & & & & & & & & & & & & & & \\
\hline 184 & & & & & & & & & & & & & & & & & & & & & & & & & & & & & & & & & & & \\
\hline 195 & & & & & & & & & 1 & & & & & & & & & & & & & & & & & & & & & & & & & 1 & \\
\hline 197 & & & & & & & & 1 & & & & & & & & & & & & & & & & & & & & & & & & & & & \\
\hline 198 & & & & 1 & & 2 & 2 & & & & & & 3 & 2 & 2 & & 2 & & & & & 2 & 2 & 2 & & & . & & & & & & & & 1 \\
\hline 199 & & 1 & & 1 & & 2 & 2 & 1 & & & & & & 2 & 1 & 2 & 2 & 1 & & & 1 & 2 & 2 & 2 & & 1 & 1 & 1 & 1 & & & . & & 1 & 1 \\
\hline 200 & & & & & & 2 & & 1 & & & & & & 2 & 2 & & & 2 & & & & 1 & & 1 & & & 2 & 1 & 1 & 1 & 2 & 2 & & & \\
\hline 201 & & & & & & & & & & & & & & & & & & & & & & & & & & & & & & & & & & & \\
\hline 203 & & & 1 & 1 & & & & & & & & & & & & & & & & & & & & & & & & & & & & & & & \\
\hline 207 & & & 1 & 1 & & & & 1 & & & & 1 & & & & 2 & & 2 & 1 & 1 & & & & & & & & & & 1 & 2 & 1 & & & \\
\hline 210 & & & & & & & & & & & & & & & & & & & & & & & & & & & & & & & & & & & \\
\hline 211 & + & 3 & 2 & 1 & 1 & 1 & 3 & 3 & & & & & & 1 & 3 & 3 & 3 & & & & & & & 3 & 3 & 3 & 3 & 3 & & & & & & & + \\
\hline
\end{tabular}




\begin{tabular}{|c|c|c|c|c|c|c|c|c|c|c|c|c|c|c|c|c|c|c|c|c|c|c|c|c|c|c|c|c|c|c|c|c|c|c|}
\hline \multicolumn{34}{|c|}{ Table 9, 1995-4 } & \\
\hline & \multicolumn{11}{|c|}{ a torar } & \multicolumn{13}{|c|}{$\mathrm{E}$} & & & & & $\mathrm{F}$ & & & & & \\
\hline & 4 & 5 & 6 & 7 & 8 & 9 & & & 12 & 13 & & 1 & 2 & 3 & 4 & 5 & 6 & & 8 & 9 & & & 12 & 13 & 1 & 2 & 3 & 4 & & & $\begin{array}{ll}7 & \varepsilon \\
\end{array}$ & & \begin{tabular}{l|l}
9 & 10 \\
\end{tabular} & \\
\hline 87 & & & & & & & & & & & & & & & & & & & & & & & & & & & & & 1 & & & & & \\
\hline 88 & & & & & & & & & & & & & & & & & & & 1 & & & & & & & & & & & 1 & 1 & 1 & 1 & \\
\hline 89 & & & & & & & & & 1 & & & & & & & & & & & & & & 1 & & & & & & & & & & 1 & \\
\hline 91 & & & & & & & & & & & & & & & & & & & & & & & & & & & & & & & & & & \\
\hline 93 & & & & & & & & 1 & 1 & & & & & & & & & & & & & & & & & & & & & & & & & \\
\hline 94 & & & & & & & & & & & & & & & & & & & & & & & & & & & & & & & & & \begin{tabular}{l|l}
1 & 1 \\
\end{tabular} & \\
\hline 95 & & & & & & & & & & & & & & & & & 1 & & & & & & & & & & & & & & 1 & & & \\
\hline 99 & & & & & 1 & & & & 1 & & & & & & & & & & & & & 1 & & & & & & & & & & 1 & \begin{tabular}{l|l}
1 & 1 \\
\end{tabular} & \\
\hline 101 & & & & & & & & & 1 & & & & & & & & & & & & & & & & & & & & & & & & & \\
\hline 103 & & & & & & & 1 & 1 & & & & & & & & & & 1 & 1 & & 1 & 1 & & 1 & & & & & & & 1 & & & \\
\hline 106 & & & & & 1 & & & & & & & & & & & & & & & & & 1 & & & & & & & & & & & & \\
\hline 107 & & & & & & & & 1 & & & & & & & & & & & 1 & 1 & & & & & & & & & & & & & 1 & \\
\hline 112 & & & & & & & & & & & & & & & & & & & & & 1 & & & & & & & & & & & & & \\
\hline 115 & & & & & & & & & & & & & & & & & & & 1 & & & & & & & & & & & & & 1 & & \\
\hline 116 & & & & & & & & 1 & & & & & & & & & & & & 1 & & & & & 1 & & & & & & & 1 & & \\
\hline 119 & & & & & & & & 1 & & & & & & & & & & & 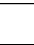 & & 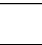 & & & & & & & & & 1 & $1 \mid 1$ & & & \\
\hline 122 & & & & & & & & & & & & & & & & & 1 & & 1 & & & & & & & & & & 1 & & 2 & 12 & \begin{tabular}{l|l}
2 & 1 \\
\end{tabular} & \\
\hline 123 & 1 & 1 & 1 & 1 & 1 & & & 1 & 1 & & & & & & & 1 & 1 & 2 & 2 & & 1 & & & 1 & & & & & 2 & 2 & 2 & 2 & \begin{tabular}{l|l}
2 & 1 \\
\end{tabular} & 1 \\
\hline 124 & 2 & 1 & 1 & 2 & 2 & 1 & 1 & 1 & 1 & & & & & 1 & 1 & 2 & 2 & 2 & 2 & & & & & & 1 & 1 & 2 & 2 & 2 & 2 & 1 & 1 & & \\
\hline 125 & & & & & & & & & & & & & & & & 1 & 1 & & & & & & & & & & & & & & & & 1 & 1 \\
\hline 126 & & & & & & & & & & & & & & & & & & & & & & & & & & & & & & & & & & \\
\hline 129 & & & & & & & & & & & & & & & & & & & & & & & & & & & & & & & & 1 & 1 & \\
\hline 130 & & & & & & & & 1 & 1 & & & & & & & 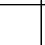 & & 1 & & 1 & 1 & & 2 & & & & & & & & 11 & 1 & \begin{tabular}{l|l|}
1 & 1 \\
\end{tabular} & \\
\hline 131 & & & & & & & & & & & & & & & & & & & & & & & & & & & & & & & 1 & 1 & 1 & \\
\hline 132 & & 1 & 1 & 1 & & 2 & 2 & 2 & 1 & 1 & & & & & & 1 & 1 & 2 & 2 & 2 & 2 & & & & & & & & 2 & & 2 & 2 & 1 & \\
\hline 141 & & & 1 & 1 & 1 & & & 2 & 1 & 1 & & & & & & 1 & & 1 & 1 & 1 & 1 & 2 & 2 & 2 & & & & & & & & 1 & \begin{tabular}{l|l}
2 & 1
\end{tabular} & \\
\hline 142 & & & & & 1 & 2 & 1 & 1 & 1 & 1 & 1 & & & & & 2 & 2 & 2 & 2 & 2 & 1 & & 2 & 1 & & & 1 & 1 & 2 & 2 & 2 & & \begin{tabular}{l|l}
22 \\
\end{tabular} & \\
\hline 143 & & & & & & & & & & & & & & & & & & & 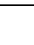 & & & & & & & & & & & & 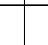 & 2 & \begin{tabular}{l|l}
2 & 1 \\
\end{tabular} & \\
\hline 144 & & & & & & & & & & & & & & & & & & & 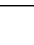 & & & & & & & & & & & & 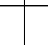 & 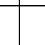 & & \\
\hline 145 & & & & & & & & & 1 & & & & & & & & & & & & & & & & & & & & & 1 & 2 & 1 & 2 & \\
\hline 146 & & & & & 1 & & & 2 & 1 & & & & & & & & 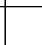 & & 1 & 2 & 2 & 2 & 1 & 1 & & & & & & & 1 & 1 & 22 & \\
\hline 149 & & & & & & & & & & & & & & & & & & & & 1 & 1 & & & & & & & & & 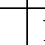 & 1 & & $\begin{array}{ll}1 & 1\end{array}$ & \\
\hline 156 & & & & & & & & & & & & & & & & & 2 & & & & & & & & & & & & & & & & & \\
\hline 158 & 1 & 1 & 1 & & 1 & & & & & & & & & & & & & 1 & & & & & & & & & & & & & & & & \\
\hline 159 & & & & & & & & 1 & & & & & & & & & & & & & & & & & & & & & & & & & 1 & \\
\hline 161 & & & & & & & & & & & & & & & & 1 & 1 & & & & 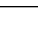 & 1 & & & & & & & 1 & 1 & 1 & 1 & & \\
\hline 168 & & & & & & & & & & & & & & & & & & & & & & & & & & & & & & & & & & \\
\hline 169 & & & & & & & & & & & & & & & & & & & & & & & & & & & & & & & 1 & 1 & & \\
\hline 173 & & & & & & & & & & & & & & & & & & & & & 1 & & 1 & & & & & & & 1 & & 1 & 1 & \\
\hline 176 & & & & & & & & & & & & & & & & & & & & & & & & & & & & & & & & & & \\
\hline 180 & & & & & 1 & 1 & 1 & 1 & 1 & 1 & & & & & & & & 1 & 1 & 1 & 1 & 2 & & & & & & & & 1 & 1 & & 2 & \\
\hline 181 & & & & & & & & & & & & & & & & & & 1 & 1 & 1 & 1 & 1 & & & & & & 1 & & 1 & 1 & & 2 & \\
\hline 182 & & & & & & & & & & & & & & & & & 1 & 1 & & 1 & 1 & & & & & & & & 1 & 1 & 1 & 1 & 1 & \\
\hline 183 & & & & & & & & & & & & & & & & - & & & & & & & & & & & & & & & & & 1 & 1 \\
\hline 184 & & & & & & & & & & & & & & & & 1 & & & & & & & & & & & & & & & & & & \\
\hline 195 & & & & & & & & & & & & & & & & & & & & & & & & & & & & & & & & & & \\
\hline 197 & & & & & & & & & & & & & & & & & 1 & 1 & & & & & & & & & & & & & & & & \\
\hline 198 & 1 & & & & & & & & & & & & 2 & 2 & 2 & & & & & & & & & & 1 & 2 & 2 & & & & & & 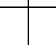 & \\
\hline 199 & 2 & 1 & & & 1 & 1 & 1 & 1 & & & & & & 1 & & 1 & 1 & 1 & & & & & & & 2 & & 1 & & & 1 & & & 1 & \\
\hline 200 & & & 1 & 1 & & 1 & & 2 & & 1 & & & & & & & & 2 & 2 & 2 & 2 & 2 & 2 & 2 & & & & 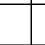 & 1 & 2 & 2 & 22 & \begin{tabular}{l|l}
22 &
\end{tabular} & \\
\hline 201 & & & & & & & & & & & & & & & & & & & & & & & & & & & & & & 1 & & & & \\
\hline 203 & & & & & & & & 1 & & & & & & & & & & & & & & & & & & & & & & & & & 1 & \\
\hline 207 & & & 1 & 11 & & & & 1 & 1 & 1 & & & & & & & 1 & 1 & 1 & 1 & 1 & & 2 & 2 & & & & & & 2 & . & 2 & 22 & \\
\hline 210 & & & & & & & & & & & & & & & & & & & & & & & 1 & & & & & & & & & & 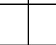 & \\
\hline 211 & 3 & & & & & & & & & 2 & & & & & & & & 2 & & & & & & & 2 & & 2 & & & & & & & \\
\hline
\end{tabular}




\begin{tabular}{|c|c|c|c|c|c|c|c|c|c|c|c|c|c|c|c|c|c|c|c|c|c|c|c|c|c|c|c|c|c|c|c|c|c|c|}
\hline \multicolumn{35}{|c|}{ Table 9, 1995-5 } \\
\hline & $A$ & & & & & & & & $\mathrm{~B}$ & & & & & & & & & $\mathrm{O}$ & & & & & & & & & & & & & & $\mathrm{D}$ & & \\
\hline & 1 & 2 & 3 & 4 & 5 & 6 & 7 & 8 & 12 & 2 & \begin{tabular}{l|l}
3 & 4 \\
\end{tabular} & $4: 5$ & 56 & $6 \quad 7$ & $\begin{array}{l}78 \\
\end{array}$ & 9 & 16 & $\begin{array}{ll}01 \\
\end{array}$ & 2 & 3 & 4 & 5 & 6 & 7 & 8 & 9 & 10 & 111 & 12 & 13 & 14 & & 23 & 3 \\
\hline 212 & & & & & & 1 & & & & & & 4 & 4 & 4 & 3 & & & 1 & 4 & & 3 & & & & 3 & & 1 & & 2 & & & & & \\
\hline 213 & & & & & & & & 2 & & & & & & & & & 2 & & & & & & & & & & & & 1 & 1 & & & & \\
\hline 214 & & 1 & 1 & & 1 & & 1 & 3 & & 1 & \begin{tabular}{l|l}
1 & 1 \\
\end{tabular} & 1 & 1 & 1 & 1 & 2 & 3 & 3 & 1 & 1 & 1 & & 1 & 1 & 2 & 3 & 4 & 3 & 4 & 3 & 4 & 1 & 1 & 1 \\
\hline 216 & & & & & & & & & & & & & & & & & & & & & & & & & & & & & 1 & & & & & \\
\hline 220 & & & & 1 & 1 & 1 & & 1 & & 2 & \begin{tabular}{l|l}
1 & 1 \\
\end{tabular} & $1]$ & $1]$ & 1 & & & 1 & 1 & 1 & 1 & 1 & & & & & & & 1 & 1 & 1 & & & & \\
\hline 221 & & & & & & & & & & & & & & & & 1 & & & & & & & & & & & & & & & & & & \\
\hline 227 & & & & & & & & & & & & & & & 2 & 21 & & & 1 & & & & 1 & 1 & & 2 & & & & & & & 1 & \\
\hline 228 & & & & & & & & & & & & & & & & & & & & & & & & & & & & & & & & & & \\
\hline 229 & 1 & 1 & 2 & 1 & 1 & 1 & 2 & 3 & & 2 & 2 & 2 & & 13 & 3 & 3 & 2 & 1 & 1 & 2 & 1 & 2 & 2 & 3 & 3 & 3 & 3 & 3 & 1 & & & 1 & $1 \mid 1$ & 1 \\
\hline 230 & & & 1 & & & & & 1 & & & & & & & 2 & 2 & 2 & & 1 & & & 1 & & 1 & 1 & 1 & 1 & 2 & & & & & & \\
\hline 232 & + & + & + & + & + & + & + & 4 & & & & . & & 1 & 11 & 12 & 1 & & 1 & & & & + & + & + & + & + & 2 & & & & + & +1 & + \\
\hline 233 & & & & & & & 1 & & & & & 1 & 1 & & & & & & & & & & & & & & & & & & & & & \\
\hline 234 & & & & 1 & & & & 1 & & & & 1 & & 1 & & & & & & & & & & & & & & & & & & & & \\
\hline 235 & & & 1 & & & & & & & & $\begin{array}{ll}1 & \end{array}$ & 1 & & & 1 & 12 & 2 & & & & & & & & & & & & & & & & & \\
\hline 240 & & & & & & & & & & & & & & & & & & & & & & & & & & & & & & & & & & \\
\hline 242 & & & & & & & & & & & & & & & & & & & 1 & & & & & & & & & & & & & & & \\
\hline
\end{tabular}

\begin{tabular}{|c|c|c|c|c|c|c|c|c|c|c|c|c|c|c|c|c|c|c|c|c|c|c|c|c|c|c|c|c|c|c|c|c|c|c|}
\hline & \multicolumn{11}{|c|}{$\mathrm{D}$} & \multicolumn{13}{|c|}{$E$} & \multicolumn{10}{|c|}{$\mathrm{F}$} \\
\hline & 4 & 5 & 6 & 7 & 8 & 9 & 10 & & 12 & & & 1 & 2 & 3 & 4 & 5 & 6 & & $8 \mathrm{~g}$ & $\begin{array}{l}9 \\
9\end{array}$ & & & 12 & 13 & 1 & 2 & 3 & 4 & 5 & & & & $\begin{array}{lll}9 & 1\end{array}$ & 10 \\
\hline 212 & & 4 & 1 & & & & & 1 & 1 & & & & & & & 4 & 4 & & 4 & & & & 4 & 2 & & & & & 4 & 42 & & & \begin{tabular}{l|l}
3 \\
\end{tabular} & \\
\hline 213 & & & & & 1 & & & 1 & 1 & & & & & & & & 1 & 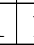 & 1 & 1 & 1 & & & & & & & & & & & 12 & 2 & \\
\hline 214 & 2 & 1 & 1 & 1 & 1 & 1 & 1 & 3 & 3 & 2 & 4 & & & & 1 & 1 & 2 & & 2 & 2 & 3 & 2 & 3 & 4 & & 1 & 1 & 1 & 1 & 2 & & 1 & 2 & \\
\hline 216 & & & & & & & & & 1 & & & & & & & & & & & & & & & & & & & & & & & & & \\
\hline 220 & & & & 1 & 1 & & & 1 & 1 & 1 & & & & & & & 1 & 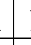 & $\begin{array}{lll}1 & 1\end{array}$ & 1 & 1 & 1 & 1 & 1 & & & & & 1 & 1 & & $\begin{array}{lll}12 \\
\end{array}$ & 2 & \\
\hline 221 & & & & & & & & & & & & & & & & & & & & & & & & & & & & & & & & & & \\
\hline 227 & 1 & & 1 & 1 & & & 1 & 1 & & & & 1 & & & & & & 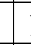 & 1 & 1 & 1 & & 1 & & & & & & & & & & & \\
\hline 228 & & & & & & & & & & & & & & & & & & & & & & 1 & & & & & & & & & & & & \\
\hline 229 & 2 & 1 & 1 & 3 & 2 & 2 & 2 & 2 & 1 & 1 & & & 2 & 2 & 3 & 3 & 3 & & $3:$ & 3 & $3 \quad$ & 3 & 2 & & 2 & 2 & 3 & 3 & 3 & 3 & 2 & 3 & 3 & \\
\hline 230 & & & & & & & & & & & & & & & & 1 & 1 & 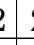 & 2 & 2 & 2 & & & & & & & 1 & 1 & & & & 1 & \\
\hline 232 & + & 1 & & 1 & & 1 & 1 & & 1 & 2 & & + & + & + & + & + & + & - & + & $t$ & & & & & 3 & + & + & + & + & & & 1 & $t$ & + \\
\hline 233 & & & & 1 & & & & & & 1 & & & & & & & & & & & & & & & & & & & 1 & & & & 11 & 1 \\
\hline 234 & & & & & & & & & & & & & & & & & & & & & & & & & & 1 & & & & & 1 & & & \\
\hline 235 & & & & & & & & & & & & & & & & & & & & & & & & & & & & & & 1 & & & 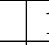 & 1 \\
\hline 240 & & & & & & & & & & & & & & & & & & & & & & & & & & & & & & & & & & 1 \\
\hline 242 & & & & & & & & & & & & & & & & & & & & & & & & & & & & & & & & & & \\
\hline
\end{tabular}




\begin{tabular}{|c|c|c|c|c|c|c|c|c|c|c|c|c|c|c|c|c|c|c|c|c|c|c|c|c|c|c|c|c|c|c|c|c|c|c|}
\hline \multicolumn{35}{|c|}{ Table 9, 1996-1 } \\
\hline & \multicolumn{8}{|c|}{$\mathrm{A}$} & \multicolumn{10}{|c|}{$\mathrm{B}$} & & & & & & & C & & & & & & & & D & \\
\hline & 1 & 2 & 3 & 4 & & 6 & 7 & 8 & 1 & 2 & 3 & 4 & 5 & \begin{tabular}{l|l}
6 & 7 \\
\end{tabular} & 78 & 3 & 1 & \begin{tabular}{l|l}
0 & 1 \\
\end{tabular} & 2 & 3 & 4 & 5 & 6 & 7 & 8 & 9 & 10 & 11 & 12 & & 14 & & & 3 \\
\hline 2 & & & & & & & & & 1 & & & & & & & & & & & & 2 & & & & & & & & & & & & & \\
\hline 3 & & & & & & & & & & & & & & & & & & & & & & & & & & & & & 1 & & & & & \\
\hline 4 & 1 & 1 & 1 & 1 & 1 & 1 & 1 & 2 & 1 & 1 & & 1 & 2 . & $1] 1$ & \begin{tabular}{l|l}
1 & 1 \\
1
\end{tabular} & 12 & 2 & & 1 & 1 & & 2 & & & 1 & 2 & 2 & & 1 & 1 & 2 & & & \\
\hline 5 & & & & & & & & 2 & & & & & 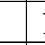 & 1 & & & & & & & & & & & 1 & 1 & 1 & & 1 & & & & & \\
\hline 6 & & & & & 1 & & & & & & & & & & & & & & & & & & & & & & & 1 & & & 1 & & & \\
\hline 7 & & & & (1) & & & & 2 & & 1 & 1 & & & & 1 & & & & & & 1 & 1 & & & (1) & 1 & & 2 & 2 & 2 & 1 & & & \\
\hline 8 & & & & & & & & 1 & & & & & & & & & & & & & & & & & & & & 1 & & 1 & & & & \\
\hline 10 & & & & & & & & 1 & & & & & & & & & & & & & & & & & 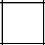 & & & 1 & & & 1 & & & \\
\hline 11 & & & & & & & & (1) & & & & & & & 1 & 1 & 1 & & & & & . & & 1 & & & 2 & & & & & & & \\
\hline 12 & 1 & 1 & 1 & & 1 & & 1 & & 1 & 1 & 1 & 1 & 1 & 1 & & & & & & 2 & 1 & 1 & & & 1 & 1 & & & & & & & & \\
\hline 13 & & & 1 & 1 & 1 & 1 & 1 & 2 & 1 & 1 & 1 & 1 & 2 & 1 & & 1 & 1 & & & 2 & 2 & 1 & & & 2 & 2 & 2 & 1 & 2 & 2 & 2 & & & 1 \\
\hline 14 & 1 & 1 & & & & 1 & & & 1 & & 1 & 1 & 1 & & & & & 1 & 1 & 1 & 1 & 1 & & & & 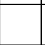 & 1 & & & & & & & 1 \\
\hline 15 & 2 & 1 & 1 & 1 & 1 & 1 & 1 & 2 & 1 & 2 & 2 & 2 & & 25 & 22 & 23 & 32 & & & 1 & 1 & 2 & 1 & 1 & 3 & 3 & 3 & 2 & 1 & 2 & 2 & & 1 & 1 \\
\hline 16 & & & & & & & & & & & & & & & & & & & & & & & & & & & & 1 & 2 & 2 & 2 & & & \\
\hline 17 & & & & & 1 & & 1 & 3 & & & & & & & 1 & 12 & 2 & & & & . & . & & 2 & & 3 & 3 & 1 & 1 & & 2 & & 1 & \\
\hline 18 & & & & & & & & & & & & & & & & & & & & & & & & & & & & & & 1 & 1 & & & \\
\hline 20 & 1 & 1 & 1 & & & & & & & & 1 & & & & & & & 1 & 1 & 1 & & & & & & & & & & & & & & \\
\hline 21 & & & & & & & & & & & & & & & & & & & & & & & & & & & & 1 & & & & & & \\
\hline 22 & 1 & 1 & & & & & & & 1 & & 2 & 2 & 1 & & & & & & & & & 2 & & & & & & & & & & & & \\
\hline 23 & & & 1 & & & & & & & & & & & & & & & & (1) & & & & & & & & & & & & & & & \\
\hline 25 & & & & & & & & & & & & & & & & & & & & & & & & & & & & & & & & & & \\
\hline 26 & & & & & & & & & & & & & & & & & & & & & & & & & & & & 1 & 1 & & & & & \\
\hline 27 & & & & & & & & & & & & & & & & & & & & & & & & & & & & 2 & 1 & 1 & & & & \\
\hline 28 & & & & & & & & 2 & & & & & & & & 2 & 22 & & & & & & & & & & 2 & 2 & 2 & & & & & \\
\hline 29 & & & & & & & & & & & & & & & & & & & & & & & & & & & & & & & & & & \\
\hline 30 & & & & & & & & & & & & & & & & & & & & & & & & & & & & & & & & & & \\
\hline 31 & & & & & & & & (1) & & & & & & & & & & & & & & & & & & & & & & & & & & \\
\hline 39 & & & & & & & & & & & & & & & & & & & & & & & & & & & & 1 & & & & & & \\
\hline 40 & & & & & & & & & & & & & & & & & & & & & & & & & & & & & & & & & & \\
\hline 41 & & & & & & & & 1 & & & & & & & & 1 & $\begin{array}{ll}1 & 1\end{array}$ & & & & & & & & & (1) & 1 & & 1 & & & & & \\
\hline 43 & & & & & & & & + & & & & & & & & & & & & & & & & & & + & + & + & & & & & & \\
\hline 44 & 1 & 1 & 1 & 1 & 1 & 1 & 1 & & 2 & 2 & 2 & 2 & 1 & $1]$ & $\begin{array}{lll}1 & 1 \\
\end{array}$ & 11 & & 2 & 22 & 2 & 3 & 2 & 2 & 2 & 2 & 2 & 1 & & & & & 1 & 1 & 1 \\
\hline 48 & & & & & & & & 2 & & & & & & & & 2 & 22 & & & & & & & & 1 & & 2 & 2 & 3 & 3 & 3 & & & \\
\hline 49 & & & & & & & & & & & & & & & & & & & & & & & & & & & & & & & 1 & & & \\
\hline 53 & & & & & & & & & & & & & & & & & & & & & & & & & & & & 2 & 2 & 1 & & & & \\
\hline 54 & & & & & & & & (1) & & & & & & & & & & & & & & & & & & & & & & & & & & \\
\hline 56 & & & & & & & & & & & & & & & & & & & & & & & & & & & & & & & & & & \\
\hline 57 & & & & & & & & & & & & & & & & & & & & & & & & & & & & & & & & & & \\
\hline 58 & & & & & & & & & & & & & & & & & & & & & & & & & & & & & & & & & & \\
\hline 60 & 2 & 2 & 1 & 1 & 1 & 1 & & & 2 & 2 & 2 & 2 & 1 & & & & & 2 & 22 & 2 & & & & & & (1) & & & & & & & & 1 \\
\hline 61 & & & & & & & & (1) & & & & & & & & & 1 & & & & & & & & & & & & & & & & & \\
\hline 62 & & & & & & & & & & & & & & & & & & & & & & & & & & & & & (1) & & & & & \\
\hline 63 & 2 & 1 & 1 & 1 & 1 & 1 & 1 & \begin{tabular}{|l|l|} 
\\
\end{tabular} & 1 & \begin{tabular}{|l|}
2 \\
\end{tabular} & 2 & 2 & 1 & 21 & 12 & 21 & 1 & 1 & 12 & 1 & & 2 & 1 & 1 & 1 & 2 & 1 & 2 & & & & 1 & 1 & 1 \\
\hline 64 & & & & 1 & 1 & 1 & 1 & & & & & & 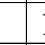 & 15 & $\begin{array}{lll}2 & 1\end{array}$ & & & 2 & & 2 & 1 & 2 & 2 & 1 & 2 & 1 & & & & & & 1 & 1 & 1 \\
\hline 69 & & & & & & & & & & & & & & & & & & & & & & & & & & & & & & & & & & \\
\hline 70 & & & & & & & & & & & & & & & & & & & & & & & & & & & & & & & & & 1 & \\
\hline 71 & & & 2 & 3 & 2 & 3 & 2 & & 1 & 3 & 3 & 4 & 3 & 2 & & & & 2 & & 1 & 1 & & & & & & & & & & & & 1 & \\
\hline 73 & 3 & 2 & 2 & 2 & 2 & 2 & 1 & & 3 & 3 & 2 & 3 & $3 \mid$ & 152 & $\begin{array}{lll}2 & 1\end{array}$ & & & 3 & \begin{tabular}{|l|l}
3 \\
\end{tabular} & 3 & 3 & 3 & 2 & 2 & 1 & (1) & & & & & & 3 & 2 & 2 \\
\hline 74 & & & & & & & & & & & & & & & & & & & & & & & & & & & & & & & & & & \\
\hline 77 & & 1 & 2 & 2 & 1 & 2 & 3 & 3 & & & + & + & 1 & 1 & 43 & $\begin{array}{l}3 \\
\end{array}$ & 42 & & & & 3 & 3 & 3 & 4 & 4 & 4 & 3 & & & & & 2 & & 2 \\
\hline 78 & 1 & & 1 & 2 & 1 & 2 & 3 & 3 & 2 & & & 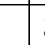 & 3 & 4 & 4 & 33 & & & & 4 & 4 & 4 & 3 & 3 & 3 & 3 & 3 & & & & & & & 2 \\
\hline 79 & 2 & & & & 1 & & 1 & & 1 & & & & 1 & 21 & \begin{tabular}{l|l}
1 & 1
\end{tabular} & 1 & & & & 1 & 3 & 2 & 2 & 1 & & & & & & & & 2 & & \\
\hline 80 & & & & & & & & & & & & & & & & & & & & & & & (1) & & & & & & & & & & & \\
\hline 81 & 2 & & 1 & & 1 & 2 & 1 & & & 1 & 1 & & 2 & 12 & 22 & $\begin{array}{ll}2 & 1\end{array}$ & & & & 1 & 1 & 2 & 2 & 2 & 3 & 2 & 1 & & & & & 3 & & 1 \\
\hline 82 & & & 1 & & & & & & & & & & & & & & & & & & & & & & & & & & & & & & & 1 \\
\hline
\end{tabular}




\begin{tabular}{|c|c|c|c|c|c|c|c|c|c|c|c|c|c|c|c|c|c|c|c|c|c|c|c|c|c|c|c|c|c|c|c|c|c|}
\hline \multicolumn{34}{|c|}{ Table 9, 1996-2 } \\
\hline & \multicolumn{11}{|c|}{ D } & \multicolumn{13}{|c|}{$E$} & & & & & $\mathrm{~F}$ & & & & \\
\hline & 4 & $5 \mid$ & $\begin{array}{ll}6 & 7 \\
6\end{array}$ & \begin{tabular}{l|l}
7 & $\varepsilon$ \\
\end{tabular} & \begin{tabular}{|l|l}
9 \\
\end{tabular} & & \begin{tabular}{l|l}
01 \\
\end{tabular} & & 121 & 13 & & 1 & 2 & 3 & 4 & $5 \mid$ & 6 & \begin{tabular}{l|l}
7 & $\varepsilon$ \\
\end{tabular} & 89 & $\begin{array}{l}91 \\
\end{array}$ & \begin{tabular}{l|l}
0 & 1
\end{tabular} & & 121 & & 1 & 2 & \begin{tabular}{|l|l|l}
3 \\
\end{tabular} & 4 & & & 8 & & 10 \\
\hline 2 & & & & & & & & & & & & & & & & & & & & & & 1 & & & & & & & 20 & \begin{tabular}{l|l}
11 & 1 \\
\end{tabular} & & & 1 \\
\hline 3 & & & & & & & & & & & & & & & & & & & & & & & & (1) & & & & & & & & 1 & \\
\hline 4 & 1 & 1 & $1]$ & 1 & 2 & 2 & 2 & 2 & 2 & 1 & 1 & & & & & 2 & 2 & 1 . & \begin{tabular}{l|l}
1 & 1 \\
1
\end{tabular} & 1 & 1 & 1 & 1 & 1 & & & & 2 & 2 & \begin{tabular}{|l|l|}
2 & 1
\end{tabular} & 1 & 1 & 2 \\
\hline 5 & & & & & & & 1 & 1 & & 1 & 1 & & & & & 1 & 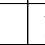 & 1 & & & & & & & & & & & & 1 & & & \\
\hline 6 & & & & & & & & & & 1 & & & & & & & & & & & & 1 & & 1 & & & & & & & & & 1 \\
\hline 7 & & 1 & & 1 & 2 & 2 & 2 & 2 & 2 & 2 & 2 & & & & & 2 & 2 & 1 & 1 & $1 \mid 1$ & 1 & 2 & 1 & 2 & & & & & & & & 2 & 2 \\
\hline 8 & & & & & & & & & & & & & & & & & & & (1) & & & & & 1 & & & & & & & & & \\
\hline 10 & & & & & & & 1 & 1 & & & 2 & & & & & 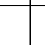 & & & 1 & 10 & 1) ( & (1) & & & & & & & & & & & 1 \\
\hline 11 & & & & (1) & & 1 & & & (1) & & & & & & & & & & 1 & 1 & 1 & & & & (1) & & & & 1 & & & & \\
\hline 12 & 1 & 1 : & $1]$ & 111 & 1 & & & & & & & 1 & & & 1 & 1 & & $1]$ & \begin{tabular}{l|l}
1 & 1 \\
\end{tabular} & 1 & & & & & & & & 1 & 1 & & & & \\
\hline 13 & & 1 & 12 & $22^{2}$ & 2 & 2 & 2 & 1 & & & 1 & & & & & 1 & 1 & 2 : & 12 & $2]$ & 1 & 1 & & & . & & & 1 & 1 & \begin{tabular}{l|l|}
2 & 2 \\
\end{tabular} & 2 & 2 & 1 \\
\hline 14 & & & & & 1 & & & & & & & & & & 1 & & 1 & & (1 & 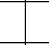 & & & & & & & (1): & 2 & \begin{tabular}{l|l}
1 \\
\end{tabular} & \begin{tabular}{|l|l|} 
\\
\end{tabular} & & & 1 \\
\hline 15 & \begin{tabular}{|l|} 
\\
\end{tabular} & 18 & 25 & $2:$ & \begin{tabular}{|l|l|}
3 &
\end{tabular} & 3 & 3 & 2 & 2 & & 2 & & & & 2 & 2 & 3 & 2 & 1 & 2 & 1 & 2 & & & 1 & & 3 & & 36 & \begin{tabular}{l|l}
2 & 2
\end{tabular} & 3 & 2 & 3 \\
\hline 16 & \begin{tabular}{|l|}
1 \\
\end{tabular} & & & & & & 2 & 2 & (1) & & 3 & & & & & & & & & & & & 1 & 2 & & & & & & & & & 2 \\
\hline 17 & & $1:$ & $1]$ & 1 & 3 & 3 & & 1 & 2 & 2 & 3 & & & & 2 & 1 & 2 & & 1 & 1 & 1 & 1 & & 1 & & & & 2 & 2 & 1 & 1 & & 2 \\
\hline 18 & & 1 & & 1 & & 1 & & & & & & & & & & & & & & & & & & & 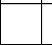 & & & 1 & & & & & \\
\hline 20 & & 1. & 1 & & (1) & & & & & & & & & & & 1 & 1 & & & & & & & & & & & 1 & & & & & \\
\hline 21 & & & & & & & & & & & & & & & & & & & (1) & & & & & & & & & & 1 & & & & \\
\hline 22 & & & & & & & & & & & & & & & & & & & & & & & & & & & & & & & & & \\
\hline 23 & & & & & & & & & & & & & & & & & & & & & & & & & & & & & & & & & \\
\hline 25 & & & & & & & & & & & & & & & & & & & & & & & & & & & & & 1 & & & & \\
\hline 26 & & & & & & & & & & & & & & & & & & & 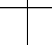 & & & 1 & & 1 & & & & & 1 & & & 1 & \\
\hline 27 & & & & & & & & & & & & & & & & & & & 1 & 1 & & 1 & & 1 & & & & & & & & & \\
\hline 28 & & 1 & & & 2 & 2 & $2(1$ & 1) & 2 & & & & & & & (1) & 1 & & 1 & 1 & 1 & 1 & 1 & 1 & & & & & 1 & \begin{tabular}{l|l}
1 & 2
\end{tabular} & 2 & 2 & 1 \\
\hline 29 & & & & & & & & & & & & & & & & & & & (1) 1 & & & 1 & & & & & & & & \begin{tabular}{l|l}
1 &
\end{tabular} & & 1 & 1 \\
\hline 30 & & & & & & & 1 & 1 & & & & & & & & & & & & & & & (1) & & & & & & & 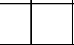 & & & 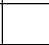 \\
\hline 31 & & & & & & & & & & & & & & & & & & & 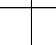 & & & & & & & & & & & $\begin{array}{ll} \\
\end{array}$ & & & \\
\hline 39 & & & & & & & & & & & & & & & & & & & & & & & & (1) & & & & & & 1 & & & \\
\hline 40 & & & & & & & & & & & & & & & & & & & & & & & & & & & & & & & & (1) & \\
\hline 41 & & 1 & & 1 & 1 & & (1) & 1) & (1) & & & & & & & & & & 1 & 1 & & 1 & & & & & & & & 1 & 1 & 1 & \\
\hline 43 & & $1:$ & 21 & 1 & & & & & & & & & & & & & 2 & & & & & & & 2 & & & & 1 & & 1 & 1 & & \\
\hline 44 & 2 & 2 & 2 & 12 & \begin{tabular}{l|l}
2 & 1 \\
\end{tabular} & & D) & & & & & 2 & 1 & \begin{tabular}{|l|}
2 \\
\end{tabular} & 2 & & 2 & 1 & \begin{tabular}{l|l}
1 & 1
\end{tabular} & $\begin{array}{ll}11 & \end{array}$ & 1 & & & & \begin{tabular}{|l|}
1 \\
\end{tabular} & 1 & & $\frac{1}{2}$ & 2 & & 1 & & 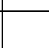 \\
\hline 48 & & & & & & 2 & 28 & 2 & 3 & 2 & 3 & & & & & & & & 1 & 12 & 2 & 1 & 2 & 2 & & & & & & 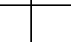 & & & 3 \\
\hline 49 & & & & & & & & & 1 & & 1 & & & & & & & & & & & & & 1 & & & & & & 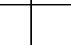 & 1 & & \\
\hline 53 & & & & (1) & 1) & & & & & & & & & & & & & & & & 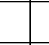 & 1 & & & & & & & & & & & \\
\hline 54 & & & & & & & & & & & & & & & & & & & & & & & & & & & & & & & & & \\
\hline 56 & & & & & & & & & & & & & & & & & (1) & & & & & & & & & & & & & & & & \\
\hline 57 & & & & & & & & & & & & & & & & & & & & & & & & & & & & & & & & 1 & \\
\hline 58 & & & & (1) & & & & & & & & & & & & & & & & & & & & & & & & & & & & & \\
\hline 60 & & 1 & 1 & & & & & & & & & & & & & 1 & 2 & & & & & & & & & & & 1 & 1 & 1 & & & \\
\hline 61 & & & & & & & & & & & & & & & & & & & & & 1 & 1 & 1 & (1) & & & & & & & & & \\
\hline 62 & & & & & & & & 1 & & & & & & & & & & & & & & 1 & & & & & & & & & & & \\
\hline 63 & 1 & 2 & 12 & 22 & 22 & 1 & 11 & 1 & & & & & & & (1) & 3 & 3 & 2 & \begin{tabular}{l|l}
1 &
\end{tabular} & \begin{tabular}{l|l}
1 & 1
\end{tabular} & 1 & & & & 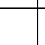 & 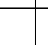 & & 2 & 2 & \begin{tabular}{l|l}
1 & 1
\end{tabular} & 1 & & \\
\hline 64 & \begin{tabular}{|l|}
1 \\
\end{tabular} & 2 & 1 & & & & & & & & & 1 & & & \begin{tabular}{|l|l|}
2 \\
\end{tabular} & & \begin{tabular}{l|l}
1 \\
\end{tabular} & & 1 & & & & & & 1 & 1 & & & & & 1 & & \\
\hline 69 & & & & & & & & & & & & & & & & & & & & & & & & & & & & & & & & 1 & \\
\hline 70 & & & & & & & & & & & & & & & & & & & & & & & & & & & & & & & & & \\
\hline 71 & & & 1 & & & & & & & & & & & & & & & & & & & & & & & & & & & & & & \\
\hline 73 & \begin{tabular}{|l|}
2 \\
\end{tabular} & 2 & 32 & 25 & 2 & & & & & & & 2 & 2 & 2 & 2 & 2 & 2 & 3 & & (1) & (1) & & & & 2 & 2 & 2 & 3 & 2 & 1 & & & \\
\hline 74 & & & & & & & & & & & & & & & & & & & & & & & & & & & & (1) & & & & & \\
\hline 77 & 2 & 3 & 3 & $2+$ & ++ & & & & & & & & 2 & & 4 & 3 & & & 22 & 2 & & & & & 2 & 3 & 3 & 3 & 3 & & 1 & & \\
\hline 78 & 2 & 3 & 2 & 1 & 3 & 3 & 4 & & & & & 4 & 3 & 4 & 4 & & & & & & & & & & 3 & 3 & 3 & & & & & & \\
\hline 79 & & & & & & & & & & & & 2 & \begin{tabular}{|l|}
2 \\
\end{tabular} & \begin{tabular}{|l|}
2 \\
\end{tabular} & & & & & & & & & & & 2 & 1 & & & & & & & \\
\hline 80 & & & & & & & & & & & & & & & & & & & & & & & & & 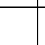 & 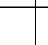 & & & & & & & \\
\hline 81 & 1 & 2 & $\begin{array}{ll}1 & \end{array}$ & 1 & 1 & & & & & & & 3 & 1 & 2 & 2 & & & & & & & & & & 2 & 2 & & & & & & & \\
\hline$\delta 2$ & & & & & & & & & & & & & & & & & & & & & & & & & & & & & & & & & \\
\hline
\end{tabular}




\begin{tabular}{|c|c|c|c|c|c|c|c|c|c|c|c|c|c|c|c|c|c|c|c|c|c|c|c|c|c|c|c|c|c|c|c|c|c|c|}
\hline \multicolumn{35}{|c|}{ Table 9, 1996-3 } \\
\hline & & \multicolumn{10}{|c|}{$\mathrm{B}$} & & & & & & & ( & $\mathrm{C}$ & & & & & & & & D & \\
\hline & \begin{tabular}{l|l}
12 \\
\end{tabular} & $2 \mid 5$ & \begin{tabular}{l|l}
3 & 4 \\
\end{tabular} & & 6 & 7 & 8 & 1 & 2 & 3 & 4 & 5 & 6 & 7 & 8 & 9 & 10 & 1 & 2 & 3 & 4 & 5 & \begin{tabular}{l|l}
6 & 1 \\
\end{tabular} & & 8 & \begin{tabular}{l|l}
91 \\
\end{tabular} & \begin{tabular}{ll|l}
10 & 1 \\
\end{tabular} & 11 & 12 & & 14 & & & 3 \\
\hline 84 & & & & & & & & & & & & & & & & & & & & & & & & & & & & & & & & & & \\
\hline 85 & & & & & & & & & & & & & & & & & & & & & & & & & & & & & & & & & & \\
\hline 86 & & & & & & & & & & & & & & & & & & & & & & & & & & & & 1 & & & & & & \\
\hline 87 & 1 & 1 & & & & & & & & 1 & & & & & & & & & & & & & & & & & & 1 & & & 2 & & & \\
\hline 88 & & & 1 & & & & 1 & & & & & & & & & & & & & & & & & & & & & 1 & & & & & & \\
\hline 89 & & & & & & & 1 & & & & & & & & & 1 & 1 & & & & & & & & & & & 1 & & 1 & & & & \\
\hline 91 & & & & & & & & & & & & & & & & & & & & & & & & & & & & & & 1 & & & & \\
\hline 93 & & & & & & & & & & & & & & & & & & & & & & & & & & & & & & & & & & \\
\hline 94 & & & & & & & 1 & & & & & & & & & & (1) & & & & & & & & & & & 1 & 1 & & & & & \\
\hline 96 & & & & & & & & & & & & & & & & & & & & & & & & & & & & & & & & & & \\
\hline 97 & & & & & & & (1) & & & & & & & & & & & & & & & & & & & & & (1) & & & & & & \\
\hline 99 & & & & & & & & & & & & & & & & & & & & & & & & & & & & 1 & 1 & (1) & & & & \\
\hline 100 & & & & & & & & & & & & & & & & & & & & & & & & & & & & (1) & & & & & & \\
\hline 101 & & & & & & & (1) & & & & & & & & & & & & & & & & & & & & & & & & & & & \\
\hline 103 & & & & & & & & & & & & & & & & & & & & & & & & & & & & & 1 & & & & & \\
\hline 107 & & & & & & & & & & & & & & & & & & & & & & & & & & & & & & (1) & & & & \\
\hline 115 & & & & & t & & & & & & & & & & & & & & & & & & & & & & & 1 & & & & & & \\
\hline 116 & & & & & 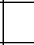 & & & & & & & & & & & & & & & & & & & & & & & & & & & & & \\
\hline 118 & & & & & & & & & & & & & & & & & & & & & & & & & & & & & & & & & 1 & \\
\hline 119 & & & & & & & & & & & . & & . & & & & & & & 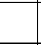 & 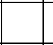 & d & & & . & & & & & & & & & \\
\hline 122 & & & & & 1 & & 2 & & & & & & & & & & & & & & & & & & & & & & & & & & & \\
\hline 123 & & & & & 1 & 1 & (1) & & & & (1) & & 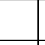 & . & & & 2 & 2 & 1 & & & & & & & (1) & 2 & 2 & 2 & & & & & \\
\hline 124 & 1.1 & $1[1]$ & \begin{tabular}{l|l|}
1 & 1 \\
\end{tabular} & 1 & 1 & 1 & & 1 & 2 & 2 & 2 & 1 & 2 & 1 & & 2 & 2 & 2 & 2 & 2 & 2 & 2 & 1 & 1 & 2 & 1 & & & & 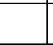 & & & & 2 \\
\hline 125 & & & & & & & (1) & & & & & & & & & & & & & & & & & & & & & & 1 & & & & & \\
\hline 126 & & & & & & & & & & & & & & & & & & & & & & & & & & & & & & & & & & \\
\hline 129 & & & & & & & & & & & & & & & & & & & & & & & & & & & & & & & & & & \\
\hline 130 & & & (1) & & & & 1 & & & & & & & & & & & & & & 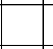 & & & & & & & 1 & 1 & 1 & & & & \\
\hline 131 & & & & & & & & & & & & & & & & & & & & & & & & & & & & 1 & & & & & & \\
\hline 132 & & & & & 1 & 1 & 2 & & & & 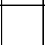 & & 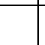 & & & & 1 & & & 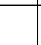 & 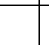 & & & & 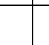 & 1 & 2 & 2 & 2 & 1 & & & & \\
\hline 133 & & & & & & & & & & & 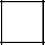 & & & & & & & & & & & & & & & & & (1) & & & & & & \\
\hline 141 & & & & 1 & 1 & & & & & & 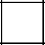 & & & & & & 1 & & & 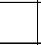 & & & & & & & & 1 & 1 & 2 & 2 & & & \\
\hline 142 & & 1 & & 1 & & & 1 & & & & 1 & 1 & 1 & 1 & & 2 & 2 & & & & 1 & & & & 1 & 1 & 2 & 2 & 1 & 2 & 2 & & & \\
\hline 143 & & & & & & & & & & & (1) & & & & & & & & & & 1 & & & & & & & & & 1 & & & & \\
\hline 144 & & & & & 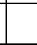 & & & & & & & & & & & & & & & & & & & & & & & & & & & & & \\
\hline 145 & & & & 1 & 1 & 1 & & & & & & 1 & & & & & & & & & & & & & (1) & (1) & & 1 & & & & & & \\
\hline 146 & & & & & & & & & & & & & & & & & & & & & & & & & & & & 1 & & & & & & \\
\hline 146 & & & & & & & & & & & & & & & & & (1) & & & & & & & & & & & & & & & & & \\
\hline 149 & & & & & & & & & & & & & & & & & & & & & & & & & & & & 1 & & & & & & \\
\hline 156 & & (1) & (1) 1 & & & & & & & & & & & & & & & & & 1 & & & & & & & & & & . & & & & \\
\hline 158 & & & & (1) & & & & & & & & & & & & & & & & 1 & & & & & & & & & & & & & 1 & \\
\hline 159 & & & & & & & & & & & & & & & & & & & & & & & & & & & & & & & & & & \\
\hline 160 & & & & & & & & & & & & & & & & & & & & & & & & & & & & & & & & & & \\
\hline 161 & & & & & & & 1 & & & & & & & & & & & & & & & & & & & & & 1 & & 1 & & & & \\
\hline 169 & & & & & & & & & & & & & & & & & & & & & & & & & & & & & & & & & & \\
\hline 173 & & & & & & & (1) & & & & & & & & & & & & & & & & & & & & & & & & & & & \\
\hline 176 & & & & & & & & & & & & & & & & & & & & & & & & & & & & & & & & & & \\
\hline 180 & & & & & & & & & & & & & & & & & & & & & & & & & & & & & & & & & & \\
\hline 181 & & & & 1 & 1 & & 1 & & & & & & & & & & (1) & & & & & & & & & & & & 1 & & & & & \\
\hline 182 & & & & & & & (1) & & & & & & & & & & & & (1) & & & & & & & & & & & & & & & \\
\hline 184 & & & & & & & & & & & & & & & & & & & & & & & & & & & & & & & & & & \\
\hline 187 & & & & & & & & & & & & & & & & & & & & & & & & ( & (1) & & & & & & & & & \\
\hline 190 & & & & & & & & & & & & & & & & & & & & & & & & & & & & & & & & & & \\
\hline 192 & & & & (1) & & & & & & & & & & & & & & & & & & & & & & & & & & & & & & \\
\hline 193 & & & & & & & & & & & & & & & & & & & & & & & & & & & & & 1 & & & & & \\
\hline 195 & & & & & & & & & & & & & & & & & & & & & & & & & & & & & & & & & & \\
\hline
\end{tabular}




\begin{tabular}{|c|c|c|c|c|c|c|c|c|c|c|c|c|c|c|c|c|c|c|c|c|c|c|c|c|c|c|c|c|c|c|c|}
\hline \multicolumn{32}{|c|}{ Table 9, 1996-4 } \\
\hline & \multicolumn{10}{|c|}{ a toror } & \multicolumn{12}{|c|}{$E$} & \multicolumn{9}{|c|}{$\mathrm{F}$} \\
\hline & \begin{tabular}{l|l}
44 & 5
\end{tabular} & 6 & 7 & 8 & \begin{tabular}{|l|}
9 \\
\end{tabular} & 10 & & 12 & 13 & & 1 & 2 & & 45 & & \begin{tabular}{l|l}
6 & 7 \\
\end{tabular} & \begin{tabular}{|l|l|}
7 & 8 \\
\end{tabular} & 9 & 10 & 11 & 12 & 13 & 1 & 2 & 3 & 4 & $5 \mid$ & \begin{tabular}{l|l}
6 & 7 \\
\end{tabular} & \begin{tabular}{l|l}
7 & 8 \\
\end{tabular} & \begin{tabular}{l|l}
8 & 9
\end{tabular} & 10 \\
\hline 84 & & & & & & & & & & & & & & & & & & & & (1) & & & & & & & & & & & \\
\hline 85 & & & & & & & & & & & & & & & & & & & & & & & & & & & & & & 1 & \\
\hline 86 & & 1 & & & & & & & & & & & & & & & & & & 1 & & (1) & & & & & & 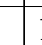 & 1 & 1 & 1 \\
\hline 87 & & & & & & & & 1 & & 1 & & & & & & & & & & & & 1 & & & & & 2 & 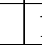 & 1 & & \\
\hline 88 & & & & & & & & & & & & & & & & & & & & 1 & & 1 & & & & 1 & 1 & & 1 & & \\
\hline 89 & & & & & & & & & & & & & & & & & & & & 1 & 1 & 1 & & & & & & & & & \\
\hline 91 & & & & & & & & & & & & & & & & & & & & (1) & & 1 & & & & & & & & & \\
\hline 93 & & & & & & & & & & & & & & & & & & & & & & (1) & & & & & & & & & \\
\hline 94 & & & & & & & & 1 & & & & & & & & & & & & 1 & & (1) & & & & & & & & & \\
\hline 96 & & & & & & & & & & & & & & & & & (1) & & & & & & & & & & & & & & \\
\hline 97 & & & & & & & & & & & & & & & & & & & & 1 & & . & & & & & & & & & \\
\hline 99 & & & & & & (1) & (1) & 1 & & & & & & & & & & & & 1 & & 1 & & & & & & & & 1 & 1 \\
\hline 100 & & & & & & & & & & & & & & & & & 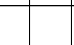 & & & & & & & & & & & & & & \\
\hline 101 & & & & & & & & & & & & & & & & & & & & & & & & & & & (1) & & & & \\
\hline 103 & & (1) & & & & & (1) & & & & & & & & & & \begin{tabular}{|l|l|}
1 & 1 \\
\end{tabular} & 1 & & 1 & & 1 & & & & & & 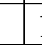 & 1 & & \\
\hline 107 & & & & & & & & & & & & & & & & & & & & & & & & & & & & & & & \\
\hline 115 & & & & & & & & & & & & & & & & & & & & 1 & & & & & & 1 & & & & & \\
\hline 116 & & & & & & & (1) & & & & & & & & & & & & & & & & 1 & & & & & & & & \\
\hline 118 & & & & & & & & & & & & & & & & & & & & & & & & & & & & & & & \\
\hline 119 & & & & 1 & & & & 1 & & & & & & & & & & & 1 & & & 1 & & & & & & & $\begin{array}{ll}12 \\
\end{array}$ & & 2 \\
\hline 122 & & & & & & & & & & & & & & & & & 1 & 1 & 1 & 1 & & & 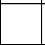 & & & 1 & 1 & & & \begin{tabular}{|l|l|}
2 & 2 \\
\end{tabular} & $\begin{array}{ll}2 \\
\end{array}$ \\
\hline 123 & & 1 & 1 & 1 & (1) & & 1 & (1) & & & & & & & & 1 & 1 & 1 & 1 & 2 & 1 & 1 & & & & 1 & 2 & 2 & 2 & \begin{tabular}{|l|l|}
22 \\
\end{tabular} & \\
\hline 124 & \begin{tabular}{l|l}
1 & 2 \\
\end{tabular} & 1 & 1 & 2 & 2 & & 1 & & & & & & & 1 & & 2 & 1 & 1 & 1 & 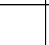 & & & 1 & 1 & $1[?$ & 2 & & 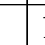 & \begin{tabular}{l|l}
1 & 1 \\
\end{tabular} & \begin{tabular}{l|l}
1 \\
\end{tabular} & 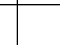 \\
\hline 125 & (1) & & & & & & & & & & & & & & & & & & & 1 & & & & & & & 1 & & & 1 & \\
\hline 126 & & & & & & & & & & & & & & & & & & & & 1 & & 1 & & & & & & & 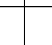 & & \\
\hline 129 & & & & & & & & & & & & & & & & & 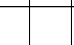 & 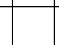 & & & (1) & (1) & & & & & & & & 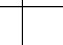 & 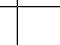 \\
\hline 130 & & & & & 1 & \begin{tabular}{|l|} 
\\
\end{tabular} & 1 & 1 & 2 & & & & & & & & 1 & 1 & 1 & 1 & 1 & 1 & & & & & 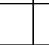 & 1 & \begin{tabular}{l|l}
1 & 1
\end{tabular} & \begin{tabular}{|l|l}
1 & 1 \\
\end{tabular} & 1 \\
\hline 131 & & & & & & & & & & & & & & & & & 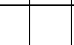 & & & & & & & & & & & & & 1 & 18 \\
\hline 132 & 2 & 1 & & 2 & 2 & 2 & 2 & 2 & 2 & & & & & & & & \begin{tabular}{|l|l}
2 & \\
\end{tabular} & 1 & 1 & 1 & 1 & 1 & & & & 1 & 2 & 2 & 2 & \begin{tabular}{|l|l}
2 & 2
\end{tabular} & \begin{tabular}{|l|l|}
2 \\
\end{tabular} \\
\hline 133 & & (1) & & & & & & & & & & & & & & & & & & & & & & & & & & & & & \\
\hline 141 & (1) & & 1 & 1 & \begin{tabular}{|l|} 
\\
\end{tabular} & & 1 & 1 & 1 & 2 & & & & & & & \begin{tabular}{l|l}
1 & 1
\end{tabular} & 1 & 1 & 1 & 1 & 2 & & & & & & 1 & 2 & & 1 \\
\hline 142 & 2 & & 1 & & 1 & 2 & 1 & 2 & & 2 & & & 1) & & & \begin{tabular}{l|l}
11 & 2 \\
\end{tabular} & \begin{tabular}{l|l}
2 & 1 \\
\end{tabular} & 1 & 1 & 1 & 2 & 1 & & & & 2 & 2 & 3 & 2 & 22 & 2 \\
\hline 143 & & & & & & & & & & & & & & & & & & & & & & & & & & & & & & & \\
\hline 144 & (1) & & & & & & & & & & & & & & & & & & & 1 & & & & & & & & & 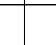 & & \\
\hline 145 & 1 & & & 2 & \begin{tabular}{|l|} 
\\
\end{tabular} & & & & \begin{tabular}{l|l} 
\\
\end{tabular} & 1 & & & & & & 1 & 1 & 1 & & & & 1 & & & & & 2 & 15 & \begin{tabular}{l|l}
2 & 2
\end{tabular} & 22 & 1 \\
\hline 146 & & & & & & (1) & & (1) & 1 & & & & & & & & & 1 & 1 & 1 & & & & & & & & & & & 1 \\
\hline 146 & & & & & & & & & & & & & & & & & & & & & & & & & & & & & & & \\
\hline 149 & & & & & & & & & & & & & & & & & & & & 1 & & & & & & & & & & & 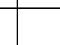 \\
\hline 156 & & & & & & & & & & & & & & & & & & & & & & & & & & & & 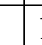 & 1 & & \\
\hline 158 & 2 & 1 & & 1 & & & & & & & & & & & & & & & & & & & & & & & & & & & \\
\hline 159 & & & & & & & & & & & & & & & & & & 1 & & & & & & & & & & & & & \\
\hline 160 & & & & & & & & & & & & & & & & & & & & 1 & & & & & & & & & & & \\
\hline 161 & & & & & & (1) & 1 & (1) & & & & & & & & & 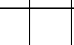 & 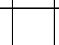 & & 1 & & 1 & & & & & 1 & 1 & & & 1 \\
\hline 169 & (1) & & & & & & & & & & & & & & & & & & & & & & & & & & & & & & \\
\hline 173 & & & & & & & & & & & & & & & & & & & & & & & & & & & 1 & & & & \\
\hline 176 & & & & & & & & & & & & & & & & & & & & & & & & & & (1) & & & & & \\
\hline 180 & 1 & & & & & & & & & & & & & & & & & 1 & & & & & & & & & & & 1 & & \\
\hline 181 & & & & (1) & & & & & & & & & & & 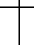 & 1 & \begin{tabular}{l|l}
1 & 1
\end{tabular} & 1 & & 1 & (1) & & & & & 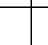 & & (1) & & \begin{tabular}{|l|l}
1 & 1
\end{tabular} & \begin{tabular}{|l}
1 \\
1
\end{tabular} \\
\hline 182 & & & & (1) & & & & & & & & & & & & \begin{tabular}{l|l}
1 & 1 \\
\end{tabular} & \begin{tabular}{l|l}
1 &
\end{tabular} & 1 & & 1 & & & & & & 2 & (1) & 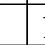 & 1 & 1 & 1 \\
\hline 184 & & & & & & & & & & & & & & & & & & & & & & & & & & & & 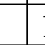 & 1 & & \\
\hline 187 & & & & & & & & & & & & & & & & & & & & & & & & & & & & & & & \\
\hline 190 & & & & & & & & & & & & & & & & (1) & & & & (1) & & & & & & & & & & & \\
\hline 192 & & & & & & & & & & & & & & & & & & & & & & & & & & & & & & & - \\
\hline 193 & & & & & & & & 1 & & & & & & & & & & & & 1 & & 1 & & & & & & & & & 1 \\
\hline 195 & & & & & & & & & & & & & & & & & (1) & & & & & & & & & & (1) & & & 1 & \\
\hline
\end{tabular}




\begin{tabular}{|c|c|c|c|c|c|c|c|c|c|c|c|c|c|c|c|c|c|c|c|c|c|c|c|c|c|c|c|c|c|c|c|c|c|c|}
\hline \multicolumn{35}{|c|}{ Table 9, 1996-5 } \\
\hline & \multicolumn{8}{|c|}{$\mathrm{A}$} & \multicolumn{9}{|c|}{ B } & & \multicolumn{13}{|c|}{ C } & \multicolumn{3}{|c|}{ D } \\
\hline & 1 & 2 & 3 & 4 & 5 & 6 & 7[ & 8 & 12 & & 3 & 4 & 5 & 6 & 78 & 9 & 1 & 01 & 12 & 3 & 4 & 5 & 6 & 7 & 8 & 9 & & 11 & 12 & 13 & 14 & 1 & & 3 \\
\hline 197 & & & & & & & & & & & & & & & & & & & & & & & & & & & & & & & & & & \\
\hline 198 & & & & & & 1 & 1 & & & & & & & 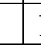 & 1 & 1 & & & & & 1 & 1 & & 1 & & & & & & & & & & \\
\hline 199 & 1 & 1 & & 1 & & 1 & & 1 & & & & & 1 & & 1 & 1 & & & & & & & & 1 & 1 & & 1 & & & & & 1 & & 1 \\
\hline 200 & & & & & & & & 2 & & & & & & & & & & & & & & & & & & & 1 & 1 & 1 & 2 & 1 & & & 1 \\
\hline 202 & & & & & & & & & & & & & & & & & & & & & & & & & & & & & & & & & & \\
\hline 205 & & & & & & & & & & & & & & & & & & & & & & & & & & & & & & & & & & \\
\hline 207 & & (1) & & (1) & 1 & & & 1 & & 1 & & 1 & & 1 & 1 & $\begin{array}{lll}1 & 1\end{array}$ & \begin{tabular}{l|l}
1 & 1
\end{tabular} & 1 & & & 1 & 1 & & & 1 & 1 & 1 & 2 & 1 & 2 & 2 & & & \\
\hline 209 & & & & & & & & & & & & & & & & & & & & & & & & & & & & & (1) & & & & & \\
\hline 210 & & & & & & & & & & & & & & & & & & & & & & & & . & . & . & & & & & & & & \\
\hline 211 & & 1 & & & 1 & & 1 & & & & & + & 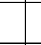 & 1 & 2 & 22 & & & & & & 1 & + & 3 & + & 4 & 3 & & & & & 2 & 2 & 2 \\
\hline 212 & & & & & & & 1 & & & & & 4 & & & & & & 3 & 34 & 3 & & & & 1 & & & & 4 & 3 & & 1 & & & \\
\hline 213 & & & & & 1 & & . & 1 & & & & (1) & & & 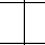 & 1 & 11 & & 1 & & & W & 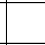 & . & 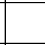 & . & & 1 & 1 & & (1) & & . & \\
\hline 214 & 1 & (1) & (1) & 1 & 1 & & 1 & & 1 & (1) & 1 & (1) & 1 & 1 & 12 & 22 & 4 & \begin{tabular}{l|l}
4 & 1 \\
\end{tabular} & & 1 & 1 & 1 & 1 & 1 & 1 & 3 & 3 & 4 & 4 & 4 & 4 & & 1 & 1 \\
\hline 216 & & & & & & & & & & & & & & & & & & & & & & & & & & & & & & & & & & \\
\hline 217 & & & & & & & & & & & & & & & & & & & & & & & & & & & & & (1) & & & & & \\
\hline 220 & & & & & 1 & 1 & & & & & & & & 1) & & & 1 & & & & & & . & & & & & 1 & 1 & (1) & & & & \\
\hline 221 & & & & & & & & & & & & & (1) & & & & & & & & & & & & & & & & & & & & & \\
\hline 222 & & & & & & & & & & & & & & & & & & & & & & & & & & & & & & & & & & \\
\hline 225 & & & & & & & & & & & & & & & & & & & & & & & & & & & & & & & (1) & & & \\
\hline 226 & & & & & & & & & & & & & & & & & & & & & & & & & & & & & & & 1 & & & \\
\hline 227 & & & & & & & & 2 & & & & & & . & 1 & & & & & 1 & & & 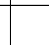 & & & 1 & & & & & & & & \\
\hline 228 & & & & & & & & & & & & & & & & & & & & & & & & & & & & & & & & & & \\
\hline 229 & 3 & 3 & 2 & 1 & (1) & 1 & 1 & 2 & $2 \mid 2$ & & 2 & 2 & & & 2 & 2 & 2 & & 1 & 1 & 1 & 2 & 2 & 3 & 3 & 3 & 3 & 3 & 2 & 1 & & 1 & 1 & 1 \\
\hline 230 & & & & & & & 1 & 2 & & & & & & & 1 & 12 & & & & & & & & 1 & & 2 & 1 & 2 & & & & & & \\
\hline 232 & & 1 & & 1 & & & 1. & + & & & + & + & & & 2 & $\begin{array}{ll}22 \\
\end{array}$ & 2 & 2 & - & + & & & & + & + & + & + & + & + & & & 2 & 2 & 2 \\
\hline 233 & & & & & & & & & & & & & & & & & & & & & & & & & & & & 1 & & & & & & \\
\hline 234 & & & & & & & & & & & & & & & & & & & & & & & & & & & & & & & & & & 1 \\
\hline 235 & (1) & & & & & & & & & & 1 & & & & & & & & & & 1 & & & & & & & & & & 1 & & 1 & \\
\hline 240 & & & & & & & & & & & & & & & & & & & & & & & & & & & & & & & & & & \\
\hline
\end{tabular}




\begin{tabular}{|c|c|c|c|c|c|c|c|c|c|c|c|c|c|c|c|c|c|c|c|c|c|c|c|c|c|c|c|c|c|c|c|c|c|c|}
\hline \multicolumn{35}{|c|}{ Table 9, 1996-6 } \\
\hline & \multicolumn{11}{|c|}{ D } & \multicolumn{13}{|c|}{$\mathrm{E}$} & \multicolumn{10}{|c|}{$\mathrm{F}$} \\
\hline & 4 & 5 & 6 & 7 & 8 & 9 & 10 & & 12 & 13 & & 1 & 2 & 3 & 4 & 5 & 6 & & 8 & 9 & 10 & & 12 & 13 & 1 & 2 & 3 & 4 & 5 & 6 & & 8 & & 10 \\
\hline 197 & & & & & & & & & & & & & & & & 1 & & & & & & & & & & & & & & & & & & \\
\hline 198 & & & 1 & & & & & & & & & 2 & 2 & & & & & & & & & & & & 1 & 1 & 1 & & & & & & & \\
\hline 199 & & 1 & 1 & 2 & & 1 & 2 & & & & & & & & & & 1 & & 1 & 1 & 1 & & & & 1 & & 1 & 1 & & & & 1 & & \\
\hline 200 & & 1 & 1 & & 2 & 1 & 1 & 1 & & & & & & & & & & & 1 & 2 & 1 & 1 & 1 & 1 & & & & & 2 & 3 & & 1 & 2 & 2 \\
\hline 202 & & & & & & & & & & & & & & & & & & & & & & & & & & & & & 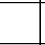 & . & & & & 1 \\
\hline 205 & & & & & & & & & & & & & & & & & & & 1 & & & 1 & & & & & & & & & & & & \\
\hline 207 & & 1 & 1 & (1) & 1 & & & 2 & 2 & 1 & 1 & & & & & (1) & 1 & & 1 & 1 & 1 & 1 & 1 & 1 & & & & 1 & 2 & 2 & 1 & & 1 & 1 \\
\hline 209 & & & & & & & & & & & & & & & & & & & & & & & & & & & & & & & & & & \\
\hline 210 & & & & & & & & & (1) & & & & & & & & & & & & & & & & & & & & & & & & & \\
\hline 211 & 2 & 2 & 2 & & + & & 2 & & & & & 3 & & & 3 & & & & & & & & & 2 & 1 & 2 & 2 & & & & & & & \\
\hline 212 & & 4 & 3 & & & & & 3 & 4 & 2 & 2 & & & & & 4 & 4 & & 3 & & & 1 & & & & & & & 3 & 4 & 4 & 4 & 3 & \\
\hline 213 & & & 1 & & (1) & 1 & & 1 & 1 & & 1 & & & & & & & & (1) & 1 & 1 & 1 & & 1 & & & & & & 1 & & & & \\
\hline 214 & 1 & 1 & 1 & 1 & & 1 & 3 & 3 & 4 & 3 & 4 & & & & (1) & 1 & 1 & 1 & 1 & 1 & 3 & 1 & 3 & 3 & & & & & 1 & & & & & 1 \\
\hline 216 & & & & & & & & & (1) & & & & & & & & & & & & 1 & & & & & & & & & & & & & \\
\hline 217 & & & & & & & & & & & & & & & & & & & & & & & & & & & & & & & & & & \\
\hline 220 & & & & & 2 & 1 & (1) & 1 & 1 & 2 & & & & & & & (1) & 1 & 1 & 1 & 1 & 1 & & 1 & & & & 1 & & 1 & 1 & 1 & & 1 \\
\hline 221 & & & & & & & & & & & & & & & & & & & & & & & & & (1) & & & & & & & & & \\
\hline 222 & & (1) & & & & & & & & & & & & & & & & & & & & & & & & & & & & & & & & \\
\hline 225 & & & & & & & & & & & & & & & & & & & & & & & & & & & & & & & & & & \\
\hline 226 & & & & & & & & & & & 1 & & & & & & & & (1) & & & & & & & & & & & & & & & \\
\hline 227 & 1 & & 1 & & 1 & & & & & & & 1 & 1 & & & 1 & & & . & & & & & 1 & & & & & & & 1 & & & \\
\hline 228 & & & & & & & & 1 & & & & & & & & & & & & & & & & & & & & & & & & & & \\
\hline 229 & \begin{tabular}{|l|}
2 \\
\end{tabular} & 2 & 2 & 3 & 3 & 3 & 3 & 3 & 3 & 2 & & 1 & 1 & 1 & 2 & 3 & 3 & 3 & 3 & 3 & 3 & 3 & 2 & & 2 & 2 & 3 & 3 & 3 & 3 & 3 & 3 & 3 & 3 \\
\hline 230 & & & & 1 & 2 & 2 & 2 & (1) & & 1 & & & & & 1 & 1 & & 1 & 1 & 1 & 1 & & & & & & & & 1 & & 1 & 2 & 2 & \\
\hline 232 & \begin{tabular}{|l|}
2 \\
\end{tabular} & 2 & 2 & 2 & + & + & + & & + & & & & & & + & + & + & + & & 1 & 1 & & & & + & + & + & + & & & + & & & \\
\hline 233 & & 1 & (1) & & & & & & (1) & & & & & & & & & & & 1 & & 1 & & & & & & & 1 & & & 1 & & \\
\hline 234 & & & & & & & & & & & & & & & & & & & & & & & & & & & & & & & & 1 & & \\
\hline 235 & & 1 & & & & & & & 1 & & & & & & & & & & & & & & & 1 & & & & & 2 & 2 & & & & \\
\hline 240 & & & & & & & & & & & & & & & & & & & & & & & & 1 & & & & & & & & & & \\
\hline
\end{tabular}




\begin{tabular}{|c|c|c|c|c|c|c|c|c|c|c|c|c|c|c|c|c|c|c|c|c|c|c|c|c|c|c|c|c|c|c|c|c|c|c|}
\hline \multicolumn{35}{|c|}{ Table 9, 1997-1 } \\
\hline & \multicolumn{8}{|c|}{$\mathrm{A}$} & \multicolumn{9}{|c|}{$\mathrm{B}$} & & & & & & & & C & & & & & & & & D & \\
\hline & 1 & 2 & 3 & 4 & & 6 & $\begin{array}{ll}78 \\
\end{array}$ & \begin{tabular}{l|l}
8 & 1 \\
1
\end{tabular} & \begin{tabular}{l|l}
12 \\
\end{tabular} & 3 & 4 & 5 & 6 & 7 & 8 & 9 & 10 & 1 & 2 & 3 & 4 & 5 & 6 & & & 9 & & 11 & 12 & & 14 & & & 3 \\
\hline 1 & & & & & & & & & & & & & & & & & & & 1 & & (1) & & & & & & & & & & & & & \\
\hline 2 & & & & 1 & & 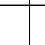 & 1 & 1 & 1 & & 1 & & & & & & & (1) & & & 1 & & & & & & & & & & & & & \\
\hline 4 & (1) & 1 & & 1 & 1 & 1 & 1 & 1 & \begin{tabular}{l|l}
1 & 1 \\
\end{tabular} & 1 & 1 & 1 & 1 & & & 2 & 2 & & & 1 & 1 & 1 & & 1 & & 1 & 1 & 1 & 1 & 1 & 1 & & & 1 \\
\hline 5 & & & (1) & & & & & & & & & & & & 1 & & & & & & & & & 1 & 1 & 1 & 1 & & 1 & 1 & 1 & & & \\
\hline 6 & & & & & & & & & & & & & & & & & & & & & & & & & & & & 1 & & & 1 & & & \\
\hline 7 & & & & & (1) & & 1 & 1 & (1 & 1 & & & 1 & & & & 1 & & & & & & & & 1 & 1 & 1 & 1 & 1 & 2 & 1 & & & \\
\hline 8 & & & & & & & & & & & & & (1) & & & & & & & & & & & & & & & (1) & & 1 & & & & \\
\hline 10 & & & & & & & & & & & & & & & & & 1 & & & & & & & & & & & & & & 1 & & & \\
\hline 11 & & & & & & & & & & & & & & & 1 & & & & 1 & & & & & & & (1) & 1 & & (1) & (1) & & & & \\
\hline 12 & 1 & 2 & 1 & 1 ( & (1) & & & 1 & \begin{tabular}{l|l}
1 & 1 \\
\end{tabular} & 1 & 1 & 1 & 1 & & 1 & 1 & & 1 & 1 & 1 & 1 & 1 & & & 1 & 1 & & & 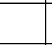 & & 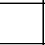 & & & 1 \\
\hline 13 & (1) & 2 & 1 & & 1 & 1 & $1 \mid 2$ & 2 & 1 & 1 & 1 & 1 & 2 & 1 & 2 & 2 & 1 & 1 & 2 & 1 & & & & 1 & 2 & 2 & & 1 & 1 & 1 & 1 & & 1 & 1 \\
\hline 14 & 1 & 2 & & & (1) & 1 & & & (1) & 1 & 1 & (1) & 1 & 1 & 1 & & & (1) & (1) & 1 & 1 & (1) & 1 & 1 & & 1 & 1 & & & & 1 & & 1 & 1 \\
\hline 15 & 2 & 3 & 1 & 2 & 2 & 2 & 23 & \begin{tabular}{l|l}
3 & 1 \\
\end{tabular} & \begin{tabular}{l|l}
1 & 1 \\
\end{tabular} & 1 & 1 & 1 & 1 & 3 & 4 & 2 & 2 & & & & 1 & & 1 & 3 & 4 & 3 & 3 & 1 & 3 & 1 & & & & 2 \\
\hline 16 & & & & & & & & & & & & & & & & & & & & & & & & & & 1 & & 2 & 3 & 2 & & & & \\
\hline 17 & & 2 & & & & & 1 & & 1 & & 1 & & & & & 3 & 2 & & & & & & (1) & & & 2 & 2 & 1 & 2 & 2 & & 1 & & 1 \\
\hline 18 & & & & & & & & & & & & & & 1 & & & & & & & & & & & 2 & 2 & & & & 2 & & & & \\
\hline 20 & 1 & & (1) & & & & & & 1 & & & & & & & & & & & & & (1) & & & & & & & & & & & & \\
\hline 21 & & & & & & & & & & & & & & & & & & & & & & & & & & & & & & & & & & \\
\hline 22 & & & & & & & (1) & 2 & $\begin{array}{lll}2 & 1 \\
2\end{array}$ & & & & & & & & & (1) & & 1 & & 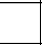 & & & & & & & & & & 2 & & \\
\hline 23 & & & & & & & & & 1 & & & & & & & & & & (1) & (1) & & & & & & & & & & & & & & (1) \\
\hline 26 & & & & & & & & & & & & & & & & & & & & & & & & & & & & 1 & & & 1 & & & \\
\hline 28 & & & & & & & & (1) & & & (1) & & & & & 2 & 1 & & & & & & & & & & & 1 & 1 & 1 & & & & \\
\hline 38 & & & & & & & & 1 & & & & & & & & & & & & 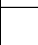 & & & & & & & & 1 & & & 1 & & & \\
\hline 39 & & & & & & & & 1 & & & & & & & & & (1) & & & & & & & & & & & & & & & & & \\
\hline 41 & & & & & & & & 1 & & & (1) & & & & & 1 & 1 & & & & 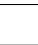 & 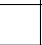 & & & & & 1 & 1 & (1) & 1 & & & & \\
\hline 43 & & & & & & & & & & 1 & & & & & & & 1 & & & & & & & & & + & & & 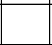 & & & & & \\
\hline 44 & 2 & & 2 & & & 1 & 1 & 2 & 2 & 1 & 11 & 1 & 1 & 1 & 1 & & & 1 & & 2 & 2 & 2 & 2 & 2 & 1 & 1 & 1 & & & & & 2 & 2 & 2 \\
\hline 47 & & & & & & & & & & & & & & & & & & & & & & & & & & & & & & & & & & \\
\hline 48 & & & & & & & & & & & & & & & & 1 & 2 & & & & 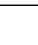 & & & & & 2 & 2 & 2 & 2 & 3 & 3 & & & \\
\hline 49 & & & & & & & & & & & & & & & & & & & & & 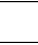 & & & & & & & + & & & 2 & & & \\
\hline 53 & & & & & & & 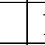 & 1 & & & & & & & & 1 & 2 & & & & 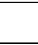 & & & & & & & & + & + & & & & \\
\hline 54 & & & & & & & 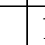 & 1 & & & & & & & & & & (1) & & & & & & & & & & & & & & & & \\
\hline 58 & & & & & & & & & & & (1) & & & & & & (1) & & & & & & & & & & & & & & (1) & & & \\
\hline 60 & 2 & 2 & 2 & 2 & & 1 & & 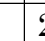 & 25 & 22 & \begin{tabular}{|l|l}
2 &
\end{tabular} & 1 & & & & & & 2 & 2 & 2 & 2 & 1 & & & & & & & & & & & 2 & 2 \\
\hline 62 & & & & & & & & & & & & & & & & & & & & & & & & & & & & & & & & & & \\
\hline 63 & 2 & 2 & 2 & 2 & 2 & 2 & 1 & 8 & 25 & 22 & 2 & 2 & 1 & 1 & 2 & 1 & 1 & 1 & 2 & 2 & 2 & 2 & 1 & 1 & 2 & 2 & & & & & & 2 & 2 & 2 \\
\hline 64 & 1 & 1 & 2 & 2 & & & & 2 & 2 & & 1 & 1 & & 1 & 1 & & & 1 & 1 & 2 & 1 & 1 & & 2 & 1 & & & & & & & 2 & 2 & \\
\hline 65 & & & & & & & & & & & & & & & & & & & & & & & & & & & & & & & & (1) & & \\
\hline 66 & & & & & & & & & & & & & & & & & & & 1 & & & & & & & & & & & & & & & \\
\hline 70 & & 2 & & & & & & & 2 & & & & & & & & & & & & & & & & & & & & & & & & 1 & \\
\hline 71 & & & & & & + & + & - & + & & + & + & + & & & & & + & & & 1 & & & & & & & & & & & & (1) & \\
\hline 73 & 3 & 3 & 2 & 3 & 3 & 3 & 1 & 3 & $\begin{array}{lll}3 & 3\end{array}$ & 33 & \begin{tabular}{|l|l}
3 \\
\end{tabular} & 2 & 1 & \begin{tabular}{|l|l} 
\\
\end{tabular} & 2 & & & 3 & 3 & 3 & 2 & & 2 & 2 & 1 & & & & & & & 3 & 3 & 3 \\
\hline 77 & + & 2 & + & + & + & 4 & 45 & 2 & & 12 & $2+$ & + & + & + & 3 & 3 & 2 & + & & & 3 & 3 & 3 & 4 & 4 & 3 & & & & & & 3 & $3 \mid$ & 4 \\
\hline 78 & 3 & & 2 & 3 & & 4 & 45 & 23 & 3 & & 1 & 3 & 3 & 3 & 3 & 3 & 1 & 3 & & 3 & 3 & 3 & 3 & 3 & 2 & 2 & 3 & & & & & 3 & 3 & 3 \\
\hline 79 & 2 & & & & & 2 & 2 & 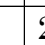 & 2 & & & 2 & 2 & 2 & 1 & & & 2 & & 2 & 2 & & 2 & 1 & & & & & & & & 2 & & 2 \\
\hline 80 & & & & 1 & & & & $(1$ & 1 & & & 1 & & & & & & (1) & & & & (1) & & (1) & & & & & & & & & & \\
\hline 81 & 3 & & 2 & 3 & & & 2 & 2 & 2 & & & 1 & 1 & 1 & 2 & 1 & & 2 & & 2 & 2 & 2 & 2 & 2 & 2 & & & & & & & 2 & 2 & 2 \\
\hline 82 & & & & & + & & + & & & & & & + & & & & & & & & & & & & & & & & & & & & & \\
\hline 86 & & & & & & & & & & 1 & & & & & & & 1 & & 1 & & & & & & & & & 1 & & & (1) & & & \\
\hline 87 & & & & & & & & & 1 & 1 & 1 & & & & & & & & & & & & & & & & & & & & 2 & & & \\
\hline 88 & & & (1) & & & & & 1 & \begin{tabular}{l|l}
1 & 1
\end{tabular} & & & & & & & & & & & & & & & & & & & & & & & & & \\
\hline 89 & & & & & & & & & & & & & & & & & & & & & & & & & & & (1) & & & & 1 & & & \\
\hline 93 & & & & & & & & & & & & & & & & & & & & & & & & & & & & & & & & & & \\
\hline 94 & & & & & & & & 1 & & 1 & & & & & & & & & & & & & & & & & & (1) & & & & & & \\
\hline 99 & & & & & & & & & & & & & & & & & 1 & & & & & & & & & & & & & (1) & & & & \\
\hline
\end{tabular}




\begin{tabular}{|c|c|c|c|c|c|c|c|c|c|c|c|c|c|c|c|c|c|c|c|c|c|c|c|c|c|c|c|c|c|c|c|c|}
\hline \multicolumn{33}{|c|}{ Table 9, 1997-2 } \\
\hline & \multicolumn{10}{|c|}{ D } & \multicolumn{13}{|c|}{$E$} & \multicolumn{9}{|c|}{$\mathrm{F}$} \\
\hline & 4 & 5 & 6 & $7 \mid 8$ & & & \begin{tabular}{l|l|l}
0 & 11
\end{tabular} & 112 & & $\begin{array}{ll}314 \\
\end{array}$ & 41 & 2 & 3 & 4 & 5 & 6 & 7 & 8 & 9 & & & 12 & & 1 & 23 & 3 & \begin{tabular}{l|l}
45 & 5 \\
4
\end{tabular} & \begin{tabular}{l|l}
5 & 6
\end{tabular} & 7 & 8 & \begin{tabular}{|l|}
9 \\
\end{tabular} & 10 \\
\hline 1 & & & & & & & & & & & & & & & & & & & & & & & & & & & & & & & & \\
\hline 2 & & & & & & & & & & & & & & & & & & & & & & (1) & & & & & & & (1) & & & \\
\hline 4 & $1: 2$ & 2. & 1 & \begin{tabular}{l|l}
1 & 1 \\
1
\end{tabular} & 2 & 1 & 1 & 2 & 2 & & & & & & 1 & 1 & 1 & 1 & 2 & 2 & 2 & & & & & & 11 & \begin{tabular}{l|l}
1 & 1 \\
\end{tabular} & 1 & 1 & 1 & \\
\hline 5 & & 1 & & 1 & 2 & 1 & & 2 & 1 & & & & & & 1 & 1 & 1 & & 1 & & & & & & & & & & & & & \\
\hline 6 & & & & & & & & & 1 & 2 & & & & & & & & & & & & 1 & 1 & & & & & (1) & & & 1 & 1 \\
\hline 7 & & 1 [1: & 1 & 1 & 1 & & & 2 & 2 & 2 & & & & & 1 & & 1 & & 2 & & 2 & 1 & & & & & & \begin{tabular}{l|l}
1 & 1 \\
\end{tabular} & 1 & 1 & 1 & \\
\hline 8 & & & & & & & (1) & & 2 & (1) & & & & & & & (1) & & & & & & & & & & & & & & & \\
\hline 10 & & & & & & & (1) & 1 & 1 & 1 & & & & & & & & & & 1 & & & & & & & & 1 & 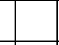 & 1 & & \\
\hline 11 & & & & & & & & & & & & & & 1 & & & & & 1 & 2 & & & & & & & & & & & 1 & 1 \\
\hline 12 & & 2 & & \begin{tabular}{l|l}
1 & 1 \\
\end{tabular} & 1 & & & & & & & & & 1 & 1 & 1 & 1 & 1 & 2 & & & & & & & (1) & 1 & \begin{tabular}{l|l}
1 & 1 \\
\end{tabular} & & & & \\
\hline 13 & 1 & 1 & 12 & \begin{tabular}{l|l}
2 & 2 \\
\end{tabular} & 2 & 2 & & 2 & 2 & & & & & & 2 & 2 & 2 & 2 & 2 & 2 & & & & (1) & & & 1 & \begin{tabular}{l|l}
1 & 1 \\
\end{tabular} & 1 & 1 & 1 & \\
\hline 14 & \begin{tabular}{|l|l}
1 & 1 \\
\end{tabular} & 1 & & 1) & & & & & & & & & & 1 & 1 & 1 & 1 & 1 & & & & & & & & & & 1 & & & & \\
\hline 15 & \begin{tabular}{|l|l}
2 & 2 \\
\end{tabular} & 25 & 25 & \begin{tabular}{l|l}
2 & 3 \\
\end{tabular} & 3 & 2 & 2 & 2 & 2 & & & & & & 2 & 2 & 2 & 3 & 3 & 2 & 2 & 2 & 2 & & & & 1 & \begin{tabular}{l|l}
1 & 1
\end{tabular} & 1 & 1 & 1 & \\
\hline 16 & & & & & & & & & 2 & 2 & & & & & & & & & 1 & 1 & 1 & & 1 & & & & & & & & 1 & \\
\hline 17 & & & 2 & & (1) & 2 & & 1 & 1 & & & & & 1 & & 1 & 2 & 1 & 1 & 2 & & & & & & & 1 & \begin{tabular}{l|l}
1 & 1
\end{tabular} & 1 & 1 & 1 & 1 \\
\hline 18 & & & & & & & & & & & & & & 1 & & & 1 & & & & 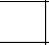 & & & & & & 1 & & & & & \\
\hline 20 & & & (1) & & & & & & & & & & & 1 & (1) & & 1 & 1 & & & & & & & & (1) & (1) & & & & & \\
\hline 21 & & & & & & & & & & & & & & & & & & & & & (1) & & & & & & & & & & & \\
\hline 22 & 1 & & & & & & & & & & & & & & & & & & & & & & & & & & & & & & & \\
\hline 23 & & & & & & & & & & & & & & & & & 1 & & & & & & & & & & & & & & & \\
\hline 26 & & & & & & & & & 1 & 2 & & & & & & & & & & & & 1 & & & & & & & & (1) & & \\
\hline 28 & & 1 & & & & & 1 & 2 & 1 & & & & & & 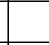 & & & 1 & 2 & 1 & 2 & 1 & & & & & (1) & 1 & & 1 & 1 & 1 \\
\hline 38 & & & & & & & & & & & & & & & & & & & & & & & & & & & & & & & & \\
\hline 39 & & & & & & & & & (1) & & & & & & & & & & & & & & (1) & & & & & & & & (1) & \\
\hline 41 & & & & & & & & & & & & & & & (1) & & & 1 & & & 1 & 1 & 1 & & & & & & 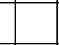 & 1 & & 1 \\
\hline 43 & & 1 & 1 & & & & & 1 & & & & & & & & & 2 & 2 & 2 & & & & & & & & & & & & & \\
\hline 44 & 25 & 25 & 21 & \begin{tabular}{l|l}
1 & 1 \\
\end{tabular} & \begin{tabular}{l|l}
1 & 1 \\
\end{tabular} & 1 & & & & & & 1 & 1 & 1 & 1 & 1 & 2 & & & & & & & 2 & 1 & 1 & 1 & & & & & \\
\hline 47 & & & & & & & & & & & & & & & & & & & & & & & & & & & & & (1) & & & \\
\hline 48 & & & & (1) & & 2 & 2 & 3 & 3 & 3 & & & & & 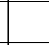 & & & & 2 & 3 & 3 & 2 & 2 & & & & & 1 & 1 & 1 & 2 & 2 \\
\hline 49 & & & & & & & & & & 2 & & & & & & & & & & & & 1 & 1 & & & & & & & & & \\
\hline 53 & & & & & & & & & 1 & & & & & & & & & & & 1 & 2 & 2 & 2 & & & & & & & & & \\
\hline 54 & & (1) & & & & & & & & & & & & & (1) & & & & & & & & & & & & & 1 & & 1 & 1 & \\
\hline 58 & & 1 & & & & & & & & & & & & & & & & & & & & & & & & & & & & & & 1 \\
\hline 60 & & 2 & & (1) & D) & & & & & & & & & & 2 & 1 & & & 1 & & & & & 1 & & 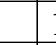 & 1 & \begin{tabular}{l|l}
1 & 1
\end{tabular} & 1 & 1 & 1 & \\
\hline 62 & & & & & & & & & & & & & & & 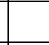 & & & (1) & & & & & & 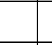 & 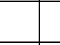 & 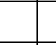 & 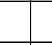 & 1 & 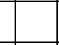 & & 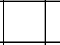 & \\
\hline 63 & 25 & 25 & 25 & $\begin{array}{ll}2 & 2 \\
\end{array}$ & $\begin{array}{l}22 \\
\end{array}$ & & & & & & & & 1 & 2 & 2 & 2 & 2 & 1 & 2 & 2 & & & & 1 & & (1) & 1 & \begin{tabular}{l|l}
1 & 1 \\
\end{tabular} & 1 & & 1 & \\
\hline 64 & $\begin{array}{ll}2 & 3 \\
\end{array}$ & 35 & $\begin{array}{lll}21 \\
\end{array}$ & \begin{tabular}{l|l}
1 & 1 \\
\end{tabular} & & & & & & & 1 & 1 & 1 & 1 & 1 & & & & & & & & & 2 & 1 & 18 & 2 & & & & & \\
\hline 65 & & & & & & & & & & & & & & & & & & & & & & & & & & & & & & & & \\
\hline 66 & & & & & & & & & & & & & & & & & & & & & & & & & & & & & & & & \\
\hline 70 & & & & & & & & & & & & & & & & & & & & & & & & & & & & & & & & \\
\hline 71 & & & & & & & & & & & & & & & & & & & & & & & & & & & & & & & & \\
\hline 73 & \begin{tabular}{|l|l}
2 & 3 \\
\end{tabular} & $3:$ & 32 & 22 & 22 & 2 & & & & & 2 & 2 & 2 & 2 & 3 & 2 & 2 & & & & & & & 2 & 1 & 1 & 2 & 3 & 2 & & & \\
\hline 77 & \begin{tabular}{|l|l}
4 & 3 \\
\end{tabular} & $3:$ & $3-$ & +3 & \begin{tabular}{|l|l}
3 \\
\end{tabular} & 3 & & & & & 4 & 4 & 4 & & 3 & 2 & & 3 & 3 & 1 & & & & + & +-1 & +- & + & + & + & & & \\
\hline 78 & \begin{tabular}{|l|l|l|}
3 & 3 \\
\end{tabular} & $3:$ & $3:$ & \begin{tabular}{l|l}
3 & 3 \\
\end{tabular} & \begin{tabular}{|l|l}
3 & 3 \\
\end{tabular} & \begin{tabular}{l|l}
3 \\
\end{tabular} & 3 & & & & 4 & 3 & 3 & 4 & 2 & 2 & 2 & 1 & 1 & & & & & 4 & 23 & 2 & & & & & & \\
\hline 79 & \begin{tabular}{|l|l|l|}
2 & 1 \\
\end{tabular} & 1 & 1 & & & & & & & & 2 & 1 & 1 & 1 & & & & & & & & & & 2 & & (1) & & & & & & \\
\hline 80 & & & 2 & 1 & & & & & & & & & & 1 & 1 & & & & & & & & & & & & & & & & & \\
\hline 81 & \begin{tabular}{|l|l}
2 & 2 \\
\end{tabular} & 25 & 25 & $\begin{array}{lll}2 & 2\end{array}$ & $\begin{array}{l}2 \\
2\end{array}$ & & & & & & 2 & 2 & & 1 & 1 & 1 & & & & & & & & 3 & 12 & 2 & & & & & & \\
\hline 82 & & 1 & & & & & & & & & & & & & & & & & & & & & & & & & & & & & & \\
\hline 86 & & 1 & & & & & & & & 1 & & & & & & 1 & & & & & 1 & & & & & & (1) & \begin{tabular}{l|l}
1 & 1 \\
\end{tabular} & 1 & 1 & & \\
\hline 87 & & & 1 & 1 & & & & & & 1 & & & & & & & & & & & & 1 & 1 & & & & & & & & & \\
\hline 88 & & & & & & & & & & 1 & & & & & & & & & & & (1) & & & & & & (1) & & & & & \\
\hline 89 & & & & & & & & & & 2 & & & & & & & & & & & & 1 & 2 & & & & & & & & & 1 \\
\hline 93 & & & & & & & & & & & & & & & & & & & & & & & & & & & & & & & & (1) \\
\hline 94 & & & & & & & (1) & 1 & & & & & & & & & & & (1) & & & (1) & (1) & & & & & & & & & \\
\hline 99 & & & & & & & & & & & & & & & & & & & & & & & 1 & & & & & & & & & \\
\hline
\end{tabular}




\begin{tabular}{|c|c|c|c|c|c|c|c|c|c|c|c|c|c|c|c|c|c|c|c|c|c|c|c|c|c|c|c|c|c|c|c|c|c|c|c|}
\hline \multicolumn{36}{|c|}{ Table 9, 1997-3 } \\
\hline & \multicolumn{8}{|c|}{$\mathrm{A}$} & & & & & $E$ & & & & & & & & & & & & & $\mathrm{C}$ & & & & & & & & D & \\
\hline & \begin{tabular}{l|l}
12 \\
1
\end{tabular} & 2 & 3 & 4 & & 6 & 7 & 8 & 1 & 2 & 3 & 4 & 5 & 6 & 7 & 8 & 9 & 10 & 1 & 2 & 3 & 4 & 5 & 6 & & & \begin{tabular}{l|l}
9 & 1 \\
\end{tabular} & & & 12 & & 14 & & & 3 \\
\hline 101 & & & & & & & & & & & & & & & & & & & & & & & & & & & & & & & & & & & \\
\hline 103 & & & & & & & & & & & & & & & & & & & & & & & & & & & & & & & & & & & \\
\hline 104 & & & & & & & & & & & & & & & & & & & & & & & & & & & & & & & & & & & \\
\hline 107 & & & & & & & & & & & & & & & & & & & & & & & & & & & & ( & (1) & & & & & & \\
\hline 111 & & & & & & & & & & & & & & & & & & & & & & & & & & & & & & & & & & & \\
\hline 115 & & & & & & & & & & & & & & & & & & & & (1) & & & & & & & & & & & & & & & \\
\hline 116 & & & & & & & & & & & (1) & & & & & & & & & & & & & & & & & & & (1) & & & & & \\
\hline 118 & & & & & & & & & (1) & & & & & & & & & & & & & & & & & & & & & & & & & & \\
\hline 119 & & & & & & & & & & & & & & & & & & & & & & & & & & & & & (1) & & & & & & \\
\hline 122 & & & & & (1) & & (1) & 1 & & (1) & 1 & 1 & & & & 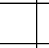 & & & & & & & & & 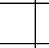 & & & & & & & & & & \\
\hline 123 & & & (1) & & & 1 & & 1 & & 1 & 1 & 1 & 1 & 1 & & & & 1 & & (1) & & & 1 & (1) & & & 1 & 2 & 2 & & & & & & \\
\hline 124 & 25 & 2 & & 2 & 2 & 1 & 2 & & 2 & & 2 & 2 & 1 & 1 & 1 & 1 & 2 & & 2 & 1 & 1 & 1 & 2 & 1 & 2 & 1 & 1 & 1 & & & & & & 2 & 2 \\
\hline 125 & & & & & & & & & & & & & & & & & & & & & & & & & & & & & & & & (1) & & & \\
\hline 127 & & & & & & & & & & & & & & & & & & & & & & & & & & & & & & & & & & & \\
\hline 129 & & & & & & & & & & & & & & & & & & & & & & & 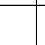 & & & - & & & & & & & & & \\
\hline 130 & & & & & & 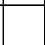 & & 1 & & & & 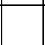 & & 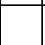 & & & & & & & & 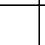 & & & & & & & 2 & 1 & 2 & (1) & & & \\
\hline 131 & & & & & & & & & & & & & & & & & & & & & & & & & & & & & & & & & & & \\
\hline 132 & & (1) & & & & 1 & 1 & 2 & & 1 & & 1 & & & & & & 2 & & & & (1) & & & & 1 & 1 & 1 & 1 & 1 & 1 & 1 & & & \\
\hline 135 & & & & & & & & & & & & & & & & & & & & & & & & & & & & & & & & & & & \\
\hline 139 & & & & & & & & & & & & & . & & & & & & & & & & & & & & & & & & & (1) & & & \\
\hline 141 & & & & & & & & & (1) & (1) & & & & & (1) & 1 & & 1 & & & & (1) & (1) & & & 1 & 1 & 1 & 1 & 1 & 1 & 2 & & & \\
\hline 142 & & & (1) & & & & & 2 & & 1 & & (1) & 1 & & & & & 2 & & (1) & & & & & & 1 & & 1 & & 1 & 1 & 2 & & & 1 \\
\hline 143 & & & & & & & & & & & & & & & & & & & & & & 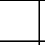 & 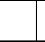 & & & & & & 1 & & & 1 & & & \\
\hline 145 & & & (1) & & & (1) & & 1 & & & & (1) & 1 & 1 & & & & 1 & & & & (1) & & & & 1 & & & 1 & & & & & & \\
\hline 146 & & & & & & & & & & & & & & & & & & & & & & 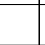 & & & & & & & & & & & & & \\
\hline 149 & & & & & & & & & & & & & & & & & & & & & & & & & & & & & & & & & & & \\
\hline 155 & & & & & & & & & & & & & & & & & & & & & & & & & & & & & & & & & & & \\
\hline 156 & & & & & & & & & (1) & & & (1) & & & & & & & & (1) & & & 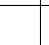 & & & & & & & & & & & & \\
\hline 158 & & & & & (1) & & & & & & (1) & 1 & 1 & & & & & & & 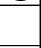 & 1 & 1 & 1 & & & & & & & & & & & & \\
\hline 161 & & (1) & & & & & & & & (1) & & & & & & & & & & & & & & & & & & & (1) & & & & & & \\
\hline 170 & & & & & & & & & & & & & & & & & & & & & & & & & & & & & & & & & & & \\
\hline 172 & & & & & & & & (1) & & & & & & & & & & & & & & & & & & & & & & & & & & & \\
\hline 173 & & & & & & & (1) & & & & & 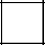 & & & & & & & & & & & & & & & & & & & & & & & \\
\hline 180 & & & & & & (1) & & & & & & & & & & & & & & & & & 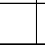 & & & & & & & & & & & & \\
\hline 181 & & & & & & & & 1 & & & & 1 & & 1 & & & & & & 1 & & & (1) & & & & & & & & & & & & 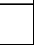 \\
\hline 182 & & & & & & & & 1 & & & & & & & & & & & & 2 & & & & & & & & & & & & & & & \\
\hline 185 & & & & & & & & & & & & & & & & & & & & & & & & & & & & & & & & & & & \\
\hline 189 & & & & & & & & & & & & & & & & & & & & & & & & & & & & & & & & & & & \\
\hline 193 & & & & & & 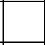 & & & & & & & & & & & & & & & & & & & & & & & & & & & & & \\
\hline 196 & & & & & & & & & & & & & & & & & & & & & & & . & & & & & & & (1) & & & & & \\
\hline 197 & & & & & & & & & & & & & & & & & & & & & & & & & & & & & & & & & & & \\
\hline 198 & & & & & & 2 & 3 & & & & & & & 2 & 2 & 1 & & & & & & 1 & 1 & 2 & 1 & & & & & & & & 1 & 2 & 2 \\
\hline 199 & & & & & & 2 & 2 & & & & (1) & & & 1 & 1 & 1 & & & & & & & 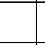 & & 1 & & & & & & & & & (1) & 1 \\
\hline 200 & & & & & & 2 & 3 & 2 & & & & & & & 2 & & 2 & & & & & & 1 & & & (1) & 1 & & & 1 & 1 & 1 & & & \\
\hline 201 & & & & & & & & & & & (1) & & & & & & & & & & & & & & & & & & & & & & & & \\
\hline 202 & & & & & & & & & & & & & & & & & & 1 & & & & & & & & & & & & & & & & & \\
\hline 203 & & & & & & & & & & & & & & & & & & & & & & & & & & & & & & & & (1) & & & \\
\hline 207 & & & & & (1) & & & & & & 1 & 1 & 1 & & & & & 2 & & 1 & 1 & & (1) & & & 1 & 1 & 1 & 1 & 2 & 1 & 2 & & (1) & \\
\hline 210 & & & & & & & & & & & & & & & & & & & & & & & & & & & (1) & & & & & & & & \\
\hline 211 & + & & + & + & + & + & + & & 1 & & + & + & + & + & + & + & + & & + & + & & + & + & & 3 & 3 & \begin{tabular}{l|}
3 \\
\end{tabular} & 4 & & & & & + & + & + \\
\hline 212 & & & & (1) & & & (1) & 4 & & & & \begin{tabular}{|l|} 
\\
\end{tabular} & & & 2 & 1 & & & 3 & 3 & 3 & & 1 & & & 1 & 1 & & 4 & 4 & 4 & 1 & & 1 & \\
\hline 213 & & 1 & & & & & (1) & 1 & & & & (1) & 1 & & & & & 1 & & & & & & & & & . & 1 & 1 & 1 & (1) & & & 7 & \\
\hline 214 & (1) & & & & & & & & (1) & (1) & 1 & & & 1 & 1 & 1 & & & 1 & & 1 & & 1 & 1 & 1 & 1 & 1 & 3 & 3 & 4 & 4 & 4 & & 1 & 1 \\
\hline 216 & & & & & & & & & & & & & & & & & & & & & & & & & & & & & & & (1) & & & & \\
\hline 218 & & & & & & & & & & & & & & & & & & & & & & & & & & & & & & & (1) & & & & \\
\hline
\end{tabular}




\begin{tabular}{|c|c|c|c|c|c|c|c|c|c|c|c|c|c|c|c|c|c|c|c|c|c|c|c|c|c|c|c|c|c|c|c|c|c|c|}
\hline \multicolumn{35}{|c|}{ Table 9, 1997-4 } \\
\hline & \multicolumn{11}{|c|}{ D } & \multicolumn{13}{|c|}{$E$} & & & & & $\mathrm{~F}$ & & & & & \\
\hline & 4 & $5 \mid$ & 6 & 7 & 8 & 9 & 10 & & 12 & 13 & 14 & & 2 & 3 & 4 & 5 & 6 & & 8 & \begin{tabular}{|c|}
9 \\
\end{tabular} & & 11 & 12 & & 1 & 2 & 3 & & & & & & \begin{tabular}{l|l}
9 & 1
\end{tabular} & \\
\hline 101 & & & & & & & & & & & & & & & & & & & & & & & & & & & & & (1) & & & & & \\
\hline 103 & & & & & & & & & & & & & & & & & & & 1 & (1) & & & 1 & & & & & & & & & & & \\
\hline 104 & & & & & & & & & & & & & & & & & & & & & & & & & & & & & & & & & (1) & \\
\hline 107 & & & & & & & & & & 1 & & & & & & & & & & & & & & & & & & & & & & & & \\
\hline 111 & & & & & & & & & & & & & & & & & & & & & & & & & & & & & (1) & & & & & \\
\hline 115 & & & & & & & & & & & & & & & & & & & & . & & & & & & & & & & 1 & & & & \\
\hline 116 & & & & & & & & & & & & & & & & & & & & & & & & & & & & & & & & & & \\
\hline 118 & & & & & & & & & & & & & & & & & & & & & & & & & & & & & & & & & & \\
\hline 119 & & + & & & & & & & & & & & & & & & & & 1 & & & & & & & & & & & & 1 & L & & \\
\hline 122 & & 1 & & & & 1 & & & & & & & & & & & 1 & & 1 & 1 & & & & & & & & & & 1 & 1 & 1 & \begin{tabular}{l|l}
1 & 1 \\
\end{tabular} & \\
\hline 123 & 1 & 2 & & & & 1 & 1 & & 2 & & & & & . & & 1 & 1 & 1 & 1 & 1 & & 1 & & & (1) & & & 1 & 1 & 1 & 1 & 11 & \begin{tabular}{l|l}
1 & 1 \\
\end{tabular} & \\
\hline 124 & 1 & 1 & 2 & 2 & 1 & 1 & & & & & & & 1 & 1 & 1 & 1 & 1 & 2 & 1 & 1 & & & & & 1 & 1 & 1 & & 1 & & t & & & \\
\hline 125 & & & & & & & & & & & & & & & & & & & & & & & 1 & & & & & & & & & & 1 & \\
\hline 127 & & & & & (1) & & & & & & & & & & & & & & & & & & & & & & & & & & & & & \\
\hline 129 & & & & & & & & & & & & & & & & & & & & & & & (1) & & & & & & (1) & & & & \begin{tabular}{l|l}
1 & 1 \\
\end{tabular} & \\
\hline 130 & & & & & & & 1 & 1 & 2 & 2 & 1 & & & & & 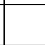 & & & 1 & 1 & 1 & 1 & 1 & 1 & & & & & & & 1 & 1 & \begin{tabular}{l|l}
1 & 1 \\
\end{tabular} & \\
\hline 131 & & & & & & & & & & & & & & & & & & & & & & & & & & & & & 1 & & & & \begin{tabular}{l|l}
1 & 1 \\
\end{tabular} & \\
\hline 132 & (1) & 2 & & 1 & 2 & 1 & 1 & 1 & 2 & 1 & & & & & & 2 & 2 & 2 & 2 & 2 & 2 & 2 & 2 & 1 & & & & & 2 & 2 & $1] 1$ & L & \begin{tabular}{l|l}
1 & 1 \\
\end{tabular} & \\
\hline 135 & & & & & & & & & & & & & & & & & & & & & & & & (1) & & & & & & & & & & \\
\hline 139 & & & & & & & & & & & & & & & & & & & & & & & & & & & & & & & & & & \\
\hline 141 & & & 1 & & & & & 1 & 1 & 1 & 2 & & & & & & & & & & 1 & 1 & 2 & 2 & & & & & & 1 & 1 & 11 & \begin{tabular}{l|l}
1 & 1
\end{tabular} & \\
\hline 142 & & & & 1 & 1 & & 1 & & 2 & 2 & 1 & & & & & & 1 & 1 & 2 & 2 & 2 & 2 & 2 & 2 & & & & 1 & & & & & \begin{tabular}{l|l}
1 & 1
\end{tabular} & \\
\hline 143 & & & & & & (1) & & & 1 & & 1 & & & & & & & & 1 & & & & & & & & & & & & & & & \\
\hline 145 & & 1 & & & & & & 1 & & 1 & & & & & & & & & 2 & & & 1 & & 1 & & & & 1 & 1 & 15 & 2 & 1 & 1 & \\
\hline 146 & & & & & & & 1 & & 1 & 1 & & & & & & & & & & & & & & 1 & & & & & & 1 & & & & \\
\hline 149 & & & & & & & & (1) & & & & & & & & & & & & & & & & & & & & & & & & & & \\
\hline 155 & & 1 & & & & & & & & & & & & & & & & & & & & & & & & & & & & & & & & \\
\hline 156 & & & & & & & & & & & & & & & & & & & & & & & & & & & & 16 & (1) & & & & & \\
\hline 158 & 1 & 1 & & & 1 & & & & & & & & & & & 1 & & & & & & & & & & & & 1 & & & & & & \\
\hline 161 & & 1 & & & & & & & & & & & & & & & & & & & & & & & & & & 1 & 1 & 1 & 1 & & 1 & \\
\hline 170 & & & & & & & & & & & & & & & & & & & & & & & & & & & & & & & (1) & & & \\
\hline 172 & & & & & & & & & & & & & & & & & & & & & & & & & & & & & & & & & & \\
\hline 173 & & & & & & & & & & & & & & & & & & & & & & & & & & & & & & (1) & & & & \\
\hline 180 & & & & & & & & & & & & & & & & & & & & & & & & & & & & 1 & & & & & & \\
\hline 181 & & (1) & & & & & & & & & & & & & & & & & & & & & & & & & & & & & & (1) & 1) & \\
\hline 182 & & & & & & & & & & & & & & & & & & & & & & & & & & & & & & & & & & \\
\hline 185 & & & & & & & & & & & & & & & & & & & (1) & & & & & & & & & & & & & & & \\
\hline 189 & & & & & & & (1) & & & & & & & & & & & & & & & & & & & & & & & & & & & \\
\hline 193 & & & & & & & & & 1 & & & & & & & & & & & & & & & & & & & & & & & & & \\
\hline 196 & & & & & & & & & & & & & & & & & & & & . & & & & & & & & & & & & & & \\
\hline 197 & & & & (1) & & & & & & & & & & & & (1) & & & . & . & & & & & & & & & & & & & & \\
\hline 198 & 2 & & 1 & & 1 & 1 & & & & & & 1 & 2 & 2 & 1 & & & & & & & & & & & & 1 & & 1 & & & & & \\
\hline 199 & 1 & 1 & 1 & 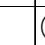 & (1) & (1) & & & & & & & & & 1 & 1 & & & 1 & 1 & & & & (1) & & 1 & 1 & & 1 & & & & & \\
\hline 200 & & & & 1 & 1 & 2 & 2 & & 2 & 2 & & & & & & & & 1 & 1 & 2 & 1 & 2 & 1 & 1 & & (1) & & & 1 & 1 & $1]$ & 11 & \begin{tabular}{l|l}
1 & 1
\end{tabular} & \\
\hline 201 & & & & & & & & & & & & & & & & & & & & & & & & & & & & & & & & & & \\
\hline 202 & & & & & & & & & & & & & & & & & & & & & & & & 1 & & & & & & & & & & \\
\hline 203 & & & & & & & & & & & & & & & & & & & & & & & & & & & & & & & & & & \\
\hline 207 & & \begin{tabular}{l|l}
1 \\
\end{tabular} & 1 & 1 & (1) & (1) & 1 & 1 & 3 & 2 & 2 & & & & 1 & & & 1 & 1 & 2 & & 1 & 2 & 2 & & & & & 1 & 1 & 1 & $\begin{array}{lll}1 & 1\end{array}$ & \begin{tabular}{l|l}
1 & 1
\end{tabular} & \\
\hline 210 & & & & & & & & & & & 1 & & & & & & & & & & & & & & & & & & & & & & & \\
\hline 211 & + & 3 & + & + & 4 & 4 & 3 & & 1 & & & 3 & & 2 & + & + & + & + & & . & & . & & & + & + & + & + & + & + & +7 & + & + & \\
\hline 212 & & 4 & & & & & 3 & 3 & 4 & 4 & 3 & & & (1) & & & 4 & 4 & 2 & 3 & 4 & 4 & 4 & 4 & & & & & 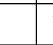 & 1 & & & & \\
\hline 213 & & & & & & & & 1 & 1 & 1 & (1) & & & & & . & & & & 1 & & & & & & & & & 1 & . & 1 & 1 & 1 & \\
\hline 214 & 1 & 1 & 1 & 2 & & 1 & 3 & 3 & 4 & 4 & 4 & & & & & 1 & 1 & & 2 & 1 & 3 & & 3 & 3 & & 1 & & & & 4 & 45 & 44 & 43 & \\
\hline 216 & & & & & & & & & & & & & & & & & & & & & & & & & & & & & & & & & & \\
\hline 218 & & & & & & & & & & & & & & & & & & & & & & & & & & & & & & & & & & \\
\hline
\end{tabular}




\begin{tabular}{|c|c|c|c|c|c|c|c|c|c|c|c|c|c|c|c|c|c|c|c|c|c|c|c|c|c|c|c|c|c|c|c|c|c|}
\hline \multicolumn{34}{|c|}{ Table 9, 1997-5 } \\
\hline & \multicolumn{8}{|c|}{$\mathrm{A}$} & \multicolumn{9}{|c|}{ B } & \multicolumn{13}{|c|}{ C } & \multicolumn{3}{|c|}{ D } \\
\hline & 1 & 2 & 3 & 4 & & & & & 2 & 3 & 4 & 5 & 6 & & 8 & & 10 & & 2 & 3 & 4 & 5 & 0 & $7 \varepsilon$ & $t$ & 1 & & \begin{tabular}{l|l}
1 & 1 \\
\end{tabular} & & 12 & & & 3 \\
\hline 219 & & & & & & & & & & & & & & & & & & & & & & & & & & & & & & & & & \\
\hline 220 & & 1 & & & & 1 & 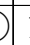 & 1 & & (1) & 1 & & 1 & & & & 1 & & & & & & & & 1 & & 1 & 1) & 1 & & & & \\
\hline 221 & (1) & & & & & & & & & & & & & & & & & & & & & 1) & & & & & & & & & & & \\
\hline 226 & & & & & & & & & & & & & & & & & & & & & & & & & & & & & & (1) & & & \\
\hline 227 & & & 1 & & & & & & & & & & . & & 1 & & 1 & & & & & & 1) & 1 & & & & 1 & & & & & \\
\hline 229 & 2 & 3 & 2 & 2 & L & 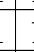 & & 2 & 2 & 2 & 2 & & 1 & 2 & 2 & 3 & 3 & 1 & 1 & 1 & 1 & 1 & 2 & 3 & 3 & & & 1 & 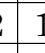 & & 2 & 2 & 2 \\
\hline 230 & & 1 & & & & & & & & & & & & 1 & & & 2 & & & & & & 1 & 1 & 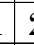 & & & 1 & 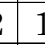 & (1) & & . & \\
\hline 232 & + & + & + & + & & & & + & & + & & + & & + & + & & + & + & & & & & & +1 & & & & + & + & & & + & + \\
\hline 233 & & & & & & & & & & & & & & & & & & & & & & & & & & & & (1) & & (1) & & & \\
\hline 234 & & & & & & & & & & & & & & & & & & & & & & & & & & & & & & & & & \\
\hline 235 & & 1 & & & & & & & & & & & & & & & 2 & & (1) & & & & & & & & & & & & & 1 & \\
\hline 236 & & & & & & & & & & & & & & & & & & & & & & & & & & & & & & & & & (1) \\
\hline 240 & & & & & & & & & & & & & & & & & & & & & & & & & & & & D & & & & & \\
\hline 242 & & & & & & & & & & & & & & & & & & & & & & & & & & & & & & (1) & & & \\
\hline
\end{tabular}

\begin{tabular}{|c|c|c|c|c|c|c|c|c|c|c|c|c|c|c|c|c|c|c|c|c|c|c|c|c|c|c|c|c|c|c|c|c|c|c|}
\hline \multicolumn{35}{|c|}{ Table 9, 1997-6 } \\
\hline & \multicolumn{11}{|c|}{$\mathrm{D}$} & \multicolumn{13}{|c|}{$E$} & \multicolumn{10}{|c|}{$\mathrm{F}$} \\
\hline & 4 & 5 & 6 & 7 & 8 & 9 & & 11 & 12 & 13 & & 1 & 2 & 3 & 4 & 5 & 6 & 7 & 8 & 9 & 10 & 11 & 12 & 13 & 1 & 2 & 3 & 4 & 5 & 6 & 7 & 8 & & 10 \\
\hline 219 & & & & & & & (1) & & & & & & & & & & & & & & & & & & & & & & & & & & & \\
\hline 220 & & & & & & & 1 & 1 & 1 & 1 & & & & & & & & & & 1 & & & 1 & & (1) & & & & & & (1) & 1 & 1 & 1 \\
\hline 221 & & & & & & & & & & & & & & & & & & & & & & & & 1 & & & & & & & & & & \\
\hline 226 & & & & & & & & & & & (1) & & & & & & & & & & & & & (1) & & & & & & & & & & \\
\hline 227 & 1 & & & & & & & & & & & & & & & & & & & & & & & & & & & & & & & & & \\
\hline 229 & 2 & 3 & 3 & 3 & 3 & 3 & 3 & 3 & 3 & 1 & & 1 & 1 & 1 & 2 & 3 & 3 & 3 & 3 & 3 & 3 & 3 & & & 2 & 1 & 2 & 2 & 2 & 2 & 2 & 2 & 1 & 3 \\
\hline 230 & 1 & 2 & 2 & 2 & 2 & 2 & 2 & 1 & 3 & 2 & & & & & (1) & & 1 & 2 & 2 & 2 & 2 & & & 1 & 2 & 2 & 2 & 2 & 2 & & 1 & 1 & & 1 \\
\hline 232 & + & + & + & & + & + & + & & + & + & & & & & + & + & + & + & + & + & + & & & & + & + & + & + & + & + & + & + & + & + \\
\hline 233 & & & & & & & & & & & & & & & & & & & & & & & & & & & & & & & (1) & 1 & 1 & 1 \\
\hline 234 & & & & & & & & & & & (1) & & & & & & & & & & & & & & & & & & & & (1) & & & 1 \\
\hline 235 & & 1 & 1 & & (1) & & (1) & & & & (1) & & & & & (1) & & 1 & & & & & & & & & & & & 1 & & & & \\
\hline 236 & & & & & & & & & & & & & & & & & & & & & & & & & & & & & & & & & & \\
\hline 240 & & & & & & & & & & & & & & & & & & & & & & & & & & & & & & & & & & \\
\hline 242 & & & & & & & & & & & & & & & & & & & & & & & & & & & & & & & & & & \\
\hline
\end{tabular}




\begin{tabular}{|c|c|c|c|c|c|c|c|c|c|c|c|c|c|c|c|c|c|c|c|c|c|c|c|c|c|c|c|c|c|c|c|c|c|c|c|}
\hline \multicolumn{36}{|c|}{ Table 9, 1998-1 } \\
\hline & \multicolumn{8}{|c|}{$\mathrm{A}$} & & & & & $E$ & & & & & & & & & & & & ( & C & & & & & & & & D & \\
\hline & 1 & 2 & 3 & 4 & & 6 & 7 & 8 & 1 & 2 & 3 & 4 & 5 & 6 & 7 & 8 & 9 & 10 & 1 & 2 & 3 & 4 & 5 & 6 & & 85 & \begin{tabular}{l|l}
9 & 1 \\
\end{tabular} & 101 & 11 & 12 & & 14 & & & 3 \\
\hline 1 & & & (1) & & & & & & & & & & & & & & & & & & & & & & & & & & & & & & & & \\
\hline 2 & & & 1 & & & & (1) & & & & & & & & & & & & & & & (1) & & & & & & & & & & & & & \\
\hline 3 & & & & & & & & & & & & & & & & & & & & & & & & & & & & & & & & & & & \\
\hline 4 & & 1 & 1 & 1 & 1 & 1 & & 2 & & 1 & 1 & 1 & 1 & (1) & & 2 & 1 & 2 & & 1 & 1 & & & & & 1 & 1 & 2 & 2 & 1 & 1 & & & & \\
\hline 5 & & & & & & & & 1 & & & & 1 & & (1) & & 2 & 1 & 2 & & & & & & & & 1 & & 2 & 1 & 1 & & & & & \\
\hline 6 & & & & & & & & & & & & & & & & & & & & & & & & & & & & & (1) & (1) & 1 & & & & \\
\hline 7 & & & & & & & (1) & 1 & & & (1) & & & & & & (1) & 1 & & & & & & & & & & 1 & & (1) & 1 & & & & \\
\hline 8 & & & & & & & & & & & & & & . & & & & & & & & & & & & & & & & & & & & & \\
\hline 10 & & & & & & & & 1 & & & & & & & & & & & & & & & & & & & & & & (1) & & 2 & & & \\
\hline 11 & & & & & & & & (1) & & & & & & & & 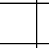 & & & & & & & & & & & & (1) & & & & & & & \\
\hline 12 & & 1 & & & & & & & & 1 & 1 & 1 & 1 & & & (1) & & & & & 1 & 1 & & & & & & & & & & & & & (1) \\
\hline 13 & 2 & 1 & 1 & 1 & 1 & 1 & 1 & 2 & 1 & 1 & 1 & 1 & 1 & 2 & 1 & 2 & 1 & & & 1 & & & (1) & & 1 & 2 & 2 & 1 & & 1 & 1 & & & & 1 \\
\hline 14 & & 1 & 1 & 1 & & 1 & & & & & & 1 & 2 & & & 1 & & & & 2 & 2 & 1 & & & 1 & & & 2 & & & & & 1 & & \\
\hline 15 & 2 & 1 & 2 & 3 & 2 & 3 & 3 & 3 & 1 & 3 & 3 & 3 & 3 & 2 & 3 & 3 & 3 & 3 & & 3 & 2 & 1 & 3 & 3 & 3 & 3 & 3 & 3 & 1 & 1 & & 2 & & 1 & 1 \\
\hline 16 & & & & & & & & & & & & & & & 1 & & & & & & 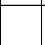 & $t$ & & & & & & 1 & & & 2 & 2 & & & \\
\hline 17 & & & & & & 1 & 2 & 2 & & 2 & 2 & 2 & 1 & & 2 & & 2 & 3 & 1 & & & 1 & & & 1 & 1 & 2 & 3 & 2 & & 2 & 1 & & & 1 \\
\hline 18 & & & & & & & & & & & & & & & & & & 2 & & & & & & & & & & 2 & & & 1 & & & & \\
\hline 19 & & & & & & & & & & & & & & & & & & & & & & & & & & & & & & & & & & & \\
\hline 20 & & & & & & & & & & & 1 & & & & & & & & & & & & & & & & & & & & & & & & \\
\hline 22 & & & 1 & & & & & & & & & & & & & & & & 1 & & & & & & & & & & & & & & & & \\
\hline 23 & & & 1 & & & & & & & & & & & & & & & & & & & & & & & & & & & & & & & & \\
\hline 26 & & & & & & & & & & & & & & & & & & 1 & & & & & & & & & & 1 & (1) & & & 1 & & & \\
\hline 27 & & & & & & & & & & & & & & & & & & & & & & & & & & & & & & & & & & & \\
\hline 28 & & & & & & & & 1 & & & & & & & & & & 2 & & & & & & & & & & 2 & 1 & 1 & & & & & \\
\hline 39 & & & & & & & & & & & & & & & & & & 1 & & & & 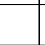 & & & & & & 1 & & & & 1 & & & \\
\hline 41 & & & & & & & & & & & & & & & & & & 1 & & & & & & & & & (1) & 1 & & (1) & & (1) & & & \\
\hline 43 & & & & & & & & & & & & & & & & & + & 1 & & 2 & & & & & & 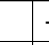 & + & 1 & & & & & & & \\
\hline 44 & & 2 & 1 & 1 & & & 1 & & & 1 & 1 & 2 & 1 & & & (1) & & & 1 & 2 & 2 & 2 & 2 & & 1 & 1 & & & & & & & 2 & & 1 \\
\hline 48 & & & & & & & & 2 & & & & & & & & & 3 & 3 & & & & & & & & 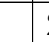 & 2 & 2 & 2 & 3 & 2 & 3 & & & \\
\hline 49 & & & & & & & & & & & & & & & & & & & & & & & & & & & & & & & & + & & & \\
\hline 53 & & & + & & & & & & & & & & & & & & & & & & & & & & & & & & & & & & & & \\
\hline 54 & & & & & & & & & & & & & & & & & & & & & & & & & & & & & & & & & & & \\
\hline 58 & & & & (1) & & & & & & & & & & & & & & & & & & & & & & & & & & & & & & & \\
\hline 60 & 1 & 2 & 1 & 2 & & 1 & & & 1 & 2 & 3 & 2 & 2 & & & & & & 2 & 2 & & 1 & 1 & & & & & & & & & & 2 & 1 & 2 \\
\hline 62 & & & & & & & & & & & & & & & & & & & & & & & 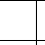 & & & & & & & & & & & & \\
\hline 63 & 2 & 2 & 2 & 2 & 2 & 2 & 1 & 2 & 1 & 2 & 2 & 3 & 3 & 2 & 2 & 2 & (1) & & 2 & 3 & 2 & 2 & 2 & 2 & 1 & 2 & 2 & 2 & & & & & 2 & 2 & 2 \\
\hline 64 & & & 1 & 2 & 1 & 1 & 1 & & & 2 & 1 & 2 & 2 & 2 & 2 & \begin{tabular}{|l|l|}
2 \\
\end{tabular} & & & & 2 & 2 & 1 & 2 & 2 & 2 & 2 & & & & & & & 2 & 2 & 2 \\
\hline 71 & & 2 & 2 & & & & & & & & & & & 2 & & & & & & & & & & & & & & & & & & & & & \\
\hline 73 & 3 & 3 & 3 & 3 & 3 & 3 & 1 & & 3 & 4 & 4 & 4 & 2 & 2 & 2 & 2 & & & 3 & 4 & 4 & 2 & 2 & \begin{tabular}{l|l}
3 & \\
\end{tabular} & 2 & 2 & & & & & & & 3 & 3 & 3 \\
\hline 77 & + & + & 3 & 3 & 2 & 3 & 4 & 3 & & 4 & $\begin{array}{ll}3 \\
\end{array}$ & 3 & 4 & 3 & 4 & $\begin{array}{l}3 \\
\end{array}$ & 3 & 4 & 2 & 4 & 4 & + & 4 & + & 4 & 3 & & 4 & & & & & + & 3 & 3 \\
\hline 78 & 3 & & & 3 & & 4 & 3 & 3 & 3 & & 1 & 3 & 4 & 4 & 4 & \begin{tabular}{|l|}
3 \\
\end{tabular} & 3 & & 3 & 1 & 4 & 4 & 3 & 3 & 4 & 2 & 3 & 3 & & & & & 4 & 2 & 3 \\
\hline 79 & 2 & & & 2 & & (1) & (1) & & & & & & 2 & 1 & & & & & 2 & & 2 & 2 & & & 1 & & & & & & & & 2 & & \\
\hline 80 & & & & 2 & & & & & & & & 1 & 1 & & & 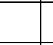 & & & & & 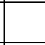 & & & & 1 & (1) & & & & & & & 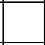 & & (1) \\
\hline 81 & 1 & & & 1 & & & 1 & & & 1 & & 2 & 2 & 2 & 2 & 2 & 1 & & 2 & & 3 & 2 & 2 & & 2 & 2 & 2 & & & & & & 2 & 2 & 1 \\
\hline 82 & 1 & & & & & & & & & & & & & & & & & & & & & & & & & & & & & & & & & & \\
\hline 86 & & & & & & & & & & & & & & & & & & & & & & & & & & & & & & 1 & & & & & \\
\hline 87 & & & & & & & & & & & & & & & & & & & 1 & & & & & & & & & & & & & 2 & & & \\
\hline 88 & & & & & & & & & & & (1) & & & & & & & & & & & & & & & & & & & & & & & & \\
\hline 89 & & & & & & & & & & & & & & & & & & & & & & & & & & & & (1) & & & & 1 & & & \\
\hline 94 & & & & & & & & & & (1) & & & & & & & & & & & & & & & & & & & & & & & & & \\
\hline 99 & & & & & & & & & & & & & & & & & & & & & & & & & & & & & & (1) & & 1 & & & \\
\hline 106 & & & & & & & & & & & & & & & & & & & & & & & & & & & 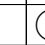 & (1) & & & & & & & \\
\hline 118 & (1) & & & & & & & & & & & & & & & & & & & & & & & & & & & & & & & & & & \\
\hline 119 & & & & & & & & & & & & & & & & & & & & & & & & & & & & & & & & & & & \\
\hline 122 & & & & & & & & & & & & & & & & & & 1 & & & & & & & & & & 1 & & & & & & & \\
\hline
\end{tabular}




\begin{tabular}{|c|c|c|c|c|c|c|c|c|c|c|c|c|c|c|c|c|c|c|c|c|c|c|c|c|c|c|c|c|c|c|c|c|c|}
\hline \multicolumn{34}{|c|}{ Table 9, 1998-2 } \\
\hline & \multicolumn{11}{|c|}{ D } & \multicolumn{13}{|c|}{$E$} & & & & & $\mathrm{~F}$ & & & & \\
\hline & 4 & $5 \mid$ & 6 & 7 & 8 & 9 & & & 12 & 13 & & 1 & 2 & 3 & $4 ! 5$ & $5 \mid$ & 6 & \begin{tabular}{l|l}
7 & $\varepsilon$ \\
\end{tabular} & 89 & \begin{tabular}{l|l}
9 & 1 \\
\end{tabular} & \begin{tabular}{l|l}
0 & 1
\end{tabular} & & 12 & & 1 & 2 & \begin{tabular}{|c|c|c|}
3 &
\end{tabular} & 45 & $5 \mid 6$ & 7 & 8 & 9 & 10 \\
\hline 1 & & & & & & & & & & & & & & & & & & & & & & & & & & & & & & & & & \\
\hline 2 & & & & & & & & & & & & & & & & & & & & & & & & & & & & & & & & & \\
\hline 3 & & & & & & & & & & & & & & & & & & & & (1) & 1) & & & & & & & & & 1 & & & \\
\hline 4 & 1 & 1 [1 & 1 & 1 & 2 & 1 & 2 & 1 & 1 & 1 & 1 & & & & (1) & 1 & $1[5$ & 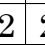 & 22 & 2 & 1 & 1 & & & & & & 1 & 2 & 2 & 2 & 2 & \\
\hline 5 & & & 1 & 1 & 1 & 1 & 1 & & & & & & & & & 1 & & & & & & 1 & & 1 & & & & (1) & 1 & & 1 & (1) & 1 \\
\hline 6 & & & & & & (1) & & & & & 1 & & & & & & & 1 & 1 & $1]$ & 1 & & 1 & & & & & 1. & 1 & 1 & 1 & (1) & 1 \\
\hline 7 & & & (1) & & 1 & 1 & & & (1) & 1 & & & & & & (1) & (1) & 2 & 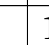 & 10 & 1) & 1 & 1 & & & & & & 1 & & & & 1 \\
\hline 8 & & & & & & & & & & & & & & & & & t. & 1 & & & & & & & & & & & & & & & \\
\hline 10 & & & & & & & & 1 & & (1) & & & & & & . & & & & & & 1 & 1 & & & & & & & & & & 2 \\
\hline 11 & & & & & & 1 & & & & & & & & & & & & & 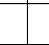 & 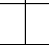 & & & & & & & & & & & & & \\
\hline 12 & & 1 : & 1 & & & 1 & & & & & & & & & (1) & 1 & 1 & 1 & & & & & & & 1 & & $1:$ & 2 & 1 & & & & \\
\hline 13 & & (1) & 2 & 1 & 2 & 2 & 2 & & & 1 & 1 & & & & 7 & 2 & 1 & 25 & 25 & 2 & 1 & 1 & & & & & (1) & 1 & 25 & 2 & 2 & 2 & \\
\hline 14 & & 1. & 1 & 1 & 1 & & & & & & & & & & & & 1 & 1 & 2 & 2 & & & & & & & & 7 & 1 & 1 & & & \\
\hline 15 & \begin{tabular}{|l|}
1 \\
\end{tabular} & 1 & 3 & 3 & 2 & 2 & 3 & 1 & 1 & 1 & & & & & 28 & 2 & 2 & 1. & 1 & & & 2 & 1 & & & & 18 & 2 & 1 & & 1 & 3 & 3 \\
\hline 16 & & & & & & & (1) & & 1 & 1 & 2 & & & & & & 18 & 21 & 12 & 25 & 2 & & & 2 & & & & & 2 & 1 & 2 & & \\
\hline 17 & (1) & 1 & 1 & 1 & 2 & 2 & 2 & 1 & 1 & 2 & & & & & 1 & 1 & 1 & 26 & 2 & 2 & & & & & 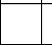 & & & 16 & & 2 & 1 & & \\
\hline 18 & & & & & & & & & & 2 & & & & & & & & & & & & & & & & & & & & & & & \\
\hline 19 & & & & & & & & & & & & & & & & & & & 1 & 1) & & & & & & & & & & 1 & & & \\
\hline 20 & & & & (1) & & & & & & & & & & & & & (1) & 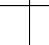 & & & & & & & & & & & & & & & \\
\hline 22 & & 1 & & & & & & & & & & & & & (1) & & & & & & & & & & & & & & & & & & \\
\hline 23 & & & & & & & & & & & & & & & & & & 8 & 2 & & & & & & & & & & & & & & \\
\hline 26 & & & & & & & & & 1 & & 1 & & & & & & & & & 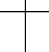 & & & 1 & 1 & & & & & & & & & 1 \\
\hline 27 & & & & & & & & & & & & & & & & & & & 1 & & & & & & & & & & & & & & \\
\hline 28 & & & & & & & 1 & 1 & & & & & & & & & 2 & 22 & 22 & 2 & 1 & & (1) & & & & & & 1 & & 2 & 1 & \\
\hline 39 & & & & & & & & & & & (1) & & & & & & & & & & & & 1 & (1) & & & & & & & & & \\
\hline 41 & & & & & & & & & & & & & & & & & & ( & (1) & & & & (1) & & & & & & & & & & (1) \\
\hline 43 & & & & + & & & & & & & & & & & & & & 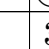 & 33 & 3 & & & & & & & 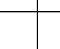 & & & & & & \\
\hline 44 & \begin{tabular}{|l|l|}
1 \\
\end{tabular} & 2 & 1 & 2 & 1 & 1 & & 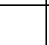 & 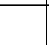 & & & (1) & & 1 & 1 & & & & & & & & & & \begin{tabular}{|l|}
1 \\
\end{tabular} & 2 & 1 & 1 & & & & & \\
\hline 48 & & & & & 1 & 2 & 1 & 2 & 2 & 2 & 3 & & & & & & & & 3 & $3:$ & \begin{tabular}{l|l}
3 &
\end{tabular} & 2 & 2 & 3 & & & (1) & (1) & & & 2 & & 2 \\
\hline 49 & & & & & & & & & & + & + & & & & & & & & & & & & & + & & & & & & & & & \\
\hline 53 & & & & & & & & & & & & & & & & & & & & & & & & & & & & & & & & & + \\
\hline 54 & & & & & & & & & & & & & & & & & & & (1) & & & & & & & & & & & & & 1 & \\
\hline 58 & & & & & & & & & & & & & & & & & & & & & & & & & & & & & & & & & (1) \\
\hline 60 & & & & & & & & & & & & & & & & & (1) & 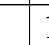 & 1 & & & & & & & & & 1 & 1 & & & & \\
\hline 62 & & & & & & & & & & & & & & & & & & & & & & & & & & & & & & & & & (1) \\
\hline 63 & 2 & 28 & 2 & 2 & 2 & 2 & 2 & & & & & & & & 1 & 2 & 2 & $3:$ & 25 & 2 & & 1 & & & 1 & 1 & 25 & 2 & 2 & 2 & 2 & & \\
\hline 64 & \begin{tabular}{|l|}
2 \\
\end{tabular} & 2 & 2 & 2 & & & & & & & & 2 & & 1 & 2 & 1 & & & & & & & & & 1 & 2 & 22 & 2 & & 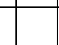 & & & \\
\hline 71 & & 1 & & & & & & & & & & & & & & & & & & & & & & & 1 & 4 & 4 & 5 & & & & & \\
\hline 73 & 3 & $3:$ & 3 & 3 & 3 & & & & & & & 2 & 2 & \begin{tabular}{|l|l|}
2 \\
\end{tabular} & $\begin{array}{l}3 \\
\end{array}$ & \begin{tabular}{|l|}
3 \\
\end{tabular} & \begin{tabular}{l|l}
3 & \\
\end{tabular} & 4 & 1 & & & & & & 3 & \begin{tabular}{l|}
3 \\
\end{tabular} & 3 & 3 & 4 & 2 & & & \\
\hline 77 & + & + & + & + & + & + & 3 & & & & & 3 & 4 & 4 & 3 & 3 & & 36 & 25 & 2 & & & & & + & + & 3 & \begin{tabular}{l|l}
3 & 3
\end{tabular} & & 2 & & & \\
\hline 78 & \begin{tabular}{|l|l|}
3 \\
\end{tabular} & $4:$ & 3 & 3 & 2 & \begin{tabular}{l|}
3 \\
\end{tabular} & 3 & & & & & 3 & 4 & \begin{tabular}{|l|l|}
3 \\
\end{tabular} & 3 & 2 & & & \begin{tabular}{l|l} 
\\
\end{tabular} & & & & & & 3 & 3 & 33 & \begin{tabular}{l|l|}
3 & \\
\end{tabular} & & & & & \\
\hline 79 & & & & & & & & & & & & 2 & & & & & & & & & & & & & 2 & & 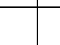 & & & & & & \\
\hline 80 & & 1 & & & & & & & & & & & & & & & & & & & & & & & & 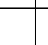 & 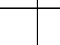 & & & & & & \\
\hline 81 & \begin{tabular}{|l|}
2 \\
\end{tabular} & 2 & 3 & \begin{tabular}{l|l}
3 \\
\end{tabular} & 2 & & & & & & & 2 & 2 & \begin{tabular}{|l|}
2 \\
\end{tabular} & 2 & 2 & & & & & & & & & 2 & 2 & \begin{tabular}{l|l}
3 & 2
\end{tabular} & 2 & & & & & \\
\hline 82 & & & & & & & & & & & & & & & & & & & & & & & & & & & & & & & & & \\
\hline 86 & & & & & & & & & & & & & & & & & & & & & & & & & & & & & & 1 & & 1 & \\
\hline 87 & & & & & & & & & & & 2 & & & & & & & & & & & & 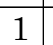 & & & & & & & & & & \\
\hline 88 & & & & & & & & & & & 1 & & & & & & & & & & & & & & & & & & & & & & \\
\hline 89 & & & & & & & & & & & & & & & & & & & & & & & & (1) & & & & & & & & & 1 \\
\hline 94 & & & & & & & & & & & & & & & & & & & & & & & & & & & & & & & & & \\
\hline 99 & & & & & & & & & 1 & 1 & & & & & & & & & & & & & 1 & 1 & & & & & & & & & 1 \\
\hline 106 & & & & & & & & & & & & & & & & & & & & & & & & & & & & & & & & & \\
\hline 118 & & & & & & & & & & & & & & & & & & & & & & & & & & & & & & & & & \\
\hline 119 & & & & & & & & & & & & & & & & & & & & 1 & 1 & & & & & & & & & & 1 & & \\
\hline 122 & & & & & 2 & & & & & & & & & & & & & 22 & 2 & & & & & & & & & & & & & 2 & 2 \\
\hline
\end{tabular}




\begin{tabular}{|c|c|c|c|c|c|c|c|c|c|c|c|c|c|c|c|c|c|c|c|c|c|c|c|c|c|c|c|c|c|c|c|c|c|c|c|}
\hline \multicolumn{36}{|c|}{ Table 9, 1998-3 } \\
\hline & \multicolumn{8}{|c|}{$\mathrm{A}$} & \multicolumn{10}{|c|}{ B } & \multicolumn{14}{|c|}{$\mathrm{C}$} & \multicolumn{3}{|c|}{$\mathrm{D}$} \\
\hline & 1 & 2 & 3 & 4 & 5 & 6 & 7 & 8 & 1 & 2 & 3 & 4 & 5 & 6 & 7 & 8 & 9 & 10 & 1 & 2 & 3 & 4 & 5 & \begin{tabular}{l|l}
6 & \\
\end{tabular} & & $8 \mathrm{~s}$ & \begin{tabular}{l|l}
9 & 1 \\
\end{tabular} & 101 & & & & 14 & & & 3 \\
\hline 123 & & & 1 & & 1 & & & & & 1 & 1 & 1 & 1 & & & & & 2 & & 2 & & & (1) & & & & & 1 & 1 & & & 2 & & & \\
\hline 124 & 1 & 2 & 2 & 2 & 1 & 2 & & & 1 & 2 & 2 & 2 & 2 & 2 & 2 & 2 & 2 & & 1 & 2 & 2 & (1) & 1 & 1 & 2 & 2.1 & 1 & & & & & & & 1 & 2 \\
\hline 125 & & & & & & & & & & & & & & & & & & & & & & & & & & & & & & & & & & & \\
\hline 129 & & & & & & & & & & & & & & & & & & & & & & & & & & & & & & & & (1) & & & \\
\hline 130 & & & & & & & & & & & 1 & & & & & & & & & & & & & & & & & & 1 & 1 & 1 & 1 & & & \\
\hline 131 & & & & & & & & & & & & & & & & & & & & & & & & & & & & & & & & & & & \\
\hline 132 & & & & 1 & 1 & 1 & 1 & 2 & & & & 1 & & & & 2 & 2 & 2 & & 2 & & 1 & & & & $1] 1$ & 1 & 2 & 1 & 2 & & & & & \\
\hline 133 & & & & & & & & & & & & & & & & & & & & & & & & & & & & & & & & & & & \\
\hline 135 & & & & & & & & & & & & & & & & & & & & & & & & & & & & & & & & 1 & & & \\
\hline 141 & & & & & & & & & & & & & & & & & & 1 & & & & & & & (1) & $1] 1$ & 1 & & 1 & 1 & 1 & 2 & & & \\
\hline 142 & & 1 & & & & & & 1 & & & & & 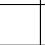 & & & 1 & 1 & 2 & & & & & & & & & 1 & 1 & 1 & 2 & 1 & & & & \\
\hline 143 & & & & & & & & & & & & & & & & & & & & & & & & & & & & & & & & 2 & & & \\
\hline 145 & & & & 1 & 1 & 1 & & & & 1 & 2 & 1 & 1 & (1) & & & & & & & & & & & & & & & & & & 1 & & & \\
\hline 146 & & & & & & & & & & & & & & & & & & & & & & & & & & & & & & & & & & & \\
\hline 156 & & & & & & & & & & & & & & & & & & & & & & & & & & & & & & & & & (1) & & \\
\hline 158 & & 2 & & & & & & & & & & 1 & & & & & & & & & 2 & 1 & 2 & & & & & & & & & & & & \\
\hline 161 & & & & & & & & & & & & & & & & & & & & & & & & & & & & (1) & & & & & & & \\
\hline 176 & & & & & & & & & & & & & & & & & & & & & & & & & & & & & & & & & & & \\
\hline 182 & & & & & & & & & & (1) & & 1 & 1 & (1) & & & & & & & & & & & & & & & & & & & & & \\
\hline 193 & & & & & & & & & & & & & & & & & & & & & & & & & & & & & & & & & & & \\
\hline 197 & & & & & & & & & & & & & & & & & & & & & & & & & & & & & & & & & & & \\
\hline 198 & & & & 1 & & 2 & & & & & & & 2 & 2 & 2 & & & & & 1 & 1 & 2 & 1 & 1 & 1 & & & 1 & & & & & & 1 & 2 \\
\hline 199 & & & & & (1) & 1 & & & & & & 1 & & & & (1) & (1) & 2 & & 1 & & & & 1 & & & 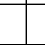 & 1 & & & & & & & (1) \\
\hline 200 & & & & & & (1) & & 1 & & & & & & & 2 & & 1 & & 1 & & & & & & 1 & 1 & 2 & & & & (1) & 2 & & & \\
\hline 203 & & & & & & & & & & & & & & & & & & & & & & & & & & & & 1 & & & & & & & \\
\hline 207 & & (1) & & & & & & & & & & & & & & & & 2 & 1 & & 1 & & & & & & & 1 & 1 & 1 & 1 & 1 & & & \\
\hline 210 & & & & & & & & & & & & & & & & & & & & & & & & & & & & & & & & 1 & & & \\
\hline 211 & & & + & 2 & + & & 3 & 3 & & 2 & 2 & 2 & & + & 3 & 3 & 3 & 3 & & & 3 & + & + & + & 4 & 3 & 3 & 3 & & & & & + & + & + \\
\hline 212 & & 3 & & & 4 & & 1 & 4 & & & & 4 & 4 & & 4 & 3 & & & & 4 & 4 & & 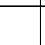 & 2 & 3 & $\begin{array}{l}3 \\
\end{array}$ & 3 & & 4 & 3 & 4 & 3 & & 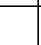 & 3 \\
\hline 213 & & & (1) & & (1) & & & (1) & & & & & & 1 & & & & 2 & & & & & (1) & & & 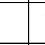 & 1 & 2 & & & & 1 & & & \\
\hline 214 & & & & 1 & & & & & & & & & & & 1 & & 1 & 4 & 2 & & 2 & 1 & 1 & 1 & 1 & 1 & 2 & 4 & 3 & 4 & 4 & 4 & & (1) & \\
\hline 220 & & & (1) & & 1 & & (1) & & & & & & & & & & & 1 & & & & & & & & & & 1 & & & & & & & \\
\hline 221 & & & & & & & & & & & & & & & & & & & & & & & & & & & & & & & & & (1) & & \\
\hline 226 & & & & & & & & & & & & & & & & & & & & & & & & & & & & & & & & (1) & & & \\
\hline 227 & & & & & & 1 & & & & & & & & & & & & & & & & & & & & & & & & & & & & & \\
\hline 229 & 2 & 3 & 2 & 2 & 1 & $\frac{1}{2}$ & 2 & 2 & 2 & 3 & 3 & 3 & 2 & 1 & 2 & 3 & 3 & 3 & 2 & 2 & 2 & 1 & 2 & 2 & 3 & 3 & 3 & 3 & 3 & 3 & 3 & & 2 & 2 & 2 \\
\hline 230 & 1 & (1) & 1 & & & (1) & & 3 & & 2 & 1 & & & (1) & 1 & 2 & 3 & & 1 & & & (1) & & 1 & 1 & 2 & 2 & 2 & 2 & 1 & 1 & (1) & \begin{tabular}{|l|}
1 \\
\end{tabular} & & \\
\hline 232 & + & + & + & + & & & & + & & + & + & + & + & & & + & + & 4 & & + & + & & + & + & + & + & & 3 & & & & & & + & \\
\hline 233 & & & & & & & & & & & & & & & & & & & & & & & & & & & & & & & & & & & \\
\hline 234 & & & & & & & & & & & & & & & & & & & & & & & & & & & & & & & & & & & \\
\hline 235 & & & & & & & & & & & & & & & & & & 1 & & & & & & & & & & 1 & & & & & & & \\
\hline 236 & & & & & & (1) & & & & & & & & & & & & & & & & & & & & & & & & & & & & & \\
\hline
\end{tabular}




\begin{tabular}{|c|c|c|c|c|c|c|c|c|c|c|c|c|c|c|c|c|c|c|c|c|c|c|c|c|c|c|c|c|c|c|c|c|c|c|}
\hline \multicolumn{35}{|c|}{ Table 9, 1998-4 } \\
\hline & \multicolumn{11}{|c|}{$\mathrm{D}$} & \multicolumn{13}{|c|}{$E$} & \multicolumn{10}{|c|}{$\mathrm{F}$} \\
\hline & 4 & 5 & 6 & 7 & 8 & 9 & & & 12 & 13 & & 1 & 2 & 3 & 4 & 5 & 6 & 7 & 8 & 9 & 10 & & & 13 & 1 & 2 & 3 & 4 & 5 & 6 & & 8 & & 10 \\
\hline 123 & & & 1 & & 1 & 1 & (1) & & & & & & & & & 1 & & 1 & 1 & & 1 & & (1) & & & & & & 1 & 2 & 2 & 2 & & 2 \\
\hline 124 & 1 & 1 & 2 & 2 & 2 & 2 & 1 & & & & & & 1 & 1 & 1 & 2 & 2 & 2 & 1 & & & (1) & & & & 1 & 1 & 2 & 1 & 1 & 1 & & & \\
\hline 125 & & & & & & & & & & & & & & & & & & & & & & & & & & & & & & & & & & \\
\hline 129 & & & & & & & & & & & & & & & & & & & & & & & & & & & & & & & & & & \\
\hline 130 & & & & & & & & 1 & 1 & 1 & & & & & & & & & & & (1) & & 1 & 2 & & & & & & & 1 & & $1: 2$ & 2 \\
\hline 131 & & & & & & & & & & & & & & & & & & & & & & & & & & & & & & & & & & 1 \\
\hline 132 & & 2 & 2 & 2 & 2 & 2 & 2 & 2 & 2 & & & & & & & 2 & 2 & 2 & 2 & 2 & 2 & 2 & 1 & 1 & & & & 1 & 2 & 2 & 2 & 2 & 2 & 2 \\
\hline 133 & & & & & & (1) & & & & & & & & & & & & & & & & & & & & & & & & & & & & \\
\hline 135 & & & & & & & & & & & & & & & & & & & & & & & & & & & & & & & & & & \\
\hline 141 & & & (1) & & 1 & 1 & & 1 & & 1 & 1 & & & & & & & (1) & & & & & 1 & 2 & & & & & & 1 & 1 & 2 & 2 & 1 \\
\hline 142 & & 2 & 1 & 1 & & 1 & (1) & 1 & 2 & 1 & 1 & & & & (1) & 1 & 1 & & & 1 & 2 & 1 & 2 & 2 & & & & (1) & 1 & 2 & 2 & 2 & 2 & 2 \\
\hline 143 & & & & & & & & & & & & & & & & & & & & & & & & & & & & & & & & & & \\
\hline 145 & & 1 & & & & & & & (1) & & & & & & & & & & & & & & & 1 & & & & & & \begin{tabular}{|l|} 
\\
\end{tabular} & 3 & & & 2 \\
\hline 146 & & & & & & & & & & & & & & & & & & & & & & & & & & & & & & & & & & (1) \\
\hline 156 & & & & & & & & & & & & & & & & & & & & & & & & & & & & & & & & & & \\
\hline 158 & & 1 & 2 & 1 & & & & & & & & & & & & 2 & & 1 & & & & & & & 2 & & & & & & & & & \\
\hline 161 & & & & & & & & & & & & & & & & (1) & & & 1 & & & & & & & & & & & 1 & & & & \\
\hline 176 & & & & & & & & & & & & & & & & 1 & & & & & & & & & & & & & & & & & & \\
\hline 182 & & & & & & & & & & & & & & & & & & & & & & & & & & & & & & & & & & \\
\hline 193 & & & & & & & & & & & (1) & & & & & 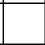 & & & & & & & & & & & & & & & & & & 1 \\
\hline 197 & & & & & & & & & & & & & & & & 1 & & 1 & & & & & & & & & & & & & & & & \\
\hline 198 & 2 & & 1 & & & & & & & & & 2 & 2 & 2 & 2 & & & & & 1 & 2 & & & & & 1 & 2 & & & & & 1 & & \\
\hline 199 & & 1 & & & & 1 & & & & & & & & & (1) & & & & 2 & 1 & & & & & & & & & 1 & 2 & 1 & 2 & & \\
\hline 200 & & & & 1 & 2 & 2 & 2 & 1 & & 1 & & & & & & & 1 & 2 & 1 & 1 & 1 & 1 & 1 & 1 & & & & 2 & 1 & 2 & 2 & 2 & 2 & 2 \\
\hline 203 & & & & & & & & & & & & & & & & & & & & (1) & & & & & & & & & & & & & & \\
\hline 207 & & & (1) & 1 & 1 & 1 & 1 & & 1 & 1 & & & & & & & (1) & 1 & & 1 & & & 1 & 1 & & & & & 1 & 1 & 1 & 2 & 1 & 1 \\
\hline 210 & & & & & & & & & & (1) & 1 & & & & & & & & & & & & & & & & & & & & & & & \\
\hline 211 & + & + & + & + & + & & + & & & & & 3 & + & 3 & 3 & 3 & & 1 & 1 & & 2 & & & & + & + & 3 & & & 1 & 1 & 1 & & \\
\hline 212 & & 4 & & 3 & & & 4 & 4 & 4 & 4 & & & & & & 4 & 4 & & & & & 4 & 4 & 4 & & & & & 4 & \begin{tabular}{|l|}
4 \\
\end{tabular} & 4 & 4 & 4 & 4 \\
\hline 213 & & (1) & 1 & & & & & & (1) & & 1 & & & & & & & & & & & & & & & & & & & & & 1 & & \\
\hline 214 & 1 & 1 & 1 & 1 & 1 & 1 & 3 & 3 & 3 & 4 & 4 & & & & 1 & & 1 & 4 & 4 & 3 & 4 & 3 & 3 & 4 & & 1 & & & & & & & & 3 \\
\hline 220 & & & & (1) & & & & & & & & & & & & (1) & & & & (1) & & & & & & & & & & & & & & \\
\hline 221 & & & & & & & & & & & & & & & & & & & & & & & & & & & & & & & & & & \\
\hline 226 & & & & & & & & & & & & & & & & & & & & & & & & & & & & & & & & & & \\
\hline 227 & & & & & & & & & & & & & (1) & & & & & & & & & & & & & & & & & & & & & \\
\hline 229 & 3 & 2 & 2 & 3 & 3 & 3 & 3 & 3 & 2 & 2 & & 1 & 2 & 2 & 3 & 3 & 3 & 3 & 3 & 3 & 3 & 3 & 1 & 1 & 2 & 2 & 2 & 3 & 3 & 2 & 2 & 2 & 2 & 3 \\
\hline 230 & 1 & 1 & 2 & 2 & 2 & 2 & 3 & 1 & 1 & 1 & & & & & 1 & 1 & 2 & 1 & 2 & 3 & 2 & 2 & & 1 & (1) & 1 & & 1 & 1 & 1 & 2 & 1 & 2 & 1 \\
\hline 232 & & + & + & + & + & + & & & & & + & + & & + & & & + & 1 & 1 & 1 & & & & & + & & + & + & 1 & 1 & & 1 & & \\
\hline 233 & & & & & & & & & (1) & & & & & & & & & & & (1) & & & & & & & & & & & 1 & & & \\
\hline 234 & & & & & & & & & & & & & & & & & & & & & & & & & & & & & & & 1 & & & \\
\hline 235 & & & & & & & & & & & & & & & & & 1 & & & & & & & & & & & (1) & & & & & & \\
\hline 236 & & & & & & & & & & & & & & & & & & & & & & & & & & & & & & & & & & \\
\hline
\end{tabular}




\begin{tabular}{|c|c|c|c|c|c|c|c|c|c|c|c|c|c|c|c|c|c|c|c|c|c|c|c|c|c|c|c|c|c|c|c|c|c|c|c|}
\hline \multicolumn{36}{|c|}{ Table 9, 1999-1 } \\
\hline & \multicolumn{8}{|c|}{$\mathrm{A}$} & & & & & $E$ & & & & & & & & & & & & & C & & & & & & & & D & \\
\hline & 1 & 2 & 3 & 4 & & 6 & 7 & 8 & 1 & 2 & 3 & 4 & 5 & 6 & 7 & 8 & 9 & 10 & 1 & 2 & 3 & 4 & 5 & 6 & & & 9 & 10 & 11 & 12 & & 14 & & & 3 \\
\hline 2 & & & (1) & & 1 & & & & & & & & & & & & & & & & & 1 & & & & & & & & & & & & & \\
\hline 4 & 1 & 1 & 1 & & 1 & 1 & 1 & 1 & & 1 & 1 & 1 & 1 & 1 & & 1 & 1 & 1 & & & & & & 1 & 1 & & 1 & 1 & & 1 & 1 & & & & \\
\hline 5 & & & & & & & & 2 & & & & & & & & 1 & 2 & 1 & & & & & & 1 & 1 & 1 & 1 & 1 & 1 & 1 & 1 & & & & \\
\hline 6 & & & & & & & & & & & & & & & & & & & & & & & & & & ( & 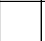 & & & & & 1 & & & \\
\hline 7 & & (1) & & & & & & 1 & & & & & & & & 1 & 2 & 1 & & & & & & & 1 & (1) & 1 & 1 & & & 1 & 1 & & & \\
\hline 8 & & & & & & & & & & & & & & & & & & & & & & & & & & 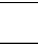 & & & & & (1) & & & & \\
\hline 10 & & & & & & & & & & & & & & & 1 & & & & & & & & & & & & & & 1 & 1 & & 1 & & & \\
\hline 11 & & & & & & & & & & & & & & 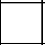 & & & (1) & & & & & & & & 1 & 1 & & (1) & & (1) & & & & & \\
\hline 12 & (1) & & 2 & 1 & 1 & 1 & & 1 & 1 & 1 & 2 & 1 & 1 & 1 & 1 & 1 & 1 & & 1 & (1) & 1 & (1) & & 1 & 1 & 1 & 1 & & & & & & (1) & 1 & 1 \\
\hline 13 & & & (1) & & 1 & 1 & 1 & 2 & (1) & 1 & 1 & 1 & 1 & 1 & 1 & 1 & 1 & & & & 1 & & & 1 & 1 & 2 & 2 & 1 & & 1 & 1 & & & 1 & 1 \\
\hline 14 & & & 1 & & 1 & & & & & & & & & & 1 & & & 1 & 1 & 1 & 1 & & & 1 & 1 & 1 & & & & & & & 1 & 1 & 1 \\
\hline 15 & & 1 & 1 & 2 & 2 & 3 & 3 & 2 & & 1 & 1 & 2 & 1 & 2 & 2 & 3 & 2 & & & 1 & 2 & 2 & 2 & 2 & 2 & 2 & 2 & 3 & & 2 & 2 & & 1 & 1 & 2 \\
\hline 16 & & & & & & & & & & & & & & & & & & 1 & & & & & & & & & & & 1 & 1 & 2 & 2 & & & \\
\hline 17 & & (1) & & & 1 & 1 & 1 & 2 & & 1 & 1 & 1 & 1 & (1) & & 1 & 1 & 1 & 1 & & & & & 1 & 1 & 1 & 1 & 2 & & & & & & & 1 \\
\hline 20 & & & 1 & & & & & & (1) & & & & & & & & & & & & (1) & 1 & & & & & & & & & & & & & \\
\hline 22 & (1) & & & & & & & (1) & 1 & 1 & 1 & & & & & & & & 1 & 1 & 1 & & & & & & & & & & & & & & \\
\hline 26 & & & & & & & & & & & & & & & & & & & & & & & & & & & (1) & & & & (1) & 1 & & & \\
\hline 27 & & & & & & & & & & & & & & & & & & & & & & & & & & & & & & & (1) & & & & \\
\hline 28 & & & & & & & & 1 & & & & & & & & 1 & & 1 & & & & & & & (1) & 1 & 1 & 1 & 1 & \begin{tabular}{|l|} 
\\
\end{tabular} & & & & & \\
\hline 29 & & & & & & & & & & & & & & & & & & & & & & & & & & & & & & & & & & & \\
\hline 39 & & & & & & & & (1) & & & & & & & & & & & & & & & & & & & & & & & & & & & \\
\hline 41 & & & & & & & & 1 & & & & & & & & & & & & & & & & & & & & & & & & & & & \\
\hline 43 & & & & & & & & + & & & & & & & & & & & & & & & & & & 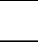 & & & & & & & & & \\
\hline 44 & 2 & 1 & 2 & 2 & 1 & & 1 & & 2 & 1 & 1 & 1 & 1 & & & 1 & 1 & & 2 & 1 & 2 & 1 & 1 & 1 & 1 & 1 & & 1 & & & & & 1 & 1 & 1 \\
\hline 48 & & & & & & & & 1 & & & & & & & & & 1 & 1 & & & & & & & & 1 & 1 & 2 & 2 & 1 & 2 & 2 & & & \\
\hline 49 & & & & & & & & & & & & & & & & & & & & & & & & & & & & L & & & & + & & & \\
\hline 53 & & & & & & & & & & & & & & (1) & & & & & & 1 & & & & & & & & & & 1 & & & & & \\
\hline 54 & (1) & & & & & & & (1) & & & & & & & & & & & & & & & & & & & & & & & & & & & \\
\hline 57 & & & & & & & & & & & & & & & & & & & & & & & & & & & & & & & & & & & \\
\hline 58 & & & & & & & & & & 1 & (1) & 1 & & & & & & & & (1) & & & & & & & & & & & (1) & & & & \\
\hline 59 & & & & & & & & & & & & & & & & & & & & & & & & & & & & & & & & & & & \\
\hline 60 & 1 & 1 & 1 & & 1 & & & & & 1 & 2 & 1 & 1 & & & & & & 1 & 1 & 1 & 1 & & & & & & & & & & & 1 & 1 & 1 \\
\hline 61 & & & & & & & & & & & & & & & & & & & & & & & & & & & & & & & & & & & \\
\hline 62 & & & & & & & & & & & & & 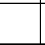 & & & & & & & & 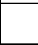 & & & & (1) & & & & & & & & & & \\
\hline 63 & 2 & 2 & 2 & 2 & 2 & 2 & 2 & 2 & 2 & \begin{tabular}{|l|}
2 \\
\end{tabular} & 2 & 2 & 2 & 2 & \begin{tabular}{|l|} 
\\
\end{tabular} & 3 & 2 & & 2 & 2 & 2 & 2 & 2 & 1 & 1 & 2 & 1 & 1 & & & & & 2 & 2 & 2 \\
\hline 64 & & & & 1 & (1) & 1 & 1 & & & & & 1 & 1 & 1 & 1 & 1 & & & & & 2 & 1 & 1 & 2 & 1 & 1 & & & & & & & & 2 & 1 \\
\hline 71 & & 3 & 2 & & 3 & 3 & 2 & & & 1 & & 2 & 2 & & & & & & 2 & 2 & & & & & & & & & & & & & & & \\
\hline 73 & 3 & 2 & 3 & 2 & 2 & 2 & & & 3 & 3 & 3 & 3 & 2 & 1 & 2 & 1 & & & 3 & 3 & 3 & 1 & 2 & 2 & 2 & 1 & & & & & & & 3 & 3 & 3 \\
\hline 77 & 3 & 2 & 3 & 4 & 3 & 4 & 4 & 3 & 1 & 1 & 2 & 3 & 3 & 3 & + & + & + & 4 & 2 & 3 & 3 & 4 & 3 & 3 & 3 & 3 & + & & & & & & & + & 3 \\
\hline 78 & 2 & & & 4 & 4 & 4 & 44 & 3 & 1 & & & 2 & 4 & 44 & \begin{tabular}{|l|}
3 \\
\end{tabular} & & 4 & & 4 & & 4 & 4 & 4 & 3 & 3 & 1 & & 3 & & & & & 3 & 3 & 3 \\
\hline 79 & 1 & & & 1 & 1 & & & & 1 & & & & 1 & & (1) & & & & & & 1 & 1 & 1 & 1 & & & & & & & & & 2 & & \\
\hline 80 & & & & & & & & & & & & & & (1) & & (1) & 1 & & & & (1) & 1 & & 1 & (1) & & & & & & & & & 1 & 1 \\
\hline 81 & & & (1) & 2 & & & 1 & & 1 & & & & 1 & 1 & \begin{tabular}{|l|}
1 \\
\end{tabular} & 1 & & & & & 1 & & 1 & 1 & 1 & 1 & & & & & & & 1 & & 1 \\
\hline 82 & & & & & & & & & 1 & (1) & 1 & 2 & & 3 & & & & & 2 & & & & & & & & & & & & & & & & \\
\hline 86 & & & & & & & & & & & & & & & & & & & & & & & & & & & & & & & & & & & \\
\hline 87 & & & & & & & & & & 1 & 1 & (1) & & & & & & & 1 & 1 & & & & & & & & & & & & 1 & & & \\
\hline 88 & & & & & & 1 & & & & 1 & 2 & & & & & & & & & (1) & & & & & & & & & & & & & & & \\
\hline 89 & & & & & & & & (1) & & & & & & & & & & & & & & & & & & & & & & & & 1 & & & \\
\hline 94 & & & & & & & & & & & & & & & & & & & & & & & & & & & & & & (1) & (1) & & & & \\
\hline 97 & & & & & & & & & & & & & & & & & & & & & & & & & & & & & & & & & & & \\
\hline 99 & & & & & & & & & & & & & & & & & & & & & & & & & & & & & (1) & & 1 & (1) & & & \\
\hline 107 & & & & & & & & (1) & & & & & & & & & & & & & & & & & & & & & (1) & 1 & & & & & \\
\hline 119 & & & & & & & & & & & & & 1 & & & & & & & & & & & & & & & & & & & & & & \\
\hline 122 & & & & & & 1 & & & & & & (1) & & & & & & & & & & & & & & & & & & & & & & & \\
\hline 123 & & & 1 & & & & & 1 & & 1 & 1 & & & & & & & 1 & & (1) & & & 1 & & 2 & 1 & 1 & 1 & 1 & 1 & & 1 & (1) & & \\
\hline
\end{tabular}




\begin{tabular}{|c|c|c|c|c|c|c|c|c|c|c|c|c|c|c|c|c|c|c|c|c|c|c|c|c|c|c|c|c|c|c|c|c|c|c|}
\hline \multicolumn{34}{|c|}{ Table 9, 1999-2 } & \\
\hline & \multicolumn{11}{|c|}{ D } & \multicolumn{13}{|c|}{$\mathrm{E}$} & & & & & 1 & $\mathrm{~F}$ & & & & \\
\hline & 4 & $5[6$ & 6 & & & & & & 12 & 13 & & 1 & 2 & 3 & 4 & 5 & 6 & 7 & 8 & 9 & 10 & & 12 & & & 2 & 3 & 4 & 5 & 6 & & 8 & & 10 \\
\hline 2 & & & & & & & & & & & & & & & & & & & & & & & & 1 & & & & & & & & & (1) & \\
\hline 4 & 1 & 1 & $1]$ & 1 & 1 & 1 & 2 & 1 & 2 & 2 & & & & & 1 & 1 & 1 & 1 & 1 & 2 & 2 & 1 & 1 & 1 & & & (1) & 2 & 2 & 2 & 1 & 2 & & 1 \\
\hline 5 & 1 & & 1 & 1 & 1 & 1 & 1 & 1 & 1 & 1 & & & & & & 1 & 1 & 1 & 1 & 1 & 1 & 1 & 1 & 1 & & & & 1 & 1 & & 1 & 2 & 2 & 1 \\
\hline 6 & & & & & & & & 1 & 1 & (1) & (1) & & & & & & & & (1) & & & & 1 & 1 & & & & & & & & & & \\
\hline 7 & & & 1 [1 1 & 1 & 1 & 1 & 1 & 1 & 1 & 1 & & & & & & 1 & (1) & 1 & & 1 & 1 & 1 & 1 & & & & & 1 & 1 & 1 & 1 & 1 & & (1) \\
\hline 8 & & & & & & & & & & (1) & & & & & & & & & & & & & & & & & & & & & & & & \\
\hline 10 & & & & & & 1 & 1 & 1 & 1 & 1 & (1) & & & & & & & & & (1) & 1 & 2 & 2 & 1 & & & & & & 1 & & 1 & & (1) \\
\hline 11 & & & (1) & & & 1 & 1 & & & 1 & & & & & & & & & & 1 & & & & & & & & & & & & & & \\
\hline 12 & 1 & 2 & $1:$ & 2 & 1 & 1 & 1 & & & & & & & & 1 & 2 & 2 & 1 & 1 & 1 & & & & & & & 1 & 1 & 1 & & 1 & & & \\
\hline 13 & 1 & 1 & 12 & 2 & 2 & 2 & 2 & 1 & 1 & 1 & & & & & 1 & 1 & 1 & 1 & 1 & 1 & 1 & 1 & & 1 & & & (1) & 1 & 1 & 10 & 1 & 1 & (1) & \\
\hline 14 & & & & & & & & & & & & & & & 1 & 1 & 1 & 1 & (1) & & & 2 & & & (1) & & & 1 & & (1) & & & 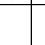 & \\
\hline 15 & \begin{tabular}{|l|l|}
2 \\
\end{tabular} & 25 & 25 & 2 & 2 & 2 & 2 & 2 & 2 & 2 & & 1 & 2 & 2 & 2 & 2 & 2 & 2 & 2 & $\begin{array}{ll}3 \\
\end{array}$ & 2 & 2 & 2 & 2 & 2 & 2 & 2 & 2 & 2 & \begin{tabular}{|l|} 
\\
\end{tabular} & 2 & 2 & \begin{tabular}{|l|}
3 \\
\end{tabular} & 3 \\
\hline 16 & & & & & & & & 1 & 2 & 1 & 1 & & & & & & & & 1 & & 1 & & & 2 & & & & & & & & & & 1 \\
\hline 17 & & 12 & $\begin{array}{ll}2 & 1\end{array}$ & 1 & 1 & 1 & 2 & & & 1 & 1 & & & (1) & & 1 & 1 & 1 & 1 & 1 & 1 & & & & & & & (1) & & (1) & (1) & & 1 & 1 \\
\hline 20 & & & 1) & & & & 1 & & & & & & & & & & 1 & 1 & & & & & & & & & & & & & & & & \\
\hline 22 & & & $1]$ & 1 & & & & & & & & & & & & & & & & & & & & & & & & & & & & & & \\
\hline 26 & & & & & & & & & & & & & & & & & & & & & & & & & & & & & & & & & & \\
\hline 27 & & & & & & & & & & & & & & & & & & & & & & (1) & & & & & & & & & & & & \\
\hline 28 & & & & & 1) ( & (1) & 2 & 1 & 1 & & & & & & & (1) & & & 1 & 1 & 1 & 1 & 1 & 1 & & & & & 1 & \begin{tabular}{|l|} 
\\
\end{tabular} & 1 & 1 & 1 & 1 \\
\hline 29 & & & & & & & & & & & & & & & & & & & & & & 1 & & & & & & & & (1) & & & & \\
\hline 39 & & & & & & & & & & & & & & & & & & & & & & & & & & & 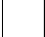 & & & & & & & \\
\hline 41 & & 1 & & & & & & & 1 & & & & & & & (1) & & & (1) & & & 1 & & & & & & & & (1) & & 1 & 1 & \\
\hline 43 & & & & & & & & & & & & & & & & 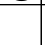 & & 1 & & & & & & & & & (1) & & (1) & & & . & & \\
\hline 44 & \begin{tabular}{|l|l|} 
\\
\end{tabular} & $2:$ & $1]$ & 1 & 1 & 1 & & & & & & 1 & & (1) & 1 & 1 & 1 & 1 & & & & & & & 2 & 1 & 1 & 1 & 1 & & & & & \\
\hline 48 & & & & & & & 2 & 2 & 2 & 2 & 2 & & & & & & & & & 1 & 2 & 2 & 1 & 2 & & & & & & & & 1 & & 1 \\
\hline 49 & & & & & & & & & & + & + & & & & & & & & & & & & & & & & & & & & & & & \\
\hline 53 & & 1 & & & & 1 & 2 & & 3 & & & & & & & + & & & & & & & & (1) & & & & & (1) & & & & & + \\
\hline 54 & & (1) & & & & & & & & & & & & & & & & & & & & & & & & & & & & & & (1) & & \\
\hline 57 & & & & & & & & & & & & & & & & & & & & & & & & & & & & & & (1) & & & & \\
\hline 58 & & (1) & & & & & & & & & & & & & & (1) & & (1) & & & & & & & & & & & & (1) & 1 & (1) & & \\
\hline 59 & & & & & & & & & & & & & & & & & & & & & & & & & & & & & & & & (1) & & \\
\hline 60 & & & & & & & & & & & & & & & & 1 & 1 & & & & & & & & & & & (1) & & & & & & \\
\hline 61 & & & & & & & & & & & & & & & & & & & & & & 1 & (1) & & & & & & & & & & & \\
\hline 62 & & & & & & & & & & & & & & & & & & & & & & & & & & & & & & & & & & \\
\hline 63 & 2 & 2 & 25 & 2 & 2 & 2 & 2 & & & & & 1 & 1 & 1 & 2 & 2 & 2 & 2 & 2 & 2 & & & & & 1 & 1 & 2 & 2 & 2 & 11 & 2 & 1 & & \\
\hline 64 & 1 & 1 & 1 & & & & & & & & & 1 & & & 1 & & & & & & & & 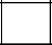 & & 1 & 1 & 1 & & & & & & & \\
\hline 71 & & & & & & & & & & & & & & & & & & & & & & & & & & & & & 1 & & & & & \\
\hline 73 & \begin{tabular}{|l|}
3 \\
\end{tabular} & 2 & 25 & 2 & 2 & 1 & & & & & & 3 & 2 & 3 & 3 & 3 & 3 & 3 & 2 & & & & 1 & & 3 & 3 & 2 & 3 & 3 & 1 & & & & \\
\hline 77 & $\begin{array}{l}3 \\
\end{array}$ & 4 & 35 & 2 & 2 & 2 & 2 & & & & & + & + & 4 & 3 & + & 3 & 2 & & & 2 & & 1 & & 1 & + & 4 & 4 & 44 & & & & & \\
\hline 78 & 4 & 4 & $\begin{array}{ll}36 \\
2\end{array}$ & 2 & 2 & 3 & 3 & & & & & 4 & 3 & 4 & & & & & & & 1 & & & & 3 & 3 & 3 & 3 & & & & & & \\
\hline 79 & & & 1) & & & & & & & & & 2 & & & & & & & & & & & & & 2 & & & & & & & & & \\
\hline 80 & & & & & & & & & & & & 1 & & & & & & & & & & & & & & & & & & & & & & \\
\hline 81 & \begin{tabular}{|l|l}
1 \\
\end{tabular} & 2 & 1 & & & & & & & & & 2 & 1 & & 1 & & & & & & & & & & 1 & 1 & 1 & & & & & & & \\
\hline 82 & & + & & & & & & & & & & & & & & & & & & & & & & & & & & & & & & & & \\
\hline 86 & & & & & & & & & & & & & & & & & & & & & & & 1 & & & & & & & & & 1 & & \\
\hline 87 & & & & & & & & 1 & & 1 & & & & & & & (1) & & & & (1) & & 1 & & & & & 2 & 2 & 1 & & 1 & 1 & 1 \\
\hline 88 & & & & & & & & & & & (1) & & & & & & & (1) & 2 & & & & 1 & (1) & & & & 2 & 1 & \begin{tabular}{|l|} 
\\
\end{tabular} & & 1 & & \\
\hline 89 & & & & & & & & & & & 1 & & & & & & & 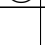 & & 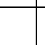 & & & 2 & 1 & & & & 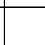 & & & 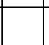 & & & 1 \\
\hline 94 & & & & & & & & & & & & & & & & & & & & (1) & & 1 & 1 & (1) & & & & & & & & 1 & (1) & \\
\hline 97 & & & & & & & & & & & & & & & & & & & & & & & & & & & & & & & (1) & & & \\
\hline 99 & & & & & 1) & & & & (1) & & & & & & & & & (1) & & & & 1 & & 1 & & & & & & & & & & \\
\hline 107 & & & & & & & & & & (1) & & & & & & & (1) & & & & & & & & & & & & & & & & & \\
\hline 119 & & & & & 1 & & & & & & & & & & & & & & & & & & & & & & & & & & 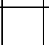 & & & \\
\hline 122 & & 1 & 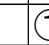 & (1) & 1 & & & 1 & & & & & & & & & 1 & 1 & 2 & 1 & 1 & 1 & & & & & & 1 & 1 & 2 & 2 & 2 & 2 & 1 \\
\hline 123 & & & & & & & & & & & & & & & & & & & & & & & & & & & & & & & & & 1 & 1 \\
\hline
\end{tabular}




\begin{tabular}{|c|c|c|c|c|c|c|c|c|c|c|c|c|c|c|c|c|c|c|c|c|c|c|c|c|c|c|c|c|c|c|c|c|c|c|c|}
\hline \multicolumn{36}{|c|}{ Table 9, 1999-3 } \\
\hline & \multicolumn{8}{|c|}{$\mathrm{A}$} & \multicolumn{10}{|c|}{$\mathrm{B}$} & \multicolumn{14}{|c|}{$\mathrm{C}$} & \multicolumn{3}{|c|}{ D } \\
\hline & 1 & 2 & 3 & 4 & & 6 & 7 & 8 & 1 & 2 & 3 & 4 & 5 & 6 & 7 & 8 & 9 & 10 & 1 & 2 & 3 & 4 & 5 & \begin{tabular}{|c|c|}
6 & \\
\end{tabular} & & $8 \mathrm{~s}$ & & & 11 & 12 & 13 & 14 & & & 3 \\
\hline 124 & & 1 & 1 & 2 & 2 & 1 & & 1 & 1 & 2 & 2 & 1 & 1 & 1 & 1 & 1 & 1 & & 2 & 1 & 1 & 1 & 2 & 1 & 1 & & & & & & & & & & 2 \\
\hline 125 & & & & & & & & & & & & (1) & & & & & & & & (1) & (1) & & & & & & & & & (1) & & & & & \\
\hline 126 & & & & & & & & & & & & & & & & & & & & & & & & & & & & & & & & & & & \\
\hline 129 & & & & & & & & & & & & & & & & & & & & & & & & & & & & & & & & & & & \\
\hline 130 & & & & & & & & 1 & & & & & & & & & & & & & & & & & & & & & 1 & & 1 & 1 & & & \\
\hline 131 & & & & & & & & & & & & & & & & & & & & & & & & & & & & & & & (1) & & & & \\
\hline 132 & & & & & & 1 & 1 & 2 & & & (1) & 1 & & & 1 & 2 & 3 & 2 & & & & & & 1 & (1) & $1]$ & 1 & 2 & 2 & 1 & & & & & \\
\hline 133 & & & & & & & & & & & & & & & & & & & & & & & & & & & & & & & & & & & \\
\hline 138 & & & & & & & & & & & & & & & & & & & & & & & & & & & & & & & & (1) & & & \\
\hline 140 & & & & & & & & & & & & & & & & & & & & & & & & & & & & & & & & & & & \\
\hline 141 & & & & & & & (1) & & & & & 1 & & & & & & & & & & & & & & (1) $(1$ & & & 1 & 1 & (1) & 1 & & & \\
\hline 142 & & (1) & & & & & & 1 & & & 1 & 2 & & 1 & 1 & 1 & 2 & 1 & & (1) & (1) & (1) & & 1 & 1 & $1]$ & 1 & 1 & 1 & 1 & 1 & 2 & & (1) & \\
\hline 143 & & & & & & & & & (1) & & & & & & & & & & & & & & & & & & & & & & & & & & \\
\hline 144 & & & & & & & & & & & & & & & & & & & & & & & & & & & & & & & & & & & \\
\hline 145 & & (1) & 1 & & & & & & & 1 & 1 & 1 & & & & & & (1) & & 1 & & & & & & & & & & & & & & & \\
\hline 146 & & & & & & & & & & & & & & & & & & & & & & & & & & & & & & & (1) & 1 & & & \\
\hline 147 & & & & & & & & & & & & & & & & & & & & & & & & & & & & & & (1) & & & & & \\
\hline 156 & & & & & & & & & & & (1) & & (1) & & & & & & & & & & & & & & & & & & & & & & \\
\hline 158 & & & & & (1) & 1 & & & & & (1) & 1 & & & & & & & & & 2 & & 1 & 1 & & 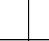 & & & & & & & & & \\
\hline 161 & & & & & & & & & & & & & & & & & & & & & & & & & & & & & & & & & & & \\
\hline 173 & & & & & & & & & & & & & & & & & & & (1) & & & & & & (1) & & & & & & & & & & \\
\hline 176 & & & & & & & & & & & & & & & & & & & & & & & & & & ( & (1) & & & & & & & & \\
\hline 181 & & & & & & & & & & & & & & & & & & & & & & & & & & & & & & & & & & & \\
\hline 182 & & & & & & & & (1) & & (1) & & & & & & & & & & & & & & & & & & & (1) & & & & & & \\
\hline 186 & & & & & & & & & & & & & & & & & & & & & & & & & & & & & & & & & & & \\
\hline 195 & (1) & & & & & & & & & & & & & & & & & & & & & & & & & & & & & & & & & & \\
\hline 197 & & & & & & & & & & & & & & & & & & & & & & & & & & & & & & & & & & & \\
\hline 198 & & & & & & 2 & 1 & & & & & & 2 & 2 & 1 & 1 & 1 & & & & 1 & 2 & \begin{tabular}{|l|}
2 \\
\end{tabular} & 2 & 1 & 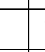 & 1 & & & & & & 2 & & 2 \\
\hline 199 & & & & 1 & (1) & 1 & 1 & 1 & & & & & 1 & 1 & 1 & 1 & 1 & & & & 1 & 1 & 1 & 1 & 1 & 1 & & 1 & & & & & 1 & 1 & 1 \\
\hline 200 & & & & & & (1) & 1 & 1 & & & & & & & 1 & 1 & & 1 & & & & & & & (1) & 1 & 1 & 1 & 1 & 1 & 1 & 2 & & & \\
\hline 206 & & & & & & & & & & & & & & & & & & & & & & & & & & & & & (1) & & & & & & \\
\hline 207 & & & & & & & & 1 & & & (1) & 1 & & & & & 1 & 1 & (1) & & 1 & & 1 & & & 1 & 1 & 1 & 1 & 1 & 1 & 1 & & & (1) \\
\hline 211 & 3 & & + & + & + & + & 3 & 3 & 1 & & 2 & & 3 & 3 & 3 & 3 & 3 & + & + & 3 & 3 & 3 & 3 & 3 & 3 & 3 & 3 & & & & & & + & 3 & 3 \\
\hline 212 & & 4 & 4 & & 4 & 3 & 3 & 4 & 1 & 4 & 4 & 4 & & & 4 & 4 & & & 4 & 4 & & & & 3 & 4 & & 3 & & 4 & 4 & 4 & 4 & & & \\
\hline 213 & & (1) & & & 1 & 1 & 1 & 1 & & & & (1) & & 1 & & & & 1 & & (1) & & & & & & 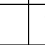 & 1 & & & & 1 & 1 & & & \\
\hline 214 & & & & 1 & & & & 1 & & & & & 1 & & (1) & (1) & 1 & 3 & & (1) & 1 & & 1 & 1 & 1 & 1 & 3 & 3 & 3 & 3 & 3 & 4 & & & \\
\hline 218 & & & & & & & & & & & & & & & & & & & & & & & & & & & & & & 1 & (1) & & & & \\
\hline 220 & & & & & & & (1) & 1 & & & & 1 & & & & & & & (1) & (1) & & & & & & & & & (1) & 1 & 1 & & & & \\
\hline 221 & & & & & & & & & 1 & & & & & & & & & & & & & & & & & & & & & & & & & & \\
\hline 222 & & & & & & & & & 1 & & & & & & & & & & & & & & & & & & & & (1) & & & & & & \\
\hline 226 & & & & & & & & & & & & & & & & & & & & & & & & & & & & & & & & & & & \\
\hline 229 & 2 & 3 & 3 & 2 & 2 & 3 & 2 & 3 & 2 & 2 & 3 & 3 & 2 & 2 & 2 & 3 & 4 & 4 & 2 & 2 & 2 & 1 & 3 & 3 & 4 & 3 & 4 & 4 & 4 & 4 & 1 & 1 & 2 & & 2 \\
\hline 230 & (1) & (1) & & & & & 1 & 3 & & & & & & 1 & 1 & 2 & 2 & 2 & & & & 1 & & 1 & 1 & 2 & 2 & 3 & 2 & 2 & 1 & & & & 1 \\
\hline 232 & + & + & + & + & + & + & & & 2 & 1 & 1 & 1 & & + & + & + & + & & + & + & & + & + & & + & + & & & & & & & & & \\
\hline 233 & & & & & & & & & & (1) & & & & & & & & (1) & & & & & & & & & & (1) & & & (1) & & & & \\
\hline 234 & & & & & & & & (1) & & & & (1) & & & & & & & & & & & & & & & & & & & & & & & \\
\hline 235 & & & & & & & & & & & & & & & & & (1) & (1) & & & & & & & & & & & & & (1) & & & & \\
\hline 237 & & & & & & & & & & & & & & & & & & & & & & & & & & & & & & & & & & & \\
\hline 242 & & & & & & & & (1) & & & & & & & & & & & & & & & & & & & & & & & & & & & \\
\hline
\end{tabular}




\begin{tabular}{|c|c|c|c|c|c|c|c|c|c|c|c|c|c|c|c|c|c|c|c|c|c|c|c|c|c|c|c|c|c|c|c|c|c|c|}
\hline & \multicolumn{11}{|c|}{ D } & \multicolumn{13}{|c|}{$E$} & \multicolumn{10}{|c|}{$F$} \\
\hline & 4 & 5 & 6 & 7 & 8 & 9 & & & & 13 & & & 2 & 3 & 4 & 5 & 6 & \begin{tabular}{|l|}
7 \\
\end{tabular} & 8 & 9 & & & & 13 & 1 & 2 & 3 & & 5 & \begin{tabular}{|l|}
6 \\
\end{tabular} & & & & 10 \\
\hline 124 & 2 & 1 & 1 & 1 & 1 & 1 & & & & & & & & (1) & 2 & 2 & 1 & 1 & 1 & 1 & & & & & & 1 & 1 & 1 & 2 & 1 & 2 & 1 & & \\
\hline 125 & & & & & & & & & & & & & & & & & & & & & & & & & & & & & & & & & & \\
\hline 126 & & & & & & & & (1) & & & & & & & & & & & & & & & & & & & & & & & & & & \\
\hline 129 & & & & & & & & & & & & & & & & & & (1) & & & & & 1 & (1) & & & & & & & & (1) & & \\
\hline 130 & & & & & & & & 1 & & 1 & & & & & & & . & & & 1 & 1 & 2 & 2 & 2 & & & & & 1 & & & 1 & 1 & 1 \\
\hline 131 & & & (1) & & & & & & & & & & & & & & & & & & & 1 & 1 & & & & & & & & & (1) & 1 & 1 \\
\hline 132 & & 2 & 1 & 1 & & 2 & 2 & 2 & 2 & 2 & & & & & & 1 & 1 & 2 & 2 & 2 & 2 & 1 & 2 & 2 & & & & 2 & 2 & 2 & 2 & 2 & 2 & 2 \\
\hline 133 & & & & & & & & & & & & & & & & & & & & & (1) & & & (1) & & & & & & & & & & \\
\hline 138 & & & & & & & & & & & & & & & & & & & & & & & & & & & & & & & & & & \\
\hline 140 & & & & & & & & & & & & & & & & & & & & & & & & (1) & & & & & & & & & & \\
\hline 141 & & 1 & & & 1 & 1 & & 1 & 1 & 1 & 1 & & & & & (1) & & 1 & & 1 & & 1 & 2 & 1 & & & & & 1 & 1 & 1 & 1 & 1 & (1) \\
\hline 142 & & 1 & 1 & 1 & 1 & 1 & 1 & 1 & 1 & 1 & 1 & & & & 1 & 1 & 1 & 2 & 2 & 2 & 2 & 1 & 2 & 2 & & & (1) & 1 & 2 & 2 & 2 & 2 & 1 & 1 \\
\hline 143 & & & & & & & & & & 1 & & & & & & & & & & & & 1 & & & & & & & & & 1 & & & \\
\hline 144 & & & & & & & & & & & & & & & & & & & & & & (1) & & & & & & & & & & & & \\
\hline 145 & & 1 & & & & & & & & & & & & & & & & & & & & & & & & & & (1) & & 1 & & 1 & 1 & \\
\hline 146 & & & & & & & & & & & & & & & & & & & & & & & & & & & & & & & & & & \\
\hline 147 & & & & & & & & & & & & & & & & & & & & & & & & & & & & & & & & & & \\
\hline 156 & & & & & & & & & & & & & & & & & & & & & & & & & & & & & & & & & & \\
\hline 158 & & 1 & 1 & (1) & & (1) & & & & & & & & & & 1 & 1 & & & & & & & & & & & (1) & & & & & & \\
\hline 161 & & & & & & & & (1) & (1) & & & & & & & & & (1) & (1) & & & & & & & & & & (1) & (1) & (1) & 1 & & \\
\hline 173 & & & & & & & & & & & & & & & & & & & & & & & & & & & & & & & & & & \\
\hline 176 & & & & & (1) & & & & & & & & & & & & & & & & (1) & & & & & & & & & & & & & \\
\hline 181 & & & & & & & (1) & & & & & & & & & & & & & & & (1) & (1) & & & & & & & & & & & \\
\hline 182 & & & & & (1) & & & & 1 & (1) & & & & & & & & & & & 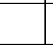 & & (1) & & & & & 1 & 1 & & 1 & & 1 & (1) \\
\hline 186 & & & & & & & & & & & & & & & & & & & & & & & & & (1) & & & & & & & & & \\
\hline 195 & & & & & & & & & & (1) & & & & & & & & & & & & & & & & & & & & & & & & \\
\hline 197 & & & & 1 & & & & & & & & & & & & 1 & (1) & & & & & & & & & & & & & & & & & \\
\hline 198 & 2 & & & & 1 & 1 & & & & & & 2 & 2 & 3 & 1 & & & & & & & & & & 1 & 1 & 2 & & & & & & & \\
\hline 199 & 1 & 1 & 1 & 1 & 1 & & 1 & & & & & 1 & 1 & 1 & 1 & 1 & & & 1 & & & & & & 2 & 1 & 1 & & 1 & & 1 & & 1 & 1 \\
\hline 200 & & & 1 & & 1 & 2 & 1 & 1 & 2 & 1 & 1 & & & & & 1 & 1 & 2 & 2 & 2 & 1 & 2 & 1 & 2 & & & & 1 & 2 & 2 & 2 & 2 & 2 & 1 \\
\hline 206 & & & & & & & & & & & & & & & & & & & & & & & & & & & & & & & & & & \\
\hline 207 & & & & & 1 & 1 & 1 & 1 & 1 & 1 & 1 & & & & & 1 & 1 & 1 & 1 & 1 & 1 & 1 & 1 & 1 & & & & 1 & 1 & 1 & 1 & 1 & 1 & (1) \\
\hline 211 & 3 & 3 & 2 & 2 & 3 & 2 & & & & & & 3 & 3 & 4 & + & & 2 & & & & & & & & 1 & 3 & 3 & 3 & & & & & & \\
\hline 212 & & 4 & 3 & 4 & 3 & & & 4 & 4 & 4 & 3 & & & & & 4 & 4 & 4 & 4 & 4 & 4 & 4 & 4 & 4 & & & & & 4 & 4 & 4 & 4 & 4 & 4 \\
\hline 213 & (1) & & & & 1 & 1 & & 1 & (1) & 1 & 1 & & & & & & & 1 & 1 & 1 & & & 1 & (1) & & & & & & (1) & 1 & 1 & & \\
\hline 214 & 1 & 1 & 1 & 1 & 1 & 1 & 3 & 3 & 3 & 3 & 4 & & & & 1 & 1 & & & 2 & 3 & 2 & & & 3 & & (1) & & (1) & & & & & & \\
\hline 218 & & & & & & & 1 & 1 & (1) & & (1) & & & & & & & & & & (1) & & & (1) & & & & & & & & & & \\
\hline 220 & & & 1 & & (1) & & & 1 & (1) & & & & & & & & & & 1 & 1 & 1 & & (1) & (1) & & & & & & (1) & & & & (1) \\
\hline 221 & & & & & & & & & & (1) & & & & & & & & & & & & & & & & & & & & & & & & \\
\hline 222 & & & & & & & & & & & & & & & & & & & & & & & & & & & & & & & & & & \\
\hline 226 & & & & & & & & & & & (1) & & & & & & & & & & & & & & & & & . & & & & & & \\
\hline 229 & 3 & 3 & 3 & 3 & 3 & 3 & 3 & 3 & 3 & 3 & & 1 & 3 & 3 & 3 & 3 & 3 & 4 & 4 & 4 & 4 & 3 & 2 & & 2 & 2 & 3 & 3 & 3 & 3 & 3 & 2 & 4 & 4 \\
\hline 230 & 1 & 1 & 1 & 2 & 2 & 2 & 2 & 1 & 1 & 1 & & & & 1 & 1 & 1 & 2 & 2 & 3 & 3 & 3 & 1 & (1) & & 1 & 1 & 1 & 1 & (1) & 2 & 1 & 2 & 2 & 2 \\
\hline 232 & + & + & 1 & + & + & & 2 & & & & & & & & + & + & + & + & + & + & + & & & & + & + & + & + & + & & & & & \\
\hline 233 & & & & & & & & (1) & & & & & & & & & & & & (1) & & & & & & & & & 1 & 1 & & & & \\
\hline 234 & & & & & & & & & & & & & & & & & & & & 1 & & & & (1) & & & & & & & & 1 & & \\
\hline 235 & & & & & & & & & & & & & & & & & & & & & & & & & & & (1) & & & (1) & (1) & & & \\
\hline 237 & & & & & & & & & & & & & & & & (1) & & & & & & & & & & & & & & & & & & \\
\hline 242 & & & & & & & & & & & & & & & & & & & & & & & & & & & & & & & & & & \\
\hline
\end{tabular}




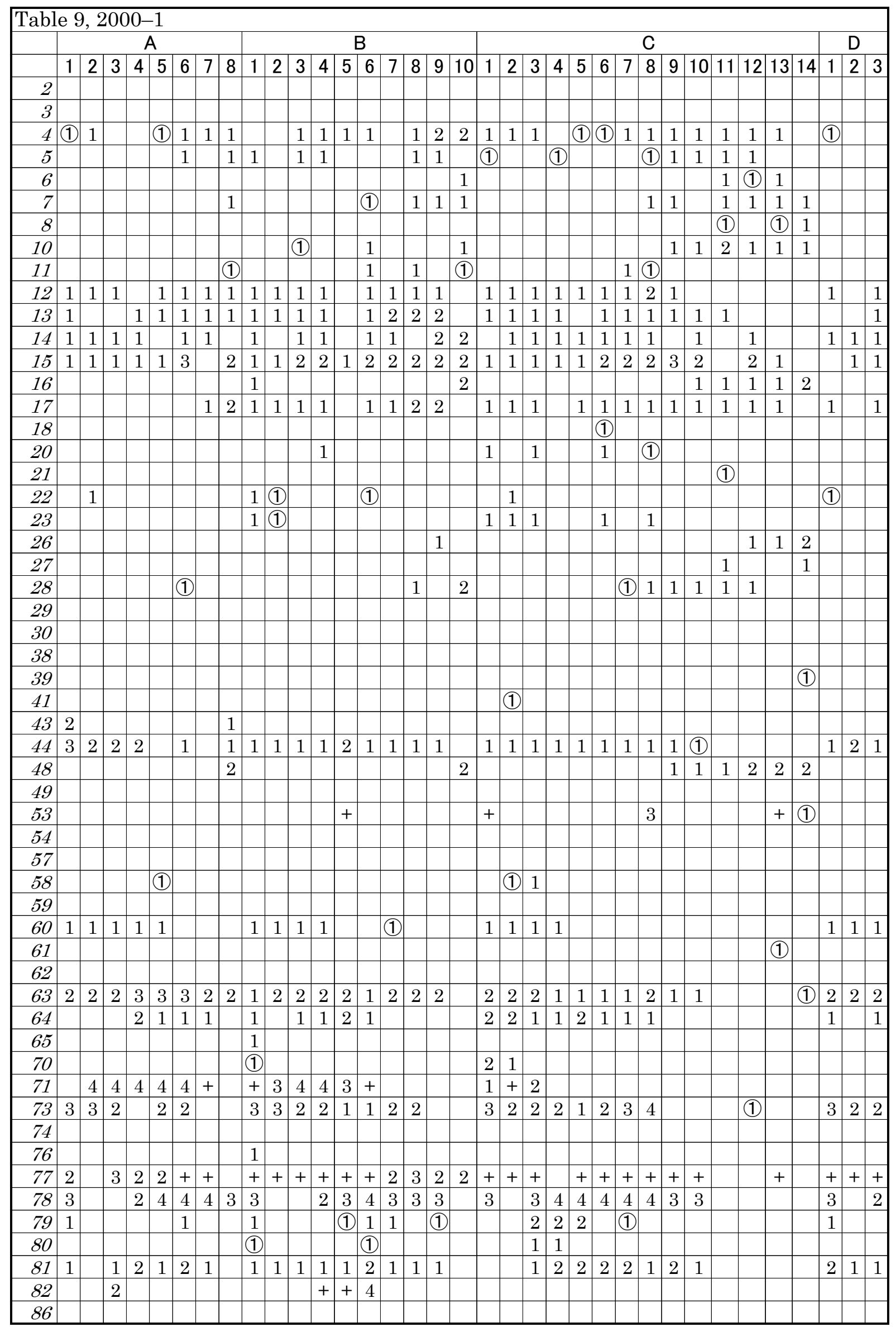




\begin{tabular}{|c|c|c|c|c|c|c|c|c|c|c|c|c|c|c|c|c|c|c|c|c|c|c|c|c|c|c|c|c|c|c|c|}
\hline \multicolumn{32}{|c|}{ Table 9, 2000-2 } \\
\hline & \multicolumn{10}{|c|}{ D } & \multicolumn{12}{|c|}{$E$} & \multicolumn{9}{|c|}{$\mathrm{F}$} \\
\hline & 4 & $5 \mid$ & 67 & \begin{tabular}{l|l}
78 & 8 \\
\end{tabular} & \begin{tabular}{|l|l}
9 \\
\end{tabular} & & & \begin{tabular}{l|l}
12 \\
12
\end{tabular} & \begin{tabular}{|l|l|}
2 & 13 \\
\end{tabular} & & 1 & 2 & 3 & 4 & 5 & & \begin{tabular}{l|l}
7 & $\varepsilon$ \\
\end{tabular} & 89 & \begin{tabular}{l|l}
9 & 10 \\
\end{tabular} & \begin{tabular}{|l|l}
0 & 11
\end{tabular} & 12 & 13 & 1 & 2 & 3 & \begin{tabular}{l|l}
44 & 5 \\
\end{tabular} & \begin{tabular}{l|l}
5 & 6
\end{tabular} & & \begin{tabular}{|l|}
8 \\
\end{tabular} & \begin{tabular}{|l|} 
\\
\end{tabular} & 10 \\
\hline 2 & & & & & & & & & & & & & & & & & & & & & & & & & & & 1 & & & & \\
\hline 3 & & & & & & & & & & (1) & & & & & & & & & & & & & & & & & (1) 1 & & (1) & & (1) \\
\hline 4 & (1) & 1 & $1]$ & 1 & 2 & 2 & 2 & 1 & 1 & & & & & & 1 & 2 . & 15 & 25 & 2 & 1 & 1 & 2 & & (1) & & 1 & \begin{tabular}{l|l}
1 & 1 \\
\end{tabular} & 2 & 1 & 1 & 1 \\
\hline 5 & & & 1 & $1] 1$ & 1 & 2 & 2 & 1 & 1 & & & & & (1) & 1 & 1 & 1 & 1 & 1 & 1 & & & & & & 1 & & 1 & 1 & & 1 \\
\hline 6 & & & & & & & & (1) & & 1 & & & & & & & & & & (1) & (1) & 1 & & & & & & 1 & 1 & 1 & \\
\hline 7 & & & $1]$ & $1] 1$ & 1 & 1 & 1 & 1 & 1 & 1 & & & & & 1 & 1 [1 & $1]$ & 1 & 1 & 1 & 1 & 1 & & & & & \begin{tabular}{l|l}
1 & 1
\end{tabular} & 1 & 1 & 1 & 1 \\
\hline 8 & & & & & & & & & & & & & & & & & & & & & (1) & 1 & & & & & & & & & \\
\hline 10 & & & & 1 & & 1 & 2 & 1 & 1 & 1 & & & & & & & (1) & (1) 2 & 2 & 2 & 1 & 1 & & & & & & & & 1 & \\
\hline 11 & & & & & 1 & 1 & & & & & & & & & & $\mathrm{c}$ & (1) & & & & & & & & & (1) & & & & & \\
\hline 12 & 1 & 1 [1 & 1 [1 & 1 & 1 & & & & & & & & & 1 & 1 & & $1]$ & 1 & & & & & 1 & & & \begin{tabular}{l|l}
1 & 1 \\
\end{tabular} & & 1 & & & \\
\hline 13 & 1 & 1 & $1]$ & 12 & 2 & 2 & 1 & (1) & 1 & & & & & 1 & 1 & 1 & $2:$ & 25 & \begin{tabular}{l|l}
2 & 1
\end{tabular} & 1 & 1 & 1 & & & & \begin{tabular}{l|l}
1 & 1
\end{tabular} & \begin{tabular}{l|l}
1 & 1
\end{tabular} & 2 & 1 & 1 & 2 \\
\hline 14 & 1 & & 1 & & & & & (1) & & & & & & 1 & 1 & & 7 & 1 & & & & & & & & \begin{tabular}{l|l|}
1 & \\
\end{tabular} & & & & 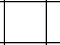 & \\
\hline 15 & 2 & 13 & 25 & 25 & 2 & 3 & 1 & 1 & 1 & & & 1 & 1 & 2 & 2 & 2 & $2:$ & 3 & \begin{tabular}{l|l}
3 & 2
\end{tabular} & 2 & 1 & & 2 & 2 & 1 & 1 & \begin{tabular}{l|l}
2 & 2
\end{tabular} & 3 & 3 & 2 & 2 \\
\hline 16 & & & & & & & 1 & & 1 & 1 & & & & & & & & & & & 1 & 1 & & & & & & & & 1 & 2 \\
\hline 17 & & & $1]$ & 1 & 2 & 2 & 1 & 1 & & 1 & & & & 1 & 2 & 1 & 16 & 25 & 2 & & & & & (1) & & (1) & & & & & \\
\hline 18 & & & & & & & & & & & & & & & & & & & & & & & & & & & & & & & \\
\hline 20 & & & $1]$ & $\begin{array}{lll}1 & 1 \\
\end{array}$ & & 1 & & & & & & & & & 1 & 1 & 10 & 1) & & & & & & & & (1) & & & & & \\
\hline 21 & & & & & & & & & & & & & & & & & & & & & & (1) & & & & & & & & & \\
\hline 22 & & & (1) & & & & & & & & & & & & & & & & & & & & & & & & & & & & \\
\hline 23 & & & & & 1 & & & & & & & & & & & & ( & 1) & & & & & & & & & & & & & \\
\hline 26 & & & & & & & (1) & & & 1 & & & & & & & & & & 1 & 1 & 2 & & & & & & (1) & & 1 & 1 \\
\hline 27 & & & & & & & & & & & & & & & & & & & 1 & & 1 & & & & & & 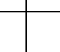 & 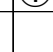 & & & (1) \\
\hline 28 & & & & 1 & 12 & 2 & $\begin{array}{lll}1 & 1\end{array}$ & 1 & 1 & & & & & & & & 2 & 2 & 2 & 1 & & & & & & & \begin{tabular}{l|l}
1 (1) & 1
\end{tabular} & 1 & & \begin{tabular}{l|l|}
1 \\
\end{tabular} & \\
\hline 29 & & & & & & & & & & & & & & & & & & & & 1 & (1) & & & & & & & & & & \\
\hline 30 & & & & & & & & & & & & & & & & & & & & & & & & & & & & & 1 & 1 & \\
\hline 38 & & & & & & & & & & 1 & & & & & & & & & & & 1 & 1 & & & & & & & & & \\
\hline 39 & & & & & & & & & & & & & & & & & & & & & & & & & & & & & & \begin{tabular}{|l|}
1 \\
\end{tabular} & (1) \\
\hline 41 & & & & & & & & & & & & & & & (1) & & & & & & (1) & 1 & & & & & & & \begin{tabular}{|l|l|}
1 \\
\end{tabular} & \begin{tabular}{|l|}
1 \\
\end{tabular} & \\
\hline 43 & & & & & & & & & & & & + & & & 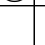 & & & & & & & & & & & & & & 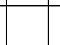 & 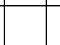 & \\
\hline 44 & \begin{tabular}{|l|}
2 \\
\end{tabular} & 1 & 11 & $\begin{array}{lll}1 & 1 \\
\end{array}$ & 12 & 21 & & & & & & & 1 & 1 & 2 & 1 & $1:$ & $\begin{array}{lll}2 & 1 \\
2\end{array}$ & 1 & & & 1 & & 2 & 2 & \begin{tabular}{l|l}
2 & 1 \\
\end{tabular} & 1 & 1 & & 1 & \\
\hline 48 & & & & 1 & & 3 & $\begin{array}{lll}3 & 2 \\
\end{array}$ & 2 & 2 & 1 & & & & & & & 1 & 12 & 2 & 2 & 2 & 2 & & & & & & 1 & 2 & 1 & 2 \\
\hline 49 & & & & & & & & & & 1 & & & & & & & & & & & & & & & & & & & & & \\
\hline 53 & & 2 & & & & + & & & + & & & & & & & & & & \begin{tabular}{l|l}
2 & 2
\end{tabular} & + & + & & & & & & & & & & \\
\hline 54 & & & & & & & & & & & & & & & & & & & & & & & & & & & 1 & (1) & 1 & & \\
\hline 57 & & & & & & & & & & & & & & & & & & & & & & & & & & & & & & (1) & \\
\hline 58 & & (1) & & & & & & & & & & & & & & & & & & & & & & & & & & 1 & 1 & & \\
\hline 59 & & & & & & & & & & & & & & & (1) & & & & & & & & & & & & & (1) & & & \\
\hline 60 & & 1 & & (1) 1 & & & & & & & & & & & 1 & & ( & (1) & (1) & & & & & & & 1 & (1) & 5 & 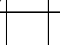 & 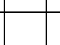 & \\
\hline 61 & & & & & & & & & & & & & & & & & & & & & 1 & 1 & & & & & & 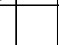 & \begin{tabular}{|l|l|}
1 \\
\end{tabular} & \begin{tabular}{|l|}
1 \\
\end{tabular} & 2 \\
\hline 62 & & & & & & & & & & & & & & & & & & & & (1) & (1) & & & & & & & & 1 & & 1 \\
\hline 63 & & 25 & 21 & 15 & 2 & $2 \mid 1$ & & & & & 1 & & & 2 & 2 & 2 & $1:$ & $\begin{array}{ll}3 \\
3\end{array}$ & \begin{tabular}{l|l}
1 & $(1)$
\end{tabular} & & & & 3 & 2 & 1 & $\begin{array}{ll}2 & 2\end{array}$ & \begin{tabular}{l|l}
2 & 1 \\
\end{tabular} & 1 & 1 & 1 & \\
\hline 64 & \begin{tabular}{|l|}
1 \\
\end{tabular} & 1 & 1 & 11 & & & & & & & 1 & 1 & & 1 & 1 & & & & & & & & 2 & 2 & & \begin{tabular}{l|l}
1 \\
\end{tabular} & (1) & & & & \\
\hline 65 & & & & & & & & & & & & & & & & & & & & & & & & & & & & & & & \\
\hline 70 & & & & & & & & & & & & & & & & & & & & & & & & & & & & & & & \\
\hline 71 & & & & & & & & & & & & & & & & + & & & & & & & & & & 1 & 1 & & & & \\
\hline 73 & 2 & $2:$ & 36 & 25 & 2 & & & & & & 4 & 2 & 1 & 3 & 2 & 2 & 3 & 1 & & & & & 2 & 3 & 3 & \begin{tabular}{l|l}
3 & 1 \\
\end{tabular} & 1 & & & & \\
\hline 74 & & & & & & & & & & & & & & & & & & & & & & & & & & & & (1) & & & \\
\hline 76 & & & & & & & & & & & & & & & & & & & & & & & 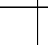 & 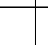 & 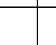 & 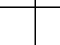 & & 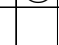 & & & \\
\hline 77 & + & 4 & +1 & +1 & $1+$ & + & & & & & + & \begin{tabular}{|l|}
3 \\
\end{tabular} & 3 & & + & +1 & +6 & 21 & + & & & & + & + & + & 4 & + & \begin{tabular}{|l|}
3 \\
\end{tabular} & 2 & & \\
\hline 78 & 4 & $3:$ & 3 & & 1 & 13 & 3 & & & & 4 & 3 & 4 & 3 & & (1) & & & & & & & 4 & & 2 & \begin{tabular}{l|l}
3 \\
\end{tabular} & & & & & \\
\hline 79 & & & & & & & & & & & 1 & 1 & (1) & & & & & & & & & & 1 & & & & & & & & \\
\hline 80 & & & & & & & & & & & (1) & & & & & & & & & & & & & & & & & & & & \\
\hline 81 & \begin{tabular}{|l|}
2 \\
\end{tabular} & 25 & 2 & & & & & & & & 1 & \begin{tabular}{|l|}
1 \\
\end{tabular} & \begin{tabular}{|l|}
2 \\
\end{tabular} & 1 & & 1 & & & & & & & 2 & 2 & 2 & 2 & & & & & \\
\hline 82 & & & & & & & & & & & & & & & + & + & & & & & & & & & & & & & & 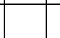 & \\
\hline 86 & & & & & & & & & & 1 & & & & & & & & & & (1) & & & & & & & & & & (1) & \\
\hline
\end{tabular}




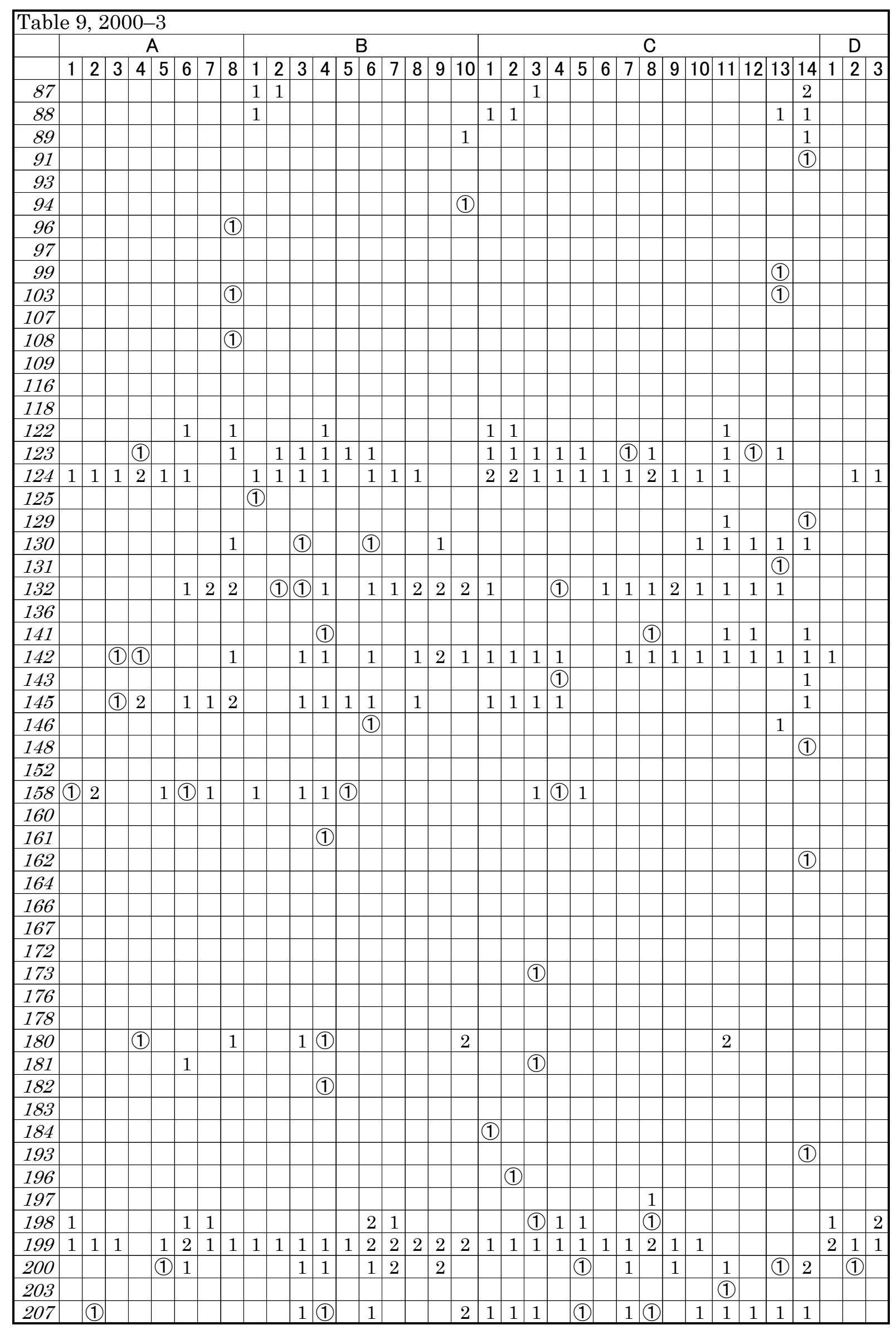




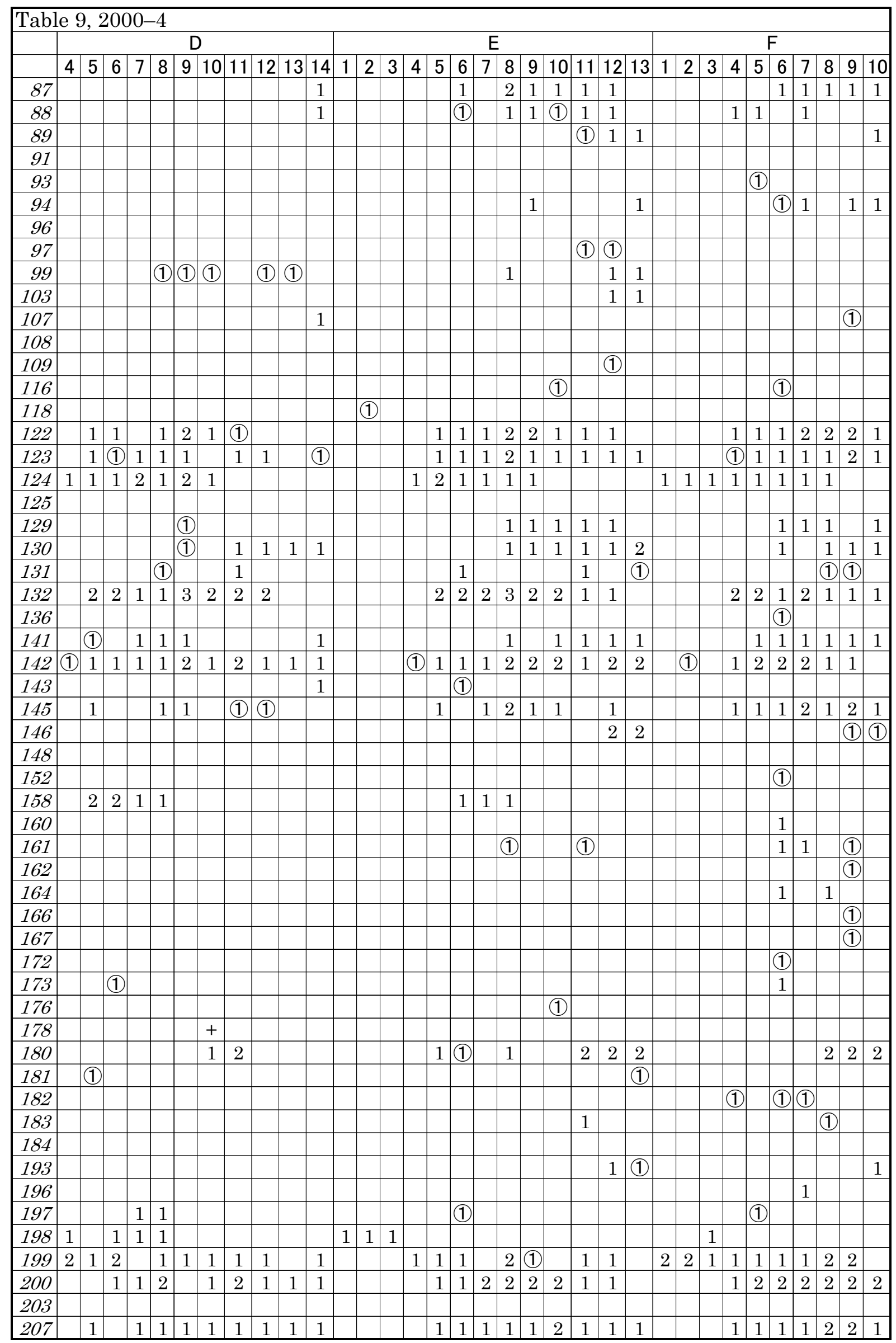




\begin{tabular}{|c|c|c|c|c|c|c|c|c|c|c|c|c|c|c|c|c|c|c|c|c|c|c|c|c|c|c|c|c|c|c|c|c|c|c|c|c|c|}
\hline \multicolumn{38}{|c|}{ Table 9, 2000-5 } \\
\hline & \multicolumn{8}{|c|}{$\mathrm{A}$} & & \multicolumn{10}{|c|}{$B$} & \multicolumn{15}{|c|}{$\mathrm{C}$} & \multicolumn{3}{|c|}{$\mathrm{D}$} \\
\hline & 1 & 2 & 3 & 4 & 5 & 6 & 7 & 8 & & 1 & 2 & 3 & 4 & 5 & 6 & 7 & 8 & 9 & 10 & 1 & 2 & 3 & 4 & 5 & 6 & 7 & 7 & 8 & 9 & 10 & 11 & 12 & 13 & 14 & 1 & 2 & 3 \\
\hline 209 & & & & & & & & & & & & & & & & & & & & & & & & & & & & & & & & & & & & & \\
\hline 211 & + & & 3 & 3 & 2 & + & 3 & 3 & & t & + & + & + & 3 & 3 & 2 & 2 & 3 & & 1 & 2 & + & + & + & + & -1 & + & + & 3 & + & & & & & 3 & 3 & 3 \\
\hline 212 & & 4 & 4 & 4 & 4 & 4 & 3 & 4 & & & 4 & 4 & 4 & 4 & 4 & 4 & 3 & 2 & & 4 & 4 & 4 & & & 3 & 3 & 4 & 4 & 4 & 4 & 4 & 4 & 4 & & & & 4 \\
\hline 213 & & & & & & (1) & & & & & & (1) & & (1) & 1 & & & & & & & 1 & & 1 & & & & & & & 1 & 1 & 1 & 1 & & & \\
\hline 214 & & & 1 & & & & & & ( & 1) & 1 & & & & 1 & (1) & (1) & & & 1 & 1 & 1 & & 1 & 1 & 1 & 1 & 1 & 2 & 2 & 3 & 3 & 3 & 4 & & 1 & 1 \\
\hline 216 & & & & & & & & & & & & & & & & & (1) & 1 & 1 & & & & & & & . & 1 & & & & & (1) & & & & & \\
\hline 220 & & & & & & & & & & & (1) & & & & & & & & (1) & 1 & 1 & & & & & & & & & & & & & & & & \\
\hline 221 & & & & & & & & & & & & & & & & (1) & & & & (1) & & & & 2 & 1 & & & & & & & & & & & & \\
\hline 222 & & & & & & & & & & & & & & & & & & & & & & & & & & & & & & & (1) & & & & & & \\
\hline 226 & & & & & & & & & & & & & & & & & & & & & & & & & & & & & & & & & & (1) & & & \\
\hline 227 & & & & & & & & & & & & & & & & & & & & & & & & & & & & & & & & & & & & & \\
\hline 229 & 3 & 3 & 3 & 2 & 2 & 3 & 3 & 3 & & 2 & 3 & 3 & 3 & 3 & 3 & 2 & 3 & 3 & 3 & 2 & 2 & 2 & 2 & 2 & 3 & 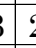 & 2 & 4 & 4 & 4 & 3 & 3 & 2 & & 2 & 2 & 2 \\
\hline 230 & & 1 & & & (1) & & 1 & 3 & & & & & & & 1 & 1 & 1 & 2 & 2 & 1 & 1 & & (1) & 1 & 1 & 1 & 1 & 1 & 3 & 3 & 1 & 2 & & & & & 1 \\
\hline 231 & & & & & & & & & & & & & & & & & & & & & & & & & & & & & & & & & & & & & \\
\hline 232 & + & + & + & + & + & & + & + & & & & & & + & + & + & + & + & & + & + & & & & + & $-1-$ & + & + & + & + & & + & + & & & + & \\
\hline 233 & & & & & & & & & & & & & & & & & & & 1 & & & & & & & & & & & & & & & & & & \\
\hline 234 & & & & & & & (1) & & & & & 1 & (1) & & & & & & & (1) & & & 1 & & & & & & & & & & & & & & \\
\hline 235 & & & & & & & & & & & & & & & & & & 1 & & & (1) & & & & & & & (1) & & & & & & & & & \\
\hline 242 & & & & & & & & & & & & & & & & & & & & & & & & & & & & & & & & & & & & & \\
\hline
\end{tabular}

\begin{tabular}{|c|c|c|c|c|c|c|c|c|c|c|c|c|c|c|c|c|c|c|c|c|c|c|c|c|c|c|c|c|c|c|c|c|c|c|c|c|c|}
\hline \multicolumn{38}{|c|}{ Table 9, 2000-6 } \\
\hline & \multicolumn{12}{|c|}{ D } & \multicolumn{14}{|c|}{$E$} & \multicolumn{11}{|c|}{$F$} \\
\hline & 4 & 5 & 6 & 7 & 8 & 9 & 1 & 1 & & 12 & 13 & 14 & 1 & 2 & 3 & 4 & 5 & 6 & & 7 & 8 & 9 & 10 & 11 & 12 & 13 & 1 & 2 & 3 & 2 & & 5 & 6 & 7 & 8 & 9 & 10 \\
\hline 209 & & & & & & & & & & & & 1 & & & & & & & & & & & & & & & & & & & & & & & & & \\
\hline 211 & 3 & 3 & 3 & 3 & + & 2 & & & & & & & 3 & 3 & 4 & 4 & 3 & + & & + & & & & & & & 3 & 3 & 4 & & & + & & + & & & \\
\hline 212 & & 4 & 3 & 4 & 3 & & & ( & & 4 & 4 & 4 & & & & & 4 & 4 & & 4 & 4 & 3 & 4 & 4 & 4 & 4 & & & & & & 4 & 4 & 4 & 4 & 4 & 4 \\
\hline 213 & & (1) & (1) & & 1 & 1 & 1 & 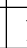 & & 1 & 1 & 1 & & & & & & 1 & & 1 & 2 & 2 & 2 & 1 & 1 & 1 & & & & (1 & & 1 & 1 & 1 & 1 & & 1 \\
\hline 214 & 1 & 1 & 1 & 1 & 1 & & 3 & 2 & & 4 & 4 & 3 & & & & 1 & 1 & & ( & 1) & 2 & 2 & 2 & 1 & 1 & 3 & & (1) & & (1 & & & & 1 & 1 & & 2 \\
\hline 216 & & & & & & & & & & & (1) & & & & & & & & & & & & & & & & & & & & & & & & & & (1) \\
\hline 220 & & & & & & & & & & & & & & & & & & & & & 1 & & & & & 1 & & & & & & (1) & & (1) & 1 & 1 & 1 \\
\hline 221 & & & & & & & & & & & & & & & & 1 & & (1 & & & & & & & & & & & & & & & & & & & \\
\hline 222 & & & & & & & & & & & & & & & & & & & & & & & (1) & & & & & & & & & & (1) & & & & \\
\hline 226 & & & & & & & & & & & & (1) & & & & & & & & & & & & & & (1) & & & & & & & & & & & \\
\hline 227 & & & & & & & & & & & & & & & & & & & & & (1) & & & & & & & & & & & & & & & & \\
\hline 229 & 2 & 3 & 3 & 3 & 3 & 4 & 4 & se & & 3 & 3 & & 2 & 2 & 3 & 3 & 4 & 3 & & 4 & 3 & 4 & 4 & 3 & 3 & 2 & 3 & 3 & 3 & 3 & & 3 & 2 & 2 & 2 & 3 & 4 \\
\hline 230 & 1 & 1 & 2 & 2 & 3 & 3 & 2 & 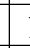 & & 1 & 1 & & & (1) & & 1 & 1 & 2 & & 3 & 3 & 3 & 3 & 1 & 2 & 1 & & 1 & 1 & 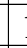 & & 1) & 1 & 2 & 2 & 2 & 2 \\
\hline 231 & & & & & & & & & & & & & & & & & & & & & (1) & & & & & & & & & & & & & & & & \\
\hline 232 & + & & + & + & + & + & + & & & & & & + & & & + & + & + & & + & + & + & + & & & & + & & + & $t$ & & + & & & & & \\
\hline 233 & & (1) & & & & & & & & & & & & & & & & & & & & & (1) & & & 1 & & & & & & & & & & & \\
\hline 234 & & & & & 1 & & 1 & & & & & & & & & & & & & & 1 & & 1 & (1) & & & & & & & & & & 1 & (1) & & \\
\hline 235 & & & 1 & 1 & & & & & & & & & & & & & & & & & & & (1) & & & & & & & & & & & & & & \\
\hline 242 & & & & & & & & & & & & & & & & & & & & & & & & & & 1 & & & & & & & & & & & \\
\hline
\end{tabular}




\begin{tabular}{|c|c|c|c|c|c|c|c|c|c|c|c|c|c|c|c|c|c|c|c|c|c|c|c|c|c|c|c|c|c|c|c|c|c|c|c|}
\hline \multicolumn{36}{|c|}{ Table 9, 2001-1 } \\
\hline & \multicolumn{8}{|c|}{$\mathrm{A}$} & & & & & $\mathrm{b}$ & $B$ & & & & & & & & & & & & C & & & & & & & & D & \\
\hline & 1 & 2 & 3 & 4 & & 6 & 7 & 8 & 1 & 2 & 3 & 4 & 5 & 6 & 7 & 8 & 9 & 10 & 1 & 2 & 3 & 4 & 5 & 6 & & 8 & 9 & 10 & 11 & 12 & & 14 & & & 3 \\
\hline 2 & & & & & & & & & & (1) & & & & & & & & & & & & & & & & & & & & & & & & & \\
\hline 4 & 1 & 1 & & & 1 & (1) & & 1 & & & 1 & 1 & & (1) & 1 & 1 & 1 & 1 & & & & & 1 & & 1 & 2 & 2 & 1 & 1 & 1 & 1 & & & (1) & 1 \\
\hline 5 & & & & & & 1 & & 2 & & & & & & & & 1 & 2 & 1 & & & & & & & & (1) & 1 & 1 & & (1) & & (1) & & & \\
\hline 6 & & & & & & & & & & & & & & & & & & & & & & & & & & . & & & 1 & (1) & 1 & & & & \\
\hline 7 & & & & & & & & 1 & & & & & & & & 1 & 1 & 1 & & & & & & & & 1 & & 1 & 1 & (1) & 1 & 1 & & & \\
\hline 8 & & & & & & & & & & & & & & & & & & & & & & & & & & & & & 1 & (1) & & & & & \\
\hline 10 & & & & & & & & 1 & & & & & & 1 & & 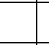 & & 2 & & & & & & & & & 1 & 1 & 1 & 2 & 1 & & & & \\
\hline 11 & & & & & & & & (1) & & & & & & & & & & & & & & & & & & & & & & & & & & & \\
\hline 12 & 1 & (1) & (1) & & 1 & & & 1 & & & & 1 & & & & (1) & (1) & & (1) & & & & 1 & & & 1 & 1 & & & & & & & & \\
\hline 13 & & 1 & & & 1 & 1 & & 2 & & 1 & 1 & 1 & & 1 & & 1 & 1 & & & 1 & (1) & & 1 & & & 1 & 2 & & & 1 & & & & 1 & \\
\hline 14 & & & 1 & (1) & & & 1 & & & 1 & 1 & (1) & (1) & & 1 & 1 & & 1 & (1) & 1 & 1 & 1 & & & & 1 & & & & (1) & & & & & \\
\hline 15 & 1 & 1 & (1) & & 1 & 1 & 3 & 3 & & & 1 & 1 & 1 & 2 & 2 & 2 & 2 & 1 & 1 & 1 & 1 & (1) & 1 & & 1 & 2 & 3 & 2 & 1 & 1 & & & 1 & 1 & 1 \\
\hline 16 & & & & & & & & & & & & & & & & & 1 & 1 & & & & & & & & & & (1) & 1 & 1 & 1 & 2 & & & \\
\hline 17 & & 1 & & & & & & 1 & & (1) & & (1) & & & & 1 & 1 & & & 1 & & & 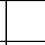 & & 1 & (1) & 1 & 2 & 1 & 1 & 1 & 1 & & & (1) \\
\hline 18 & & & & (1) & & & & (1) & & & & & & . & & & (1) & & & & & & . & & & & & & (1) & & 1 & & & & \\
\hline 19 & & & & & & & & & & & & & & & & & & & & & & & & & & & & & & & & & & & \\
\hline 20 & & & & & & & & & & & & & & & & & & & & & & & & & & & & & & & & & & & (1) \\
\hline 21 & & & & & & & & & & & & & & & & & & & & & & & & & & & & & & & & & & & \\
\hline 22 & & & & & & & & & & 1 & 1 & & & & & & & & (1) & 1 & 1 & & & & & & & & & & & & & & \\
\hline 23 & & & (1) & 1 & & & & & & 1 & (1) & & & & & & & & & & 1 & 1 & & & & & & & & & & & & & \\
\hline 26 & & & & & & & & & & & & & & & & & & & & & & & & & & & & & & (1) & & & & & \\
\hline 27 & & & & & & & & & & & & & & & & & & & & & & & & & & & & & & & & & & & \\
\hline 28 & & & & & & & & 1 & & & & & & & & (1) & 1 & 1 & & & & (1) & & & & & 1 & 2 & 1 & 1 & & & & & (1) \\
\hline 29 & & & & & & & & & & & & & & & & & & & & & & & & & & & & & & & & & & & \\
\hline 41 & & & & & & & & & & & & & & & & & & & & & & & & & & & & & (1) & & (1) & & & & \\
\hline 43 & & & & & & & & & & & & & & & & & & & & & & & & & & & + & & & & & & & 2 & \\
\hline 44 & 1 & 1 & 1 & 1 & 1 & 1 & 1 & 1 & & 1 & 1 & 1 & 1 & & & 1 & & & & 1 & 2 & 2 & 1 & & & & 1 & & & & & & & & (1) \\
\hline 48 & & & & & & & & 2 & & & & & & & & & & 1 & & & & & & & 1 & & & 1 & 2 & 2 & 2 & 2 & & & \\
\hline 49 & & & & & & & & & & & & & & & & & & & & & & & & & & & & & & & & + & & & \\
\hline 53 & & & & & & & & (1) & & & & & & & & & & & & & & & & & & & & & & & & & & & \\
\hline 54 & & & & & & & & & & & & & & & & & & & & & & & & & & & & & & & & & & & \\
\hline 58 & & & & & & & & & & & & & & & & & & & & & & & & & & & & & & & & & & & \\
\hline 60 & 1 & 2 & 1 & 1 & & & & & 1 & 1 & 1 & 2 & & & & & & (1) & 1 & 1 & 2 & 2 & & & & & & & & & & & 1 & 1 & 1 \\
\hline 61 & & & & & & & & & & & & & & & & & & & & & & & & & & & & & & (1) & & & & & \\
\hline 62 & & & & & & & & & & & & & & & & & & & & & & & & & & & & & & & & & & & \\
\hline 63 & 2 & 2 & 2 & 3 & 3 & 3 & 2 & 2 & 1 & 3 & 2 & 3 & 3 & 2 & 1 & 2 & 2 & & 2 & 2 & 3 & 3 & 2 & 2 & 1 & 2 & 2 & 1 & & & & & 2 & 2 & 1 \\
\hline 64 & 1 & (1) & 1 & 1 & 1 & 1 & 1 & (1) & & & & 2 & 2 & 1 & 1 & 2 & & & 2 & & 2 & 2 & 2 & 2 & 2 & 2 & & & & & & & & 1 & 1 \\
\hline 65 & & & & & & & & & & & & & & & & & & & & & & & & & & & & & & & & & (1) & & \\
\hline 70 & & & & & & & & & & & & & & & & & & & (1) & 2 & & & & & & & & & & & & & (1) & & \\
\hline 71 & & + & + & + & 4 & & + & & 1 & 4 & 4 & 4 & + & 4 & & & & & 3 & 4 & 3 & 3 & & & & . & & & & & & & & & \\
\hline 73 & 3 & 2 & 2 & 2 & 2 & 1 & 1 & 1 & 1 & \begin{tabular}{|l|}
3 \\
\end{tabular} & 3 & 3 & 2 & 1 & 2 & 3 & & & 3 & 3 & 2 & 2 & 3 & 2 & 2 & 2 & 1 & & & & & & 2 & 3 & 3 \\
\hline 74 & & & & & & & & & & & & & & $\square$ & & & & & & & & 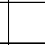 & & & & & 1 & & & & & & & & \\
\hline 77 & + & + & + & + & 4 & 3 & 4 & + & & + & + & + & + & 4 & \begin{tabular}{|l|}
4 \\
\end{tabular} & \begin{tabular}{|l|}
3 \\
\end{tabular} & 3 & + & + & + & + & + & + & + & + & + & + & + & & & & & + & + & + \\
\hline 78 & 3 & & 2 & 3 & 4 & 3 & 4 & 3 & & & 2 & 2 & 4 & 4 & 44 & 3 & 3 & & 3 & & 3 & 4 & 4 & 3 & 3 & 3 & 3 & 3 & & & & & 2 & 3 & 3 \\
\hline 79 & & & & 1 & 2 & 1 & & & & & & & 2 & 2 & & (1) & & & 2 & & 2 & 2 & 1 & 1 & 1 & & & & & & & & 2 & & (1) \\
\hline 80 & & & & & & & & & & & & & & (1) & & & & & & & & & & & & & & & & & & & & & \\
\hline 81 & 2 & 2 & 1 & 2 & 1 & 1 & & & & 1 & 1 & 1 & 2 & 1 & 1 & 2 & 2 & & 3 & 1 & 3 & 3 & 2 & 2 & 2 & & 1 & 1 & & & & & & 2 & 2 \\
\hline 82 & & & & & & & & & & + & & & & + & & & & & & & & & & & & & & & & & & & & & \\
\hline 86 & & & & & & & & & & & & & & & & & & & & & & & & & & & & & & & & & & & \\
\hline 87 & & & & & & & & (1) & & & & & & & & & & & (1) & & & & & & & & & & & & & 1 & & & \\
\hline 88 & & (1) & & & & (1) & & & & & & & & & & & & & & 1 & & & & & & & & & & & & & & (1) & \\
\hline 89 & & & & & & & & & & & & & & & & & & & & & & & & & & & & & & & & & & & \\
\hline 94 & & & & & & & & 1 & & & & & & & & & (1) & & & & & & & & & & & & & & & & & & \\
\hline 99 & & & & & & & & & & & & & & & & & & & & & & & & & & & & & & 1 & & & & & \\
\hline 103 & & & & & & & & & & & & & & & & & & & & & & & & & & & & & & & (1) & & & & \\
\hline
\end{tabular}




\begin{tabular}{|c|c|c|c|c|c|c|c|c|c|c|c|c|c|c|c|c|c|c|c|c|c|c|c|c|c|c|c|c|c|c|c|c|}
\hline \multicolumn{33}{|c|}{ Table 9, 2001-2 } \\
\hline & \multicolumn{11}{|c|}{ D } & \multicolumn{12}{|c|}{$E$} & \multicolumn{9}{|c|}{$\mathrm{F}$} \\
\hline & 4 & $5 \mid$ & 6 & 7 & 8 & $9 \mid 1$ & & & 12 & 13 & & 1 & 2 & 3 & $4 !$ & $5 \mid$ & $\begin{array}{ll}6 & 7 \\
6\end{array}$ & \begin{tabular}{l|l}
7 & $\varepsilon$ \\
\end{tabular} & 89 & $\begin{array}{l}91 \\
\end{array}$ & \begin{tabular}{l|l|}
0 & 1
\end{tabular} & & \begin{tabular}{|l|l|}
2 & 13 \\
\end{tabular} & 1 & \begin{tabular}{|l|l}
2 \\
\end{tabular} & \begin{tabular}{l|l}
3 & 4 \\
4
\end{tabular} & 4 & $5 \mid 6$ & \begin{tabular}{l|l}
6 & 7
\end{tabular} & 8 & 9 & 10 \\
\hline 2 & & & (1) & & & & & & & & & & & & & & & & & & & & & & & & & & & & & \\
\hline 4 & & 1 & 1 & 1 & 1 & 1 & 1 & 2 & 1 & 1 & & & & & 1 & 1 & 2 & $1]$ & 1 & 1 & 2 & 1 & & & & \begin{tabular}{l|l}
1 & 1 \\
\end{tabular} & 1 & 1 & \begin{tabular}{l|l}
1 & 1
\end{tabular} & 1 & 1 & 1 \\
\hline 5 & & (1) & & 1 & 1 & 1 & 2 & 1 & 1 & & & & & & & 1 & 1 & & 1 & 1 & 1 & 1 & & & & & & & 1 & & 1 & 1 \\
\hline 6 & & & & & & & & & (1) & & & & & & & & & & & & & 1 & 1 & & & & & & & & & \\
\hline 7 & & 1 & & 1 & 1 & 1 & 1 & 1 & & & & & & & & & 1 & 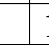 & \begin{tabular}{l|l}
1 & 1 \\
\end{tabular} & \begin{tabular}{l|l}
1 & 1 \\
1
\end{tabular} & 1 & & & & & & 1 & & \begin{tabular}{l|l|}
1 & 1
\end{tabular} & & 1 & 1 \\
\hline 8 & & & & & & & & & & & & & & & & & & & & & & & & & & & & & & & 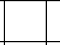 & \\
\hline 10 & & & & & & (1) & & 2 & 1 & 1 & & & & & & (1) & & 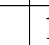 & 1 & . & 1 & 1 & (1) & & & & & & & & 1 & 1 \\
\hline 11 & & & & & & 1 & & & & & & & & & & & & & & & & & & & & & & & & & & \\
\hline 12 & & 1 : & 1 & 1 & & 1 & & & & & & & & & 1 & 1 & 1 & 1 & & & & & & & 1 & 1 & 1 & 1 & 1 & & & \\
\hline 13 & & 16 & (1) & 1 & 1 & 1 & 2 & & 1 & 1 & & & 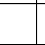 & & & 1 & 1 & $1] 1$ & 1 & 1 & 1 & 1 & & & & & 1 & 1 & \begin{tabular}{l|l|}
1 & 1 \\
\end{tabular} & 1 & 1 & 1 \\
\hline 14 & & (1) 1 & (1) & & & & & & & & & & & & & 1 & & & & & & & & (1) & & 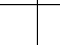 & & & t & a & (a) & \\
\hline 15 & & 1. & 1 & 2 & 3 & 3 & 3 & 2 & 2 & & & & 1 & 2 & 2 & 2 & 2 & 25 & 23 & 35 & 2 & 1 & 1 & 1 & \begin{tabular}{|l|}
1 \\
\end{tabular} & 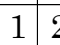 & 2 & \begin{tabular}{l|l}
3 & 2 \\
\end{tabular} & \begin{tabular}{|l|l}
2 & 3 \\
\end{tabular} & 3 & \begin{tabular}{|l|}
3 \\
\end{tabular} & 1 \\
\hline 16 & & & & & & & & 1 & 1 & 2 & 2 & & & & & & & & & & 1 & & 1 & & & & & & & & & \\
\hline 17 & & & 1 & (1) & 1 & 1 & 1 & 1 & 1 & & & (1) & & & 1 & 1 & 1 & 1 & 1 & 1 & 1 & & & & (1) & (1) & (1) & 1 & 1 & 1 & 1 & \\
\hline 18 & & & & & & & & (1) & & & & & & & & & & & & & & & & & & & & & & & & \\
\hline 19 & & & & & & & & & & & & & & & & & & & (1) & 1) & & & & & & & & & & & & \\
\hline 20 & & (1) & & (1) & (1) & (1) & & & & & & & & & & 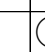 & (1) & ( & (1) & & & & & & & & & & & & & \\
\hline 21 & & & & & & & & & & & & & & & & & (1) & & & & & & & & & & & & & & & \\
\hline 22 & & 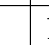 & 1 & & & & & & & & & & & & & & & & & & & & & & & & & & & & & \\
\hline 23 & & ( & (1) & & & & & & & & & & & & & & & & & & & & & & & & & & & & & \\
\hline 26 & & & & & & & & & & (1) & & & & & & & & 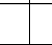 & 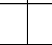 & & & (1) (1) & & & & & & & & & & (1) \\
\hline 27 & & & & & & & & & & & & & & & & & & & & & & & 1 & & & & & & & & & \\
\hline 28 & & & & (1) & (1) & \begin{tabular}{l|}
1 \\
\end{tabular} & 1 & 1 & 1 & 1 & & & & & & & & (1) 1 & 1 & & 1 & & & & & & & (1) & (1) & 1 & & \\
\hline 29 & & & & & & & & & & & & & & & & & & & & & & & & & & & & & (1) (1) & & & (1) \\
\hline 41 & & & & & & & & & & & & & & & & & & & & & & (1) & (1) & & & & & & & 1 & 1 & \\
\hline 43 & & & & & & & & & & & & & & & & & & & & -1 & + & & & & & & & & & & & \\
\hline 44 & (1) & 1 & 1 & 1 & 1 & 1 & 1 & & & & & & & & 1 & 1 & 1 & 16 & (1) 1 & (1) & & & & 2 & & \begin{tabular}{ll|}
12 \\
\end{tabular} & 2 & 1 & & & & \\
\hline 48 & & & & & & 2 & 2 & 2 & 2 & 2 & 2 & & & & & & & $(1)$ & (1) 2 & 25 & \begin{tabular}{l|l}
2 &
\end{tabular} & \begin{tabular}{|l|l}
1 & 1
\end{tabular} & 2 & & & 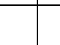 & & & 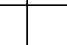 & (1) & & 2 \\
\hline 49 & & & & & & & & & & & + & & & & & & & & 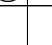 & & & & & & & & & & & & & \\
\hline 53 & & & & & & & & & & & & & & & & & & & & & & & & & & & & & & & & \\
\hline 54 & & & & & & & & (1) & & & & & & & & & & & & & & & & & & & & & & & & \\
\hline 58 & & & & & & & & & & & & & & & & (1) & & & & & & & & & & & & & & & (1) & \\
\hline 60 & & 1 & & & & & 1 & & & & & & & & & 1 & 1 & & 1 & 161 & (1) & & & (1) & & & $\begin{array}{ll}2 & \end{array}$ & 1 & \begin{tabular}{l|l}
1 & 1 \\
\end{tabular} & & 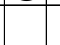 & \\
\hline 61 & & & & & & & & & & & & & & & & & & & & & & (1) & & & & & & & & & & \\
\hline 62 & & & & & & & & & & & & & & & & & & & & & & & & & & & & & & & (1) & \\
\hline 63 & \begin{tabular}{|l|l|}
1 \\
\end{tabular} & 25 & 2 & 1 & 1 & 2 & 2 & & & & & (1) & 1 & (1) & 1 & 1 & 1 & 12 & 2 & 2 & & & & 3 & 1 & 1 & 1 & 2 & 2 & 1 & & \\
\hline 64 & \begin{tabular}{|l|}
1 \\
\end{tabular} & 2 & 1 & 1 & & & & & & & & 1 & 1 & & 2 & & & & & & & & & 2 & \begin{tabular}{|l|l|} 
\\
\end{tabular} & 1 & & & & & & \\
\hline 65 & & & & & & & & & & & & & & & & & & & & & & & & & & & & & & & & \\
\hline 70 & & & & & & & & & & & & & & & & & & & & & & & & & & & & & & & & \\
\hline 71 & & & & & & & & & & & & & & & & & & & & & & & & & & & 2 & 1 & + & & & \\
\hline 73 & \begin{tabular}{|l|l|}
2 \\
\end{tabular} & 2 & 3 & 2 & 1 & 2 & & & & & & 3 & 2 & 1 & 1 & 2 & 2 & 2 & & 1 & & & & 2 & 2 & 23 & 3 & 3 & 1 & & & \\
\hline 74 & & & & & & & & & & & & & & & & & & & & & & & & & 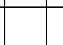 & & & 1 & 1 & & & \\
\hline 77 & +1 & + & + & + & +1 & + & + & + & & & & + & + & + & + & 3 & + & $+t$ & $+t$ & + & & & & + & + & +3 & 3 & + & + & & & \\
\hline 78 & \begin{tabular}{|l|}
2 \\
\end{tabular} & 3 & 3 & 2 & \begin{tabular}{l|}
2 \\
\end{tabular} & 2 & & & & & & 4 & \begin{tabular}{|l|}
3 \\
\end{tabular} & \begin{tabular}{|l|}
3 \\
\end{tabular} & 3 & $\begin{array}{l}3 \\
\end{array}$ & 2 & 1 & & & & & & 3 & \begin{tabular}{|l|l|}
2 \\
\end{tabular} & $\begin{array}{ll}43 \\
\end{array}$ & 3 & & & & & \\
\hline 79 & (1) & 1 & & & & & & & & & & 2 & & & & & & & & & & & & 2 & & & & & & & & \\
\hline 80 & & & & & & (1) & & & & & & & (1) & & & & (1) & & & & & & & & & & & & & & & \\
\hline 81 & \begin{tabular}{|l|}
3 \\
\end{tabular} & 18 & 2 & 1 & & 1 & & & & & & \begin{tabular}{l|}
3 \\
\end{tabular} & \begin{tabular}{|l|l|}
2 \\
\end{tabular} & \begin{tabular}{|l|}
2 \\
\end{tabular} & 3 & & 1 & & & & & & & 3 & \begin{tabular}{|l|}
3 \\
\end{tabular} & 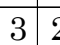 & 2 & & & & & \\
\hline 82 & & & & & & & & & & & & & & & & & & & & & & & & & & & & & & & & \\
\hline 86 & & & & & & & & & & & & & & & & & & & & & & (1) & & & & & & & & & & \\
\hline 87 & & & & & & & & (1) & & & 1 & & & & & & & & & & & & & & & & & & & & & \\
\hline 88 & & (1) & & & & & & & (1) & & & & & & & & & (1) & & & & & & & & & 2 & 1 & & 1 & & \\
\hline 89 & & & & & & & & & & & & & & & & & & & & & & (1) & (1) & & & & & & & & & \\
\hline 94 & & & & & & & & & & & & & & & & & & & & & & & & & & & & & & & & \\
\hline 99 & & & & & (1) & & & & 1 & & & & & & & & & & & 1 & (1) & & 1 & & & & & & & & & \\
\hline 103 & & & & & & & & & & & & & & & & & & & & & & & & & & & & & & & & \\
\hline
\end{tabular}




\begin{tabular}{|c|c|c|c|c|c|c|c|c|c|c|c|c|c|c|c|c|c|c|c|c|c|c|c|c|c|c|c|c|c|c|c|c|c|c|c|}
\hline \multicolumn{36}{|c|}{ Table 9, 2001-3 } \\
\hline & \multicolumn{8}{|c|}{$A$} & \multicolumn{10}{|c|}{$B$} & \multicolumn{14}{|c|}{ C } & \multicolumn{3}{|c|}{ D } \\
\hline & 1 & 2 & 3 & 4 & & 6 & 7 & 8 & 1 & 2 & 3 & 4 & 5 & 6 & 7 & 8 & 9 & 10 & 1 & 2 & 3 & 4 & 5 & 6 & & 8 & 9 & & 11 & & & 14 & & & 3 \\
\hline 115 & & & & & & & & & & & & & & & & & & & & & & & & & & & & & (1) & & & & & & \\
\hline 118 & (1) & & & & & & & & & & & & & & & & & & & & & & & & & & & & & & & & & & \\
\hline 122 & & & & & & 1 & 1 & 1 & & & & & & & & & & & & & 1 & & & & & & & & & & & & & (1) & \\
\hline 123 & & & & & & 1 & 1 & (1) & & & & & & & & & & & 1 & 1 & 1 & & 1 & & & (1) & & 1 & 1 & & & & & & \\
\hline 124 & 1 & 1 & 1 & 1 & 1 & 1 & & & & 1 & 1 & 1 & & 1 & 1 & 1 & 2 & & 2 & & 1 & 1 & 1 & & & 1 & 1 & & & & & & & & 1 \\
\hline 129 & & & & & & & & & & & & & & & & & & & & & & & & & & & & & & & & & & & \\
\hline 130 & & & & & & & & 1 & & & & & & & & & (1) & 1 & & & & & & & & . & & & 1 & 1 & 1 & 1 & & & \\
\hline 131 & & & & & & & & & & & & & & & & & & & & & & & & & & & & & (1) & & & & & & \\
\hline 132 & & & & & 1 & 1 & 2 & 2 & & & & & & 1 & 1 & 3 & 2 & 2 & 1 & 1 & & (1) & & & & 1 & 2 & 2 & 2 & 2 & & & & 1 & \\
\hline 138 & & & & & & & & & & & & & & & & & & & & & & & & & & & & & & & & & & & \\
\hline 141 & & & & & . & & (1) & & & & & . & & . & & (1) & & & ( & . & 1 & & & & & 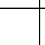 & ( & & 1 & 1 & 1 & & & & \\
\hline 142 & (1) & 1 & & 1 & 1 & 1 & & 1 & & & & 1 & 1 & (1) & & 1 & 1 & 1 & 1 & 1 & 1 & & & & & 1 & 1 & 1 & 2 & 2 & 1 & 1 & & & (1) \\
\hline 143 & & & & & & & & & & & & & & & & & & & & & & & & & & & & & & & 1 & & & & \\
\hline 145 & & & & & 1 & & & 1 & & & & & & & & & & & (1) & & & & & & & & 1 & & 1 & 1 & & & & & \\
\hline 146 & & & & & & & & (1) & & & & & & & & & & & & & & & & & & & & & & & (1) & & & & \\
\hline 156 & & & & & & & & & & & & & & & & & & & & (1) & & & & & & & & & & & & & & & \\
\hline 158 & & & & & & & & & & (1) & & & & & & & & & & & 1 & & & & & & & & & & & & & & \\
\hline 161 & & & & & & & & & & & & & & & & & & & & & & & & & & & & & & & & & & & (1) \\
\hline 162 & & & & & & & & (1) & & & & & & & & & & & & & & & & & & & & & & & & & & & \\
\hline 176 & & & & & & & & & & & & & & & & & & & & & & & & & & & & & & & & & & & \\
\hline 180 & & & & & & & & & & & & & & & & & & & (1) & & & & & & & & & & & & & & & & \\
\hline 181 & & & & 1 & 1 & 1 & & & & & & (1) & & 1 & & & & & & 1 & (1) & & & & & & & & & & & & & & \\
\hline 182 & & & & & & (1) & & & & & & & & & & & & & & & & & & & & & & & & & & & & & \\
\hline 183 & & & & & & & & & & & & & & & & & & & & & & & & & & & & & & & & & & & \\
\hline 188 & & & & & & & & & & & & & & & & & & & & & & & & & & & & & & & & & & & \\
\hline 190 & & & & & & & & & & & & & & & & & & & & & & & & & & & & & & & & & & & \\
\hline 193 & & & & & & & & & & & & & & & & & & & & & & & & & & & & & & & & & & & \\
\hline 194 & & & & & & & & & & & & & & & & & & & & & & & & & & & & & & & & & & & (1) \\
\hline 197 & & & & & & & & & & & & & & & (1) & & & & & & & & & & & & & & & & & & & & (1) \\
\hline 198 & & & & & & & 1 & & & & & & 1 & 1 & \begin{tabular}{|l|} 
\\
\end{tabular} & 1 & 1 & & 1 & & & & & & 2 & 2 & & & & & & & & 1 & 1 \\
\hline 199 & & & & & & 1 & 1 & & & & & & & 1 & \begin{tabular}{|l|} 
\\
\end{tabular} & 1 & & \begin{tabular}{|l|} 
\\
\end{tabular} & & & 1 & & & (1) & 1 & 1 & & 1 & 1 & & & & & (1) & 1 \\
\hline 200 & & & & & & (1) & 1 & & & & & & & & 1 & 1 & & & & & & (1) & & & & 2 & 2 & & 1 & 1 & & 1 & & & \\
\hline 207 & & & & & & & & 1 & & & & 1 & & & & & (1) & 1 & & (1) & & & & & & 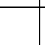 & 1 & & 1 & 1 & 1 & (1) & & & \\
\hline 210 & & & & & & & & & & & & & & & & & & & & & & . & & & & 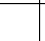 & 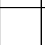 & & (1) & (1) & & & (1) & & \\
\hline 211 & & + & + & + & + & + & 3 & 3 & & + & + & + & + & 3 & 3 & 3 & 4 & & 3 & 3 & + & 3 & 3 & 2 & 3 & 3 & 3 & 2 & & & . & & 3 & 3 & 3 \\
\hline 212 & & 4 & 4 & \begin{tabular}{l|}
4 \\
\end{tabular} & 4 & & \begin{tabular}{|l|}
4 \\
\end{tabular} & & & 4 & 4 & \begin{tabular}{|l|} 
\\
\end{tabular} & 4 & 4 & 4 & 4 & & & 4 & 4 & 4 & & & 3 & 4 & 4 & \begin{tabular}{|l|} 
\\
\end{tabular} & 3 & 4 & 4 & 4 & 4 & & & 4 \\
\hline 213 & & & & & & (1) & & 1 & & & 1 & & & 1 & & & & 1 & & & & 1 & & & & & & 2 & (1) & 1 & 1 & 1 & & & \\
\hline 214 & & & (1) & & & & & & & & & & & & & 1 & & 3 & 1 & 1 & 1 & & & 1 & 1 & 1 & 3 & 2 & 3 & 4 & 4 & 4 & & & 1 \\
\hline 218 & & & & & & & & & & & & & & & & & & & & & & & & & & & & & & & & & & & \\
\hline 220 & & & & & & & & & & & & & & & & & & & (1) & & & & & & & & & & & & & & & & \\
\hline 221 & & & & & & & & & & & & & & & & & & & & & & & & & & & (1) & & & & & & & & \\
\hline 226 & & & & & & & & (1) & & & & & & & & & & & & & & & & & & & & & & & (1) & & & & \\
\hline 229 & 3 & 3 & 3 & 3 & 2 & 3 & 3 & 3 & & 3 & 3 & 3 & 3 & 3 & 3 & 3 & 3 & 3 & 2 & 2 & 3 & 2 & 3 & 3 & 3 & 3 & 3 & 3 & 3 & 3 & 1 & & 2 & 3 & 2 \\
\hline 230 & & 1 & & (1) & & & & 3 & & & (1) & & & & & 2 & 2 & 2 & & & & & & & & 1 & 3 & 2 & & 1 & & & & & \\
\hline 231 & & & & & & & & & & & & & & & & & 1 & & & & & & & & & & 2 & & & & & & & & \\
\hline 232 & & & & & & & & + & & & & & + & & + & & + & & + & & & & + & & & & + & & & & & & & & + \\
\hline 233 & & & & & & (1) & & & & & & & & & & & & & & (1) & & & & & & & & & & & & & & & \\
\hline 234 & & & & & & & & & & & & & & & & & & & & & & & & & & & & & & & & & & & \\
\hline 235 & & & & & & & & & & & & & & & & & & & & & & & & & & & & & & & & & & & \\
\hline
\end{tabular}




\begin{tabular}{|c|c|c|c|c|c|c|c|c|c|c|c|c|c|c|c|c|c|c|c|c|c|c|c|c|c|c|c|c|c|c|c|c|c|c|}
\hline \multicolumn{35}{|c|}{ Table 9, 2001-4 } \\
\hline & \multicolumn{11}{|c|}{ D } & \multicolumn{13}{|c|}{$E$} & \multicolumn{10}{|c|}{$F$} \\
\hline & 4 & 5 & 6 & 7 & 8 & 9 & & & & 13 & & & 2 & 3 & 4 & 5 & 6 & 7 & 8 & 9 & 10 & & & 13 & 1 & 2 & 3 & 4 & 5 & 6 & 7 & 8 & & 10 \\
\hline 115 & & & & & & & & & & & & & & & & & & & & & & & & & & & & & & & & & & \\
\hline 118 & & & & & & & & & & & & & & & & & & & & & & & & & & & & & & & & & & \\
\hline 122 & & & 1 & & & 1 & (1) & & & & & & & & & & 2 & 1 & 2 & 1 & 1 & 1 & & & & & & & 2 & 2 & 2 & 2 & 1 & 1 \\
\hline 123 & & 1 & 1 & & 1 & (1) & & 1 & & & & & & & & (1) & 1 & & (1) & & & & & & & & & (1) & 1 & 1 & & & 1 & \\
\hline 124 & (1) & 1 & 1 & 1 & 1 & 1 & 1 & & & & & & & & 1 & 1 & 1 & 1 & 1 & 1 & & & & & 1 & (1) & 1 & 1 & 1 & 1 & (1) & & & \\
\hline 129 & & & & & & & & & & & & & & & & & & & & & & & & & & & & & & & & & 1 & \\
\hline 130 & & & & & & & & 1 & 1 & 1 & & & & & & & & & 1 & & 1 & & 1 & 1 & & & & & (1) & & & & & 1 \\
\hline 131 & & & & & & (1) & & & & & & & & & & & 1 & & & . & & & (1) & & & & & & & & & (1) & 1 & 1 \\
\hline 132 & & 1 & 2 & 1 & 1 & 2 & 2 & 2 & 2 & & & & & & & 1 & 1 & 2 & 2 & 2 & 2 & 2 & 2 & & & & & 2 & 2 & 2 & 2 & 2 & 1 & 1 \\
\hline 138 & & & & & & & & & (1) & (1) & & & & & & & & & & & & & & & & & & & & & & & & \\
\hline 141 & & & 1 & (1) & & (1) & & 1 & & 1 & (1) & & & & & & & (1) & & (1) & & 1 & 1 & 1 & & & & & & & 1 & 1 & 1 & 1 \\
\hline 142 & & 1 & 1 & 1 & 1 & 1 & 1 & 1 & 1 & 1 & 1 & & & & 1 & 1 & 2 & 1 & 1 & & 1 & 1 & 1 & 1 & & & 1 & 1 & 2 & 2 & 1 & 1 & & 1 \\
\hline 143 & & & & & & & & & & (1) & & & & & & & & & & 1 & & & & & & & & & 1 & & & & & \\
\hline 145 & & 1 & (1) & & (1) & 1 & (1) & 1 & & (1) & & & & & & & & & & & & 2 & 1 & 1 & & & & (1) & & (1) & & 1 & 2 & 1 \\
\hline 146 & & & & & & & & & & & & & & & & & & & & & & & (1) & & & & & & & & & & & \\
\hline 156 & & & & & & & & & & & & & & & & & & & & & & & & & & & & & & & & & & \\
\hline 158 & & 1 & 1 & & 1 & 1 & & & & & & & & & & & & & & & & & & & 1 & & & & & & & & & \\
\hline 161 & & & & & & & & & & & & & & & & & & & & & & & & & & & & & 1 & & & & & \\
\hline 162 & & & & & & & & & & & & & & & & & & & & & & & & & & & & & & & & & & 1 \\
\hline 176 & & & & & & & & & & & & & & & & & & & & & & & & & & & & & & & & & (1) & \\
\hline 180 & & & & & & & & & & & & & & & & & & & & & & & 2 & (1) & & & & & & & & & & 2 \\
\hline 181 & & & & & & & & & & & & & & & & & & & (1) & & (1) & & 1 & & & & & & (1) & & & & & \\
\hline 182 & & & & & & & & & & & & & & & & & 1 & 1 & 1 & & & 1 & & & & & & & & (1) & & 1 & & \\
\hline 183 & & & & & & & & & & & (1) & & & & & & & & & & & & & & & & & & & & & & & \\
\hline 188 & & & & & & & & & & & & & & & & & & & & & & & 1 & & & & & & & & & & & \\
\hline 190 & & & & & & & & & & & & & & & & & & & & & & & (1) & & & & & & & & & & & \\
\hline 193 & & & & & & & & & & & & & & & & & & & & & & & & & & & & & & & & & & 1 \\
\hline 194 & & & & & & & & & & & & & & & & & & & & & & & & & & & & & & & & & & \\
\hline 197 & & & & & (1) & & & & & & & & & & & & & & & & & & & & & & & & & & & & & \\
\hline 198 & (1) & & 1 & 1 & 1 & & & & & & & 1 & 2 & 1 & & & & & & & & & & & & 1 & & & & & & & & \\
\hline 199 & (1) & 1 & 1 & 1 & 1 & 1 & 1 & & & & & 1 & & 1 & 1 & 1 & 1 & 1 & 1 & 1 & & & & & & 1 & & 1 & & & (1) & 1 & 1 & \\
\hline 200 & & 1 & & 1 & 3 & 2 & 2 & 1 & 1 & 1 & 1 & & & & & & 2 & 2 & 2 & 2 & 1 & 1 & 1 & 1 & & & & 2 & 3 & 2 & 2 & 2 & 2 & \\
\hline 207 & & 1 & & (1) & 1 & (1) & & 1 & (1) & (1) & & & & & 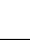 & & 1 & 1 & (1) & 1 & 1 & (1) & 1 & (1) & & & & 1 & 1 & & 1 & 1 & & (1) \\
\hline 210 & & & & & & & & & & & (1) & & & & & & & & & & & & & & & & & & & & & & & \\
\hline 211 & 3 & 2 & + & + & + & + & & & & & & 3 & 3 & 3 & 3 & + & + & + & & & & & & & + & 3 & 3 & 3 & + & & & & & \\
\hline 212 & 1 & 3 & 3 & 4 & & & & 4 & 4 & 3 & 4 & & & & 4 & 4 & 4 & 4 & 4 & 4 & 3 & 4 & 4 & 4 & & & & 4 & 4 & 4 & 4 & 4 & & 3 \\
\hline 213 & & & 1 & & 1 & 1 & 1 & 1 & (1) & & & & & & & & 1 & 1 & 1 & 1 & 1 & (1) & & 1 & & & & 1 & 1 & 1 & & & & \\
\hline 214 & 1 & 1 & 1 & 1 & 1 & 1 & 2 & 3 & 4 & 4 & 4 & & & & 1 & & (1) & 1 & 2 & 3 & 3 & & & 3 & & (1) & & (1) & 1 & & & & & 1 \\
\hline 218 & & & & & & & & & & & & & & & & & & & (1) & & & & & & & & & & & & & & & \\
\hline 220 & & & & & (1) & & & & & & & & & & & & & & & & & & & & & & & & & & & & & \\
\hline 221 & & 1 & & & & & & & & & & & & 1 & & (1) & & & & & & & & & & & & & & & & & & \\
\hline 226 & & & & & & & & & & & 1 & & & & & & & & & & & & & & & & & & & & & & & \\
\hline 229 & 2 & 3 & 3 & 3 & 4 & 4 & 4 & 3 & 3 & 2 & & 2 & 2 & 2 & 3 & 3 & 3 & 4 & 3 & 3 & 3 & 3 & 3 & & 3 & 3 & 3 & 3 & 3 & 2 & 3 & 2 & 3 & 3 \\
\hline 230 & & 1 & 2 & 2 & 2 & 2 & 2 & 1 & 2 & 1 & & & & & 1 & 1 & 2 & 3 & 3 & 3 & 3 & & & & & (1) & (1) & 1 & 1 & 1 & 2 & 2 & 2 & 2 \\
\hline 231 & & & & & & & & & & & & & & & & & & & & & & & & & & & & & & & & & & \\
\hline 232 & + & + & & & & + & & & & & & & & & & + & + & + & + & + & + & & & & + & + & + & + & & + & & & & \\
\hline 233 & & & & & (1) & & & & & & & & & & (1) & & & & & & & & & & & & & & & & & & & \\
\hline 234 & & & & & 1 & & (1) & & & & & & & & & & & & & & & & (1) & & & & & & & & & & & \\
\hline 235 & & & & & & & & & & & & & & & & & & & & & & & & & & & & & & & & & & \\
\hline
\end{tabular}




\begin{tabular}{|c|c|c|c|c|c|c|c|c|c|c|c|c|c|c|c|c|c|c|c|c|c|c|c|c|c|c|c|c|c|c|c|c|c|c|}
\hline \multicolumn{35}{|c|}{ Table $9,2002-1$} \\
\hline & & & & A & & & & & & & & & $B$ & & & & & & & & & & & & C & & & & & & & & D & \\
\hline & \begin{tabular}{l|l}
12 & 29
\end{tabular} & 2 & 3 & 4 & & 6 & $7[\varepsilon$ & 8 & 12 & 3 & 4 & 5 & 6 & 7 & 8 & 9 & 10 & 1 & 2 & 3 & 4 & 5 & 6 & & 8 & & & & 12 & & 14 & 1 & & 3 \\
\hline 1 & & & & & & & & & & & & & & & & & & & & & & & & & & & & & & & & & & \\
\hline 2 & & & & & & & & & & & & & & & & & & & & (1) & & & & & & & & & & & & & & \\
\hline 4 & & 1 & 1 & & 1 & 1 & 1 & 1 & & 1 & 1 & & & & 1 & 1 & 1 & & & & 1 & & & 1 & 1 & & & 2 & 1 & 1 & & & & \\
\hline 5 & & & & & 1 & & & 2 & & & & & & & 1 & 1 & 1 & & & & & & & 1 & 1 & 1 & & & 1 & 1 & 1 & & & \\
\hline 6 & & & & & & & & (1) & & & & & & & & & & & & & & & & & & & (1) & & & & & & & \\
\hline 7 & & & & (1) & & & & 1 & & & & & & & & 1 & (1) & & & & & & & & 16 & (1) & & 1 & & & 1 & & & \\
\hline 8 & & & & & & & & & & & & & & & & & & & & & & & & & & & & & & (1) & (1) & & & \\
\hline 10 & & & & & & & 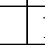 & 1 & & & & & & & & & 2 & & & & & & & 1 & 1 & 1 & 1 & 2 & 1 & 1 & 1 & & & \\
\hline 11 & & & & & & & & (1) & & & & & & & & & & & & & & & & & & (1) & & & & & (1) & & & \\
\hline 12 & & 1 & 1 & & & & & & (1) & ) & & 1 & & & & & & & & & & & & & 1 & 1 & & & & & & & & \\
\hline 13 & & 1 & 1 & 1 & 1 & & $1: 2$ & 2 & & 1 & 1 & 1 & & & 2 & 2 & & 1 & 1 & 1 & 1 & 1 & & 1 & 1 & 2 & 1 & & 1 & 1 & 1 & & 1 & 1 \\
\hline 14 & 1 & 1 & 1 & 1 & 1 & & 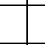 & & 1 & & 1 & & & 1 & 1 & & & 1 & 1 & & & & & (1) & 1 & 1 & & & & & & & & 1 \\
\hline 15 & 1 & 1 & 1 & 1 & 1 & 2 & 1 & 1 & 1 & 1 & 1 & 1 & 1 & 2 & 2 & 2 & 1 & 1 & 1 & 1 & & & & 2 & 2 & 3 & 2 & 2 & 2 & 2 & & & & (1) \\
\hline 16 & & & & & & & & 1 & & & & & & & & & 1 & & & & & 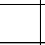 & 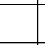 & & & & & 1 & 1 & 2 & 1 & & & \\
\hline 17 & & & 1 & & & & 1 & 1 & & & & & & 1 & 1 & 1 & 1 & & & 1 & & $t$ & & 1 & 1 & 1 & & 1 & 1 & 1 & 1 & (1) & & \\
\hline 18 & & & & & & & & & & & & & & & & & & & & & & & & & & & & & & & 1 & & & \\
\hline 20 & & & & & & & & & & & & & & & & & & & & & & & & & & & & & & & & & & \\
\hline 22 & & 1 & & & & & & 1 & 1 & 1 & & & & & & & & 1 & 2 & 1 & & (1) & & & & & & & & & & & & \\
\hline 23 & & (1) & & (1) & & & & 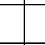 & 1 & 1 & & & & & 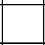 & 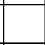 & & 2 & 2 & 2 & & & & & 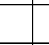 & & & & & & 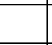 & & & \\
\hline 26 & & & & & & & & & & & & & & & & & (1) & & & & & 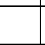 & & & & & & & & 1 & 1 & & & \\
\hline 27 & & & & & & & & & & & & & & & & & & & & & & & & & & & & & & & & & & \\
\hline 28 & & & & & & & & 1 & & & & & & & (1) & 1 & 1 & & & 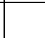 & & 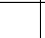 & & & 1 & & & 1 & 1 & & & & & \\
\hline 29 & & & & & & & & & & & & & & & & & & & & & & & & & & & & & & (1) & & & & \\
\hline 30 & & & & & & & & & & & & & & & & & & & & & & & & & & & & & & & (1) & & & \\
\hline 33 & & & & & & & & & & & & & & & & & & & & & & & & & (1) & & & & & & & & & \\
\hline 34 & & & & & & & & & & & & & & & & & & & & & & & & & & & & & & & & & & \\
\hline 38 & & & & & & & & & & & & & & & & & & & & & & & & & & & & & & & & & & \\
\hline 39 & & & & & & & & & & & & & & & & & & & & & & & & & & & & & & & (1) & & & \\
\hline 41 & & & & & & & & 1) & & & & & & & 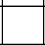 & & & & & & & 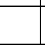 & & & & & 1 & (1) & & (1) & 1 & & & \\
\hline 42 & & & & & & & & & & & & & & & & & & & & & & & & & & & & & & & & & & \\
\hline 43 & & & & & & & & & & & & & & & & & & & & & & & & & & 1 & & & & & & & & \\
\hline 44 & 1 & 1 & 1 & 1 & 2 & 1 & 1 & 1 & 1 & 1 & 2 & 1 & 1 & 1 & 2 & 1 & 1 & 2 & 2 & 2 & 2 & 1 & 1 & 1 & 2 & 2 & 1 & & & & & 2 & 2 & 2 \\
\hline 45 & & & & & & & & & & & & & & & & & & 1 & & & & & & & & & & & & & & & & \\
\hline 48 & & & & & & & (1) & 2 & & & & & & & & 2 & 1 & & & & & & & & & 2 & 2 & 2 & 2 & 3 & 2 & & & \\
\hline 49 & & & & & & & & (1) & & & & & & & & & & & & & & & & & & & & & & & & & & \\
\hline 53 & & & & & & & & & & & & & & & & & & & & & & & & & & & & & & & & & & \\
\hline 54 & & & & & & & & & & & & & & & & & & & & & & & & & & & & & & & & & & \\
\hline 57 & & & & & & & & & & & & & & & & & & & & & & & & & & & & & & & & & & \\
\hline 58 & & & & & & & & & & & & & & & & & & & & & & & & & & & & & & & & & & \\
\hline 59 & & & & & & & & & & & & & & & & & & & & & & & & & & & & & & & & & & \\
\hline 60 & & 1 & 1 & 1 & 1 & 1 & & & 2 & 22 & 1 & 1 & 2 & & 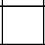 & & & 2 & 2 & 1 & 1 & & & (1) & 1 & 1 & & & & & & & & 2 \\
\hline 62 & & & & & & & & & & & & & & & & & & & & & & & & & & & & & & & & & & \\
\hline 63 & 1 & 1 & 1 & 1 & 1 & 3 & 2 & 213 & 12 & 2 & 2 & 1 & 2 & 2 & 2 & 2 & & 2 & 2 & 2 & 2 & 1 & 1 & 1 & 2 & 2 & 1 & & & & & 1 & 2 & 2 \\
\hline 64 & 1 & & 1 & 1 & & 1 & 1 & 1 & 1 & 1 & 1 & 1 & 1 & 1 & 2 & & & 1 & 1 & & & 2 & 2 & 2 & (1) & & & & & & & 1 & 1 & \\
\hline 70 & & & & & & & & & & & & & & & & & & & 1 & & & & & & & & & & & & & & & \\
\hline 71 & & 3 & 3 & 4 & 4 & 3 & 3 & & 3 & 3 & 4 & 3 & 2 & & & & & & & & & & & & & & & & & & & & & \\
\hline 73 & 1 & 2 & 3 & 3 & 3 & 3 & 2 & 15 & $\begin{array}{l}33 \\
3\end{array}$ & 2 & 3 & 1 & 2 & 2 & 3 & 1 & & 3 & 2 & 2 & 2 & 2 & 3 & 3 & 3 & 2 & & & & & & 2 & 3 & 2 \\
\hline 77 & 3 & & 3 & 4 & + & 3 & $4:$ & $3-$ & $+t$ & + & + & + & 3 & 4 & 3 & 3 & 4 & & & 3 & 3 & 2 & 4 & 4 & 4 & 3 & 3 & & & & & + & + & + \\
\hline 78 & & & 1 & 4 & 4 & 4 & 4 & 4 & & & 1 & 3 & 4 & 4 & 3 & 3 & & & & 3 & 3 & 3 & 4 & 4 & 3 & 3 & 3 & & & & & 3 & 3 & 3 \\
\hline 79 & & & (1) & 1 & & 1 & 1 & & & & & 1 & 2 & & & & & & & 1 & 2 & 1 & 2 & 1 & & & & & & & & & & (1) \\
\hline 80 & & & & & & (1) & & & & & & & & & & & & & & & & & & & & & & & & & & & & (1) \\
\hline 81 & 2 & 1 & 1 & 1 & & 1 & 1 & 15 & $\begin{array}{lll}2 & 1 \\
\end{array}$ & 1 & & 1 & 1 & 1 & 2 & 1 & & 1 & 1 & 2 & 2 & 2 & 2 & 2 & 1 & \begin{tabular}{l|l}
3 \\
\end{tabular} & 1 & & & & & 4 & 3 & 2 \\
\hline 82 & & & & & & & & & & & & & & & & & & & & & & & & & & & & & & & & & & \\
\hline 84 & & & & & & & & & & & & & & & & & & & & & & & & & & & & & & & & & & \\
\hline 86 & & & 1 & & & & & & & & & & & & & & & & & & & & & & & & & & & & & & & \\
\hline 87 & & 1 & & 1 & & & & & & & & & & & & & & & & & & & & & & & & & & & & & & 1 \\
\hline 88 & & & & (1) & & 1 & & & & & & & & & & & & & & & & & & & & & & & & & & & & \\
\hline 91 & & & & & & & & & & & & & & & & & & & & & & & & & & & & & & & 1 & & & 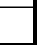 \\
\hline
\end{tabular}




\begin{tabular}{|c|c|c|c|c|c|c|c|c|c|c|c|c|c|c|c|c|c|c|c|c|c|c|c|c|c|c|c|c|c|c|c|c|c|c|}
\hline \multicolumn{35}{|c|}{ Table 9, 2002-2 } \\
\hline & & & & & & $\mathrm{D}$ & & & & & & & & & & & & $\mathrm{E}$ & $E$ & & & & & & & & & & $\mathrm{~F}$ & & & & & \\
\hline & 4 & 5 & 6 & 7 & 8 & & 10 & & 12 & 213 & & & 12 & 23 & 34 & 5 & 6 & 7 & 8 & 9 & 10 & 11 & & 13 & 1 & 2 & 3 & 4 & 5 & 6 & & 8 & & 10 \\
\hline 1 & & (1) & & & & & & & & & & & & & & & & & & & & & & & & & & & & & & & & \\
\hline 2 & & & & & & & & & & & & & & & & & & & & & & & & & & & & & 1 & & & & (1) & \\
\hline 4 & & & 1 & 1 & 1 & 1 & 2 & 2 & 2 & 1 & & & & & (1) & 2 & 1 & 2 & 2 & 2 & 2 & 2 & 1 & 1 & & & & 1 & 1 & 1 & 1 & 1 & 1 & 2 \\
\hline 5 & & & 1 & 1 & 1 & 2 & 1 & 1 & 1 & 1 & 1 & & & & & & 1 & 1 & 1 & 2 & 1 & 1 & & & & & & & 1 & 1 & & 1 & 1 & 1 \\
\hline 6 & & & & & & & & & (1) & & & & & & & & & & & & & & & 1 & & & & & & & & & & (1) \\
\hline 7 & & (1) & (1) & & 1 & 1 & 1 & 1 & 1 & 1 & 1 & & & & & 1 & & (1) & & 2 & & 1 & 1 & & & & & & & (1) & 1 & 1 & 1 & 1 \\
\hline 8 & & & & & & & & & & & 1 & & & & & & & & & & & & & & & & & & & & & & & (1) \\
\hline 10 & & & & & & & 1 & 3 & 2 & 1 & 1 & 1 & 1 & & & & & & (1) & 1 & 2 & 2 & 2 & 1 & & & & & & (1) & & & & \\
\hline 11 & & & & & & 1 & 1 & & & & & & & & & & & & (1) & & & -1 & & & & & & 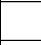 & & 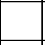 & & & & \\
\hline 12 & & 1 & 1 & 1 & 1 & & (1) & & & & & & & & & 1 & 1 & 1 & & & & & & & & & & 1 & & & 1 & & & \\
\hline 13 & 1 & 1 & 1 & 1 & 2 & 2 & 2 & 1 & 1 & 1 & & & & & 1 & 1 & 1 & 1 & 1 & 2 & 1 & & & & & & 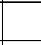 & & 1 & 18 & 1 & 1 & 2 & 1 \\
\hline 14 & 1 & 1 & & & 1 & 1 & 1 & & & & & & & & 1 & 1 & & 1 & 1 & & & & & & & & & & & & & & & \\
\hline 15 & & & 1 & 2 & 3 & 3 & 3 & 2 & 2 & 2 & 1 & & & & 1 & 2 & 2 & 2 & 2 & 3 & 2 & 2 & & & & & & (1) & 1 & 1 & 3 & 3 & 1 & 1 \\
\hline 16 & & & & & & & & 1 & 1 & 2 & 1 & & & & & & (1) & (1) & & 1 & 1 & & & 1 & & & & & & & & (1) & 1 & 2 \\
\hline 17 & & 1 & 1 & & & 1 & 2 & 1 & 1 & 1 & 1 & & & & 1 & 1 & & 1 & 1 & 2 & 1 & & & & & & & & & & 1 & & & \\
\hline 18 & & & & & & & & & & & 1 & & & & & & & & & & & & & & & & & & & & & & & \\
\hline 20 & & & & & & 1 & 1 & & & & & & & & & & & 1 & & & & & & & & & & & & & & & & \\
\hline 22 & & & & & & & & & & & & & & & & & & & & & & & & & & & & & & & & & & \\
\hline 23 & & 2 & & & & & & & & & & & & & & 1 & & & (1) & & & & & & & & & & & & & & & \\
\hline 26 & & & & & & & & & & & 1 & & & & & & & & & & & & & & & & & & & & & & & \\
\hline 27 & & & & & & & & & & & (1) & & & & & & & & 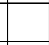 & & & & & & & & & & & & & & & \\
\hline 28 & & & (1) & (1) & 1 & & 1 & 1 & 2 & 1 & & & & & & & & & 1 & 1 & 1 & & 1 & & & & & (1) & & & 1 & 1 & 1 & 1 \\
\hline 29 & & & & & & & & & & & & & & & & & & & & & & & & (1) & & & & & & & & & & \\
\hline 30 & & & & & & & & & & & (1) & & & & & & & & & & & & & & & & & & & & & & & \\
\hline 33 & & & & & & & & & & & & & & & & & & & & & & & & & & & & & & & & & & \\
\hline 34 & & & & & & & & & & & & & & & & & & & & & & & & & & & & & & & & & & (1) \\
\hline 38 & & & & & & & & & & & (1) & & & & & & & & & & & & (1) & & & & & & & & & & & \\
\hline 39 & & & & & & & & & & & & & & & & & & & & & & & & & & & & & & & & & & \\
\hline 41 & & & & & & & & & & & & & & & & & & & 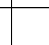 & & & (1) & (1) & 1 & & & & & & & & & 1 & \\
\hline 42 & & & & & & & & & & & & & & & & & & & & & & & & & & & & & (1) & & & & & \\
\hline 43 & & & & & & 1 & & & & & & & & & & & & & & 1 & & & & & & & & 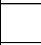 & & & & + & & \\
\hline 44 & 1 & 1 & 1 & 1 & 2 & 2 & 1 & & 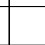 & & & & 1 & 1 & 1 & 12 & 2 & 2 & 2 & 2 & 1 & & & & & (1) & & 1 & 2 & & 1 & & & \\
\hline 45 & & & & & & & & & & & & & & & & & & & & & & & & & & & & & & & & & & \\
\hline 48 & & & & & 2 & 2 & 3 & 2 & 2 & 3 & 1 & & & & & & & & 2 & 2 & 3 & 2 & 2 & 2 & & & & & & (1) & 1 & 1 & 1 & 2 \\
\hline 49 & & & & & & & & & & & & & & & & & & & & & & & & & & & & & & & & & & \\
\hline 53 & & & & & + & & & 2 & & & (1) & & & & & & & 2 & & & & & & & & & & & + & & & & & \\
\hline 54 & & & & & & & & & & & & & & & & & & & & & & (1) & & & & & & & & & & & & \\
\hline 57 & & & & & & & & & & & & & & & & & & & & & & & & & & & & 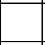 & (1) & & & & & \\
\hline 58 & & & & & & & & & & & (1) & & & & & & & & & & & & & & & & & & & & & & & \\
\hline 59 & & & & & & & & & & & & & & & & & & & (1) & & & & & & & & 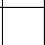 & 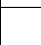 & & & & & & \\
\hline 60 & 1 & 1 & & 1 & 1 & & & & & & & & & & & 2 & 1 & 1 & & & & & & & & & 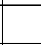 & 1 & 1 & \begin{tabular}{|l|} 
\\
\end{tabular} & (1) & & & \\
\hline 62 & & & & & & & & & & & & & & & & & & & & & & & (1) & & & & & & & & & & & \\
\hline 63 & 1 & 2 & 2 & 11 & \begin{tabular}{|l|}
2 \\
\end{tabular} & 2 & 1 & & & & & & 1 & & (1) & 1) & 1 & 1 & 2 & 1 & 1 & & & & 1 & 1 & 1 & 2 & 1 & \begin{tabular}{|l|} 
\\
\end{tabular} & 2 & & 1 & \\
\hline 64 & & & 1 & & & & & & & & & & & & 1 & 11 & 1 & 1 & & & & & & & (1) & 2 & & 1 & 1 & & & & & \\
\hline 70 & & & & & & & & & & & & & & & & & & & & & & & & & & & & & & & & & & \\
\hline 71 & & & & & & & & & & & & & & & & & & & & & & & & & & & & 1 & & \begin{tabular}{|l|l} 
\\
\end{tabular} & & & & \\
\hline 73 & 3 & 3 & 3 & 3 & 3 & 2 & 1 & & & & & & 25 & 2 & 4 & 43 & 3 & 3 & 3 & 1 & & & & & 1 & 2 & 3 & 3 & 3 & 1 & 2 & & & \\
\hline 77 & + & + & + & + & + & + & + & & & & & & $1-$ & 1 & $3+$ & +1 & + & & & & & & & & 1 & 4 & 4 & 4 & + & & & & & \\
\hline 78 & 4 & 4 & 3 & 3 & 2 & 3 & 3 & & & & & & 42 & 4 & \begin{tabular}{l|l}
35 \\
\end{tabular} & \begin{tabular}{|l|l}
4 & 1
\end{tabular} & & & & & & & & & 4 & 4 & 4 & 3 & & & & & & \\
\hline 79 & & (1) & & & & & & & & & & & $1]$ & 11 & & & & & & & & & & & 1 & 1 & & & & & & & & \\
\hline 80 & & & & & & & (1) & & & & & & & & & & & & & & & & & & & & & & & & & & & \\
\hline 81 & 3 & 2 & 2 & 2 & 2 & 3 & 1 & & & & & & $\begin{array}{lll}3 & 3\end{array}$ & 3 & 3 & $\begin{array}{l}3 \\
3\end{array}$ & 1 & & & & & & & & 4 & 3 & 3 & 3 & 1 & & & & & \\
\hline 82 & & 3 & & & & & & & & & & & & & & 3 & & & & & & & & & & & & & + & & & & & \\
\hline 84 & & & & & & & & & & & & & & & & & & & & & & & & & & & & & (1) & & & & & \\
\hline 86 & & & & & & & & & & & & & & & & & & & & (1) & & & & & & & & & & (1) & & & 1 & \\
\hline 87 & & & & & & & & & 1 & & (1) & & & & & & & & & & & & & & & & & & (1) & \begin{tabular}{|l|}
2 \\
\end{tabular} & & & & \\
\hline 88 & (1) & & & & & & & & & & 1 & & & & (1) & 1) & & & 1 & & & & & & & & & (1) & (1) & (1) & & (1) & 1 & \\
\hline 91 & & & & & & & & & & & 1 & & & & & & & & & & & & & & & & & & 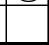 & & 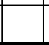 & & & \\
\hline
\end{tabular}




\begin{tabular}{|c|c|c|c|c|c|c|c|c|c|c|c|c|c|c|c|c|c|c|c|c|c|c|c|c|c|c|c|c|c|c|c|c|c|c|c|}
\hline \multicolumn{36}{|c|}{ Table 9, 2002-3 } \\
\hline & & & & A & & & & & & & & & $\mathrm{E}$ & & & & & & & & & & & & & C & & & & & & & & D & \\
\hline & \begin{tabular}{l|l}
12 & 2
\end{tabular} & 2 & 3 & 4 & & 6 & 7 & 8 & 1 & 2 & 3 & 4 & 5 & 6 & 7 & 8 & 9 & 10 & 1 & 2 & 3 & 4 & \begin{tabular}{|l|l|}
5 & \\
\end{tabular} & \begin{tabular}{l|l}
6 & 1 \\
\end{tabular} & & 8 & 9 & & 11 & 12 & & 14 & & & 3 \\
\hline 94 & & & & & & & & 1 & & & & & & & & & & & & & & & & & & & & & & (1) & & & & & \\
\hline 97 & & & & & & & & & & & & & & & & & & & & & & & & & & & & & & & & & & & \\
\hline 99 & & & & & & & & & & & & & & & & & & & & & & & & & & & & & & (1) & (1) & (1) & & & \\
\hline 103 & & & & & & & & & & & & & & & & & & & & & & & & & & & & & & & (1) & & & & \\
\hline 104 & & & & & & & & & & & & & & & & & & & & & & & & & & & & & & & & & & & \\
\hline 107 & & & & & & & & & & & & & & & & & & & & & & & & & & & & & & & & & & & \\
\hline 110 & & & & & & & & & & & & & & & & & & (1) & & & & & & & & & & & & & & & & & \\
\hline 122 & & (1) & & & & & (1) & 1 & & & 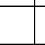 & & & & & 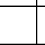 & & & & & 1 & 1 & & & & & & & & & & & & & \\
\hline 123 & & & 1 & 1 & & & & & & & & & 1 & & & & & & & 1 & & & & & & & & & & & & (1) & & & \\
\hline 124 & (1) 1. & 1 & 1 & 1 & 1 & 1 & 1 & 1 & (1) & 1 & 2 & 2 & 1 & 2 & 1 & 1 & 1 & & 1 & 1 & 1 & (1) & 1 & & 1 & 1 & 1 & & & & & & & 1 & 1 \\
\hline 129 & & & & & & & & & & & & & & & & & & & & & & & & & & & & & & & (1) & 1 & & & \\
\hline 130 & & & & & & & & 1 & & & & & & & & & & 1 & & & & & & & & & & & 1 & 1 & 1 & 1 & & & \\
\hline 131 & & & & & & & & & & & & & & & & & & & & & & & 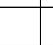 & & & & 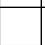 & & 1 & 1 & 1 & & & & \\
\hline 132 & & 1 & & & 1 & 1 & 1 & 2 & & & & & 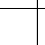 & & 1 & 3 & 2 & 2 & & 1 & & & 1 & & 1 & 1 & 2 & 2 & 2 & 2 & & & & & \\
\hline 138 & & & & & & & & & & & & & & & & & & & & & & & & & & & & & & & (1) & 1 & & & \\
\hline 141 & & & & 1 & 1 & (1) & & & & & & & 1 & 1 & & (1) & & & & 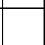 & & & & (1) & & & . & & 1 & 1 & 1 & 1 & & & \\
\hline 142 & & 1 & 1 & 1 & 1 & 1 & & 1 & & & 1 & 1 & 1 & & 1 & 1 & 2 & 1 & 1 & 1 & 1 & & 1 & 1 & & 1 & 1 & 1 & 1 & 2 & 2 & 1 & & & 1 \\
\hline 143 & & & & (1) & & & & & & & & & & & & & & & & & & & & & & & & & 1 & & 1 & 1 & & & \\
\hline 145 & & & & 1 & 1 & 1 & 1 & 1 & & & & & 1 & & & & & 1 & & & & & 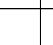 & & & & 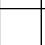 & & 1 & 1 & 1 & & & & \\
\hline 146 & & & & & & & & & & & & & & & & & & & & & & & & & & & & & & (1) & 1 & & & & \\
\hline 149 & & & & & & & & & & & & & & & & & & & & & & & & & & & & & & & & & & & \\
\hline 155 & & & & & & & & & & (1) & & & & & & & & & 1 & 1 & & & & & & & & & & & & & & & \\
\hline 158 & & 1 & & & (1) & & & & & & & & & & & & & & & & 2 & & 1 & & & & & & & & & & & & \\
\hline 161 & & & & & & & & (1) & & & & & & & & & & & & & & & & & & & & (1) & & & & & & & \\
\hline 162 & & & & & & & & & & & & & & & & & & & & & & & & & & & & & & & & & & & \\
\hline 174 & & & & & & & & & & & & & & & & & & & & & & & & & & & & & & & & & & & \\
\hline 176 & & & & & & & & & & & & & & & & & & & & & 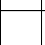 & & 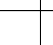 & & & & 1 & & & & & & & & \\
\hline 180 & & & & & & & & & & & & & & & & & & & & & & & & & & & & & 1 & & & & & & \\
\hline 181 & & & & & & & & & & & & & & & & & & & & & 1 & & & & & & & & & & & & & & \\
\hline 182 & & & & & & & & & & & & & & & & & & & & & (1) & & & & & & 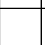 & & & & & & & & \\
\hline 192 & & & & & & & & & & & & & & & & & & & & & & & & & & & & & & & & & & & \\
\hline 193 & & & & & & & & & & & & & & & & & & & & & & & & & & & & & & & & & & & \\
\hline 197 & & & & & & & & & & & & & & & & & & & & & & & & & & & & & & & & & & & \\
\hline 198 & & & & & 1 & 1 & & & & & & & 1 & 1 & 2 & 1 & & & & & 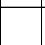 & & & & 1 & 1 & & & & & & & & 1 & 1 \\
\hline 199 & $\begin{array}{lll}1 & 1\end{array}$ & & 1 & 1 & & & & 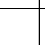 & & & & 1 & 1 & & 1 & 1 & & & (1) & & 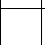 & & 1 & & 1 & 1 & 1 & 1 & (1) & & & 1 & & 1 & 1 \\
\hline 200 & & & & & 1 & & & & & & & & 1 & 1 & 1 & 1 & 1 & 1 & & & 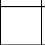 & & & & & 1 & 2 & & & & (1) & (1) & & & \\
\hline 203 & & & & & & & & & & & & & & & & & & & & & & & 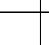 & & & & & & & & (1) & & & & \\
\hline 205 & & & & & & & & & & & & & & & & & & & & & & & & & & & & & & & & (1) & & & \\
\hline 207 & & (1) & (1) & 1 & & & & 1 & & & & 1 & 1 & & & & & 1 & & (1) & 1 & & 1 & & & & & (1) & 1 & & 1 & 1 & & & (1) \\
\hline 210 & & & & & & & & & & & & & & & & & & & & & & & & & & & & & & & & & & & \\
\hline 211 & $\begin{array}{ll}16 \\
\end{array}$ & 2 & 2 & 2 & \begin{tabular}{|l|}
3 \\
\end{tabular} & 2 & 3 & 3 & & & & 1 & 1 & 3 & 3 & 3 & 4 & & 2 & 1 & 2 & 3 & 2 & \begin{tabular}{|l|l|}
3 & \\
\end{tabular} & \begin{tabular}{|l|}
3 \\
\end{tabular} & 4 & \begin{tabular}{|l|} 
\\
\end{tabular} & 2 & & & & 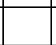 & 3 & \begin{tabular}{|l|}
3 \\
\end{tabular} & 4 \\
\hline 212 & & 3 & 1 & 2 & 4 & & 3 & 2 & & 4 & 3 & 4 & 3 & 4 & 4 & 4 & 3 & & 4 & 4 & 4 & & 2 & \begin{tabular}{|l|l|}
3 &
\end{tabular} & 4 & 4 & 4 & 4 & 4 & 4 & 4 & & & & \\
\hline 213 & & (1) & 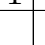 & & (1) & & 1 & 1 & & & 1 & & & & & & & 3 & & & & & & & & & & 2 & 1 & 1 & 1 & 1 & & & (1) \\
\hline 214 & & & 1 & 1 & & & & & & & & & (1) & & & & & 1 & & & & & & 1 & 1 & & 2 & 3 & 3 & 4 & 4 & 4 & & & (1) \\
\hline 216 & & & & & & & & & & & & & & & & & & & & & & & & & & & & & & & & 1 & & & \\
\hline 218 & & & & & & & & (1) & & & & & & & & & & & & & & & & & & & & & & & & (1) & & & \\
\hline 220 & & & & (1) & & & (1) & (1) & & (1) & & & & & & & & & & & & & & & & & & & & & & & & & \\
\hline 221 & & & 1 & & & & & & (1) & & & & & & & & & & & & & & & & & & & & & & & & & & \\
\hline 222 & & & & & & & & & & & & & & & & & & & & & & & & & & & & & & & (1) & & & & \\
\hline 226 & & & & & & & & & & & & & & & & & & & & & & & & & & & & 1 & & & & & & & \\
\hline 229 & 25 & 2 & 3 & 1 & 2 & 3 & 3 & 3 & 2 & 3 & 3 & 3 & 1 & 2 & 3 & 3 & 3 & & 2 & 3 & 2 & 2 & 2 & \begin{tabular}{|l|l|}
3 \\
\end{tabular} & 3 & 3 & 4 & 4 & 3 & 4 & 2 & & 3 & 3 & 3 \\
\hline 230 & & & & & (1) & & 1 & 3 & & & & (1) & & & & 2 & 3 & 2 & & & & 1 & & 1 & 1 & 2 & 3 & 2 & 2 & 2 & 1 & & & & \\
\hline 231 & & & & & & & & & & & & & & & & & & 2 & & & & & & & & & & & & & & & & & \\
\hline 232 & & & & & & & & & & & & & & & & & & & & & & & & & & & & & & & & & & & \\
\hline 233 & & & & & & & & & & & & & & & & & & & & & & & & & & & & & & & & & & & \\
\hline 234 & & & & 1 & 1 & 1 & 1 & & & & & & & & & & & & & & & & & & & & & & & & & & & & \\
\hline 235 & & & & & & & & & & & & & & & & & & (1) & & & & & & & & & & & & & & 1 & & & \\
\hline 240 & & & & & & & & & & & 1 & & & & & & & & & & & & & & & & & & & & & & & & 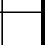 \\
\hline
\end{tabular}




\begin{tabular}{|c|c|c|c|c|c|c|c|c|c|c|c|c|c|c|c|c|c|c|c|c|c|c|c|c|c|c|c|c|c|c|c|c|c|c|}
\hline \multicolumn{35}{|c|}{ Table 9, 2002-4 } \\
\hline & & & & & & $\mathrm{D}$ & & & & & & & & & & & & $\mathrm{E}$ & $E$ & & & & & & & & & & 1 & $F$ & & & & \\
\hline & 4 & 5 & 6 & 7 & 8 & & 10 & & 12 & 21 & & & 12 & 23 & 34 & 5 & 6 & 7 & 8 & 9 & 10 & 11 & & 13 & 1 & 2 & 3 & 4 & & 6 & & 8 & & 10 \\
\hline 94 & & & & & & & & & (1) & & & & & & & & & & & & & & & & & & & & & & & & & \\
\hline 97 & & & & & & & & & & & & & & & & & & & & & & (1) & & & & & & & & & & & & \\
\hline 99 & & & & & & & & (1) & (1) & & & & & & & & & & & & & (1) & & & & & & & & & & & & (1) \\
\hline 103 & & & & & & & & & & & & & & & & & & & & & & & & & & & & & & & & & & \\
\hline 104 & & & & & & & & & & & & & & & & & & & & & & & & & & & & & & & & & & (1) \\
\hline 107 & & & & & & & & & & & & & & & & & & & & & & & & & & & & & & & & & & (1) \\
\hline 110 & & & & & & & & & & & & & & & & & & & & & & & & & & & & & & & & & & \\
\hline 122 & & & & & 1 & 2 & 1 & 1 & & & & & & & & 1 & 1 & 1 & 2 & 2 & 1 & 1 & 1 & & & & & & 1 & 1 & 2 & 2 & 1 & 1 \\
\hline 123 & & 1 & 1 & 1 & 1 & & & 1 & 1 & & & & & & 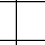 & & & (1) & 1 & 1 & & 1 & 1 & & & & & 1 & 1 & 1 & & 1 & 1 & \\
\hline 124 & 1 & 1 & 1 & 1 & 1 & 1 & 1 & & & & & & & & 1 & 1 & 1 & 1 & 1 & & & & & & & & & 1 & 1 & 1 & & & & \\
\hline 129 & & & (1) & & & & & & & & & & & & & & & & & & & & & & & & & & & (1) & & & & \\
\hline 130 & & & & & & (1) & & 1 & 1 & 1 & & & & & & & & & & & 1 & 1 & & 1 & & & & & t & t & & & & 2 \\
\hline 131 & & & & & & & & (1) & & & & & & & & (1) & & & & 1 & & 1 & & 1 & & & & & & & & (1) & 1 & 1 \\
\hline 132 & & 2 & 2 & 2 & 2 & 2 & 2 & 2 & 3 & 2 & & & & & & 2 & 2 & 2 & 2 & 3 & 2 & 2 & 2 & 2 & & & & 1 & 2 & 2 & 1 & 2 & 1 & 1 \\
\hline 138 & & & & & & (1) & & & & & & & & & & & & & & & & & & & & & & & & & & & & \\
\hline 141 & & & & & (1) & & & & 1 & & 1 & & & & & & & & & & & 1 & 1 & 1 & & & & & & & & & 1 & 1 \\
\hline 142 & & & 1 & 1 & 1 & 1 & 1 & 1 & 2 & 2 & 1 & & & & 1 & 1 & 1 & 1 & 1 & 1 & 1 & 2 & 2 & 2 & & & 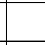 & 1 & 1 & 2 & 2 & 1 & 1 & 1 \\
\hline 143 & & & & & & & & & & 1 & 1 & & & & & & & & & & & & 1 & 1 & & & & & & 1 & (1) & & (1) & \\
\hline 145 & & & & & & & & 1 & 1 & 1 & (1) & & & & & & & & & & & 2 & 2 & 2 & & & & & (1) & 1 & & 2 & 2 & 2 \\
\hline 146 & & & & & & & & & & & 1 & & & & & & & & & & & & & & & & & & & & & & & \\
\hline 149 & & & & & & & & & & & 1 & & & & & & & & & & & & & & & & & & & & & & & \\
\hline 155 & & & & & & & & & & & & & & & & & & & & & & & & & & & & & & & & & & \\
\hline 158 & & 1 & 1 & & & & & & & & & & & & & & & & & & & & & & & & & & & & & & & \\
\hline 161 & & & & & & & & & & & & & & & & & & & & & & & & & & & & & & & & & & (1) \\
\hline 162 & & & & & & & & & & & & & & & & & & & & & & & (1) & & & & & & & & & & & \\
\hline 174 & & & & & & & & & & & & & & & & & & & & & & & & & & & & & & & & & & (1) \\
\hline 176 & & & & & & & & & & & & & & & & & & & & & & & & & & & & & & & & & & \\
\hline 180 & & & & & & & & & & & & & & & & & & & & & & & & & & & & & & & & & & (1) \\
\hline 181 & & & & & & & & & & & & & & & & & & (1) & & & & & & (1) & & & & & & & & & & 1 \\
\hline 182 & & & & & & & & & (1) & & & & & & & & & & & & & & & & & & & 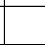 & & (1) & & & & (1) \\
\hline 192 & & & & & & & & & & & & & & & & & & & & & & & & & & & & & & & & & (1) & (1) \\
\hline 193 & & & & & & & & & & & & & & & & & & & & & & & & & & & & & & & & & & 1 \\
\hline 197 & & & & & & & & & & & & & & & & & (1) & (1) & & & & & & & & & & & & (1) & & & & \\
\hline 198 & (1) & & & 1 & 1 & & & & & & & & 1 & 1) & & & & (1) & & & & & & & & & & & & & & & & \\
\hline 199 & & (1) & 1 & 1 & 1 & 1 & 1 & & & & & & 1 & (1) & & & (1) & 1 & & 1 & & . & & & (1) & & & L & & & 1 & & & \\
\hline 200 & & & & 1 & \begin{tabular}{|l|l|}
2 \\
\end{tabular} & 2 & 1 & 1 & 2 & 1 & 1 & & & & & (1) & 1 & 1 & 1 & 2 & 1 & 2 & & & & & 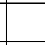 & 2 & 2 & 2 & 3 & 2 & 2 & 2 \\
\hline 203 & & & & & & & & & & & & & & & & & & & & & & & & & & & & & & & & & & \\
\hline 205 & & & & & & & & & & & & & & & & & & & & & & & & & & & & & & & & & & \\
\hline 207 & & & & 1 & \begin{tabular}{|l|}
1 \\
\end{tabular} & (1) & & 1 & 1 & & 1 & & & & & (1) & ) 1 & 1 & 1 & 1 & 1 & 1 & 1 & 1 & & & & & 1 & 1 & 1 & 1 & \begin{tabular}{l|l|} 
\\
\end{tabular} & 1 \\
\hline 210 & & & & & & & & & & & (1) & & & & & & & & $1-$ & & & & & & & & & & & & & & & \\
\hline 211 & 3 & 3 & 3 & \begin{tabular}{|l|}
2 \\
\end{tabular} & \begin{tabular}{|l|l}
2 \\
\end{tabular} & 3 & & & & & & & $\begin{array}{ll}3 & 5\end{array}$ & 34 & 44 & & & & & & & & & & 1 & 4 & 4 & 4 & & & & & & \\
\hline 212 & & & 4 & 4 & \begin{tabular}{|l|l|}
3 \\
\end{tabular} & & & 3 & 4 & 4 & & & & & & 4 & 4 & 4 & 4 & & & 4 & 4 & 4 & & & & & 4 & 4 & 4 & 4 & 1 & 2 \\
\hline 213 & & 1 & & & (1) & & & 1 & 1 & 1 & (1 & & & & & & & 1 & & 1 & 1 & & 1 & 1 & & & & & & 1 & 1 & 1 & (1) & \\
\hline 214 & (1) & (1) & & 2 & \begin{tabular}{|l|} 
\\
\end{tabular} & 1 & 3 & 3 & 4 & 4 & 4 & & & & & & & 1 & 2 & 1 & 2 & & & 3 & & & & (1) & & & & & & \\
\hline 216 & & & & & & & & & & & (1) & & & & & & & & & & & & & & & & & & & & & & & \\
\hline 218 & & & & & & & & (1) & & (1) & & & & & & & & & & & & (1) & & & & & & & & & & & & \\
\hline 220 & & & & & & & & & & & (1 & & & & & & & & & & & & & & & & & & & & (1) & & (1) & \\
\hline 221 & & & & & & & & & & & & & & & & & & & & & & & & & & & & & & & & & & \\
\hline 222 & & & & & & & & & & (1) & & & & & & & & & & & & & & & & & & & & & & & & \\
\hline 226 & & & & & & & & & & & & & & & & & & & & & & & & & & & & & & & 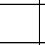 & & & \\
\hline 229 & 3 & 3 & 3 & 4 & 4 & 4 & 4 & 3 & 4 & 3 & 1 & & $2:$ & $\begin{array}{l}3 \\
3\end{array}$ & 33 & 33 & 3 & 4 & 3 & 4 & 3 & 3 & 2 & & 3 & 3 & 3 & 3 & 3 & 2 & 3 & 1 & & 2 \\
\hline 230 & & 2 & 2 & 2 & \begin{tabular}{|l|}
3 \\
\end{tabular} & 3 & 3 & 2 & 2 & & & & & & & 1 & 2 & 3 & 3 & 3 & 3 & & & & & & & 1 & & 1 & 3 & 2 & 2 & 2 \\
\hline 231 & & & & & & & & & & & & & & & & & & & 1 & & & & & & & & & & & & & & (1) & \\
\hline 232 & & & & & & & + & & & & & & & & & & & & & & & & & & & & & & & & & & & \\
\hline 233 & & & & & & & & & & & & & & & & & & & & & & & & & & & & & 1 & (1) & & & & \\
\hline 234 & & & & & & & & & & & & & & & & & & & & & & & & & & & & & & & & & 1 & \\
\hline 235 & & & & & & & & & & & & & & & & & & & & & & & & & & & & & & & & & & \\
\hline 240 & & & & & & & & & & & & & & & & & & & & & & & & & & & & & & & & & 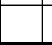 & \\
\hline
\end{tabular}




\begin{tabular}{|c|c|c|c|c|c|c|c|c|c|c|c|c|c|c|c|c|c|c|c|c|c|c|c|c|c|c|c|c|c|c|c|c|c|c|c|}
\hline \multicolumn{36}{|c|}{ Table 9, 2003-1 } \\
\hline & \multicolumn{8}{|c|}{$\mathrm{A}$} & & & & & $\mathrm{E}$ & & & & & & & & & & & & & $\mathrm{C}$ & & & & & & & & D & \\
\hline & 1 & 2 & 3 & 4 & & 6 & 7 & 8 & 1 & 2 & 3 & 4 & 5 & 6 & 7 & 8 & 9 & 10 & 1 & 2 & 3 & 4 & 5 & 6 & & 8 & \begin{tabular}{l|l}
9 & 1 \\
\end{tabular} & 101 & 111 & 12 & & 14 & & & 3 \\
\hline 2 & & & & & & & & & & & & & & & & & & & & & & & & & & & & & & & & & & & \\
\hline 4 & & 1 & & & 1 & & 1 & 1 & & 1 & 1 & & & & & 1 & 2 & 1 & & & (1) & & (1) & & 1 & 1 & 1 & 2 & 2 & 1 & 1 & & & & \\
\hline 5 & & & & & (1) & & & 2 & & & & & & & & 1 & 2 & 1 & & & & 1 & & & 1 & 1 & 2 & 1 & & & & & & & \\
\hline 6 & & & & & & & & & & & & & & & & & & & & & & & & & & & & & & & & & & & \\
\hline 7 & & & & & (1) & & & 1 & & & (1) & (1) & & 1 & & & 1 & 1 & & & & & & & & & 1 & 1 & (1) & & & & & & \\
\hline 8 & & & & & & & & & & & & & & & & & & & & & & & & & & & & & & & & & & & \\
\hline 10 & & & & & & & 1 & 1 & & & & & & & & & 1 & 1 & & & & & & & (1) & 1 & 1 & 1 & & 2 & 2 & (1) & & & \\
\hline 11 & & & & & & & & & & & & & & & & & (1) & & & & & & & & & & & & & & & & & & \\
\hline 12 & & 1 & & 1 & 1 & & (1) & & & & (1) & 1 & (1) & (1) & & 1 & 1 & & & & & & 1 & & & (1) & 1) & & & & & & & & 1 \\
\hline 13 & & & 1 & 1 & 1 & 1 & 2 & 3 & & 1 & & 1 & 1 & & 1 & 1 & 2 & & & 1 & 1 & 1 & 1 & & 1 & 1 & 2 & 1 & & 1 & 1 & & & 1 & (1) \\
\hline 14 & & 1 & 1 & & 1 & & & & & & & 1 & 1 & 1 & 1 & 1 & & & 1 & 1 & 1 & 2 & 1 & 1 & 1 & 1 & & & & 1 & & & & 1 & 1 \\
\hline 15 & & 1 & 1 & 1 & 2 & 2 & 2 & 2 & & & 1 & 1 & 2 & 2 & 2 & 2 & 3 & 1 & 1 & 1 & 1 & 1 & 2 & 1 & 2 & 2 & 2 & 2 & 2 & 1 & & & 1 & 1 & 1 \\
\hline 16 & & & & & & & & & & & & & & & & & & & & & & & & & & & & 1 & & 1 & 2 & 1 & & & \\
\hline 17 & (1) & 1 & 1 & 1 & 1 & & 1 & 1 & & & 1 & & & 1 & & 1 & 1 & & (1) & & 1 & & 1 & & 1 & 1 & 1 & 1 & & 1 & 1 & & 1 & & 1 \\
\hline 18 & & & & & & (1) & & & & & & & & & & & & & & & & & & & & (1) & & & & & & 1 & & & \\
\hline 20 & & (1) & & & & & & & & & 1 & & & & & & & & & & & & & & & & & & & & & & & (1) & \\
\hline 22 & & 2 & 1 & 1 & 1 & & (1) & 1 & & 2 & & (1) & (1) & & & & & & 2 & 1 & 2 & & & & & & & & & & & & & & \\
\hline 23 & & & & & & & & & & & (1) & & 1 & & & & & & & & & & 1 & & & & & & & & & & & & \\
\hline 26 & & & & & & & & & & & & & & & & & & & & & & & & & & & & & & (1) & & & & & \\
\hline 28 & & & & & & & & 1 & & & & & & & & & (1) & 1 & & & & & & & (1) & 1 & 1 & 2 & 1 & 1 & 1 & & & & \\
\hline 29 & & & & & & & & & & & & & & & & & & & & & & & & & & & & & & & & & & & \\
\hline 39 & & & & & & & & & & & & & & & & & & & & & & & & & & & & & & & & & & & \\
\hline 41 & & & & & & (1) & & & & & & (1) & & & & & (1) & 1 & & & & & 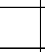 & & 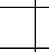 & 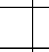 & & & & & 1 & & & & \\
\hline 43 & & (1) & & & & & & & & & & & & & & & & 1 & & & & & & & & & & & & & & & & & \\
\hline 44 & & 2 & 2 & 1 & 2 & 1 & (1) & (1) & & 2 & 1 & 2 & 2 & 1 & 1 & & 1 & & 2 & 2 & 1 & 1 & 1 & 1 & 1 & 1 & 1 & & & & & & 1 & 2 & 1 \\
\hline 48 & & & & & & & & 1 & & & & & & & & & 1 & 1 & & & & & & & & & 2 & 2 & 2 & 2 & 2 & 2 & & & \\
\hline 49 & & & & & & & & & & & & & & & & & & & & & & & & & & & & & & & & 1 & & & \\
\hline 53 & & & (1) & & & & & & & & & & & & & & & & & & & & & & & & & & & & & & & & \\
\hline 54 & & & & & & & & (1) & & & & & & & & & & & & & & & & & & & & & & & (1) & & & & \\
\hline 58 & & & & & & & & & & & & & & & & & & & & & & & & & & & & & & & & & & & \\
\hline 60 & & 2 & 2 & 2 & 2 & 1 & 1 & & & 2 & 2 & 1 & 1 & & 2 & 1 & & & 2 & 2 & 2 & 1 & (1) & & 1 & 1 & & 1 & & & & & & 1 & 2 \\
\hline 62 & & & & & & & & & & & & & & & & & & & & & & & & & & & & & & & & & & & \\
\hline 63 & & 2 & 2 & 3 & 2 & 3 & 2 & 1 & & 2 & 2 & 2 & 2 & 2 & 1 & 2 & 1 & & 2 & 2 & 2 & 2 & 2 & 1 & 1 & 1 & 1 & 1 & & & & & 2 & 3 & 2 \\
\hline 64 & & & (1) & 1 & (1) & 2 & 1 & & & & (1) & & 2 & 1 & 1 & 1 & & & (1) & & 2 & 2 & 2 & 2 & 2 & 1 & & & & & & & 2 & 1 & 1 \\
\hline 70 & & & & & & & & & & & & & & & 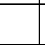 & & & & & 3 & & 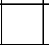 & 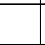 & 4 & & & & & & & & & & & \\
\hline 71 & & & 1 & 2 & 1 & 3 & 3 & & & 1 & 1 & 1 & 2 & 3 & & & & & & & & & & & & & & & & & & & & & \\
\hline 73 & 3 & 3 & 4 & 3 & 3 & 3 & & 2 & & 3 & \begin{tabular}{l|}
3 \\
\end{tabular} & 3 & 2 & 2 & 3 & 2 & 1 & & 3 & 4 & 3 & 2 & 2 & 2 & 3 & 3 & 1 & 1 & & & & & 2 & 3 & 3 \\
\hline 77 & & 2 & 3 & 4 & 2 & 3 & 4 & 4 & & 2 & 2 & 3 & 4 & 4 & 4 & 3 & 3 & 1 & 2 & 3 & 3 & 3 & 4 & 4 & 3 & 2 & 3 & 3 & 1 & & 1 & & 2 & 4 & 3 \\
\hline 78 & 1 & & & 4 & 4 & 4 & 4 & 3 & & & & 3 & 4 & 4 & 4 & & 3 & & & & 4 & 4 & 4 & 4 & 3 & 3 & 3 & 3 & & & & & 2 & 2 & 3 \\
\hline 79 & & & & & 2 & & 1 & & & & & & 1 & & & & & & & & 2 & 2 & 1 & (1) & & & & & & & & & & & \\
\hline 80 & & & & & & & & & & & & 1 & & & & & & & & & & (1) & & & & & & & & & & & & & \\
\hline 81 & 1 & 2 & 2 & 2 & 2 & 1 & 1 & & & & & 1 & 1 & 1 & 1 & & & & & & 1 & 1 & 2 & 1 & 1 & & & & & & & & 3 & 1 & 1 \\
\hline 82 & & & & & & & & & & & & & & & & & & & & & 1 & & & & & & & & & & & & & & \\
\hline 86 & & & & & & & & & & & & & & & & & & & & & & & & & & & & & & & & & & & \\
\hline 87 & & & 1 & & & & & & & 1 & & & & & & & & & & & & & & & & & & & & & & & & & \\
\hline 88 & & & 1 & 1 & & 1 & & & & 1 & 1 & & & & & & & & & & & & & & & & & & (1) & & & & & & \\
\hline 89 & & & & & & & & & & & & & & & & & & & & & & & & & & & & & & & & & & & \\
\hline 94 & & & & & & & & (1) & & & & & & & & & & & & & & & & & & & & & & & & & & & \\
\hline 99 & & & & & & & & & & & & & & & & & & & & & & & & & & & & & & (1) & (1) & & & & \\
\hline 100 & & & & & & & & & & & & & & & & & & & & & & & & & & & & & & & & & & & \\
\hline 103 & & & & & & & & & & & & & & & & & & & & & & & & & & & & & (1) & & & & & & \\
\hline 122 & & & & & 1 & 1 & 1 & 1 & & & & & 1 & 1 & & & & & & & & & & & & & & & & & & & & & \\
\hline 123 & & & & 1 & & & & (1) & & & & 1 & 1 & & & & & & & & & & & & & & & & & & & & & & \\
\hline 124 & & 1 & 1 & 2 & 1 & 1 & 1 & 1 & & 1 & 1 & 1 & 2 & 1 & 1 & 1 & 2 & & 1 & 1 & 1 & 1 & 1 & 1 & 1 & 1 & & 1 & & & & & & & \\
\hline 129 & & & & & & & & & & & & & & & & & & & & & & & & & & & & & & & & & & & \\
\hline
\end{tabular}




\begin{tabular}{|c|c|c|c|c|c|c|c|c|c|c|c|c|c|c|c|c|c|c|c|c|c|c|c|c|c|c|c|c|c|c|c|c|}
\hline \multicolumn{33}{|c|}{ Table 9, 2003-2 } \\
\hline & \multicolumn{11}{|c|}{ D } & \multicolumn{12}{|c|}{$E$} & \multicolumn{9}{|c|}{$\mathrm{F}$} \\
\hline & 4 & $5 \mid$ & $\begin{array}{ll}6 & 7 \\
6\end{array}$ & \begin{tabular}{l|l}
7 & $\varepsilon$ \\
\end{tabular} & \begin{tabular}{|l|l}
9 \\
\end{tabular} & & \begin{tabular}{l|l}
0 & 1
\end{tabular} & & & & & 1 & 2 & 3 & 4 & $5 \mid$ & & & \begin{tabular}{l|l}
8 & 9 \\
\end{tabular} & 10 & 11 & 12 & 13 & 1 & 2 & \begin{tabular}{l|l}
3 & 4 \\
\end{tabular} & \begin{tabular}{l|l}
4 & 5 \\
\end{tabular} & 6 & 7 & 8 & 9 & 10 \\
\hline 2 & & & & & 1 & & & & & & & & & & & & & & & & & & & & & & & & & & & \\
\hline 4 & & & 1 & 11 & 1 & 2 & 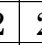 & 2 & 1 & 1 & & & & & & 12 & 2 & 2 & \begin{tabular}{l|l}
211 \\
\end{tabular} & 2 & 1 & 1 & 1 & & & (1) 1 & \begin{tabular}{l|l}
1 & 1 \\
\end{tabular} & 1 & 1 & 1 & 1 & 2 \\
\hline 5 & & & 1 & 11 & 1 & 2 & & & 1 & & (1) & & & & & 1 & 1. & 1 & 1 & & 1 & & & & & & (1) & & (1) & & & \\
\hline 6 & & & & & & & & & (1) & (1) & & & & & & & & & & & & & 1 & & & & & & & & & \\
\hline 7 & & 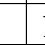 & $1]$ & 1 & 1 & 1 & 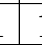 & 1 & 1 & & & & & & & $1:$ & 1 [1 1 & $1] 1$ & \begin{tabular}{l|l}
1 & 1 \\
\end{tabular} & \begin{tabular}{l|l}
1 & 1 \\
\end{tabular} & 1 & 1 & 1 & & & & & 1 & & & (1) & \\
\hline 8 & & & 1) & & & (1) & & & & & & & & & & & & & & & & & & & & & & & & & & \\
\hline 10 & & & & 1 & 2 & 1 & 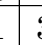 & 3 & 2 & 2 & (1) & & & & & & & $2 \sqrt{2}$ & 25 & \begin{tabular}{l|l}
22 \\
\end{tabular} & 2 & 1 & 1 & & & & & 1 & 1 & 1 & 3 & 2 \\
\hline 11 & & & & & 1 & 1 & & & (1) & & & & & & & & & & & & & & & & & & & & & & & \\
\hline 12 & & 1 & 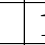 & 1 & (1) & & & & & & & & & & & & 1 & & & & & & & & & (1) & & & & & & \\
\hline 13 & & 1 : & $1] 1$ & $1] 1$ & 2 & 1 & ( & 1) & 2 & & & & & & & $1:$ & $1[2$ & 2 & 2 & \begin{tabular}{l|l}
2 & 1 \\
\end{tabular} & & 1 & & & & (1) 1 & \begin{tabular}{l|l}
1 & 1 \\
\end{tabular} & 1 & 1 & 1 & & \\
\hline 14 & 1 & 1. & 1 & 1 & & & & & & & & & & & (1) ( & (1). & 1 & 1 & 1) & & & & & & & 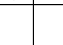 & & & & & & \\
\hline 15 & 1 & $1]$ & 1 & 13 & 3 & 3 & 3 & 1 & 1 & 2 & & 1 & 2 & 2 & 2 & 2 & \begin{tabular}{l|l}
2 & 3
\end{tabular} & 33 & $\begin{array}{ll}32 \\
\end{array}$ & \begin{tabular}{l|l}
2 \\
\end{tabular} & & 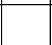 & & & (1) & \begin{tabular}{l|l}
2 & 1
\end{tabular} & 1 & 2 & 2 & \begin{tabular}{|l|}
3 \\
\end{tabular} & 2 & 1 \\
\hline 16 & & & & & & & 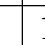 & 1 & 1 & 1 & & & & & & & & & & & 1 & & & & & & & & & & 1 & \\
\hline 17 & 1 & 1. & 11 & 1 & 1 & 1 & & & 1 & 1 & & & 1 & 1 & 1 & $1:$ & \begin{tabular}{l|l}
1 & 1
\end{tabular} & $\begin{array}{lll}1 & 1\end{array}$ & 1 & \begin{tabular}{l|l}
1 & 1
\end{tabular} & & & & (1) & (1) & (1) & (1) & & & & 1 & \\
\hline 18 & & & & & & & & & & 1 & & & & & & & & & & & & & 1 & & & & & & & & & \\
\hline 20 & & 16 & 1). & 11 & 1 & & & & & & & & & & & 1 & 1 & . & & & & & & & & & & & & & & \\
\hline 22 & & & & & & & & & & & & & & & & & & & & & & & & & & & & & & & & \\
\hline 23 & (1) & & & & & & & & & & & & & & & & & 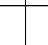 & 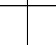 & & & & & & & & (1) & & & & & \\
\hline 26 & & & & & & & & & & (1) & & & & & & & & & & & & 1 & 1 & & & & & & & & & \\
\hline 28 & & & & 1 & 1 & 1 & 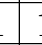 & 1 & 1 & 1 & & & & & & & & 1 & \begin{tabular}{l|l}
1 & 1 \\
\end{tabular} & \begin{tabular}{l|l}
1 & 1 \\
\end{tabular} & 1 & & & & & & (1) & (1) & & & & \\
\hline 29 & & & & & & & & & & & & & & & & & & & & & & (1) & & & & & & & & & & \\
\hline 39 & & & & & & & & & & & & & & & & & & & & & & (1) & 1 & & & & & & & & & \\
\hline 41 & & & & & & & & & (1) & & & & & & & & & & 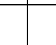 & & (1) & 1 & 1 & & & - & 1 & & & & & \\
\hline 43 & & & & & 1 & 2 & & & & & & & & & & & 1 & 1 & & & & & & & & & & & & & & \\
\hline 44 & 2 & 2 & 17 & $\begin{array}{lll}1 & 1\end{array}$ & 1 & (1) & & & & & & (1) & 1 & 1 & 1 & $1:$ & \begin{tabular}{l|l}
1 & 1
\end{tabular} & (1) & (1) & & & & & 1 & 2 & \begin{tabular}{l|l}
2 & 1
\end{tabular} & \begin{tabular}{l|l}
1 & 1
\end{tabular} & & & & & \\
\hline 48 & & & & & 2 & 2 & 2 & 2 & 2 & 2 & 3 & & & & & & & & 1 & 2 & 1 & 1 & 2 & & & & & & & & 1 & \\
\hline 49 & & & & & & & & & & & & & & & & & & & & & & & (1) & & & & & & & & & \\
\hline 53 & & & & & & & & & 1 & & & & & & & & & (1) & 1) & 1 & & & & & & & (1) & & & & & \\
\hline 54 & & & & & & & & & & & & & & & & & & & & & & & & & & & & (1) & & & & \\
\hline 58 & & & & & & & & & & & & & & & & & & & & & & & & & & & (1) & (1) & & (1) & & \\
\hline 60 & & 1 & 11 & 11 & 1 & & & & & & & 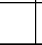 & & & & $2:$ & \begin{tabular}{l|l}
2 & 1 \\
\end{tabular} & & & (1) & & & & & & & 1 & 1 & 1 & & & \\
\hline 62 & & & & & & & & & & & & & & & & & & & & & & (1) & & & & & & & & & & \\
\hline 63 & \begin{tabular}{|l|}
1 \\
\end{tabular} & 23 & 21 & 15 & 25 & 2 & & & & & & 1 & 1 & 1 & 1 & 2 & \begin{tabular}{l|l}
11 &
\end{tabular} & 25 & 22 & 2 & & & & 1 & \begin{tabular}{l|l|}
1 \\
\end{tabular} & \begin{tabular}{l|l}
1 & 1 \\
\end{tabular} & $\begin{array}{ll}12 \\
\end{array}$ & & & & & \\
\hline 64 & 2 & 13 & 21 & 1 & & & & & & & & (1) & 1 & 1 & 1 & & 1 & & & & & & & 1 & 2 & \begin{tabular}{l|l}
1 & 1
\end{tabular} & 1 & & & & & \\
\hline 70 & & & & & & & & & & & & & & & & & & & & & & & & & & & & & & & & \\
\hline 71 & & 2 & & & & & & & & & & & & & & & & & & & & & & & & 2 & 2 & 2 & 2 & & & \\
\hline 73 & \begin{tabular}{|l|l|}
3 \\
\end{tabular} & 4 & 42 & 44 & 2 & & & & & & & 2 & 3 & \begin{tabular}{l|l}
2 \\
\end{tabular} & \begin{tabular}{|l|}
3 \\
\end{tabular} & 3 & \begin{tabular}{l|l}
2 & 2
\end{tabular} & 211 & 1 & & & & & 3 & 2 & \begin{tabular}{|l|l}
3 & 2
\end{tabular} & 22 & & & & & \\
\hline 77 & \begin{tabular}{|l|}
3 \\
\end{tabular} & 2 & 41 & 15 & 2 & 2 & & & & & & 2 & 4 & 4 & \begin{tabular}{|l|}
3 \\
\end{tabular} & 2 & & & 1 & 1 & & & & 1 & 3 & \begin{tabular}{l|l}
4 & 3 \\
\end{tabular} & \begin{tabular}{l|l}
3 & \\
\end{tabular} & & & & & \\
\hline 78 & \begin{tabular}{|l|}
3 \\
\end{tabular} & 3 & 2 & 3 & 35 & 33 & & & & & & 3 & 3 & 4 & 4 & & & & 1 & & & & & 3 & $\begin{array}{ll}3 \\
\end{array}$ & \begin{tabular}{l|l}
3 & 3 \\
\end{tabular} & 3 & & & & & \\
\hline 79 & & & (1) & & & & & & & & & 1 & 1 & & & & & & & & & & & 1 & & & & & & & & \\
\hline 80 & & & & & & & & & & & & & & & & & 1 & & & & & & & & & & & & & & & \\
\hline 81 & \begin{tabular}{|l|}
1 \\
\end{tabular} & & 1 & & & & & & & & & & 2 & \begin{tabular}{l|l}
1 \\
\end{tabular} & 2 & & & & & & & & & 2 & 3 & \begin{tabular}{l|l}
2 & 1
\end{tabular} & 1 & & & & & \\
\hline 82 & & 3 & & & & & & & & & & & & & & 3 & & & & & & & & & & (1) & (1) & & & & & \\
\hline 86 & & & & & & (1) & & & & & & & & & & & & & & & & & & & & & & & & & & \\
\hline 87 & & & & & & & 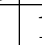 & 1 & & & 1 & & & & & & & & & & & & 1 & & & & & & & & & \\
\hline 88 & & & & & & & & & & & & & & & & & (1) & 1) & & & & & & & & 1 & \begin{tabular}{l|l}
1 & 1
\end{tabular} & 1 & (1) & 1 & & \\
\hline 89 & & & & & & & & & & & & & & & & & & & & & & (1) & & & & & & & & & & \\
\hline 94 & & & & & & & & & & & & & & & & & & & & & & & & & & & & & & & & \\
\hline 99 & & & & & & & & & & (1) & & & & & & & & & & & (1) & 1 & 1 & & & & & & & & & \\
\hline 100 & & & & & & & & & & & & & & & & & & & & & & & & & & & & & & & (1) & \\
\hline 103 & & & & & & & & & & & & & & & & & & & & & & & & & & & & & & & & \\
\hline 122 & & 1 & 11 & $\begin{array}{lll}1 & 1 \\
\end{array}$ & \begin{tabular}{l|l}
1 & 1 \\
\end{tabular} & 11 & 1 & 1 & & & & & & & & 1. & \begin{tabular}{l|l}
1 & 2 \\
\end{tabular} & 25 & \begin{tabular}{|l|l}
2 & 1 \\
\end{tabular} & \begin{tabular}{|l|l}
1 & 1 \\
\end{tabular} & 1 & & & & & & 1 & 2 & 2 & 2 & 1 & 1 \\
\hline 123 & & & & 1 & 1 & & & & & & & & & & & & \begin{tabular}{l|l} 
& 1
\end{tabular} & 1 & & & $\begin{array}{ll} \\
\end{array}$ & 1 & & & & & \begin{tabular}{l|l} 
(1) & 1
\end{tabular} & 1 & & & \begin{tabular}{|l|l|}
1 \\
\end{tabular} & \\
\hline 124 & \begin{tabular}{|l|}
1 \\
\end{tabular} & 1 & 1 & 1 & 16 & (1) & & & & & & & & & (1) & 2 & 1 & 1 & 1 & & & & & & & 1 & $\begin{array}{ll}11 \\
\end{array}$ & 1 & & & 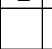 & \\
\hline 129 & & & & & & & & & & & & & & & & & & & & & & & & & & & & & & & (1) & \\
\hline
\end{tabular}




\begin{tabular}{|c|c|c|c|c|c|c|c|c|c|c|c|c|c|c|c|c|c|c|c|c|c|c|c|c|c|c|c|c|c|c|c|c|c|c|c|}
\hline \multicolumn{36}{|c|}{ Table 9, 2003-3 } \\
\hline & \multicolumn{8}{|c|}{$\mathrm{A}$} & \multicolumn{10}{|c|}{ B } & \multicolumn{14}{|c|}{ C } & \multicolumn{3}{|c|}{ D } \\
\hline & 1 & 2 & 3 & 4 & 5 & 6 & $7 \mid \varepsilon$ & 8 & 1 & 2 & 3 & 4 & $5 \mid 6$ & \begin{tabular}{l|l}
6 & 7 \\
\end{tabular} & \begin{tabular}{l|l}
7 & 8 \\
\end{tabular} & 3 & & 10 & 1 & 2 & 3 & 4 & 5 & \begin{tabular}{|l|l}
6 & \\
\end{tabular} & & \begin{tabular}{|c|c|c|}
8 \\
8
\end{tabular} & \begin{tabular}{l|l}
9 & 1
\end{tabular} & & & 12 & 13 & 14 & & & 3 \\
\hline 130 & & & & & & & & & & & & & & & & & & & & & & & & & & & & & 1 & 1 & 1 & 1 & & & \\
\hline 131 & & & & & & & & & & & & & & & & & & & & & & & & & & & & & 2 & & & & & & \\
\hline 132 & & (1) & & & 2 & & 2 (5 & 2 & & (1) & (1) & 1 & 1 & $1 \mid 1$ & 1 & 2 & 2 & 2 & & & 1 & (1) & & 1 & & 2 & 2 & 2 & 2 & 2 & & & & & \\
\hline 133 & & & & & & & & & & & & & & & & & & & & & & & & & & & & & & & & & & & \\
\hline 138 & & & & & & & & & & & & & & & & & & & & & & & & & & & & & (1) & & (1) & & & & \\
\hline 141 & & & & & (1) & & & & & & & & & & & & & & & & & & & & & (1) & & & 1 & & 1 & (1) & & & \\
\hline 142 & & 1 & & 1 & 1 & & & 1 & & 1 & 1 ( & (1) & & 1 & 1 & 1 & 1 & 1 & & & 1 & & & & & & 1 & 1 & 2 & 1 & 1 & & & (1) & 1 \\
\hline 143 & & & 1 & & & & & & & & & & & & & & & & & & & & & & & & & & & & & & & & \\
\hline 145 & & & & 1 & 1 & 1 & & (1) & & 1 & 1 & 1 & 1 & & & & 1) & & & & & & & & & & & & & & & & & & \\
\hline 155 & & & & & 1 & & 1 & & & (1) & & & & & & & & & & & & & & & & & & & & & & & & & \\
\hline 156 & & & & (1) & (1) & & & & & & & & & & & & & & & & & & & & & & & & & & & & & & \\
\hline 157 & & & & & & & & & & & & & & & & & & & & & & & & & & & & & & & & & & & \\
\hline 158 & & 1 & & & & 1 & & & & & & & & & & & & & & & 2 & 1 & (1) & & & & & & & & & & & & \\
\hline 161 & & & & & & & & & & & & & & & & & 1) & & & & & & & & & & & & & & & & & & \\
\hline 162 & & & & & & & & & & & & & & & & & & & & & & & & & & & & & & & & & & & \\
\hline 165 & & & & & & & & & & & & & & & & & & & & & & & & & & & & & & & & & & & \\
\hline 176 & & & & & & & & & & & & & & & & & & & & & & & & & & & & & & & & & & & \\
\hline 181 & & & & & & & & & & & & & & & & & & & & & & & & & & & & & & & & & & & \\
\hline 182 & & & & & & & & & & 1 & & & & & & & & & & & & & & & & & & & 1 & & & & & & \\
\hline 193 & & & & & & & & & & & & & & & & & & & & & & & & & & & & & & & & & & & \\
\hline 197 & & & & & & & & & & & & & & ( & 1) & & & & & & & & & & & (1) & & & & & & & & & \\
\hline 198 & & & & & 1 & 1 & 1 & & & & & & 1 & 1 & 1 & 1 & & & & & & 1 & 1 & & 1 & 1 & 1 & & & & & & & 1 & 2 \\
\hline 199 & & & (1) & 1 & 1 & 1 & 1 & 1 & & & & (1) & (1) & (1) & 1 & 1 & 1 & & & (1) & & & (1) & & 2 & 1 & 1 & (1) & & & & & 1 & 1 & 1 \\
\hline 200 & & & & & (1) & & 1 & 1 & & & & & & (1) & 1 & 1 & 1 & 1 & & & & & & & 1 & 1 & & & & 1 & 1 & & & & \\
\hline 207 & & & & & & & & & & & (1) & & 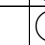 & (1) & & & & & & (1) & & 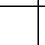 & 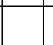 & 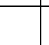 & 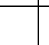 & 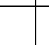 & 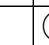 & (1) & 1 & 1 & 1 & & & & \\
\hline 211 & 1 & 1 & & 1 & & 2 & 3 & 3 & & 1 & & 2 & 2 & 1 & 2 & 4 & 3 & 1 & & & 3 & 2 & 3 & 3 & 3 & 4 & 3 & 3 & & & & & 3 & 3 & 2 \\
\hline 212 & & 4 & 4 & 3 & 4 & & 3 & 3 & & 4 & & 4 & 4 & 4 & 4 & 4 & 3 & 3 & 4 & 4 & 4 & & & 4 & 4 & 4 & 4 & & 4 & 4 & 4 & 4 & & & 4 \\
\hline 213 & & & (1) & (1) & & 1 & 1 & 1 & & & & (1) & 1 & 1 & & & 1 & 2 & & & & & (1) & & & 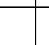 & & 2 & & & (1) & & & & . \\
\hline 214 & & & & (1) & & & & & & & & & (1) & & & & 1) & 1 & (1) & & & & & (1) & 1 & & 2 & 3 & 2 & 3 & 3 & 3 & & & \\
\hline 218 & & & & & & & & & & & & & & & & & & & & & & & & & & & & & & & & & & & \\
\hline 226 & & & & & & & & & & & & & & & & & & & & & & & & & & & & & & & & & & & \\
\hline 229 & 3 & 3 & 3 & 3 & 3 & 3 & 3 & 3 & & 3 & 3 & 4 & 3 & $3:$ & 3 & 4 & 4 & 3 & 2 & 3 & 3 & 2 & 3 & 3 & 3 & 3 & 3 & 3 & 3 & 3 & 2 & & 2 & 3 & 2 \\
\hline 230 & & (1) & & 1 & & & (1) & 3 & & & & & & & 1) 2 & & & 3 & & & & & & & 2 & 2 & 3 & 3 & 2 & 2 & & & & & \\
\hline 231 & & & & & & & & & & & & & & & & & 2 & & & & & & & & & & & & & & & & & & \\
\hline 232 & & & & & & & & & & & & & & & & & & & & & & & & & & & & & & & & & & & \\
\hline 233 & & & & & & & & & & & & & & & & & & & & & & & & & & & & & & & & & & & \\
\hline 234 & & & & & & & & (1) & & & & & & & & & & & & & & & & & & & & & (1) & & & & & & \\
\hline 235 & & & & & & & & & & & & & & & & & & & & & & & & & & & & & & & & & & & \\
\hline 236 & & & & & & & & & & (1) & & & & & & & & & & & & & & & & & & & & & & & & & 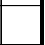 \\
\hline
\end{tabular}




\begin{tabular}{|c|c|c|c|c|c|c|c|c|c|c|c|c|c|c|c|c|c|c|c|c|c|c|c|c|c|c|c|c|c|c|c|c|c|c|}
\hline \multicolumn{35}{|c|}{ Table 9, 2003-4 } \\
\hline & \multicolumn{11}{|c|}{$\mathrm{D}$} & \multicolumn{13}{|c|}{$\mathrm{E}$} & \multicolumn{10}{|c|}{$\mathrm{F}$} \\
\hline & 4 & 5 & 6 & 7 & 8 & 9 & & 11 & 12 & 13 & & 1 & 2 & 3 & 4 & 5 & 6 & 7 & 8 & 9 & & 11 & 12 & 13 & 1 & 2 & 3 & 4 & 5 & 6 & 7 & 8 & & 10 \\
\hline 130 & & & & & & (1) & & 1 & 1 & 1 & & & & & & & & & & & & & 2 & 2 & & & & & & & & & & \\
\hline 131 & & & & & 1 & & (1) & & & & & & & & & & & & (1) & & & (1) & (1) & & & & & & & (1) & 1 & 1 & 1 & (1) \\
\hline 132 & & 2 & 2 & 2 & 3 & 3 & 2 & 2 & 2 & 2 & & & & & & 1 & 1 & 2 & 2 & 2 & 2 & 2 & 2 & & & & & 1 & 2 & 2 & 2 & 2 & 1 & 1 \\
\hline 133 & & & & & & & & & & & & & & & & & & & & & & (1) & & & & & & & & & & & & \\
\hline 138 & & & & & & & & & & & & & & & & & & & & & & & & (1) & & & & & & & & & & \\
\hline 141 & & & & (1) & (1) & (1) & & (1) & & 1 & & & & & & & & & & & & 1 & 1 & 2 & & & & & (1) & & & (1) & 1 & 1 \\
\hline 142 & & 1 & 1 & 1 & 1 & 1 & 1 & 1 & 1 & 1 & & & & 1 & (1) & 1 & 1 & 1 & 1 & 1 & 1 & 1 & 2 & 2 & & & & 1 & 1 & 2 & & 1 & 1 & 2 \\
\hline 143 & & & & & & & & & & & & & & & & & & & & & & & (1) & & & & & & & (1) & & & & \\
\hline 145 & & & & & & 1 & & & & & & & & & & (1) & & & & & & & 1 & 1 & & & & & 1 & (1) & & & 1 & 1 \\
\hline 155 & & & & & & & & & & & & & & & & & & & & & & & & & & & & & & & & & & \\
\hline 156 & & & & & & & & & & & & & & & & & & & & & & & & & & & & & & & & & & \\
\hline 157 & & & & & & (1) & & & & & & & & & & & & & & & & & & & & & & & & & & & & \\
\hline 158 & & & (1) & & & & & & & & & & & & & & & & & & & & & & & & & & & & & & & \\
\hline 161 & & & & & & & & & & & & & & & & & 1 & & & & & & & & & & & & & (1) & & & & \\
\hline 162 & & & & & & & & & & & & & & & & & & & & & & (1) & & & & & & & & & & & & \\
\hline 165 & & & & & & & & & & & & & & & & & & & & & & & (1) & & & & & & & & & & & \\
\hline 176 & & & & & & & & & & & & & & & & & & & & & (1) & & & & & & & & & & & & & \\
\hline 181 & & & & & & & & & & & & & & & & & & & & & & & & & & & & & & & & & (1) & \\
\hline 182 & & & & & (1) & & & & & & & & & & & & (1) & & & & & & & & & & & & & & & (1) & & (1) \\
\hline 193 & & & & & & & & & & & & & & & & & & & & & & & (1) & & & & & & & & & & & \\
\hline 197 & & & & & & & & & & & & & & & & & & & & & & & & & & & & & & & & & & \\
\hline 198 & (1) & & (1) & & & & & & & & & & 1 & 2 & & 1 & & & & & & & & & & 1 & 2 & & & & & & & \\
\hline 199 & 1 & & 1 & 1 & 1 & & (1) & & 1 & 1 & & (1) & 1 & 1 & 1 & 1 & 1 & 10 & 1 & & 1 & 1 & & & 2 & 1 & 1 & (1) & (1) & 1 & & 1 & 1 & \\
\hline 200 & & 1 & & 1 & 2 & 2 & & 1 & 1 & 1 & & & & & & 1 & 1 & 2 & 2 & 2 & 1 & 1 & 1 & & & & & 1 & 1 & 2 & 3 & 2 & \begin{tabular}{|l|}
2 \\
\end{tabular} & 1 \\
\hline 207 & & (1) & (1) & & 1 & 1 & 1 & 1 & 1 & 1 & 1 & & & & & & 1 & (1) & (1) & & & 1 & 1 & 1 & & & & & 1 & 1 & (1) & & (1) & \\
\hline 211 & 3 & 3 & 2 & 2 & 2 & & 1 & & & & & 3 & 3 & 2 & 3 & 2 & & & 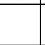 & & & & & & 1 & 1 & 2 & 3 & 2 & & 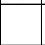 & 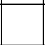 & - & \\
\hline 212 & & 4 & 4 & 4 & 4 & & 4 & 4 & 4 & 4 & 4 & & & & & 4 & 4 & 4 & 4 & 4 & 3 & 4 & 4 & 4 & & & & (1) & 4 & 44 & 4 & 44 & 4 & 4 \\
\hline 213 & & & (1) & & 1 & 1 & 1 & 1 & 1 & 1 & (1) & & & & & & & & 1 & 1 & 1 & 1 & 1 & & & & & (1) & 1 & 1 & & 1 & 1 & \\
\hline 214 & & & (1) & 1 & & & 2 & 2 & 2 & 3 & 3 & & & & & & & & & & & & & 2 & & & & & & & & & & \\
\hline 218 & & & & & & & & & & (1) & & & & & & & & & & & & & & & & & & & & & & & (1) & \\
\hline 226 & & & & & & & & & & & & & & & & & & & & & & (1) & (1) & & & & & 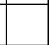 & & & & & & \\
\hline 229 & 3 & 3 & 3 & 3 & 3 & 3 & 4 & 2 & 3 & 3 & & 2 & 2 & 3 & 3 & 3 & 3 & 3 & 2 & 2 & 2 & 2 & 2 & 2 & 2 & 3 & 3 & 3 & 3 & 1 & 2 & 1 & 2 & 2 \\
\hline 230 & & 2 & 2 & 3 & 3 & 3 & 3 & 2 & 3 & 2 & & & & & (1) & 2 & 3 & 3 & 3 & 3 & 3 & & & & & & (1) & 1 & 1 & 1 & 2 & 2 & 3 & 1 \\
\hline 231 & & & & & & & & & & & & & & & & & & & & & & & & & & & & & & & & & & \\
\hline 232 & & & & & & & & & & & & & & & & & & & & & & & & & & 2 & & & & & & & & \\
\hline 233 & & & & & & & & & & (1) & & & & & & & (1) & & & & & & & & & & & & & & & & & \\
\hline 234 & & & & & (1) & & (1) & & & & & & & & & & & & (1) & & & & & & & & & & & & & & & \\
\hline 235 & & (1) & & & & & & & & & & & & & & & & & & & & & & & & & & & & & & & & \\
\hline 236 & & & & & & & & & & & & & & & & & & & & & & & & & & & & & & & & & & \\
\hline
\end{tabular}




\begin{tabular}{|c|c|c|c|c|c|c|c|c|c|c|c|c|c|c|c|c|c|c|c|c|c|c|c|c|c|c|c|c|c|c|c|c|c|c|c|}
\hline \multicolumn{36}{|c|}{ Table 9, 2004-1 } \\
\hline & \multicolumn{8}{|c|}{$\mathrm{A}$} & & & & & $E$ & & & & & & & & & & & & $\mathrm{c}$ & 5 & & & & & & & & D & \\
\hline & 1 & 2 & 3 & & & 6 & 7 & 8 & 1 & 2 & 3 & 4 & 5 & 6 & 7 & 8 & 9 & 10 & 1 & 2 & 3 & 4 & 5 & \begin{tabular}{l|l}
6 \\
\end{tabular} & & & \begin{tabular}{l|l}
9 & 1 \\
\end{tabular} & & 11 & 12 & & 14 & & & 3 \\
\hline 2 & & & & (1) & & & (1) & & & & & & & & & & & & & & (1) & (1) & & & & & & & & & & & & & \\
\hline 4 & & 1 & & (1) & & (1) & 1 & 1 & & & 1 & 1 & (1) & & 1 & 1 & 2 & 1 & & & 1 & & 1 & 1 & 1 & 2 & 2 & & 1 & 1 & 1 & & & & \\
\hline 5 & & & & & & 1 & & 2 & & & & 1 & & & 1 & 1 & 1 & & & & 1 & & 1 & 1 & 2 & 1 & 1 & 1 & 1 & & & & & & (1) \\
\hline 6 & & & & & & & & & & & & & & & & & & & & & & & & & & & & & & & & (1) & & & \\
\hline 7 & & (1) & 1 & (1) & 1 & & & 1 & & & 1 & & & & 1 & 1 & 1 & 1 & & & (1) & & (1) & & 1 & $1]$ & & & 1 & & 1 & & & & \\
\hline 8 & & & & & & & & & & & & & & & & & & & & & & & & & & & & & & & (1) & & & & \\
\hline 10 & & & & & & & & 2 & & & & & & & & & & 1 & & & & & & & & 1 & & 2 & 1 & 1 & 1 & 1 & & & \\
\hline 11 & & & & & & (1) & & 1 & & & & & & & & & & & & & & & & & & & & & & & & & & & \\
\hline 12 & & 1 & (1) & 1 & (1) & 1 & 1 & & & & 1 & 1 & (1) & & 1 & 1 & 1 & & (1) & & 1 & & & 1 & 1 & $1] 1$ & 1 & & & & & & & (1) & (1) \\
\hline 13 & (1) & 1 & 1 & 2 & 1 & 1 & 2 & 2 & & 1 & 1 & 1 & 2 & 2 & 2 & 2 & 2 & 1 & (1) & 1 & 1 & & 1 & 1 & 1 & 2 & 2 & 1 & 1 & 1 & 1 & & 1 & 1 & 2 \\
\hline 14 & & & 1 & 1 & & 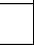 & 1 & & & 1 & & & & & & 4 & & & & 1 & 1 & & & & & & & & & & & & 1 & & \\
\hline 15 & 1 & 1 & 1 & 2 & 1 & 3 & 2 & 3 & & 1 & 1 & 1 & 2 & 2 & 2 & 2 & 3 & 3 & 1 & 1 & 1 & 2 & 2 & 2 & 2 & $23:$ & 3 & 3 & 2 & 2 & 1 & & 1 & 2 & 2 \\
\hline 16 & & & & & & & & (1) & & & & & & & & & 1 & 1 & & & & & & & & 1 & & & & 1 & 1 & 1 & & & \\
\hline 17 & 1 & 1 & 1 & & & 1 & 1 & 1 & & 1 & & (1) & 1 & & 2 & 1 & 1 & & & 1 & & & 1 & 1 & 2 & $2]$ & 1 & 2 & 1 & 1 & 1 & 1 & & 1 & 1 \\
\hline 18 & & & & & & & & & & 1 & & & & & & & & & & & & & & & & & & & & 1 & & & & & \\
\hline 20 & & & & & & (1) & & (1) & & & (1) & & & & & & & & & & & & (1) & & 1 & 1 & & & & & & & & (1) & \\
\hline 22 & & & & & & & & & (1) & & & & & & & & & & 1 & 1 & 1 & & & & & & & & & & & & & & \\
\hline 26 & & & & & & & & & & & & & & & & & & 1 & & & & & 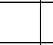 & & & & & & (1) & & 1 & 1 & & & \\
\hline 27 & & & & & & & & & & & & & & & & & & & & & & & & & & & & & & & & & & & \\
\hline 28 & & & & & & & 1 & 1 & & & & & & & 1 & 2 & 1 & 1 & & & & & 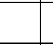 & & 1 & $1]$ & 1 & 2 & & 1 & 1 & 1 & & & \\
\hline 33 & & & & & & & & & & & & & & & & & & & & (1) & & & & & & & & & & & & & & & \\
\hline 39 & & & & & & & & & & & & & & & & & & & & & & & & & & & & & & & & & & & \\
\hline 41 & & & & & & & (1) & & & & & & & & & & & & & (1) & & & . & & | & & & & & & & & & & \\
\hline 43 & & & & & & & & 2 & & & & & & & & & & & & & & & & & & & & & & & & & & & \\
\hline 44 & 2 & 1 & 1 & 1 & & & 1 & & 2 & 2 & 1 & 1 & 1 & 1 & 1 & (1) & 1 & & 2 & 1 & 1 & 2 & 1 & 1 & 1 & 1 & 1 & 1 & & & & & 2 & 1 & 1 \\
\hline 48 & & & & & & & & 1 & & & & & & & & & 2 & 1 & & & & & & & & & 2 & 1 & 1 & 2 & 2 & 3 & & & \\
\hline 53 & & & & & & + & & & & & & & & & & & & & & & & & & & & 1 & & & 2 & \begin{tabular}{l|l}
1 \\
\end{tabular} & & & & & \\
\hline 54 & & & & & & & & & & & & & & & & & & & & & & & & & & & & & & & & & & & (1) \\
\hline 58 & & & & & & & & & & & & & & & & (1) & & & & & & & & & & & & & & & & & & & \\
\hline 60 & 1 & 2 & 1 & 2 & & & & & 2 & 2 & 2 & 1 & 1 & & & (1) & & & 2 & 2 & 2 & (1) & 1 & & (1) & 1 & & & & & & & & 1 & 1 \\
\hline 61 & & & & & & & & & & & & & & & & & & & & & & & & & & & & & & & & & & & \\
\hline 62 & & & & & & & & & & & & & & & & & & & & & & (1) & (1) & & & & & & & & & & & & \\
\hline 63 & 2 & 2 & 3 & 3 & 2 & 2 & 2 & 1 & 2 & 3 & 2 & 2 & 2 & 2 & 2 & 2 & 2 & & 3 & 2 & 2 & 2 & 2 & 1 & 2 & 1 & 2 & 1 & & & & & 1 & 2 & 2 \\
\hline 64 & 1 & & & 1 & 1 & 1 & 1 & & 1 & & & 1 & 1 & & 1 & 1 & & & 2 & 1 & 2 & 1 & 1 & 1 & 1 & 1 & & & & & & & 2 & 1 & 1 \\
\hline 65 & & & & & & & & & & & & & & & & & & & & & & & 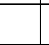 & & & & & & & & & & (1) & & \\
\hline 67 & & & & & & & & & & & & & & & & & & & & & & & (1) & & & & & & & & & & & & \\
\hline 70 & & & & & & & & & & & & & & & & & & & 1 & 1 & & & & & & & & & & & & & & & \\
\hline 71 & & & 2 & & + & & & & 3 & 3 & 2 & & & & & . & & & 3 & & & & & & & & & & & & & & & & \\
\hline 73 & 3 & 3 & 3 & 3 & 3 & 3 & 1 & 2 & 3 & 3 & 3 & 2 & 2 & 2 & 2 & 2 & 1 & & 3 & 3 & 2 & 2 & 2 & 2 & 2 & 3 & 2 & & & & & & 3 & 3 & 3 \\
\hline 74 & & & & & & & & & & & & & & & & & & & & & & & & & & & & & & & & & & & \\
\hline 75 & & & & & & & & & & & & & & & & & & & (1) & & & & & & & & & & & & & & & & \\
\hline 76 & (1) & & & & & & & & (1) & & & & & & . & 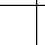 & & & & & & . & . & 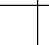 & & & & & & & & & & . & \\
\hline 77 & 2 & 3 & \begin{tabular}{|l|}
3 \\
\end{tabular} & 4 & 4 & 4 & 4 & 2 & 2 & & \begin{tabular}{l|l}
2 \\
\end{tabular} & 4 & 2 & \begin{tabular}{|l|}
3 \\
\end{tabular} & 3 & 2 & 3 & 4 & 3 & 2 & 3 & + & + & + & + & 3 & 3 & 3 & 3 & & & & 2 & 4 & 4 \\
\hline 78 & 3 & & 2 & 3 & 4 & 4 & 4 & 3 & 3 & & & 2 & 3 & 3 & 3 & 2 & 3 & & 3 & & 4 & 3 & 3 & 3 & 3 & 3 & 3 & 3 & & & & & 4 & 3 & 3 \\
\hline 79 & 1 & & & 2 & 2 & 2 & 1 & & 1 & & & 1 & 2 & 2 & 2 & 2 & & & 1 & & 2 & 2 & 2 & 2 & 2 & 1 & & & & & & & 2 & & \\
\hline 80 & & & & & & 2 & & (1) & 1 & & & & & & & & & & 1 & & (1) & & & 2 & 2 & & 1 & 1 & & & & & & & \\
\hline 81 & 1 & 1 & 1 & 2 & & 1 & 1 & & 2 & & & 1 & 1 & 1 & 1 & 1 & 1 & & 1 & & 1 & 2 & 3 & 3 & 2 & 1 & & & & & & & 2 & & 1 \\
\hline 82 & & 3 & 3 & & + & & & & & 2 & & & & & & & & & & & & & & & & & & & & & & & & & \\
\hline 86 & & & & & & & & & & & & & & & & & & & & & (1) & & & & & & & & & & & & & & \\
\hline 87 & & & & & (1) & 2 & & & & 1 & 1 & & & & & & & & & (1) & & & 1 & & & & & & & & & & & & \\
\hline 88 & & & (1) & & & & & & & & (1) & & & & & & & & & & & & & & & & & & & & & (1) & & & \\
\hline 94 & & & & & & & & & & & & & & & & & & & & (1) & & & & & & & & & & & & & & & \\
\hline 99 & & & & & & & & & & & & & & & & & & & & & & & & & & & & & (1) & & (1) & & & & \\
\hline 122 & & (1) & (1) & & 2 & 1 & 2 & 1 & & 1 & 1 & & & & & & & & & 1 & & & (1) & & & & & & & & & & & (1) & \\
\hline 123 & & & (1) & & & 1 & & 1 & 1 & & \begin{tabular}{|l|} 
\\
\end{tabular} & & 1 & & & & & & 1 & 1 & & & 1 & & & (1) & & & & & & & & & (1) \\
\hline
\end{tabular}




\begin{tabular}{|c|c|c|c|c|c|c|c|c|c|c|c|c|c|c|c|c|c|c|c|c|c|c|c|c|c|c|c|c|c|c|c|c|}
\hline \multicolumn{33}{|c|}{ Table 9, 2004-2 } \\
\hline & \multicolumn{11}{|c|}{ D } & \multicolumn{12}{|c|}{$E$} & \multicolumn{9}{|c|}{$\mathrm{F}$} \\
\hline & 4 & 56 & \begin{tabular}{l|l}
6 & 7 \\
\end{tabular} & \begin{tabular}{l|l}
78 & 8 \\
\end{tabular} & & & & & & & & 1 & 2 & 3 & 45 & 56 & & \begin{tabular}{|l|l|}
7 & 8
\end{tabular} & 9 & 10 & 11 & 12 & & 1 & 2 & 3 & 4 & & \begin{tabular}{l|l}
6 & 7
\end{tabular} & 8 & 9 & 10 \\
\hline 2 & & & & 1 & & & & & & & & & & & & & & & & & & & & & & & & & & & & \\
\hline 4 & & & \begin{tabular}{l|l}
211 \\
\end{tabular} & 11 & 2 & 2 & 2 & 2 & 2 & 1 & & & & & 1 & 12 & 25 & \begin{tabular}{l|l|}
2 & 2 \\
\end{tabular} & 2 & 2 & 2 & 1 & 1 & & & & 1 & 1 & 1 & 1 & 2 & 1 \\
\hline 5 & & & 2 & $1]$ & 2 & 2 & 2 & 1 & 1 & 1 & & & & & & 11 & \begin{tabular}{l|l}
1 & 1 \\
\end{tabular} & 1 & 1 & 1 & 1 & & & & & (1) & 1 & & & 1 & & \\
\hline 6 & & & & & & & & & & & (1) & & & & & & & & & & (1) & (1) & 1 & & & & & & & & & \\
\hline 7 & & 11 & \begin{tabular}{l|l}
1 & 1 \\
1
\end{tabular} & 1 & 1 & 1 & 1 & 1 & 1 & 1 & (1) & & & & & 11 & $1[1$ & \begin{tabular}{l|l|}
1 & 1
\end{tabular} & 1 & 1 & 1 & 1 & 1 & & & & (1) & 1 & \begin{tabular}{l|l|}
1 & 1
\end{tabular} & 1 & & 1 \\
\hline 8 & & & & & & & & (1) & & & & & & & & & & 5 & 4 & & (1) & & & & & & & & t & 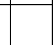 & & \\
\hline 10 & & & \begin{tabular}{l|l}
1 & 1 \\
1
\end{tabular} & $1]$ & 2 & & & 2 & 2 & 3 & 1 & & & & & 1 & $1]$ & \begin{tabular}{l|l|}
1 & 1 \\
\end{tabular} & 2 & 2 & 2 & 2 & 2 & & & & 1 & & \begin{tabular}{l|l|}
1 & 1 \\
\end{tabular} & 2 & 3 & 2 \\
\hline 11 & & & & & 1 & & & & & & & & & & & & & & & & & & & & & & & & & & & \\
\hline 12 & 1 & \begin{tabular}{l|l}
1 & 1 \\
\end{tabular} & \begin{tabular}{l|l}
1 & 1 \\
\end{tabular} & 1 (1 & (1) & & & & & & & & (1) & (1) & (1) & 1 & 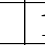 & 1 & & & & & & & & 1 & 1 & 1 & & & & \\
\hline 13 & 1 & 12 & $2 \sqrt{2}$ & 21 & 2 & 2 & & 1 & 1 & 1 & 1 & & & & 1 & 21 & 15 & \begin{tabular}{l|l}
2 & 2 \\
\end{tabular} & 2 & 1 & 1 & 1 & & (1) & & 1 & 1 & 1 & \begin{tabular}{l|l}
1 & 1
\end{tabular} & 1 & 1 & 1 \\
\hline 14 & (1) & (1) & 1) & & & 1 & & & & & & & & & & 1 & & 1 & & & & & & & 1 & & 1 & 1 & 1 & 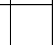 & 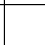 & \\
\hline 15 & \begin{tabular}{|l|l|} 
\\
\end{tabular} & 1 & 2 & 23 & \begin{tabular}{|l|l}
3 \\
\end{tabular} & 3 & 3 & 2 & 2 & 1 & & 1 & 1 & 2 & 2 & 2 & 23 & \begin{tabular}{l|l}
3 & 2 \\
\end{tabular} & 3 & 3 & 2 & 2 & & 2 & 1 & 1 & 2 & 15 & \begin{tabular}{l|l}
2 \\
\end{tabular} & 3 & 3 & \\
\hline 16 & & & & & & & . & 1 & 2 & 1 & 1 & & & & & & & & & (1) & 1 & & 1 & & & & & & (1) & & & \\
\hline 17 & 10 & (1) 1 & \begin{tabular}{l|l}
1 & 1
\end{tabular} & $\begin{array}{lll}1 & 1\end{array}$ & 2 & 2 & 2 & 1 & 1 & 1 & 1 & & & & 1 & 1 1] & 11 & $\begin{array}{lll}1 & 2\end{array}$ & 2 & 2 & & 1 & 1 & & 1 & 1 & 1 & 1 & 2 & 2 & 1 & 1 \\
\hline 18 & & & & & & & & & & 1 & & & & & & & & 1) & 1 & 1 & & & 1 & & & & & & & & & \\
\hline 20 & & $1]$ & \begin{tabular}{l|l}
1 & 1 \\
\end{tabular} & $\begin{array}{lll}1 & 1\end{array}$ & 1 & & & & & & & & & & (1) & (1) & 1 & 1 & (1) & & 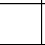 & & & & & 1 & 1 & & & & & \\
\hline 22 & & & & & & & & & & & & & & & & & & & & & & & & & & & & & & & & \\
\hline 26 & & & & & & & & & & (1) & (1) & & & & & & & & 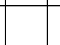 & & (1) & & 1 & & & & & & & & & \\
\hline 27 & & & & & & & & & & & & & & & & & & & & & & & (1) & & & & & & & & & \\
\hline 28 & & & 1 & 1 & 2 & 2 & 2 & 1 & 1 & & & & & & & & 1 & $\begin{array}{lll}1 & 1\end{array}$ & 1 & 1 & & 1 & 1 & & & & & & 1 & 1 & & \\
\hline 33 & & & & & & & & & & & & & & & & & & & & & & & & & & & & & & & & \\
\hline 39 & & & & & & & & & & & & & & & & & & & & & & & 1 & & & & & & & & & \\
\hline 41 & & & & & & & & & & & & & & & & & & & & & & & (1) & & & & & & & & & \\
\hline 43 & & & & 2 & 2 & 1 & & & & & & & & & & & & & 2 & 1 & & & & & & & & & 1 & & & \\
\hline 44 & 1 & 21 & $\begin{array}{lll}1 & 1\end{array}$ & 1 & 1 & 1 & & & & & & 1 & 1 & (1) & 1 & 1 & 1 & 1 & (1) & & & & & 1 & 1 & 1 & 1 & & & & & \\
\hline 48 & & & & 1 & 1 & 2 & 2 & 2 & 2 & 2 & 2 & & & & & & & (1) 1 & 1 & 2 & 1 & 2 & 2 & & & & & & & & & 1 \\
\hline 53 & & & & & 2 & $t$ & + & + & + & + & & & & & & 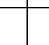 & & & + & 2 & & & 1 & & & & & & & 2 & & + \\
\hline 54 & & & & & & & & & & & & & & & & & & & & & & & & & & & & 1 & & (1) & (1) & \\
\hline 58 & & 1 & & & & & & & & & & & & & & (1) ( & & & & & & & & & & & & & \begin{tabular}{l|l} 
1) & 1
\end{tabular} & & & \\
\hline 60 & & 1 & & 1) & & & & & & & & & & & & 21 & \begin{tabular}{l|l}
1 & 1
\end{tabular} & 1 & & & & & & 2 & & & 1 & 1 & & & & \\
\hline 61 & & & & & & & & & & & & & & & & & & & & & & (1) & & & & & & & & & & \\
\hline 62 & & & & & & & & & & & & & & & & & & & & & & & & & & & & & & & (1) & \\
\hline 63 & \begin{tabular}{|l|}
2 \\
\end{tabular} & 22 & 25 & 25 & 2 & 2 & 20 & (1) & & & & & 1 & 1 & 2 & 25 & 25 & $\begin{array}{ll}2 & 2\end{array}$ & 2 & & & & & 2 & 1 & 1 & 2 & 1 & $\begin{array}{ll}1 & 2\end{array}$ & 1 & & \\
\hline 64 & 1 & 12 & 22 & 2 & & & & & & & & 1 & 1 & & 1 & $1]$ & & & & & & & & 1 & 1 & 1 & 1 & & & & & \\
\hline 65 & & & & & & & & & & & & & & & & & & & & & & & & & & & & & & & & \\
\hline 67 & & & & & & & & & & & & & & & & & & & & & & & & & & & & & & & & \\
\hline 70 & & & & & & & & & & & & & & & & & & & & & & & & & & & & & & & & \\
\hline 71 & & & & & & & & & & & & & & & & & & & & & & & & & & 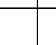 & & & & & & \\
\hline 73 & \begin{tabular}{|l|}
3 \\
\end{tabular} & 35 & 33 & 33 & 35 & & & & & & & 3 & \begin{tabular}{l|l|}
3 \\
\end{tabular} & 2 & 3 & 23 & $\begin{array}{lll}3 & \end{array}$ & 3 & & & & & & 3 & 3 & 3 & 3 & 2 & & & & \\
\hline 74 & & & & & & & & & & & & & & & & & & (1) & & & & & & & & & & & & & & \\
\hline 75 & & & & & & & & & & & & & & & & & & & & & & & & & & & & & & & & \\
\hline 76 & & & & & & & & & & & & & & & & & & & & & & & & (1) & & & & & & & & \\
\hline 77 & 4 & 43 & 33 & $3:$ & 2 & 2 & 2 & & & & & 3 & 4 & 4 & 3 & 3 & 45 & 2 & & 1 & & & & 3 & 2 & 4 & 4 & 3 & 3 & 2 & & \\
\hline 78 & 4 & 35 & $\begin{array}{lll}3 & 1\end{array}$ & 15 & 23 & 3 & 3 & & & & & \begin{tabular}{|l|}
3 \\
\end{tabular} & 3 & 3 & \begin{tabular}{|l|}
3 \\
\end{tabular} & 11 & 1 & & & & & & & 4 & 4 & 3 & 3 & & & & & \\
\hline 79 & & (1) & & & & & & & & & & 2 & 1 & (1) & & & & & & & & & & 2 & 2 & & 1 & & & & & \\
\hline 80 & & & 2 & 2 & 1 & & & & & & & (1) & & & & 1 & 1 & & & & & & & & & & & & & & & \\
\hline 81 & & & 2 & 2 & & & & & & & & 1 & 1 & & 1 & 1 & 1 & & & & & & & 1 & 1 & & 1 & & & & & \\
\hline 82 & & 2 & & & & & & & & & & & & & & & 6 & $2+$ & & & & & & & & & 2 & 2 & & & & \\
\hline 86 & & & & & & & & & & & & & & & & 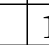 & 1 & & & & & (1) & & & & & & & (1) & & & (1) \\
\hline 87 & & & & & & & 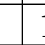 & 1 & 1 & & 1 & & & & & 1 & 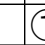 & (1) & & 1 & & 1 & 1 & & & & & 1 & 1 & (1) & & \\
\hline 88 & & & & & & & & & & & & & & & & 1 & 1 & 1 & & (1) & & & & & & & & 1 & 1 & 1 & & \\
\hline 94 & & & & & & & & 1) & & & & & & & & & & & (1) & & (1) & & (1) & & & & & (1) & & & & \\
\hline 99 & & & & & & & ( & 1) & & & & & & & & & & 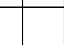 & 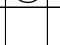 & & & (1) & (1) & & & & & & 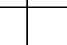 & (1) & 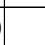 & \\
\hline 122 & (1) & 1 & 1 & 1 & 1 & & & & & & & & (1) & & (1) & 1 & 1 & $\begin{array}{lll}2 & 1\end{array}$ & 1 & 1 & & & & & & & 1 & 2 & $\begin{array}{ll}2 & 2\end{array}$ & 2 & 2 & 1 \\
\hline 123 & & & & & & & & & & & & & & & & & & & 1 & & & 1 & & & & & & & & & & 1 \\
\hline
\end{tabular}




\begin{tabular}{|c|c|c|c|c|c|c|c|c|c|c|c|c|c|c|c|c|c|c|c|c|c|c|c|c|c|c|c|c|c|c|c|c|c|c|}
\hline \multicolumn{35}{|c|}{ Table 9, 2004-3 } \\
\hline & \multicolumn{8}{|c|}{$\mathrm{A}$} & \multicolumn{9}{|c|}{$\mathrm{B}$} & \multicolumn{14}{|c|}{$\mathrm{C}$} & \multicolumn{3}{|c|}{$\mathrm{D}$} \\
\hline & 125 & & 3 & $4[1$ & & 6 & 7 & 8 & 1 & 2 & 3 & 4 & & \begin{tabular}{l|l}
6 & 7 \\
\end{tabular} & 8 & 9 & 10 & 01 & 2 & 3 & 4 & 5 & 6 & & 8 & 9 & & 11 & 12 & & 14 & & & 3 \\
\hline 124 & $1[1]$ & & 1 & 2 & 1 & 1 & 1 & 1 & 1 & 1 & 1 & 1 & 16 & 1) 1 & 1 & 1 & & 1 & 1 & 1 & & 2 & 1 & 1 & 1 & 1 & 1 & & & & & 1 & & 1 \\
\hline 129 & & & & & & & & & & & & & & & & & & & & & & & & & & & & & & & & & & \\
\hline 130 & & & & & & & & (1) & & & & & & 1 & 1 & & 1 & & & & & & & (1) & & (1) & 1 & 1 & 1 & 1 & 1 & & & \\
\hline 131 & & & & & & & & (1) & & & & & & & & & (1) & & & & & & 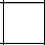 & 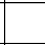 & & & & & & (1) & & & & \\
\hline 132 & & 1 & 1 & 1 & 2 & 1 & 2 & 2 & & 1 & 1 & 1 & 1 & \begin{tabular}{l|l}
2 & 1
\end{tabular} & 12 & 2 & 2 & & 1 & 1 & & 2 & 1 & 1 & 2 & 2 & 2 & 2 & 1 & & & (1) & & 1 \\
\hline 141 & & & & & & & & & & & & & & & & & & & & & & & & (1) & 1 & & & & (1) & 1 & 1 & & & \\
\hline 142 & & & (1) & & & & 1 & 1 & & 1 & 1 & 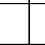 & 1 & \begin{tabular}{l|l}
1 & 1
\end{tabular} & 1 & 1 & 1 & & & & & 1 & & 1 & 1 & 1 & 1 & 2 & 1 & 1 & 1 & & & \\
\hline 143 & & & & & (1) & & & & & & & & & & & & & & & (1) & & & & & & & (1) & 1 & 1 & 1 & 1 & & & \\
\hline 145 & & & & 1 & 1 & 1 & 1 & 1 & & & & 1 & 1 & & & & & & & & & & & & 1 & & & (1) & & & & & & \\
\hline 150 & & & 2 & & & & & & & & & & & & & & & & & & & & & & & & & & & & & & & \\
\hline 158 & & & & & & & (1) & & & & & & & & & & & & & 1 & & 1 & & & & & & & & & & & & \\
\hline 161 & & & & & & & & & & & & & & & & & & & & & & & & & & & & & & & & & & \\
\hline 176 & & & & & (1) & & & & & & & & & & & & & & & & & & & & & & & & & & & & & \\
\hline 180 & & & & & & & & & & & & & & & & & & & & & & & & & & & & & & & & & & \\
\hline 181 & & & (1) & & & & & & & & & & & & & & & & & & & & & & & & & & & & & & & \\
\hline 182 & & & & (1) & (1) & & & & & (1) & & & 1)( & 1) & & & & (1) & & & & & & & & & & & (1) & & & & & \\
\hline 183 & & & & & & & & & (1) & & & & 1) & & & & & & & & & & & & & & & & & & & & & \\
\hline 197 & & & & & & & & & & & & & & & & & & & & & & & & & & & (1) & & & & & & & \\
\hline 198 & & & & 1 & & 1 & 1 & & & & & & 2 & 25 & & & & & & 1 & 1 & 2 & 1 & 1 & 1 & & & & & & & 1 & 1 & 2 \\
\hline 199 & 1 & 1 & 1 & 1 & 1 & 1 & 1 & 1 & & (1) & & 1 & 2 & 1 & 21 & 1 & & (1) & 1 & 1 & & 2 & 2 & 2 & 2 & 1 & 1 & & & & & 1 & 2 & 1 \\
\hline 200 & & & & & & 1 & & 2 & & & 1 & & 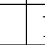 & $1]$ & 1 & 1 & 1 & & & 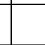 & & 1 & & 2 & 1 & 2 & 1 & 1 & 1 & 1 & 1 & & & \\
\hline 207 & & & & & 1 & & & & & & (1) & & & & & & 1 & & & 1 & & 1 & & 1 & (1) & 1 & 1 & 1 & 1 & 1 & 1 & & & \\
\hline 211 & & & & 2 & 2 & 2 & 3 & 3 & & 2 & & 1 & 2 & 2 & 2 & 3 & & 1 & & 2 & 2 & 2 & 2 & 3 & 2 & 3 & & & & & 1 & 3 & 2 & 3 \\
\hline 212 & & 4 & 4 & & 4 & 3 & 3 & 4 & & 4 & 4 & 3 & 2 & 3 & $\begin{array}{l}14 \\
\end{array}$ & 3 & & 4 & 4 & 4 & & -1 & 44 & 44 & 44 & 44 & & 4 & 4 & 4 & 3 & & & 4 \\
\hline 213 & & & & & 1 & $\begin{array}{ll}1 \\
\end{array}$ & 2 & 1 & & & (1) & (1) & & & & & 2 & & & & & 1 & & & & (1) & & & 1 & 1 & & & & \\
\hline 214 & & & & & & & & & & & & & & & & & 1 & & & 1 & & (1) & 1 & 1 & 1 & 2 & 2 & 2 & 3 & 3 & 4 & (1) & & \\
\hline 218 & & & & & & & & & & & & & & & & & & & & & & & & & & & & & & & & & & \\
\hline 220 & & & & & & & & & & & & & & & & & & & & & & & & & & & & & & & & & & \\
\hline 221 & & & & & & & & & & & & & & & & & & & & & & 1 & & & (1) & & & & & & & & & \\
\hline 226 & & & & & & & & & & & & & & & & & & & & & & & & & & & & & & & & & & \\
\hline 229 & 3 & & 3 & 2 & 2 & 3 & 3 & 4 & & 3 & 3 & 3 & 2 & 23 & 3 & 4 & 4 & +2 & 3 & 2 & 2 & 3 & 3 & 3 & 3 & 4 & 4 & 4 & 3 & 1 & & 3 & 3 & 3 \\
\hline 230 & & & & & & (1) & & 3 & & & & & & 1 & 2 & 3 & 2 & & & & & (1) & & 1 & 2 & 3 & 3 & 2 & 2 & & & & & (1) \\
\hline 232 & & & + & & + & + & + & + & + & & & + & & + & + & + & + & $-1+$ & + & + & & + & + & + & + & + & + & & & & & + & + & + \\
\hline 233 & & & & & & & & & & & & & & & & & & & & & & & & & & & & & (1) & & & & & \\
\hline 234 & & & & & & & & & & & & 1 & & & & & & & & & & & & & & & & & & & & & & \\
\hline 235 & & & & & & & & & & & & & & & & & & & & & & & & & & & & (1) & & & & & & \\
\hline
\end{tabular}




\begin{tabular}{|c|c|c|c|c|c|c|c|c|c|c|c|c|c|c|c|c|c|c|c|c|c|c|c|c|c|c|c|c|c|c|c|c|c|c|c|}
\hline \multicolumn{36}{|c|}{ Table 9, 2004-4 } \\
\hline & \multicolumn{12}{|c|}{, } & \multicolumn{13}{|c|}{$E$} & \multicolumn{10}{|c|}{$\mathrm{F}$} \\
\hline & 4 & 5 & 6 & 7 & 8 & & 10 & & & 21 & & & 1 & 2 & 3 & 4 & 5 & 6 & 7 & 8 & 9 & & & & 13 & 1 & 2 & 3 & 4 & & \begin{tabular}{|c|}
6 \\
\end{tabular} & & 8 & 9 & 10 \\
\hline 124 & 1 & 1 & 1 & 1 & 1 & 1 & 1 & & & & & & & 1 & 1 & 1 & 1 & 1 & 1 & 1 & 1 & & & & & & 1 & & & & & 1 & & & \\
\hline 129 & & & & & (1) & & & & & & & & & & & & & & & & & & & & & & & & & & & & (1) & & (1) \\
\hline 130 & & & & & 1 & 1 & 1 & 1 & 1 & 2 & & & & & & & & & (1) & & (1) & 1 & 1 & 1 & 1 & & & & & & & & & & 1 \\
\hline 131 & & & & & (1) & & & & & & & & & & & & & & & & (1) & & (1) & & 1 & & & & & & & 1 & 1 & 1 & 1 \\
\hline 132 & 1 & 2 & 2 & 2 & 2 & 3 & 2 & 2 & 2 & 1 & & & & & & & 2 & 2 & 2 & 2 & 2 & 2 & 2 & 1 & & (1) & & 1 & 1 & 2 & 2 & 2 & 2 & 2 & 2 \\
\hline 141 & & & & & 1 & 1 & 1 & & 1 & & 1 & & & & & & & 1 & 1 & (1) & & & (1) & 1 & & & & & & & 1 & 1 & & 1 & 1 \\
\hline 142 & (1) & 1 & & 1 & 1 & 1 & 1 & 2 & 1 & & & & & & & & 1 & 1 & 1 & 1 & (1) & 1 & 1 & 1 & 2 & & & 1 & 1 & 1 & 2 & 2 & 2 & 2 & 1 \\
\hline 143 & & & 1 & (1) & & & & (1) & (1) & 1 & & & & & & & & & (1) & & & & 1 & & 1 & & & & & & 1 & & & & 1 \\
\hline 145 & & & & & & 1 & & & & & & & & & & & & & & 1 & & & 1 & & & & & & & & & & 1 & 1 & \\
\hline 150 & & & & & & & & & & & & & & & & & & & & & & & & & & & & & & & & & & & \\
\hline 158 & & 1 & \begin{tabular}{|l|} 
\\
\end{tabular} & & & & & & & & & & & & & & & & & & & & & & & & & & & & & & & & \\
\hline 161 & & & & & & & & & & & & & & & & & & & & & & & & & & & & & & & 1 & & & & \\
\hline 176 & & & & & & & & & & & & & & & & & & & & & & & & & & & & & & & & & & & \\
\hline 180 & & & & & & & & & & & & & & & & & & & & & & & & & & & & & & & & & 1 & & \\
\hline 181 & & & & & & & & & & & & & & & & & & & & & & & & & & & & & & & & & & (1) & \\
\hline 182 & & & & & & (1) & & & & & & & & & & & & (1) & & 1 & 1 & & & & & & 1 & & & & 1 & (1) & (1) & & \\
\hline 183 & & & & & & & & & & & & & & & & & & & & & & & & & & & & & & & & & & & \\
\hline 197 & & & & & & & & & & & & & & & & & & & (1) & & & & & & & & & & & & & & & & \\
\hline 198 & 1 & 1 & 1 & (1) & & & & & & & & & & 2 & 2 & & & & & & & & & & & 2 & 1 & 2 & (1) & & & & & & \\
\hline 199 & 1 & 1 & 1 & 1 & & 1 & 1 & 1 & 1 & 1 & & & & 1 & 1 & & 1 & 1 & 1 & 1 & 1 & 1 & & & & 2 & 1 & 1 & 1 & 1 & 1 & 1 & 1 & & \\
\hline 200 & & (1) & 1 & 1 & 1 & 2 & 2 & 1 & 2 & 1 & 1 & 1 & & & & & 1 & 1 & 2 & 3 & 2 & 2 & 2 & 1 & 1 & & & & 2 & 2 & 2 & 3 & 3 & 2 & 2 \\
\hline 207 & & & \begin{tabular}{|l|} 
\\
\end{tabular} & & 1 & & 1 & 1 & 1 & 1 & 1 & 1 & & & & & & 1 & 1 & 1 & 1 & 1 & 1 & 1 & 1 & & & & & 1 & 1 & 1 & \begin{tabular}{|l|} 
\\
\end{tabular} & 1 & 1 \\
\hline 211 & 3 & 3 & 2 & 2 & & & & & & & & & 3 & 4 & 3 & 3 & & & 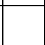 & & & & & & & & 3 & 4 & 2 & & 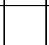 & 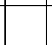 & 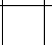 & 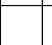 & \\
\hline 212 & & 4 & 4 & 4 & 4 & & & 4 & 4 & 4 & 43 & 36 & & & & & 4 & 4 & 4 & 4 & 4 & 3 & 4 & 4 & 4 & (1) & & & & 4 & \begin{tabular}{|l|} 
\\
\end{tabular} & 4 & 4 & 4 & 4 \\
\hline 213 & & 1 & 1 & & 1 & 1 & 2 & 1 & 1 & & & & & & & & & 1 & 1 & 2 & 1 & 2 & & 1 & & & & & & 1 & 1 & 1 & 1 & 1 & \\
\hline 214 & & (1) & (1) & & & (1) & (1) & 1 & 3 & 4 & 4 & 4 & & & & & & & & & & & & & 2 & & & & & & & & & & \\
\hline 218 & & & & & & & & & & & & & & & & & & & & & & (1) & & & & & & & & & & & & & (1) \\
\hline 220 & & & & & & & & & & & & & & & & & & & & & & & & & & & & & & (1) & & & & (1) & \\
\hline 221 & & & & & & & & & & & & & & & & & & (1) & & & & & & & & & & & & & & & & & \\
\hline 226 & & & & & & & & & & & 0 & 1) & & & & & & & & & & & & & (1) & & & & & & & & & & \\
\hline 229 & 3 & 3 & 3 & 4 & 4 & 4 & 4 & 2 & 2 & 2 & & & 2 & 3 & 3 & 3 & 3 & 3 & 4 & 2 & 2 & 2 & 2 & 2 & 2 & 3 & 3 & 3 & 3 & 3 & 1 & 1 & 1 & & 1 \\
\hline 230 & & 2 & 2 & 3 & \begin{tabular}{|l|}
3 \\
\end{tabular} & 3 & 3 & 2 & 2 & 1 & & & & & & & 2 & 3 & 3 & 3 & 3 & 3 & & & 1 & & & & 1 & 1 & 1 & 2 & 2 & 2 & 2 \\
\hline 232 & + & & + & + & + & + & + & + & & + & & & & & + & + & + & + & + & + & & & & & & & + & + & + & & & & & & \\
\hline 233 & & & & & & & & & & & & & & & & & & & & & & & & & & & & & & & & & & & \\
\hline 234 & & & & & & & & & & & & & & & & & & & & & & & & & & & & & & & & & & & \\
\hline 235 & & & & & & & (1) & & & & & & & & & & (1) & & & & & & & & & & & & & & & & (1) & & \\
\hline
\end{tabular}




\begin{tabular}{|c|c|c|c|c|c|c|c|c|c|c|c|c|c|c|c|c|c|c|c|c|c|c|c|c|c|c|c|c|c|c|c|c|c|c|}
\hline \multicolumn{35}{|c|}{ Table 9, 2005-1 } \\
\hline & \multicolumn{7}{|c|}{$\mathrm{A}$} & & \multicolumn{9}{|c|}{$\mathrm{B}$} & & & & & & & & C & & & & & & & & D & \\
\hline & 12 & 2 & & 4 & & 6 & $7 \mid 8$ & $\begin{array}{ll}31 \\
\end{array}$ & \begin{tabular}{l|l}
12 \\
\end{tabular} & 3 & 4 & 5 & 6 & 7 & 8 & 9 & 10 & 1 & 2 & 3 & 4 & 5 & 6 & 7 & & 9 & & 11 & 12 & & 14 & & & 3 \\
\hline 2 & & & & (1) & & & & & & & & & & & & & & & & (1) & & & & & & & & & & & & & & \\
\hline 4 & & 1 & 1 & & & & 1. & 1 & & (1) & & & & 1 & 1 & 1 & 2 & & & & & & & 1 & & 1 & 1 & 1 & 1 & 1 & & & & \\
\hline 5 & & (1) & & & & & 2 & 2 & & & (1) & & & & 1 & 2 & 1 & & & & & (1) & & & (1) & 2 & 1 & (1) & 1 & & & & & \\
\hline 6 & & & & & & & & 1) & & & & & & & & & & & & & & & & 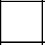 & 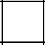 & 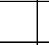 & & & & & 1 & & & \\
\hline 7 & & & & & & & & 1 & $1 \mid 1$ & 1 & & & & & & 1 & 2 & (1) & & & & (1) & & & 1 & 1 & 1 & 1 & 1 & 1 & 1 & & & \\
\hline 8 & & & & & & & & & & & & & & & & & (1) & & & & & & & & & & & & & & & & & \\
\hline 10 & & & & & & & 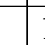 & 1 & & & & & & & & (1) & 1 & & & & & & & & & & & (1) & & & & & & \\
\hline 11 & & & & & & & & & & & & & & & & & & & & & & & & & & & & & & & & & & \\
\hline 12 & (1) 1 & $1]$ & 1) & & & 1) & & 1 & $1 \mid 1$ & 1 & 1 & 1 & & & 1 & 1 & & 1 & 1 & & & 1 & & 1 & 1 & 1 & & & & & & & & \\
\hline 13 & $2[1$ & 1 & 1 & 2 & 2 & 1 & $2[5$ & $2[1$ & \begin{tabular}{l|l}
1 & 1 \\
1
\end{tabular} & 2 & 2 & 2 & 2 & & 1 & 2 & 1 & 1 & 1 & 1 & 1 & 1 & & 2 & 2 & 2 & 2 & 1 & 1 & 1 & 2 & & 1 & 1 \\
\hline 14 & & & 1 & & & & 1 & 71 & \begin{tabular}{l|l}
1 & 1 \\
\end{tabular} & & & & -1 & & 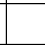 & 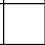 & & & (1) & & & - & & $\square$ & & 1 & & 1 & & & & 1 & & \\
\hline 15 & 11 & 1 & 1 & 1 & & 1 & 1 & 3 & 1 & 1 & 1 & 1 & 1 & 2 & 2 & 3 & 2 & & & & 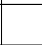 & & 1 & 2 & 2 & 2 & 2 & 2 & 1 & & 1 & & (1) & 1 \\
\hline 16 & & & & & & & & & & & & & & & & (1) & 1 & & & & & & & & & & & & 1 & 1 & 2 & & & \\
\hline 17 & 1 & 1 & 1 & (1) & 1 & & 1 & 1 & & 1 & 1 & 1 & & & 1 & 1 & 2 & & & & 1 & & & (1) & 1 & 1 & 2 & 1 & 1 & 1 & 1 & & & \\
\hline 18 & & & (1) & & & & & & & & & & & & (1) & & & & & & & & & & & & & 1 & & 1 & & & & \\
\hline 19 & & & & & & & & & & & & & & & & & & & & & & & & & & & & & & & & & & \\
\hline 20 & 1 & 1 & 1 & & & & & & & 1 & & & & & & & & & & & & (1) & & & & & & & & & & (1) & & \\
\hline 21 & & & 1 & & & & 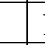 & 1 & & & & & & & & & & (1) & & & 1 & & & & & & & & & & & & & \\
\hline 22 & & & 1 & & & & & & 1 & & & & & & & & & & 1 & & & & & & & & & & & & & & & \\
\hline 26 & & & & & & & & & & & & & & & & & (1) & & & & & & & & 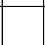 & & & & (1) & 1 & & & & \\
\hline 28 & & & & & & & 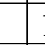 & 1 & & & & & & & & 1 & 2 & & & & & & & & 1 & 2 & 1 & 1 & & & & & & \\
\hline 29 & & & & & & & & & & & & & & & & & & & & & & & & & & & & & & & & & & \\
\hline 38 & & & & & & & & (1) & 1) & & & & & & & & & & & & & & & & & & & & & & & & & \\
\hline 41 & & & & & & & & & & & & & & & & & & & & & & & & & & & & & & & & & & \\
\hline 43 & & & + & & 1 & & & & & & & & & & & & & & & & & & & & & & & & & & & & & \\
\hline 44 & 22 & 2 & 1 & & 1 & 1 & & 2 & $\begin{array}{lll}2 & 1\end{array}$ & 11 & 1 & 1 & 1 & & & & & 1 & 1 & 1 & 2 & 1 & & 1 & & & & & & & & 1 & 1 & 1 \\
\hline 48 & & & & & & & & 2 & & & & & & & & 2 & 22 & & & & & & & & & 3 & 2 & 1 & 2 & 2 & 2 & & & \\
\hline 49 & & & & & & & & & & & & & & & & & & & & & & & & & & & & & & & + & & & \\
\hline 53 & & & + & + & & & & + & & & & & & & & & & & & & & & & & & & & & & & & & & \\
\hline 54 & & & & & & & & & & & & & & & & & & & & & & & & & & & & & & & & & & \\
\hline 58 & & & 1) & & & & & & & (1) & & & & & & & & & & & & & & & & & & & & & & & & \\
\hline 60 & 12 & 2 & 1 & & & & & 2 & $\begin{array}{lll}2 & 1 \\
2\end{array}$ & 1 & 1 & & & & & & & 1 & 2 & 1 & 1 & & & & & & & & & & & 1 & & 1 \\
\hline 63 & 3 & 2 & 2 & 2 & 2 & 1 & 1 & 12 & \begin{tabular}{l|l}
2 & 1
\end{tabular} & 12 & 2 & 2 & 2 & 2 & 1 & 1 & & 2 & 2 & 2 & 2 & 2 & 2 & 1 & 2 & 2 & 1 & & & & & 2 & 2 & 2 \\
\hline 64 & 1 & & 1 & & 1 & & 1 & 7 & 1 & & 2 & 1 & 1 & 1 & (1) & & & 2 & & 1 & 1 & 1 & 1 & 1 & & & & & & & & 2 & 2 & 1 \\
\hline 65 & (1) & & & & & & & & & & & & & & 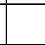 & & & & & & & & & & & & & & & & & & & \\
\hline 71 & & & 1 & & & & & & & & & & & & & & & & & & & & & & & & & & & & & & & \\
\hline 73 & $\begin{array}{ll}36 \\
\end{array}$ & 2 & 2 & 2 & 1 & (1) & & 3 & $\begin{array}{ll}3 & 2\end{array}$ & 22 & 2 & 1 & & 1 & (1) & & & 2 & & 2 & 2 & 2 & 3 & 3 & 2 & & & & & & & 2 & 2 & 1 \\
\hline 77 & 3 & \begin{tabular}{l|}
3 \\
\end{tabular} & 4 & 4 & 4 & 4 & 4 & +5 & $3+$ & +3 & 3 & 3 & 3 & 3 & 3 & & & 4 & 3 & 3 & 4 & 3 & 3 & 3 & 2 & & 3 & & & & & 3 & 4 & 4 \\
\hline 78 & 4 & & 3 & 3 & 4 & 3 & 2 & $2:$ & \begin{tabular}{l|l}
3 \\
\end{tabular} & 1 & 3 & 3 & 3 & 3 & 3 & 2 & & 3 & & 4 & 4 & 3 & 3 & 3 & 2 & 2 & 3 & & & & & 44 & 3 & 3 \\
\hline 79 & 1 & & & & & & & 7 & 1 & & & 2 & 1 & & & & & 2 & & 1 & 3 & & 1 & & & & & & & & & 2 & & \\
\hline 80 & (1) & & & & & & & & & & 1 & 1 & & & & & & (1) & & & & 1 & & & & & & & & & & & & \\
\hline 81 & 2 & & 2 & 1 & 1 & 1 & 1 & 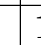 & 1 & & 1 & 1 & 1 & 2 & 1 & & & & & & 1 & 1 & 2 & 2 & 1 & & & & & & & 1 & & 1 \\
\hline 84 & & & & & & & & & & & & & & & & & & & & & & & & & & & & & & & & & & \\
\hline 86 & & & & & & & & & & & 1 & & & & & & & & & & & & & & & & & & & & & & & \\
\hline 87 & & 2 & & (1) & & & & & & 1) 1 & & & (1) & & & & 1 & & & & & & & & & & & & & 1 & & & & 1 \\
\hline 88 & & & & & & & & & & & & & & & & & & & & & & & & & & & & & & & & & & \\
\hline 91 & & & & & & & & & & & & & & & & & & & & & & & & & & & & & & & & & & \\
\hline 94 & & & & & & & & 1 & 1) & & (1) & & & & & & 1 & & & & & & & & & & & & & & & & & \\
\hline 101 & & & & & & & & 1) & & & & & & & & & & & & & & & & & & & & & & & & & & \\
\hline 122 & & 1 & 1 & & 1 & 1 & & & & & 1 & & & & & & & & 1 & & & & & & & & & & & & & & & \\
\hline 123 & & 1 & 1 & & & & & & 1 & 1 & 1 & & & & & & & & 1 & & & & & & & & & & & & & & & \\
\hline 124 & $1]$ & 1 & 1 & 2 & 1 & 1 & 1 . & 1 & \begin{tabular}{l|l}
1 & 1
\end{tabular} & \begin{tabular}{l|l}
1 & 1
\end{tabular} & 12 & 1 & 1 & 1 & 1 & & & 1 & 1 & & & 1 & 1 & 1 & & & & & & & & & & 1 \\
\hline 130 & & & & & & & $t$ & 1 & & & & & & & & & & & & & & & & & & & & (1) & & & & & & \\
\hline 131 & & 1 & & & (1) & & & (1) & & & & & & & & & & & & & & & & & & & & & & & & & & \\
\hline 132 & & & 1 & & 2 & 1 & & 3 & & $\begin{array}{lll}1 & 1\end{array}$ & 11 & 2 & & 2 & 2 & 2 & 2 & & 1 & & & 1 & & 1 & 1 & 3 & 2 & 1 & 1 & & & & & 1 \\
\hline
\end{tabular}




\begin{tabular}{|c|c|c|c|c|c|c|c|c|c|c|c|c|c|c|c|c|c|c|c|c|c|c|c|c|c|c|c|c|c|c|c|c|c|c|}
\hline \multicolumn{35}{|c|}{ Table 9, 2005-2 } \\
\hline & \multicolumn{11}{|c|}{ D } & \multicolumn{13}{|c|}{$E$} & & & & & $\mathrm{~F}$ & & & & & \\
\hline & 4 & $5 \mid$ & 6 & 7 & 8 & & & & 12 & 13 & & 1 & 2 & 3 & 4 & 5 & 6 & 7 & 8 & 9 & 10 & & 12 & 13 & 1 & 2 & 3 & 4 & & & & 89 & \begin{tabular}{l|l}
9 & 10 \\
\end{tabular} & \\
\hline 2 & & (1) & & & & & & & & & & & & & & & & & & & & & & & & & & & & 1 & & & & \\
\hline 4 & & & 1 & 1 & 1 & 1 & 1 & 1 & 1 & 1 & & & & & 1 & 1 & 1 & 1 & 1 & 2 & 2 & 1 & & & & & 1 & 1 & 1 & 1 & 1 & & \begin{tabular}{l|l}
1 & 1 \\
\end{tabular} & \\
\hline 5 & & 1 : & 1 & 1 & 1 & 1 & 2 & 1 & 1 & 1 & & & & & & 1 & & 1 & 1 & 1 & 1 & 1 & (1) & & & & & & & & 1 & 1 & 1 & \\
\hline 6 & & & & & & & & & & & & & & & & & & & & & & 1 & & (1) & & & & & & & & & & \\
\hline 7 & & 1 & 1 & 1 & 1 & 1 & 1 & 1 & 1 & 1 & 1 & & & & & & & 1 & 1 & 1 & 1 & 1 & & 1 & & & & (1) & 1 & & 1 & 1 & 1 & \\
\hline 8 & & & & & & & & 1 & & & & & & & & & & & & (1) & & & & & & & & & & & & & & \\
\hline 10 & & & & & & & 1 & 2 & 1 & 1 & 1 & & & & & & & & & 1 & 2 & 1 & 2 & 2 & & & & & & & & 1 & \begin{tabular}{l|l}
1 & 1
\end{tabular} & \\
\hline 11 & & & & & & & & & & & & & & & & & & & & (1) & & & & & & & & (1) & & & & & & \\
\hline 12 & & 1 : & 1 & 1 & & (1) & & & & & & & & & 1 & 1 & 1 & 1 & 1 & & & & & & & & (1) & 1 & 1 & & & & & \\
\hline 13 & & 1 & 2 & 2 & 2 & 2 & 2 & 2 & 2 & 2 & 1 & & & & & 1 & 1 & 2 & 1 & 3 & 2 & 1 & 1 & 1 & & & & 1 & 1 & 1 & 1 & 2 & \begin{tabular}{l|l}
2 & 1 \\
\end{tabular} & \\
\hline 14 & & & & & 1 & 1 & & (1) & & & & & & & 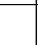 & & 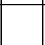 & & 1 & & & & & & \begin{tabular}{|l|l|} 
& \\
\end{tabular} & 1 & & & & & & & & \\
\hline 15 & 1 & & 2 & 2 & 2 & 2 & 3 & 3 & 3 & 1 & & & & 2 & 1 & 3 & 1 & 2 & 2 & 2 & 2 & 2 & 3 & \begin{tabular}{l|l}
2 \\
\end{tabular} & 1 & $\begin{array}{ll}1 \\
\end{array}$ & 1 & 1 & 2 & 2 & 32 & 2 & \begin{tabular}{l|l}
3 & 3 \\
\end{tabular} & \\
\hline 16 & & & & & & & & 1 & 1 & 1 & 2 & & & & & & & & & & 1 & 1 & (1) & 2 & & & & & & & & & & \\
\hline 17 & & & 1 & 1 & 1 & 2 & 1 & 1 & 1 & 1 & 1 & & & & 1 & 1 & 1 & 1 & 1 & 2 & 2 & & 1 & & & 1 & (1) & 1 & & & 1 & 1 & 1 & \\
\hline 18 & & & & & & & & & & 1 & & & & & & & & & & 1 & & & 1 & & & & & & 1 & & & & & \\
\hline 19 & & & & & & & & & & & & & & & 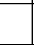 & & & & & (1) & & & & & & & & & & & & & & \\
\hline 20 & & & & & & 1 & 1 & & & & & & & & 1 & 1 & 1 & 1 & & & & & & & & & & 1 & & & & & & \\
\hline 21 & & & & & & & & & & & & & & & & 1 & & & & 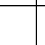 & & & & & & & & & 1 & & & 1 & & \\
\hline 22 & & 1 & & & & (1) & & & & & & & & & & (1) & & & & & & & & & & & & & & & & & & \\
\hline 26 & & & & & & & & & 1 & (1) & & & & & & & & & & & 1 & & (1) & 1 & & & & & & & & & & \\
\hline 28 & & & & 1 & 1 & 2 & 1 & 2 & 1 & & & & & & & & (1) & 1 & 1 & 1 & 1 & & 1 & & & & & & & & & & & \\
\hline 29 & & & & & (1) & & & & & & & & & & & & & & & & & & & & & & & & & & & & & \\
\hline 38 & & & & & & & & & & & & & & & & & & & & & & & & & & & & & & & & & & \\
\hline 41 & & & & & & & & & & & & & & & & & & & & & & & & & & & & & & & $\begin{array}{lll}1 & 1\end{array}$ & & & \\
\hline 43 & & & & & & & & & & & & & & & & & & 1 & 1 & & & & & & & & & & & & & & & \\
\hline 44 & 1 & 1 & 1 & 1 & & & (1) & & & & & & & & & 2 & 1 & & & & & & & & 1 & 1 & 1 & 1 & & & & & & \\
\hline 48 & & & & (1) & & 2 & 2 & 2 & 2 & 2 & 2 & & & & & & & & 1 & 2 & 3 & 1 & 1 & 2 & & & & & 1 & & & \begin{tabular}{l|l}
1 & 1 \\
\end{tabular} & \begin{tabular}{l|l}
1 & 1 \\
\end{tabular} & \\
\hline 49 & & & & & & & & & & & + & & & & & & & & & & & & & & & & & & & & & & & \\
\hline 53 & & & & & + & + & + & & & & & & & & & & & & & 2 & & & & & & & & + & & & 1 & & & \\
\hline 54 & & & & & & & & & & & & & & & & & & & & & & & & & & & & & & & & & (1) & \\
\hline 58 & & & & & & & & & & & & & & & & (1) & & & & & & & & & & & & & & & 1) & & & \\
\hline 60 & & (1) & & & & & & & & & & & & (1) & & 1 & 1 & & & & & & & & 1 & & & 1 & & & & & & \\
\hline 63 & \begin{tabular}{|l|}
1 \\
\end{tabular} & 18 & 2 & 2 & 1 & 2 & & & & & & & & (1) & 1 & 2 & 1 & 1 & 1 & 2 & & & & & 2 & 1 & 1 & 1 & & & & & & \\
\hline 64 & & 18 & 2 & & & & & & & & & & 1 & & 1 & & 1 & & & & & & & & 2 & 1 & (1) & 1 & & & & & & \\
\hline 65 & & & & & & & & & & & & & & & & & & & & & & & & & & & & & & & & & & \\
\hline 71 & & & & & & & & & & & & & & & & & & & & & & & & & & & & & & & & & & \\
\hline 73 & 2 & 2 & 3 & 2 & 1 & & & & & & & 3 & 2 & 2 & 2 & 1 & 1 & 1 & & & & & & & 3 & 2 & 1 & 2 & & & & & & \\
\hline 77 & 3. & + & 3 & & + & & + & & & & & 4 & 4 & + & + & + & 3 & + & & + & 3 & & & & 4 & 4 & \begin{tabular}{l|l}
4 \\
\end{tabular} & & 3 & - & + & & & \\
\hline 78 & 3 & 3 & 3 & & 2 & 2 & 1 & & & & & 4 & 4 & 3 & 3 & & 2 & \begin{tabular}{|l|} 
\\
\end{tabular} & & 1 & & & & & 4 & 3 & 2 & 2 & & & & & & \\
\hline 79 & \begin{tabular}{|l|} 
\\
\end{tabular} & 1 & & & & & & & & & & 2 & & & & & & & & & & & & & 2 & & & & & & & & & \\
\hline 80 & & & & & & & & & & & & & & & & & & & & & & & & & & (1) & & & & & & & & \\
\hline 81 & 1 & & 2 & & & & & & & & & & 2 & 2 & 1 & 1 & 1 & & & & & & & & 2 & 1 & 1 & 1 & & & & & & \\
\hline 84 & & & & & & & & & & & & & & & & & & & & & & & & & & & & & ( & (1) & & & & \\
\hline 86 & & & & & & & & & & & & & & & & & & & & & & (1) & & & & & & & $\bar{C}$ & (1) & & & & \\
\hline 87 & & 1 & & 1 & (1) & & & & 1 & & & & & & & 1 & 1 & 1 & 1 & & 1 & 1 & 1 & & & & & & 1 & 1 & & & 1 & \\
\hline 88 & & & & & & & & & & & & & & & & & 1 & (1) & 1 & & & & & & & & & 1 & 1 & 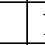 & 11 & 1 & & \\
\hline 91 & & & & & & & & & 1 & & & & & & & & & & & & & & & & & & & & & & & & & \\
\hline 94 & & & & & & & & (1) & & & & & & & & & & & & & & & & & & & & & & & & & & \\
\hline 101 & & & & & & & & & & & & & & & & & & & & & & & & & & & & & & & & & & \\
\hline 122 & & 1 & 1 & 1 & 1 & & & & & & & & & & & 1 & 2 & 1 & & & & & 1 & & & & & 2 & 2 & 2 & 2 & & 1 & \\
\hline 123 & & 1 & & & & & & & & & & & & & & & & 1 & & & & & & & & & & & 1 & 1 & $1]$ & & (1) & \\
\hline 124 & 1 & 1 & 1 & 1 & 1 & & & & & & & & & 1 & 1 & 1 & 1 & 1 & 1 & & & & & & & & & 1 & & 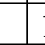 & 1 & & & \\
\hline 130 & & & & & & & & & 1 & & & & & & & & & & & & & (1) & 1 & & & & & & & (1) & 1) & 1 & 1 & \\
\hline 131 & & 1 & & & & & & & & & & & & & & & & & & & & & & & & & & & & 1. & 1) & & \begin{tabular}{l|l}
1 & 1 \\
\end{tabular} & \\
\hline 132 & 1 & & & & 2 & & & & 1 & & & & & & & & & & & & & 2 & 1 & & & & & & & & & & \begin{tabular}{l|l}
1 & 1
\end{tabular} & \\
\hline
\end{tabular}




\begin{tabular}{|c|c|c|c|c|c|c|c|c|c|c|c|c|c|c|c|c|c|c|c|c|c|c|c|c|c|c|c|c|c|c|c|c|c|c|}
\hline \multicolumn{35}{|c|}{ Table $9,2005-3$} \\
\hline & \multicolumn{8}{|c|}{$\mathrm{A}$} & \multicolumn{9}{|c|}{$\mathrm{B}$} & \multicolumn{14}{|c|}{ C } & \multicolumn{3}{|c|}{ D } \\
\hline & 1 & 2 & 3 & 4 & & 6 & 7 & 8 & 1 & & & & & \begin{tabular}{l|l}
6 \\
\end{tabular} & 88 & 9 & 10 & \begin{tabular}{l|l}
01 \\
\end{tabular} & 2 & 3 & 4 & 5 & 6 & 7 & 8 & 9 & & 11 & 12 & 13 & 14 & & & 3 \\
\hline 136 & & & & & & & & & & & & & & & & & & & & & & & & & & & & & & (1) & & & & \\
\hline 137 & & & & & & & & & & & & & & & & & & & & & & & & & & & & & & & & & & \\
\hline 141 & & 1 & (1) & & & & & & & & 1 & & & & & & & & & & (1) & (1) & & & & & & 1 & 1 & 1 & 1 & & & \\
\hline 142 & & 1 & & & & & & 1 & & & 1 & $\mathrm{~L}$ & & 1 & & 1 & 1 & 1 & 1 & & & 1 & & (1) & 1 & 1 & 1 & 2 & 1 & 1 & 1 & & 1 & \\
\hline 143 & & (1) & (1) & & & & & & 1 & & & & & & & & & & & & & & & & 1 & & & & & & (1) & & & \\
\hline 145 & & 1 & 2 & 2 & 2 & 2 & 1 & & 1 & 1 & 1 & L & & 1 & & & & 1 & 1 & & & & & & & & & & & & & & & \\
\hline 158 & & & & & & (1) & & & & & & & & & & & & & & (1) & & & & & & & & & & & & & & \\
\hline 161 & & (1) & (1) & & & & & & & & & & & & & & & & & & & & & & & & & & & & & & & \\
\hline 173 & & & & & & & & & & 1 & 1 & & & & & & & & & (1) & & & & & & & & & & & & & & \\
\hline 180 & & & & & & & & & & & & & & & & & & & & & & & & & & & & & & & & & & \\
\hline 181 & & 1 ( & (1) & 1 & 1 & 1 & & 1 & 1 & & 1 & & & 1 . & & (1) & & & & & & & & & & & & & & & & & & \\
\hline 182 & (1) & & & 1 & 1 & 1 & & & & & & 1 & & & & & & & & & & & & & & & (1) & & & & & & & \\
\hline 183 & & & & 1 & (1) & (1) & & & & & & 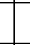 & & & & & & & & & & & & & & & & & & & & & & \\
\hline 191 & & & & & & & & 1) & & & & & & & & & & & & & & & & & & & & & & & & & & \\
\hline 193 & & & & & & & & & & & & & & & & & & & & & & & & & & & & & & & & & & \\
\hline 197 & & (1) & & & & & & & & & & & & & & & & & & & & & & & 1 & & & & & & & & & \\
\hline 198 & 1 & & & 1 & 1 & 1 & 1 & & & & 1 & 1 & 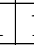 & $1: 2$ & & 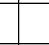 & & & & & & 1 & 1 & 2 & (1) & & . & 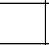 & & & & 1 & & 2 \\
\hline 199 & 1 & 1 & 1 & & 1 & & & 1 & & 1 & 2 & 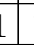 & & 1 & 1 & 1 & 1 & & & & & & & 1 & 2 & 2 & 2 & & & & & & & \\
\hline 200 & (1) & & & (1) & 1 & & 1 & 1 & & & & 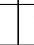 & & 1 & 2 & 21 & 2 & & & (1) & & & & 2 & 1 & 2 & 2 & 1 & 1 & 1 & 2 & & & \\
\hline 207 & & & & & & & & 1 & & & & & & & & & 1 & & & & & & & (1) & & (1) & & 1 & 1 & 1 & 1 & & & \\
\hline 210 & & & & & & & & & & & & & & & & & & & & & & & & & T & & & & (1) & (1) & & & & \\
\hline 211 & & & 3 & 3 & 3 & 3 & 3 & 3 & & & & 2 & & 3 & $3+$ & 3 & & 2 & & 3 & 3 & 3 & 3 & 2 & 1 & & & 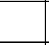 & & 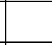 & & 3 & 3 & 3 \\
\hline 212 & & 4 & 4 & & 4 & 3 & 3 & & & 4 & 3 & 3 & & \begin{tabular}{l|l}
3 & \\
\end{tabular} & 13 & & 3 & 33 & 34 & 4 & & & 4 & 4 & 4 & 3 & & & 3 & & 3 & 3 & 4 & 4 \\
\hline 213 & & (1) & (1) & & & 1 & & 1 & & & & & & & & & 2 & & & & & & & & & & & (1) & 1 & 1 & 1 & & & \\
\hline 214 & & & & & & & & & & & & & & & & & 2 & & & & & & 1 & (1) & & 1 & 2 & 2 & 4 & 4 & 4 & & & \\
\hline 216 & & & & & & & & & & & & & & & & & & & & & & & & & & & & & (1) & 1 & & & & \\
\hline 220 & (1) & & & & & & & & (1) & & & & & & & & & & & & (1) & & & & & & & & & & & & & \\
\hline 221 & & & & & & & & & & & & & & & & & & & & & & 1 & & & & & & & & & & & & \\
\hline 222 & & & & & & & & & & & & & & & & & & & & & & & & & & & & & & & & & & \\
\hline 229 & 3 & 3 & 3 & 3 & 3 & 2 & 2 & 2 & 3 & 3 & 3 & & & 2 & 34 & 44 & 3 & 32 & 2 & 2 & 2 & 3 & 3 & 3 & 3 & 4 & 3 & 3 & 3 & 1 & & & & 3 \\
\hline 230 & & & 1 & 1 & & & 1 & 3 & & & & & & & D 1 & 12 & 3 & & & & & & & 2 & 2 & 3 & 3 & 2 & 2 & 1 & & & & \\
\hline 232 & & & & & & & & + & & & & & & & & & & & & & & & & & & & & & & & & & & \\
\hline 233 & & & & & & & & & & & 1) & & & & & & (1 & & & & & & & & & & & & & & & & & \\
\hline 234 & & & & & & & & 1) & & & & & & & & & & & & & & & & & & & & (1) & & & & & & \\
\hline 235 & & & & & & & & & & & & & & & & & & & & & & & & & & 1 & & & & (1) & & & & \\
\hline
\end{tabular}




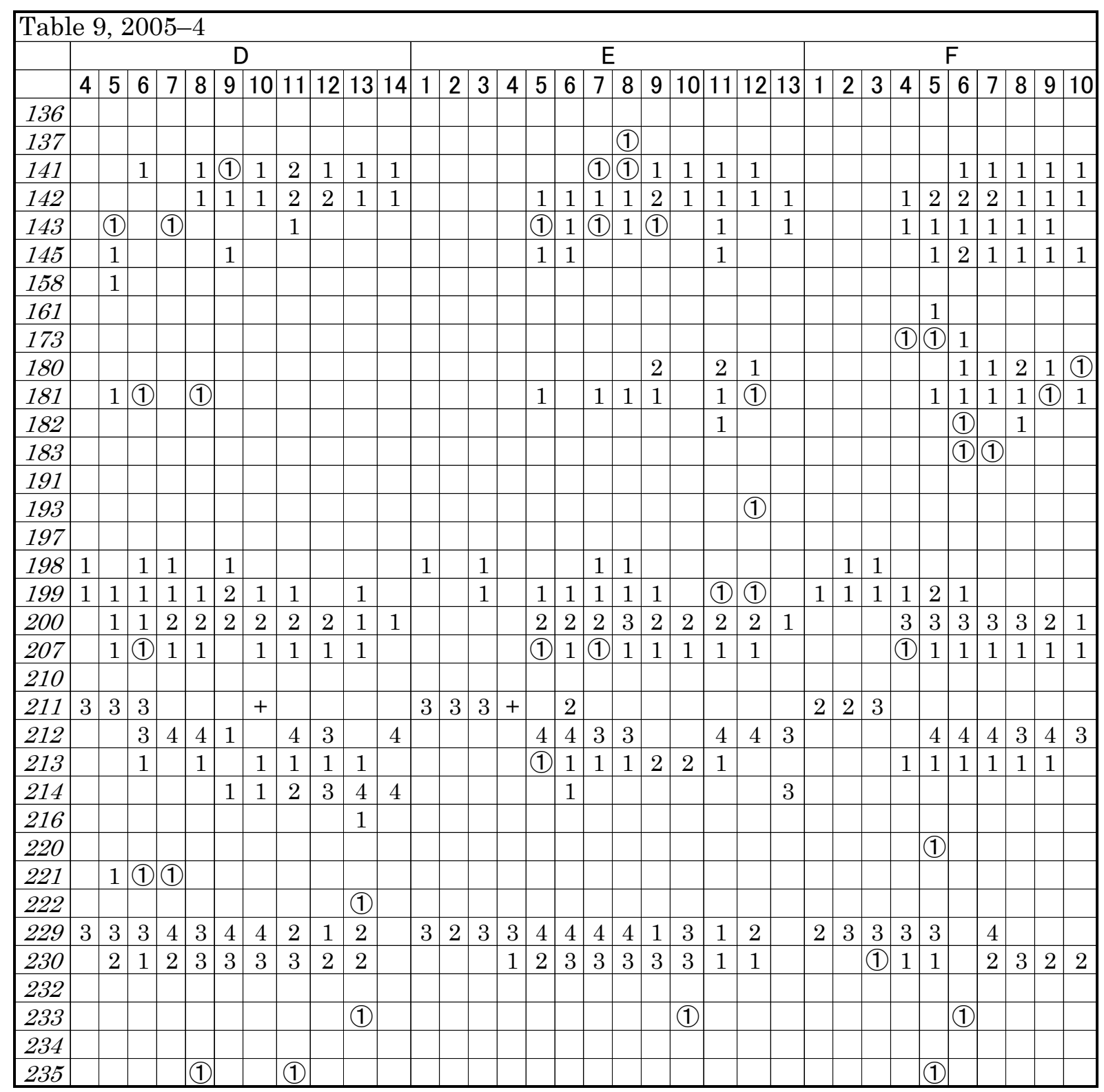




\begin{tabular}{|c|c|c|c|c|c|c|c|c|c|c|c|c|c|c|c|c|c|c|c|c|c|c|c|c|c|c|c|c|c|c|c|c|c|c|c|}
\hline \multicolumn{36}{|c|}{ Table 9, 2006-1 } \\
\hline & \multicolumn{8}{|c|}{$\mathrm{A}$} & & & & & $E$ & & & & & & & & & & & & & $\mathrm{C}$ & & & & & & & & D & \\
\hline & 1 & 2 & 3 & 4 & & 6 & 7 & 8 & 1 & 2 & 3 & 4 & 5 & 6 & 7 & 8 & 9 & 10 & 1 & 2 & 3 & 4 & 5 & 6 & & 8 & \begin{tabular}{l|l}
9 & 1 \\
\end{tabular} & & & 12 & & 14 & & & 3 \\
\hline 2 & & & 1 & & 1 & 1 & & (1) & & & (1) & & & & & & & & & & & & & & & & & & & & & & & & \\
\hline 3 & & & & & & & & & & & & & & & & & & & & & & & & & & & & & & & & & & & \\
\hline 4 & & 1 & (1) & & 1 & & (1) & 1 & & & 1 & 1 & & & & 1 & 2 & 1 & 1 & & & & & & 1 & 1 & 2 & 2 & 1 & 1 & 1 & & & & \\
\hline 5 & & & & & & & 1 & 2 & & 1 & & & & 1 & 1 & 2 & 2 & 1 & & & & & (1) & & 1 & 1 & 2 & 1 & & & & & & & \\
\hline 6 & & & & & & & & & & & & & & & & (1) & & & & & & & & & & & & & 1 & 1 & & 1 & & & \\
\hline 7 & & & 1 & & & & & 1 & & & & & (1) & & & 1 & 1 & 1 & & & & & & & 1 & 1 & 1 & 1 & 1 & 1 & 1 & & & & \\
\hline 8 & & & & & & & & & & & & & & & & & & & & & & & & & & & & & & & & & & & \\
\hline 10 & & & & & & & 1 & 1 & & & & & & & & 1 & 1 & 1 & & & & & & & & & 2 & 1 & 1 & 2 & (1) & 1 & & & \\
\hline 11 & & & & & & & (1) & 1 & & & & & & & & 1 & 1 & & & & & & & & (1) & 16 & 1) & 1 & & 1 & & & & & \\
\hline 12 & 1 & 1 & (1) & & & ( & (1) & (1) & 1 & (1) & & 1 & & & & 1 & 1 & & & & & 1 & 1 & & 1 & 1 & & & & & & & & & \\
\hline 13 & 2 & & 2 & 2 & 1 & 1 & 2 & 2 & 1 & 1 & 1 & 1 & 1 & 1 & 1 & 2 & 2 & 1 & 1 & 1 & 1 & 1 & 1 & 1 & 2 & 2 & & 2 & 1 & 1 & 1 & 1 & & & \\
\hline 14 & & & 1 & 1 & 1 & 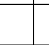 & 1 & 1 & 1 & & 1 & 1 & & 1 & 1 & 1 & 1 & & & & 1 & 1 & & & 1 & 1 & & & & & & & & & (1) \\
\hline 15 & 1 & 1 & 1 & 1 & 1 & 1 & 1 & 3 & 1 & 1 & 1 & 1 & & 1 & 1 & 2 & 2 & 1 & 1 & & & 1 & 1 & & 1 & 2 & 3 & 2 & 2 & 2 & 1 & & & (1) & (1) \\
\hline 16 & & & & & & & & 1 & & & & & & & & & & & & & 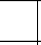 & & & & & & & & & 2 & 1 & 1 & & & (1) \\
\hline 17 & & 1 & 1 & & & & 1 & 2 & 1 & 1 & 1 & 1 & & & 1 & 1 & 2 & 1 & & & 1 & & & 1 & 1 & 1 & 1 & 2 & 1 & 1 & 1 & & & (1) & 1 \\
\hline 18 & & & & & & & & 1 & & & 1 & & & & & 1 & & 1 & & & & & & & & & 1 & 1 & 1 & 1 & 1 & & & & (1) \\
\hline 20 & & & 1 & & & & & & 1 & & & & & & & & 16 & (1) & & & & & 1 & & (1) & & & 1 & & (1) & & & & & \\
\hline 21 & & & & & & & & 1 & & 1 & 1 & & & & & & & & & & & & & & & & & & & & & & & & \\
\hline 22 & 1 & & & & & & & & 1 & 1 & 1 & 1 & & & & & & & & & 1 & & (1) & & 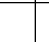 & | & . & & & 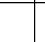 & & & & & \\
\hline 23 & & & & & & & & & (1) & & & & & & & 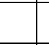 & & & & & & & . & & & 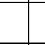 & & & & & & & & & \\
\hline 26 & & & & & & & & & & & & & & & & & & & & & & & 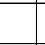 & & & & & & 1 & 1 & 1 & 1 & & & \\
\hline 28 & & & & & & (1) & 1 & 2 & & & & 1 & & & & 1 & 1 & 1 & & & & & & & & 1 & 2 & 1 & & 1 & & & & & \\
\hline 29 & & & & & & & & & & & & & & & & & & & & & & & & & & & & & & & & & & & \\
\hline 33 & & & & & & & & & & & & & & & & & & & & & & & & & & & & & & & & & & & \\
\hline 34 & & & & & & & & (1) & & & (1) & & & & & & & & & & & & & & & & & & & & & & & & \\
\hline 36 & & & & & & & & (1) & & & & & & & & & & & & & & & & & & & & & & & & & & & \\
\hline 39 & & & & & & & & (1) & & & & & & & & & & & & & & & & & & & & & & & & & & & \\
\hline 41 & & & & & & & & & & & & & 1 & & & & & & & & & & & & & & & & & & & & & & \\
\hline 43 & & & & & & & 1 & 1 & & - & & & & & & & 1 & & & & & & & & & & & & 1 & & & & & & \\
\hline 44 & 2 & 2 & 1 & 2 & 2 & 1 & & (1) & 1 & 2 & 1 & 2 & 2 & 1 & 1 & 1 & 1 & & 1 & 1 & 2 & 2 & 1 & 2 & 2 & 1 & 1( & (1) & & & & & 1 & 1 & 1 \\
\hline 48 & & & & & & & & 3 & & & & & & & & (1) & 2 & 2 & & & & & & & & & 2 & 2 & 2 & 2 & 2 & 2 & & & \\
\hline 49 & & & & & & & & & & & & & & & & & & & & & & & & & & & & & & & & 1 & & & \\
\hline 53 & & & & & & 1 & & & & & & & & & & & 1 & & & & & & & & & & & & 2 & 1 & & & & & \\
\hline 54 & & & & & & & & & & & & & & & & & & & & & & & & & & & & & & & & & & & \\
\hline 58 & & & & & & & & & & & & & (1) & & & & & & & & & & 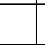 & & & (1) & & & & & & & & & \\
\hline 60 & 1 & 1 & \begin{tabular}{|l|l|} 
& \\
\end{tabular} & 1 & & 1 & & & 1 & 1 & 1 & 1 & & & & & & & 1 & 2 & 1 & (1) & (1) & & & & & & & & & & & & \\
\hline 63 & 2 & 1 & \begin{tabular}{|l|}
2 \\
\end{tabular} & 1 & 2 & 2 & & & 2 & 1 & 2 & 1 & 1 & 1 & 1 & 1 & 1 & & 2 & 2 & 2 & 2 & 1 & 1 & 1 & 1 & 1 & & & & & & & (1) & \\
\hline 64 & 1 & 1 & & 1 & 1 & 1 & 1 & & 1 & 1 & & \begin{tabular}{|l|} 
\\
\end{tabular} & 1 & 2 & 2 & 1 & & & 2 & & 1 & 2 & 1 & 1 & 2 & 1 & & & & & & & & (1) & (1) \\
\hline 71 & 3 & 3 & \begin{tabular}{|l|}
3 \\
\end{tabular} & 1 & 3 & & 1 & & 4 & \begin{tabular}{|l|}
4 \\
\end{tabular} & 3 & 2 & 2 & 3 & & & & & 4 & 4 & 3 & & 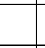 & $t$ & 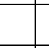 & & & & & & & & & & \\
\hline 73 & 3 & 1 & \begin{tabular}{|l|}
2 \\
\end{tabular} & 2 & 1 & 1 & 1 & & 2 & \begin{tabular}{|l|}
2 \\
\end{tabular} & 2 & 3 & 1 & 1 & 1 & 2 & 1 & & 2 & 2 & 2 & 1 & 2 & 3 & \begin{tabular}{|l|}
3 \\
\end{tabular} & 2 & 1 & & & & & & 2 & 2 & 2 \\
\hline 77 & 4 & 3 & \begin{tabular}{|l|}
3 \\
\end{tabular} & 4 & 4 & 4 & 4 & 3 & 4 & 4 & 2 & 3 & 4 & 4 & 4 & 4 & \begin{tabular}{|l|l|}
3 &
\end{tabular} & 1 & 4 & & 4 & 4 & 4 & 4 & 4 & 4 & 1 & 2 & 2 & & & & 4 & 4 & 4 \\
\hline 78 & 3 & & \begin{tabular}{|l|}
3 \\
\end{tabular} & 4 & \begin{tabular}{|l|}
4 \\
\end{tabular} & 4 & 3 & 3 & 3 & \begin{tabular}{|l|}
2 \\
\end{tabular} & 1 & \begin{tabular}{|l|} 
\\
\end{tabular} & 4 & 4 & 4 & 4 & \begin{tabular}{|l|l|}
3 & \\
\end{tabular} & & 3 & & 4 & 4 & 4 & 4 & 4 & 3 & 3 & 3 & & & & & 4 & 4 & 4 \\
\hline 79 & 2 & & & \begin{tabular}{|l|}
1 \\
\end{tabular} & \begin{tabular}{|l|}
1 \\
\end{tabular} & 1 & 1 & & 2 & & & 2 & 2 & 11 & 1 & & & & 2 & & 2 & 3 & 1 & 2 & & & & & & & & & 2 & 1 & \\
\hline 80 & & & & & & (1) & & 2 & & & & & & & & & & & & & & & & & & 1 & (1) & & & & & & & & \\
\hline 81 & 2 & & & 2 & 1 & 1 & 1 & & 2 & & 1 & 1 & 2 & 2 & 1 & 2 & 2 & & 1 & & 2 & 2 & 2 & 2 & 2 & 1 & 2 & 1 & & & & & & 1 & 2 \\
\hline 82 & & & 1 & & & & & & & & (1) & & & 2 & & & & & & & & & & & & & & & & & & & & & \\
\hline 86 & & & & & & & & & & & & & & & & & & & & & & & & & & & & & & & & & & & \\
\hline 87 & & & & & 1 & & & 1 & & & & & & & & & & & & & & & & & & & & & (1) & 1 & & & & & \\
\hline 88 & & & & & & & & & & 1 & & & & & & & & (1) & & & & & & & & & & & & & & & & & \\
\hline 91 & & & & & & & & & & & & & & & & & & & & & & & & & & & & & (1) & 1 & & (1) & & & \\
\hline 93 & & & & & & & & & & & & & & & & & & & & & & & & & & & & & & & & & & & \\
\hline 94 & & & & & & & & & (1) & & & & 1 & 1 & & & 1 & & & & & & & & & 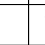 & 1( & (1) & 1 & & & & & & \\
\hline 107 & & & & & & & & & & & & & & & & & & & & & & & & & & & & & & & & & & & \\
\hline 122 & & & 1 & 1 & 2 & 1 & & 1 & & & (1) & 1 & 1 & & & & & & & 1 & & 1 & & & & & & & & & & & & & \\
\hline 123 & & & & & \begin{tabular}{|l|} 
\\
\end{tabular} & 1 & & & & & & & 1 & & & & & & & & & & & & & (1) & & & & & & & & & \\
\hline
\end{tabular}




\begin{tabular}{|c|c|c|c|c|c|c|c|c|c|c|c|c|c|c|c|c|c|c|c|c|c|c|c|c|c|c|c|c|c|c|c|c|}
\hline \multicolumn{33}{|c|}{ Table 9, 2006-2 } \\
\hline & \multicolumn{11}{|c|}{ D } & \multicolumn{12}{|c|}{$E$} & \multicolumn{9}{|c|}{$\mathrm{F}$} \\
\hline & 4 & $5 \mid$ & $\begin{array}{ll}6 & 7 \\
& \end{array}$ & \begin{tabular}{l|l}
7 & $\varepsilon$ \\
\end{tabular} & \begin{tabular}{|l|l}
9 \\
\end{tabular} & & & & \begin{tabular}{l|l}
2 & 1 \\
\end{tabular} & $3 \mid$ & & 1 & 2 & 3 & 4 & 5 & & & \begin{tabular}{|l|l}
8 & 9
\end{tabular} & 10 & & 12 & & 1 & 2 & \begin{tabular}{l|l}
3 & 4 \\
\end{tabular} & 45 & & 7 & 8 & & 10 \\
\hline 2 & & & & & & & & & & & & & & & & & & & & & & & & & & & 1 & 1 & & & & \\
\hline 3 & & & & & & & & & & & & & & & & & & & & & & & & & & & 1 & & & & & \\
\hline 4 & 1 & (1) & $1]$ & $1]$ & 1 & 2 & 1 & 1 & & 2 & & & & & 1 & 1 & 1 & 12 & \begin{tabular}{|l|}
22 \\
\end{tabular} & 2 & 1 & 1 & 2 & & & & \begin{tabular}{l|l|}
1 & 1 \\
\end{tabular} & 1 & 1 & 1 & 1 & 1 \\
\hline 5 & & & 1 & 1 & 1 & 1 & 1 & 1 & 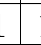 & 1 & & & & & & 1 & 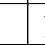 & 1 & \begin{tabular}{l|l}
1 & 1 \\
\end{tabular} & 1 & & & & & & & 1 & 1 & 1 & & & \\
\hline 6 & & & & & & & & & & 1 & 1 & & & & & & & & & & & 1 & 1 & & & & & & & & 1 & (1) \\
\hline 7 & & 1 [. & $1] 1$ & $1] 1$ & 1 & 1 & 1 & 1 & & 1 & 2 & & & & & 1 & 1 & & 1 & 1 & 1 & 1 & 1 & & & & \begin{tabular}{l|l}
1 & 1 \\
\end{tabular} & 1 & 1 & 1 & 1 & 1 \\
\hline 8 & & & & & & (1) & & & & & & & & & & & & & & & & (1) & & & & & & & & & & \\
\hline 10 & & & 1 & 1 & 1 & 1 & 1 & & 2 & 1 & 1 & & & & & & 12 & \begin{tabular}{l|l}
2 & 1 \\
\end{tabular} & \begin{tabular}{l|l|}
1 & 2
\end{tabular} & 2 & & 2 & 1 & & & & (1) & 1 & 2 & 3 & 2 & 2 \\
\hline 11 & & 16 & 1) & & & 1 & & & & & & & & & 1 & 1 & 1 & 1 & & & & & & & 1 & & 2 & & & & & \\
\hline 12 & 1 & 2 & $1] 1$ & $1]$ & 1 & & & & & & & & & & 1 & 1 & 1 & $1(1$ & 1) & & & & & & 1 & \begin{tabular}{l|l}
1 & 1 \\
\end{tabular} & 11 & & & & & \\
\hline 13 & 2 & 25 & 25 & $2 \sqrt{2}$ & 2 & 2 & 1 & & L & 2 & 1 & & & 1 & 2 & 2 & 2 & 25 & \begin{tabular}{|l|l}
22 \\
\end{tabular} & 2 & 1 & 1 & 1 & 1 & 1 & \begin{tabular}{l|l}
1 & 2 \\
\end{tabular} & \begin{tabular}{l|l}
2 & 1
\end{tabular} & 1 & 1 & 2 & 1 & 2 \\
\hline 14 & \begin{tabular}{|l|}
1 \\
\end{tabular} & 1 & 11 & 1 & & & & & & 1 & & & & 1 & (1) & & 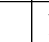 & 1 & 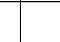 & 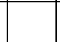 & & & & & 1 & \begin{tabular}{l|l}
1 & 1 \\
\end{tabular} & \begin{tabular}{l|l}
1 & 1 \\
\end{tabular} & 1 & 1 & & & \\
\hline 15 & \begin{tabular}{|l|} 
\\
\end{tabular} & 1 & 1 & 26 & 2 & 3 & 2 & & & 2 & & 1 & & 1 & 1 & 2 & 2 & 25 & \begin{tabular}{|l|l|}
2 \\
\end{tabular} & 2 & 3 & 2 & 1 & 1 & 1 & (1) 2 & \begin{tabular}{l|l}
2 & 2
\end{tabular} & 1 & 3 & 3 & 3 & 3 \\
\hline 16 & & & & 1 & 1) & & 1 & 1 & & 2 & 2 & & & & & & & & 1 & & & & 1 & & & & & & & & & \\
\hline 17 & 1 & 1 & 15 & $\begin{array}{ll}21 \\
21\end{array}$ & 1 & 2 & & 1 & & 1 & & 1 & 1 & 1 & 1 & 2 & 1 & 2 & 2 & 2 & & & 1 & & 1 & \begin{tabular}{l|l}
1 & 1 \\
\end{tabular} & \begin{tabular}{l|l}
1 & 1 \\
\end{tabular} & & 2 & 1 & 1 & 1 \\
\hline 18 & & (1) & $1]$ & 1 & & & & 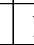 & & & 1 & & & (1) & 1 & & & & 1 & 1 & & 1 & & & 4 & & & 1 & & & 1 & \\
\hline 20 & & & (1). & $\begin{array}{ll}11 \\
\end{array}$ & \begin{tabular}{l|l}
1 & 1 \\
\end{tabular} & & & & & & & & & & 1 & & 1 & 1 & & & & & & & 1 & \begin{tabular}{l|l}
1 & 1 \\
\end{tabular} & 1 & & & & & \\
\hline 21 & & 1 & & & & & & & & & & & & & & 1 & & & & & & & & & & & 1 & 1 & & & & \\
\hline 22 & & & & & & & & & & & & & & & 1 & & & & & & & & & & & & 1 & & & & & \\
\hline 23 & & & & & & & & & & & & & & & & & & 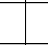 & & & & & & & 1 & & & & & & & \\
\hline 26 & & & 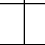 & & & & & & D) & 1 & 1 & & & & & & & 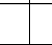 & 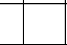 & 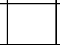 & 1 & 1 & 1 & & & & & & 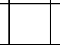 & & & (1) \\
\hline 28 & & 1. & 11 & 111 & \begin{tabular}{l|l}
1 & 1
\end{tabular} & 1 & (1) & 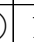 & & & & & & & & & & \begin{tabular}{l|l}
1 & 1
\end{tabular} & \begin{tabular}{|l|l}
1 & 1
\end{tabular} & 1 & & & & & & & & & 1 & & 1 & \\
\hline 29 & & & & & & & & & & & & & & & & & & & & & & & & & & & (1) & & & & & \\
\hline 33 & & & & & & (1) & & & & & & & & & & & & & & & & & & & & & & & & & & \\
\hline 34 & & & & & & & & & & & & & & & & & & & & & & & & & & & & & & & & \\
\hline 36 & & & & & & & & & & & & & & & & & & & & & & & & & & & & & & & & \\
\hline 39 & & & & & & & & & & & & & & & & & & & & & & & & & & & & & & & & \\
\hline 41 & & & & & & & & & & & & & & & & & & & & & & & (1) & & & & & & & & & \\
\hline 43 & & & & 2 & 2 & & & & & & & & & & & & & & 1 & 1 & & & & & & & 1 & 2 & & & & \\
\hline 44 & 2 & 2 & 21 & \begin{tabular}{l|l}
1 & 1 \\
\end{tabular} & 1 & & & & & & & & & & 1 & 1 & 2 & $\begin{array}{lll}2 & 1\end{array}$ & 1 & & & & & 2 & 1 & & 1 & & & & & \\
\hline 48 & & & 1) & 1 & 1 & 2 & 22 & & 2 & 3 & 3 & & & & & & & (1) 2 & \begin{tabular}{|l|l|}
2 & 1 \\
\end{tabular} & 2 & 1 & 2 & 2 & & & & \begin{tabular}{l|l}
$1(1$ \\
\end{tabular} & 1 & 1 & 1 & 1 & 2 \\
\hline 49 & & & & & & & & & & & 1 & & & & & & & & & & & & & & & & & & & & & \\
\hline 53 & & & & & & & & & & & & & & & & & & & & & & 1 & & & & & & & 1 & 1 & & \\
\hline 54 & & & & & & & & & & & & & & & & & & & & & & & & & & & (1) & & & & & \\
\hline 58 & & (1) & & & & & & & & & & & & & & (1) & & & & & & & & & & & & & & (1) & & \\
\hline 60 & (1) & & & & & & & & & & & & & & & 1 & (1) & & & & & & & 1 & & & \begin{tabular}{l|l}
1 & 1 \\
\end{tabular} & & & & & \\
\hline 63 & \begin{tabular}{|l|l|}
2 \\
\end{tabular} & 2 & 2 & $\begin{array}{ll}111 \\
\end{array}$ & 1 & & & & & & & & & & 1 & $\frac{1}{2}$ & 1 & \begin{tabular}{l|l}
1 & 1
\end{tabular} & 1 & & & & & 1 & & & $\begin{array}{ll}1 & 1 \\
\end{array}$ & 1 & 1 & & & \\
\hline 64 & \begin{tabular}{|l|}
2 \\
\end{tabular} & 2 & 26 & 2 & 1 & & & & & & & 1 & 1 & \begin{tabular}{|l|}
1 \\
\end{tabular} & 2 & (1) & (1) & & & & & & & 1 & 2 & \begin{tabular}{l|l}
1 & 1
\end{tabular} & 1 & & & & & \\
\hline 71 & & & & & & & & & & & & & & & & & & & & & & & & 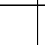 & & & & & & & & \\
\hline 73 & 3 & 2 & 25 & 25 & 22 & & & & & & & 2 & 2 & 2 & 2 & 3 & 2 & $\begin{array}{lll}2 & 1\end{array}$ & 1 & & & & & 2 & 2 & \begin{tabular}{l|l}
3 & 2 \\
\end{tabular} & \begin{tabular}{l|l}
2 & 2 \\
\end{tabular} & & & & & \\
\hline 77 & 4 & $\begin{array}{ll}4 \\
\end{array}$ & 42 & 4 & 4 & & & 1 & & & & 3 & 4 & \begin{tabular}{|l|}
4 \\
\end{tabular} & 4 & 4 & & & & & & & & 4 & 4 & \begin{tabular}{l|l}
44 & 4 \\
\end{tabular} & \begin{tabular}{|l|l}
4 & 3 \\
\end{tabular} & & & & & \\
\hline 78 & \begin{tabular}{|l|}
4 \\
\end{tabular} & 4 & $\begin{array}{ll}32 \\
\end{array}$ & 2 & 3 & 2 & & & & & & 4 & 4 & 4 & 4 & 3 & & & & 1 & & & & 4 & 4 & \begin{tabular}{|l|l}
4 & 4
\end{tabular} & 4 & & & & & \\
\hline 79 & & 1 & & & & & & & & & & 1 & \begin{tabular}{|l|}
1 \\
\end{tabular} & \begin{tabular}{|l|} 
\\
\end{tabular} & & & & & & & & & & 2 & 1 & & 1 & & & & & \\
\hline 80 & & & 7 & 1 & 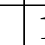 & & & & & & & & & & & & 1 & & & & & & & & (1) & & & & & & & \\
\hline 81 & 2 & & 1 & & (1) & & & & & & & 2 & 2 & \begin{tabular}{|l|l|} 
\\
\end{tabular} & 2 & & & & & & & & & 2 & 2 & \begin{tabular}{l|l}
2 & 2 \\
\end{tabular} & 2 & & & & & \\
\hline 82 & & & & & & & & & & & & & & & & & & & & & & & & & & & & & & & & \\
\hline 86 & & & & & & & & & & & & & & & & & & & & & & & & & & & & & & & 1 & \\
\hline 87 & & & & & & & (1) & & & & & & & & & 1 & 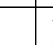 & 11 & (1) & 1 & 1 & 1 & & & & & 1 & 2 & & & & (1) \\
\hline 88 & & & & & & & & & & & & & & & & & 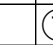 & 1) 1 & & & & 1 & & & & & \begin{tabular}{l|l}
1 & 1 \\
\end{tabular} & 1 & 1 & 1 & & \\
\hline 91 & & & & & & & & & & & & & & & & & & & & & & & & & & & & & & & & \\
\hline 93 & & & & & & & & & & & & & & & & & & & & & & (1) & & & & & & & & & & \\
\hline 94 & & & & 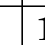 & 1 & (1) & 1) 1 & & & (1) & & & & & & & & & 1 & 1 & 1 & & 1 & & & & 1 & 1 & \begin{tabular}{|l|}
1 \\
\end{tabular} & 1 & 1 & \\
\hline 107 & & & & & & & & & & & & & & & & & & & (1) & & & & & & & & & & 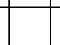 & 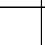 & 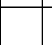 & \\
\hline 122 & & 1 & & 1 & 1 ( & & & & & & & & & & & 1 & & 1 & & 1 & & & & & & & \begin{tabular}{l|l}
1 & 2 \\
\end{tabular} & 2 & 2 & 2 & 2 & \\
\hline 123 & & & & & & & & & & & & & & & & & & & & & & & & & & & & & & & & \\
\hline
\end{tabular}




\begin{tabular}{|c|c|c|c|c|c|c|c|c|c|c|c|c|c|c|c|c|c|c|c|c|c|c|c|c|c|c|c|c|c|c|c|c|c|c|c|}
\hline \multicolumn{36}{|c|}{ Table 9, 2006-3 } \\
\hline & \multicolumn{8}{|c|}{$\mathrm{A}$} & \multicolumn{10}{|c|}{$\mathrm{B}$} & \multicolumn{14}{|c|}{$\mathrm{C}$} & \multicolumn{3}{|c|}{$\mathrm{D}$} \\
\hline & 1 & 2 & 3 & 4 & & 6 & 7 & 8 & 1 & 2 & 3 & 4 & 5 & & 7 & 8 & 9 & 10 & 1 & 2 & 3 & 4 & 5 & \begin{tabular}{|l|l|}
6 \\
\end{tabular} & & 8 & 91 & & & 12 & & 14 & & & 3 \\
\hline 124 & 1 & 1 & 1 & 2 & 1 & 1 & & 1 & 1 & 1 & 1 & 1 & & 1 & & 1 & 1 & & 1 & 1 & 1 & & 1 & 1 & 1 & & & & & & & & & & 1 \\
\hline 130 & & & & & & & & (1) & & & & & & & & & & & & & & & & & & & & & & & & & & & \\
\hline 131 & & & & & & & & & & & & & & & & & & & & & & & & & & & & & (1) & (1) & & & & & \\
\hline 132 & & 1 & 1 & 1 & 3 & 2 & 2 & 2 & & & 1 & 1 & 1 & 1 & 1 & 2 & 2 & 2 & 1 & 1 & 1 & 1 & 1 & & (1) & 1 & 2 & 2 & 2 & 1 & & & & & \\
\hline 137 & & & & & & & & & & & & & & & & & & & & & & & & & & & & & & & & & & & \\
\hline 141 & & & & (1) & 1 & 1 & & & & & 1 & 1 & & (1) & (1) & 1 & & & & 1 & 1 & & 1 & & (1) & 1 & 1 & & 1 & 1 & 1 & 1 & & & (1) \\
\hline 142 & 1 & 1 & 1 & & & 2 & 1 & 1 & 1 & (1) & 1 & 1 & 1 & 1 & 1 & 1 & 2 & 1 & 1 & 1 & 1 & 1 & 1 & (1) & 1 & 1 & 1 & 2 & 2 & 1 & 2 & 1 & & & \\
\hline 143 & & & & & & & & & & & & (1) & & & & (1) & & (1) & & & & (1) & & & & & 1 & & (1) & 1 & 1 & 1 & & & \\
\hline 145 & & & 1 & 2 & 2 & 2 & 2 & 1 & & 1 & 2 & 2 & 2 & 1 & & (1) & & & & & & 1 & & & & & & & 1 & 1 & & & & & \\
\hline 146 & & & & & & & & & & & & & & & & & & & & & & & & & & & & & & & & & & & \\
\hline 149 & & & & & & & & 1 & & & & & & & & & & & & & & & & & & & & & & & & & & & \\
\hline 155 & & & & (1) & & & & & & & & & & & & & & & & & & & & & & & & & & & & & & & \\
\hline 158 & & & & & (1) & (1) & & & & & & & & & & & & & & & 1 & 1 & & & & & & & & & & & & & \\
\hline 161 & & (1) & & & & & & & & & & (1) & & & & & & & & & & & & & & & & & & & & & & & \\
\hline 175 & & & (1) & & & & & & & & & & & & & & & & & & & & & & & & & & & & & & & & \\
\hline 178 & & & & & & & & & & 1 & 2 & & & & & & & & & & 1 & & & & & & & & & & & & & & \\
\hline 180 & & & & & & (1) & & & & & & & & & & & & & & & & & & & & & & & & & & & & & \\
\hline 192 & & & & & & & & & & & & (1) & & & & & & & & & & & & & & & & & & & & & & & \\
\hline 193 & & & & & & & & & & & & & & & & & & & & & & & & & & & & & & & & & & & \\
\hline 195 & & & & & & & & & (1) & & & & & & & & & & & & & & & & & & & & & & & & & & \\
\hline 197 & & & & & & & & & & & & & & & 1 & (1) & & & & & & & & & (1) & 1 & & & & & & & & & \\
\hline 198 & & & (1) & 1 & 1 & 1 & 1 & & & & & 1 & 2 & 2 & 1 & 1 & 1 & & & & 1 & 1 & 1 & 2 & 1 & 1 & & & & & & & & 1 & 1 \\
\hline 199 & 2 & 1 & & 1 & 1 & 1 & 1 & 1 & 1 & & 1 & 1 & & 1 & 2 & 2 & 2 & 1 & 1 & & 1 & & 1 & 1 & 2 & 2 & 2 & 2 & 1 & & & & 1 & & (1) \\
\hline 200 & & & 1 & & 1 & 1 & 1 & 2 & 1 & & 1 & 1 & 1 & 1 & 1 & 2 & 1 & 1 & & & & & 1 & 1 & 1 & 1 & 2 & 1 & 1 & & 1 & 1 & & & (1) \\
\hline 203 & & & & & & & & & & & & & & & & & & & & & & & & & & & & & & (1) & & & & & \\
\hline 205 & & & & & & & & & & & & & & & & & & & & & & & & & & & & & & & & & & & \\
\hline 207 & & & & & & (1) & & 1 & & & (1) & & & (1) & & (1) & & 1 & & & & (1) & & & & & 1 & (1) & 1 & 1 & 1 & 1 & & & \\
\hline 209 & & & & & & & & & & & & & & & & & & & & & & & & & & & & & & & & 1 & & & \\
\hline 210 & & & & & & & & & & & & & & & & & & & & & & & & & & & & & & & & & & & \\
\hline 211 & & & 2 & 2 & 2 & & 3 & 3 & 1 & & & 2 & 1 & 2 & 2 & 3 & 3 & & 2 & & 1 & 2 & & 3 & 2 & 3 & 2 & 1 & & & . & & 1 & & \begin{tabular}{|l|l} 
\\
\end{tabular} \\
\hline 212 & & 4 & 4 & & 2 & & 3 & & & 4 & \begin{tabular}{|l|}
4 \\
\end{tabular} & 4 & & 3 & 4 & 44 & 3 & 3 & 4 & 4 & 4 & & 2 & 4 & 4 & 4 & 3 & & 4 & 4 & 4 & 3 & & & \\
\hline 213 & 1 & & 1 & & 2 & 1 & 1 & 1 & & & & & 1 & 1 & & & & 2 & & 1 & & & (1) & & & & 1 & 3 & 1 & & & 1 & & & \\
\hline 214 & & & & & & & & & & & & & & & & (1) & 1 & 3 & & & (1) & & 1 & & (1) & (1) & 1 & 2 & 3 & 4 & 4 & 4 & & & \\
\hline 220 & & & & & & & & & & & & & & & & & & & & & & & & & & & & & & (1) & & & & & \\
\hline 221 & & & & & & & & (1) & & & & & & & & & & & 1 & & & & & 1 & & & & & & & & & & & \\
\hline 222 & & & & & & & & & & & & & & & & & & & & & & & & & & & & & & & & & & & \\
\hline 226 & & & & & & & & & & & & & & & & & & & & & & & & & & & & & & & & & & & \\
\hline 228 & & & & & & & & & & & & & & & & & & & & & & & & & & & & & & & & & & & \\
\hline 229 & 3 & 2 & 2 & 2 & 2 & 2 & 2 & 2 & 1 & 2 & 2 & 3 & 2 & 1 & 2 & 3 & 3 & 3 & 2 & 2 & 1 & & 3 & 3 & 3 & 4 & 3 & 4 & 3 & 4 & 1 & & 1 & 1 & 2 \\
\hline 230 & & & 1 & & & & 1 & 3 & & & & & & & 1 & 2 & 2 & 3 & & & & 1 & & (1) & 1 & 2 & 2 & 3 & 2 & 2 & & & & & \\
\hline 231 & & & & & & & & & & & & & & & & & & 1 & & & & & & & & & & 1 & & & & & & & \\
\hline 232 & & & & 1 & & & & & & & & & & & & & & & & & & & & & & & & & & & & & & & \\
\hline 233 & & & & & & & & & & & & & & & & & & & & & & & & & & & & & & (1) & & & & & \\
\hline 234 & & & & & & & & & & & & & & & & & & & & & & & & & & & & & & & & & & & \\
\hline 235 & & & & & & & & & & & & & & & & & & & & & & & & & & & & & & & & & & & \\
\hline
\end{tabular}




\begin{tabular}{|c|c|c|c|c|c|c|c|c|c|c|c|c|c|c|c|c|c|c|c|c|c|c|c|c|c|c|c|c|c|c|c|c|c|c|}
\hline \multicolumn{35}{|c|}{ Table 9, 2006-4 } \\
\hline & \multicolumn{11}{|c|}{ D } & \multicolumn{13}{|c|}{$E$} & \multicolumn{10}{|c|}{$\mathrm{F}$} \\
\hline & 4 & 5 & 6 & 7 & 8 & 9 & & & 12 & 13 & & 1 & 2 & 3 & 4 & 5 & 6 & 7 & 8 & 9 & 10 & & & & 1 & 2 & 3 & 4 & 5 & 6 & 7 & 8 & & 10 \\
\hline 124 & & 1 & 1 & 1 & 1 & 1 & 1 & & & & & 1 & & 1 & 1 & 1 & 1 & 1 & 1 & (1) & & & & & 1 & & & 1 & 1 & & 1 & & & \\
\hline 130 & & & & & & & & 1 & 1 & & & & & & & & & & 1 & & 1 & & 1 & 1 & & & & . & & & & & 1 & 1 \\
\hline 131 & & 1 & & & 1 & 1 & & 1 & (1) & (1) & & & & & & 1 & 1 & (1) & 1 & 1 & & 1 & (1) & & & & & & 1 & 1 & 1 & 1 & 1 & 1 \\
\hline 132 & & 2 & 2 & 2 & 2 & 3 & 2 & 2 & 2 & 1 & & & & & & 2 & 2 & 2 & 2 & 2 & 2 & 1 & 1 & & (1) & & & 1 & 2 & 2 & 2 & 2 & 2 & 1 \\
\hline 137 & & & & & & & & & & & & & & & & & & & & & & (1) & & & & & & & & & & & & \\
\hline 141 & & 1 & & 1 & & 1 & & 1 & 1 & 1 & 1 & & & & & 1 & & 1 & 1 & 1 & 1 & 1 & 1 & 1 & & & & & 2 & 1 & 1 & 1 & 1 & 1 \\
\hline 142 & 1 & 1 & 1 & 1 & 1 & 1 & 1 & 1 & 2 & 2 & 1 & & & 1 & 1 & 1 & 2 & 2 & 1 & 1 & 1 & 1 & 1 & 2 & & & 1 & 2 & 2 & 2 & 2 & 1 & 1 & 1 \\
\hline 143 & & & (1) & (1) & (1) & (1) & & & 1 & 1 & & & & (1) & & (1) & (1) & & & (1) & 1 & & 1 & 1 & & & & & & 1 & 1 & 1 & & \\
\hline 145 & & 1 & & & 1 & 1 & & & & & & & & & & 1 & & & 1 & 1 & & 1 & & & & & & 1 & & 1 & 1 & 1 & 1 & \\
\hline 146 & & & & & & & & (1) & & & & & & & & & & & & & & & & & & & & & (1) & & & & & \\
\hline 149 & & & & & & & & & & & & & & & & (1) & & & & & & & & & & & & & & & & & & \\
\hline 155 & & & & & & & & & & & & & & & & & & & & & & & & & & & & & & & & & & \\
\hline 158 & & 2 & 1 & & & & & & & & & & & & & 1 & & & & & & & & & & & & & & & & & & \\
\hline 161 & & & & & & & & & & & & & & & & & & & & & & & & & & & & & 1 & & & & & \\
\hline 175 & & & & & & & & & & & & & & & & & & & & & & & & & & & & & & & & & & \\
\hline 178 & & & & & & & & & & & & & & & & & & & & & & & & & & & & & & & & & & \\
\hline 180 & & & & & & & & & & & & & & & & & & & & & & & & & & & & & & & & & & 1 \\
\hline 192 & & & & & & & & & & & & & & & & & & & & & & & & & & & & & & & & & & \\
\hline 193 & & & & & & & & & & & & & & & & & & & & & & (1) & 1 & & & & & & & & & & & \\
\hline 195 & & & & & & & & & & & & & & & & & & & & & & & & & & & & & & & & & & \\
\hline 197 & & & & (1) & & & & & & & & & & & & & & & & & & & & & & & & & & & & & & \\
\hline 198 & & & 1 & & & & & & & & & 1 & 1 & 1 & 1 & & & 1 & & & & & & & & & 1 & & & & & & & \\
\hline 199 & 1 & & 1 & 1 & 2 & 2 & 1 & 1 & & & & (1) & 1 & 1 & 1 & 1 & & 1 & 1 & 2 & & & & & 1 & 1 & 1 & 1 & 1 & 1 & & 1 & & 2 \\
\hline 200 & (1) & 1 & 1 & 1 & 2 & 1 & 1 & 2 & 1 & 1 & (1) & & 1 & & 1 & 1 & 2 & 2 & 2 & 2 & 2 & 2 & 1 & 2 & & & & 3 & 3 & 2 & 3 & \begin{tabular}{|l|l|}
2 \\
\end{tabular} & 2 & 2 \\
\hline 203 & & & & & & & & & & & & & & & & & & & & & & & & & & & & & & & & & & \\
\hline 205 & & & & & & & & & & & & & & & & & & & & & & & (1) & & & & & & & & & & & \\
\hline 207 & & & (1) & 1 & 1 & 1 & 1 & 1 & 1 & 1 & 1 & & & & & & & & 1 & 1 & 1 & 1 & 1 & 1 & & & & & 1 & \begin{tabular}{|l|} 
\\
\end{tabular} & 1 & 1 & 1 & 1 \\
\hline 209 & & & & & & & & & (1) & & (1) & & & & & & & & & & & & & & & & & & & & & & & \\
\hline 210 & & & & & & & & 1 & & & & & & & & & & & & & & & & & & & & & & & & & & \\
\hline 211 & 1 & 1 & 2 & 1 & & 1 & & & & & & & 1 & 2 & 2 & 1 & 1 & 1 & & & & & & & 1 & 1 & 1 & 1 & & & & & & \\
\hline 212 & & 4 & 4 & 4 & 4 & & & & 4 & 3 & 2 & & & & & 4 & 4 & 4 & 4 & & & 3 & 3 & 3 & & & & & 4 & \begin{tabular}{|l|} 
\\
\end{tabular} & 4 & \begin{tabular}{|l|l|}
3 \\
\end{tabular} & \begin{tabular}{|l|l|}
3 \\
\end{tabular} & 3 \\
\hline 213 & & 1 & & & 1 & 1 & 1 & 1 & 1 & (1) & 1 & & & & & & & 1 & 1 & 1 & 1 & 1 & & 1 & & & & 1 & 1 & 11 & & 1 & 1 & \\
\hline 214 & (1) & & & (1) & (1) & 1 & 2 & 2 & 4 & 4 & 4 & & & & & & & 1 & 1 & 1 & 1 & 1 & 3 & 3 & & & (1) & & & & & & & 1 \\
\hline 220 & & & & & & & & & & & & & & & & & & & & & & & & & & & & & & & & (1) & & \\
\hline 221 & & & & (1) & & & & & & & & & & & & & & & & & & & & & & & & & & & & & & \\
\hline 222 & & & & & & & & & & & (1) & & & & & & & & & & & 1 & & & & & & & & & & & & \\
\hline 226 & & & & & & & & & & (1) & & & & & & & & & & & & & & & & & & & & & & & & \\
\hline 228 & & & & & & & & (1) & & & & & & & & & & & & & & & & & & & & & & & & & & \\
\hline 229 & 3 & 2 & 3 & 4 & 4 & 4 & 3 & 3 & 4 & 2 & & 1 & 1 & 2 & 3 & 4 & 3 & 4 & 3 & 3 & 2 & 3 & 3 & 3 & 3 & 2 & 3 & 3 & 3 & 1 & 3 & 1 & 1 & 2 \\
\hline 230 & 1 & 1 & 2 & 2 & 2 & 3 & 3 & 2 & 2 & 2 & & & & & 1 & 2 & 2 & 2 & 3 & 3 & 3 & 2 & & (1) & & & & 1 & 1 & 1 & 2 & \begin{tabular}{|l|l|}
3 \\
\end{tabular} & \begin{tabular}{|l|l|}
3 & \\
\end{tabular} & 2 \\
\hline 231 & & & & & (1) & & & & & (1) & (1) & & & & & & & & (1) & & & 1 & & & & & & & & & & & & \\
\hline 232 & & & & & & & & & & & & & (1) & & & & & & & & & & & & & & & 2 & & & & & & \\
\hline 233 & & & & & & & & & & & & & & & & & & & & & & (1) & & & & & & & & & & & & \\
\hline 234 & & & & & & & & & & & & & & & & & & & & & & & & 1 & & & & & & (1) & & & & \\
\hline 235 & & & & & & & & & & & & & & & & & & & & & & & & & & & & & (1) & & & & & \\
\hline
\end{tabular}




\begin{tabular}{|c|c|c|c|c|c|c|c|c|c|c|c|c|c|c|c|c|c|c|c|c|c|c|c|c|c|c|c|c|c|c|c|c|c|c|}
\hline \multicolumn{35}{|c|}{ Table 9, 2007-1 } \\
\hline & \multicolumn{8}{|c|}{$\mathrm{A}$} & \multicolumn{9}{|c|}{ B } & & & & & & & & 5 & & & & & & & & D & \\
\hline & 1 & 2 & 3 & 4 & & 6 & $7 \mid \varepsilon$ & 8 & \begin{tabular}{l|l}
12 \\
\end{tabular} & 3 & 4 & 5 & 6 & 7 & 8 & 9 & 10 & 1 & 2 & 3 & 4 & 5 & \begin{tabular}{l|l}
6 \\
\end{tabular} & & & \begin{tabular}{l|l}
9 & 1 \\
\end{tabular} & & & 12 & & 14 & & & 3 \\
\hline 2 & & & (1) & 1 & 1 & & & 1 & & & 1 & & & & & & & & & & & & & & & & & & & & & & & \\
\hline 3 & & & & & & & & & & & & & & & & & & & & & & & & & & & & & & & & & & \\
\hline 4 & & 1 & & & (1) & 1 & 1 . & 1 & & (1) & & & 1 & 1 & 1 & 1 & 2 & & & & & & & 2 & 25 & & 2 & & 2 & & & & & \\
\hline 5 & & & & (1) & & & 1 & 2 & & & 1 & 1 & & 1 & 1 & 1 & 2 & & & (1) & & & (1) & 1 & 1 & 1 & 2 & & & 1 & & & & (1) \\
\hline 6 & & & & & & & & & & & & & & & & & & & & & & & & & & & & & (1) & & & & & \\
\hline 7 & (1) & & & & & (1) & 1 & 1 & & & & (1) & (1) & & & & 1 & & & & & & & (1) & (1) $(1)$ & 1) ( & (1) & & & & (1) & & & \\
\hline 8 & & & & & & & (1) & & & & & & & & & & & & & & & & & & & & & & & & & & & \\
\hline 10 & & & & & & & 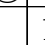 & 1 & & & & & & & & & & & & & & (1) & & & & & & & 1 & 1 & 1 & & & \\
\hline 11 & & & & & & & & (1) & & & & & & & & & & & & & & & & & & (1) & & & & & & & & \\
\hline 12 & 1 & 1 & & & 1 & 1 & 1 & 1 & 1 & & 1 & & 1 & 1 & (1) & 1 & & & & (1) & & 1 & & 1 & $1]$ & & & & & & & & & \\
\hline 13 & 1 & 1 & 1 & & 2 & 2 & 25 & $2] 1$ & \begin{tabular}{l|l}
1 & 1 \\
\end{tabular} & 1 & 1 & 2 & 2 & 2 & 2 & 2 & & & 1 & 1 & 1 & 1 & & 2 & 26 & 2 & 2 & & 1 & & 1 & & 1 & 1 \\
\hline 14 & 1 & & 1 & 1 & 1 & & & 2 & \begin{tabular}{l|l}
2 & 1 \\
\end{tabular} & 1 & 1 & & & & & & & 2 & 2 & 1 & 1 & 1 & 1 & & & & & & & & & 1 & 2 & 1 \\
\hline 15 & 2 & 1 & 1 & 1 & 2 & 1 & 25 & 211 & 1 & 1 & 2 & & 1 & 2 & 2 & 2 & 1 & & 1 & 1 & 1 & \begin{tabular}{|l|l|} 
\\
\end{tabular} & 1 & 2 & 26 & 2 & 3 & & 1 & & & & 1 & 1 \\
\hline 16 & & & & & & & 1 & 1 & & & & & & & & 1 & & & & & & & & & & & & & 1 & 1 & 1 & & & \\
\hline 17 & & 1 & & & & 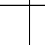 & 1 & 21 & 1 & & & & & & & 1 & & & 1 & & (1) & 1 & & 1 & & & & & & 1 & 1 & & & 1 \\
\hline 18 & 1 & 1 & 1 & & & 1 & 1 & & & 1 & 1 & & & & & 1 & & & & & & & & & & & & 1 & & & & & & \\
\hline 20 & (1) & & & & (1) & & . & 1 & & & & & & & & & & & & & & & & & & & & & & & & & & \\
\hline 21 & & & 1 & & & & & & (1) & & & & & & & & & & & & & & & & & & & & & & & & & \\
\hline 22 & 2 & 1 & & & & & 1 & 1 & \begin{tabular}{l|l}
15 \\
\end{tabular} & & 1 & & & & & & & 2 & 1 & 1 & & & & . & & & & & & & & & 1 & \\
\hline 26 & & & & & & & & & & & & & & & & & & & & & & & & & & & & 1 & & (1) & (1) & & & \\
\hline 28 & & & & & & & 1 & 1 & & & (1) & & & 1 & & 1 & 2 & & & & & & & 1 & & & 2 & 1 & 1 & & & & & \\
\hline 36 & & & & & & & & & & (1) & & & & & & & & & & & & & & & & & & & & & & & & \\
\hline 39 & & & & & & & 1 & & & & & & & & & & & & & & & & & & & & & (1) & & (1) & & & & \\
\hline 41 & & & & & & & (1) & & & & & & & & & & & & & & & & & & & & & & & & & & & \\
\hline 43 & 2 & & & & & & 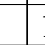 & 1 & & & & & & & & & & & & & & & & & & & & & & & & & & \\
\hline 44 & 1 & 1 & 1 & 2 & 1 & 1 & 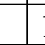 & 12 & 22 & 21 & 2 & 2 & 1 & 1 & 1 & 1 & & 2 & 1 & 1 & 1 & 2 & 1 & 2 & 1 & 1 & 1 & & & & & 1 & 1 & 1 \\
\hline 48 & & & & & & & & 2 & & & & & & & & 2 & 2 & & & & & & & & & 2 & & 2 & 2 & 2 & 2 & & & \\
\hline 53 & & & & & & & & & & & & & & & & & & & & & & & & & & & & & & & & & & \\
\hline 54 & & & & & & & & & & & & & & & & & & & & & & & & & & & & & & & & & & \\
\hline 58 & & & & & & & & & & & & & & & & & & & & & & & & & & & & & & & & & & \\
\hline 60 & 2 & 2 & 2 & 2 & 1 & & & s & 22 & 1 & 2 & 1 & 1 & & & & & 2 & 2 & 1 & 2 & 2 & & 1 & 1 & & & & & & & & 2 & 1 \\
\hline 63 & 2 & 2 & 1 & 2 & 2 & 2 & 1 & 16 & \begin{tabular}{l|l}
2 & 2
\end{tabular} & 1 & 1 & 2 & 1 & \begin{tabular}{|l|}
1 \\
\end{tabular} & 1 & 1 & & 1 & 1 & 1 & 11 & 1 & 1 & 1 & 1 & 1 & & & & & & & 1 & 1 \\
\hline 64 & & & & & 1 & & 1 & 1 & 1 & 1 & 1 & 1 & 1 & 1 & 1 & & & 1 & & & 1 & 1 & 1 & 1 & 1 & & & & & & & 1 & 1 & 1 \\
\hline 65 & & & & & & & & & & & & & & & & & & & & & & & & & & & & & & & & 1 & & \\
\hline 70 & & & & & & & & (1) & (1) & & & & & & & & & & 1 & & & & & & & & & & & & & & & \\
\hline 71 & 2 & 4 & 4 & 4 & 3 & 2 & 4 & 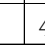 & \begin{tabular}{l|l}
4 & 4 \\
\end{tabular} & 44 & 3 & 4 & 3 & & & & & 3 & 4 & 4 & & 2 & & & & & & & & & & & 3 & 3 \\
\hline 73 & 2 & 2 & 2 & 1 & 2 & 3 & 2 & 3 & \begin{tabular}{l|l}
3 & 2 \\
\end{tabular} & 22 & 3 & & 2 & 2 & 2 & 1 & & 3 & 2 & 2 & 2 & 2 & 2 & 3 & 1 & 2 & & & & & & 2 & 2 & 2 \\
\hline 77 & 4 & 4 & 4 & 4 & 4 & 4 & 4 & 42 & $\begin{array}{l}43 \\
\end{array}$ & 33 & 4 & 4 & 4 & 4 & 3 & 3 & & 4 & 4 & 4 & 4 & 4 & 4 & 3 & & & & & & & & 3 & 4 & 4 \\
\hline 78 & 3 & & 4 & 4 & 3 & 4 & $3:$ & $3:$ & \begin{tabular}{|l|l}
3 & 1 \\
\end{tabular} & & 3 & 4 & 4 & 3 & 3 & & & 4 & 2 & 4 & 4 & 4 & 3 & 3 & 2 & 2 & & & & & & 4 & 2 & 3 \\
\hline 79 & 1 & & & & (1) & 1 & & 75 & 2 & & (1) & 1 & & 1 & & & & 2 & & 2 & 3 & (1) & 1 & 1 & & & & & & & & 1 & & \\
\hline 80 & & & & & & & & & & & 2 & & & & & & & & & & & & & & & & & & & & & & & \\
\hline 81 & 2 & 1 & 1 & 2 & 1 & 1 & 2 & 2 & 2 & 1 & 1 & 2 & 1 & 1 & & & & 2 & 2 & 1 & 1 & 2 & 2 & 1 & 2 & 2 & & & & & & 3 & 1 & 2 \\
\hline 82 & & & 2 & 2 & & & & & 72 & 22 & 2 & & & & & & & & & & & & & & & & & & & & & & & \\
\hline 84 & & & & & & & & & & & & & & & & & & & & & & & & & & & & & & & & & & \\
\hline 87 & & & & & & & & & & & & & & & & & (1) & & & & & (1) & & & & & & 1 & & & & & & \\
\hline 88 & & (1) & (1) & & & 1 & & & 1 & 11 & & & & & & & & & & & & & & & & & & (1) & & & & & & \\
\hline 94 & & & & & 1 & & 1 & & & 1 & & 1 & 1 & & & 1 & 1 & & & & & & & & & & & & & & & & & \\
\hline 99 & & & & & & & & & & & & & & & & & & & & & & & & & & & & & & & & & & \\
\hline 113 & (1) & & & & & & & $(1$ & (1) & & & & & & & & & & & & & & & & & & & & & & & & & \\
\hline 122 & & (1) & 1 & & 2 & 2 & 2 & 1 & & & 2 & 1 & & & & & & & & & 1 & & & & & & & & & & & & & \\
\hline 123 & & & & & 1 & & & & & & & 1 & & & & & & & & & & & & 1 & & & & & & & & & & \\
\hline 124 & 1 & 1 & 1 & 1 & 2 & 1 & 1 & 1 & \begin{tabular}{l|l}
1 & 1
\end{tabular} & \begin{tabular}{l|l}
1 & 1
\end{tabular} & 1 & & 1 & 1 & 1 & 1 & & 1 & 1 & 1 & & (1) & 1 & 2 & 1 & 1 & 1 & & & & & & 1 & 1 \\
\hline 129 & & & & & & & & & & & & & & & & & & & & & & & & & & & & (1) & & & & & & \\
\hline 130 & & & & & & & 1 & & & & & & & & & & & & & & & & & & & & & (1) & & & & & & \\
\hline 131 & & & & & & & & & & & & & & & & & & & & & & & & & & & & 1 & & & & & & \\
\hline
\end{tabular}




\begin{tabular}{|c|c|c|c|c|c|c|c|c|c|c|c|c|c|c|c|c|c|c|c|c|c|c|c|c|c|c|c|c|c|c|c|c|c|}
\hline \multicolumn{34}{|c|}{ Table 9, 2007-2 } \\
\hline & \multicolumn{11}{|c|}{ D } & \multicolumn{13}{|c|}{$E$} & & & & & $\mathrm{~F}$ & & & & \\
\hline & 4 & $5 \mid$ & 6 & 7 & 8 & & & & 12 & 13 & & 1 & 2 & 3 & 4 & 5 & 6 & 7 & 8 & 9 & 10 & & 12 & & 1 & 2 & 3 & 45 & & & 8 & 9 & 10 \\
\hline 2 & & & & & & & & & & & & & & & & & & & & & & & & & & & & & & & & & \\
\hline 3 & & & & & & & & & & & & & & & & & & & & & & & & & & & & & (1) & & & & \\
\hline 4 & 1 & 1 : & 1 & 1 & 1 & 2 & 2 & 2 & 1 & 1 & & & & & & 1 & 1 & 1 & 2 & 1 & 1 & 1 & 1 & (1) & & & & 1 & 1 & \begin{tabular}{l|l|}
1 & 1 \\
\end{tabular} & 1 & 1 & \\
\hline 5 & 1 & & 2 & 2 & 1 & 2 & 2 & 1 & & 1 & & & & & & 1 & 1 & 1 & 1 & 1 & 1 & & & & & & & & 1 & & 1 & & 1 \\
\hline 6 & & (1) & & & & & & & & & (1) & & & & & & & & & & & & & & & & & & & & & & (1) \\
\hline 7 & & & 1 & 1 & 1 & 1 & 1 & 1 & & 1 & 1 & & & & & 1 & 1 & 1 & 1 & 1 & 1 & 1 & (1) & (1) & & & & & (1) & \begin{tabular}{l|l|}
1 & 1 \\
\end{tabular} & & 1 & 1 \\
\hline 8 & & & & & & & & & & & & & & & & & & & & & & & & & & & & & & & & & \\
\hline 10 & & & & 1 & 1 & 2 & & 1 & 1 & 1 & 1 & & & & & & (1) & 1 & 1 & 1 & 1 & 1 & 1 & 1 & & & & & & 2 & 2 & 1 & 1 \\
\hline 11 & & & 1 & & & 1 & & & & & & & & & & & & & & & & & & & & & & 1 & & & & & \\
\hline 12 & & 2 & 1 & 1 & 1 & (1) & & & & & & & & & (1) & 1 & & 1 & & & & & & & (1) & & & 1 & & & & & \\
\hline 13 & 1 & 18 & 2 & 2 & 2 & 2 & 2 & & 1 & 1 & 1 & & & & & 1 & 2 & 2 & 1 & 2 & 1 & & & & & & & 1 & 1 & & 1 & 1 & \\
\hline 14 & 1 & 2 & & + & & (1) & 2 & 1 & & & & & & & 1 & 1 & & 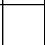 & 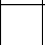 & 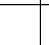 & & & & & 1 & (1) & & \begin{tabular}{l|l}
1 \\
\end{tabular} & & 1 & & 4 & \\
\hline 15 & \begin{tabular}{|l|} 
\\
\end{tabular} & 1 & 2 & 2 & 2 & 3 & 3 & 2 & 1 & 1 & & & & 1 & 1 & 3 & 1 & 2 & 3 & 2 & 2 & 2 & 2 & 1 & 1 & 1 & 1 & 2 & 1 & \begin{tabular}{l|l}
2 & 3
\end{tabular} & 3 & 4 & 3 \\
\hline 16 & & & 2 & & & & & 1 & 1 & 1 & 1 & & & & & & & & & & & 1 & & 1 & & & & & & & & 1 & 1 \\
\hline 17 & 1 & 1 & 1 & 1 & 1 & 2 & & & & 1 & 1 & & & & 1 & 1 & 1 & 1 & & & 1 & & 1 & (1) & & 1 & 1 & 1 & 1 & 1. & 1 & & 1 \\
\hline 18 & 1 & & 1 & 1 & & & & & 1 & 1 & & & & & 1 & & 1 & & 1 & 2 & & & & 1 & & & & & & 1 & & 1 & 1 \\
\hline 20 & & & & & & & & & & & & & & & & (1) & 1 & 1 & & 1 & & & & & & & & & 1 & & & & \\
\hline 21 & & 1 & & & & & & & & & & & & & & & & & & & & & & & & & & & & & & & \\
\hline 22 & & 1 & & & & & & & & & & 1 & & & & & & & & & & & & & & & & & & & & & \\
\hline 26 & & & & & & & & & (1) & & & & & & & & & & & & & & & & & & & & & & & & 1 \\
\hline 28 & & & 1 & 1 & 1 & 1 & 2 & 2 & 1 & & & & & & & 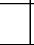 & & 1 & 1 & 1 & 1 & 1 & & 1 & & & & & & \begin{tabular}{l|l}
1 & 1 \\
\end{tabular} & 1 & 1 & \\
\hline 36 & & & & & & & & & & & & & & & & & & & & & & & & & & & & & & & & & \\
\hline 39 & & & & & & & & & & & & & & & & & & & & & & & & (1) & & & & & & & & & \\
\hline 41 & & & & & & & & & & & & & & & & & & & & & & (1) & & & & & & & & & & (1) & \\
\hline 43 & & & & & & 1 & & & & & & & & & & 2 & & 1 & 2 & 1 & 1 & & & & & & & 1 & & 1 & & & \\
\hline 44 & \begin{tabular}{|l|l|}
1 \\
\end{tabular} & 18 & 2 & 1 & 1 & (1) & & & & & & & 1 & & 1 & 2 & 2 & 1 & & 1 & & & & & 1 & 1 & 1 & 1 & 1 & 1 & & & \\
\hline 48 & & & & (1) & & 2 & 2 & 2 & 2 & 2 & 2 & & & & & (1) & & 1 & 1 & 1 & 2 & 2 & 2 & 2 & & & & & & 1 & & \begin{tabular}{l|l}
2 \\
\end{tabular} & 1 \\
\hline 53 & & & & 1 & & & & & & & & & & & & & & & & & 2 & 1 & & (1) & & & & & & \begin{tabular}{l|l}
1 & 1
\end{tabular} & & - & \\
\hline 54 & & & & & & & & & & & & & & & & & & & (1) & & & & & & & & & & & & & & \\
\hline 58 & & & & & & & & & & & & & & & & & & & & & & (1) & & & & & & & & & & & \\
\hline 60 & \begin{tabular}{|l|}
1 \\
\end{tabular} & 1 & 1 & 1 & 1 & & & & & & & & & & & 1 & (1) & 1 & & & & & & & 2 & 1 & & 1 & 2 & 1 & & (1) & \\
\hline 63 & \begin{tabular}{|l|} 
\\
\end{tabular} & 2 & 2 & 1 & 1 & (1) & & & & & & & & & 1 & 2 & \begin{tabular}{|l|}
1 \\
\end{tabular} & \begin{tabular}{|l|}
1 \\
\end{tabular} & & & & & & & 1 & 1 & & 1 & 1 & 1 & & & \\
\hline 64 & (1) & 1 & 1 & 1 & & & & & & & & & & & \begin{tabular}{l|l}
1 \\
\end{tabular} & & & & & & & & & & 2 & 1 & 11 & (1) & & & & & \\
\hline 65 & & & & & & & & & & & & & & & & & & & & & & & & & & & & & & & & & \\
\hline 70 & & & & & & & & & & & & & & & & & & & & & & & & & & & & & & & & & \\
\hline 71 & & 2 & & & & & & & & & & & & & & 2 & & & & & & & & & & & & & & & & & \\
\hline 73 & \begin{tabular}{|l|}
2 \\
\end{tabular} & 2 & 3 & 3 & 3 & 2 & & & & & & $\begin{array}{ll}3 \\
\end{array}$ & 2 & \begin{tabular}{|l|l|}
2 \\
\end{tabular} & 2 & 3 & 2 & 2 & 1 & 1 & & & & & 3 & 3 & 3 & 2 & 2 & & & & \\
\hline 77 & \begin{tabular}{|l|}
4 \\
\end{tabular} & \begin{tabular}{l|l}
3 \\
\end{tabular} & 4 & 3 & 2 & 3 & & & & & & 4 & 4 & 4 & 4 & 3 & 3 & & 1 & \begin{tabular}{|l|} 
\\
\end{tabular} & 2 & & & & 2 & 3 & 4 & 4 & 2 & & & & \\
\hline 78 & \begin{tabular}{|l|}
3 \\
\end{tabular} & 1 & 3 & 2 & \begin{tabular}{l|}
3 \\
\end{tabular} & \begin{tabular}{l|}
3 \\
\end{tabular} & 1 & & & & & 4 & 3 & \begin{tabular}{|l|}
3 \\
\end{tabular} & 3 & 2 & 1 & & & & & & & & 4 & $\begin{array}{ll}3 \\
\end{array}$ & 2 & 2 & & & & & \\
\hline 79 & & & & & & & & & & & & 1 & & & & & & & & & & & & & 2 & & & & & & & & \\
\hline 80 & (1) & & & & & & & & & & & & & & & & & & & & & & & & (1) & & & & & & & & \\
\hline 81 & \begin{tabular}{|l|l|}
1 \\
\end{tabular} & 11 & 1 & 1 & & & & & & & & 2 & 2 & \begin{tabular}{|l|}
2 \\
\end{tabular} & 1 & & & & & & & & & & 2 & 2 & 3 & 1 & & & & & \\
\hline 82 & & & & & & & & & & & & & & & & & & & 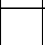 & & & & & & & & & 3 & & & & & \\
\hline 84 & & & & & & (1) & & & & & & & & & & & & & & & & & & & & & & & & & & (1) & \\
\hline 87 & & & & & & (1) & & & & & 1 & & & & & & & & & & & 1 & & & & & & & & (1) & & & \\
\hline 88 & & & & & & & & & & & & & & & & & (1) & & (1) & & & & & & & & & 2 & 1 & 1 & (1) & & (1) \\
\hline 94 & & & & & & & 1 & 1 & (1) & & & & & & & (1) & & (1) & & (1) & & 1 & (1) & & & & & (1) & (1) & 1 & 1 & & \\
\hline 99 & & & & & & & & & & & (1) & & & & & 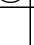 & 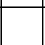 & & & 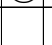 & & & & & & & & 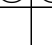 & & 7 & & & \\
\hline 113 & & & & & & & & & & & & & & & & & & & & & & & & & & & & & & & & & \\
\hline 122 & & 1 & 1 & & 1 & & & & & & & & & & & 1 & 1 & 1 & 1 & 1 & & & & & & & & \begin{tabular}{l|l}
1 & 6
\end{tabular} & 2 & \begin{tabular}{l|l}
2 & 2 \\
\end{tabular} & 2 & 1 & \\
\hline 123 & & & & & & (1) & & & & & & & & & & (1) & & (1) & & & & & & & & & & & (1) & \begin{tabular}{l|l}
1 &
\end{tabular} & & & \\
\hline 124 & \begin{tabular}{|l|}
1 \\
\end{tabular} & 1 & 1 & 1 & 1 & \begin{tabular}{|l|l|}
1 \\
\end{tabular} & 1 & & & & & & & (1) & & 1 & 1 & $\begin{array}{ll} \\
\end{array}$ & & 1 & & & & & & & & 1 & & \begin{tabular}{|l|} 
\\
\end{tabular} & & & \\
\hline 129 & & & (1) & & & & & & & & & & & & & & & & & & & & & & & & & & & & & 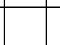 & \\
\hline 130 & & & & & & & & 1 & 1 & & & & & & & (1) & & & 1 & & (1) & 1 & 1 & & & & & 1 & & & & & (1) \\
\hline 131 & & & & & & & & & & & & & & & & & & & & & & 1 & & & & & & & & \begin{tabular}{l|l}
1 & 1
\end{tabular} & & 1 & \\
\hline
\end{tabular}




\begin{tabular}{|c|c|c|c|c|c|c|c|c|c|c|c|c|c|c|c|c|c|c|c|c|c|c|c|c|c|c|c|c|c|c|c|c|c|}
\hline \multicolumn{34}{|c|}{ Table 9, 2007-3 } \\
\hline & \multicolumn{7}{|c|}{ A } & & \multicolumn{9}{|c|}{$\mathrm{B}$} & \multicolumn{13}{|c|}{$\mathrm{C}$} & \multicolumn{3}{|c|}{ D } \\
\hline & 1 & $2[5$ & 3 & 4 & & & $7 \mid \varepsilon$ & \begin{tabular}{l|l}
3 & 1 \\
\end{tabular} & 12 & 3 & 4 & 5 & 6 & 7 & 8 & 9 & 10 & 1 & 2 & 3 & 4 & 5 & \begin{tabular}{|c|c|}
6 \\
\end{tabular} & & $8 \mathrm{~g}$ & \begin{tabular}{l|l}
9 & 10 \\
\end{tabular} & 11 & 12 & 13 & 14 & & & 3 \\
\hline 132 & & $1]$ & 1 & & 1 & & $2[5: 5 x-5$ & & & 1 & 2 & & 1 & 1 & 2 & 2 & 2 & & 1 & 1 & 1 & (1) & & 1 & & \begin{tabular}{|l|l|}
2 & 2 \\
\end{tabular} & & & & & & & (1) \\
\hline 138 & & & & & & & & & & & & & & & & & & & & & & & & & & & & & & & & & \\
\hline 140 & & & & & & & & & & & & & & & & & & & & & & & & & & & & & & & & & \\
\hline 141 & & & & & & & & & & & & & & & & & 1 & & & & & & & & & & 2 & & (1) & (1) & & & \\
\hline 142 & 1 & 1 1] 1 & 1 & 1 & & & 15 & 2 & & (1 & 1 & & 1 & 1 & 1 & 2 & 2 & & & 1 & & & & 1 & \begin{tabular}{l|l}
1 & 1 \\
1 &
\end{tabular} & \begin{tabular}{l|l}
1 & 2 \\
\end{tabular} & 2 & 1 & 1 & 1 & & (1) & 1 \\
\hline 143 & & & & & & & & & & & & & & & & & 1 & & & & & & & & & & 2 & & & 1 & & & \\
\hline 145 & & & & & 2 & 1 & 2 & 1 & & & 1 & 2 & 1 & & & & 1 & & & & 1 & & & & & & (1) & & & & & & \\
\hline 146 & & & & & & & & & & & & & & & & & & & & & & & & & & & & & & & & & \\
\hline 149 & & & & & & & & 1) & & & & & & & & & & & & & 1 & & & & & & & & & & & & \\
\hline 155 & & & & & & & & & & 1 & & 1 & & & & & & & & & & & & & & & & & & & & & \\
\hline 156 & & (1) & & & & & & & & & & & & & & & & & & (1) & & & & & & & & & & & & & \\
\hline 158 & & 1 & & & 2 & & & & & & & & & & & & & & & 2 & 1 & & & & & & & & & & & & \\
\hline 161 & & & & & & & & & & & & & & & & & & & & & & & & & & & & & & & & & \\
\hline 168 & & & & & & & & & & & & 1 & & & & & & & & & & & & & & & & & & & & & \\
\hline 173 & & & & & & & & & & & & & & & & & & & (1) & & & & & & & & & & & & & & \\
\hline 175 & & (1) & & & & & & & & & & & & & & & & & & & & & & & & & & & & & & & \\
\hline 179 & & & & & & & & & & & & & (1) & & & & & & & & & & & & & & & & & & & & \\
\hline 180 & & & & & & & & & & & & & & & & & & & & & & & & & & & & & & & & & \\
\hline 182 & & & & 1) ( & & & & & & & & & 1 & & & & & & & & (1) & & & & & & & & (1) & & & & \\
\hline 193 & & & & & & & & & & & & & & & & & & & & . & & & & & & & & & & & & & \\
\hline 197 & & & & & & & & & & & & & & 2 & 1 & & & & & & & & & & 2 & & & & & & & & \\
\hline 198 & 1 & 13 & 1 & 1 & 2 & $2]$ & 2 & & & & 1 & 2 & 2 & 2 & 1 & & & & & 1 & 2 & 2 & 2 & 2 & 2 & & & & & & & & 2 \\
\hline 199 & 2 & $1] 1$ & 1 & 1 & 1 & & 1 & 1 & \begin{tabular}{l|l}
1 & 1 \\
\end{tabular} & 11 & 1 & 2 & 1 & 1 & 1 & & & 1 & 1 & 1 & & 1 & \begin{tabular}{l|l|}
1 & \\
\end{tabular} & 1 & 1 & 1 & & & & & & 1 & \\
\hline 200 & & & & & 1 & & & & & & 1 & & 2 & 2 & 2 & 2 & & & & & & 1 & 1 & 1 & 22 & \begin{tabular}{l|l}
2 & 1 \\
\end{tabular} & & (1) & 1 & 1 & & & \\
\hline 207 & & & & & & & & 1) & & & 1 & (1) & & & & & 1 & & & 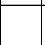 & 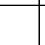 & & & (1) & (1) 1 & 1 & 1 & (1) & (1) & 1 & & & \\
\hline 210 & & & & & & & & & & & & & & & & & & & & & & & & 1 & & (1) & & & & & & & \\
\hline 211 & 2 & 26 & 2 & 2 & & 1 & 45 & 25 & 2 & 1 & 2 & 1 & 1 & & & & 2 & 2 & 2 & 2 & 2 & 2 & 1 & 2 & 2 & & & & & & 1 & 2 & 2 \\
\hline 212 & & $\begin{array}{ll}4 & 4\end{array}$ & 4 & 2 & & 4 & 3 & 36 & 1) 4 & $\begin{array}{l}44 \\
\end{array}$ & 4 & & 3 & 4 & 4 & 2 & 3 & 4 & 4 & 4 & & & 4 & 4 & 3 & & 4 & 4 & 4 & 4 & 3 & 4 & 4 \\
\hline 213 & & 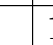 & 1 & & 2 & & 2 & 1 & & 1 & & 1 & 1 & & & 1 & 2 & & & 1 & 1 & (1) & & & 1 & \begin{tabular}{l|l}
11 & 2
\end{tabular} & 2 & 1 & 1 & & & & \\
\hline 214 & & & & & & & 10 & (1) & & & & 1 & (1) & & & 1 & 3 & & 1 & & & & 1 & 1 & 1 & \begin{tabular}{l|l}
1 & 2 \\
\end{tabular} & 3 & 44 & 4 & 4 & & (1) & \\
\hline 218 & & & & & & & & & & & & & & & & & (1) & & & & & & & & & & 1 & & & & & & \\
\hline 220 & & & & & & & & & & & & (1) & & & & & & & & & & & & & & & & & & & & & \\
\hline 221 & & & & & & & 1 & 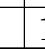 & 1 & & & & & & & & & & & & (1) & & 1 & (1) & & & & & & & & & \\
\hline 222 & & & & & & & & & & & & & & & & & & & & & & & & & & & 1 & & & (1) & & & \\
\hline 225 & & & & & & & (1) & & & & & & & & & & & & & & & & & & & & & & & & & & \\
\hline 226 & & & & & & & & & & & & & & & & & & & & & & & & & & & & & & & & & \\
\hline 229 & 3 & 2 & 2 & 2 & 2 & 2 & 2 & 35 & $\begin{array}{lll}2 & 1\end{array}$ & 12 & 3 & 2 & 2 & 2 & 3 & 4 & 4 & 2 & 2 & 2 & 2 & 2 & 3 & 3 & & \begin{tabular}{l|l}
4 & 3
\end{tabular} & 3 & 4 & 1 & 1 & 3 & 2 & 3 \\
\hline 230 & & & & (1) & & & $1]$ & 3 & & & & & 1 & 1 & 1 & 2 & 2 & & & & 1 & & 1 & & & \begin{tabular}{l|l}
2 & 3 \\
\end{tabular} & 2 & 2 & (1) & & & & \\
\hline 231 & & & & & & & & & & & & & & & & & & & & & & & & & & (1) & & & & & & & \\
\hline 232 & 1 & & & & & & & & & & & & & & & & & & & & & & & & & & & & & & & & \\
\hline 233 & & & & & & & & & & & & & & & & & & & & & & & & & & & (1) & & & & & & \\
\hline 234 & & & & & & & 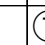 & (1) & & & & & & & & & & & & & & & & & & & & & & & & & \\
\hline 235 & & & & & & & & & & & & & & & & & & & & & & & & & & (1) & & & & & & & \\
\hline & & & & & & & & & & & & & & & & & & & & & & & & & & & & & & & & & \\
\hline
\end{tabular}




\begin{tabular}{|c|c|c|c|c|c|c|c|c|c|c|c|c|c|c|c|c|c|c|c|c|c|c|c|c|c|c|c|c|c|c|c|c|c|c|}
\hline \multicolumn{35}{|c|}{ Table 9, 2007-4 } \\
\hline & \multicolumn{11}{|c|}{$\mathrm{D}$} & \multicolumn{13}{|c|}{$E$} & \multicolumn{10}{|c|}{$\mathrm{F}$} \\
\hline & 4 & 5 & 6 & 7 & 8 & & & & 12 & 13 & & 1 & 2 & 3 & 4 & 5 & 6 & & 8 & 9 & 10 & & 12 & 13 & 1 & 2 & 3 & 4 & & & & 8 & & 10 \\
\hline 132 & (1) & 2 & 2 & 2 & 2 & 3 & 2 & 2 & 2 & & & & & & & 1 & 2 & 2 & 2 & 2 & 2 & 2 & 2 & & & & & 1 & 2 & 2 & 2 & 2 & 2 & 2 \\
\hline 138 & & & & & & & & & (1) & & & & & & & & & & & & & & & & & & & & & & & & & \\
\hline 140 & & & & & & & (1) & & & & & & & & & & & & & & & & & & & & & & & & & & & \\
\hline 141 & & & 1 & & 1 & 1 & & 2 & & 1 & & & & & & & & 1 & & & (1) & 1 & 1 & 1 & & & & & & 1 & 1 & 1 & 1 & 1 \\
\hline 142 & & 1 & 1 & 1 & 1 & 1 & 2 & 2 & 1 & 1 & 1 & & & & 1 & 1 & 1 & 1 & 1 & 1 & 1 & 1 & 1 & 2 & & 1 & & 1 & 2 & 2 & 1 & 1 & 1 & 1 \\
\hline 143 & & & & & & & & 1 & & & 1 & & & & & & (1) & & (1) & & 1 & 1 & 1 & 1 & & & & & & 1 & 1 & 1 & & 1 \\
\hline 145 & & & & & (1) & 1 & & & & & & & & & & & & & & & & 1 & & & & & & & 1 & 1 & (1) & & 1 & 1 \\
\hline 146 & & & & & & & & 1 & & & & & & & & & & & & & & & & & & & & & & & & & & \\
\hline 149 & & & & & & & & & & & & & & & & & & & & & & & & & & & & & & (1) & & & & \\
\hline 155 & & & & & & & & & & & & & & & & & & & & & & & & & & & & & & & & & & \\
\hline 156 & & & & & & & & & & & & & & & & & & & & & & & & & & & & & & & & & & \\
\hline 158 & & (1) & & & 1 & & & & & & & & & & & & & & & & & & & & & & & & & & & & & \\
\hline 161 & & & & & & & & & & & & & & & & & (1) & & & & & & & & & & & & & (1) & (1) & & & \\
\hline 168 & & & & & & & & & & & & & & & & & & & & & & & & & & & & & & & & & & \\
\hline 173 & & & & & & & & & & & & & & & & & & & & & & & & & & & & & & & & & & \\
\hline 175 & & & & & & & & & & & & & & & & & & & & & & & & & & & & & & & & & & \\
\hline 179 & & & & & & & & & & & & & & & & & & & & & & & & & & & & & & & & & & \\
\hline 180 & & & & & & & & & & & & & & & & & & & & & & & & & & & & & & & & & (1) & \\
\hline 182 & & & & & & & & (1) & & & & & & & & & & & & & & & & & & & & 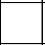 & & & & & 4 & \\
\hline 193 & & & & & & & & & & & & & & & & & & & & & & & 1 & & & & & 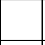 & & & & & 1 & \\
\hline 197 & & & & & & & & & & & & & & & & & & & & & & & & & & & & & & & & & & \\
\hline 198 & 2 & & 2 & 1 & 1 & & & 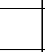 & & & & 2 & 2 & 2 & 2 & & & 1 & & & & & & & 1 & 1 & 1 & 1 & & & & & & \\
\hline 199 & & 1 & 2 & 1 & & 1 & 1 & & & & & & (1) & (1) & & 1 & 1 & 1 & & 1 & 1 & & & (1) & 1 & 1 & & 1 & 1 & 1 & 1 & 1 & & \\
\hline 200 & 1 & & 2 & 1 & 2 & 2 & 2 & 2 & 1 & 1 & & & & & & 3 & 1 & 2 & 2 & 2 & 2 & 2 & & 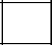 & & & & 1 & 2 & 2 & 3 & 2 & \begin{tabular}{|l|l|}
3 \\
\end{tabular} & 3 \\
\hline 207 & & (1) & & (1) & 1 & (1) & 1 & 1 & 1 & 1 & 1 & & & & & & (1) & 1 & 1 & 1 & 1 & 1 & 1 & 1 & & & & 1 & 1 & 1 & 1 & 1 & 1 & 1 \\
\hline 210 & & & & & & & & & & & & & & & & & & & & & & & & & & & & (1) & & & & & & \\
\hline 211 & 2 & 2 & 2 & 2 & 2 & & & 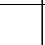 & & & & 2 & 2 & 2 & 2 & & 2 & & & & & & & & & 2 & 2 & 1 & & & 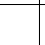 & & & \\
\hline 212 & & 4 & 4 & 4 & 4 & (1) & & 3 & 4 & 3 & 1 & & & & & 4 & 4 & 4 & 3 & & (1) & 4 & 4 & 4 & & & & & 4 & 4 & 4 & 4 & 2 & 3 \\
\hline 213 & & 1 & (1) & & 1 & 1 & 1 & 1 & 1 & 1 & & & & & & & 1 & 1 & 1 & & 2 & 1 & & 1 & & & & 1 & 2 & 1 & 1 & 1 & 1 & \\
\hline 214 & (1) & (1) & 1 & (1) & & (1) & 2 & 3 & 4 & 4 & 3 & & (1) & & & (1) & 1 & & & & 1 & & (1) & 4 & & & & & & & & & & \\
\hline 218 & & & & & & & & 1 & & & & & & & & & & & (1) & & (1) & (1) & & & & & & & & & & & & \\
\hline 220 & & & & & & & & & & & & & & & & & & & & & & & (1) & & & & & & & & & & & \\
\hline 221 & & (1) & (1) & & & & & & & & & & & & & & & & & & & & & & & & & (1) & & & & & & \\
\hline 222 & & & & & & & & & & & & & & & & & & & & & & & & & & & & & (1) & & & & & \\
\hline 225 & & & & & & & & & & & & & & & & & & & & & & & & & & & & & & & & & & \\
\hline 226 & & & & & & & & & & & 1 & & & & & & & & & & & & & & & & & & & & & & & \\
\hline 229 & 2 & 3 & 3 & 3 & 3 & 4 & 4 & 3 & 4 & 2 & & 1 & 2 & 2 & 3 & 3 & 4 & 3 & 1 & 2 & 3 & 4 & 4 & 3 & 2 & 3 & 3 & 4 & 3 & 1 & 3 & 1 & 2 & 4 \\
\hline 230 & & 1 & 1 & 2 & 2 & 3 & 3 & 2 & & 1 & & & 1 & 1 & 1 & 2 & 2 & 3 & 3 & 3 & 3 & & & & 1 & (1) & 1 & 1 & 1 & 1 & 2 & 2 & 3 & 1 \\
\hline 231 & & & & & & & & & & & & & & & & & & & & & & & & & & & & & & & & & & \\
\hline 232 & & & & & & & & & & & & & & & 1 & & & & & & & & & & & 1 & 2 & 1 & & & & & & \\
\hline 233 & & & & & & & & & & & & & & & & & & & & & & & & & & & & & & & & & & \\
\hline 234 & & & & & & & & & & & & & & & & & & & & & & & & & & & & & & & & & & \\
\hline 235 & & & & & & (1) & & 1 & & & & & & & & & & & & & & & & & & & & & & & & & & \\
\hline 242 & & & & & & & & & & & & & & & & & & & & & & & & (1) & & & & & & & & & & \\
\hline
\end{tabular}




\begin{tabular}{|c|c|c|c|c|c|c|c|c|c|c|c|c|c|c|c|c|c|c|c|c|c|c|c|c|c|c|c|c|c|c|c|c|c|c|}
\hline \multicolumn{35}{|c|}{ Table 9, 2008-1 } \\
\hline & \multicolumn{7}{|c|}{$\mathrm{A}$} & \multicolumn{10}{|c|}{ B } & & & & & & & & C & & & & & & & & D & \\
\hline & 1 & & 3 & 4 & & 6 & 78 & $\begin{array}{l}3 \cdot 1 \\
\end{array}$ & & & & 4 & 5 & & 78 & 9 & 10 & 01 & 2 & 3 & 4 & 5 & 6 & 7 & 8 & 9 & & 11 & 12 & 13 & 14 & & 2 & 3 \\
\hline 2 & & & (1) & & 1 & & & 1 & & & & & & & & & & & & & & & & & & & & & & & & & & \\
\hline 3 & & & & & & & & & & & & & & & & & & & & & & & & & & & (1) & & & & & & & \\
\hline 4 & & 1 & 1 & 1 & & (1) & 1) 1. & 1 & & & 1) & 1 & & 1 & 1 & 1 & 2 & 1 & & (1) & & & & 1 & 1 & 2 & 2 & 1 & 1 & 1 & & & & \\
\hline 5 & (1) & & & & & (1) & 1): & 2 & & 1 & & 1 & 1 & 1 & 1 & 2 & & & & & & 1 & & 1 & 1 & 2 & 1 & 1 & 1 & 1 & & & & \\
\hline 6 & & & & & & & & & & & & & & & & & & & & & & & & . & . & & & & & 1 & & & & \\
\hline 7 & (1) & 1 & & & & & & 1 & & & 1) & 1 & & & & 1 & 1 & & (1 & & & & & 1 & & 1 & & 1 & 1 & 1 & 1 & & & (1) \\
\hline 10 & & & & & & & & & & & & & & & & & & & & & & & & & & 1 & & 1 & 1 & 1 & 1 & & & \\
\hline 11 & & & & & & & & & & & & & & & & . & & & & & & & & . & & & & & & 1 & & & & \\
\hline 12 & (1) & 1 & 1 & & & (1) & & 1 & & 1) & 1 & 1 & 10 & 1) & 1 & 1 & & 1 & & (1 & (1) & & & 1 & 1 & & & & & & & & & \\
\hline 13 & 1 & 1 & 1 & 1 & 1 & 1 & $1: 2$ & 213 & & 1 & & 1 & $1:$ & $2[1$ & 1 & 2 & 1 & & & & & 1 & & 2 & 2 & 2 & 2 & 1 & 1 & 1 & 1 & & 1 & 1 \\
\hline 14 & 1 & 1 & 1 & & 1 & & 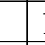 & 1 & & 1 & & 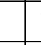 & 16 & & & 1 & & 1 & 1 & 2 & 1 & (1) & 1 & (1) & & & & & & & & & 1 & \\
\hline 15 & 1 & 2 & 1 & 1 & & 1 & 1 & 2 & & & 1 & 2 & 1 & 21 & 12 & 3 & 1 & 1 & 1 & 1 & 1 & 1 & 1 & 2 & 3 & 3 & 3 & 2 & 2 & 2 & & & 1 & 2 \\
\hline 16 & & & & & & & & 1 & & & & & & & & & 1 & & & & & & & 4 & $\square$ & & & 1 & 1 & 2 & & & & \\
\hline 17 & & 1 & 1 & & & & & 17 & & 1 & & & & & 1 & 1 & 1 & & 1 & 1 & & & 1 & & 1 & 1 & 1 & 1 & 1 & & & & (1) & (1) \\
\hline 18 & 1 & 1 & 1 & & & & & 1 & & & & & & & & & 1 & & & & & & 1 & 1 & 1 & 1 & 1 & 1 & 1 & 1 & 2 & & & (1) \\
\hline 20 & & 1 & & & & & & (1) & D) & & & & & & & & & & & (1) & & & & (1) & (1) & & & & & & & & 1 & \\
\hline 21 & & & & & & & & $(1$ & 1 & 1) & & & & & & & & 1 & (1) & 1 & & & & & & & & & & & & & & \\
\hline 22 & (1) & & 1 & & & & & 1 & & 1 & 1 & & & & & & & 1 & 1 & 1 & & 1 & & & & & & & & & & & & \\
\hline 23 & & & & & & & & & & & & & & & & & & & & & & & & & & & & & & & & & & \\
\hline 26 & & & & & & & & & & & & & & & & (1) & & & & & & & & & & & & 1 & (1) & 1 & 1 & & & \\
\hline 28 & & & & & & & & 1 & & & & 1 & & & 1 & 1 & 1 & & & & & & & & 1 & 1 & 2 & 1 & 1 & & (1) & & & \\
\hline 29 & & & & & & & & & & & & & & & & & & & & & & & & & & & & & & & (1) & & & \\
\hline 39 & & & & & & & & & & & & & & & & & & & & & & & & & & & & & 1 & & & & & \\
\hline 41 & & (1) & & & & & & & & 1 & & & & & & (1) & & & (1) & & & & & & & & & & & & & & & \\
\hline 43 & & & 1 & & & & & 1 & & . & & & & & 2 & & & & . & & & & & & 1 & & 1 & & & & & & & \\
\hline 44 & 2 & 1 & 1 & 1 & & & (1) & 2 & & 1 & 1 & 2 & 1 & & $D$ & & & 1 & 1 & 1 & 1 & & (1) & & (1) & (1) & & & & & & 2 & 1 & 1 \\
\hline 48 & & & & & & & 2 & 2 & & & & & & & & 2 & 2 & & & & & & & & & 2 & 2 & 2 & 2 & 3 & 2 & & & \\
\hline 53 & & & & & & & & & & & & & & & & & & & & & & & & & & & & & & & & & & \\
\hline 54 & & & & & & (1) & & & & & & & & & & & & & & & & & & & & & & & & & & & & \\
\hline 58 & & & & & & & & & & & & & & & & & & & & & & & & & & & & & & & (1) & & & \\
\hline 60 & 1 & 2 & 2 & 2 & 1 & & & 2 & & 2 & 1 & 1 & 1 & (1) & D) & & & 3 & 3 & 1 & 2 & 1 & & & & & & & & & & 2 & 2 & 2 \\
\hline 61 & & & & & & & & & & & & & & & & & (1) & & & & & & & & & & & & & & & & & \\
\hline 62 & & & & & & & & & & & & & & & (1) & & & & & & & & & & & & & & & & & & & \\
\hline 63 & 1 & 2 & 2 & 1 & 1 & 2 & 1 & 1) 1 & & 1 & 1 & 1 & 1 & 1 & 11 & 1 & & 1 & 1 & 2 & 1 & 1 & 1 & 1 & 1 & 1 & (1) & & & & & & & 1 \\
\hline 64 & (1) & & & (1) & & 1 & & & & & & & 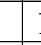 & 11 & 12 & & & 1 & & & & 1 & 1 & 1 & 1 & & & & & & & 1 & 1 & 1 \\
\hline 65 & & & & & & & & & & & & & & & & & & & & & & & & & & & & & & & & & & \\
\hline 70 & (1) & & & & & & & & & & & & & & & & & & & & & & & & & & & & & & & & & \\
\hline 71 & 3 & 4 & 4 & 1 & 1 & . & 1 & 2 & & 4 & 4 & 1 & $1:$ & 3 & & & & 4 & 4 & 4 & & & & & & & & & & & & & & \\
\hline 73 & 3 & 2 & 2 & 2 & 2 & 3 & 1 & 25 & & 2 & & 2 & 2 & 25 & 2 & 1 & & 3 & 1 & 1 & 3 & 2 & 3 & 2 & 2 & 1 & & & & & & 3 & 3 & 2 \\
\hline 77 & 3 & 3 & 3 & 4 & 4 & 4 & $4:$ & $3:$ & & 3 & & 3 & 4 & 4 & 1 & 34 & & 4 & 3 & 3 & 4 & 3 & 2 & 3 & 3 & 1 & 1 & & & & & 4 & 4 & 4 \\
\hline 78 & \begin{tabular}{|l|l|}
3 \\
\end{tabular} & & 3 & 4 & 3 & 3 & $4:$ & & & 2 & & 3 & 4 & 35 & 32 & 2 & & 4 & & 4 & 4 & 4 & 4 & 3 & 2 & 2 & 3 & & & & & 4 & 3 & 3 \\
\hline 79 & & & & & 1 & 2 & 1 & & & & & & 1 & 21 & 11 & & & 1 & & 2 & 3 & 2 & 1 & & & & & & & & & 2 & & 1 \\
\hline 80 & & & 1 & 2 & & & 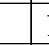 & 1 & & & & & & 1 & D) & (1) & & 1 & & 1 & & & & & & (1) & (1) & & & & & & & \\
\hline 81 & 3 & 1 & 2 & 1 & 1 & 1 & 1 & 1 & & 1 & 1 & & 1 & 11 & 12 & 1 & & 1 & & 1 & 1 & 2 & 2 & 2 & 1 & 1 & (1) & & & & & 2 & 2 & 1 \\
\hline 86 & & & & & & & & & & & & & & & & & & & & & & & & & & & & & & & & & & \\
\hline 87 & & 1 & & & & & & 1) & & & & 1 & & & & & & & 1 & & & & & & & & & & & (1) & 1 & & & \\
\hline 88 & & 1 & & & & & & & & & 1) & & & & & & & & & & & & & & & & & & & & & & & \\
\hline 91 & & & & & & & & & & & & & & & & & & & & & & & & & & & & & & & & & & \\
\hline 92 & & & & & & & & & & & & & & & & & & & & & & & & & & & & & & & & & & \\
\hline 94 & & & 1 & & (1) & & & 1 & & 1 & & 1 & 1 & & & & 1 & & 1 & (1) & & & & & & (1) & (1) & & & & & & & \\
\hline 122 & & (1) & (1) & 1 & 2 & 1 & 1 & 1 & & 1 & & 2 & 1 & 1 & & & & & 1 & 1 & 1 & & & & & & & & & & & & & \\
\hline 123 & & & & & & & & & & (1) & & & & & & & & & & & & & & & & & & & & & & & & \\
\hline 124 & & 1 & 2 & 2 & 1 & 1 & & 1. & & 1 & & 2 & 1 & $1]$ & 11 & 2 & & 1 & 1 & 1 & (1) & 1 & 2 & 2 & 1 & 2 & 1 & & & & & & & 1 \\
\hline 128 & & & & & & & & 1) & & & & & & & & & & & & & & & & & & & & & & & & & & \\
\hline 130 & & & & & & & & 1 & & & & & & & & & & & & & & & & & & & & & & 1 & & & & \\
\hline
\end{tabular}




\begin{tabular}{|c|c|c|c|c|c|c|c|c|c|c|c|c|c|c|c|c|c|c|c|c|c|c|c|c|c|c|c|c|c|c|c|c|c|c|}
\hline \multicolumn{35}{|c|}{ Table 9, 2008-2 } \\
\hline & \multicolumn{11}{|c|}{ D } & \multicolumn{13}{|c|}{$E$} & & & & & $\mathrm{~F}$ & & & & & \\
\hline & 4 & 5 & 6 & 7 & 8 & 9 & & & 12 & 13 & & 1 & 2 & 3 & 4 & 5 & 6 & 7 & 8 & 9 & 10 & & & 13 & 1 & 2 & 3 & 4 & 5 & 6 & 7 & 8 & & 10 \\
\hline 2 & & & & & & & & & & & & & & & & & & & & & & & & & & & & & & & & & & \\
\hline 3 & & & & & & & & & & & & & & & & & & & & & & & & & & & & & & & & & & \\
\hline 4 & 1 & 1 & 1 & 1 & 1 & 1 & 1 & 1 & 1 & 1 & & & & & 1 & 1 & 1 & 1 & 1 & 1 & 2 & 1 & 1 & & & & & 1 & 1 & 1 & 1 & 1 & 1 & 1 \\
\hline 5 & 1 & 1 & 1 & 1 & 1 & 1 & 2 & 1 & 1 & 1 & & & & & 1 & 1 & 1 & 2 & 1 & 2 & 1 & 1 & 1 & 1 & & & & & & & & & 1 & 1 \\
\hline 6 & & & & & & & & & & & 1 & & & & & & & & & & & & & & & & & & & & & & & (1) \\
\hline 7 & & & & & 1 & 1 & 1 & 1 & 1 & 1 & 1 & & & & & 1 & (1) & 1 & & 1 & 1 & 1 & 1 & & & & & & 1 & 1 & 1 & 1 & & 1 \\
\hline 10 & & & & & & 1 & 1 & 1 & 1 & 1 & 1 & & & & (1) & & 1 & 1 & & & 1 & & 1 & 1 & & & & & & 1 & & & & \\
\hline 11 & & & & & & & & & & (1) & & & & & & & & & & & & & & & & & & & & & & & & \\
\hline 12 & 1 & 1 & 1 & 1 & & & & & & & & & & & 1 & 1 & (1) & (1) & & & & & & & & & & 1 & & & & & & \\
\hline 13 & (1) & 1 & 2 & 2 & 2 & 2 & 2 & 1 & 1 & 2 & 1 & & & & 1 & 2 & 1 & 2 & 2 & 2 & 2 & 1 & 1 & & 1 & & & 1 & 1 & 1 & 1 & 1 & 1 & 1 \\
\hline 14 & 1 & 1 & 1 & & & 1 & & 1 & & & & & & & 1 & & & 1 & & 1 & & & & & & & & 1 & & (1) & & & & \\
\hline 15 & 2 & 2 & 2 & 2 & 2 & 3 & 3 & 2 & 1 & 2 & 2 & (1) & (1) & 1 & 1 & 2 & 2 & 2 & 2 & 2 & 3 & 3 & 2 & & 1 & 1 & 1 & 1 & 2 & 2 & 2 & 2 & \begin{tabular}{|l|}
3 \\
\end{tabular} & 3 \\
\hline 16 & & & & & & & & 1 & 1 & 2 & 2 & & & & & & & & & & 1 & 1 & 1 & & & & & & & & & & & (1) \\
\hline 17 & (1) & & 1 & 2 & & 1 & 1 & 1 & 1 & 1 & & & & & 1 & 1 & 1 & 1 & 1 & 2 & 1 & & & & & & (1) & 1 & 1 & 1 & & 1 & 1 & 1 \\
\hline 18 & & & & & & 1 & 1 & 1 & 1 & 1 & & & & & & & & & 1 & 1 & 1 & 1 & 1 & 1 & & & & 1 & 1 & & & & 1 & \\
\hline 20 & & & (1) & & & & & & & & & & & & (1) & 1 & (1) & 1 & & & & 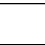 & & & & & 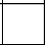 & (1) & & 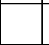 & & 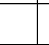 & 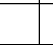 & 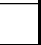 \\
\hline 21 & & & & & & & & & & & & & & & & 1 & & & & & & & & & & & & & & & & & & \\
\hline 22 & & (1) & & & & & & & & & & & & & & & & & & & & & & & & & & & & & & & & \\
\hline 23 & & & (1) & & & & & & & & & & & & & & & & & & & & & & & & & & & & & & & \\
\hline 26 & & & & & & & & & & (1) & & & & & & & & & 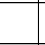 & & & 1 & (1) & 1 & & & & & & & & & (1) & \\
\hline 28 & & 1 & & 1 & 2 & 1 & 1 & 1 & 1 & 1 & & & & & & (1) & 1 & 2 & 1 & 1 & 1 & 1 & 1 & 1 & & & & & 1 & 1 & 1 & 1 & \begin{tabular}{l|l|}
1 \\
\end{tabular} & 1 \\
\hline 29 & & & & & & & & & & & & & & & & & & & & & & & & & & & & & & & & & & \\
\hline 39 & & & & & & & & & & & & & & & & & & & & & & & & & & & & & & & & & & \\
\hline 41 & & & & & & & & & & & & & & & & & & & & & & & & & & & & & & & & & & (1) \\
\hline 43 & & 1 & 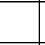 & & & & & 1 & & & & & & & & 2 & 2 & 2 & & 1 & 2 & & & & & & & & 1 & 1 & & 2 & & \\
\hline 44 & (1) & 2 & 1 & 1 & & & (1) & & & & & & & & (1) & 2 & 11 & 1 & 1 & & & & & & 2 & (1) & & 1 & 1 & . & & 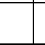 & 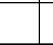 & \\
\hline 48 & & & & & & (1) & 2 & 2 & 3 & 2 & 2 & & & & & & & 1 & 1 & 2 & 2 & 2 & 2 & \begin{tabular}{|l|}
3 \\
\end{tabular} & & & & 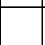 & & 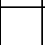 & & (1) & \begin{tabular}{l|l}
1 \\
\end{tabular} & 1 \\
\hline 53 & & & & & & 1 & & & & & & & & & & & & & 2 & & 1 & & & & & & & 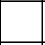 & & & & & & \\
\hline 54 & & (1) & & & & & & & & & & & & & & & & & & & & & & & & & & & & & & (1) & & \\
\hline 58 & & & & & & & & & & & & & & & & & & & & & & & & & & & & (1) & & (1) & & & & \\
\hline 60 & 1 & 1 & (1) & 1 & (1) & & & & & & & & & & & 1 & & 1 & & & & & & & 1 & & & 1 & 1 & 1 & & & & \\
\hline 61 & & & & & & & & & & & & & & & & & & & & & & & & & & & & 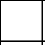 & & & & & & \\
\hline 62 & & & & & & & & & & & & & & & & & & & & & & & & & & & & & & & & & & \\
\hline 63 & 2 & 2 & 1 & 1 & 1 & (1) & (1) & & & & & & & & & 1 & 1 & 1 & 1 & & & & & & 1 & (1) & & 1 & 1 & & (1) & & & \\
\hline 64 & 1 & 1 & 1 & & & & & & & & & & & 1 & 1 & & (1) & 1 & & & & & & & 2 & 1 & 1 & & & & & & & \\
\hline 65 & & & & & & & & & & & & & & & & & & & & & & & & & (1) & & & & & & & & & \\
\hline 70 & & & & & & & & & & & & & & & & & & & & & & & & & 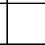 & & & & & & & & & \\
\hline 71 & & & & & & & & & & & & & & & & & & & & & & & & & & & & & & & & & & \\
\hline 73 & 2 & 2 & 3 & $\begin{array}{ll}3 \\
\end{array}$ & 2 & 2 & & & & & & 3 & 3 & 3 & 3 & 2 & 3 & 3 & 1 & 1 & & & & & 3 & 2 & 2 & 1 & 1 & & & & & \\
\hline 77 & 4 & 3 & 4 & & & 1 & 1 & & 1 & & & 3 & 4 & 4 & & 4 & 4 & & & & & & & & 4 & 4 & 4 & 4 & 3 & 2 & 2 & & & \\
\hline 78 & 4 & 4 & 2 & 2 & 2 & 3 & 2 & & & & & 4 & 4 & 4 & 4 & 2 & 1 & & & 1 & 1 & & & & 4 & 4 & 3 & 3 & & & & & & \\
\hline 79 & & & & & & & & & & & & 1 & & & (1) & & & & & & & & & & 2 & & & & & & & & & \\
\hline 80 & & 1 & & & & & & & & & & & 1 & & & 1 & 1 & & & & (1) & & & & & & & & & & & & & \\
\hline 81 & 2 & 2 & 1 & & & & & & & & & 2 & 1 & 1 & 1 & & & & & & & & & & 2 & 2 & 2 & & & & & & & \\
\hline 86 & & & & & 1 & & & & & & & & & & & & & & & & & & & 1 & & & & & & & & & (1) & \\
\hline 87 & & & & & & & & & & & & & & & & & & & & & & & & 1 & & & & 2 & 2 & & & & & \\
\hline 88 & & & & (1) & & & & & & & & & & & & & & 1 & & & & (1) & & & & & & 2 & 1 & \begin{tabular}{|l|} 
\\
\end{tabular} & 1 & (1) & (1) & \\
\hline 91 & & & & & & & & & (1) & & & & & & & & & & & & & & & & & & & & & & & & & \\
\hline 92 & & & & & & & & & 1 & & & & & & & & & & & & & & & & & & & & & & & & & \\
\hline 94 & & (1) & & & & & 1 & 1 & 1 & & & & & & & 1 & & (1) & 1 & 1 & 1 & 1 & & & & & & (1) & & 1 & 1 & 1 & & (1) \\
\hline 122 & 1 & 1 & 1 & 1 & 2 & & & & & & & & & & & 1 & 1 & 2 & 1 & 1 & & (1) & & & & 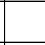 & & 1 & 2 & 2 & 2 & 1 & 1 & 1 \\
\hline 123 & & & & & & & & & & & & & & & & & & & & & & & & & & & & & & & & & & \\
\hline 124 & 1 & 1 & 1 & 2 & 1 & 1 & (1) & & & & & & & 1 & 1 & 2 & 1 & 2 & 1 & (1) & & & & 2 & 1 & 1 & (1) & 1 & 1 & & & & & \\
\hline 128 & & & & & & & & & & & & & & & & & & & & & & & & & & & & & & & & & & \\
\hline 130 & & & & & & & 1 & (1) & 1 & & & & & & & & & & & (1) & & 1 & 1 & & & & & & & & 1 & & & 1 \\
\hline
\end{tabular}




\begin{tabular}{|c|c|c|c|c|c|c|c|c|c|c|c|c|c|c|c|c|c|c|c|c|c|c|c|c|c|c|c|c|c|c|c|c|c|c|c|}
\hline \multicolumn{36}{|c|}{ Table 9, 2008-3 } \\
\hline & \multicolumn{8}{|c|}{$\mathrm{A}$} & \multicolumn{10}{|c|}{$\mathrm{B}$} & \multicolumn{14}{|c|}{$\mathrm{C}$} & \multicolumn{3}{|c|}{ D } \\
\hline & 1 & 2 & & 4 & & 6 & 7 & 8 & 1 & 2 & 3 & 4 & 5 & 6 & 7 & 8 & \begin{tabular}{l|l}
9 & 1 \\
\end{tabular} & 10 & 1 & 2 & 3 & 4 & 5 & \begin{tabular}{l|l}
6 & 1 \\
\end{tabular} & & $8 \mathrm{~s}$ & & & & \begin{tabular}{l|l}
12 & 1 \\
1
\end{tabular} & & 14 & & & 3 \\
\hline 131 & & & & & & & & & & (1) & & & & & & & & & & & & & & & & & & & (1) & & & (1) & & & \\
\hline 132 & & 2 & 2 & 1 & 1 & 1 & 2 & 2 & (1) & 1 & 2 & 2 & & 2 & 1 & 2 & 2 & 2 & 1 & 1 & 2 & 2 & (1) & & 1 & $13:$ & 3 & 2 & 1 & 1 & & & & & (1) \\
\hline 136 & & & & & & & & & & & & & & & & & & & & & & & & & & & & & & & & & & & \\
\hline 138 & & & & & & & & & & & & & & & & & & & & & & & & & & & & & & (1) ( & (1) & & & & \\
\hline 141 & & & & & & & & & & & (1) & & & 1 & & & & & & & & & & & & $1]$ & 1 & & 1 & 1 & 2 & 1 & & & \\
\hline 142 & & 1 & 1 & 1 & & (1) & 1 & 1 & (1) & & 1 & 1 & & (1) & 1 & 1 & 1 & 1 & (1) & 1 & & (1) & & 1 & 1 & 15 & 2 & 2 & 2 & 2 & 1 & 1 & & (1) & 1 \\
\hline 143 & & (1) & & & & & & & & & 1 & & & & & & & & & & & & & & & & & & & (1) & & 1 & & & \\
\hline 145 & & & & & & 1 & 1 & & & (1) & (1) & 1 & 1 & 1 & & & & (1) & & & & & & & & $\mathrm{I}$ & 1 & & & & & & & & \\
\hline 146 & & & & & & & & & & & & & & & & & & & & & & & & & & & & & & & & & & & \\
\hline 150 & & & & & & & & & (1) & & & & & & & & & & & & & & & & & & & & & & & & & & \\
\hline 156 & & & (1) & & & & & & & & & & & & & & & & & & & & & & & & & & & & & & & & \\
\hline 158 & & & & & & 1 & & & & & & & (1) & & & & & & & & 1 & 2 & & & & & & & & & & & & & \\
\hline 159 & & & & & & & & & & & & & & & & & & & & & & & & & & & & & & & & & & & \\
\hline 161 & & & & & & & & & & & & & & & & & & & & & & & & & (1) & & & & & & & & & & \\
\hline 173 & & & & & & & & & & & & & (1) & & & & & & & & & & & & & | & & & & & & & & & \\
\hline 175 & 1 & & & & & & & & & & & & & & & & & & & & & & & & & & & & & & & & & & \\
\hline 176 & & & & & & & & & & & & & & & & & & & & & & & & & & & & & & & & & & & \\
\hline 180 & & & & & & & & (1) & & & & & & & & & & & & & & & & & & & & & & & & & & & \\
\hline 181 & & & & & & & & & & & & & & & & & & & & & & & & & & & & & & & & & & & \\
\hline 182 & & & & & & & & & (1) & & & & & & & & & & & & & & & & & & & & & & (1) & & & & \\
\hline 183 & & & & & & & & & & & & & & & & & & & & & & & & & & & & & & & & & & & \\
\hline 197 & & & & & & & & & & & & & & & & & & & & & & & & & (1) ( & (1) & & & & & & & & & \\
\hline 198 & & & & (1) & & 2 & 2 & & & & & & 1 & 2 & 2 & 16 & (1) & & (1) & 1 & 1 & 2 & 1 & 2 & 1 & $1]$ & 1 & & & & & & & 1 & 2 \\
\hline 199 & 1 & 1 & 1 & & & & 1 & 1 & & & & (1) & (1) & & 1 & 1 & (1) & 1 & & & (1) & 1 & (1) & 1 & 1 & 1 & 1 & 1 & 1 & 1 & & & & 1 & 1 \\
\hline 200 & & & & & & & 1 & \begin{tabular}{|l|} 
\\
\end{tabular} & & & & & 1 & 1 & 1 & 2 & 1 & & & & & & 1 & 1 & 2 & 2 & 2 & 1 & 2 & 2 & 2 & 2 & & & \\
\hline 205 & & & & & & & & & & & & & & & & & & & & & & & & & & & & & & & & & & & \\
\hline 207 & & & & & & & & & & & & & (1) & 1 & & & (1) & 1 & & & . & & (1) & & & 1 & 1 & (1) & 1 & 1 & 1 & 1 & & (1) & \\
\hline 209 & & & & & & & & & & & & & & & & & & & & & & & & & & & & & & & (1) & & & & \\
\hline 211 & 2 & & 2 & 2 & 1 & 2 & 2 & 2 & & & & 2 & 2 & 2 & 2 & 2 & 2 & & 2 & 2 & 2 & 2 & 2 & 2 & 1 & 2 & 1 & 1 & & & & & 3 & 3 & 2 \\
\hline 212 & & 4 & 4 & 2 & & 4 & 3 & 3 & & & 4 & 4 & & 3 & 4 & 4 & & & 4 & 4 & 4 & & & \begin{tabular}{|l|}
3 \\
\end{tabular} & 4 & 3 & & & 4 & 4 & 4 & & 3 & 4 & 4 \\
\hline 213 & & (1) & 1 & 1 & 1 & 2 & 2 & 2 & 1 & & (1) & 1 & 1 & 2 & & 1 & 1 & 2 & & 1 & 1 & 1 & 1 & & & & 1 & 2 & 1 & 1 & 2 & & & & \\
\hline 214 & & & & & & & & & & & & & & (1) & & & 1 & 3 & & & & & & 1 & 1 & 16 & & 3 & 3 & \begin{tabular}{|l|l|}
3 \\
\end{tabular} & 4 & 3 & & (1) & \\
\hline 218 & & & & & & & & & & & & & & & & & & & & & & & & & & & & & & & & & & & \\
\hline 220 & & & & & & & & & & & & & 1 & & & & & & & & & (1) & & & & & & & & & & & & & \\
\hline 221 & & & & & & & & 1 & 1 & (1) & & & & & (1) & 1 & & & & & & & & 1 & & & & & & & & & & & \\
\hline 222 & & & & & & & (1) & & & & & & & & & & & & & & & & & & & & & & & 1 & 1 & (1) & & & \\
\hline 226 & & & & & & & & & & & & & & & & & 1 & 1 & & & & & & & & & & 1 & & & & & & & \\
\hline 227 & & & & & & & & & & & & & & & & & & & & & & & & & & & & & & (1) & & & & & \\
\hline 229 & 3 & 2 & 3 & 2 & 2 & 2 & 2 & 3 & 2 & 3 & 2 & 3 & 2 & 2 & 3 & 3 & 3 & 3 & 2 & 2 & 2 & 2 & 2 & \begin{tabular}{|l|l|}
3 \\
\end{tabular} & 3 & 3 & 3 & 4 & 3 & 3 & 1 & 1 & 3 & & 3 \\
\hline 230 & 1 & 1 & 1 & (1) & & & 2 & 3 & & & & & & & 1 & 1 & 2 & \begin{tabular}{|l|l|}
3 \\
\end{tabular} & & & & 1 & 1 & 1 & 1 & 2 & 2 & 2 & 1 & 2 & 1 & & & & \\
\hline 231 & & & & & & & & & & & & & & & & & & & & & & & & & & & & & & & & & & & \\
\hline 232 & & & 1 & & & & & & & 1 & & & & & & 2 & & & & & 1 & & & & & & & & & & & & & & \\
\hline 233 & & & & & & & & & & & & & & & & & & & & & & & & & & & & & & 1 & & & & & \\
\hline 234 & & & & & & (1) & & & & & & & & & & & & & & & & & & & & & & & & & & & & & \\
\hline 235 & & & & & & & & & & & & & & & & & & & & & & & & & & & & & & & & & & & \\
\hline 241 & & & & & & & & & & & & & & & & & & & & & & & & & & & & & & (1) & & & & & \\
\hline
\end{tabular}




\begin{tabular}{|c|c|c|c|c|c|c|c|c|c|c|c|c|c|c|c|c|c|c|c|c|c|c|c|c|c|c|c|c|c|c|c|c|c|c|c|}
\hline \multicolumn{36}{|c|}{ Table 9, 2008-4 } \\
\hline & \multicolumn{12}{|c|}{ D } & \multicolumn{13}{|c|}{$E$} & \multicolumn{10}{|c|}{$\mathrm{F}$} \\
\hline & 4 & 5 & 6 & 7 & 8 & 9 & & 11 & 12 & 21 & & & 1 & 2 & 3 & 4 & 5 & 6 & 7 & 8 & 9 & 10 & & & 13 & 1 & 2 & 3 & 4 & 5 & \begin{tabular}{|l|}
6 \\
\end{tabular} & & 8 & & 10 \\
\hline 131 & & (1) & & & 1 & & (1) & & (1) & & & & & & & & & (1) & (1) & 1 & (1) & & (1) & & & & & & & & & 1 & 1 & 1 & 1 \\
\hline 132 & 1 & 2 & 2 & 2 & 2 & 3 & 2 & 2 & 2 & 1 & & & & & & & 2 & 2 & 2 & 2 & 2 & 2 & 1 & 1 & & & & & 1 & 2 & 2 & 2 & 2 & 2 & 2 \\
\hline 136 & & & & & & & & & & & & & & & & & & & & & & & & & (1) & & & & & & & & & & \\
\hline 138 & & & & & & & & & & & & & & & & & & & & & & & & (1) & (1) & & & & & & & & & & \\
\hline 141 & & & & & 1 & (1) & (1) & 1 & 1 & 1 & $\mathrm{~L}$ & & & & & & & (1) & (1) & & & (1) & 1 & 1 & 2 & & & & 1 & & 1 & (1) & 1 & 1 & 1 \\
\hline 142 & 1 & (1) & 1 & 1 & 2 & 1 & 1 & 1 & 1 & 1 & & & & & (1) & 1 & 1 & 2 & 1 & 1 & 1 & 1 & 1 & 1 & 2 & & & 1 & 1 & 2 & 2 & 1 & 2 & 1 & 1 \\
\hline 143 & & (1) & & & & (1) & & 1 & 1 & 1 & 1 & & & & & & (1) & 1 & & & & & 1 & & 1 & & & & & 1 & 1 & 1 & 1 & & \\
\hline 145 & & 1 & & & 1 & & (1) & & & & & & & & & & 1 & & & 1 & & & & & & & & & (1) & & & & & (1) & \\
\hline 146 & & & & & & & & & & & & & & & & & & & & & & & 1 & & & & & & & & & & & & \\
\hline 150 & & & & & & & & & & & & & & & & & & & & & & & & & & & & & & & & & & & \\
\hline 156 & & & & & & & & & & & & & & & & & & & & & & & & & & & & & & & & & & & \\
\hline 158 & & & 1 & & & & & & & & & & & & & & & (1) & & & & & & & & & & & & & & & & & \\
\hline 159 & & & & & & & (1) & & & & & & & & & & & & & & & & & & & & & & & & & & & & \\
\hline 161 & & & & & & & & & & & & & & & & & & & & & & & (1) & & & & & & & & & & & & \\
\hline 173 & & & & & & & & & & & & & & & & & & & & & & & & & & & & & & & & & & & \\
\hline 175 & & & & & & & & & & & & & & & & & & & & & & & & & & & & & & & & & & & \\
\hline 176 & & & & & & & & & & & & & & & & & & & & & & & & & & & & & & & 1 & & & & \\
\hline 180 & & & & & & & & & & & & & & & & & & & & & & & & & & & & & & & & & & & \\
\hline 181 & & & & & & & & & & & & & & & & & & & & & & & 2 & & & & & & & & & & & & \\
\hline 182 & & & & & & & & & & & & & & & & & & & & & & & & & & & & & 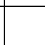 & & & & & & \\
\hline 183 & & & & & & & & & & & & & & & & & & & & & (1) & & & & & & & & . & & & & & & \\
\hline 197 & & 1 & & 1 & & & & & & & & & & & & & (1) & & & & . & & & & & & & & & & & & & & \\
\hline 198 & 1 & & 1 & 1 & 1 & & & & & & & & 1 & 1 & 2 & 2 & (1) & (1) & 1 & & & & & & & & 1 & 2 & (1) & & & & & & \\
\hline 199 & & & & 1 & 1 & 1 & 1 & 1 & 1 & 1 & & & & & & (1) & (1) & & 1 & 1 & 1 & 1 & 1 & & & 1 & 1 & (1) & 1 & & (1) & & & & \\
\hline 200 & & 1 & 1 & 2 & 2 & 2 & 2 & 2 & 1 & 1 & 12 & & & & & & 2 & 2 & 2 & 2 & 2 & 2 & 2 & 2 & 1 & & & & 2 & 2 & \begin{tabular}{|l|}
2 \\
\end{tabular} & 3 & 3 & 3 & 3 \\
\hline 205 & & & & & & & & & & & & & & & & & & & & & & & & (1) & & & & & & & & & & & \\
\hline 207 & & (1) & (1) & 1 & 1 & & 1 & 1 & 1 & 1 & I & & & & & & & & 1 & 1 & & & 1 & 1 & & & & & & 1 & 1 & 1 & 1 & 1 & 1 \\
\hline 209 & & & & & & & & & & & & & & & & & & & & & & & & & & & & & & & & & & & \\
\hline 211 & 2 & 2 & 1 & & 2 & & & & & & & & 2 & 3 & 3 & 2 & 1 & 1 & & & & & & & & 1 & 2 & 2 & 2 & & 2 & & & & \\
\hline 212 & & 4 & 4 & 4 & 3 & & & 4 & 4 & 4 & & & & & & & 3 & 4 & 4 & 4 & 3 & & 4 & 4 & 4 & & & & & 4 & 4 & 4 & 4 & 4 & 3 \\
\hline 213 & 1 & 2 & 1 & 1 & 2 & & 1 & & 1 & 1 & 1 & & & & & & (1) & 1 & 1 & 1 & 2 & 2 & 1 & 1 & & & & & 1 & 2 & 2 & 2 & 1 & & \\
\hline 214 & & & (1) & & 1 & 1 & 2 & 2 & 3 & 4 & 44 & & & & & & & & & & 1 & & 2 & 3 & & & & & & & & & & & \\
\hline 218 & & & & & & & & & & (1) & & & & & & & & & & & & & & & & & & & & & & & & & \\
\hline 220 & & & & & & & 1 & & (1) & & & & & & & & & & & & & 1 & & & & & & & & & & & & & \\
\hline 221 & & & (1) & & (1) & & (1) & & & & & & & & & & & (1) & & & & & & & & & & (1) & & (1) & & & & & \\
\hline 222 & & & & & & & & & & 1 & & & & & & & & & & & & & & & & & & & & & & & & & \\
\hline 226 & & & & & 1 & & & & & & 1 & & & & & & & & & & & & (1) & & 1 & & & & & & & & & & \\
\hline 227 & & & & & & & & & & & & & & & & & & & & & & & & & & & & & & & & & & & \\
\hline 229 & 3 & 3 & 3 & 3 & 3 & 3 & 3 & 4 & 4 & 3 & & & 2 & 3 & 3 & 3 & 3 & 3 & 3 & 3 & 2 & & 3 & 4 & & 3 & 3 & 2 & 3 & 3 & & & & 2 & 3 \\
\hline 230 & & 2 & 2 & 2 & 2 & 3 & 3 & 2 & 2 & 2 & & & & 1 & & 1 & 2 & 2 & 3 & 3 & 3 & 3 & & 2 & & & 2 & 1 & 1 & 1 & 1 & 2 & 2 & 2 & \\
\hline 231 & & & & & & & & & & & & & & & & & & & & (1) & & & & & & & & & & & & & & & \\
\hline 232 & & & & & & & & & & & & & & & & & & & & & & & & & & & & & & & & & & & \\
\hline 233 & & & & & & & & & & (1) & & & & & & & (1) & & & & & & & & & & & & & & & & & & \\
\hline 234 & & & & & & & & & & & & & & & & & & & & & & & & & & & & & & (1) & & & & & \\
\hline 235 & & & & & & (1) & & & & & & & & & & & & & & (1) & & & & & & & & & & & & & & (1) & \\
\hline 241 & & & & & & & & & & & & & & & & & & & & & & & & & & & & & & & & & & 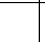 & \\
\hline
\end{tabular}




\begin{tabular}{|c|c|c|c|c|c|c|c|c|c|c|c|c|c|c|c|c|c|c|c|c|c|c|c|c|c|c|c|c|c|c|c|c|c|c|c|}
\hline \multicolumn{36}{|c|}{ Table 9, 2009-1 } \\
\hline & \multicolumn{8}{|c|}{$\mathrm{A}$} & & & & & $E$ & & & & & & & & & & & & $\mathrm{c}$ & 5 & & & & & & & & D & \\
\hline & 1 & 2 & 3 & 4 & & 6 & 7 & 8 & 1 & 2 & 3 & 4 & 5 & 6 & 7 & 8 & 9 & 10 & 1 & 2 & 3 & 4 & 5 & 6 & & 85 & \begin{tabular}{l|l}
9 & 1 \\
\end{tabular} & $\begin{array}{ll}01 \\
\end{array}$ & 11 & 12 & & 14 & & & 3 \\
\hline 2 & & & (1) & (1) & & 1 & & 1 & & 1 & (1) & & & & & & & & (1) & (1) & (1) & 1 & & & & & & & & & & & & & \\
\hline 4 & & 1 & & (1) & & (1) & 1 & 1 & & & & 1 & & & & 1 & 1 & 1 & & & & & & (1) & 1 & 1 & 1 & 1 & 1 & 1 & 1 & & & & \\
\hline 5 & & & & & & & 2 & 2 & & & (1) & 1 & & (1) & (1) & 1 & 2 & 1 & & & & & & & 1 & 2 & 2 & 1 & 1 & 1 & & 1 & & & (1) \\
\hline 6 & & & & & & & & & & & & & & & & & & & & & & & & & & & & & (1) & & & & & & \\
\hline 7 & & & & & & & 1 & (1) & & & & & & & & & & & & & & & & & & 1 ( & 1) & 1 & (1) & 1 & 1 & & & & \\
\hline 8 & & & & & & & & & & & & & & & & & & & & & & & & & & & & & & & & & & & \\
\hline 10 & & & & & & & & 1 & & & & & & & & & & & & & & & & & & 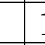 & 1 & 1 & 2 & 1 & 1 & & & & \\
\hline 11 & & & & & & & & (1) & & & & & & & & & & & & & & & & & & & & & & & & & & & \\
\hline 12 & (1) & 1 & (1) & & & (1) & 1 & 1 & (1) & (1) & 1 & 1 & & (1) & & & & & (1) & 1 & & & (1) & & (1) & 1 & 1 & & & & & & & & \\
\hline 13 & & (1) & (1) & 1 ( & (1) & 1 & 1 & 2 & & 1 & 1 ( & (1) & (1) & 1 & & 1 & 2 & & & & & 1 & & & 1 & 2 & 2 & 1 & & 1 & 1 & 1 & & & \\
\hline 14 & 1 & 1 & 1 & 1 & & & & 1 & 1 & 1 & 1 & & & & & & & & 1 & 1 & 1 & 1 & & & 1 & $1]$ & 1 & 1 & & & & & 1 & & \\
\hline 15 & 1 & 1 & 1 & 1 & & 1 & 1 & 1 & 1 & 1 & 1 & 2 & & & 2 & 2 & 3 & 1 & 1 & 1 & 1 & 1 & 2 & 1 & 2 & 2 & 3 & 3 & 2 & 2 & 1 & 1 & & & 1 \\
\hline 16 & & & & & & & & & & & & & & & & & & & & & & & & & & 1 & & 1 & 1 & 1 & 1 & 1 & & & \\
\hline 17 & 1 & 1 & 1 & & & & & 1 & 1 & 1 & 1 & 1 & & & & & 1 & 1 & & 1 & & & 1 & & 1 & $1]$ & 1 & 1 & 1 & 1 & 2 & 1 & & & \\
\hline 18 & & & & & & & & 1 & & & & & & & & & & & & & & & & & & & & & 1 & & 1 & 1 & & & \\
\hline 20 & & 1 & & & & & & (1) & (1) & & & & & & & & & & & & & & & & & & & & & & & & & & \\
\hline 21 & & & & & & & & & & & (1) & & & & & & & & & 1 & & & & & & & & & & & & & & & \\
\hline 22 & & 1 & 1 & 1 & & & & & (1) & 1 & 1 & & (1) & & & & & & 2 & 1 & 1 & (1) & (1) & & & & & & & & & & 1 & & 1 \\
\hline 23 & & & & (1) & & & & & (1) & & & & & & & & & & & 1 & & & 1 & & & 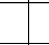 & 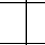 & & & & & & & & \\
\hline 26 & & & & & & & & (1) & & & & & & & & & (1) & & & & & & & & & & & & & & & (1) & & & \\
\hline 28 & (1) & & & & & & & 1 & & & & & & & & 1 & 1 & 1 & & & & & & & 1 & 2 & & 1 & 1 & 1 & 1 & & & & \\
\hline 29 & & & & & & & & & & & & & & & & & & & & & & & & & & & & & & & & (1) & & & \\
\hline 38 & & & & & & & & 1 & & & & & & & & & & & & & & & & & & & & & & & & 1 & & & \\
\hline 39 & & & & & & & & 1 & & & & & & & & & & & & & & & & & & & & & & & & & & & \\
\hline 41 & & & & & & & & 1 & & 1 & (1) & & (1) & & & & & & & 1 & & & & & & & & & & & (1) & & & & \\
\hline 43 & & & & & & & & & & & & & & & & & & 2 & & & & & & & & & & & & & & & & & \\
\hline 44 & 2 & 1 & 1 & 1 & & & & 1 & 1 & 1 & 1 & 1 & 1 & & & & & & 2 & 1 & 1 & 2 & 1 & & 1 & 1 & 1 & & & & & & 2 & 1 & 1 \\
\hline 48 & & & & & & & & & & & & & & & & & 2 & & & & & & & & & & & 2 & 2 & 2 & 2 & 2 & & & \\
\hline 49 & & & & & & & & & & & & & & & & & & & & & & & & & & & & & & & & 1 & & & \\
\hline 51 & & & & & & & & & & & & & & & & & & & & & & & & & & & & & & & & & & & \\
\hline 53 & & & & & & & & & & & & & & & & & & & & & & & & & & & & & & & & 1 & & & \\
\hline 54 & & & & & & & & & & & & & & & & & & & & & & & & & & & & & & & & & & & \\
\hline 60 & & 2 & 2 & 2 & 1 & & & & 2 & 1 & 2 & 1 & 1 & & (1) & & & & 2 & 2 & 1 & 2 & 1 & & & & & & & & & & 1 & 2 & 2 \\
\hline 61 & & & & & & & & & & & & & & & & & & & & & & & & & & & & & & & & & & & \\
\hline 63 & & 1 & 1 & 1 & 2 & 2 & 1 & 1 & 1 & 1 & 2 & 2 & 1 & (1) & (1) & 1 & 1 & & 1 & 2 & 1 & 2 & 2 & 1 & 1 & (1) & 1 & & & & & & (1) & 1 & 1 \\
\hline 64 & 1 & 1 & 1 & 1 & 1 & & & & 1 & & & & 1 & 1 & 1 & 2 & & & 1 & & (1) & 1 & 1 & 2 & 1 & 1 & & & & & & & & 2 & (1) \\
\hline 65 & & & & & & & & & & & & & & & & & & & & & & & & & & & & & & & & & & & \\
\hline 70 & & & & & & & & & & (1) & & & & & & & & & 2 & & & & & & & & & & & & & & & & \\
\hline 71 & & 3 & 2 & & & & & & 4 & 4 & 4 & & 1 & 4 & & & & & 4 & 4 & 4 & & & & & & & & & & & & & & 2 \\
\hline 73 & 3 & 2 & 1 & 2 & 2 & 2 & 1 & 1 & 3 & 2 & 2 & 2 & 2 & 1 & 1 & 1 & 1 & & 3 & 2 & 2 & 2 & 2 & 2 & 2 & 2 & 2 & 1 & & & & & 3 & 3 & 2 \\
\hline 77 & 3 & 4 & \begin{tabular}{|l|} 
\\
\end{tabular} & 4 & 4 & 4 & 4 & & 3 & 3 & \begin{tabular}{|l|l|}
3 \\
\end{tabular} & 4 & 4 & 4 & 4 & 4 & 4 & & 3 & 3 & 3 & 4 & 4 & 4 & 4 & 4 & 4 & 3 & & 2 & 1 & & \begin{tabular}{|l|}
3 \\
\end{tabular} & 4 & 4 \\
\hline 78 & 2 & 1 & 3 & 4 & 4 & 4 & 4 & 3 & & & & 2 & 4 & 4 & 4 & 2 & 2 & & 3 & 2 & 4 & 4 & 4 & 4 & 3 & 3 & 2 & 2 & & & & & 4 & 2 & 3 \\
\hline 79 & & & & & & & 1 & & & & & & & 1 & (1) & & & & (1) & & 2 & 2 & 2 & 18 & (1) & & & & & & & & 2 & (1) & \\
\hline 80 & 1 & & 1 & & & & & 1 & & & & & & & & & & & 1 & (1) & & & & & 1 & & & & & & & & & & \\
\hline 81 & 3 & & 1 & 1 & 1 & 1 & 1 & 1 & 1 & & & & 1 & 1 & 2 & & 1 & & 2 & & 1 & 1 & 1 & 1 & 1 & 1 & 1 & & & & & & 2 & 1 & 1 \\
\hline 82 & & & & & & & & & & & & & & & & & & & & & 2 & & & & & & & & & & & & & & \\
\hline 87 & & & & & & & & & & (1) & (1) & 1 & & & & & & & & & & & & & & & & & (1) & & & (1) & & & \\
\hline 88 & & (1) & & & & & & & & (1) & (1) & & & & & & & (1) & & & & & & & & & & & & (1) & & & & & \\
\hline 89 & & & & & & & & & & & & & & & & & & & & & & & & & & & & & (1) & & & & & & \\
\hline 91 & & & & & & & & & & & & & & & & & & & & & & & & & & & & & & & & (1) & & & \\
\hline 94 & & & 1 & & (1) & & & 1 & & & 1 & & 1 & (1) & & & (1) & & 1 & 1 & & & & & & & & (1) & & & & & & & \\
\hline 99 & & & & & & & & & & & & & & & & & & & & & & & & & & & & & & & & & & & \\
\hline 107 & & & & & & & & & & & & & & & & & & & & & & & & & & & & & & & & & & & \\
\hline 122 & & 2 & 1 & 1 & 1 & 1 & 2 & 1 & & & 1 & & & 1 & & & & & & 1 & & 2 & & & & & & & & & & & & & \\
\hline 123 & & & & & & & & (1) & & (1) & & & & & & & & & & & (1) & & & & & & & & & & & & & & \\
\hline
\end{tabular}




\begin{tabular}{|c|c|c|c|c|c|c|c|c|c|c|c|c|c|c|c|c|c|c|c|c|c|c|c|c|c|c|c|c|c|c|c|c|c|c|c|}
\hline \multicolumn{36}{|c|}{ Table 9, 2009-2 } \\
\hline & \multicolumn{11}{|c|}{$\mathrm{D}$} & & & & & & & 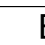 & $E$ & & & & & & & & & & & $F$ & & & & & \\
\hline & 4 & & 6 & 7 & 8 & & 10 & 11 & 12 & 13 & $3 \mid 12$ & \begin{tabular}{l|l}
4 & 1 \\
\end{tabular} & 2 & 3 & 4 & 5 & 6 & 7 & 8 & 9 & & 1 & & \begin{tabular}{l|l|}
121 & 1 \\
\end{tabular} & 13 & 12 & & 3 & 4 & 5 & & & 89 & \begin{tabular}{l|l}
9 & 1 \\
\end{tabular} & 10 \\
\hline 2 & & & & & & (1) & & & & & & & & & & & & & & & & & & & & & & & & & & & & & \\
\hline 4 & (1) & 1 & 1 & 2 & 2 & 2 & 2 & 2 & 1 & 1 & & & & & 1 & 2 & 1 & 1 & 1 & 2 & 2 & 1 & 2 & 2 & 1 & & & (1) & 1 & 1 & 1 & 1 & 12 & & 2 \\
\hline 5 & & 1 & 1 & 1 & 1 & 1 & 1 & & 1 & 1 & & & & & & 1 & 1 & 1 & 1 & 1 & 1 & 2 & & & & & & & 1 & 1 & & & 1 & 1 & \\
\hline 6 & & & & & & & & & & & 1 & & & & & & & & & & & & & & & & & & & & & & & & 1 \\
\hline 7 & & & & 1 & 1 & 1 & 1 & 1 & 1 & 1 & (1) & & & & & 1 & 1 & 1 & 1 & 1 & 1 & 1 & $1(1$ & (1) & 1 & & & & 1 & 1 & 1 & 1 & 11 & & 1 \\
\hline 8 & & & & & & & & & & & (1) & & & & & & & & & & & & & & & & & & & & & & & & \\
\hline 10 & & & & & & 1 & & 1 & 1 & 1 & 1 & & & & & & 1 & 1 & & 1 & 1 & & & 1 & 1 & & & & & & & & 1 & & 1 \\
\hline 11 & & & & & & & & & & & & & & & & & & & & & & & & & & & & & & & & & & & \\
\hline 12 & (1) & 1 & 1 & 1 & 1 & & 1 & & & & & & & & & 1 & 2 & 1 & & & & & & & & & & & 1 & & & & & & \\
\hline 13 & (1) & 1 & 1 & 2 & 2 & 2 & 2 & & 1 & 1 & (1) & & & & & 1 & 1 & 2 & 1 & 2 & 1 & & & & & & & & & & & & $1(1$ & 1) & \\
\hline 14 & 1 & 1 & & 1 & 1 & 1 & & 1 & & & & & & & (1) & & 1 & & & & & & & & & (1) & & & & & & & 1) & & \\
\hline 15 & 2 & 2 & 2 & 2 & 2 & 2 & 2 & 1 & 2 & 2 & 1 & & & (1) & 2 & 2 & 1 & 2 & 2 & 3 & 2 & 2 & & 1 & & 1 & 1 & 1 & 2 & 1 & & & 3 & & 3 \\
\hline 16 & & & & & & & 1 & 1 & 1 & 1 & 2 & & & & & & & & & & & & & & 2 & & & & & & & & & & 1 \\
\hline 17 & & & & & 1 & 1 & 1 & & 1 & 1 & 1 & & & & 1 & 2 & 1 & 1 & 1 & 1 & 1 & 1 & & & & & (1) & & 1 & 1 & & & & & 1 \\
\hline 18 & & 1 & & & 1 & & 1 & 1 & 1 & & 1 & & & & & & & 1 & 1 & 1 & & & 1 & 1 & 1 & & & & & 1 & & & & & \\
\hline 20 & (1) & & & & & & & & & & & & & & & & (1) & 1 & & & & & & & & & & & & (1) & & & & & \\
\hline 21 & & & & & & (1) & & & & & & & & & & & & & & & & & & & & & & & & & & & & & \\
\hline 22 & & (1) & & & & & & & & & & & & & & & & & & & & & & & & & & & & & & & & & \\
\hline 23 & & & & & & & & & & & & & & & & & & 1 & & - & & & & & & & & & & & & & & & \\
\hline 26 & & & & & . & & . & & & & & & & & & & & & & & & 1 & & 1 & 1 & & & & (1) & & & & & & \\
\hline 28 & (1) & & & 2 & 1 & 2 & 2 & 2 & 1 & 1 & & & & & (1) & 1 & (1) & 1 & 1 & 1 & 1 & 2 & 1 & 1 & 1 & & & & & (1) & & 10 & 1) 1 & & 1 \\
\hline 29 & & & & & & & & & & & & & & & & & & & & & & & & & & & & & & & & & & & \\
\hline 38 & & & & & & & & & & & & & & & & & & & & & & & 1 & 1 & & & & & & & & (1) & & & \\
\hline 39 & & & & & & & & & & & & & & & & & & & & & & & & & & & & & & & & & & & \\
\hline 41 & & & & & 1 & 1 & & 1 & & & & & & & & (1) & & & (1) & & & 1 & & & & & & & & & & & & (1) & \\
\hline 43 & & & & 1 & & & & & & & & & & & & 1 & 2 & & & 2 & & & & & & & & & 2 & 1 & & 2 & 1 & 1 & \\
\hline 44 & 2 & 1 & 1 & 1 & 1 & & 1 & & & & & (1) & & (1) & & 2 & 1 & 1 & 11 & $\sqrt{11}$ & & & & & & \begin{tabular}{l|l}
2 & 1
\end{tabular} & 1 & \begin{tabular}{l|}
1 \\
\end{tabular} & 1 & 1 & & & & & \\
\hline 48 & & & & & & 1 & 2 & 1 & 2 & 2 & 2 & & & & & & & & & 2 & 1 & 2 & 22 & 28 & 2 & & & & & & & & & & 2 \\
\hline 49 & & & & & & & & & & & & & & & & & & & & & & & & & & & & & & & & & & & 1 \\
\hline 51 & & & & & & & & & & & & & & & & & & & & & & & & & & & & & & & (1) & & & & \\
\hline 53 & & & & & & & & & & & 1 & & & & & & & & & & 1 & & & & & & & & & & & & & & \\
\hline 54 & & & & & & & & & & & & & & & & & & & & & & & & & & & & & & & & & 1) & & \\
\hline 60 & \begin{tabular}{|l|}
1 \\
\end{tabular} & 2 & & & (1) & & & & & & & & & & & 1 & & 1 & & & & & & & & 2 & & & 1 & 1 & & & & & \\
\hline 61 & & & & & & & & & & & (1) & & & & & & & & & & & & & & & & & & & & & & & & \\
\hline 63 & 2 & 2 & 2 & 2 & 2 & 2 & & & & & & & & & & 1 & 2 & 2 & 21 & & & & & & & & (1) & & 1 & 2 & & 1 & 1 & & \\
\hline 64 & 1 & 1 & 1 & 1 & & & - & & & & & (1) & & 1 & 1 & (1) & & & 1 & & & & & & & 2 & 2 & 1 & 1 & & & & & & \\
\hline 65 & & & & & & & & & & & & & & & & & & & & & & & & & & (1) & & & & & & & & & \\
\hline 70 & & & & & & & & & & & & & & & & & & & & & & & & & & & & & & & & & & & \\
\hline 71 & & & & & & & & & & & & & & & & & & & & & & & & & & & & & & & & & & & \\
\hline 73 & 2 & 1 & 2 & 3 & 2 & 2 & & & & & & 2 & 2 & 2 & 1 & 2 & 2 & 3 & 32 & & & & & & & 3 & 2 & 2 & 1 & 1 & & 1 & & & \\
\hline 77 & 4 & 3 & 3 & 4 & 3 & 2 & & & & & & 3 & \begin{tabular}{|l|l|} 
&
\end{tabular} & 4 & 3 & 4 & 4 & 3 & \begin{tabular}{|l|l}
3 & 1 \\
\end{tabular} & & 1 & & & & & & 4 & 4 & 4 & & & & & & \\
\hline 78 & 3 & 4 & 3 & 2 & 1 & 2 & 2 & & & & & 4 & 4 & 4 & 4 & 1 & & & & & 1 & & & & & 4 & 4 & \begin{tabular}{l|}
3 \\
\end{tabular} & 3 & & & & & & \\
\hline 79 & & & & & & & & & & & & 1 & & & & & & & & & & & & & & 26 & (1) & & & & & & & & \\
\hline 80 & & (1) & 1 & & & & & & & & & & & & & & & & 1 & & & & & & & 1 & (1) & & & & & & & & \\
\hline 81 & 1 & 1 & 2 & & 1 & & & & & & & 2 & 1 & 1 & 1 & 1 & & & & & & & & & & 3 & & 1 & 1 & & & & & & \\
\hline 82 & & & & & & & & & & & & & & & & & & & & & & & & & & & & & & & & & & & \\
\hline 87 & & & & & & & & & & (1) & 1 & & & & & 1 & & & & & & & (1) & (1) & & & & & & 1 & 1 & 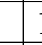 & 1 & & 1 \\
\hline 88 & & & & & & & & & & & & & & & & 1 & & 1 & 1 & & & & & & & & & & 1 & 1 & 10 & (1) & 17 & (1) & \\
\hline 89 & & & & & & & & & & & & & & & & & & & & & & & (1) & (1) & & & & & & & & & & & \\
\hline 91 & & & & & & & & & & & & & & & & & & & & & & & & & & & & & & & & & & & \\
\hline 94 & & & & & 1 & 1 & 1 & 1 & 1 & 1 & & & & & & (1) & & 1 & 11 & 1 & 1 & 1 & 1 & 1 & & & & & & 1 & (1) & & 11 & & 1 \\
\hline 99 & & & & & & & & & & & & & & & & & & & & & & & (1) & & & & & & & & & & & & \\
\hline 107 & & & & & & & & & & & (1) & & & & & & & & & & & & & & & & & & & & & & & & \\
\hline 122 & 1 & 1 & & & 1 & & 1 & 1 & & & & & & & & 1 & 1 & 1 & 11 & 12 & 1 & 1 & & & & & & & 1 & 2 & & & & & 1 \\
\hline 123 & & & & & & & & & & (1) & & & & & & & & & & & & & & & & & & & & 4 & & & & & \\
\hline
\end{tabular}




\begin{tabular}{|c|c|c|c|c|c|c|c|c|c|c|c|c|c|c|c|c|c|c|c|c|c|c|c|c|c|c|c|c|c|c|c|c|c|c|c|}
\hline \multicolumn{36}{|c|}{ Table 9, 2009-3 } \\
\hline & \multicolumn{8}{|c|}{$\mathrm{A}$} & \multicolumn{10}{|c|}{$\mathrm{B}$} & \multicolumn{14}{|c|}{ C } & \multicolumn{3}{|c|}{ D } \\
\hline & 1 & 2 & 3 & 4 & & 6 & 7 & 8 & 1 & 2 & 3 & 4 & 5 & & 7 & 8 & 9 & 10 & 1 & 2 & 3 & 4 & 5 & \begin{tabular}{l|l}
6 & 1 \\
\end{tabular} & & 8 & 9 & 10 & 11 & 12 & & 14 & & & 3 \\
\hline 124 & & 1 & 1 & 1 & 1 & 1 & & & 1 & 2 & 1 & 1 & 1 & 1 & 1 & 1 & 1 & & 1 & 2 & 1 & 1 & 1 & 1 & 1 & 1 & 1 & 1 & & & & & & & 1 \\
\hline 130 & & & & & & & 1 & 1 & & & & & & & & & & 1 & & & & & & & & & & 1 & 1 & 1 & 1 & & & & \\
\hline 131 & & & & & & & & & & & & & & & & & & & & & & & & & & & & & & & & & & & \\
\hline 132 & (1) & 1 & 2 & 1 & & 1 & 2 & 2 & & 1 & 1 & 2 & & 2 & 1 & 2 & 2 & 2 & & 2 & 2 & 1 & 1 & & 1 & & 2 & 2 & 2 & (1) & & & & & (1) \\
\hline 136 & & & & & & & & & & & & & & & & & & & & & & & & & & & & & & & & & & & \\
\hline 138 & & & & & & & & & & & & & & & & & & & & & & & & & & & & & & & & & & & \\
\hline 141 & & & & & & & & (1) & & & & & & & (1) & (1) & & & & & & & & & & (1) & & & 1 & 1 & 1 & 1 & & & \\
\hline 142 & & 1 & 1 & (1) & 1 & & 1 & 1 & (1) & & & 1 & (1) & & & & 1 & 2 & & (1) & (1) & & & & (1) & & 1 & 1 & 2 & 1 & 2 & 1 & & & \\
\hline 143 & & & & & & & & (1) & & (1) & & & & & (1) & & (1) & & & & & & & & & & & & (1) & 1 & 1 & 1 & & & \\
\hline 145 & & & & & 1 & 1 & & 1 & & & (1) & & 1 & & & & & & & & & & & & & & (1) & & & & & (1) & & & \\
\hline 146 & & & & & & & & & & & & & & & & & (1) & & & & & & & & & & & & & & & & & & \\
\hline 149 & & & & & & & & (1) & & & & & & & & & & & & & & & & & & & & & & & & & & & \\
\hline 153 & & & & & & & & & & & & & & & & & & & (1) & & & & & & & & & & & & & & & & \\
\hline 155 & & (1) & & & & & & & & & & & & & & & & & & & & & & & & & & & & & & & & & \\
\hline 158 & & & & & & & 1 & & & & & & & & & & & & & & 1 & 2 & & & & & & & & & & & & & \\
\hline 161 & & & & & & & & & & & & & & & & & & & & & & & & & & & & & & & & & & & \\
\hline 162 & & & & & & & & & & & & & & & & & & & & & & & & & & & & & & & & & & & \\
\hline 164 & & & & & & & & & & & & & & & & & & & & & & & & & & & & & & & & & & & \\
\hline 176 & & & & & & & & & & & & & & & & & & & & & & & & & & & (1) & & & & & & & & \\
\hline 180 & & & & & & & & & & & & & & & & & & & & & & & & & & & & & & & & & & & \\
\hline 193 & & & & & & & & & & & & & & & & & & & & & & & & & & & & & & & & & & & \\
\hline 197 & & 1 & & & & & & & & & & & & & 1 & & & & & & & & & & 2 & 2 & & & & & & & & & \\
\hline 198 & & & 1 & & & 2 & 2 & 1 & & & & & & & 1 & 2 & 1 & & & & & 1 & 1 & 1 & 1 & 1 & 1 & & & & & & 1 & 1 & 2 \\
\hline 199 & 1 & 1 & 1 & & & & (1) & & & & 1 & (1) & & & 1 & 1 & 1 & 1 & & (1) & (1) & & 1 & 1 & 1 & 1 & 1 & 1 & 1 & 1 & 1 & & & & \\
\hline 200 & & & & & & & 1 & 2 & & & & & & & 1 & 1 & 1 & (1) & & 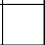 & & 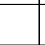 & & (1) & 2 & 2 & 2 & 2 & 2 & 1 & (1) & & & & \\
\hline 207 & & & & & & & & 1 & & & & . & & & & & (1) & 1 & & & & 1 & & . & & (1) & 1 & & 1 & 1 & 1 & 1 & & & \\
\hline 211 & 2 & 1 & 2 & 2 & & 2 & 3 & 2 & 1 & 2 & & 2 & 2 & 2 & 2 & 3 & 2 & & 1 & 3 & 3 & 2 & 2 & 2 & 3 & 2 & 2 & 1 & & & & & 3 & 3 & 2 \\
\hline 212 & & 4 & 4 & 2 & & & 3 & 3 & & 4 & 4 & 4 & & & 4 & 4 & 2 & 3 & 4 & 4 & 4 & & & \begin{tabular}{l|l|}
3 & \\
\end{tabular} & 4 & 4 & & & 4 & 4 & 4 & 4 & & 4 & 4 \\
\hline 213 & & (1) & 2 & 1 & 1 & 1 & 2 & 2 & & & 1 & \begin{tabular}{|l|} 
\\
\end{tabular} & 1 & 1 & & & 1 & 2 & & 1 & & 1 & & & & & 1 & 2 & 1 & 1 & \begin{tabular}{|l|l|} 
\\
\end{tabular} & 1 & & & \\
\hline 214 & & & & & & & & & & & & & & & & & & 2 & & & & & & 1 & (1) & & (1) & 2 & 2 & 4 & 4 & 4 & & & \\
\hline 218 & & & & & & & & & & & & & & & & & & (1) & & & & & & & & & & & & & & & & & \\
\hline 220 & & & & & & & & (1) & & & & & (1) & & & & (1) & & & & & & & & & & & & & & & & & & \\
\hline 221 & (1) & (1) & & (1) & & & & 1 & 1 & & & & (1) & 1 & 1 & 1 & & & & & (1) & (1) & 1 & 1 & 1 & (1) & (1) & & & & & & (1) & & (1) \\
\hline 222 & & & & & & & & & & & & & & & & & & & & & & & & & & & & & & & & & & & \\
\hline 226 & & & & & & & & & & & & & & & & & & & & & & & & & & & & & & & & & & & \\
\hline 228 & & & & & & & & & & & & & & & & & & & & & & & & & & & & & & & & & & & \\
\hline 229 & 3 & 2 & 3 & 3 & 3 & 2 & 2 & 2 & 2 & 3 & 2 & 3 & 2 & 2 & 3 & 3 & 4 & 2 & 2 & 3 & 2 & 2 & 2 & 3 & 3 & 3 & 4 & 3 & 3 & 4 & 1 & (1) & 3 & & 2 \\
\hline 230 & & 1 & & 1 & & 1 & 2 & 3 & & & 1 & & 1 & 1 & & \begin{tabular}{|l|l|}
2 \\
\end{tabular} & 3 & 3 & & & & 1 & 1 & 1 & 1 & 1 & 2 & 2 & 2 & 2 & & (1) & & 1 & \\
\hline 231 & & & & & & & & (1) & & & & & & & & & 1 & & & & & & & & & & & & & & & & & & \\
\hline 232 & & & & & & & & & & & & & & & & & & & 1 & & & & & & & & & & & & & & & & \\
\hline 233 & & & & & & & (1) & & & & & & & & & & & & & & & & & & & & & & (1) & & & & & & \\
\hline 234 & & & & & & & & 1 & & & & & & & & & & & & & & & & & & & & & & & & & & & \\
\hline
\end{tabular}




\begin{tabular}{|c|c|c|c|c|c|c|c|c|c|c|c|c|c|c|c|c|c|c|c|c|c|c|c|c|c|c|c|c|c|c|c|c|c|c|}
\hline \multicolumn{35}{|c|}{ Table 9, 2009-4 } \\
\hline & \multicolumn{11}{|c|}{ D } & \multicolumn{13}{|c|}{$E$} & \multicolumn{10}{|c|}{$\mathrm{F}$} \\
\hline & 4 & 5 & 6 & 7 & 8 & & & & & 13 & 14 & 1 & 2 & 3 & 4 & 5 & 6 & & 8 & 9 & 10 & 11 & & 13 & 1 & 2 & 3 & 4 & 5 & & 7 & & & 10 \\
\hline 124 & 1 & 1 & 1 & 2 & 2 & 1 & 2 & & & & & & & & & 2 & 1 & 1 & 1 & 1 & & & & & 1 & & & 1 & (1) & & 1 & & & \\
\hline 130 & & & & & (1) & 1 & 1 & 1 & 1 & 1 & & & & & & & & & (1) & & 1 & 1 & 2 & 1 & & & & & & (1) & & 1 & 1 & 1 \\
\hline 131 & & (1) & & & (1) & 1 & 1 & 1 & & (1) & & & & & & (1) & & . & & & & 1 & 1 & & & & & & (1) & 1 & 1 & 1 & 1 & 1 \\
\hline 132 & & 2 & 2 & 3 & 2 & 2 & & 2 & 2 & (1) & & & & & & 2 & 2 & 2 & 2 & 2 & 2 & 2 & 1 & & & & & 2 & 2 & 2 & 2 & & 2 & 1 \\
\hline 136 & & & & & & & & & & & (1) & & & & & & & & & & & & & & & & & & & & & & & \\
\hline 138 & & & & & & & & (1) & & & & & & & & & & & & & & & & (1) & & & & & & & & & & \\
\hline 141 & & & & & & & & & 1 & 1 & 1 & & & & & & (1) & & & & & 1 & 1 & 1 & & & & & & (1) & 1 & 1 & 1 & 1 \\
\hline 142 & & 2 & (1) & 1 & 1 & 2 & 2 & 2 & 2 & 1 & 1 & & & & & 1 & 1 & 1 & 1 & 1 & 1 & 2 & 2 & 2 & & & & 1 & 2 & 1 & 1 & 2 & 1 & 1 \\
\hline 143 & & & & & 1 & & & 1 & 1 & 1 & 1 & & & & & & (1) & & & (1) & & & 1 & 1 & & & & & & & (1) & 1 & 1 & 1 \\
\hline 145 & & 1 & & & 1 & 1 & & & & & & & & & & 1 & (1) & & 1 & & & 1 & (1) & & & & & & & & & 1 & & \\
\hline 146 & & & & & & & (1) & (1) & & & & & & & & & & & & & & & & & & & & & & & & & & \\
\hline 149 & & & & & & & & & & & & & & & & & & & & & & & & & & & & & & & & & & \\
\hline 153 & & & & & & & & & & & & & & & & & & & & & & & & & & & & & & & & & & \\
\hline 155 & & & & & & & & & & & & & & & & & & & & & & & & & & & & & & & & & & \\
\hline 158 & & (1) & & & & & & & & & & & & & & & & 1 & & & & & & & & & & & & & & & & \\
\hline 161 & & & & & & & & & & & & & & & & & & & & & & & & & & & & & & (1) & & (1) & & \\
\hline 162 & & & & & & (1) & & & & & & & & & & & & & & & & & (1) & (1) & & & & & & & & (1) & & \\
\hline 164 & & & & & & & & & & & & & & & & & & & & & & & & & & & & & & & & (1) & & \\
\hline 176 & & & & & & & & & & & & & & & & & & & & & & & & & & & & & 1 & & & & 1 & \\
\hline 180 & & & & & & & & & & & & & & & & & & & & & & & & (1) & & & & & & & & & & \\
\hline 193 & & & & & & & & & & & & & & & & & & & & & & & 1 & & & & & & & & & & & 1 \\
\hline 197 & & & 2 & 1 & 1 & & & & & & & & & & & & & & & & & & & & & & & & & & & & & \\
\hline 198 & 1 & 1 & 1 & & 1 & & & & & & & & & (1) & 1 & 1 & & (1) & & & & & & & (1) & & & (1) & & & & & & \\
\hline 199 & 1 & 1 & 1 & 1 & 2 & 1 & 1 & & 1 & & & & & & & 1 & 1 & 1 & 1 & 1 & 1 & & & & 1 & 1 & & 1 & & & & & & 1 \\
\hline 200 & & & 1 & 3 & 2 & 1 & 2 & 1 & 1 & 1 & & & & & (1) & 1 & 1 & 1 & 3 & 2 & 2 & 2 & 1 & & & & & 2 & 2 & 1 & 2 & 3 & 3 & 2 \\
\hline 207 & 1 & & & 1 & 1 & & 1 & 1 & 1 & & 1 & & & & & & (1) & 1 & 1 & 1 & 1 & 1 & 1 & 1 & & & & & 1 & 1 & & & 1 & 1 \\
\hline 211 & 3 & 2 & 2 & & & & & & & & & 2 & 2 & 2 & 3 & 1 & & & & & & & & & 1 & 3 & 3 & 2 & & & & 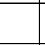 & 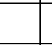 & \\
\hline 212 & & 3 & 4 & 4 & 3 & & & 4 & 4 & 4 & 4 & & & & & 3 & 4 & 4 & 4 & & 3 & 4 & 4 & 4 & & & & . & & 4 & 4 & 4 & \begin{tabular}{|l|}
4 \\
\end{tabular} & 4 \\
\hline 213 & 1 & 2 & 1 & 1 & 1 & 1 & 1 & 2 & 2 & 1 & 1 & & & & & 1 & 1 & 2 & 1 & 1 & 2 & 1 & 1 & & & & & 1 & 2 & 2 & 1 & 2 & 1 & \\
\hline 214 & & & 1 & & & & 2 & 3 & 3 & 3 & 4 & & & & & & & & (1) & & & (1) & & 2 & & & & & & & & & & \\
\hline 218 & & & & & & & (1) & (1) & & & & & & & & & & & & & & & & & & & & & & & & & & (1) \\
\hline 220 & & & & & 1 & (1) & & & & & & & & & & & & & & & & & & & & & & & 1 & & (1) & 1 & & \\
\hline 221 & & 1 & (1) & & & & & & & & & & (1) & (1) & (1) & & & & & & & & & & & 1 & & & & & & & & \\
\hline 222 & & & & & & & & & & (1) & & & & & & & & & & & & & & (1) & & & & & & & & (1) & & \\
\hline 226 & & & & & & & & & & & 1 & & & & & & & & & & & & 1 & 2 & & & & & & & & & & (1) \\
\hline 228 & & & & & & & & & (1) & & & & & & & & & & & & & & & & & & & & & & & & & \\
\hline 229 & 3 & 3 & 3 & 3 & 4 & 4 & 3 & 4 & 3 & 3 & & 2 & 3 & 2 & 3 & 3 & 3 & 2 & & 2 & (1) & 4 & 3 & 4 & 3 & 3 & 3 & 3 & 3 & 1 & 2 & & & 3 \\
\hline 230 & 1 & 2 & 2 & 2 & 2 & 2 & 3 & 3 & 2 & 1 & & 1 & 1 & 1 & 1 & 2 & 2 & 3 & 3 & 3 & 3 & 1 & 1 & & & (1) & 1 & 1 & 1 & 1 & 2 & 3 & \begin{tabular}{|l|l|}
3 \\
\end{tabular} & 2 \\
\hline 231 & & & & & 1 & & & & & & & & & & & & & & & & & & & & & & & & & & & & & \\
\hline 232 & & & & & & & & & & & & & & & & 1 & & & & & & & & & & 1 & & & 1 & & & & & \\
\hline 233 & & & & & & & & & (1) & & & & & & & & & & & & & & & & & & & & & & & & & \\
\hline 234 & & & & & & & & & & & & & & & & & & & & & & & & & & & & & & & & & & \\
\hline
\end{tabular}




\begin{tabular}{|c|c|c|c|c|c|c|c|c|c|c|c|c|c|c|c|c|c|c|c|c|c|c|c|c|c|c|c|c|c|c|c|c|c|c|}
\hline \multicolumn{35}{|c|}{ Table 9, 2010-1 } \\
\hline & \multicolumn{7}{|c|}{$\mathrm{A}$} & \multicolumn{10}{|c|}{$\mathrm{B}$} & & & & & & & & C & & & & & & & & D & \\
\hline & 1 & 2 & \begin{tabular}{l|l}
34 \\
3
\end{tabular} & & 6 & 7 & 8 & 1 & 2 & 3 & 4 & 5 & 6 & 7 & 8 & 9 & 10 & 1 & 2 & 3 & 4 & 5 & \begin{tabular}{l|l}
6 \\
\end{tabular} & & & \begin{tabular}{l|l}
9 & 1 \\
\end{tabular} & & & \begin{tabular}{l|l}
12 & 1 \\
\end{tabular} & & 14 & & & 3 \\
\hline 2 & & & 1 & & 1 & & 2 & & & & (1) & (1) & & & & & & & & (1) & (1) & & & & & & & & & & & (1) & & \\
\hline 3 & & & & & & & & & & & & & & & & & & & & & & & & & & & & & & (1) & & & & \\
\hline 4 & (1). & $1]$ & (1) & & (1) & 1 & 1 & & (1) & (1) & 1 & & 1 & 1 & 1 & 1 & 1 & & & & & & (1) & 1 & $\begin{array}{ll}15 \\
2\end{array}$ & 2 & 2 & 1 & 1 & & & & & \\
\hline 5 & & & (1) 1 & & & 1 & 2 & & & & & (1) & 1 & 1 & 1 & 1 & 1 & & & & & (1) & & 1 & 1 & & 2 & 1 & & & & & & \\
\hline 6 & & & & & & & & & & & & & & & & & (1) & & & & & & & & & & & 1 & & 1 & 1 & & & \\
\hline 7 & & 1 (c) & (1) & & & & (1) & & & & & & & & & 1 & 1 & & & & & & & & 11 & 1 : & & 1 & 1 & 1 & (1) & & & \\
\hline 8 & & & & & & & & & & & & & & & & & & & & & & & & & & & & & & & & & & \\
\hline 10 & & & & & & & & & & & & & & & & 1 & 1 & & & & & & & & & & 1 & 1 & 1 & 1 & & & & \\
\hline 11 & & & & & & (1) & & & & & & & & & & & & & & & & & & & & & & & & & & & & \\
\hline 12 & & 2 & $1]$ & (1) & 1 & 1 & 1 & (1) & (1) & 1 & 1 & (1) & (1) & 1 & 1 & 1 & & (1) & (1) & 1 & 1 & (1) & (1) & 1 & 1.1 & 1 & & & & & & & 1 & 1 \\
\hline 13 & & 1 & 1 & 2 & 2 & 2 & 2 & 1 & 1 & 1 & 2 & 1 & 2 & 2 & 2 & 2 & & (1) & 1 & 1 & 1 & 1 & 1 & 1 & 2 & 2 & 1 & & 1 & 1 & 1 & & 1 & 2 \\
\hline 14 & & 1 & $1]$ & 1 & 1 & 1 & & 1 & 1 & 1 & 1 & 1 & 1 & 1 & 2 & 1 & & 1 & 1 & 1 & 1 & (1) & 1 & 1 & 1 & & 1 & & & & & 1 & 1 & \\
\hline 15 & 1 & 1 & $\begin{array}{ll}2 & 1\end{array}$ & 1 & 2 & 2 & 3 & & & 1 & 2 & 2 & 2 & 2 & 2 & 2 & 1 & & & 1 & 1 & \begin{tabular}{|l|}
2 \\
\end{tabular} & 1 & 2 & 2 & 2 & 3 & 1 & 2 & 2 & & & 1 & \\
\hline 16 & & & & & & & & & & & & & & & & & 1 & & & & & & & (1) & & (1) & & 1 & 1 & 1 & 2 & & & \\
\hline 17 & 1 & 1 & 1 & 1 & & 1 & 1 & & 1 & 1 & & 1 & & 1 & 1 & 2 & 1 & & & 1 & 1 & & 1 & 1 & 1 & 1 & 2 & 1 & 1 & 2 & 1 & 1 & 1 & 1 \\
\hline 18 & & & & 1 & & & 1 & & & & 1 & & & & & 1 & & & & & 1 & & 1 & & 1 & & & & 1 & 1 & & & 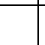 & \\
\hline 19 & & & & & & & & & & & & & & & & & & & & & & & & & & & & & & & & & & \\
\hline 20 & & 1 & 1 & & & (1) & & & & 1 & & & & & & 1 & & & & 1 & (1) & & & & 1 & & & & & & & & 1 & \\
\hline 21 & & & & (1) & & & (1) & & & & & & & & & & & & 1 & & & & & & & & & & & & & & & \\
\hline 22 & & & 1 & & & & & (1) & 2 & 1 & & & & & & & & 2 & & 2 & & & & & & & & & & & & 1 & 1 & \\
\hline 23 & & & & & & & & & & 1 & & & & & & & & & & 1 & & & & & & & & & & & & & & \\
\hline 25 & & & & & & (1) & & & & & & & & & & & & & & & & & & & & & & & & & & & & \\
\hline 26 & & & & & & & (1) & & & & & & & & & (1) & & & & & & & & & & & & & & 1 & 1 & & & \\
\hline 28 & & (1) & (1) & & & & 1 & & & & & & & & 1 & 1 & 1 & & & & & (1) & & 1 & 12 & 2 & 1 & 1 & 1 & 1 & 1 & & & \\
\hline 29 & & & & & & & & & & & & & & & & & & & & & & & & & & & & & & & & & & \\
\hline 33 & & & & & & & & & (1) & & & & & & & & & & & & & & & & & & & & & & & & & \\
\hline 38 & & & & & & & (1) & & & & & & & & & & & & & & & & & & & & & & & 1 & & & & \\
\hline 39 & & & & & & & (1) & & & & & & & & & & & & & & & & & & & & & & & & & & & \\
\hline 41 & & & (1 & & & & & & & & & (1) & & & & & & & & & & & & & & & & & & & & & & \\
\hline 42 & & & & & & & & & & & & & & & & & & & & & & & & & & & & & & & & & & \\
\hline 43 & & 1 & & & & 1 & 1 & & & & & & & & & & & & & & & & 1 & & & 2 & 1 & & & & & & & \\
\hline 44 & \begin{tabular}{|l|}
1 \\
\end{tabular} & 1 & \begin{tabular}{l|l}
1 & 1
\end{tabular} & & 1 & 1 & & & 2 & 1 & & 1 & 1 & 1 & 1 & & & 1 & 1 & 2 & 2 & 2 & 1 & 1 & 1 & 1 & & & & & & 2 & 2 & 1 \\
\hline 48 & & & & & & & 2 & & & & & & & & (1) & 3 & 2 & & & & & & & & 1 & 2 & 1 & 2 & 2 & 2 & 2 & & & \\
\hline 49 & & & & & & & & & & & & & & & & & & & & & & & & & & & & & & & & & & \\
\hline 53 & & & 1 & & & 1 & & & & & & & & & & & & & & & & & & & & & & & & 1 & & & & \\
\hline 54 & & & & & & & & & & & & & & & & & & & (1) & & & & & & & & & & & & & & & \\
\hline 58 & & & & & & & & & & & & (1) & & & & & & & (1) & & & & & & & & & & & & & & & \\
\hline 59 & & & & & & & & & & & & & & & & & & & 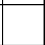 & 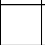 & & & & & & & & & & & & & & (1) \\
\hline 60 & (1) & 2 & 22 & 21 & & & & 2 & 2 & 1 & 2 & 1 & & (1) & & & & 2 & 2 & 2 & 2 & 1 & & (1) & & & & & & & & 2 & 2 & 2 \\
\hline 61 & & & & & & & & & & & & & & & & & & & & & & & & & & & & & & & & & & \\
\hline 63 & & 1 & 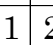 & \begin{tabular}{l|l}
2 & 1
\end{tabular} & 2 & 1 & 1 & 1 & 1 & 1 & 2 & 1 & 2 & 1 & 2 & 1 & & 1 & 2 & 1 & 2 & 1 & 1 & 1 & 1 & & (1) & & & & & 1 & 1 & 1 \\
\hline 64 & & 1 & & & 1 & & & (1) & & & & 1 & & 2 & 1 & & & 1 & & 1 & 1 & 2 & 2 & 2 & 1 & & & & & & & 1 & 1 & 2 \\
\hline 65 & & & & & & & & & & & & & & & & & & (1) & & & & & & & & & & & & & & & & \\
\hline 68 & & & & & & & & & & & & & & & & & & & & & & & & & & & & & & & & & & \\
\hline 70 & & (1) & & & & & & & 1 & & & & & & & & & & 2 & & & & & & & & & & & & & 1 & 2 & (1) \\
\hline 71 & & 2 & 21 & 1 & 1 & & & & 3 & 4 & & 1 & & & & & & & 1 & & & & & & & & & & & & & & & \\
\hline 73 & 2 & 2 & 2 & 2 & 2 & 2 & 1 & 3 & 2 & 2 & 2 & 2 & 2 & 3 & 3 & 2 & & 2 & 1 & 2 & 2 & 2 & 3 & 2 & 3 & 2 & & & & & & 2 & 2 & 2 \\
\hline 77 & 2 & 3 & \begin{tabular}{l|l}
3 & 4 \\
\end{tabular} & 3 & 4 & 4 & 3 & 2 & 3 & 3 & 4 & 4 & 4 & 3 & 2 & 3 & 2 & 3 & 3 & 4 & 4 & 2 & 4 & 3 & 2 & 2 & 3 & & & 1 & & 3 & 3 & 4 \\
\hline 78 & 2 & & 3 & \begin{tabular}{|l|l}
3 & 4 \\
\end{tabular} & 4 & 4 & 3 & & & & 3 & 4 & 3 & 3 & 3 & 2 & & 3 & 1 & 4 & 4 & 4 & 3 & 3 & 2 & 2 & & & & & & 4 & 2 & 3 \\
\hline 79 & & & & 1 & 1 & & & & & & & 1 & 1 & 1 & & & & 1 & & 2 & 2 & & 1 & & & & & & & & & 1 & (1) & (1) \\
\hline 80 & & & & 1 & 1 & & & & & & & 1 & & & 1 & & & & & 1 & 1 & & & & & & 1 & & & & & & & \\
\hline 81 & 2 & 1 & 21 & \begin{tabular}{|l|l}
1 & 1 \\
\end{tabular} & 1 & 1 & 1 & 1 & & 1 & & 1 & 1 & 2 & 2 & 1 & & 2 & & 1 & 1 & 2 & 1 & 1 & 1 & 2 & & & & & & 2 & 1 & 1 \\
\hline 82 & & 3 & \begin{tabular}{|l|}
3 \\
\end{tabular} & & & & & & 3 & 3 & & & & & & & & & 2 & & & & & & & & & & & & & & & \\
\hline 86 & & & & & & & & & & & & & & & & & & & & & & & & & & & & & & & & & & \\
\hline 87 & & 1 & 1 & & & & & & & (1) & & & & & & & & & & 1 & & & & & & & & & & & (1) & & & \\
\hline
\end{tabular}




\begin{tabular}{|c|c|c|c|c|c|c|c|c|c|c|c|c|c|c|c|c|c|c|c|c|c|c|c|c|c|c|c|c|c|c|c|c|}
\hline \multicolumn{33}{|c|}{ Table 9, 2010-2 } \\
\hline & \multicolumn{11}{|c|}{ 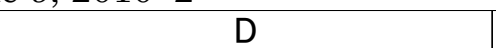 } & \multicolumn{12}{|c|}{$E$} & \multicolumn{9}{|c|}{$\mathrm{F}$} \\
\hline & 4 & $5 \mid$ & 67 & \begin{tabular}{l|l}
78 & 8 \\
\end{tabular} & \begin{tabular}{|l|l}
9 \\
\end{tabular} & & & & \begin{tabular}{|l|l}
2 & 1 \\
\end{tabular} & \begin{tabular}{l|l}
3 & 1
\end{tabular} & & 12 & 23 & 34 & 5 & 6 & 7 & 8 & 9 & 10 & & 12 & & 1 & 2 & 3 & \begin{tabular}{l|l}
44 & 5 \\
\end{tabular} & & & 8 & 9 & 10 \\
\hline 2 & & & & & & & & & & & & & & & & & & & & & & & & & & & & & & 1 & & \\
\hline 3 & & & & & & & & & & & & & & & & & & & & & & & & & & & & & & & & \\
\hline 4 & 1 & & $1]$ & $1]$ & 2 & 2 & 2 & 1 & 1 & & & & & (1) & 1 & 2 & 1 & 2 & 1 & 1 & 1 & 1 & 1 & & & & 1 & 1 & \begin{tabular}{l|l|}
2 & 1 \\
\end{tabular} & 2 & 1 & 1 \\
\hline 5 & & $1:$ & 2 & 2 & 1 & 2 & 2 & 1 & 1 & & & & & & & 2 & 1 & 1 & 2 & 1 & 1 & & 1 & & & & 1 & & \begin{tabular}{l|l|}
1 & 1 \\
\end{tabular} & & 1 & 1 \\
\hline 6 & & & & & & & & & & 1 & 1 & & & & & & & & & & 1 & & & & & & & & & & & 1 \\
\hline 7 & & & & 1 & 12 & 1 & 1 & ( & (1) & (1) 1 & & & & & & 1 & 1 & 1 & 1 & 1 & 1 & (1) & (1) & & & & & 1 (c) & \begin{tabular}{l|l} 
1) & 1 \\
\end{tabular} & 1 & & 1 \\
\hline 8 & & & & & & & & & (1) & 1) & & & & & & & & & (1) & & & & & & & & & & & & & \\
\hline 10 & & & & & & & & 1 & L & . & 1 & & & & 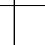 & 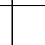 & & & 1 & & 1 & 1 & 1 & & & & & & & & 1 & 1 \\
\hline 11 & & & & & & (1) & & & & & & & & & & & & & & & & & & & & & (1) & & & & & \\
\hline 12 & & 15 & 2 . & $1]$ & 1 & 1 & & & & & & & (1) & 1 & 1 & 2 & 1 & & & & & & & (1) & (1) & 1 & \begin{tabular}{l|l}
2 & 1 \\
\end{tabular} & 1 & & & & \\
\hline 13 & 1 & $1:$ & 25 & $2 \sqrt{2}$ & 1 & 2 & & 1 & 1 & 10 & & & 1 & 1 & 2 & 2 & 2 & 2 & 2 & 1 & & & $1 \mid c$ & (1) & & & \begin{tabular}{l|l}
1 & 1 \\
\end{tabular} & 16 & \begin{tabular}{l|l} 
1) & 2 \\
\end{tabular} & 1 & 1 & 1 \\
\hline 14 & & & & & 1 & & & & & & & & & & & & 1 & & & 1 & & & & 1 & 1 & & 1 & 1 & 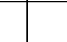 & & 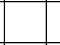 & \\
\hline 15 & \begin{tabular}{|l|} 
\\
\end{tabular} & 18 & 25 & 25 & 23 & 3 & 2 & 1 & 2 & & & 1 1. & 12 & 2 & 2 & 2 & 2 & 3 & 3 & 2 & 2 & & 1 & 1 & 1 & 2 & 2 & 1 & \begin{tabular}{l|l}
2 & 3
\end{tabular} & 3 & 3 & 3 \\
\hline 16 & & & & & & & 1 & 1 & 1 & 1 & 2 & & & & & & & & & 1 & 1 & 1 & 2 & & & & & & 1 & & & 1 \\
\hline 17 & & & 1 & 1 & 2 & 1 & & 1 & 1 & 1 & & 1 & 1 & 1 & 1 & & 1 & 1 & 1 & & & & & 1 & & 1 & 1 & 1 & 1 & 1 & 1 & 1 \\
\hline 18 & 1 & & 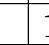 & $\begin{array}{lll}1 & 1\end{array}$ & 1 & 1 & 1 & & 1 & 1 & & & & & 1 & 1 & 1 & 1 & 1 & & 1 & & & & 1 & 1 & & 1 & 1 & 1 & & \\
\hline 19 & & & & & & & & & & & & & & & & & & & 1 & & & & & & & & & & & & & \\
\hline 20 & 1 & & & & 1 & & & & & & & & (1) 1 & 1 & & & & & 1 & & & & & & & 1 & \begin{tabular}{l|l}
1 & 1
\end{tabular} & 1 & & 1 & & \\
\hline 21 & & & & & & & & & & & & & & & & & & & & & & & & & & & & & & 1 & & \\
\hline 22 & & & & & & & & & & & & & & & & 1 & & & & & & & & (1) & & & & (1) & & & & \\
\hline 23 & & & & & & & & & (1) & 1) & & (1) & & & & & & & & & & & & & & & & 1 & & & & \\
\hline 25 & & & & & & & & & & & & & & & & & & & & & & & & & & & & & & & & \\
\hline 26 & & & & & & & & & & ( & 1) & & & & & & & & & & (1) & 7 & (1) & & & & & & & & & (1) \\
\hline 28 & & $1:$ & 1 & 1 & \begin{tabular}{l|l}
1 & 1
\end{tabular} & 1 & 1 & 1 & 1 & & & & & & 1 & 1 & 1 & 1 & 1 & 1 & 1 & 1 & 1 & (1) & & & $1(1$ & (1) & \begin{tabular}{l|l}
1 1) & 1
\end{tabular} & 1 & 1 & 1 \\
\hline 29 & & & & & & & & & & & & & & & & & & & & & & & (1) & & & & & & & & & \\
\hline 33 & & & & & & & & & & & & & & & & & & & & & & & & (1) & & & & & & & & \\
\hline 38 & & & & & & & & & & & & & & & & & & & & & & & & & & & & & & & & \\
\hline 39 & & & & & & & (1) & & & & & & & & & & & & & & & & & & & & & & & (1) & & \\
\hline 41 & & & & (1) & & (1) & & & & & & & & & & & & & & 1 & (1) & & & & & & & & & 1 & 1 & \\
\hline 42 & & & & & & & & & & & & & & & & & & & & & & & & & & & & & (1) & (1) & & \\
\hline 43 & & & & & & & & & & & & & & & & & & 2 & 1 & & & & & & & & & & & 2 & & \\
\hline 44 & \begin{tabular}{|l|}
2 \\
\end{tabular} & 18 & 2 & 11 & 1 & & & & & & & (1) & 2 & 22 & 21 & 2 & 1 & & $\begin{array}{l} \\
\end{array}$ & 1 & & & & 2 & \begin{tabular}{l|l}
1 \\
\end{tabular} & 1 & $\overline{1}$ & 1 & & & & \\
\hline 48 & & & & 1 & $D 1$ & 2 & 22 & & 22 & 22 & 2 & & & & & & 1 & 1 & 2 & 2 & 2 & 2 & 3 & & & & & & & (1) & 2 & 1 \\
\hline 49 & & & & & & & & & & 1 & 1 & & & & & & & & & & & & 1 & & & & & & & & & \\
\hline 53 & & & & & & & & & & & & & & & & & & 2 & & 3 & & & & & & & & & & 2 & & 2 \\
\hline 54 & & (1) & & & & & & & & & & & & & & & & & & & & & & & & & & & & & (1) & \\
\hline 58 & & & & & & & & & & & & & & & & & & & & & & & (1) & & & & $(1$ & & & (1) & & \\
\hline 59 & & & & & & & & & & & & & & & & & & & & & & & & 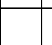 & & & 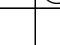 & & & 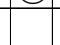 & & \\
\hline 60 & \begin{tabular}{|l|l} 
\\
\end{tabular} & & & & & (1) & D) & & & & & & 1 & 11 & 12 & (1) & & & & & & & & 2 & & & \begin{tabular}{l|l}
1 & 1
\end{tabular} & 1 & & & & \\
\hline 61 & & & & & & & & & & & & & & & & & & & & & & & & & & & & & & (1) & & \\
\hline 63 & \begin{tabular}{|l|l|}
2 \\
\end{tabular} & 2 & 28 & 25 & 2 & & & & & & & (1) & (1) & 1) 2 & 2 & 2 & 1 & (1) & 1 & & & & & 1 & 1 & (1) & & 1 & & & & \\
\hline 64 & \begin{tabular}{|l|}
1 \\
\end{tabular} & 1 & 2 & (1) & & & & & & & & 1 & 11 & 12 & 21 & & & & & & & & & 2 & 2 & 1 & 2 & (1) & & & & \\
\hline 65 & & & & & & & & & & & & & & & & & & & & & & & & (1) & & & & & & & & \\
\hline 68 & & (1) & & & & & & & & & & & & & (1) & & & & & & & & & & & & & & & & & \\
\hline 70 & & & & & & & & & & & & & & & & & & & & & & & & & & & & & & & & \\
\hline 71 & & & & & & & & & & & & & & & & & & & & & & & & & & & & & & & & \\
\hline 73 & 3 & 25 & 32 & 25 & 23 & & & & & & & 2 & 32 & 23 & 32 & 2 & 2 & 2 & & & & & & 2 & \begin{tabular}{|l|}
3 \\
\end{tabular} & 2 & 2 & 1 & & & & \\
\hline 77 & 4 & 3 & 2 & 23 & 32 & & & & & & & 42 & 44 & 43 & 33 & 32 & 2 & 1 & 2 & 2 & & & & 3 & 4 & 4 & 3 & & & & & 2 \\
\hline 78 & \begin{tabular}{|l|l}
3 \\
\end{tabular} & 25 & 28 & 2 & 3 & & & & & & & 4 & 3 & 33 & & & & & & & & & & 4 & 4 & 3 & 3 & & & & & \\
\hline 79 & & & & & & & & & & & & 1 & 1 & & & & & & & & & & & 2 & & & 1 & & & & & \\
\hline 80 & & & & & & & & & & & & & 1 & & & & & & & & & & & & & & & 1 & & & & \\
\hline 81 & \begin{tabular}{|l|}
1 \\
\end{tabular} & 2 & 27 & 1 & & & & & & & & 2 & \begin{tabular}{l|l}
1 & 1
\end{tabular} & 12 & & & & & & & & & & 2 & 2 & 2 & \begin{tabular}{l|l}
1 \\
\end{tabular} & & & & & \\
\hline 82 & & & & & & & & & & & & & & & & & & & & & & & & & & & & & & & & \\
\hline 86 & & & & & & & & (1) & & & & & & & & & & & & & & & & & & & & & 1 & & & \\
\hline 87 & & & & & & & & & & & & & & & & & & & & & & & & & & & & & & 1 & & \\
\hline
\end{tabular}




\begin{tabular}{|c|c|c|c|c|c|c|c|c|c|c|c|c|c|c|c|c|c|c|c|c|c|c|c|c|c|c|c|c|c|c|c|c|c|c|c|}
\hline \multicolumn{36}{|c|}{ Table 9, 2010-3 } \\
\hline & \multicolumn{8}{|c|}{$\mathrm{A}$} & & & & & $\mathrm{b}$ & $B$ & & & & & & & & & & & ( & C & & & & & & & & D & \\
\hline & 1 & 2 & 3 & 4 & & 6 & 7 & 8 & 1 & 2 & 3 & 4 & 5 & 6 & 7 & 8 & 9 & 10 & 1 & 2 & 3 & 4 & 5 & 6 & & & 9 & & 11 & 12 & & 14 & & & 3 \\
\hline 88 & & 2 & 2 & & & & & & & (1) & 1 & & & & & & & & & & & & & & & & & & & & & & & & \\
\hline 89 & & & & & & & & (1) & & & & & & & & & & & & & & & & & & & & & & & 1 & & & & \\
\hline 94 & (1) & 1 & 1 & & (1) & & & 1 & & 1 & 1 & 1 & 1 & 1 & & 16 & (1) & & 1 & 1 & 1 & & & & & & 1 & & & (1) & & & & & \\
\hline 99 & & & & & & & & & & & & & & & & & & & & & & & & & & & & & (1) & & & & & & \\
\hline 104 & & & & & & & & & & & & & & & & & & & & & & & & & & & & & & & & & & & \\
\hline 122 & & 1 & 2 & 1 & 2 & 1 & 1 & 2 & & & 1 & 2 & 1 & 1 & & t & & & & 1 & & 1 & 1 & & & & & & & & & & & & 1 \\
\hline 123 & & & & & & & & & & & & (1) & & & & & & & & & & & & & & & & & & & & & & & \\
\hline 124 & 1 & 1 & 1 & 2 & 1 & 1 & (1) & & & 1 & 1 & 1 & & 1 & 1 & 2 & 1 & & 1 & 1 & 1 & 1 & 1 & 1 & 1 & 2 & 1 & & & & & & & 1 & 1 \\
\hline 129 & & & & & & & & & & & & & & & & & & & & & & & & & & & & & & & & & & & \\
\hline 130 & & & & (1) & & & 1 & 1 & & & & (1) & & & & 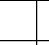 & & & & & 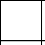 & - & $\rightarrow$ & 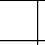 & & 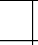 & & & 1 & 1 & 1 & (1) & & & \\
\hline 131 & & & & & & & & & & & & & & & & & & & & & & & & & & 1 & & & & & 1 & & & & \\
\hline 132 & & 2 & 2 & 1 & 1 & 2 & 2 & 2 & & & 2 & 1 & 1 & 1 & 2 & 2 & 2 & 3 & & 1 & 1 & 2 & 1 & 1 & 1 & 2 & 2 & 2 & 2 & 2 & & & (1) & & 1 \\
\hline 136 & & & & & & & & & & & & & & & & & & & & & & & & & & & & & (1) & & & & & & \\
\hline 140 & & & & & & & & & & & & & & & & & & & & & & & & & & & & & & & & & & & \\
\hline 141 & & & & (1) & & & & & & & & & & & & & & & & & & & & & & (1) & & & (1) & & 1 & (1) & & & \\
\hline 142 & & 1 & 1 & 1 & 1 & (1) & 1 & 2 & & & 1 & & & & 1 & 1 & 2 & 2 & 1 & 1 & 1 & & (1) & & 1 & 2 & 2 & 1 & 2 & 1 & 1 & 1 & & & 1 \\
\hline 143 & & & (1) & & & & & & & & & & & & & & & & & & & & & & & & & & (1) & & & & & & \\
\hline 144 & & & & & & & & & & & & & & & & & & & & & & & & & & & & & & & & & & & \\
\hline 145 & & & & & 1 & 1 & & 1 & & & (1) & (1) & 1 & (1) & & & & & & & & & & & & & & & & & & & & & \\
\hline 154 & & & & & & & & & & & & & & & & & & & & & & & & & & & & & & & & & & & \\
\hline 158 & & & & & 1 & & & & & & & (1) & & & & & & & & & & 1 & & & & & & & & & & & & & \\
\hline 160 & & & & & (1) & & & & & & & & & & & & & & & & & & & & & & & & & & & & & & \\
\hline 161 & & & & & & & & & & & & & & & & & & & & & & & & & & & & & & & & & & & \\
\hline 162 & & & & & & & & 1 & & & & & & & & & & & & & & & & & & & & & & & & & & & \\
\hline 163 & & & & & & & & & & & & & & & & & & & & & & & & & & & & & & & & & & & \\
\hline 166 & & & & & & & & (1) & & & & & & & & & & & & & & & & & & & & & & & & & & & \\
\hline 167 & & & & & & & & & & & & & & & & & & & & & & & & & & & & & & & & & & & \\
\hline 178 & & & & & 1 & & & & & & & & & & & & & & & & & & & & & & & & & & & & & & \\
\hline 182 & & & & & & & & & & & & & & & & & & & & & & & & & & & & & (1) & & & & & & \\
\hline 183 & & & & & & & & & & & & & & & & & & & & & & & & & & & & & & (1) & & & & & \\
\hline 193 & & & & & & & & & & & & & & & & & & & & & & & & & & & & & & & & & & & \\
\hline 196 & & & & & & & & & & & & & & & & & & & & & & & & & & & & 1 & & & & & & & \\
\hline 197 & & & & & & & & & & (1) & & & & & 1 & 2 & & & & & & & & & 2 & 2 & & & & & & & & & \\
\hline 198 & & & (1) & 1 & 1 & 2 & 2 & & & & & 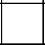 & 2 & 2 & 2 & 1 & 1 & & & & 1 & 2 & 1 & 2 & 1 & 1 & & . & & & & & 1 & (1) & 2 \\
\hline 199 & & 1 & 1 & (1) & 1 & 1 & 1 & 1 & & & 1 & 1 & 1 & 1 & 2 & 1 & 1 & (1) & & (1) & & & 1 & & 1 & 1 & & 1 & & & & & 1 & 1 & 1 \\
\hline 200 & & (1) & (1) & & & 1 & 1 & 1 & & & & & & (1) & 2 & \begin{tabular}{|l|l|}
2 \\
\end{tabular} & 1 & & & & & & 1 & (1) & 2 & 1 & 2 & 1 & 1 & 1 & 1 & 1 & & & \\
\hline 203 & & & & & & & & & & & & & & & & & & 1 & & & & & & & & & & & & & & & & & \\
\hline 205 & & & & & & & & (1) & & & & & & & & & & & & & & & & & & & & & & & & & & & \\
\hline 207 & & & & & & & & 1 & & & & (1) & & 1 & & & (1) & 1 & & & & & & & (1) & & 1 & 1 & 1 & 1 & 1 & (1) & & & \\
\hline 211 & 1 & & 2 & 3 & 1 & 1 & 2 & 3 & & & & 2 & 2 & 2 & 3 & 2 & 3 & 1 & 3 & 3 & 3 & 2 & 2 & 2 & 2 & 2 & 2 & 2 & & & & & 3 & 3 & 3 \\
\hline 212 & & 4 & 4 & 3 & & 3 & 3 & & & 4 & 4 & 4 & & & 4 & 4 & 1 & & 4 & 4 & 4 & & 3 & 4 & 4 & 4 & & & 4 & 4 & 4 & 4 & & 4 & 4 \\
\hline 213 & & 1 & 1 & 1 & 2 & 2 & 2 & 2 & & & & \begin{tabular}{|l|} 
\\
\end{tabular} & 1 & 1 & & 1 & 2 & 3 & & 1 & & 1 & 1 & & (1) & & 1 & 1 & 1 & 1 & 1 & 1 & & & 1 \\
\hline 214 & & & & & & & & & & & & & & (1) & & & & 1 & & & & & (1) & 1 & & & 1 & 3 & 3 & 4 & 4 & 4 & & & \\
\hline 218 & & & & & & & & & & & & & & & & & & & & & & & & & & & & & & (1) & & & & & \\
\hline 220 & & & & 1 & & & & (1) & & & (1) & 1 & (1) & & & & & & & & & & & & & & & & & & & & & & \\
\hline 221 & (1) & & & & 1 & & 1 & & 1 & & & & 1 & 1 & 1 & & & & & & & (1) & 1 & 2 & & 1 & & & & & & & 1 & & 1 \\
\hline 222 & & & & & & & & & & & & & & & & & & & & & & & & & & & & & (1) & (1) & 1 & & & & \\
\hline 226 & & & & & & & & 1 & & & & & & & & & & & & & & & & & & & & & & & & & & & \\
\hline 228 & & & & & & & & & & & & & & & & & & (1) & & & & & & & & & & & & & & & & & \\
\hline 229 & 3 & 3 & 2 & 3 & 2 & 1 & 2 & & 2 & 3 & 2 & 3 & 2 & 2 & 2 & 3 & 3 & 2 & 2 & 2 & 2 & 2 & 2 & 3 & 3 & 4 & 4 & 4 & 3 & 3 & & 1 & 2 & & 2 \\
\hline 230 & 1 & & 1 & 1 & & 1 & 2 & 3 & & (1) & & 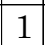 & 1 & 1 & 2 & \begin{tabular}{|l|l|}
2 \\
\end{tabular} & 2 & 3 & & 1 & & 2 & 1 & 1 & 1 & 2 & 2 & 2 & 2 & 2 & & & 1 & & \\
\hline 232 & & & & & & & & & & & & & & & & + & & & & & & & & & & & + & 2 & & & & & 1 & & \\
\hline 233 & & & & & & & & 1 & & & & & & & & & & & & & & & & & & & & & & (1) & & & & & \\
\hline 234 & & & & & & & & (1) & & & & (1) & & & & & & & & & & & & & & & & & (1) & & & & & & \\
\hline 236 & & & & & & & & & & & & (1) & & & & & & & & & & & & & & & & & & & & & & & \\
\hline
\end{tabular}




\begin{tabular}{|c|c|c|c|c|c|c|c|c|c|c|c|c|c|c|c|c|c|c|c|c|c|c|c|c|c|c|c|c|c|c|c|c|}
\hline \multicolumn{33}{|c|}{ Table 9, 2010-4 } \\
\hline & \multicolumn{11}{|c|}{ D } & \multicolumn{12}{|c|}{$\mathrm{E}$} & \multicolumn{9}{|c|}{$\mathrm{F}$} \\
\hline & 4 & $5 \mid$ & $\begin{array}{ll}6 & 7 \\
6\end{array}$ & \begin{tabular}{l|l}
7 & $\varepsilon$ \\
\end{tabular} & 9 & & & & 21 & 13 & & 1 & 2 & 3 & $4 !$ & $5 \mid$ & 6 & \begin{tabular}{l|l}
7 & $\varepsilon$ \\
\end{tabular} & 89 & 10 & & 12 & 13 & 1 & 2 & \begin{tabular}{|l|l}
3 & \\
\end{tabular} & \begin{tabular}{|l|l|}
4 & \\
\end{tabular} & \begin{tabular}{|l|l}
5 & $(6-2)$ \\
\end{tabular} & \begin{tabular}{l|l}
6 & 7 \\
\end{tabular} & \begin{tabular}{l|l|}
7 & 8
\end{tabular} & & 10 \\
\hline 88 & & & & & & & & $(1$ & 1) & & & & & & & (1) ( & & & 1) & & & & & & & & \begin{tabular}{|l|l|l|}
1 &
\end{tabular} & 1 & & 1 & & \\
\hline 89 & & & & & & & & & & & & & & & & & & & & & & (1) & & & & & & & & & & \\
\hline 94 & & (1) & & 1 & 1 & 1 & 1 & 1 & 1 & & & & & & & (1) & 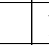 & 1 : & \begin{tabular}{l|l}
1 & 1 \\
\end{tabular} & 1 & 1 & 1 & & & & & & $1:$ & \begin{tabular}{l|l}
1 & 1 \\
\end{tabular} & 1 (1) & 1 & 1 \\
\hline 99 & & & & & & & (1) & & & & & & & & & (1) & & & & & & & & & & & & & & & (1) & \\
\hline 104 & & & & & & & & & & & & & & & & & & & & & & (1) & & & & & & & & & & \\
\hline 122 & 1 & 1 [. & 11. & $1]$ & 1 & 1 & 1 & & & & & & & & & 2 & 2 & 25 & $\begin{array}{ll}2 & 2 \\
\end{array}$ & \begin{tabular}{|l|l|}
2 & 1 \\
\end{tabular} & 1 & (1) & & & & & 1 & $2:$ & 25 & \begin{tabular}{l|l}
2 & 2 \\
\end{tabular} & 2 & 2 \\
\hline 123 & & & & & & & & & & & & & & & & & & & & & & & & & & & & & & & & \\
\hline 124 & & 1 : & 1 [1 1 & 11 & 1 & & & & & & & & & 1 & 1 & 1 & 1 & 1 & 1 & & & & & 1 & (1) & & 1 & (1) & & & & \\
\hline 129 & & (1) & & & & & 1 & & & & & & & & & & & & & & & & & & & & & & & & & \\
\hline 130 & & & 1 & 1 & (1) & & 1 & & 1 & 1 & & & & & & & & 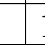 & 1 & 1 & 1 & 1 & 2 & & & & & 1 & & 1 & 1 & 1 \\
\hline 131 & & & 1) & 1 & (1) & 1 & 1 & (1) & (1) & 1 & & & & & & & & 2 & 1 & 1 & 2 & & (1) & & & & & & 1 & 1 & 1 & 1 \\
\hline 132 & 1 & 25 & 25 & 25 & 2 & 2 & 2 & & 2 & & & (1) & & 1 & 1 & 2 & 2 & 25 & 22 & 2 & 2 & 2 & (1) & & 1 & (1) & \begin{tabular}{|l|l|}
2 \\
\end{tabular} & 2 & & \begin{tabular}{l|l}
2 & \\
\end{tabular} & 2 & 2 \\
\hline 136 & & & & & & & & & & & & & & & & & & & & & & & & & & & & & & & & \\
\hline 140 & & & & & & & & & & & & & & & & & & & & & & (1) & & & & & & & & & & \\
\hline 141 & & (1) & 1 & 1 & (1) & 1 & 1 & & 1 & (1) & (1) & & & & & (1) & & 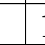 & 1 & & 1 & 1 & 1 & & & & & & & 1 & 1 & 1 \\
\hline 142 & 1 & 1 & 25 & $\begin{array}{ll}2 & 1\end{array}$ & 2 & 1 & & 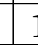 & 1 & (1) & & & & 1 & 2 & 1 & 2 & 2 & 1 & 12 & 1 & 1 & 2 & 1 & (1) & \begin{tabular}{|l|l|} 
\\
\end{tabular} & 2 & 1 & & \begin{tabular}{l|l}
2 & 1 \\
\end{tabular} & 1 & 1 \\
\hline 143 & & & & (1) & $\sqrt{1}$ & & & & (1) & (1) & & & & & & & & & & & 1 & 1 & 1 & & & & & & & & & 1 \\
\hline 144 & & & & & & & (1 & & & & & & & & & & & & & & & & & & & & & & & & & \\
\hline 145 & & 2 & & & (1) & & & & & & & & & & & (1) & 1 & 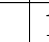 & 1 & & & & & & & & & & & 1 & 1 & (1) \\
\hline 154 & & & (1) & & & & & & & & & & & & & & & & & & & & & & & & & & & & (1) & \\
\hline 158 & & 1 & & & & & & & & & & & & & & & & & & & & & & 1 & & & & & & & & \\
\hline 160 & & & & & & & & & & & & & & & & & & & & & & & & & & & & & & & & \\
\hline 161 & & & & & & & & & & & & & & & & (1) & & & & & & & & & & & & (1) & & (1) & & \\
\hline 162 & & & & & & & & & & & & & & & & & & & & & & & & & & & & & & & & \\
\hline 163 & & & & & & & & & & & & & & & & & & & & & & & & & & & & & & & (1) & \\
\hline 166 & & & & & & & & & & & & & & & & & & & & & & & & & & & & & & & & \\
\hline 167 & & & & & & & & & & & & & & & & & & & & & & & & & & & & & & & (1) & \\
\hline 178 & & & & & & & & & & & & & & & & & & & & & & & & & & & & & & & & \\
\hline 182 & & & & & & & & & & & & & & & & & & & & & & & & & & & & & & & & \\
\hline 183 & & & & & & & & & & & & & & & & & & & & & & & & & & & & & & & & \\
\hline 193 & & & & & & & & & & & & & & & & & & & & & 1 & & & & & & & & & & & \\
\hline 196 & & & & & & & & & & & & & & & & & & & & & & & & & & & & & & & & \\
\hline 197 & & & $1)($ & (1) 1 & 1 & & & & & & & & & & & (1) & & & & & & & & & & & & & & & & \\
\hline 198 & 2 & 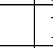 & 1 & 1 & & & & & & & & 1 & 1 & 2 & 1 & 1 & & & & & & & & (1) & & 2 & & & & & & \\
\hline 199 & 1 & 1 & 1 & & 2 & & & & & & & 1 & & 1 & 1 & 1 & & 1 . & 1 & \begin{tabular}{l|l}
1 & 1 \\
\end{tabular} & & & & 1 & 1 & 1 & 1 & 1 & (1) & (1) & 1 & 1 \\
\hline 200 & & & 16 & 22 & 2 & 1 & 12 & 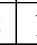 & 1 & 1 & & & & & & 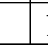 & 1 & 28 & \begin{tabular}{|l|l}
2 & \\
\end{tabular} & \begin{tabular}{|l|l}
2 & \\
\end{tabular} & 2 & 1 & 1 & & & & 1 & \begin{tabular}{ll|}
2 & \\
\end{tabular} & \begin{tabular}{l|l}
1 & 2 \\
\end{tabular} & \begin{tabular}{l|l}
2 \\
\end{tabular} & 2 & 2 \\
\hline 203 & & & & & & & & & & & (1) & & & & & & & & & & & & & & & & & & & & & \\
\hline 205 & & & & & & & & & & & & & & & & & & & & & & & & & & & & & & & & \\
\hline 207 & & & 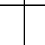 & 1 & 1 & & 1 & & 16 & (1) & & & & & & (1) & 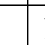 & 1 & \begin{tabular}{l|l}
1 & 1 \\
\end{tabular} & 1 & 1 & 1 & 1 & & & 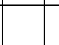 & & 11 & \begin{tabular}{l|l}
1 & 1 \\
\end{tabular} & \begin{tabular}{l|l}
1 & 1 \\
\end{tabular} & 1 & 1 \\
\hline 211 & 3 & 3 & 2 & & & & & & & & & 2 & 2 & 2 & 3 & & & & & & & & & 2 & 2 & 2 & 2 & & 2 & 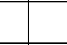 & & \\
\hline 212 & 2 & 3 & 32 & 44 & 4 & 2 & 4 & & 4 & 4 & 3 & & & & & 1 & 4 & $3:$ & 3 & & 4 & 3 & 3 & & (1) & & & & 4 & \begin{tabular}{|l|l}
3 & 4 \\
\end{tabular} & 4 & 3 \\
\hline 213 & & 18 & 2 & 2 & \begin{tabular}{l|l}
2 & 1 \\
\end{tabular} & 2 & 2 & & 1 & 1 & & & & & 1 & 1 & 2 & 18 & $\begin{array}{ll}22 \\
\end{array}$ & \begin{tabular}{l|l}
2 \\
\end{tabular} & 1 & & 1 & & & & & \begin{tabular}{l|l}
2 & 5 \\
\end{tabular} & 2 & \begin{tabular}{l|l}
11 & 2 \\
\end{tabular} & 1 & 1 \\
\hline 214 & & & & & 1 & 52 & 2 & & 4 & 3 & 4 & & & & & & & 7 & $\begin{array}{ll}1 \\
\end{array}$ & & & & 2 & & & & & & & \begin{tabular}{l|l}
1 &
\end{tabular} & & \\
\hline 218 & & & & & & & & & & & & & & & & & & & & 1 & & & & & & & & & & & & \\
\hline 220 & & & & 1 & 1 & (1) & & & & & & & & & & & & 0 & 1) & 1 & & & & & & & & ( & (1) & (1) & & \\
\hline 221 & \begin{tabular}{|l|} 
\\
\end{tabular} & 10 & 1) & & & & & & & & & & (1) & 1 & (1) & 1 & & & & & & & & & & (1) & (1) & (1) & & & & \\
\hline 222 & & & & & & & & & & & & & & & & & & & & & & & & & & & & & & & & \\
\hline 226 & & & & (1) & 1) & & & & & & & & & & & & & & & & & & 1 & & & & & & & & & \\
\hline 228 & & & & & & & & & & (1) & & & & & & & & & & & & & & & & & & & & & & \\
\hline 229 & 3 & 2 & $3:$ & 3 & 3 & 3 & 3 & & 3 & 3 & & 2 & 2 & 2 & 3 & 3 & 3 & 1 & & & 3 & 3 & 3 & 3 & 3 & \begin{tabular}{|l|l|}
3 \\
\end{tabular} & 3 & 2 & 1 & 1 & & \\
\hline 230 & & 2 & 26 & 25 & 23 & 3 & 3 & & 2 & 1 & & 1 & 1 & 1 & 1 & 1 & 2 & $3:$ & $\begin{array}{l}3 \\
3\end{array}$ & $\begin{array}{ll}3 & 2\end{array}$ & 2 & & & 1 & 1 & 1 & \begin{tabular}{|l|l|} 
\\
\end{tabular} & 1 & 1 & $\begin{array}{ll}2 & 2\end{array}$ & 2 & 2 \\
\hline 232 & & & & & & & & & & & & & 1 & & & & & & & & & & & & & & & & & & & \\
\hline 233 & & & & & & & & & & & & & & & & & & & & & & & & & & & & (1) & (1) & (1) & & \\
\hline 234 & & (1) & & & & & & & & & & & & & & & & & & & & & & & & & & & & & & \\
\hline & & & & & & & & & & & & & & & & & & & & & & & & & & & & & & & & \\
\hline
\end{tabular}

